\title{
A BENCHMARK ANALYSIS OF RADIATION FLUX DISTRIBUTION FOR BORON NEUTRON CAPTURE THERAPY OF CANINE BRAIN TUMORS
}

JEAN M. MORAN

Published february 1992

IDAHO NATIONAL ENGINEERING LABORATORY EG\&G IDAHO, INC. IDAHO FALLS, ID 83415

Prepared for the

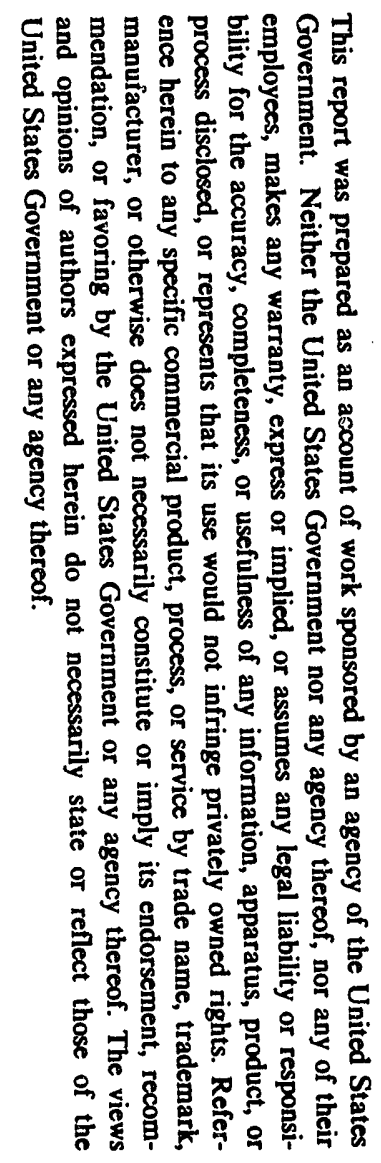

U. S. Department of Energy

Office of Energy Research Under DOE Idaho Field Office Contract DE-AC07-76ID01570 


\section{A BENCHMARK ANALYSIS OF RADIATION FLUX DISTRIBUTIONS}

FOR BORON NEUTRON CAPTURE THERAPY OF CANINE BRAIN TUMORS

by

JEAN M. MORAN

Submitted to the Department of Nuclear Engineering on March 2, 1992 in partial fulfillment of the requirements for the Degrees of

Bachelor of Science and Master of Science in

Nuclear Engineering

\section{ABSTRACT}

Calculations of radiation flux and dose distributions for Boron Neutron Capture Therapy (BNCT) of brain tumors are typically performed using sophisticated three-dimensional analytical models based on either a homogeneous

approximation or a simplified few-rerion approximation to the actual highly-heterogeneous geome $r y$ of the irradiation volume. Such models should be validated by comparison with calculations using detailed models in which all significant macroscopic tissue heterogeneities and geometric structures are explicitly represented as faithfully as possible. This work describes a validation exercise for BNCT of canine brain tumors.

Geometric measurements of the canine anatomical structures of interest for this work were performed by dissecting and examining two essentially identical Labrador Retriever heads. Chemical analyses of various tissue samples taken during the dissections were conducted to obtain measurements of elemental compositions for tissues of interest. The resulting geometry and tissue composition data were then used to construct a detailed heterogeneous calculational model of the Labrador Retriever head.

Calculations of three-dimensional radiation flux distributions pertinent to BNCT were performed for the model using the TORT discrete-ordinates radiation transport code. The calculations were repeated for a corresponding volumeweighted homogeneous tissue model. Comparison of the results showed that the peak neutron and photon flux magnitudes were quite similar for the two models (within 5\%), but that the spatial flux profiles were shifted in the heterogeneous model such that the fluxes in some locations 
away from the peak differed from the corresponding fluxes in the homogeneous model by as much as 10-20\%. Diffexences of this magnitude can be therapeutically significant, emphasizing the need for proper validation of simplified treatment planning models.

Thesis Supervisor: Jacquelyn C. Yanch

Title: Associate Professor of Nuclear Engineering 


\section{ACKNOWLEDGEMENTS}

This project could not have been completed without the participation and support of a number of individuals.

Dave Nigg served as mentor and advisor for this work. Dr. Rhonda Davis, DVM, provided guidance and assistance in connection with the canine head sections. The preparation of the head sections could not have been accomplished without Jim Hall, taxidermist. The section photographs were taken by Chris Morgan. Bill Bauer, with the assistance of Kyle Messick and John Wey, measured the chemical compositions of the tissues. Several of the figures were prepared by Allen Haroldsen and Mary Harris. Floyd Wheeler directed the canine head sectioning process. Last, but not least, Professor Jacqueline Yanch served as the resident MIT acivisor for this work.

The author of this report has been on assignment to the INEL in connection with the Massachusetts Institute of Technology Engineering Internship Program. This study was performed under the auspices of the U.S. Department of Energy, Office of Energy Research, DOE Field Office, Idaho, Contract No. DE-ACO7- 
CONTENTS

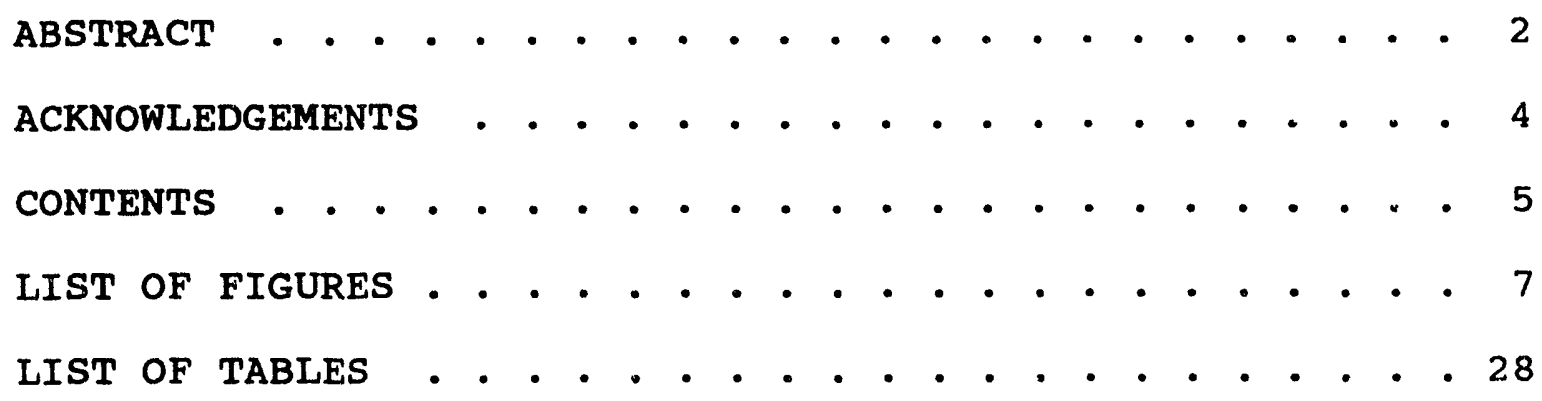

1. INTRODUCTION . . . . . . . . . . . . . . . 30

2. BASIS FOR STUDY . . . . . . . . . . . . . . 35

2.1 Glioblastoma Multiforme . . . . . . . . 35

2.2 Cell Biology . . . . . . . . . . . . . . 40

2.3 Considerations in Boron Distribution . . . . 44

2.4 Interactions of Particles in Tissue . . . . 48

2.4 .1 Neutrons ............. . 52

2.4.2 Alpha Particles $\left({ }^{4} \mathrm{He}\right)$. . . . . . 54

2.4.3 Gamma Rays . . . . . . . . . . 55

2.5 Radiobiology . . . . . . . . . . . 56

2.6 summary . . . . . . . . . . . . . . 59

3. REASONS FOR STUDY . . . . . . . . . . . . . 61

3.1 Types of Heterogeneity Effects . . . . . . 61

3.2 Canine Head Model . . . . . . . . . . 63

3.3 A Review of other Models for BNCT . . . . . 65 
4. EXPERIMENTAL METHODS . . . . . . . . . . . 70

4.1 Review of Data in Literature . . . . . . . 71

4.2 Dissection and Examination . . . . . . . 73

4.3 Chemical Analysis . . . . . . . . . . 77

4.4 Comparison of Chemical Compositions with

Literature . . . . . . . . . . . . . . . . . 80

5. ANALYTICAL MODELING AND RESULTS • . • • • • • . . 87

5.1 Geometric Modeling . . . . . . . . . . 87

5.2 GIP and TORT . . . . . . . . . . . . . 98

5.3 Presentation and Discussion of Results . . . 99

6. CONCLUSIONS AND RECOMMENDATIONS FOR FUTURE WORK • - 146

6.1 Conclusions . . . . . . . . . . . . . 146

6.2 Recommendations for Future Work . . . . . 150

7. REFERENCES . . . . . . . . . . . . . . . . 153

APPENDIX A-TABLE OF MEASURED ELEMENTAL COMPOSITIONS • 159

APPENDIX B--SAGITTAL SECTION PHOTOGRAPHS . • . . . • 175

APPENDIX C--DETAILED SAGITTAL MESH SECTIONS USED IN

MODELING . . . . . . . . . . . . . . 191

APPENDIX D--AXIAL SECTION PHOTOGRAPHS . . . . . . . . 199

APPENDIX E--GIP AND TORT INPUT . . . . . . . . . . . 227

APPENDIX F--ADDITIONAL CONTOUR PLOTS . . . . . . . . 279

APPENDIX G--RAW DATA-CHEMICAL ANALYSES • • • . • . . 361 
FIGURES

1-1. Boron activation by capture of neutrons. . . .

2-1. Components of a typical animal cell (from

Wessells and Hopson, p. 103). . . . . . . .

2-2. A diagram of the blood-brain barrier, which is formed by the endothelial cells that line the brain capillaries (from Vander, Sherman, and Luciano, p. 103) . . . . . . . . . . . . 46

2-3. Relationship of RBE and LET. Sparsely ionizing radiations require more than one hit for cell kill. Densely ionizing radiations result in a waste of energy because more energy than is needed to kill the cell is deposited (from Hall, p. 172).

2-4. An illustration of the indirect and direct action of photons on DNA. Indirect action dominates for sparsely ionizing radiation and works through the formation of a hydroxyl radical (from Hall, p. 11). . . . . . . . . . . . . . . . .

4-1. Photograph of the right side of the center sagittal slice. . . . . . . . . . . . .

4-2. Photograph of the cenier sayittal slice depicting the locations where samples were taken. . . . .

5-1. Axial Labrador Retriever head section through a plane just behind the axial midplane of the brain at approximately $\mathrm{x}=10 \mathrm{~cm}$. The grid marks are 
spaced $10 \mathrm{~mm}$ center to center. . . . . . . .

5-2. Detailed axial mesh through the brain

corresponding to the $x=12.25 \mathrm{~cm}$ plane. . . . . . 90

5-3. Detailed axial mesh corresponding to the $x=17.25$

cm plane. This plane includes the eyes and the nasal cavities. . . . . . . . . . . . . .

5-4. Detailed dorsal mesh through the brain at $z=11.5$ $\mathrm{cm}$.

5-5. Detailed sagittal mesh used in the TORT model corresponding to the sagittal midplane of the head of the Labrador Retriever. . . . . . . . . . .

5-6. As-built aluminum oxide epithermal neutron filter installed in the Brookhaven Medical Research Reactor. . . . . . . . . . . . . . .

5-7. Thermal neutron flux contours at $y=-0.125 \mathrm{~cm}$ for the heterogeneous case $(10 \mathrm{~cm} \times 10 \mathrm{~cm}$ beam aperture). Percentage contours are shown normalized to a peak thermal flux of $7.00 \times 10^{8}$ neutrons $/ \mathrm{cm}^{2} / \mathrm{s}$ at a BMRR power of $1 \mathrm{MW}$. . . . 101 5-8. Thermal neutron flux contours at $y=-0.125 \mathrm{~cm}$ for the homogeneous case. Percentage contours are shown normalized to a peak thermal flux of $6.92 \times$ $10^{8}$ neutrons $/ \mathrm{cm}^{2} / \mathrm{s}$. . . . . . . . . . . . 102

5-9. Calculated thermal neutron flux along the BMRR 10 $\mathrm{cm} \times 10 \mathrm{~cm}$ beam centerline for the Labrador Retriever head models. . . . . . . . . . 105 
5-10. Fast neutron flux contours at $y=-0.125 \mathrm{~cm}$ for the hetergeneous case. Percentage contours are shown normalized to a peak fast flux of $1.61 \times 10^{7}$

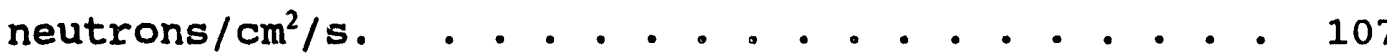

5-11. Fast flux contours at $y=-0.125 \mathrm{~cm}$ for the homogeneous case. Percentage contours are shown normalized to a peak fast flux of $1.62 \times 10^{7}$ neutrons $/ \mathrm{cm}^{2} / \mathrm{s}$. . . . . . . . . . . . . . 108

5-12. Calculated fast neutron flux along the BMRR 10 $\mathrm{cm} \times 10 \mathrm{~cm}$ beam centerline for the Labrador Retriever head models. . . . . . . . . . . 110

5-13. Gamma flux contours at $y=-0.125 \mathrm{~cm}$ for the hetergeneous case. Percentage contours are shown normalized to a peak gamma flux of $5.63 \times 10^{7}$

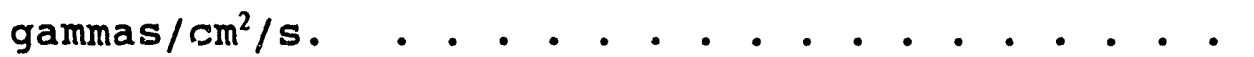

5-14. Gamma flux contours at $y=-0.125 \mathrm{~cm}$ for the homogeneous case. Percentage contours are shown normalized to a peak gamma flux of $5.61 \times 10^{7}$

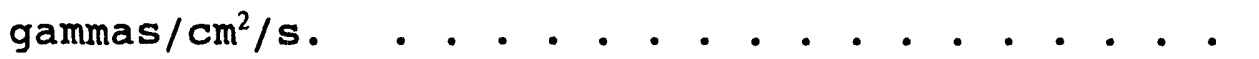

5-15. Thermal neutron flux contours at $y=-0.375 \mathrm{~cm}$ for the heterogeneous case. Percentage contours are shown normalized to a peak thermal flux of $7.00 \mathrm{x}$ $10^{8} \mathrm{n} / \mathrm{cm}^{2} / \mathrm{s}$. . . . . . . . . . . . . . . .

5-16. Thermal neutron flux contours at $y=-0.375$ for the homogeneous case. Percentage contours are shown normalized to a peak thermal flux of $6.92 \mathrm{x}$ 


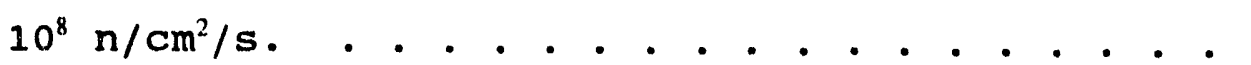

5-17. Fast neutron flux contours at $y=-0.375 \mathrm{~cm}$ for the heterogeneous case. Percentage contours are shown normalized to a peak fast flux of $1.61 \times 10^{7}$ neutrons $/ \mathrm{cm}^{2} / \mathrm{s}$.

5-18. Fast neutron flux contours at $y=-0.375 \mathrm{~cm}$ for the homogeneous case. Percentage contours are shown normalized to a peak fast flux of $1.62 \times 10^{7}$ neutrons $/ \mathrm{cm}^{2} / \mathrm{s}$.

5-19. Gamma flux contours at $y=-0.375 \mathrm{~cm}$ for the heterogeneous case. Percentage contours are shown normalized to a peak gamma flux of $5.63 \times 10^{7}$

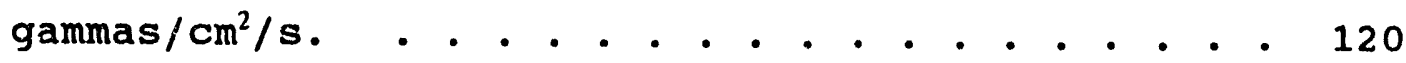

5-20. Gamma flux cortours at $y=-0.375 \mathrm{~cm}$ for the homogeneous case. Percentage contours are shown normalized to a peak gamma flux of $5.61 \times 10^{7}$ gammas $/ \mathrm{cm}^{2} / \mathrm{s}$.

5-21. Thermal neutron flux contours at $y=-2.5 \mathrm{~cm}$ for the heterogeneous case. Percentage contours are shown normalized to a peak thermal flux of $7.00 \times$ $10^{8}$ neutrons $/ \mathrm{cm}^{2} / \mathrm{s}$. . . . . . . . . . . . .

5-22. Thermal neutron flux contours at $y=-2.5 \mathrm{~cm}$ for the homogeneous case. Percentage contours are shown normalized to a peak thermal flux of $6.92 \mathrm{x}$

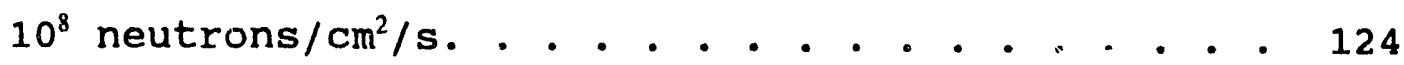

5-23. Thermal neutron flux contours at $y=-4.5 \mathrm{~cm}$ for 
the heterogeneous case. Percentage contours are shown normalized to a peak thermal flux of $7.00 \mathrm{x}$ $10^{8}$ neutrons $/ \mathrm{cm}^{2} / \mathrm{s}$. . . . . . . . . . . . . 126

5-24. Thermal neutron flux contours at $y=-4.5 \mathrm{~cm}$ for the homogeneous case. Percentage contours are shown normalized to a peak thermal flux of $6.92 \mathrm{x}$ $10^{8}$ neutrons $/ \mathrm{cm}^{2} / \mathrm{s}$. . . . . . . . . . . . . 127

5-25. Thermal neutron flux contours at $x=17.25 \mathrm{~cm}$ for the heterogeneous case. Percentage contours are shown normalized to a peak thermal flux of $7.00 \mathrm{x}$ $10^{8}$ neutrons $/ \mathrm{cm}^{2} / \mathrm{s}$. . . . . . . . . . . . . 128

5-26. Thermal neutron flux contours at $x=17.25 \mathrm{~cm}$ for the homogeneous case. Percentage contours are shown normalized to a peak thermal flux of $6.92 \mathrm{x}$ $10^{8}$ neutrons $/ \mathrm{cm}^{2} / \mathrm{s}$. . . . . . . . . . . . . . 129

5-27. Gamma flux contours at $x=17.25 \mathrm{~cm}$ for the heterogeneous case. Percentage contours are shown normalized to a peak gamma flux of $5.63 \times 10^{7}$ gammas $/ \mathrm{cm}^{2} / \mathrm{s}$. . . . . . . . . . . . . . . . .

5-28. Gamma flux contours at $x=17.25 \mathrm{~cm}$ for the homogeneous case. Percentage contours are shown normalized to a peak gamma flux of $5.61 \times 10^{7}$ gammas $/ \mathrm{cm}^{2} / \mathrm{s}$.

5-29. Thermal neutron flux contours at $x=17.25 \mathrm{~cm}$ for the heterogeneous case with the $5 \mathrm{~cm} \times 10 \mathrm{~cm}$ beam aperture. Percentage contours are shown 
normalized to a peak thermal flux of $4.14 \times 10^{8}$ $\mathrm{n} / \mathrm{cm}^{2} / \mathrm{s}$. . . . . . . . . . . . . . . . . . .

5-30. Thermal neutron flux contours at $x=17.25 \mathrm{~cm}$ for the homogeneous case with the $5 \mathrm{~cm} \times 10 \mathrm{~cm}$ beam aperture. Percentage contours are shown normalized to a peak thermal flux of $4.04 \times 10^{8}$

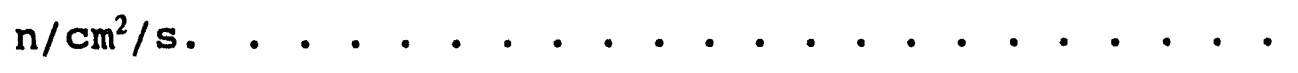

5-31. Thermal neutron flux contours at $x=12.25 \mathrm{~cm}$ for the heterogeneous case with the $5 \mathrm{~cm} \times 10 \mathrm{~cm}$ beam aperture. Percentage contours are shown normalized to a peak thermal flux of $4.14 \times 10^{8}$ $\mathrm{n} / \mathrm{cm}^{2} / \mathrm{s}$. . . . . . . . . . . . . . . . . .

5-32. Thermal neutron flux contours at $x=12.25 \mathrm{~cm}$ for the homogeneous case with the $5 \mathrm{~cm} \times 10 \mathrm{~cm}$ beam aperture. Percentacye contours are shown normalized to a peak thermal flux of $4.04 \times 10^{8}$ $\mathrm{n} / \mathrm{cm}^{2} / \mathrm{s}$. . . . . . . . . . . . . . . . .

5-33. Fast neutron flux contours at $z=13.25 \mathrm{~cm}$ for the heterogeneous case with the $10 \mathrm{~cm} \times 10 \mathrm{~cm}$ beam aperture. Percentage contours are shown normalized to a peak fast flux of $1.61 \times 10^{7}$ $\mathrm{n} / \mathrm{cm}^{2} / \mathrm{s}$. . . . . . . . . . . . . . . . . .

5-34. Fast neutron flux contours at $z=13.25 \mathrm{~cm}$ for the homogeneous case with the $10 \mathrm{~cm} \times 10 \mathrm{~cm}$ beam aperture. Percentage contours are shown normalized to a peak fast flux of $1.62 \times 10^{7}$ 
$\mathrm{n} / \mathrm{cm}^{2} / \mathrm{s}$. . . . . . . . . . . . . . . .

5-35. Fast neutron flux contours at $z=13.25 \mathrm{~cm}$ for the heterogeneous case with the $5 \mathrm{~cm} \times 10 \mathrm{~cm}$ beam aperture. Percentage contours are shown normalized to a peak fast flux of $1.26 \times 10^{7}$ $\mathrm{n} / \mathrm{cm}^{2} / \mathrm{s}$. . . . . . . . . . . . . . . . .

5-36. Fast neutron flux contours at $z=13.25 \mathrm{~cm}$ for the homogeneous case with the $5 \mathrm{~cm} \times 10 \mathrm{~cm}$ beam aperture. Percentage contours are shown normalized to a peak fast flux of $1.27 \times 10^{7}$ $\mathrm{n} / \mathrm{cm}^{2} / \mathrm{s}$. . . . . . . . . . . . . . . .

5-37. Depth profile of BMRR (1.0 MW) thermal flux for dog-head phantom and Labrador Retriever models along the beam centerline. . . . . . . . . . 144

5-38. Closer look at the depth profile of BMRR (1.0 MW) thermal flux for dog-head phantom and Labrador Retriever models along the beam centerline. . . 145 B-1. Photograph of sagittal slice 7 right at $y=0 \mathrm{~cm}$. 176 B-2. Photograph of sagittal slice 7 left at $y=1 \mathrm{~cm}$. 177 B-3. Photograph of sagittal slice 6 right at $y=1 \mathrm{~cm} . \cdot 178$ B-4. Photograph of sagittal slice 6 left at $y=2 \mathrm{~cm}$. . 179 B-5. Photograph of sagittal slice 5 right at $y=2 \mathrm{~cm} .+180$ B-6. Photograph of sagittal slice 5 left at $y=3 \mathrm{~cm} . \quad 181$ B-7. Photograph of sagittal slice 4 right at $y=3 \mathrm{~cm} . .182$ B-8. Photograph of sagittal slice 4 left at $y=4 \mathrm{~cm}$. 183 B-9. Photograph of sagittal slice 3 right at $y=4 \mathrm{~cm}$. 184 
B-10. Photograph of sagittal slice 3 left at $y=5 \mathrm{~cm}$. 185

B-11. Photograph of sagittal slice 2 right at $y=5 \mathrm{~cm}$. 186

B-12. Photograph of sagittal slice 2 left at $y=6 \mathrm{~cm}$. . 187

B-13. Photograph of sagittal slice 1 right at $y=6 \mathrm{~cm}$. 188

B-14. Photograph of sagittal slice 1 left at $y=7 \mathrm{~cm}$. 189

C-1. Detailed mesh for sagittal slice 1. . . . . . . 192

C-2. Detailed mesh for sagittal slice 2. . . . . . . 193

C-3. Detailed mesh for sagittal slice 3. . . . . . . 194

C-4. Detailed mesh for sagittal slice 4. . . . . . . 195

C-5. Detailed mesh for sagittal slice 5. . . . . . 196

C-6. Detailed mesh for sagittal slice 6. . . . . . . 197

C-7. Detailed mesh for sagittal slice 7. . . . . . 198

D-1. Photograph of the neck at $x=7 \mathrm{~cm}$. . . . . . . 200

D-2. Photograph of axial slice 1 back at $x=7 \mathrm{~cm}$. . . 201

D-3. Photograph of axial slice 1 front at $x=8 \mathrm{~cm}$. . 202

D-4. Photograph of axial slice 2 back at $x=8 \mathrm{~cm}$. . . 203

D-5. Photograph of axial slice 2 front at $x=9 \mathrm{~cm}$. . 204

D-6. Photograph of axial slice 3 back at $x=9 \mathrm{~cm}$. . . 205

D-7. Photograph of axial slice 3 front at $x=10 \mathrm{~cm}$. . 206

D-8. Photograph of axial slice 4 back at $x=10 \mathrm{~cm}$. . 207

D-9. Photograph of axial slice 4 front at $x=11 \mathrm{~cm}$. . 208

D-10. Photograph of axial slice 5 back at $x=11 \mathrm{~cm}$. . 209

D-11. Photograph of axial slice 5 front at $x=12 \mathrm{~cm}$. 210

D-12. Photograph of axial slice 6 back at $x=12 \mathrm{~cm}$. . 211

D-13. Photograph of axial slice 6 front at $x=13 \mathrm{~cm}$. 212

D-14. Photograph of axial. sljce 7 back at $x=13 \mathrm{~cm}$. . 213 
D-15. Photograph of axial slice 7 front at $x=14 \mathrm{~cm}$. 214

D-16. Photograph of axial slice 8 back at $x=14 \mathrm{~cm}$. . 215

D-17. Photograph of axial slice 8 front at $x=15 \mathrm{~cm}$. 216

D-18. Photograph of axial slice 9 back at $x=15 \mathrm{~cm}$. . 217

D-19. Photograph of axial slice 9 front at $x=16 \mathrm{~cm}$. 218

D-20. Photograph of axial slice 10 back at $x=16 \mathrm{~cm}$. 219

D-21. Photograph of axial slice 10 front at $x=17 \mathrm{~cm}$. 220

D-22. Photograph of axial slice 11 back at $x=17 \mathrm{~cm}$. 221

D-23. Photograph of axial slice 11 front at $x=18 \mathrm{~cm}$. 222

D-24. Photograph of axial slice 12 back at $x=18 \mathrm{~cm}$. 223

D-25. Photograph of axial slice 12 front at $x=19 \mathrm{~cm}$. 224

D-26. Photograph of snout extending from $x=19 \mathrm{~cm}$ to approximately $30 \mathrm{~cm}$. . . . . . . . . . . . 225

F-1. Fast neutron flux contours at $y=-2.5 \mathrm{~cm}$ for the heterogeneous case. Percentage contours are shown normalized to a peak fast flux of $1.61 \times 10^{7}$

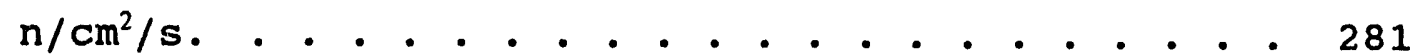

F-2. Fast neutron flux contours at $y=-2.5 \mathrm{~cm}$ for the homogeneous case. Percentage contours are shown normalized to a peak fast flux of $1.62 \times 10^{7}$ $\mathrm{n} / \mathrm{cm}^{2} / \mathrm{s}$. . . . . . . . . . . . . . . . 282

F-3. Fast neutron flux contours at $y=-4.5 \mathrm{~cm}$ for the heterogeneous case. Percentage contours are shown normalized to a peak fast flux of $1.61 \times 10^{7}$

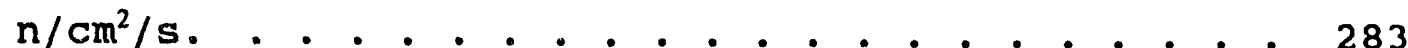
F-4. Fast neutron flux contours at $y=-4.5 \mathrm{~cm}$ for the 
homogeneous case. Percentage contours are shown normalized to a peak fast flux of $1.62 \times 10^{7}$

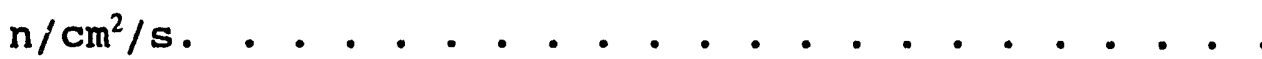

F-5. Gamma flux contours at $y=-2.5 \mathrm{~cm}$ for the

heterogeneous case. Percentage contours are shown normalized to a peak gamma flux of $5.63 \times 10^{7}$ gammas $/ \mathrm{cm}^{2} / \mathrm{s}$.

F-6. Gamma flux contours at $y=-2.5 \mathrm{~cm}$ for the homogeneous case. Percentage contours are shown normalized to a peak gamma flux of $5.61 \times 10^{7}$ gammas $/ \mathrm{cm}^{2} / \mathrm{s}$.

F-7. Gamma flux contours at $y=-4.5 \mathrm{~cm}$ for the heterogeneous case. Percentage contours are shown normalized to a peak gamma flux of $5.63 \times 10^{7}$

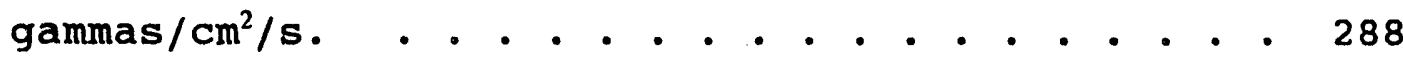

F-8. Gamma flux contours at $y=-4.5 \mathrm{~cm}$ for the homogeneous case. Percentage contours are shown normalized to a peak gamma flux of $5.61 \times 10^{7}$

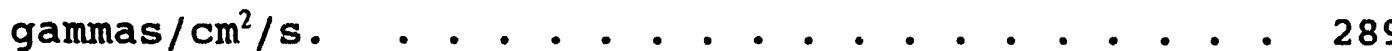

F-9. Thermal neutron flux contours at $x=7.25 \mathrm{~cm}$ for the heterogeneous case. Percentage contours are shown normalized to a peak thermal flux of $7.00 \mathrm{x}$ $10^{8} \mathrm{n} / \mathrm{cm}^{2} / \mathrm{s} . . . . . . . . . . . . . .290$

F-10. Thermal neutron flux contours at $x=7.25 \mathrm{~cm}$ for the homogeneous case. Percentage contours are shown normalized to a peak thermal flux of $6.92 \times$ 


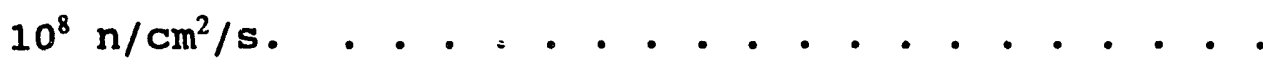

F-11. Thermal neutron flux contours at $x=12.25 \mathrm{~cm}$ for the heterogeneous case. Percentage contours are shown normalized to a peak thermal flux of $7.00 \times$

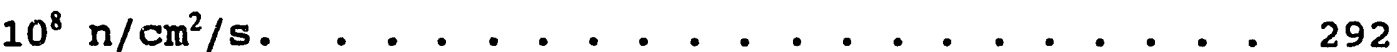

F-12. Thermal neutron flux contours at $x=12.25 \mathrm{~cm}$ for the homogeneous case. Percentage contours are shown normalized to a peak thermal flux of $6.92 \mathrm{x}$ $10^{8} \mathrm{n} / \mathrm{cm}^{2} / \mathrm{s}$.

F-13. Fast neutron flux contours at $x=7.25 \mathrm{~cm}$ for the heterogeneous case. Percentage contours are shown normalized to a peak fast flux of $1.61 \times 10^{7}$

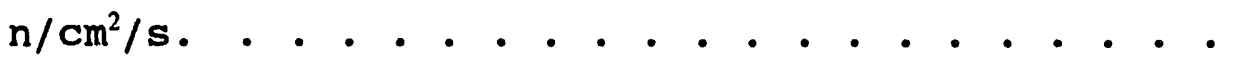

F-14. Fast neutron flux contours at $x=7.25 \mathrm{~cm}$ for the homogeneous case. Percentage contours are shown normalized to a peak fast flux of $1.62 \times 10^{7}$ $\mathrm{n} / \mathrm{cm}^{2} / \mathrm{s}$. . . . . . . . . . . . . . . . . . 296

F-15. Fast neutron flux contours at $x=12.25 \mathrm{~cm}$ for the heterogeneous case. Percentage contours are shown normalized to a peak fast flux of $1.61 \times 10^{7}$

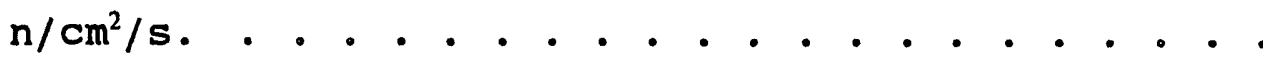

F-16. Fast neutron flux contours at $x=12.25 \mathrm{~cm}$ for the homogeneous case. Percentage contours are shown normalized to a peak fast flux of $1.62 \times 10^{7}$

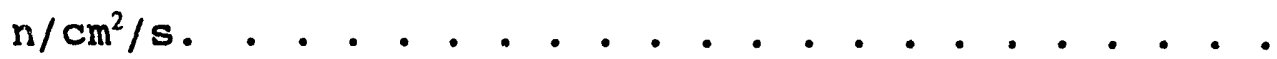

F-17. Fast neutron flux contours at $x=17.25 \mathrm{~cm}$ for the 
heterogeneous case. Percentage contours are shown normalized to a peak fast flux of $1.61 \times 10^{7}$

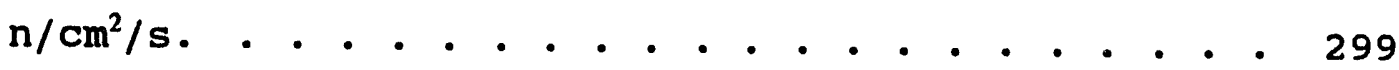

F-18. Fast neutron flux contours at $x=17.25 \mathrm{~cm}$ for the homogeneous case. Percentage contours are shown normalized to a peak fast flux of $1.62 \times 10^{7}$

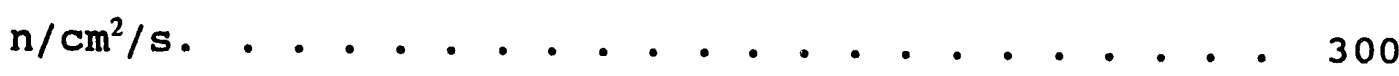

F-19. Gamma flux contours at $x=7.25 \mathrm{~cm}$ for the heterogeneous case. Percentage contours are shown normalized to a peak gamma flux of $5.63 \times 10^{7}$ gammas $/ \mathrm{cm}^{2} / \mathrm{s}$.

F-20. Gamma flux contours at $x=7.25 \mathrm{~cm}$ for the homogeneous case. Percentage contours are shown normalized to a peak garnma flux of $5.61 \times 10^{7}$ gammas $/ \mathrm{cm}^{2} / \mathrm{s}$. 303

F-21. Gamma flux contours at $x=12.25 \mathrm{~cm}$ for the heterogeneous case. Percentage contours are shown normalized to a peak gamma flux of $5.63 \times 10^{7}$ gammas $/ \mathrm{cm}^{2} / \mathrm{s}$. 304

F-22. Gamma flux contours at $x=12.25 \mathrm{~cm}$ for the homogeneous case. Percentage contours are shown normalized to a peak gamma flux of $5.61 \times 10^{7}$ gammas $/ \mathrm{cm}^{2} / \mathrm{s}$.

F-23. Thermal neutron flix contours at $z=13.25 \mathrm{~cm}$ for the heterogeneous case with the $10 \mathrm{~cm} \times 10 \mathrm{~cm}$ beam aperture. Percentage contours are shown 
normalized to a peak thermal flux of $7.00 \times 10^{8}$ $\mathrm{n} / \mathrm{cm}^{2} / \mathrm{s}$. . . . . . . . . . . . . . . . 307

F-24. Thermal neutron flux contours at $z=13.25 \mathrm{~cm}$ for the homogeneous case with the $10 \mathrm{~cm} \times 10 \mathrm{~cm}$ beam aperture. Percentage contours are shown normalized to a peak thermal flux of $6.92 \times 10^{8}$

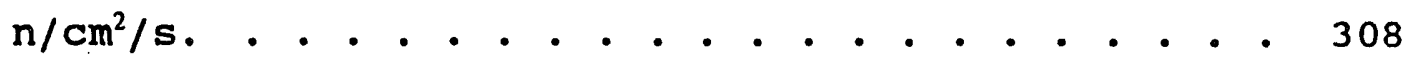

F-25. Gamma flux contours at $z=13.25 \mathrm{~cm}$ for the heterogeneous case with the $10 \mathrm{~cm} \times 10 \mathrm{~cm}$ beam aperture. Percentage contours are shown normalized to a peak gamma flux of $5.63 \times 10^{7}$

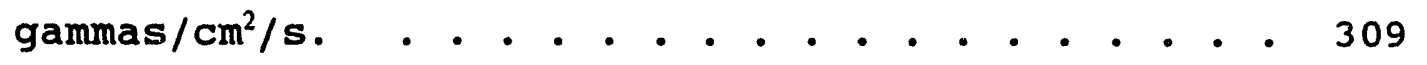

F-26. Gamma flux contours at $z=13.25 \mathrm{~cm}$ for the homogeneous case with the $10 \mathrm{~cm} \times 10 \mathrm{~cm}$ beam aperture. Percentage contours are shown normalized to a peak gamma flux of $5.61 \times 10^{7}$

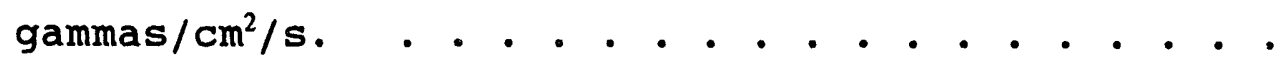

F-27. Thermal neutron flux contours at $y=-0.125 \mathrm{~cm}$ for the heterogeneous case with the $5 \mathrm{~cm} \times 10 \mathrm{~cm}$ beam aperture. Percentage contours are shown normalized to a peak thermal flux of $4.14 \times 10^{8}$ $\mathrm{n} / \mathrm{cm}^{2} / \mathrm{s}$. . . . . . . . . . . . . . . . . .

F-28. Thermal neutron flux contours at $y=-0.125 \mathrm{~cm}$ for the homogeneous case with the $5 \mathrm{~cm} \times 10 \mathrm{~cm}$ beam aperture. Percentage contours aje shown normalized to a peak thermal flux of $4.04 \times 10^{8}$ 


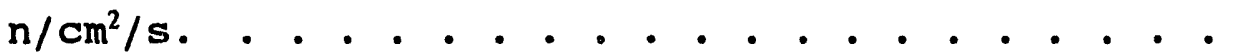

F-29. Fast reutron flux contours at $y=-0.125 \mathrm{~cm}$ for

the heterogeneous case with the $5 \mathrm{~cm} \times 10 \mathrm{~cm}$ beam

aperture. Percentage contours are shown

normalized to a peak fast flux of $1.26 \times 10^{7}$

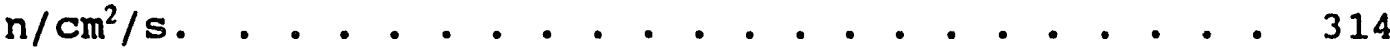

F-30. Fast neutron flux contours at $y=-0.125 \mathrm{~cm}$ for

the homogeneous case with the $5 \mathrm{~cm} \times 10 \mathrm{~cm}$ beam

aperture. Percentage contours are shown

normalized to a peak fast flux of $1.27 \times 10^{7}$

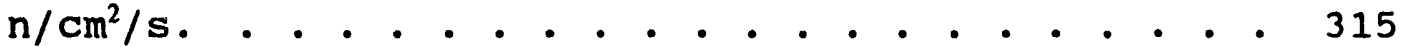

F-31. Gamma flux contours at $y=-0.125 \mathrm{~cm}$ for the

heterogeneous case with the $5 \mathrm{~cm} \times 10 \mathrm{~cm}$ beam

aperture. Percentage contours are shown

normalized to a peak gamma flux of $2.98 \times 10^{7}$

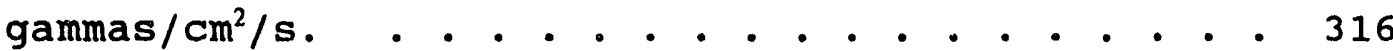

F-32. Gamma flux contours at $y=-0.125 \mathrm{~cm}$ for the

homogeneous case with the $5 \mathrm{~cm} \times 10 \mathrm{~cm}$ beam

aperture. Percentage contours are shown

normalized to a peak gamma flux of $2.97 \times 10^{7}$

gammas $/ \mathrm{cm}^{2} / \mathrm{s}$.

F-33. Thermal neutron flux contours at $y=-2.5 \mathrm{~cm}$ for

the heterogeneous case with the $5 \mathrm{~cm} \times 10 \mathrm{~cm}$ beam

aperture. Percentage contours are shown

normalized to a peak thermal flux of $4.14 \times 10^{8}$

$\mathrm{n} / \mathrm{cm}^{2} / \mathrm{s}$. 
F-34. Thermal neutron flux contours at $y=-2.5 \mathrm{~cm}$ for the homogeneous case with the $5 \mathrm{~cm} \times 10 \mathrm{~cm}$ beam aperture. Percentage contours are shown normalized to a peak thermal flux of $4.04 \times 10^{8}$

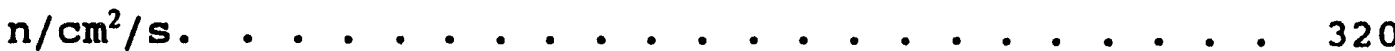

F-35. Fast neutron flux contours at $y=-2.5 \mathrm{~cm}$ for the heterogeneous case with the $5 \mathrm{~cm} \times 10 \mathrm{~cm}$ beam aperture. Percentage contours are shown normalized to a peak fast flux of $1.26 \times 10^{7}$

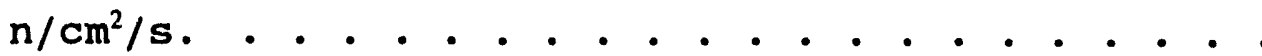

F-36. Fast neutron flux contours at $y=-2.5 \mathrm{~cm}$ for the homogeneous case with the $5 \mathrm{~cm} \times 10 \mathrm{~cm}$ beam aperture. Percentage contours are shown normalized to a peak fast flux of $1.27 \times 10^{7}$

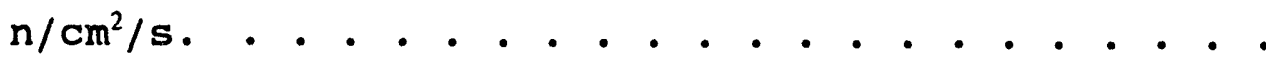

F-37. Gamma flux contours at $y=-2.5 \mathrm{~cm}$ for the heterogeneous case with the $5 \mathrm{~cm} \times 10 \mathrm{~cm}$ beam aperture. Percentage contours are shown normalized to a peak gamma flux of $2.98 \times 10^{7}$ gammas $/ \mathrm{cm}^{2} / \mathrm{s}$.

F-38. Gamma flux contours at $y=-2.5 \mathrm{~cm}$ for the homogeneous case with the $5 \mathrm{~cm} \times 10 \mathrm{~cm}$ beam aperture. Percentage contours are shown normalized to a peak gamma flux of $2.97 \times 10^{7}$ gammas $/ \mathrm{cm}^{2} / \mathrm{s}$.

F-32. Thermal neutron flux contours at $y=-4.5 \mathrm{~cm}$ for 
che heterogeneous case with the $5 \mathrm{~cm} \times 10 \mathrm{~cm}$ beam aperture. Percentage contours are shown normalized to a peak thermal flux of $4.14 \times 10^{8}$ $\mathrm{n} / \mathrm{cm}^{2} / \mathrm{s}$. . . . . . . . . . . . . . . . . . 326

F-40. Thermal neutron flux contours at $y=-4.5 \mathrm{~cm}$ for the homogeneous case with the $5 \mathrm{~cm} \times 10 \mathrm{~cm}$ beam aperture. Percentage contours are shown normalized to a peak thermal flux of $4.04 \times 10^{8}$

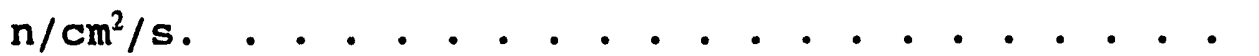

F-41. Fast neutron flux contours at $y=-4.5 \mathrm{~cm}$ for the heterogeneous case with the $5 \mathrm{~cm} \times 10 \mathrm{~cm}$ beam aperture. Percentage contours are shown normalized to a peak fast flux of $1.26 \times 10^{7}$

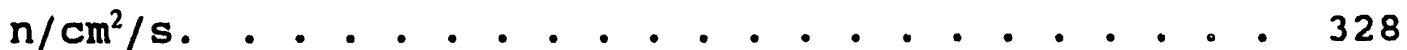

F-42. Fast neutron flux contours at $y=-4.5 \mathrm{~cm}$ for the homogeneous case with the $5 \mathrm{~cm} \times 10 \mathrm{~cm}$ beam aperture. Percentage contours are shown normalized to a peak fast flux of $1.27 \times 10^{7}$ $\mathrm{n} / \mathrm{cm}^{2} / \mathrm{s}$. . . . . . . . . . . . . . . . .

F-43. Gamma flux contours at $y=-4.5 \mathrm{~cm}$ for the heterogeneous case with the $5 \mathrm{~cm} \times 10 \mathrm{~cm}$ beam aperture. Percentage contours are shown normalizei to a peak gamma flux of $2.98 \times 10^{7}$ gammas $/ \mathrm{cm}^{2} / \mathrm{s}$.

F-44. Gamma flux contours at $y=-4.5 \mathrm{~cm}$ for the homogeneous case with the $5 \mathrm{~cm} \times 10 \mathrm{~cm}$ beam 
aperture. Percentage contours are shown

normalized to a peak gamma flux of $2.97 \times 10^{7}$

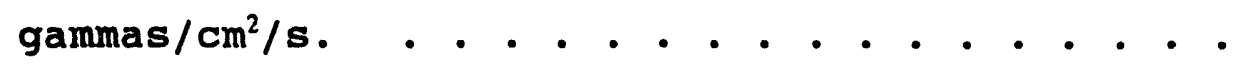

F-45. Thermal neutron flux contours at $y=2.5 \mathrm{~cm}$ for

the heterogeneous case with the $5 \mathrm{~cm} \times 10 \mathrm{~cm}$ beam

aperture. Percentage contours are shown

normalized to a peak thermal flux of $4.14 \times 10^{8}$

$\mathrm{n} / \mathrm{cm}^{2} / \mathrm{s}$. . . . . . . . . . . . . . . . .

F-46. Thermal neutron flux contours at $y=2.5 \mathrm{~cm}$ for the homogeneous case with the $5 \mathrm{~cm} \times 10 \mathrm{~cm}$ beam aperture. Percentage contours are shown normalized to a peak thermal flux of $4.04 \times 10^{8}$ $\mathrm{n} / \mathrm{cm}^{2} / \mathrm{s}$. . . . . . . . . . . . . . . . 334

F-47. Fast neutron flux contours at $y=2.5 \mathrm{~cm}$ for the heterogeneous case with the $5 \mathrm{~cm} \times 10 \mathrm{~cm}$ beam aperture. Percentage contours are shown normalized to a peak fast flux of $1.26 \times 10^{7}$ $\mathrm{n} / \mathrm{cm}^{2} / \mathrm{s}$. . . . . . . . . . . . . . . . .

F-48. Fast neutron flux contours at $y=2.5 \mathrm{~cm}$ for the homogeneous case with the $5 \mathrm{~cm} \times 10 \mathrm{~cm}$ beam aperture. Percentage contours are shown normalized to a peak fast flux of $1.27 \times 10^{7}$

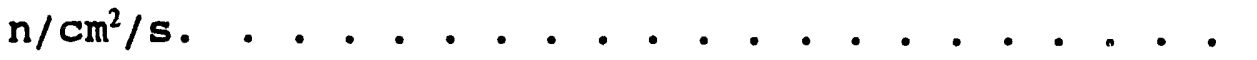

F-49. Gamma flux contours at $y=2.5 \mathrm{~cm}$ for the heterogeneous case with the $5 \mathrm{~cm} \times 10 \mathrm{~cm}$ beam aperture. Percentage contours are shown 
normalized to a peak gamma flux of $2.98 \times 10^{7}$

gammas $/ \mathrm{cm}^{2} / \mathrm{s}$. . . . . . . . . . . . . . . . 337

F-50. Gamma flux contours at $y=? .5 \mathrm{~cm}$ for the

homogeneous case with the $5 \mathrm{~cm} \times 10 \mathrm{~cm}$ beam

aperture. Percentage contours are shown

normalized to a peak gamma flux of $2.97 \times 10^{7}$

gammas $/ \mathrm{cm}^{2} / \mathrm{s}$. . . . . . . . . . . . . . . 338

F-51. Thermal neutron flux contours at $x=7.25 \mathrm{~cm}$ for

the heterogeneous case with the $5 \mathrm{~cm} \times 10 \mathrm{~cm}$ beam

aperture. Percentage contours are shown

normalized to a peak thermal flux of $4.14 \times 10^{8}$

$\mathrm{n} / \mathrm{cm}^{2} / \mathrm{s}$. . . . . . . . . . . . . . . . . . 340

F-52. Thermal neutron flux contours at $x=7.25 \mathrm{~cm}$ for

the homogeneous case with the $5 \mathrm{~cm} \times 10 \mathrm{~cm}$ beam

aperture. Percentage contours are shown

normalized to a peak thermal flux of $4.04 \times 10^{8}$

$\mathrm{n} / \mathrm{cm}^{2} / \mathrm{s}$. . . . . . . . . . . . . . . .

F-53. Fast neutron flux contours at $x=7.25 \mathrm{~cm}$ for the

heterogeneous case with the $5 \mathrm{~cm} \times 10 \mathrm{~cm}$ beam

aperture. Percentage contours are shown

normalized to a peak fast flux of $1.26 \times 10^{7}$

$\mathrm{n} / \mathrm{cm}^{2} / \mathrm{s}$. . . . . . . . . . . . . . . . . .

F-54. Fast neutron flux contours at $x=7.25 \mathrm{~cm}$ for the homogeneous case with the $5 \mathrm{~cm} \times 10 \mathrm{~cm}$ beam aperture. Percentage contours are shown normalized to a peak fast flux of $1.27 \times 10^{7}$ 


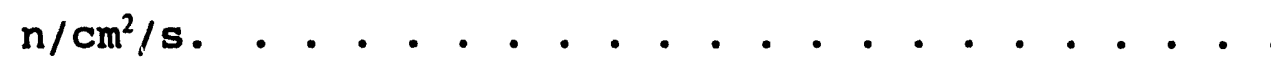

F-55. Gamma flux contours at $x=7.25 \mathrm{~cm}$ for the heterogeneous case with the $5 \mathrm{~cm} \times 10 \mathrm{~cm}$ beam aperture. Percentage contours are shown normalized to a peak gamma flux of $2.98 \times 10^{7}$ gammas $/ \mathrm{cm}^{2} / \mathrm{s}$.

F-56. Gamma flux contours at $x=7.25 \mathrm{~cm}$ for the homogeneous case with the $5 \mathrm{~cm} \times 10 \mathrm{~cm}$ beam aperture. Percentage contours are shown normalized to a peak gamma flux of $2.97 \times 10^{7}$ gammas $/ \mathrm{cm}^{2} / \mathrm{s}$.

F-57. Fast neutron flux contours at $x=12.25 \mathrm{~cm}$ for the heterogeneous case with the $5 \mathrm{~cm} \times 10 \mathrm{~cm}$ beam aperture. Percentage contours are shown normalized to a peak fast flux of $1.26 \times 10^{7}$

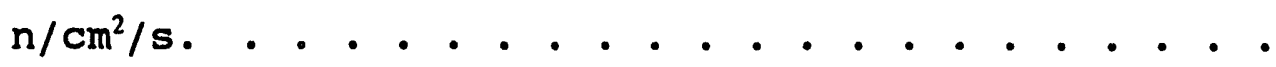

F-58. Fast neutron flux contours at $x=12.25 \mathrm{~cm}$ for the homogeneous case with the $5 \mathrm{~cm} \times 10 \mathrm{~cm}$ beam aperture. Percentage contours are shown normalized to a peak fast flux of $1.27 \times 10^{7}$

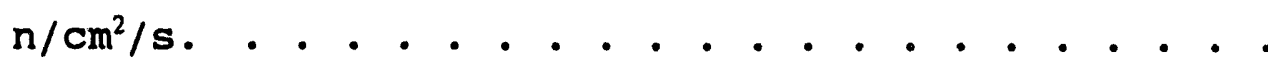

F-59. Gamma flux contours at $x=12.25 \mathrm{~cm}$ for the heterogeneous case with the $5 \mathrm{~cm} \times 10 \mathrm{~cm}$ beam aperture. Percentage contours are shown normalized to a peak gamma flux of $2.98 \times 10^{7}$ gammas $/ \mathrm{cm}^{2} / \mathrm{s}$. 
F-60. Gamma flux contours at $x=12.25 \mathrm{~cm}$ for the homogeneous case with the $5 \mathrm{~cm} \times 10 \mathrm{~cm}$ beam aperture. Percentage contours are shown normalized to a peak gamma flux of $2.97 \times 10^{7}$ gammas $/ \mathrm{cm}^{2} / \mathrm{s}$. . . . . . . . . . . . . . 349

F-61. Fast neutron flux contours at $x=17.25 \mathrm{~cm}$ for the heterogeneous case with the $5 \mathrm{~cm} \times 10 \mathrm{~cm}$ beam aperture. Percentage contours are shown normalized to a peak fast flux of $1.26 \times 10^{7}$ $\mathrm{n} / \mathrm{cm}^{2} / \mathrm{s}$. . . . . . . . . . . . . . . . . .

F-62. Fast neutron flux contours at $x=17.25 \mathrm{~cm}$ for the homogeneous case with the $5 \mathrm{~cm} \times 10 \mathrm{~cm}$ beam aperture. Percentage contours are shown normalized to a peak fast flux of $1.27 \times 10^{7}$ $\mathrm{n} / \mathrm{cm}^{2} / \mathrm{s}$. . . . . . . . . . . . . . . . . . .

F-63. Gamma flux contours at $x=17.25 \mathrm{~cm}$ for the heterogeneous case with the $5 \mathrm{~cm} \times 10 \mathrm{~cm}$ beam aperture. Percentage contours are shown normalized to a peak gamma flux of $2.98 \times 10^{7}$ gammas $/ \mathrm{cm}^{2} / \mathrm{s}$.

F-64. Gamma flux at $x=17.25 \mathrm{~cm}$ for the homogeneous case with the $5 \mathrm{~cm} \times 10 \mathrm{~cm}$ beam aperture. Percentage contours are shown normalized to a peak gamma flux of $2.97 \times 10^{7}$ gammas $/ \mathrm{cm}^{2} / \mathrm{s}$.

F-65. Thermal neutron flux contours at $z=13.25 \mathrm{~cm}$ for the heterogeneous case with the $5 \mathrm{~cm} \times 10 \mathrm{~cm}$ beam 
aperture. Percentage contours are shown

normalized to a peak thermal flux of $4.14 \times 10^{8}$

$\mathrm{n} / \mathrm{cm}^{2} / \mathrm{s}$. . . . . . . . . . . . . . . 355

F-66. Therma: veutron flux contours at $z=13.25 \mathrm{~cm}$ for

the homogereous case with the $5 \mathrm{~cm} \times 10 \mathrm{~cm}$ beam

aperture. Percentage contours are shown

normalized to a peak thermal flux of $4.04 \times 10^{8}$

$\mathrm{n} / \mathrm{cm}^{2} / \mathrm{s}$. . . . . . . . . . . . . . 356

F-66. Gamma flux contours at $z=13.25 \mathrm{~cm}$ for the

heterogeneous case with the $5 \mathrm{~cm} \times 10 \mathrm{~cm}$ beam

aperture. Percentage contours are shown

normalized to a peak gamma flux of $2.98 \times 10^{7}$

gammas $/ \mathrm{cm}^{2} / \mathrm{s}$. . . . . . . . . . . . . 358

F-67. Gamma flux contours at $z=13.25 \mathrm{~cm}$ for the

homogeneous case with the $5 \mathrm{~cm} \times 10 \mathrm{~cm}$ beam

aperture. Percentage contours are shown

normalized to a peak gamma flux of $2.97 \times 10^{7}$

gammas $/ \mathrm{cm}^{2} / \mathrm{s}$. . . . . . . . . . . . . 359 
TABLES

2-1. TYPICAL SYMPTOMS OF SITE-SPECIFIC BRAIN TUMORS WITH THEIR RELATIVE FREQUENCY OF OCCURRENCE (FROM J. R. CHOI, p. 32) . . . . . . . . . . . . 38

2-2. SURVIVAL WITH GLIOBLASTOMA MULTIFORME (FROM R. V. DORN et al., p. 137) . . . . . . . . . . . . 39

4-1. MASS TISSUE DENSITIES $\left(\mathrm{g} / \mathrm{cm}^{3}\right)$ AND VOLUME FRACTIONS FOR THE TORT CALCULATIONS . . . . . . 72

4-2. PERCENT WATER OF TISSUE SAMPLES . . . . . . . . . 78

4-3. ELEMENTAL COMPOSITIONS FOR COMPARISON WITH AVAILABLE CANINE DATA (atoms $/ \mathrm{cm}^{3} \times 10^{-24}$ ) . . . . 81

4-4. ELEMENTAL COMPOSITIONS FOR COMPARISON WITH SELECTED HUMAN DATA . . . . . . . . . . . . . 83

5-1. MATERIAL COMPOSITIONS FOR BMRR DELIMITER COMPONENTS • . • . . . . . . . . . . . . . . 96

A1. MEASURED TISSUE COMPOSITION FOR TEMPORALIS MUSCLE 160

A2. MEASURED TISSUE COMPOSITION FOR LOWER SKIN • • • . 161

A3. MEASURED TISSUE COMPOSITION FOR UPPER SKIN • • • 162

A4. MEASURED TISSUE COMPOSITION FOR SKULL . • • . • . 163

45. MEASURED TISSUE COMPOSITION FOR WHITE MATTER • • . 164

A6. MEASURED TISSUE COMPOSITION FOR GRAY MATTER • • • 165

A7. MEASURED TISSUE COMPOSITION FOR SPINAL CORD • • 166

A8. MEASURED TISSUE COMPOSITION FOR TEETH . • • • • . 167

A9. MEASURED TISSUE 1:OMPOSITION FOR EYE • • • • • . . 168

A10. MEASURED TISSUE COMPOSITION FOR : ONGUE . . . . 169

A11. MEASURED TISSUE COMPOSITION FOR CONCHA . . . . 170 
A12. MEASURED TISSUE COMPOSITION FOR ETHMOID

LABYRINTH . . . . . . . . . . . . . 171

A13. MEASURED TISSUE COMPOSITION FOR GENIO GLOSSUS . . 172

A14. MEASURED TISSUE COMPOSITION FOR ORAL MUCOSA . . . 173

A15. MEASURED TISSUE COMPOSITION FOR EYE LENS AND OUTER SKIN RETINA . . . . . . . . . . . . 174

G-1. SUMMARY OF CARBON, HYDROGEN, NITROGEN, AND

OXYGEN CONTENT IN DOG 21 SAMPLES . . . . . . . . 363

G-2. SUMMARY OF TRACE ELEMENT CONTENT IN DOG 21

SAMPLES . . . . . . . . . . . . . 369

G-3. SUMMARY OF CARBON, HYDROGEN, NITROGEN, AND

OXYGEN CONTENT IN DOG 22 SAMPLES . . . . . . . . 370

G-4. SUMMARY OF TRACE ELEMENT CONTENT IN DOG 22

SAMPLES . . . . . . . . . . . . . . . 376 


\section{INTRODUCTION}

Boron Neutron Capture Therapy (BNCT) is an experimental treatment for cancer. The programs in the United states primarily focus on its application to a currently incurable brain tumor -- glioblastoma multiforme -- and for metastatic malignant melanoma. The idea of BNCT, the selective delivery of boron to cancer cells followed by neutron irradiation resulting in neutron capture and then the fissioning of the boron nucleus, was first presented by Locher in $1936^{1}$, only four years after Chadwick discovered the neutron. Boron-10 is used because of its high cross section for capture of thermal neutrons (3838 barns). The resulting fission particles are ${ }^{7} \mathrm{Li}$ and ${ }^{4} \mathrm{He}$. These particles deposit their combined energy of $2.33 \mathrm{MeV}$ over ranges of about 10 microns, approximately the diameter of the cell. In Figure 1-1, a thermal neutron bombards a tumor cell that contains boron- 10. Boron-10 captures the neutron, fissioning into ${ }^{7} \mathrm{Li}$ and ${ }^{4} \mathrm{He}$. A gamma ray may also be emitted. Ideally, these reaction products cause the reproductive death of the cell.

In the 1940s, in vivo experiments were carried out on mice demonstrating the possibilities of neutron capture therapy. Studies on boron and lithium compounds as capture agents were also performed. A beam powerful enough to treat 


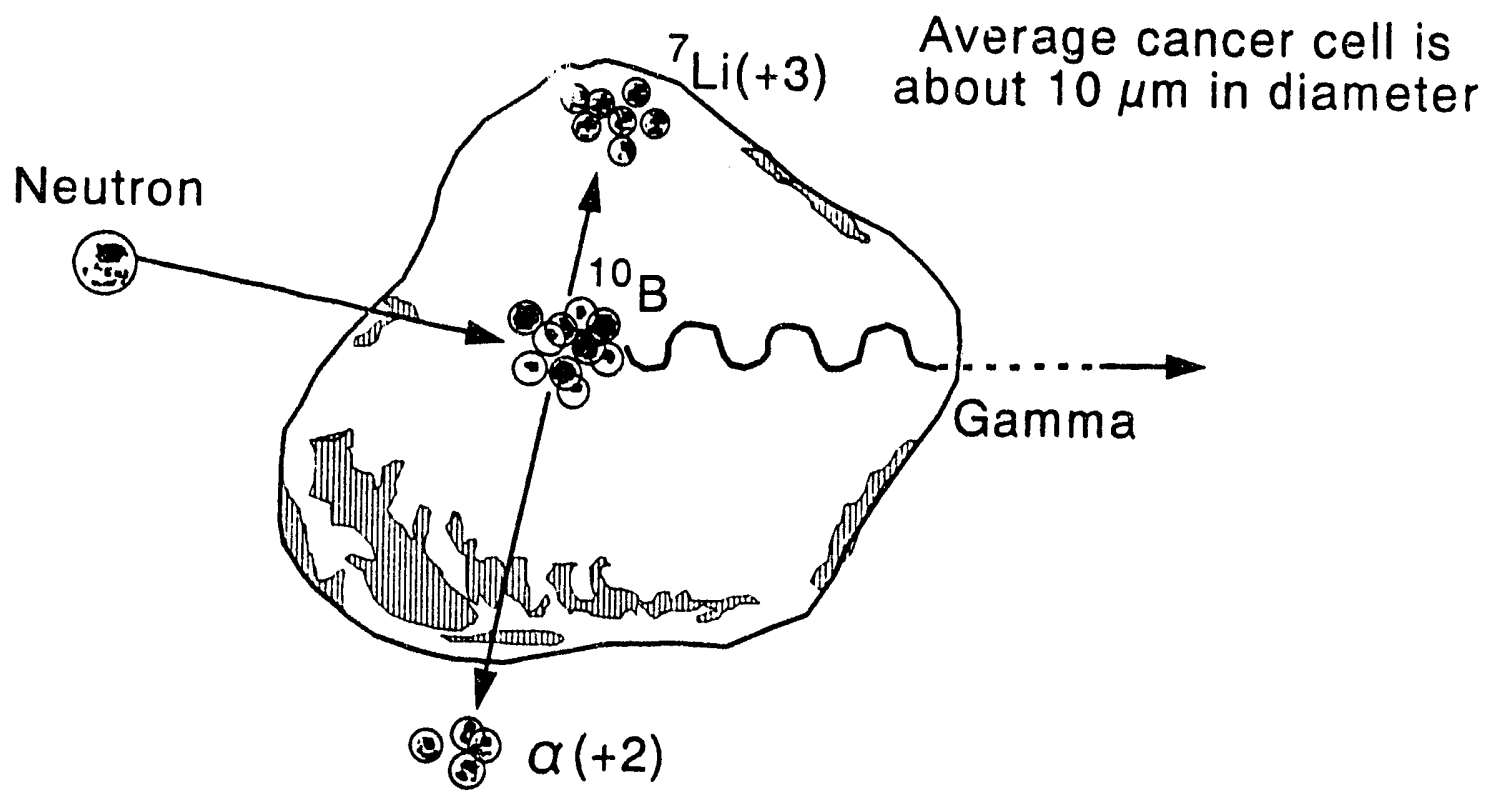

Figure 1-1. Boron activation by capiure of neutrons. 
humans was not available until 1951 when the Brookhaven National Laboratory graphite research reactor was in operation. Between 1951 and 1961, human clinical trials took place at the graphite research reactor, the Brookhaven Medical Research Reactor (BMRR), and the Massachusetts Institute of Technology Reactor (MITR). ${ }^{2}$ These trials were unsuccessful for a number of reasons which are dealt with, for example, in References 3 and 4. However, since 1968 Hiroshi Hatanaka has used BNCT as a treatment with some apparent success resulting in a resurgence of world interest in the field.

One area which has been investigated in depth is dosimetry in BNCT. Calculation of radiation flux and absorbed dose profiles is a complex three-dimensional problem. The various dose components (e.g. the incident fast neutron dose, the hydrogen capture gamma dose, the boron capture dose) all have different spatial distributions and relative biological effectiveness (RBE). Both stochast $i c$ and deterministic techniques may be employed to accurately perform the necessary calculations to determine spatial distributions. ${ }^{5.9}$ In both cases, however, the typical approach has been to approximate the highlyheterogeneous calculational geometry of the treatment volume by either a spatially homogenized model ${ }^{6,7}$ or by a simplified few-region model. ${ }^{8,9}$ The accuracy of such models for patient 
treatment planning should ideally be validated by comparison with calculated results obtained by modeling the actual heterogeneous geometry and tissue compositions as faithfully as possible.

The purpose of this study is to quantify macroscopic heterogeneity effects in a Labrador Retriever head utilizing TORT, ${ }^{10}$ a three-dimensional discrete ordinates transport code developed by Oak Ridge National Laboratory and adapted at the INEL for BNCT. Data on the spatial distributions of fluxes within a canine head were obtained for the BMRR epithermal beam for a heterogeneous case and a volumeweighted homogenized model enabling comparison of fluxes for the two models. From this data, a determination can be made of the accuracy of the homogeneous approximation for use in experimental therapy programs such as the one coordinated by the INEL.

In the following chapter, a brief review of glioblastoma multiforme, cell biology, particle interactions, and radiobiology provides a basis for this study. Heterogeneity effects, the canine head model used in this study, and other models for BNCT are then considered. A review of the available elemental composition data is followed by the methods of dissection and examination used to obtain the composition and geometric data. The results 
of the chemical analysis are presented along with a comparison with the available data in the literature. The methods of analytical modeling are discussed. Finally, the results are presented and discussed followed by conclusions and recommendations for future work in the area of patient treatment planning in BNCT. 


\section{BASIS FOR STUDY}

In order for BNCT to be an effective treatment, many different aspects of the therapy need to be improved and integrated. The program requires experts in many fields to study areas such as the biology, chemistry, and physics of the body. In addition, BNCT research also pertains to the physics and engineering of designing and implementing an effective neutron beam plus the necessary participation of the medical field.

Because BNCT is an interdisciplinary field, it is useful to have some knowledge of the disease and the biology, chemistry, and physics involved. By understanding particle interactions in tissue, the need for examining macroscopic heterogeneity effects such as those due to passage through bone or the nasal cavity rather than soft tissue becomes clear. However, it is important to first understand how this disease challenges the scientific field and why BNCT has been identified as a potential treatment.

\subsection{Glioblastoma Multiforme}

Glioblastoma multiforme is an invasive brain tumor which has finger-like projections that extend from the tumor into the surrounding healthy tissues. These extensions 
prevent effective treatment through surgery since all viable tumor cells cannot be removed without causing unacceptable brain damage. In addition, all of the tumor cells must be killed to prevent tumor regrowth. These cells are heterogeneous, meaning that properties characteristic of one cell may not be characteristic of another, adding to the difficulty in treatment. As a result, conventional therapies, including surgery, chemotherapy, radiation therapy, and combination therapies, do not offer definitive treatment for this cancer.

\section{Glioblastoma multiforme is a member of a group of} neoplasms known as gliomas or astrocytomas. A glioma is a cancer of the glial cells, which comprise about 908 of the cells in the central nervous system and aid in regulating the extracellular environment of this system." Due to diffuse tumor growth, the invasive extensions of this cancer result in a hard to define tumor edge. Based on cell histology, these tumors are graded I through I.V, including the extent of cellularity and pleomorphism, degree of anaplasia, number of mitotic cells seen, evidence of endothelial proliferation, and extenc of necrosis. ${ }^{12}$ Grade I and II gliomas are usually considered benign. Grade I gliomas are characterized by an increase in the number of normal astrocytes which can usually only be detected with a microscope, whereas in Grade II gliomas some abnormalities 
in the cell structure are also evident. If diagnosed at the early stages of the disease, these can be effectively treated with conventional therapy.

As the disease progresses into the malignant stages, more changes are seen in the cells. Grade III and IV gliomas are considered malignant. These gliomas are characterized by multiple nuclei in cells, a decrease in cytoplasm, and other changes in cell structure and composition with more extreme abnormalities in Grade IV gliomas. Grade IV gliomas and sometimes Grade III gliomas are known as glioblastoma multiforme.

The disease is very difficult to detect in its early stages due to the nature of its symptoms. These symptoms are often site-specific depending on the location of the tumor in the brain. ${ }^{3}$ Table 2-1 includes some symptoms with their origin and relative occurrence. Headaches and mood swings are common due to the increased pressure on the brain as a result of tumor growth. Local paralysis may also occur. Table $2-2^{13}$ shows the survival rate of patients with the disease in the III and IV stages. BNCT has been shown by Dr. Hatanaka in Japan to be a potential treatment, and in some cases possibly a cure, for these patients despite their currently poor prognosis with conventional therapy. 
TABLE 2-1. TYPICAL SYMPTOMS OF SITE-SPECIFIC BRAIN TUMORS WITH THEIR RELATIVE FREQUENCY OF OCCURRENCE (FROM J. R. CHOI, p. 32)

\begin{tabular}{l|l|r} 
Bymtoms & Location & f \\
\hline Disturbance in memory and & Frontal/Temporal & $30-60$ \\
judgement. & lobes & 30 \\
Disturbance of personality & Frontal lobes & 11 \\
Contralateral weakness and & $\begin{array}{l}\text { Frontoparietal } \\
\text { cortex }\end{array}$ & 5 \\
numbness & Occipital lobes & -- \\
Contralateral visual fields & $\begin{array}{l}\text { Cerebellar } \\
\text { hemisphere } \\
\text { Ipsilateral incoordination of } \\
\text { limbs }\end{array}$ & \\
Incoordination of gait & &
\end{tabular}


TABLE 2-2. SURVIVAL WITH GLIOBLASTOMA MULTIFORME (FROM R. V. DORN et al., p. 137)

\begin{tabular}{|c|c|c|c|c|c|}
\hline \multirow{2}{*}{ Grade } & \multirow{2}{*}{$\begin{array}{c}\text { surgery } \\
\end{array}$} & \begin{tabular}{c} 
Radiation \\
Therapy \\
\cline { 4 - 6 }
\end{tabular} & \pm & \multicolumn{3}{|c|}{ Survival $(8)$} \\
\hline III & + & - & $10-15$ & $5-10$ & 0 \\
& + & + & 40 & 30 & $15-20$ \\
& + & - & 5 & 0 & 0 \\
& + & + & $25-50$ & 10 & $0-3$ \\
\hline
\end{tabular}




\subsection{Cell Biology}

To understand how BNCT is able to kill tumor cells, it is useful to have some knowledge of the cell and its functions. Figure $2-1^{14}$ depicts a typical animal cell. A plasma membrane surrounds the entire cell. Inside this membrane, numerous organelles are suspended in the cytoplasm. The nucleus, which controls the functions of the cell, and its membrane are depicted in the center of the cell. The cell's genetic information is stored here in the form of deoxyribonucleic acid (DNA). Chains of DNA make up the chromosomes seen in Figure 2-1 in the nucleus. The nucleus is the primary target for boron-10. If the capture reaction occurs within the nucleus, then cell death will more than likely occur. The cell will be unable to replicate if its genetic information is destroyed. However, if the boron is concentrated in the cytoplasm, the "hit," the place where particles deposit their energy, may not be lethal and the cell may be able to repair itself and continue proliferating. The mechanisms of cell repair and the number of hits required to kill the cell are not entirely understood at this time.

The cell is composed of many different organelles, each with a unique function or product which contributes to the call. Some of the organelles in the cytoplasm, as shown in 
Figure 2-1, are the mitochondria, where energy for the cell is processed zind stored in forms like adenosine trjphosphate (ATP), ready to meet the cells energy needs; the endoplasmic reticulum, responsible for the production of some proteins and fats in addition to their transport; the ribosomes, responsible for protein synthesis; and the Golgi complex, which packages and distributes molecules such as proteins to other areas of the cell, including the cell membrane. ${ }^{14}$ The cel: membrane, which regulates transport into and out of the cell, is composed of lipid and protein molecules. The membrane is important in the search for a boron compound which concentrates in the cell since the compound must be able to cross this barrier. Within the cell, mechanisms exist for transporting necessary substances across the plasma membrane to maintain ion concentrations and to get nourishment (oxygen) from blood. It is also possible that one of these mechanisms can be used to transport the boron compound into the ccll in a concentration that is sufficient for successful therapy.

Division of normal cells occurs in phases. After cell division is complete the cell is in interphase during which DNA is replicated. Then mitosis occurs; the nucleus divides into two equal daughter nuclei. For cell division to be complete, cytokinesis must occur after mitosis. Cytokinesis is the division of the cytoplasm. Multinucleated cells, 


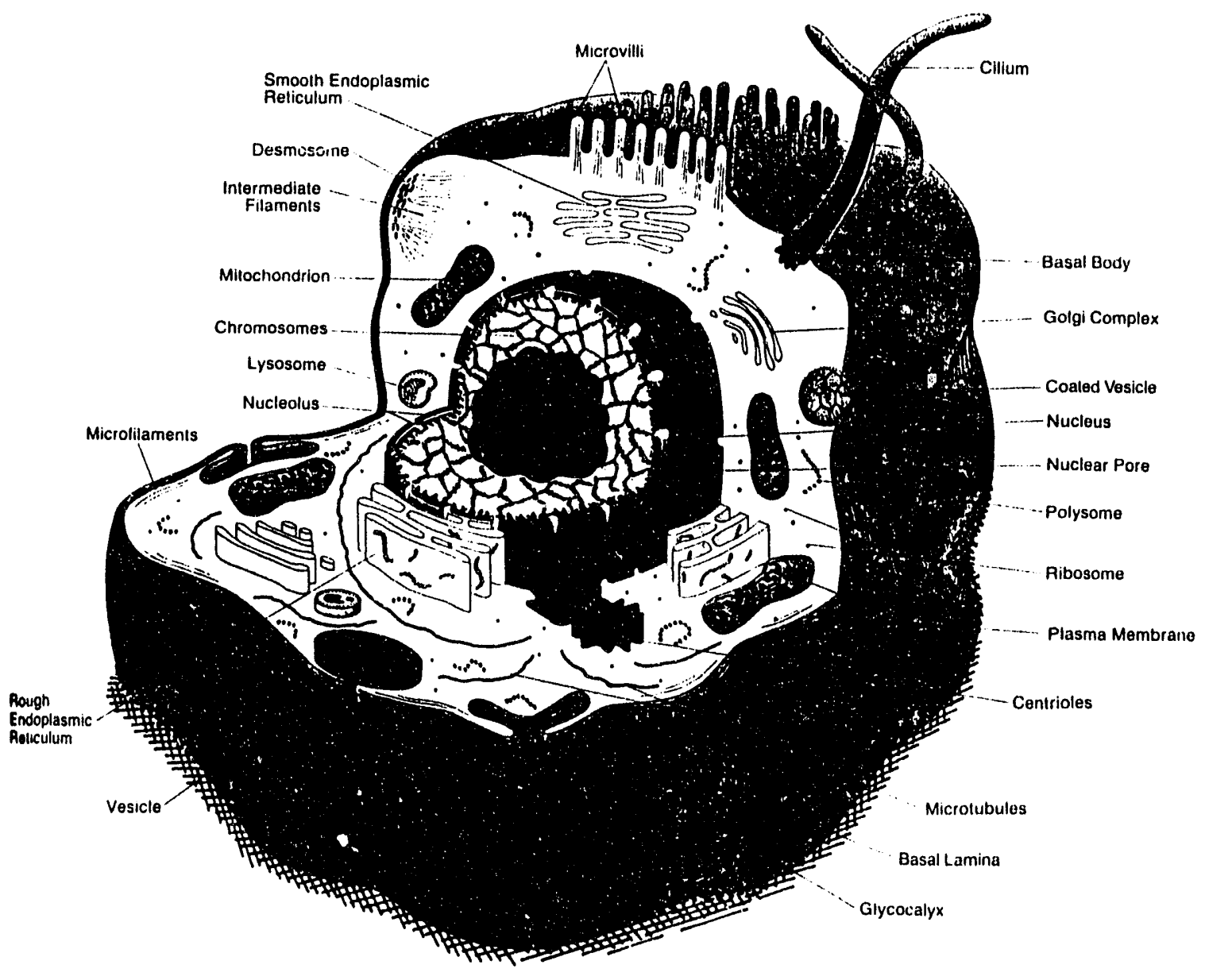

Figure 2-1. Components of a typical animal cell (from Wessells and Hopson, p. 103). 
such as those characteristic of Grade III and IV gliomas, are produced when mitosis is complete but cytokinesis does not take place. In the body, the production of new cells is balanced unless a cancer develops.

When the controls which regulate cell multiplication fail, a cancer may begin. Cell growth occurs at a rapid rate even though the body no longer needs these cells. Each new cell lacks the ability to regulate multiplication and growth; therefore, each cell continues multiplying, forming clones, resulting in a tumor such as glioblastoma multiforme.

Cancer cells can be distinguished from normal cells in a variety of ways. Such cells contain an abnormal and unstable amount of chromosomes while losing their differentiating traits. Differentiation is the specialization of cells to perform certain functions; examples of differentiated cells include red blood cells and glial cells. The cancer cells lack a specialized structure and grow at an abnormally rapid rate, affecting the two main stages of cell growth, the duplication of DNA and the physical division of the cell into two cells. It is possible that one or more mutations in DNA causes the cancer to develop. If a mutation is not fixed by the cell's repair mechanisms, mutations in a single cell can lead to a tumor. 


\subsection{Considerations in Boron Distribution}

One of the necessary components for BNCT is the development of a non-toxic compound to deliver boron-10 preferentiaily to the tumor. The method of delivery of the compound depends on the subject and the scientist. Rats are given the compound orally. For larger animals and humans, the compound may be given through an artery or vein. The boron compound is allowed enough time to circulate throughout the body and accumulate in the tumor before irradiation. The typical boron-10 compounds have a biological half-life of about 250 minutes, but the half-life variess with the compound. Optimum neutron irradiation occurs at the optimum boron concentration in the tumor.

As the boron concentration in the tumor is optimized, a less intense irradiation field will be necessary, and normal tissue will receive less dose. There are many considerations in defining a boron compound, including delivery mechanisms, boron carriers, and the distribution within the cells. The blood-brain barrier, located in the endothelial cells which line the capillaries of the brain, aids in preventing the drug from getting into healthy cells. This barrier controls the transport of substances into the extracellular fluid of the brain and the rate at which transport occurs. In designing a boron delivery agent, this 
selectivity is often relied upon because the barrier is often damaged at the tumor site.

Figure 2-2 depicts the blood-brain barrier. Tight junctions seal the endothelial cells together, preventing large molecules from getting into the extracellular fluid through these two cells. Blood-brain barrier selectivity is monitored by both physical structures and physiological systems which regulate the entrance of substances from the blood into the extracellular space of the brain. The normal blood-brain barrier prevents the boron compound from concentrating in the healthy cells, whereas since the barrier is damaged near tumor cells, boron will be able to concentrate in the extracellular fluid around those cells. Once in the extracellular fluid, the compound needs to be transported across the plasma membrane and possibly the nuclear membrane for concentration either in the cytoplasm or the nucleus.

The location of boron in the brain and within the cells is vital, as mentioned previously. ${ }^{15}$ This is because the biological response of the cells is dependent upon the charged particles produced in a capture reaction which have a short range in tissue (6-8 microns) and are high LET radiation. The boronated agent should be highly selective and concentrate mainly in the tumor cells, so that when the 


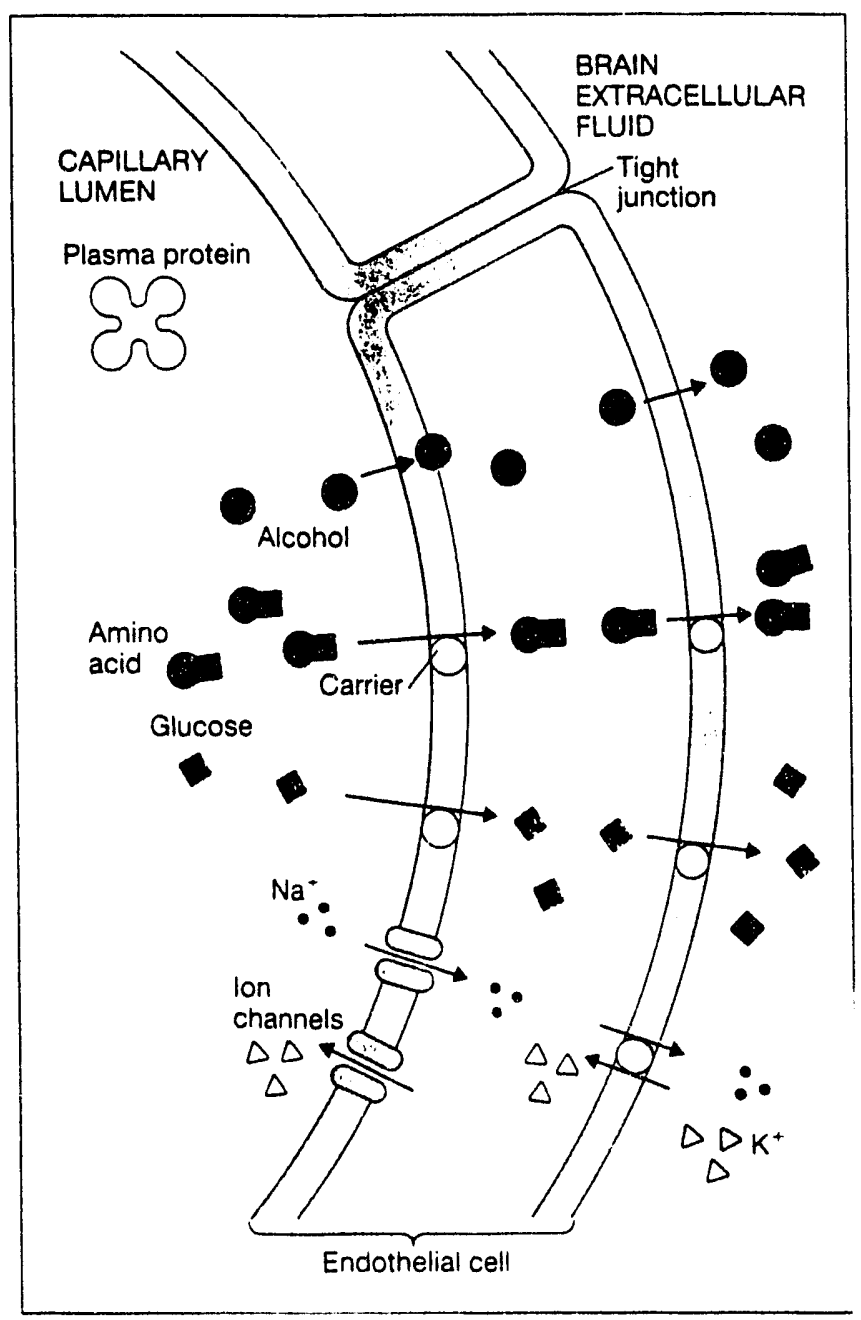

Figure 2-2. A diagram of the blood-brain barrier, which is formed by the endothelial cells that line the brain capillaries (from Vander, Sherman, and Luciano, p. 103). 
neutron beam is applied and a reaction takes place, the alpha and lithium particles will be localized within the tumor cells. Because the range of these heavy particles is approximately one cell diameter, cells without the boron agent, which should be the normal cells, will not be exposed to the additional dose from the charged particles of the boron reaction. However, healthy cells may be exposed by the gamma rays produced during the capture reaction in a tumor cell due to the range of the gamma rays (on the order of $\mathrm{cm})$. Healthy cells also receive radiation dose from hydrogen capture gammas, proton recoil, nitrogen $(n, p)$ reactions, and other unavoidable background reactions.

A number of compounds have been studied for boron delivery, and new compounds are under development. Two groups of compounds for boron delivery are low molecular weight substances and biopolymers or higher molecular weight substances. Reference 16 contains an overview of compounds in these two classes and their applicability to BNCT. In the low molecular weight group of substances is disodium mercaptoundecahydro-closo-dodecaborate $\left(\mathrm{Na}_{2} \mathrm{~B}_{12} \mathrm{H}_{11} \mathrm{SH}\right)$. It is a member of sulfhydryl-containing polyhedral boranes. Hatanaka has been using this compound for his BNCT treatments in Japan.

A variety of biopolymers are also being developed. 
Some methods for delivery include attaching boron compounds to monoclonal antibodies and antibody fragments, boronated peptides, and attachment to cell surface receptors. Another promising method is the use of encapsulating complexes. The boron would be enclosed in a liposome or low density lipoprotein. The amount of a compound needed depends on the localization of that compound once in the tumor. For example, if the boron localizes on the cell surface (e.g. delivered by antibodies), six to seven times more boron is necessary achieve the same dose that is given to the nucleus when the boron is uniformly distributed. A cell which does not accumulate boron receives about half of the dose that a cell with boron receives, depending on the amount of boron accumulation. ${ }^{17}$ Consequently, these methods of boron delivery need to compensate for the lack of boron in the nucleus by having much more accumulation on the cell surface or in the cytoplasm than is needed with a compound that concentrates boron in the nucleus.

\subsection{Interactions of Particles in Tissue}

The main particles in the BNCT reaction are neutrons, alpha particles, gamma rays, and the recoil nucleus of ${ }^{7} \mathrm{Li}$. Recoil protons are generated by interactions with fast neutrons. Protons and gamma rays are also produced as a result of the nitrogen and hydrogen capture reactions. The 
interactions of these particles in tissue are dependent upon the size, charge, and energy of each particle. As a result of these factors, some particles are more effective in killing the cell.

particles lose energy upon collision through excitation and ionization of the medium through which they travel. Charged particles, such as alpha particles, are directly ionizing. These particles are able to produce chemical and biological changes by altering the atomic structure in the material they traverse. Indirectly ionizing radiations are not able to produce biological and chemical damage without first creating a charged particle. Indirect ionization, such as that caused by neutrons and gamma rays, results in a transfer of energy in the material which generates fastmoving charged particles. These resultant particles cause biological and chemical damage in the cell.

When comparing different radiations, it is useful to discuss the concepts of relative biological effectiveness (RBE) and linear energy transfer (LET). The RBE is defined as the ratio of the absorbed dose of a reference radiation to the absorbed dose of a test radiation to produce the same level of biological effects, other conditions being equal. ${ }^{18}$ It allows one to compare, for example, the amount neutron and photon radiation needed to cause the same biological 
effect. There is some question with regard to the proper definition of $\mathrm{RBE}$ and its values for the BNCT reaction, because of certain microdosimetric factors that are unique to BNCT. Classically, RBE is based on the LET, radiation dose, dose rate, the number of dose fractions, and the biological system or endpoint. A determination can be made as to the effectiveness of a radiation in killing tumor cells taking into account the dose to healthy cells. This is termed the therapeutic gain factor which is defined for a cell as the ratio of the RBE of the tumor to the RBE of the normal tissue. ${ }^{18}$ In the evaluation of a treatment modality, such as BNCT for clinical use, it is important that the therapeutic gain factor be as high as possible. This value also allows comparison and evaluation of different therapies to determine the most useful method.

Another term used in discussing different particles is linear enurgy transfer (LET). As each particle travels through tissue it transfers energy along its path by collision with other particles. LET is an average of the energy transferred per unit length along the track. It is represented in units of $\mathrm{keV}$ per micron of unit density material. RBE and LET are related for different types of radiation. RBE can be plotted against LET as shown in Figure 2-3.18 Sparsely ionizing radiations, such as photons, have a low LET. They are usually inefficient at killing 


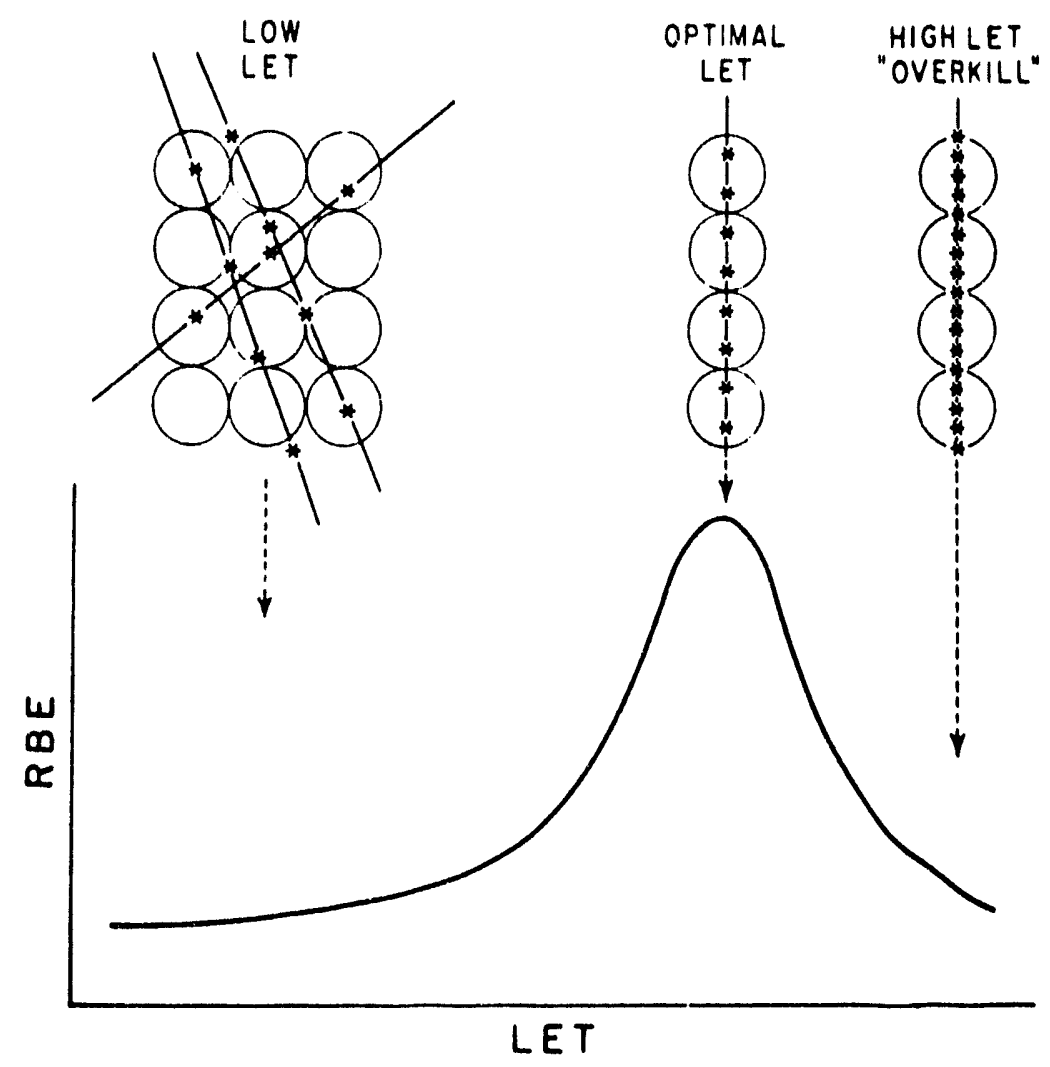

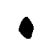

Figure 2-3. Relationship of RBE and LET. sparsely ionizing radiations require more than one hit for cell kill. Densely ionizing radiations result in a waste of energy because more energy than is needed to kill the cell is deposited (from Hall, p. 172). 
killing cells because enough energy is not deposited in one cell implying that two or more hits may be necessary for cell death. Also related to LET is the attenuation of particles as they travel through the medium. The amount ofenergy lost depends on the mass density of the material being traveled through in addition to the mass of the particle and interaction probabilities. For heavy particles, the amount of energy loss is approximately equal to the stopping power of the material.

\subsubsection{Neutrons}

Any achievable neutron beam for BNCT will contain neutrons having a range of energies including thermal 10 $0.414 \mathrm{eV})$, epithermal $(0.414-10 \mathrm{keV})$, and fast (>10 keV). Radiative capture reactions as well as scattering interactions can occur with neutrons in matter. Each element has a cross section for neutron scattering and capture. The cross section defines the ability of an atom to capture a neutron. cross sections are greatest for thermal neutrons because, due to their slower velocity, particles stay in the vicinity of the atom long enough for a capture event to happen.

The $2200 \mathrm{~m} / \mathrm{s}$ capture cross section of boron-10 for thermal capture is 3838 barns. The capture cross sections 
for hydrogen ( 0.333 barns) and nitrogen (1.91 barns) are not high compared to that of boron-10, but hydrogen and nitrogen exist in much greater quantities in cells leading to a substantial dose due to these reactions. The dose from hydrogen and nitrogen capture is probably the dose-limiting factor to the healthy tissue as far as background dose is concerned unless the neutron beam has an unduly high fast neutron component which then becomes the dose-limiting factor to the skin.

A thermal neutron beam can be used to treat a tumor with a depth up to three to four cm. As a result, this beam is only effective if the tumor is on or near the surface or if the skin and skull flap is reflected before irradiation. A well-designed epithermal beam allows penetration to a deep-seated tumor while, at the same time, delivering a dose which the skin can tolerate.

Epithermal neutrons interact primarily through elastic collisions. They are not as harmful as fast neutrons; this leads to some skin sparing where the beam enters the patient. These neutrons are able to penetrate much deeper through the skin and skull due to their increased energy over thermal neutrons, becoming thermalized for capture at the tumor. 
As mentioned previously, fast neutrons are also present in BNCT. They are considered contamination and beam designs aim at minimizing the number of fast neutrons. Fast neutrons can cause radiobiologically-significant skin and central nervous system damage as a result of hydrogen recoil. Since it has essentially the same mass, a fast neutron can lose all of its energy in a single collision with a hydrogen atom. Because fast neutron interactions are independent of boron concentration, hydrogen recoil deposits energy in healthy and neoplastic cells equally. The radiobiology of the interactions will be discussed further in section 2.5 .

\subsubsection{Alpha Particles ( $\left.{ }^{4} \mathrm{He}\right)$}

Alpha particles lose energy by collision with atoms and electrons in the material being traversed. They are densely ionizing and follow a straight path, especially when compared with an electron's path. Due to its large mass relative to an electron, the alpha particle will transfer only a tiny portion of its energy each time it collides with an electron. With each collision the alpha particle slows down. The amount of energy transferred per collision depends on energy and the size of the other particle. The alpha particles produced as a result of boron capture deposit all their energy within 10 microns. 


\subsubsection{Gamma Rays}

There are two sources of gamma rays in the BNCT procedure. Gamma rays are incident in the beam but are held to insignificant levels. The main source of gamma rays is from reactions occurring within the patient such as from hydrogen capture. Hydrogen is a major component of tissue and is in far more abundance than boron in the cell. Gamma rays are also emitted part of the time as neutron capture in boron. Gamma rays can interact with matter by three main mechanisms. One type of gamma ray interaction is the photoelectric effect, whereby the incoming gamma photon interacts with a bound electron. The second form is the Compton effect, in which the gamma photon interacts with a free electron, losing only part of its energy. The third gamma ray interaction is pair production. There is a 1.022 MeV threshold for this reaction, but the $2.2 \mathrm{MeV}$ gamma formed by hyarogen capture is above the threshold for this interaction. In pair production, a positron-electron pair is produced which then annihilates jtself, giving off two gamma rays in opposite directions with equivalent energies of $0.511 \mathrm{MeV}$. These gamma rays are now free to interact in the tissue. Both the photcelectric and compton effects liberate fast, high energy electrons, setting them in motion through the tissue. Pair production leads to additional gamma rays. Because gamma rays do not interact in a dense 
pattern like alpha particles, the range of these particles in tissue, or any material, is much greater than that of alpha particles and electrons.

\subsection{Radiobiology}

A number of physical, chemical, and biological events take place upon ionization and excitation of atoms within cells which affect the RBE associated with a type of radiation. If ar interaction takes place, the first events relate to the physics of the absorption process. Ionizing radiation causes ionization and excitation of atoms within $10^{-15}$ seconds. ${ }^{18}$ Following these events, free radicals are formed and chemical bonds are broken. Between $10^{-12}$ and $10^{-6}$ seconds, chemical events such as reaction of radicals with solutes take place. The biochemical and cellular processes take longer to occur. Cellular repair mechanisms may become activated; the metabolism may be obstructed; mitosis may be delayed; chromosomal damage may occur; and reproductive capacity may be lost. Later effects of ionizing radiation include tissue damage (days to months); late somatic effects, such as cancer; and genetic effects which may not be seen for generations.

Interactions of radiation with tissue lead to biological effects which are dependent on the type of 
radiation. In section 2.4 different types of ionizing radiation were discussed. The important concept resides in the understanding that the probability of a direct interaction between the particle track and the target molecule (possibly the DNA) increases with increasing density of ionization. ${ }^{18}$ Direct action dominates for high-LET radiation, such as alpha particles, and indirect action, a process by which a product of radiolysis of water, such as a hydroxyl radical, interacts with and damages the DNA, dominates for low-LET radiation such as gamma rays. Figure 2-4 demonstrates the indirect and direct actions of photons on the double helix of DNA.

High-LET radiations such as heavy particles formed by neutron collisions are dominated by direct action, the process by which radiation interacts directly with the DNA to cause cellular damage. The probability of direct action increases with the density of ionization.

There are two ways of damaging the cell. One effect is indirect in which structures necessary for the cell's survival are damaged by chemical products formed as a result of the high LET emission within the cell. For example, the mitochondria are responsible for generating energy compounds for use by the cell. If the cell is unable to change an energy compound into a form it can utilize because of a 


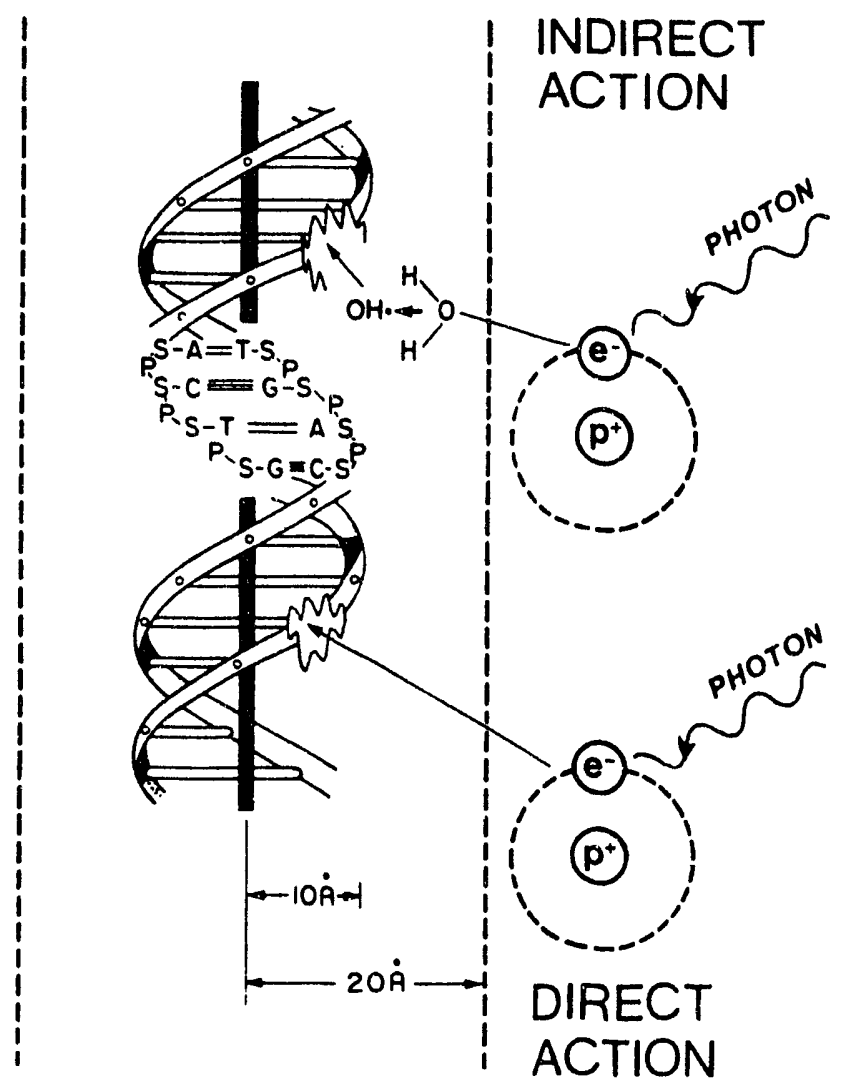

Figure 2-4. An illustration of the indirect and direct action of photons on DNA. Indirect action dominates for sparsely ionizing radiation and works through the formation of a hydroxyl radical (from Hall, p. 11). 
chemical block, cell death will occur with no source of energy. In contrast, a directly damaging effect occurs when ionization and excitation of atoms within the cell occurs as a direct result of an energy transfer from an external source, such as radiation.

\subsection{Summary}

High LET particles, such as alpha particles and electrons, deposit their energy within a limited area. Thus, more damage occurs locally in tissue as a result of high LET radiation. By using high LET emissions in BNCT to treat glioblastoma multiforme, irradiation is more likely to directly cause major cell damage or even cell death, decreasing the chances of cell repair in tumor cells. However, the average dose to individual nuclei in a tumor can vary by a factor of ten or more, ${ }^{19}$ a result which depends upon the location of boron within the cell structure, as discussed earlier. It is therefore important to develop models to better understand the irradiation process and to avoid unwanted cell damage at both the microscopic and macroscopic levels.

The study described in this thesis will aid in the preparation for human clinical trials in the United States by evaluating the accuracy of typically homogeneous 
calculational models for BNCT dosimetry. This study includes calculations of fluxes in an anatomically and chemically detailed Labrador Retriever head and in a volumeweighted homogeneous model. As a result, perturbations in the flux between the two cases are easily identified and can be traced directly back to areas such as bone or the nasal cavity based on chemical composition or attenuation of the material being traversed. 


\section{REASONS FOR STUDY}

In studying heterogeneity effects, one must first identify the effects of interest, such as those due to differing transport properties because of geometric structures, individual tissue variations, or tissue densities. The methods to examine and possibly quantify the extent of the effects of interest must be selected. Three types of both macroscopic and microscopic heterogeneity effects on radiation dose distributions for BNCT exist. ${ }^{20}$ This work examines one type of heterogeneity effect. It is also necessary to review other computer models for patient treatment planning in BNCT. A number of these studies include experimental data obtained with different phantoms. These studies will be examined further in this section.

\subsection{Types of Heterogeneity Effects}

The first type of heterogeneity effect to be discussed is macroscopic, occurring on the millimeter to centimeter scale. Effects on the spatial distribution of the absorbed radiation dose can be seen when comparing the local scalar neutron and gamma fluxes in heterogeneous and homogeneous models, all other factors being equal. Perturbations in the heterogeneous case are due to individual tissue variations 
such as those due to geometry and chemical composition. Each tissue has different scattering and absorption properties which in turn affect particle interactions in that tissue. This results in a change in the neutron and gamma flux distributions in the region of interest. Local variations in the neutron and gamma fluxes are most noticeable in void regions, such as in the nasal cavity.

The second type of heterogeneity effect occurs as a result of tissues having different KERMA (Kinetic Energy Release in Matter) factors, which are dependent on the elemental composition of the tissue. The resulting spatial variations in fluxes are seen on a macroscopic scale. For example, the absorbed dose in a given neutron flux field directly increases with increasing nitrogen content in a tissue, all other compositions being equal in the tissues. This is due to the nitrogen $(n, p)$ interaction.

The third type of heterogeneity effect, local secondary charged-particle imbalance, occurs on a macroscopic and/or microscopic scale. It is more pronounced near material interfaces, external surfaces, and internal voids. studied in standard photon therapy, local secondary charged-particle imbalance is also evident in BNCT on a macroscopic scale for the photon-induced component. As a result of this effect, the radiation dose distribution does not necessarily follow 
the photon flux due to the production and interactions of charged particles. ${ }^{20}$

The study presented here was designed to examine Type 1 heterogeneity effects. Type 2 heterogeneity effects are accounted for by employing KERMA response functions for each tissue. Type 3 effects have been investigated on both the macroscopic and microscopic scale. Those that occur on a microscopic level are studied in the field of microdosimetry. For example, references 22 and 23 examine these effects.

\subsection{Canine Head Model}

Calculations models are typically homogeneous or consist of a few different regions. Approximations of shape are often made, such as using cylinders or ellipses to represent a human head. The accuracy of such models should be determined for use in patient treatment planning. One way of analyzing the use of such a model is by comparing a carefully constructed heterogeneous model with a volumeweighted homogeneous model. Stochastic and deterministic methods can both be used to solve the three-dimensional problem of calculating the various fluxes and doses inherent to BNCT. 
It was established that the deterministic method would be better suited to examine heterogeneity effects. The random statistical variations associated with a Monte carlo code might mask the effects due to inhomogeneities unless allowed to run for an impractical length of time. A deterministic method would assure that any perturbations in fluxes between the heterogeneous and homogeneous cases would be due to Type 1 effects.

By using the discrete ordinates method of TORT, 10 valuable information can be extracted. Thermal and fast neutron fluxes and gamma fluxes can be evaluated in one-, two-, or three-dimensions. The two-dimensional plots can be overlaid on a sketch of the outline of the brain and skin for that plane. Sagittal, dorsal, and axial planes can all be examined. These plots aid in the evaluation of flux distributions and the identification of differences due to Type 1 heterogeneity effects.

A computer model of the head of a Labrador Retriever was made to evaluate the use of a homogeneous model for the canine irradiation program jointly conducted by the INEL and Washington State University (WSU) ${ }^{23}$ Measurements of specific structures in the canine head were accomplished by dissection and examination of two similar Labrador Retriever heads. Also, various tissue samples were taken during the 
dissections to acquire data on the elemental compositions of these tissues. Subsequently, three-dimensional calculations of the radiation flux distributions were performed for a detailed Labrador Retriever head model irradiated in an epithermal neutron beam.

Before presenting in detail the model mentioned above, a review of other work in the area of patient treatment planning is necessary to understand how this work expands and supports this area of BNCT research.

\subsection{A Review of Other Models for BNCT}

Working in the field of BNCT, much information is found through the use of computer modeling. Approximations are made to simplify the irradiation volume, often decreasing not only the amount of time necessary to model, but also computer run time. This can amount to valuable savings and possibly lead to the extension of a study to include other areas of interest, for example, evaluating two neutron beams instead of one. Computer models are validated in a number of ways. Calculations are validated through comparison with experimental results. Modeling volumes in BNCT are usually confined to homogeneous or few-region approximations. In the area of patient treatment planning, calculations and/or measurements have been made for a variety of reactors, 
linear accelarators, and modeling volumes. These studies should be noted.

In Japan, T. Matsumoto and O. Aizawa took measurements at the Musashi Institute of Technology reactor with an epithermal beam for a cylindrical phantom. ${ }^{24}$ Calculations were made using DOT $3.5,25$ an Oak Ridge National Laboratory two-dimensional discrete ordinates transport code. Good agreement was seen between experimental values and calculations. At the Kyoto University Research Reactor (KUR) Institute, T. Kobayashi, M. Ono, and K. Kanda studied the flux distributions of the KUR beam. ${ }^{17}$ They made measurements and calculations for a cylindrical heavy water phantom. These studies also had good agreement between experimental and calculational values.

At the Massachusetts Institute of Technology Research Reactor, MITR-II, a number of efforts have been made to advance the field of patient treatment planning. $0 . L$. Deutsch and B. W. Murray designed a more specific model by defining the human skull and brain regions as ellipsoids. ${ }^{26}$ The regions were homogeneous approximations of the size of an average brain. They also included the cavity formed as a result of the craniotomy procedure performed on all patients prior to neutron irradiation. Calculations were done for a thermal neutron beam with a general purpose Monte Carlo 
code, ANDY, from the LOs Alamos Scientific laboratory. Data obtained included dose-depth plots for different B-10 concentrations. Several years later 0 . K. Harling et al made measurements for epithermal beams with a polyethylene cylindrical phantom. ${ }^{27}$ Calculations were performed with MCNP, a Monte Carlo code used for coupled neutron and photon transport. Also, MCNP was used for the same model of the human brain and skull that had been used by Deutsch and Murray with the ANDY code at the MITR-II. ${ }^{28}$ This time the model was used to examine epithermal beams. Homogenized gray and white matter were used for the brain composition. The skull composition had a density of $1.5 \mathrm{~g} / \mathrm{cc}$. To perform measurements, a gelatin-based phantom was used. This brain and skull model was further utilized by R. G. Zamenhof et al to develop a Monte Carlo dosimetry and treatment planning program for BNCT that allows input of a number of treatment parameters to arrive at a treatment protocol specific for each patient. ${ }^{29}$ The planning program, NCTPLAN, calculates one- and two-dimensional dose distributions in addition to other calculations. This work explores various parameters further such as the advantage depth and advantage ratio. These parameters are used by the authors to evaluate epithermal beams at the MITR-II.

Calculations have been made for other neutron sources as well. Modeling a linear accelerator, calculations were 
performed and analyzed by J. C. Yanch, X-L. Zhou, and G. L. Brownell.9 Their analysis included two phantoms, a cylindrical phantom made of human brain-equivalent material and an elliptical phantom made of the brain and skull material for humans used by Deutsch and Murray as reported above. MCNP was used for calculations, and results were presented in terms of the advantage ratio and advantage depth as used by zamenhof et al above. These calculations were performed to evaluate a variety of neutron energies and source diameters.

other work in the field of BNCT treatment planning has been performed by D. W. Nigg, P. D. Randolph, and F. J. Wheeler. ${ }^{6}$ Measurements of thermal flux were made using a homogeneous lucite dog-head phantom for an epithermal beam at the Brookhaven Medical Research Reactor (BMRR). Calculations were made with TORT, the same code used for calculations in this work. Agreement was within the known uncertainties of the calculations and measurements.

Each of these models has furthered the understanding of the scientific community on the flux and dose distributions of neutron beams for use in BNCT. Both thermal and epithermal beams have been characterized and analyzed throughout the world. Linear accelerators are being investigated as an alternative neutron source. A variety of 
models have been used ranging from homogeneous cylinders and dog head phantoms to mudels which include the human skull and brain as ellipsoids. This work strives to understand the neutron and gamma flux distributions still further by examining a heterogeneous canine head model. 


\section{EXPERIMENTAL METHODS}

The TORT code requires information describing the geometry and chemical composition of each tissue within the irradiation volume. In order to make a clinically relevant heterogeneous model, it was necessary for the model to be both anatomically and chemically accurate. Because of the limited information available in the literature, a detailed model could not be made based on those sources. Another modeling method was necessary. One possible approach to part of the problem is to reconstruct the required geometric information directly from medical imagery. 5 This method is under development at the INEL, but it was not available for use in this study. It was determined that the most accurate way to model the heterogeneity of a canine head would be to experimentally obtain all the required geometric and composition data by using an actual dog instead of relying on inadequate literature sources. Following this, the data obtained could serve the purpose of validating software designed to reconstruct geometric data from medical imagery.

The TORT code requires input data describing the chemical composition and mass density of each tissue. These data, specified by region, make the model heterogeneous. When these data are combined with the geometry, any shifts in the magnitudes and/or shapes of the fluxes due to 
inhomogeneities can be seen.

\subsection{Review of Data in Literature}

A limited amount of information was found in the literature on elemental compositions of canine tissues. J. R. Mehta et al analyzed total lipid content, muscle water, and muscle elements in six healthy adult dogs and three mature Labrador Retrievers with hereditary muscular dystrophy. ${ }^{30}$ Another study by Mehta et al analyzed peripheral nerves, the spinal cord, and the brain for various elements, total tissue water, and total lipid content. ${ }^{31}$ Five healthy adult mixed breed dogs were used for this study. Most elemental compositions were determined by atomic absorption spectroscopy in both works. The samples analyzed were fat free solids. Other data on canine tissues were found in References 32 and 33. Both references do not provide sources for the data, information on breed, number of samples taken, or the method of analysis.

No information was found in the literature for mass densities of canine tissues. As a result, data for humans was used for modeling with the assumption in this work that corresponding human and canine tissues have similar mass densities (but not necessarily the same elemental composition). In Table 4-1, the densities of the tissues 
TABLE 4-1. MASS TISSUE DENSITIES $\left(\mathrm{g} / \mathrm{cm}^{3}\right)$ AND VOLUME FRACTIONS FOR THE TORT CALCULATIONS

\begin{tabular}{lcc}
\multicolumn{1}{c}{ Tissue } & Mass Density $\left(\mathrm{g} / \mathrm{cm}^{3}\right)$ & Volume Fraction \\
\cline { 2 - 2 } & 1.041 & 0.603 \\
Skin & 1.1 & 0.130 \\
Bone & 1.61 & 0.120 \\
Brain & 1.036 & 0.045 \\
Spinal Cord & 1.038 & 0.00438 \\
Teeth & 2.165 & 0.00948 \\
Eye & 1.026 & 0.00297 \\
Tongue & 1.041 & 0.0471 \\
Nasal Cavity & 0.5 & 0.0250 \\
\hline a. See Reference 34. & & \\
b. Volume fractions of tissue do not sum to 1.0 because of \\
$\quad$ void regions.
\end{tabular}


used in the TORT model are shown. All densities except for that of bone and the nasal cavity were taken from Reference 34 and represent an average where a range is presented. The range was between 1-2\& for eyes, muscle, and brain and between 7-98 for teeth and skin. The mass density of bone was taken from the value of white and woodard. The density of the nasal cavity was judged by visual examination to be approximately $0.5 \mathrm{~g} / \mathrm{cc}$. Table $4-1$ also shows the volume fractions used for the TORT input. These fractions do not add to one because of void regions.

\subsection{Dissection and Examination}

To obtain chemical and anatomical data, the heads of two Labrador Retrievers, euthanized for reasons unrelated to this study, were dissected and examined. Both heads were severed at the third cervical vertebra, shaved, and frozen at approximately -20 degrees celsius. Prior to sectioning, two dowels were inserted to provide markers for use in measuring. One centimeter sagittal slices were cut from the left hemisphere of the first head. Approximately $1 \mathrm{~mm}$ of tissue was lost from each slice due to the thickness of the saw blade. To prevent deterioration, each slice was immediately refrozen after being cut. Photographs of each side of each slice were taken with the slice placed on a 
background grid with $10 \mathrm{~mm}$ x $10 \mathrm{~mm}$ squares. The slices were first cleaned with a damp cloth to remove saw cuttings and frost. Figure 4-1 depicts the right (inside) surface of the center sagittal slice, which corresponds to the sagittal midplane.

Two 1-gram samples were taken from the sagittal midplane to be analyzed. These areas were marked by white pins as shown in Figure 4-2. To obtain the brain composition, both white and gray matter were sampled. Temporalis muscle and spinal cord samples were taken. The dermis and epidermis layers of the skin were analyzed. Reçions of the nasal and sinus cavities and mouth were also saypled. From these regions, concha, ethmoid labyrinth, Genio glossus, oral mucosa, and teeth samples were obtained. one eye was analyzed. For more information on canine brain anatomy, see Reference 35 .

The above procedure was repeated for the axial sections of the second dog. However, concha samples were not taken from this dog and both eyes were analyzed whole. The sections were cut from the back of the neck towards the nose. 


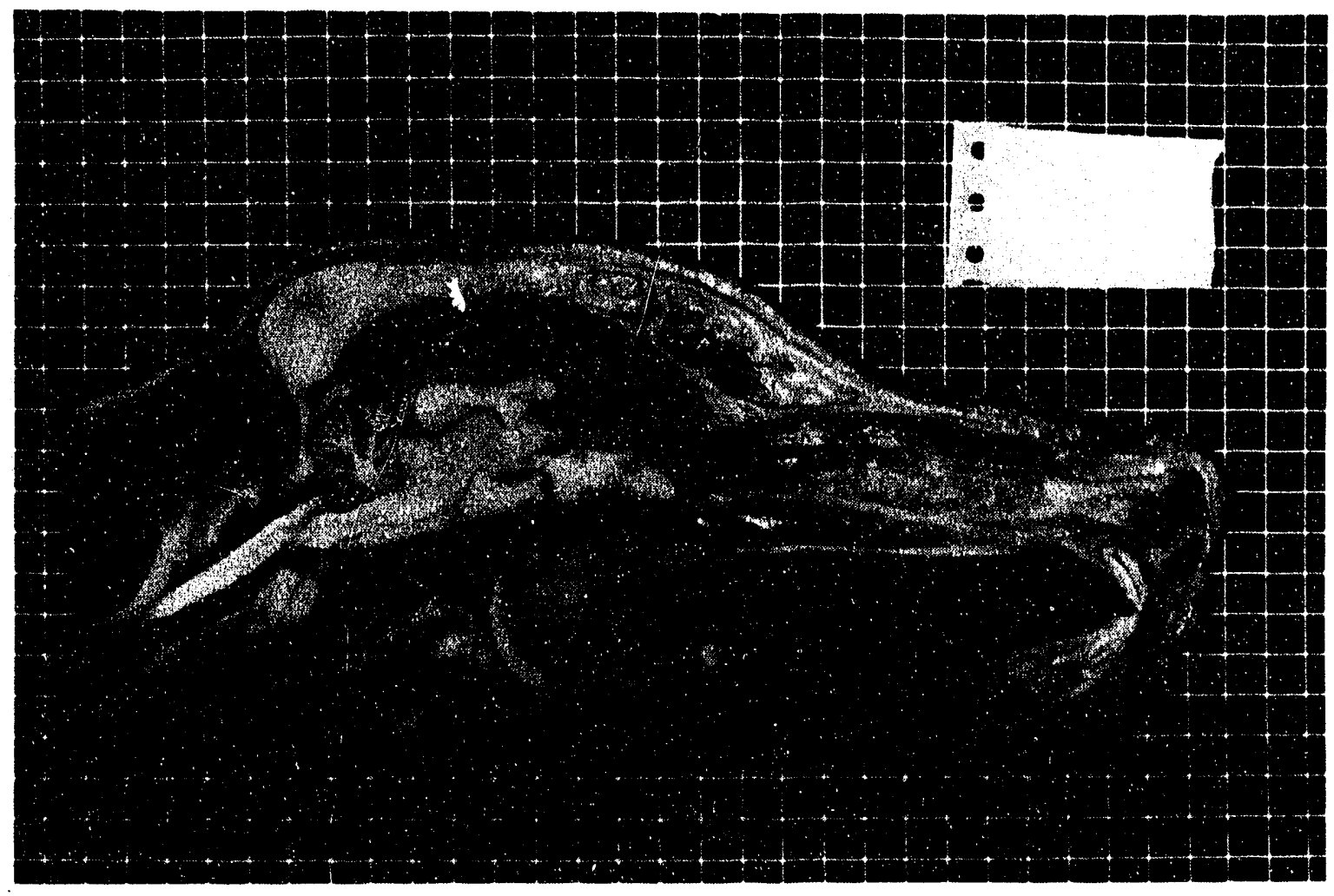

Figure 4-1. Photograph of the right side of the center sagittal slice. 


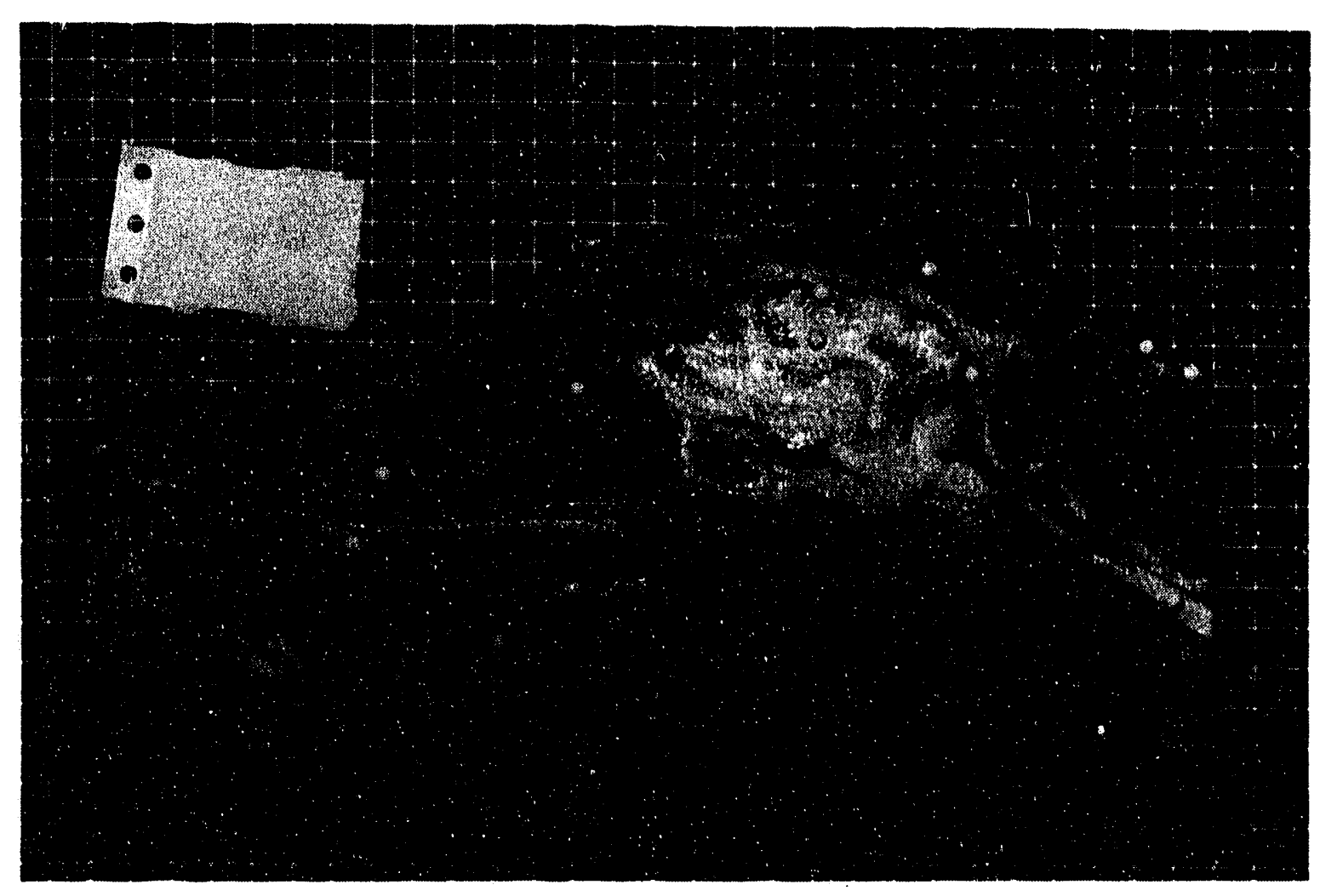

Figure 4-2. Photograph of the center sagittal slice depicting the locations where samples were taken. 


\subsection{Chemical Analysis}

Both sets of tissue samples were analyzed to determine the volume-average elemental composition for each. William F. Bauer was responsible for obtaining these data. ${ }^{20}$ The analysis was performed to determine the water content and the values of hydrogen, carbon, oxygen, nitrogen, sulfur, phosphorous, magnesium, calcium, sodium, and potassium. Chlorine was also of interest but was not analyzed in any of the samples.

First, water content was determined by mass lost upon drying at 100 degrees Celsius for at least 16 hours. Table 4-2 presents the water percentage of all samples. Using a Carlo Erba Instruments EA1108 elemental analyzer, values in parts per million were obtained for hydrogen, carbon, and nitrogen for $1-2 \mathrm{mg}$ of dried sample. The value for hydrogen was determined by adding the hydrogen contents of both the elemental analysis and the water lost upon drying.

Inductively coupled plasma-atomic emission spectroscopy (ICP-AES) was used to determine values for all other elements of interest with the exception of oxygen and chlorine. ICP-AES was performed after a closed vessel microwave digestion procedure. For this procedure, $0.5-1.0$ $\mathrm{g}$ of wet sample and $7.5 \mathrm{ml}$ of concentrated $\mathrm{HNO}_{3}$ were placed 


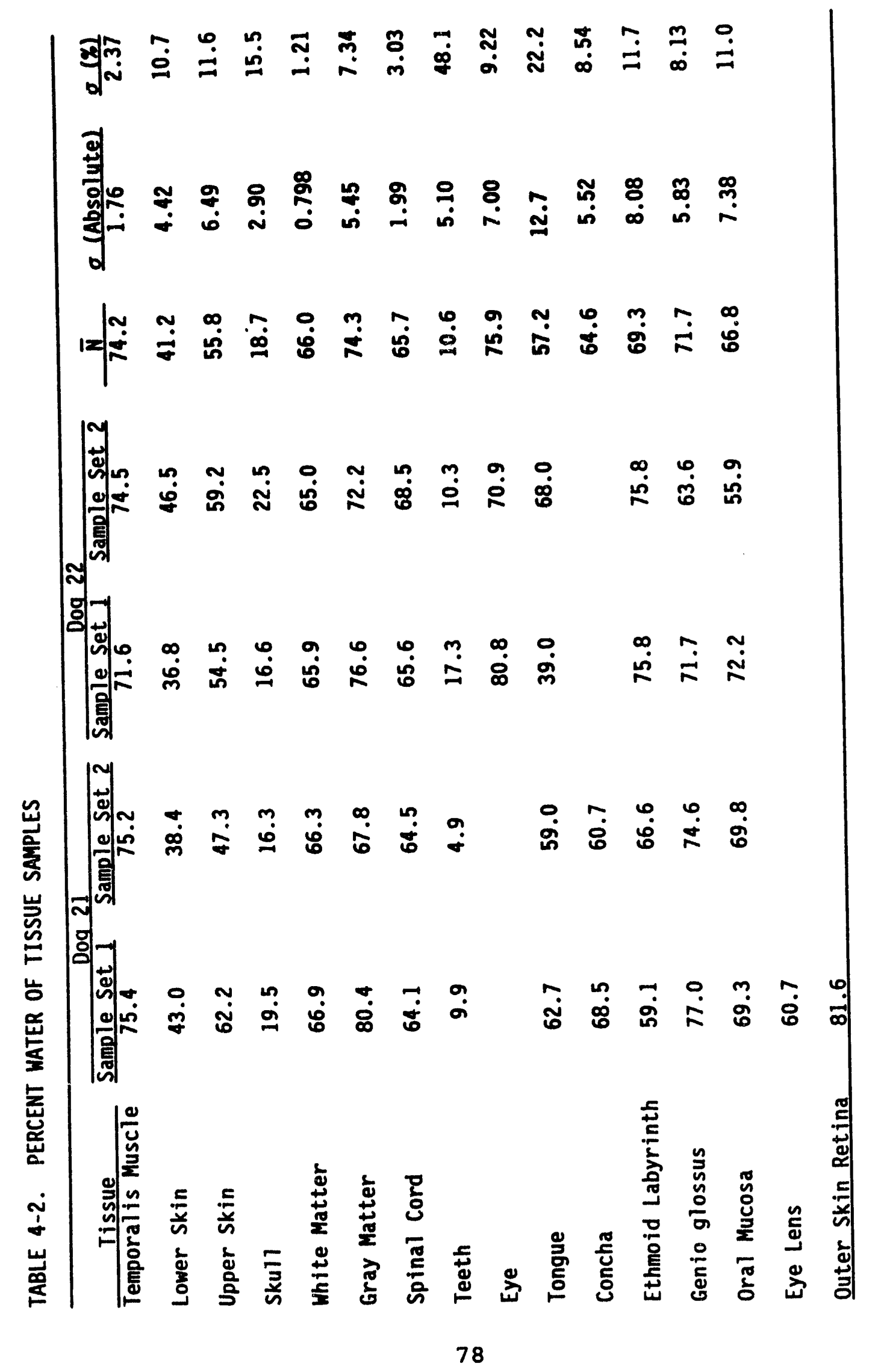


in a Tefion digestion bomb, and then the sealed bombs were heated in a microwave oven for approximately 30 minutes. The power was controlled by constant feedback from a pressure monitor set to maintain a maximum of $20 \mathrm{psi}$ inside the bombs Following this, the bombs were cooled, vented, and an additional $1 \mathrm{ml}$ of $\mathrm{HNO}_{3}$ was added before resealing. The bombs were then heated again for approximately 15 minutes. After cooling, the contents of each bomb were diluted to $25 \mathrm{ml}$ for background $B$ analysis described in Reference 37. A $1 \mathrm{ml}$ aliquot of this sample was then diluted to $50 \mathrm{ml}$ for the analyses of all other elements. oxygen was estimated from the water content and by assuming all phosphorous was present as $\mathrm{PO}_{4}^{-3}$.

The results were measured in parts per million and then converted into units of atoms per cubic centimeter with a unit mass density of $1.0 \times 10^{-24}$ grams per cubic centimeter assumed. The measured elemental compositions for each tissue are presented in Appendix A. Averages and standard deviations are also included. The standard deviation $(\sigma)$ was calculated from the following formula:

$$
\sigma^{2}=\Sigma(s-\bar{s})^{2} /(N-1)
$$




\subsection{Comparison of Chemical compositions with Literature}

Although limited information was found on canine elemental compositions, this data can be presented for comparison with the results obtained in this work. Most of the information concerned the concentrations of trace elements in tissue. These results are shown in Table 4-3. A unit mass density $\left(1 \mathrm{~g} / \mathrm{cm}^{3}\right)$ was assumed for all tissue types for these data. For the trace elements, the measurements taken for this study correlate fairly well with previous measurements for the elements represented.

More information was available on human tissue compositions for a number of elements. Hence, the data presented here can also be compared with human data from selected sources such as ICRU, ${ }^{37} \mathrm{Kim},{ }^{38}$ Awschalom et al, ${ }^{39}$ Brooks et al, ${ }^{40}$ and works by Woodard and White. ${ }^{41,42}$ These sources are not the only ones available, but they are the sources most commonly employed in radiation dosimetry. It should be noted that values for certain elements were not represented in each sample. It is possible that certain elements existed in a quantity below the detection limits of the time. The values are presented in Table 4-4 in percentages, consequently these numbers are not as accurate as those presented in Table 4-3. In Table 4-4, the results obtained from this work are presented with any relevant 
TABLE 4-3. ELEMENTAL COMPOSITIONS FOR COMPARISON WITH AVAILABLE CANINE DATA (atoms $/ \mathrm{cm}^{3} \times 10^{.24}$ )

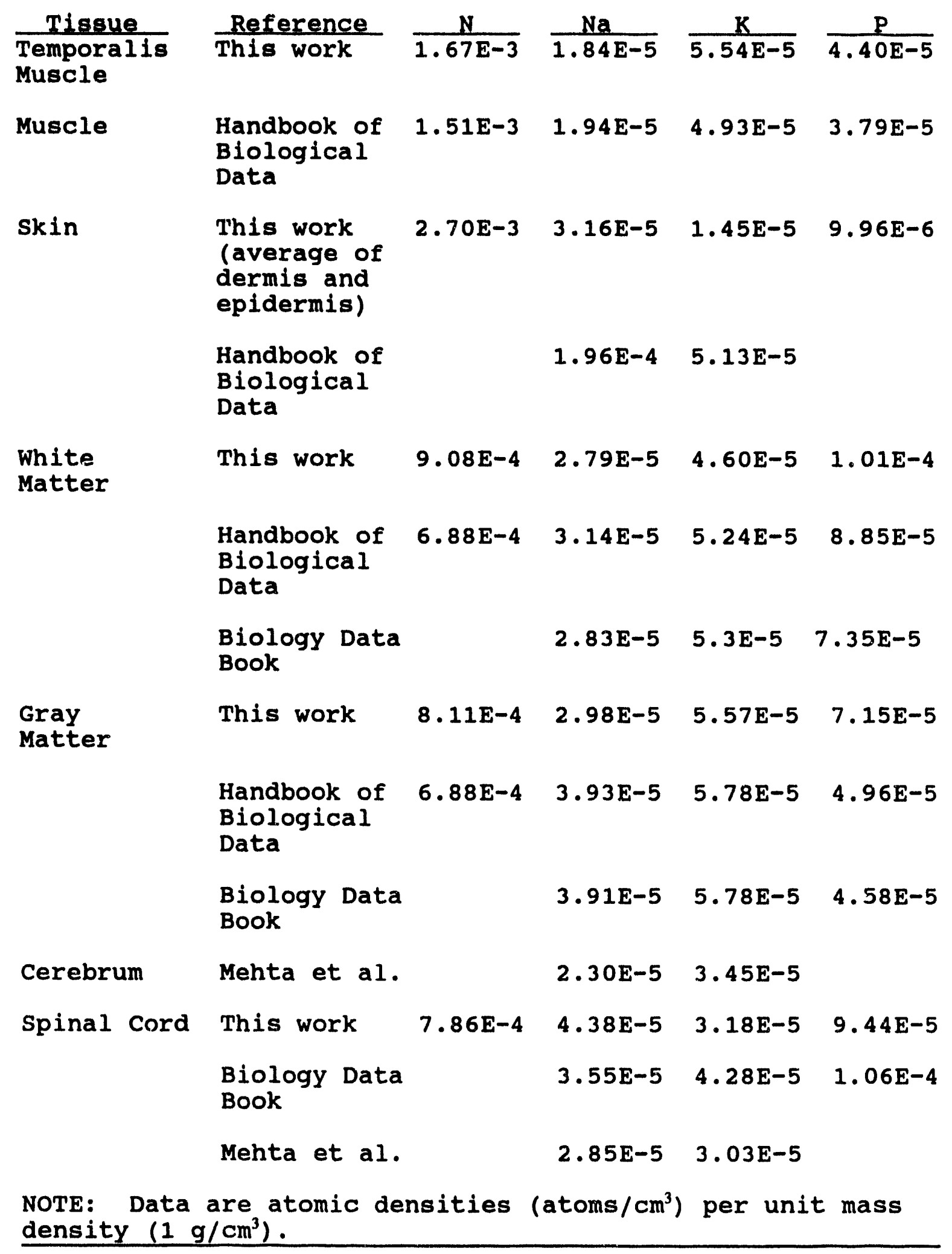


TABLE 4-3. CONTINUED

\begin{tabular}{|c|c|c|c|c|}
\hline Tisgue & Reference & $\mathrm{Mg}$ & $\mathrm{Ca}$ & $\mathrm{Cl}$ \\
\hline $\begin{array}{l}\text { Temporalis } \\
\text { Muscle }\end{array}$ & This work & $7.13 E-6$ & $7.64 \mathrm{E}-6$ & \\
\hline Muscle & $\begin{array}{l}\text { Handbook of } \\
\text { Biological } \\
\text { Data }\end{array}$ & $1.09 E-5$ & $4.96 \mathrm{E}-7$ & $1.06 \mathrm{E}-5$ \\
\hline \multirow[t]{2}{*}{ skin } & $\begin{array}{l}\text { This work } \\
\text { (average of } \\
\text { dermis and } \\
\text { epidermis) }\end{array}$ & $1.64 \mathrm{E}-6$ & $4 \cdot 33 E-6$ & \\
\hline & $\begin{array}{l}\text { Handbook of } \\
\text { Biological } \\
\text { Data }\end{array}$ & $5.58 E-6$ & $6.68 \mathrm{E}-6$ & $1.82 \mathrm{E}-4$ \\
\hline \multirow{3}{*}{$\begin{array}{l}\text { White } \\
\text { Matter }\end{array}$} & This work & $3.76 \mathrm{E}-6$ & $3.74 \mathrm{E}-6$ & \\
\hline & $\begin{array}{l}\text { Handbook of } \\
\text { Biological } \\
\text { Data }\end{array}$ & & & $2.12 \mathrm{E}-5$ \\
\hline & $\begin{array}{l}\text { Biology Data } \\
\text { Book }\end{array}$ & & & 2. $11 E-5$ \\
\hline \multirow{3}{*}{$\begin{array}{l}\text { Gray } \\
\text { Matter }\end{array}$} & This work & $3.84 \mathrm{E}-6$ & & \\
\hline & $\begin{array}{l}\text { Handbook of } \\
\text { Biological } \\
\text { Data }\end{array}$ & & $2.63 E-5$ & \\
\hline & $\begin{array}{l}\text { Biology Data } \\
\text { Book }\end{array}$ & & $2.65 \mathrm{E}-5$ & \\
\hline Cerebrum & Mehta et al. & $7.32 \mathrm{E}-6$ & $3.20 \mathrm{E}-6$ & $1.48 \mathrm{E}-5$ \\
\hline \multirow[t]{3}{*}{ Spinal Cord } & This work & $3.29 E-6$ & $1.29 \mathrm{E}-6$ & \\
\hline & $\begin{array}{l}\text { Biology Data } \\
\text { Book }\end{array}$ & & & $2.17 E-5$ \\
\hline & Mehta et al. & $8.70 E-6$ & $3.05 E-6$ & $1.66 \mathrm{E}-5$ \\
\hline
\end{tabular}


TABLE 4-4. ELEMENTAL COMPOSITIONS FOR COMPARISON WITH SELECTED HUMAN DATA (atoms $/ \mathrm{cm}^{3} \times 10^{-24}$ )

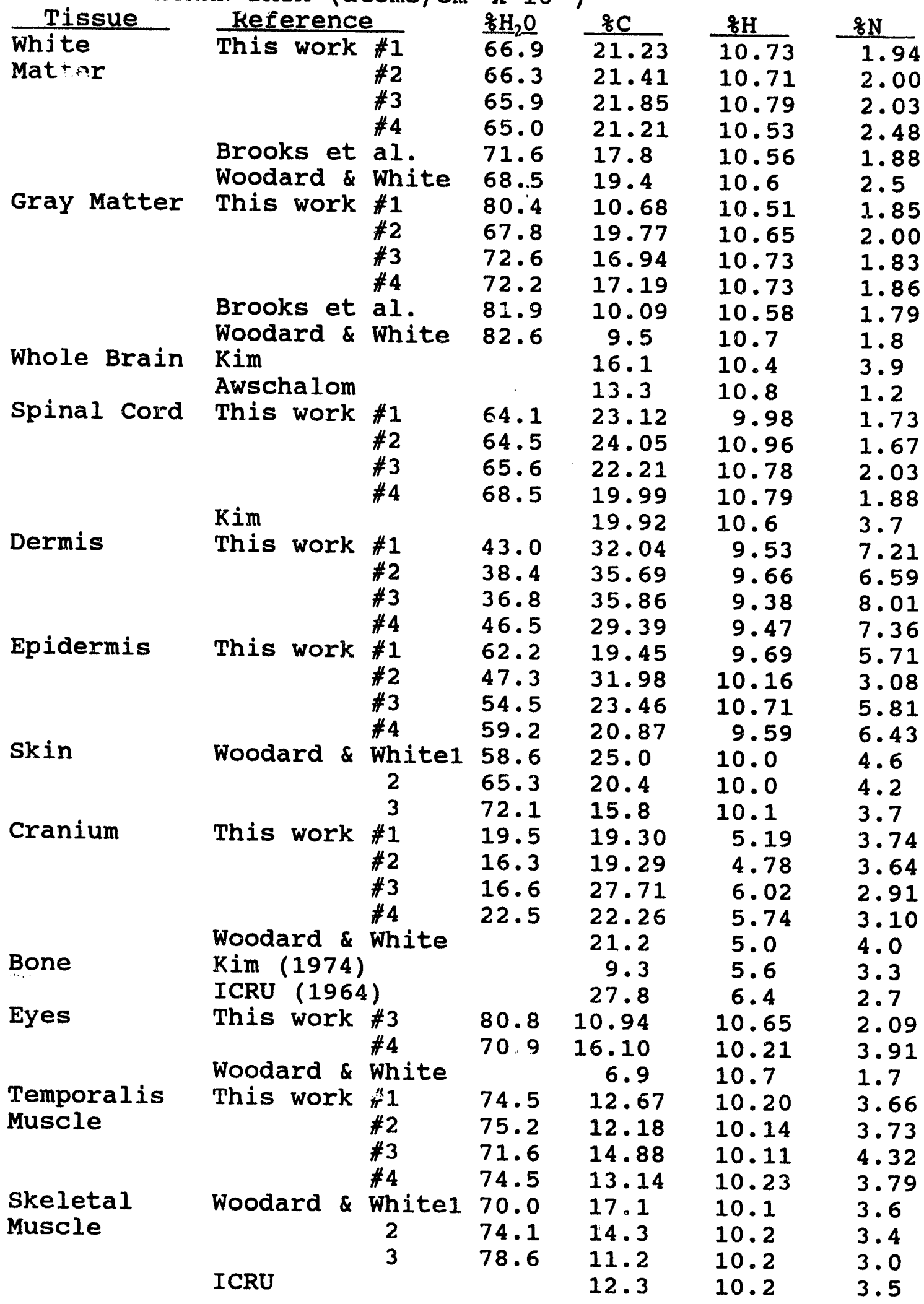


TABLE 4-4. CONTINUED

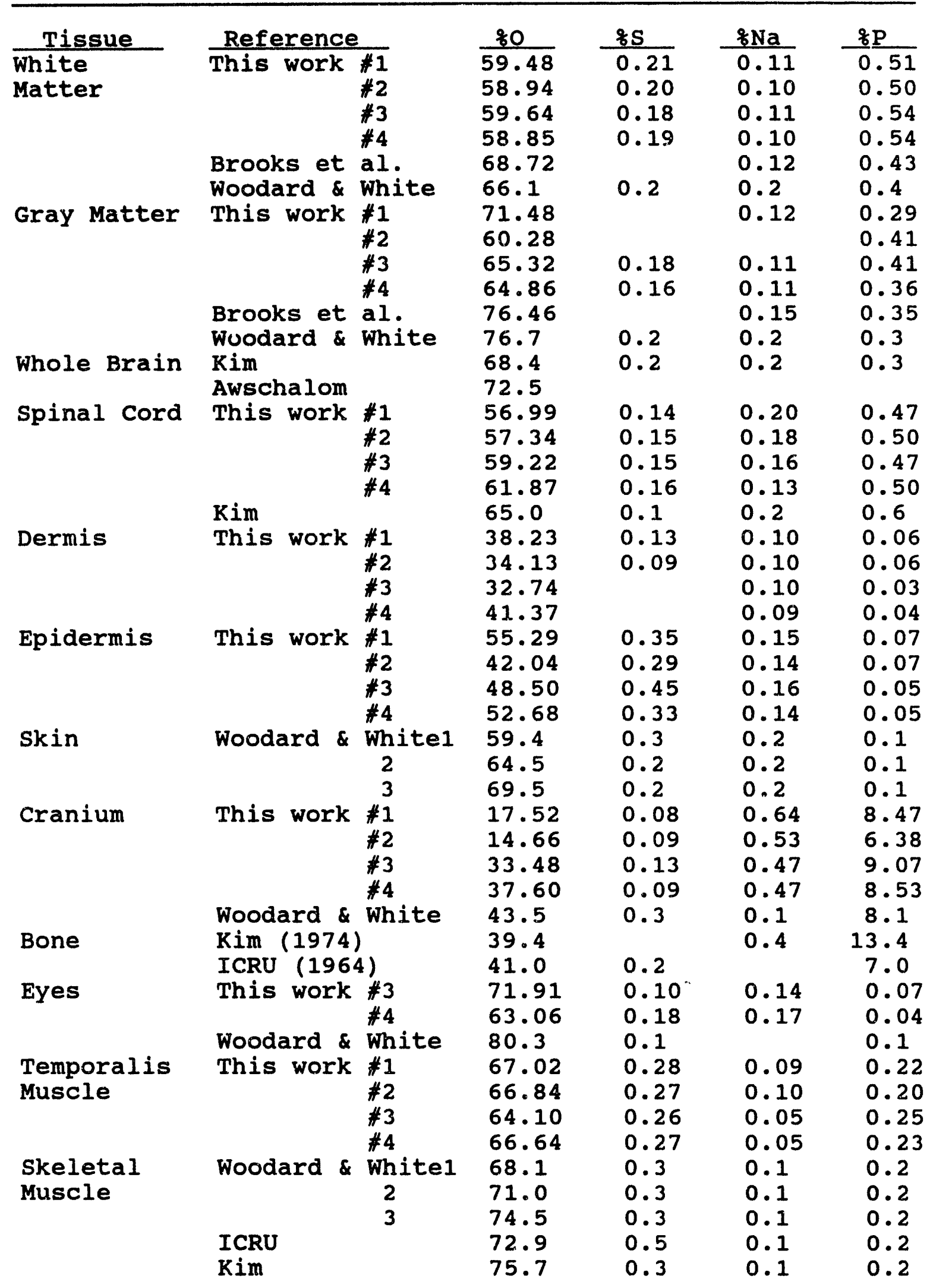


TABLE 4-4. CONTINUED

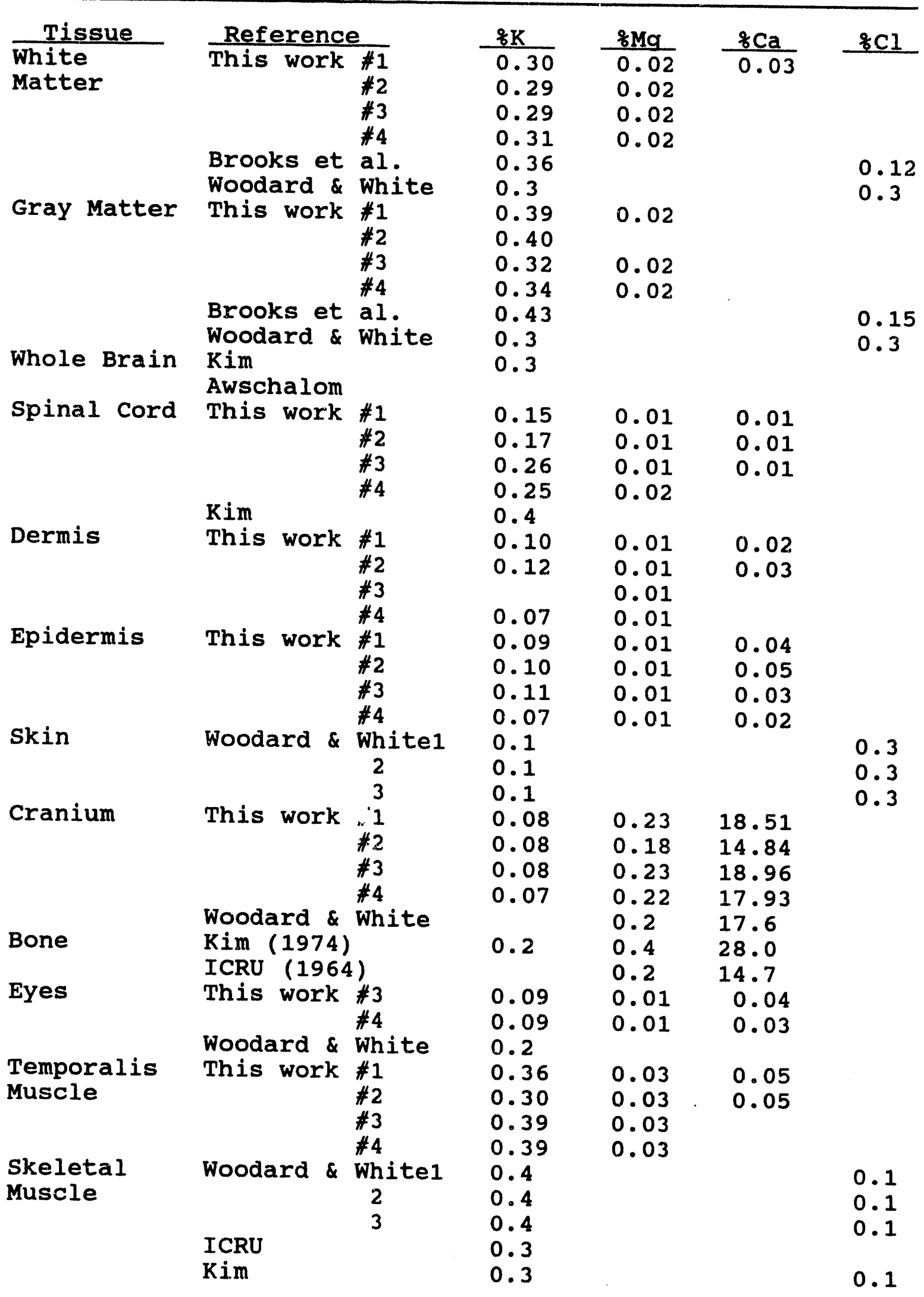


information from other sources below for easy comparison with each tissue type. References 38 and 39 presented data for whole brain rather than white and gray matter. Reference 41 measured the values for the skin as a whole, whereas in this work two distinct layers of skin, epidermis and dermis, were analyzed. The type of bone measured in References 37 and 38 is not specified. It can be seen that the values in Reference 42 for the cranium compare fairly well with the measured results except for the percentage of oxygen. Overall, the values obtained in this work are within $20 \%$ of the corresponding data available in the literature. Thus, the comparison supports the accuracy of the measurements. The results obtained here contribute data to a field deficient of reference values for the elemental compositions of canine tissues.

In dosimetry, reliable composition and density data are required to minimize the uncertainties currently involved in patient treatment planning. This information aids in the formation of truly tissue-equivalent phantoms increasing the importance of measured and calculated doses in treatment. To improve dosimetry, more work is necessary in the field of elemental compositions and tissue densities. 
For a complete heterogeneous model, the chemical composition data, as presented in the previous chapter, are combined with the geometric information available from the sagittal and axial photographs. This combined information serves as input to the GIP and TORT codes. A homogeneous model consistent with the elemental composition data is also needed to assess the differences in flux distribution caused by heterogeneities. Spatial flux distributions from the heterogeneous and volume-weighted homogeneous models in the axial, sagittal, and dorsal planes have been examined for both a $10 \mathrm{~cm} \times 10 \mathrm{~cm}$ and a $5 \mathrm{~cm} \times 10 \mathrm{~cm}$ beam delimiter in this section at the Brookhaven Medical Research Reactor (BMRR), and results are presented here.

\subsection{Geometric Modeling}

The geometric data were largely compiled from the sagittal photographs. The axial photographs were used to refine and verify the sagittal section model. The rectangular mesh structure employed 46,34 , and 20 intervals in the $x, y$, and $z$ directions, respectively. In the $x$ direction, the mesh was in $10 \mathrm{~mm}$ intervals from $0 \mathrm{~cm}$ to 5 $\mathrm{cm}$. The mesh was then decreased to $5 \mathrm{~mm}$ intervals. This was done to better calculate the fluxes beneath the beam port. 
The $y$-mesh was in $10 \mathrm{~mm}$ intervals correlating directly to the sagittal slices except for the center slice. After examination of the axial photographs, it was determined that the occipital crest would be incorrectly represented as two centimeters wide with such a mesh structure. Intervals were added at $2.5 \mathrm{~mm}$ and at $5.0 \mathrm{~mm}$ to more accurately represent the crest at the midplane as seen in Figure 5-1. The mesh in the $z$ direction was in intervals of $5.0 \mathrm{~mm}$. A complete set of sagittal and axial photographs are in Appendices $B$ and $D$. The figures containing the detailed mesh, corresponding to the sagittal photographs, are in Appendix c.

Figures 5-2 and 5-3 show the axial mesh at $x=12.25 \mathrm{~cm}$ and $x=17.25 \mathrm{~cm}$ respectively. These figures correspond to photographs - and - in Appendix D. In order to better visualize the three-dimensional model, the dorsal mesh at $z$ $=11.5 \mathrm{~cm}$ is shown in Figure 5-4.

The regions modeled were air (void) outside the dog head, muscle, skin, bone, brain, spinal cord, internal voids (such as regions of the ear and the nasal cavity), teeth, eye, tongue, and nasal cavity. Soft tissue that was not one of these tissues was modeled as muscle. This was considered a good approximation since muscle was judged to be the most prevalent type of tissue. The skin was represented by a 


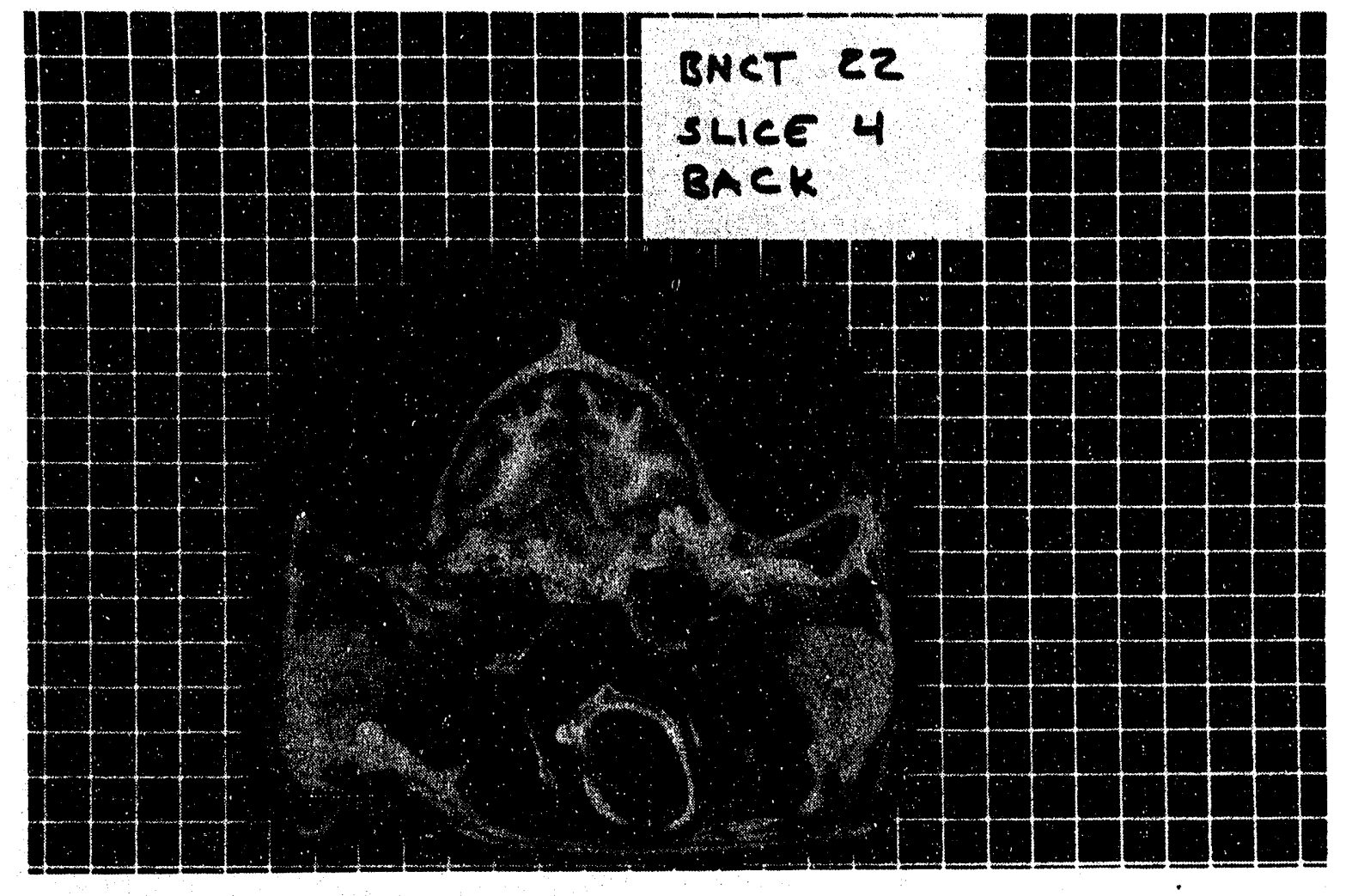

Figure 5-1. Axial Labrador Retriever head section through a plane just behind the axial midplane of the brain at approximately $x=10 \mathrm{~cm}$. The grid marks are spaced $10 \mathrm{~mm}$ center to center. 


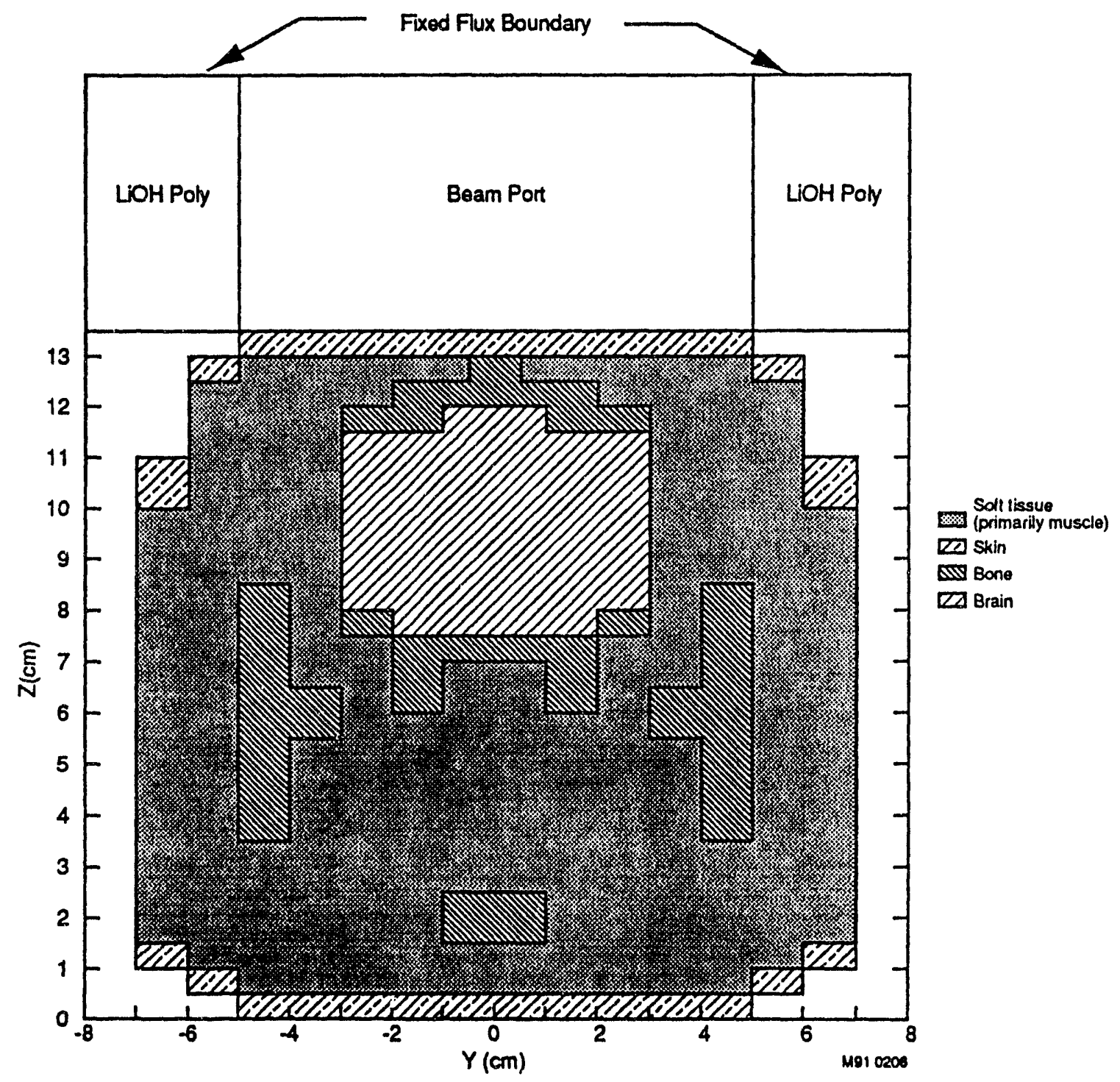

Figure 5-2, Detailed axial mesh through the brain corresponding to the $x=12.25 \mathrm{~cm}$ plane. 


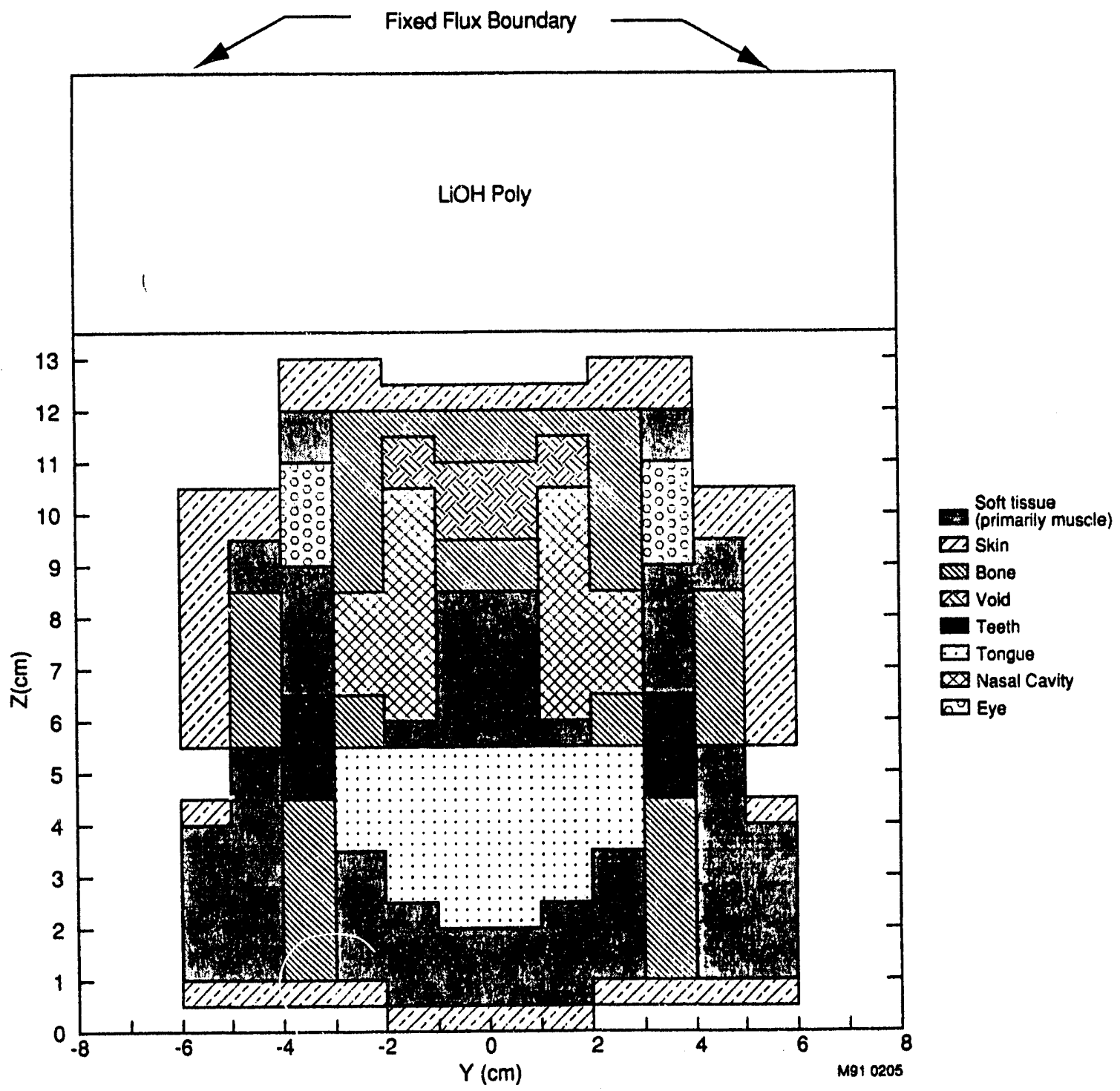

Figure 5-3. Detailed axial mesh corresponding to the $x=17.25 \mathrm{~cm}$ plane. This plane includes the eyes and the nasal cavities. 


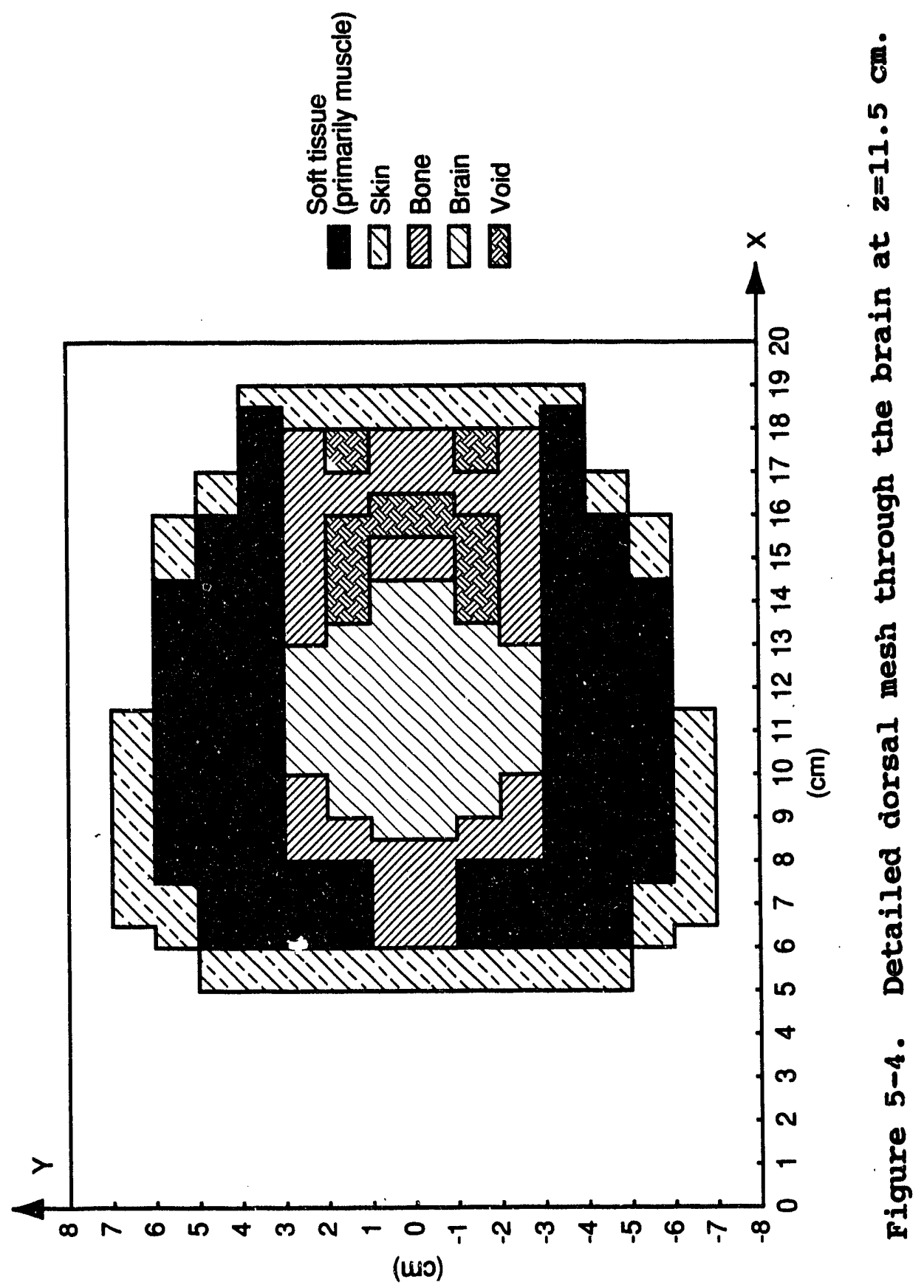


homogeneous mixture of dermis and epidermis. A homogeneous mixture of gray and white matter, in equal parts, was used to represent brain in the model. The data for concha was used for the nasal cavity. The compositions for the skull were also used for the jawbone.

It should be noted that the region above the tongue is part of the nasal cavity. In Figure 5-5, one can see that it was modeled as muscle. For the midplane section, the area was judged to have a density closer to that of muscle than that of the nasal cavity. However, in the next sagittal slice the nasal cavity is clearly seen to be less dense and is modeled as such.

The epithermal neutron filter available at the BMRR facility, shown in Figure 5-6, was designed and constructed in a collaborative INEL-Brookhaven effort ${ }^{43}$. The BMRR epithermal beam has been used extensively in connection with the INEL canine irradiation program. The regions for the BMRR delimiter were LiOH-Poly, bismuth, masonite, Rx-215, and the beam port. The material compositions for these regions are seen in Table 5-1. The information is presented in terms of density (atoms $/ \mathrm{cm}^{3} \times 10^{-24}$ ). Figure 5-5 also depicts the regions of the beam delimiter and their boundaries as included in the computational model. 
㐘

总窟

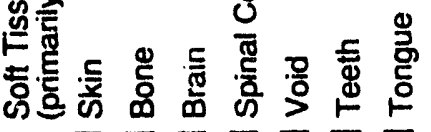

岱

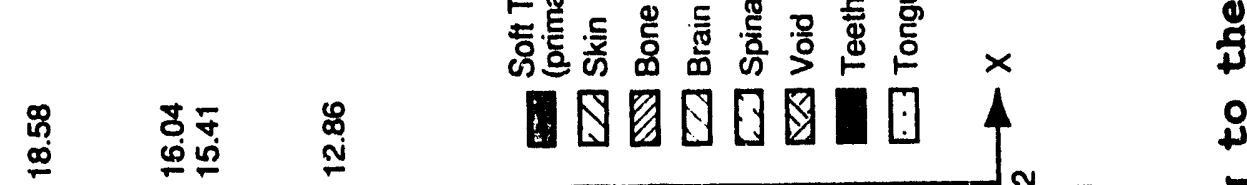
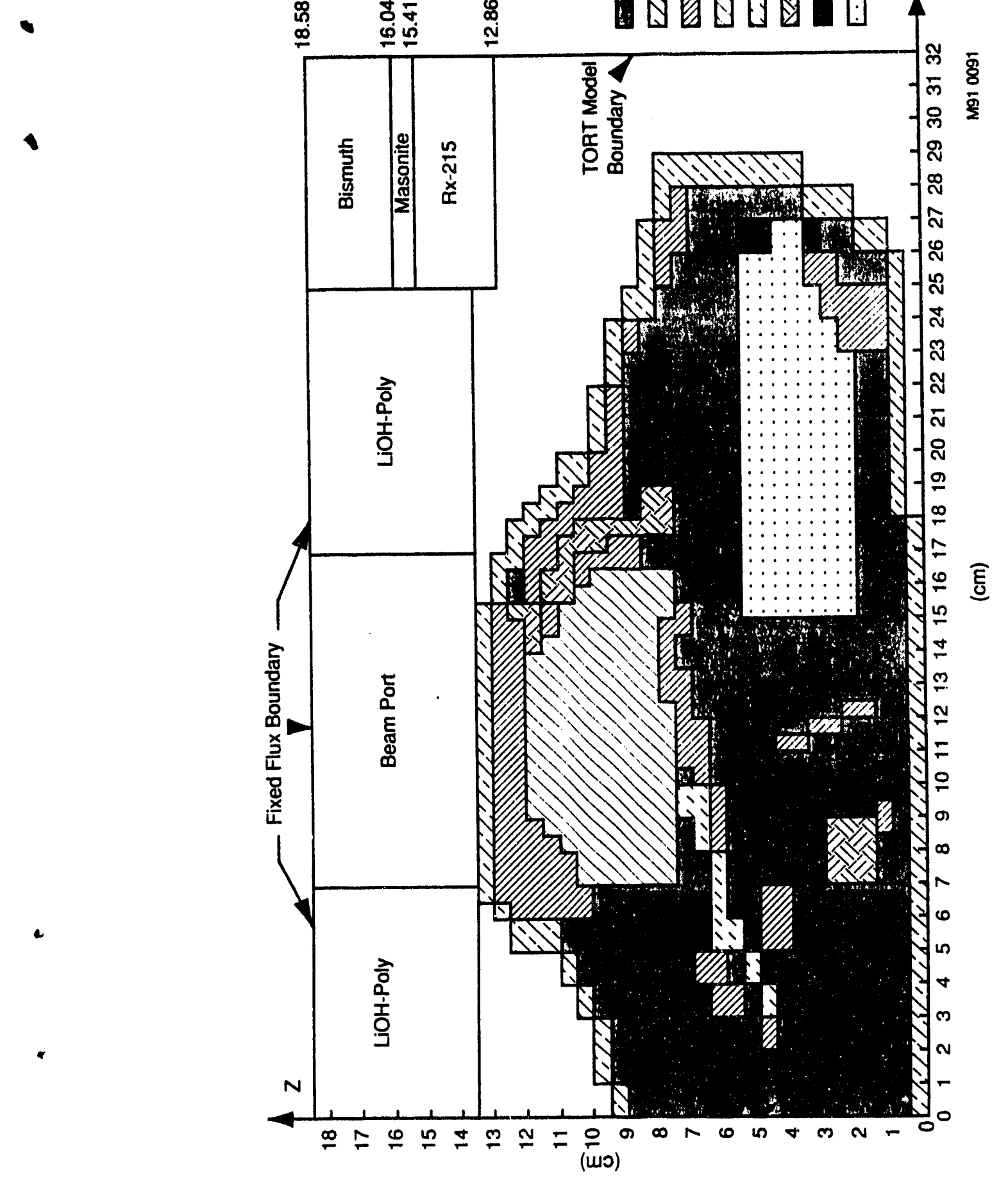

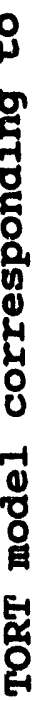

岂

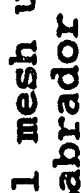

\%.

$+$

ơ

ơ 4

g)

-

.

路

in 0

!n

(1)

()

믐

舟年 


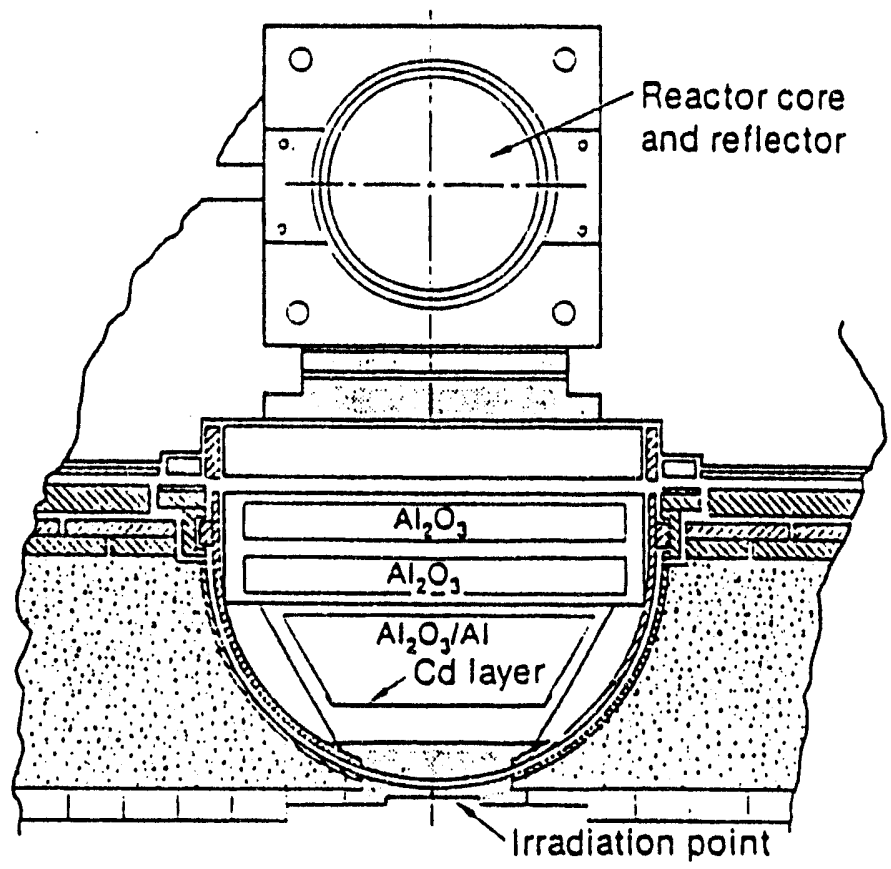

Figure 5-6. As-built aluminum oxide epithermal neutron filter installed in the Brookhaven Medical Research Reactor. 
TABLE 5-1. MATERIAL COMPOSITIONS FOR BMRR DELIMITER COMPONENTS

Material

Lithiated-polyethylene

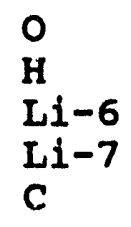

Bismuth

Masonite

Rx-215 Shielding

$\mathrm{Bi}$

0

H
Isotope Density (atoms $/ \mathrm{cm}^{3} \times 10^{-24}$ )

$$
\begin{aligned}
& 0.01183 \\
& 0.07065 \\
& 0.006432 \\
& 0.0003385 \\
& 0.02656
\end{aligned}
$$

0.02824

0.01189

0.02378

0.01426

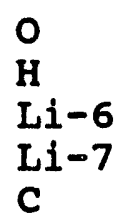

0.01043

0.05445

0.0005025

0.006198

0.03073 
Hemispherical symmetry was assumed, and the seven sagittal slices were measured and reconstructed on the other side of the head. It was also determined that in therapy the dog is located further forward (in the $x$ direction) under the beam. Thus, the model was shifted forward one $\mathrm{cm}$ in the $x$ direction. The addition consisted mainly of muscle and skin. For example, in Figure 5-5 skin was added from $0.0 \mathrm{~cm}$ to $0.5 \mathrm{~cm}$ in $z$, and muscle was added from $0.5 \mathrm{~cm}$ to 9 $\mathrm{cm}$. With this addition to each slice, the model would more accurately simulate an actual treatment.

To examine Type 1 heterogeneity effects, a homogeneous model needed to be constructed which would preserve the total elemental compositions throughout the irradiation volume. For this model, the macroscopic cross section data used in the heterogeneous model for each discrete region was weighted by the calculated volume fraction of that region and summed over all regions (including voids). This pseudomaterial was assigned to all anatomical regions within the head. The exterior boundary of the head remained exactly the same as in the heterogeneous model. Through this process, the total quantity of all elements was preserved while at the same time each element was distributed uniformly throughout the interior of the head geometry. The beam delimiter regions and angular flux boundary condition that simulates the incident beam remained the same in this 
model. As a result, any observed differences in the calculated neutron and gamma fluxes obtained with the heterogeneous model as opposed to the homogeneous model may be attributed specifically to Type 1 heterogeneity effects.

\subsection{GIP and TORT}

The radiation transport calculations presented here were performed using an INEL adaptation of the TORT threedimensional discrete ordinates transport code, originally developed at Oak Ridge National Laboratory. ${ }^{10}$ The code calculates the flux of neutral particles incident upon a two- or three-dimensional geometric system. TORT numerically solves the Boltzmann transport equation for neutral-particles by the discrete ordinates method. This is accomplished by partitioning the spatial geometry into a three-dimensional finite difference mesh and approximating all scattering integrals over direction space as weighted sums over a set of prespecified discrete angular directions. A numerical solution for the direction-, space-, and energydependent particle intensity is iteratively calculated and integrated over direction space to provide the scalar flux.10

All material cross sections were represented with data from the BUGLE-80 47-neutron-20-gamma-group coupled library ${ }^{44}$ 
to obtain discrete ordinates solutions for the flux of neutrons and secondary gammas. Scattering cross sections are approximated as functions of the scattexing angle on the BUGLE-80 1ibrary by a P3 Legendre polynomial expansion. This expansion was retained in the TORT calculations. The GIP code was used to process the cross section data from the BUGLE-80 library to provide the macroscopic cross section data required by TORT. The GIP and TORT input are located in Appendix E. The GIP input is the same for both the $5 \mathrm{~cm}$ x $10 \mathrm{~cm}$ and $10 \mathrm{~cm} \times 10 \mathrm{~cm}$ beam port cases and heterogeneous and homogeneous cases. The TORT input is the same with the exception of a few parameters, which are noted in each case in Appendix E. Accurate, numerically well-behaved calculated results for three-dimensional BNCT dosimetryrelated applications have been previously demonstrated for the TORT code, in conjunction with the BUGLE- 80 cross section library. ${ }^{6}$

\subsection{Presentation and Discussion of Results}

The results presented here compare calculated radiation fluxes from the heterogeneous and homogeneous models of a Labrador Retriever head. Information was obtained through the calculations on the spatial distribution of the fluxes in the canine head. Any differences noted between the heterogeneous and homogeneous cases are due to Type 1 
heterogeneity effects, that is, due to the explicit representation of inhomogeneities in the model. The results are for a $10 \mathrm{~cm} \times 10 \mathrm{~cm}$ beam port at the BMRR unless noted otherwise.

The calculated thermal neutron flux contours at $y=-$ $0.125 \mathrm{~cm}$ are depicted in Figures 5-7 and 5-8 for the heterogeneous and homogeneous cases, respectively. The flux contours are overlaid on a sketch of the outline of the brain and outer surface of the head to better visually represent the location of the flux contours with respect to the head geometry. The detailed sagittal mesh corresponding to this data is in Figure 5-5. In each case, the flux data are shown as percentages of the peak values per megawatt of indicated BMRR reactor power. Flux distributions, rather than kerma or absorbed dose distributions, are examined because the scalar particle flux is the fundamental quantity that is perturbed by Type 1 heterogeneity effects. The spatial distribution of the thermal neutron flux, with the exception of the fast neutron (hydrogen recoil) and gamma ray components, is representative of the spatial distribution of essentially all of the absorbed dose in BNCT. For this reason, the thermal neutron, fast neutron, and gamma fluxes are examined in this work for selected sagittal, axial, and dorsal planes. 


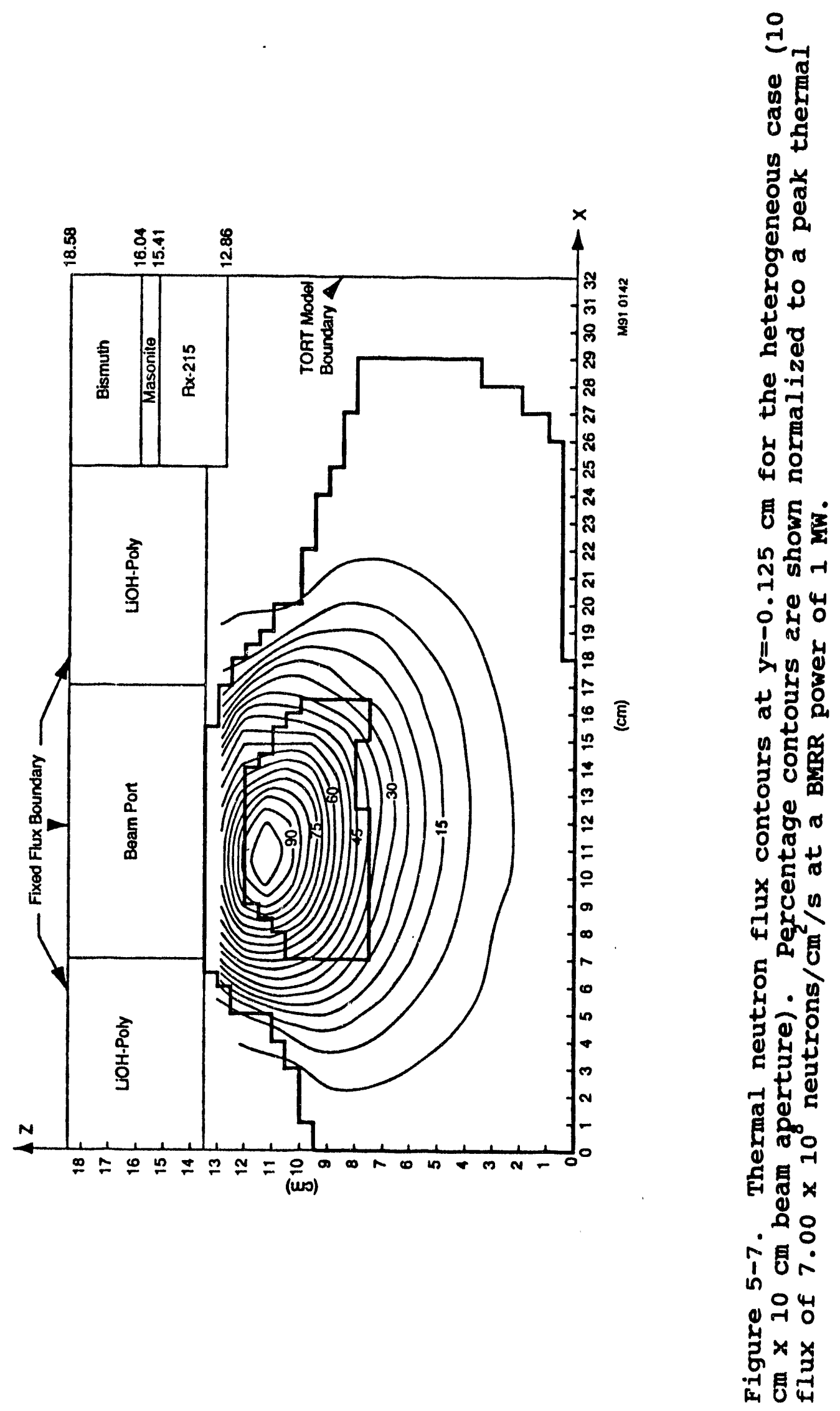




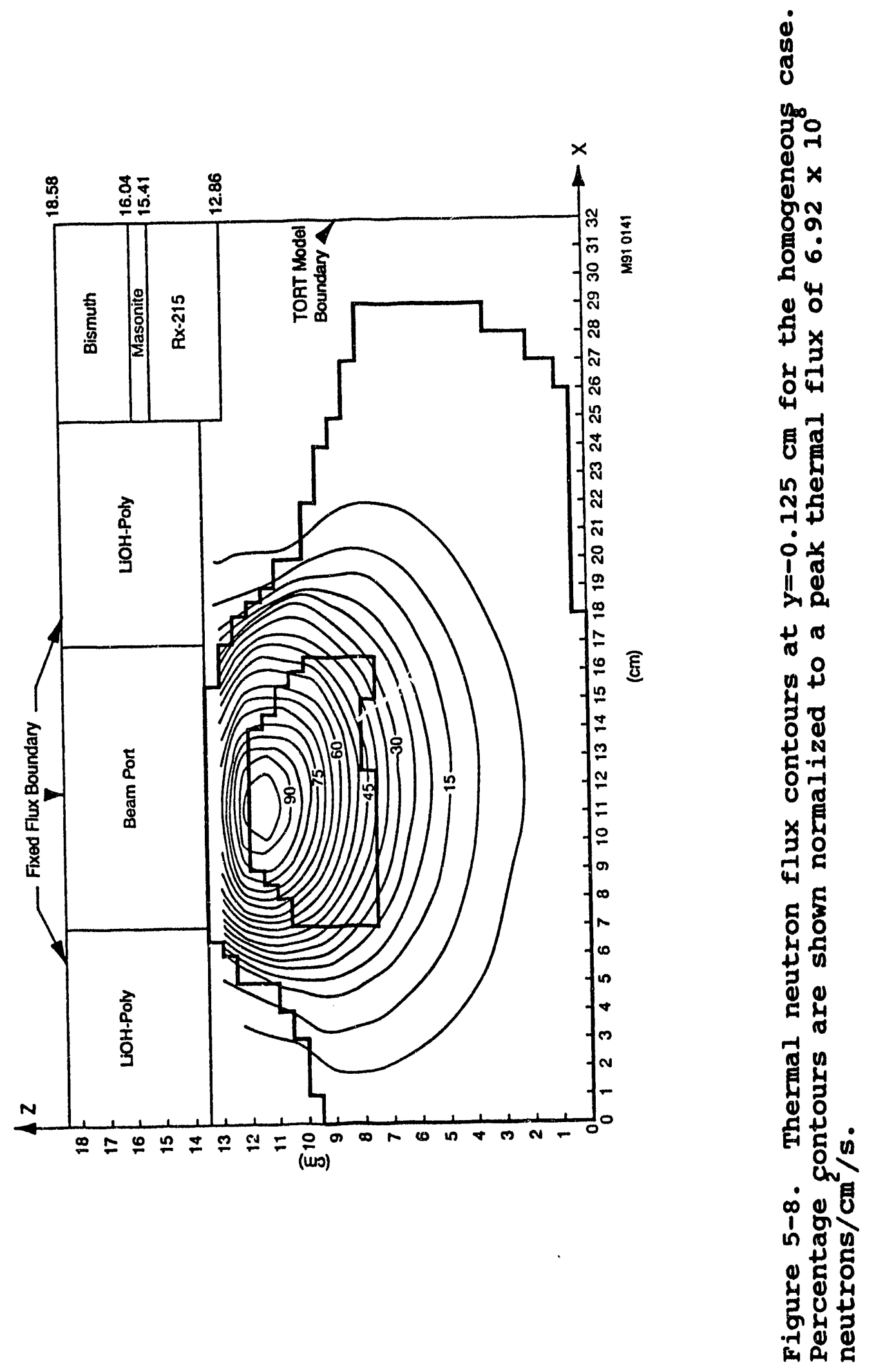


The absolute magnitude of the thermal neutron flux peak differs between the two models by only about $1 \%$. The location of the peak shifts about $10 \mathrm{~mm}$ in the heterogeneous model relative to the corresponding location in the homogeneous model. The shift is in the ventral and caudal directions, as expected. This shift is primarily due to the explicit representation of the sinus voids and other low density regions in the heterogeneous model.

In Figures 5-7 and 5-8, one notes that the spatial distributions between the two cases differ slightly. The thermal flux developed in the homogeneous case (Figure 8) is higher in the rostral direction (towards the nose) in the flux range of 20-95\%. It is in these regions where bone, the nasal cavity, and brain regions are predominating and the fluxes are most pertinent for therapy. At the top of the head, just below the skin, is the occipital crest. The brain is below this region. Forward of the brain, more bone is present above and below the void areas of the nasal cavity present in this section. The densities of these regions are bone, $1.61 \mathrm{~g} / \mathrm{Cc}$; nasal cavity $0.5 \mathrm{~g} / \mathrm{cc}$; and brain, $1.036 \mathrm{~g} / \mathrm{cc}$. Passage of particles through these distinct regions changes the attenuation and absorption of particles which affects the spatial distribution of the thermal flux. This can be seen upon examination of the differing distributions between the homogeneous and 
heterogeneous cases.

Although the magnitude of the peak thermal neutron flux is not significantly changed by the explicit representation of inhomogeneities, the spatial shift of the peak corresponds to a shift of all of the flux contours. Local, potentially significant, variations of the thermal flux are evident. For example, in the regions of the brain near the sinus cavities there is a 10-15\% decrease in thermal flux at some points relative to what is calculated with the homogeneous model. The difference could be therapeutically significant if the BNCT target volume (tumor) was located in this region, since the absorbed dose from boron interactions in the tumor would be affected by essentially the same percentage. Use of a homogeneous tissue model that has not been validated for treatment planning in this particular situation could lead to an overestimate of the boron dose relative to what would actually be delivered.

The behavior of the thermal neutron flux can be further examined along the beam centerline. Figure 5-9 shows detailed linear traverses along the beam centerline through the contours plotted in Figures 5-7 and 5-8. In this figure, one can observe a few minor oscillations in the thermal flux data as a function of depth below the top surface of the head for the heterogeneous case relative to 


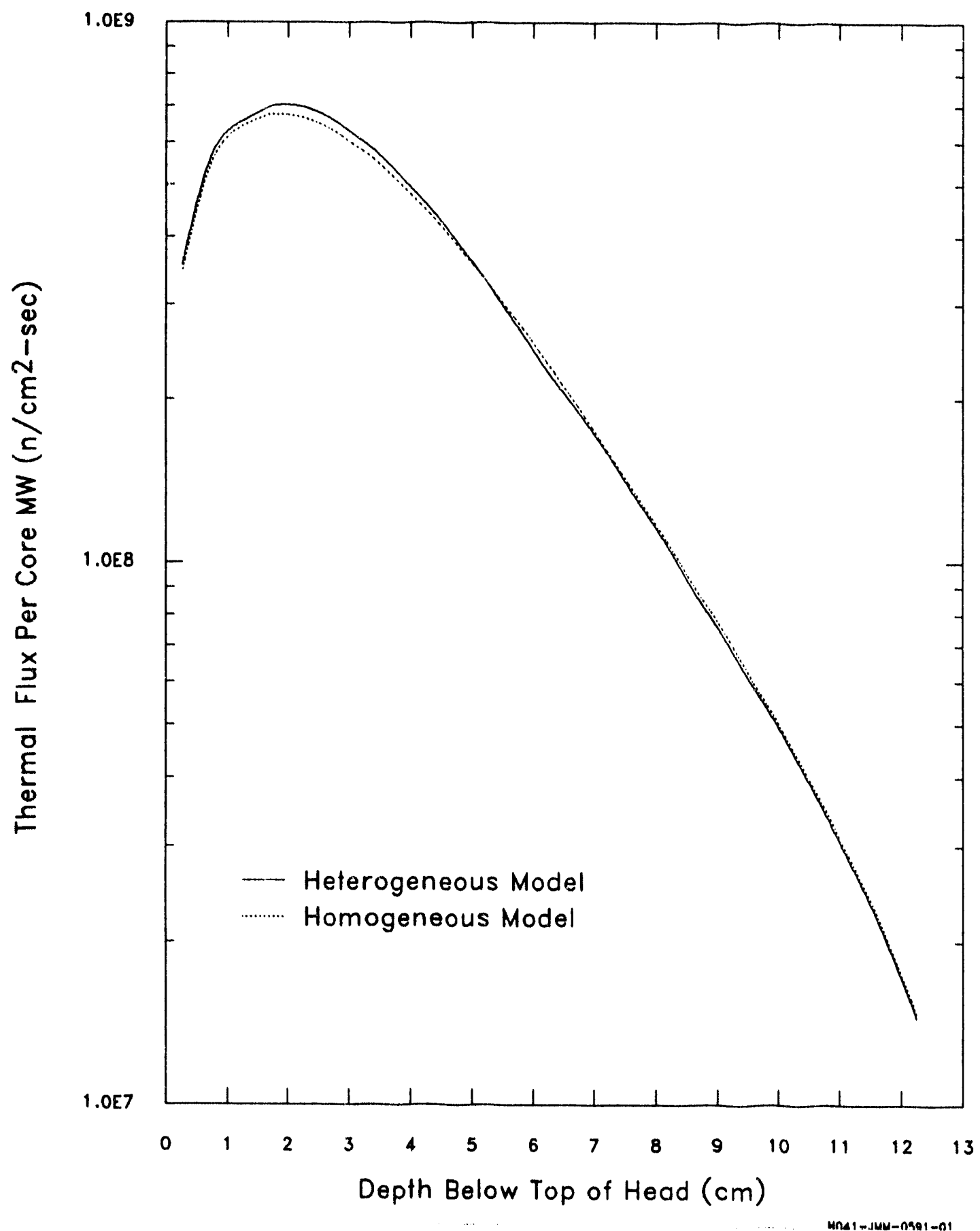

Figure 5-9. Calculated thermal neutron flux along the BMRR $10 \mathrm{~cm} \times 10 \mathrm{~cm}$ beam centerline for the Labrador Retriever head models. 
the smooth behavior of the calculated results from the homogeneous model. These oscillations are small and tend to occur in regions containing bone, where the neutron transport properties are somewhat different than in soft tissue. It must be emphasized that the information in Figure 5-9 is for a traverse along the beam centerline. In regions away from the beam centerline the effect of tissue heterogeneities may be larger. The analyst must carefully examine the calculated results at all locations within the irradiation volume.

Figures 5-10 and 5-11 show the calculated fast flux contours at $y=-0.125 \mathrm{~cm}$ for the two models. As mentioned earlier, fast neutrons are an undesirable component of therapy, but are unavoidably present to some extent in BNCT. The differences between the calculated results from the two models are small, as expected, although there is some streaming of fast neutrons in the regions behind the eyes in the heterogeneous calculations. This Type 1 heterogeneity effect is due to the sinus voids. In an actual canine irradiation, there could be a slight effect on the fast neutron dose to the retina of the eye, and perhaps other nearby regions because of the streaming, which would not be predicted with a homogeneous model.

Figure 5-12 depicts the calculated fast neutron fluxes 


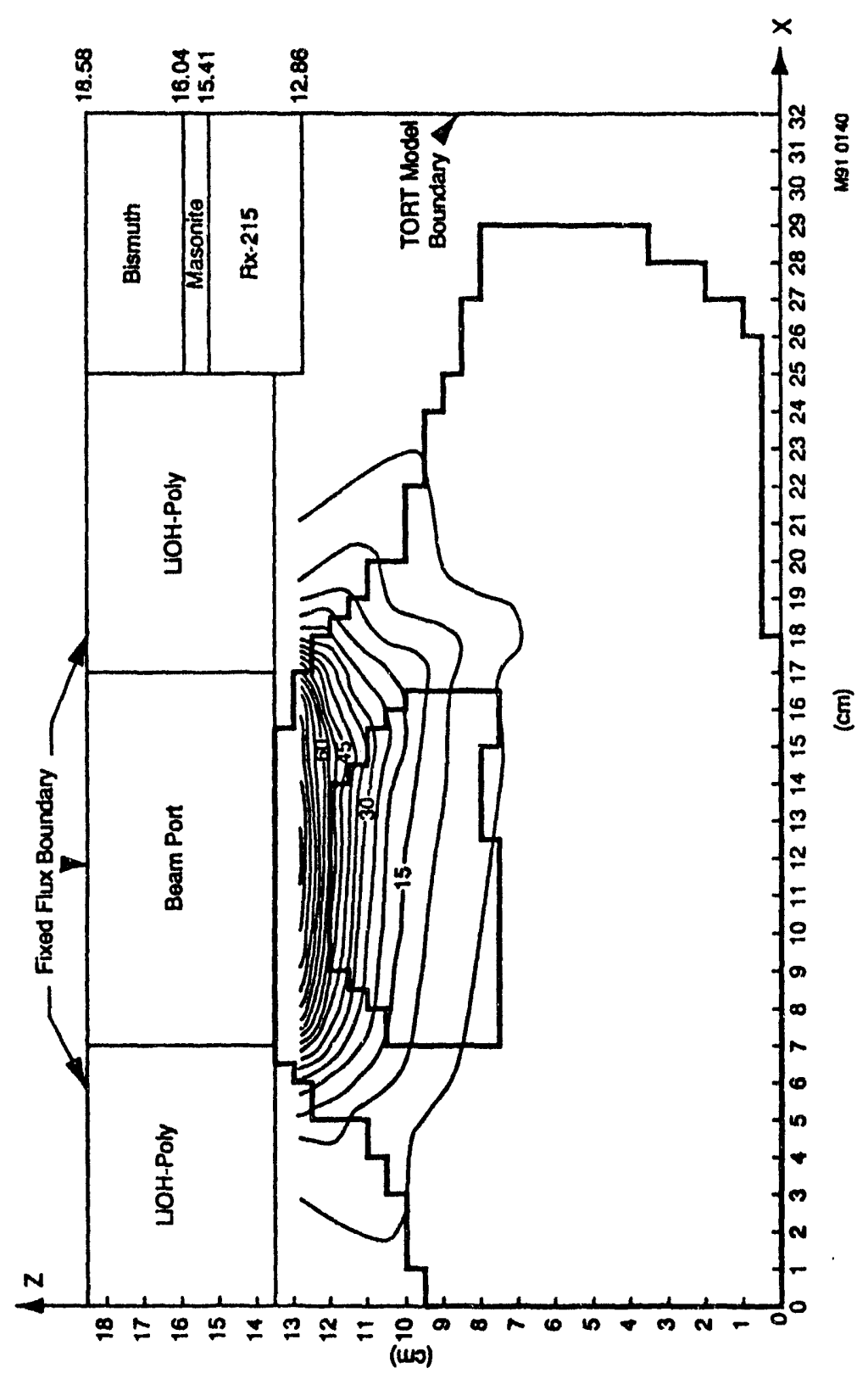

造 

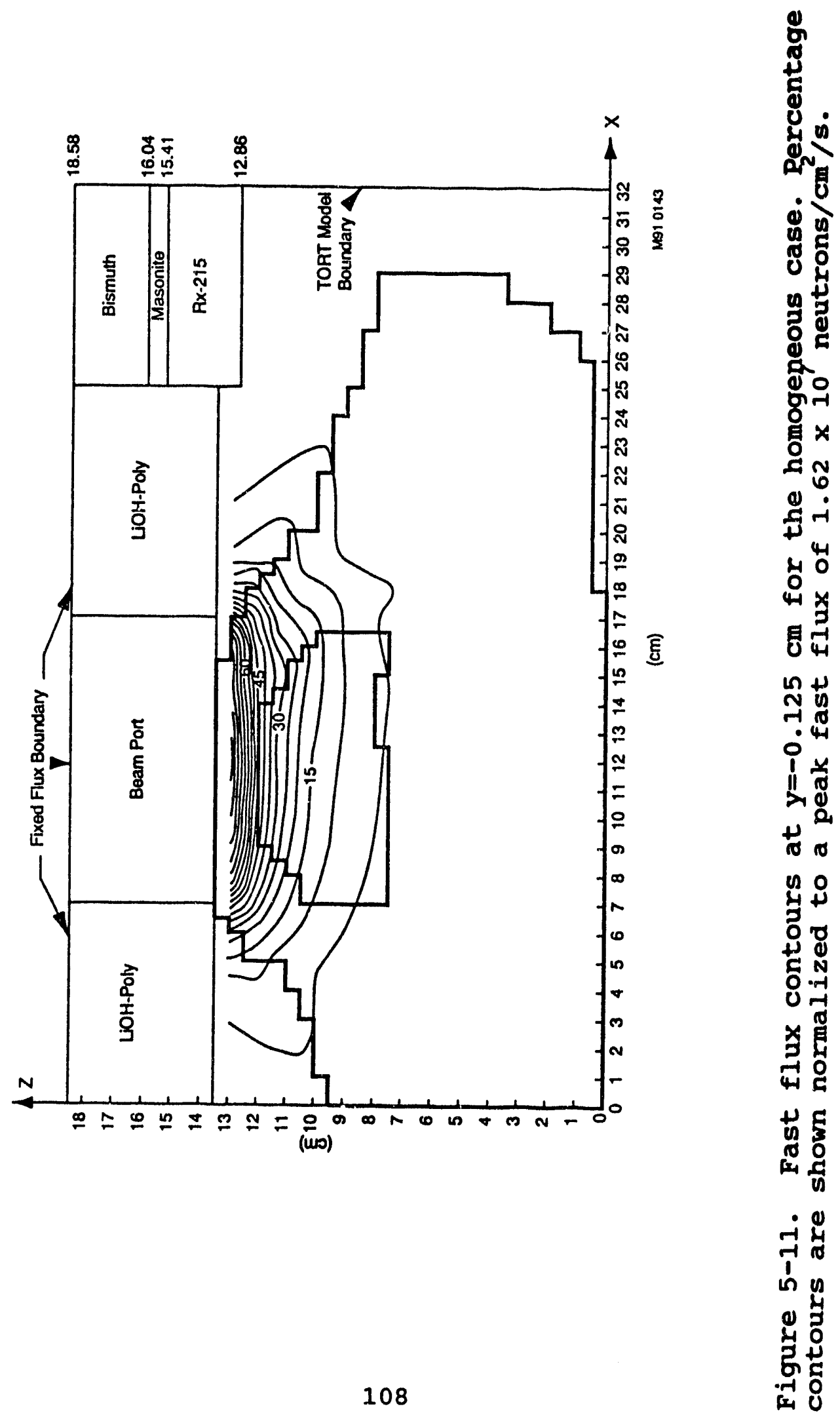
along the beam centerline for both cases. The effects of differing hydrogen content in the various heterogeneous regions can be seen. For the heterogeneous case, the fast neutrons develop slightly farther near the surface but are attenuated slightly more rapidly in the brain relative to the homogeneous results. It should be noted again that this behavior of fast neutron flux along the centerline is not representative of the flux in the entire irradiation volume.

The gamma flux is generally perturbed by Type 1 heterogeneity effects similar to those affecting the thermal neutron flux. This result is expected since capture gammas in tissue are produced primarily by thermal neutron interactions with the tissue constituents. There is also a second-order effect because the capture gamma source per unit thermal neutron flux at each point changes when the tissue at that point is represented by the actual composition rather than by the volume-weighted homogeneous average tissue composition for the irradiation volume.

The calculated gamma fluxes for the heterogeneous and homogeneous cases at $y=-0.125 \mathrm{~cm}$ are shown in Figures $5-13$ and 5-14, respectively. As noted for the thermal neutron flux, tine peak flux shifts in the caudal and ventral directions for the gamma flux. As mentioned previously, the photon-induced absorbed dose distribution can also be 


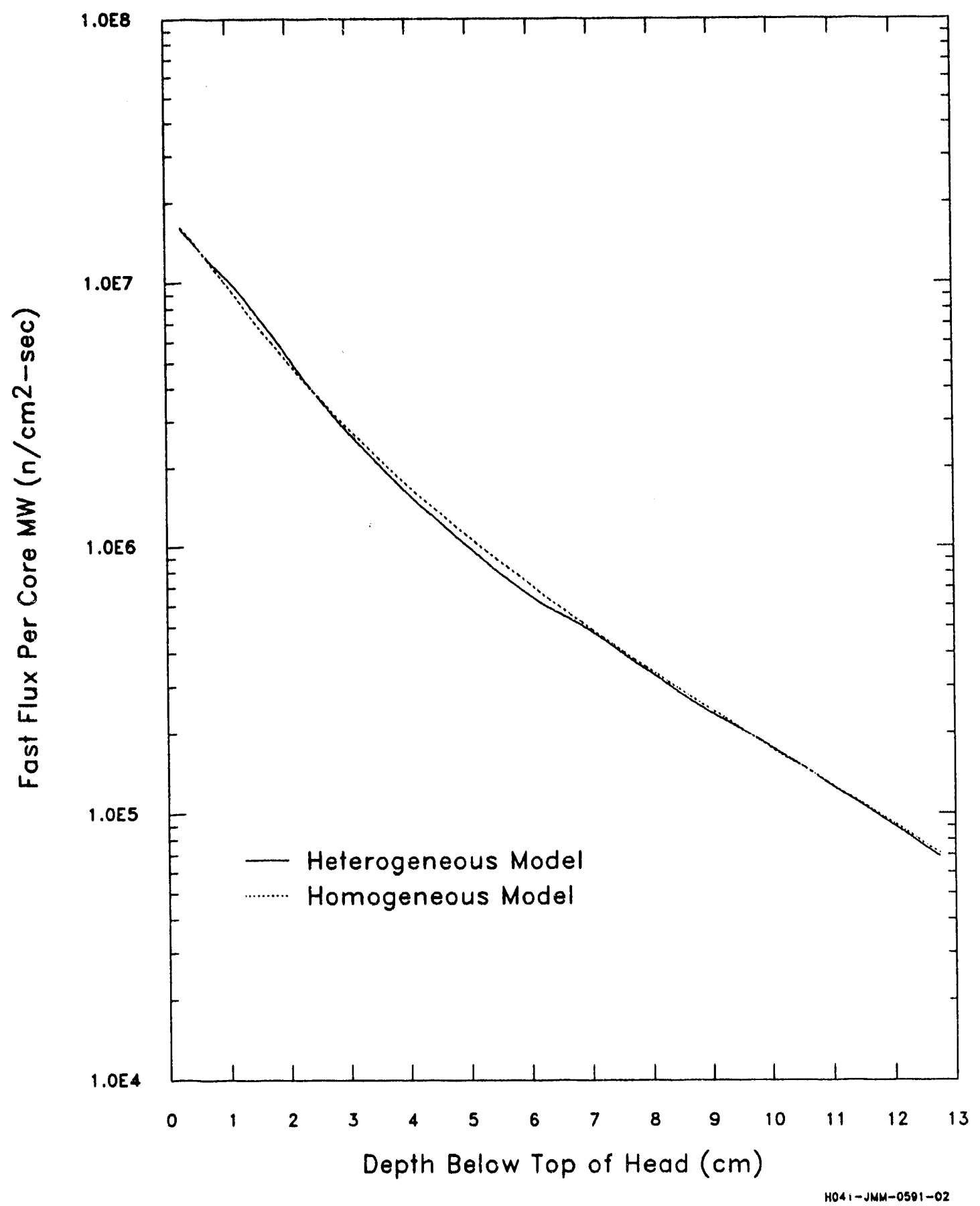

Figure 5-12. Calculated fast neutron flux along the BMRR 10 $\mathrm{cm} \times 10 \mathrm{~cm}$ beam centerline for the Labrador Retriever head models. 


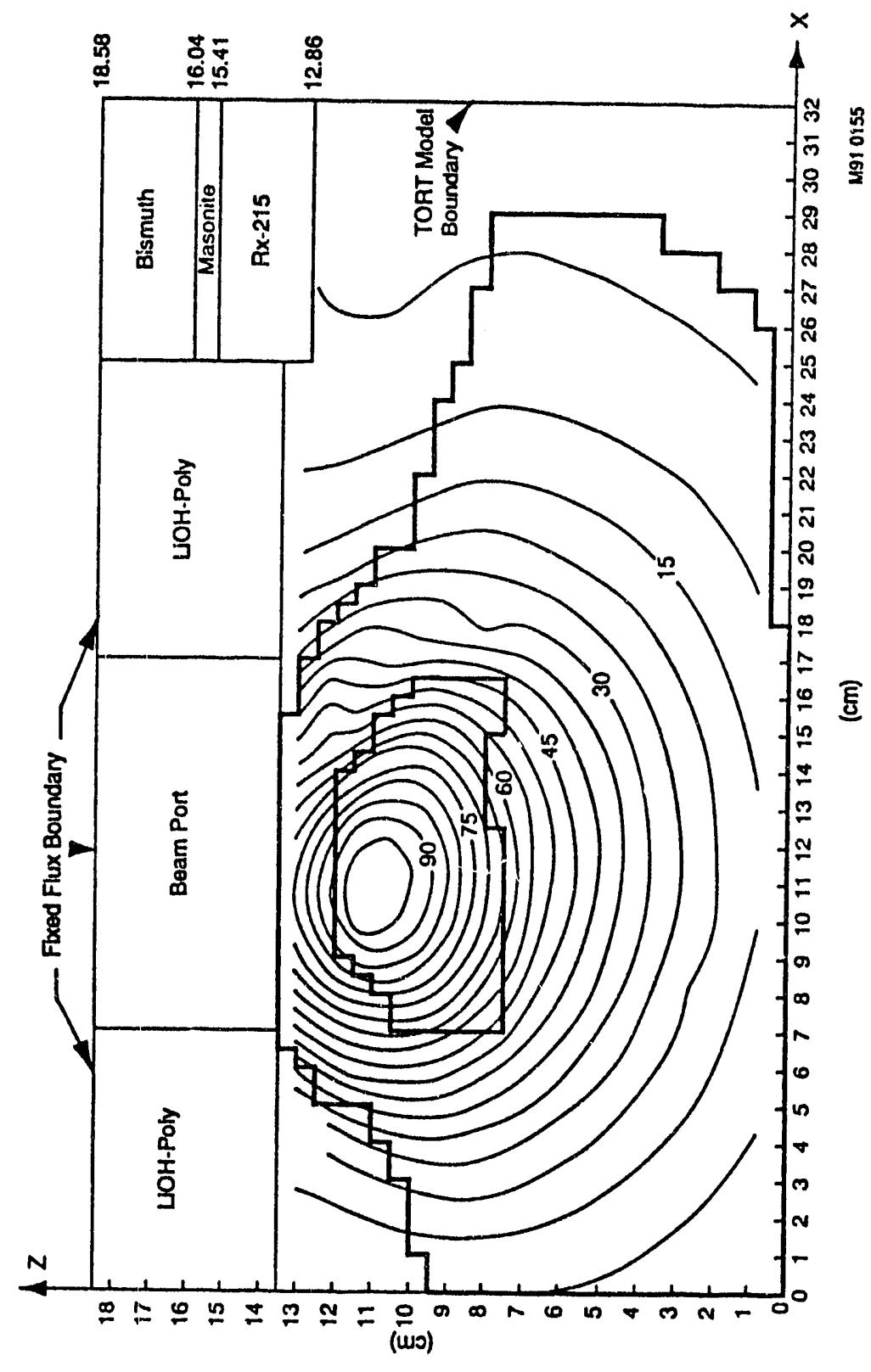

111

号

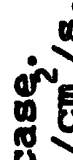

8

号慁

ô.

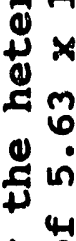

留

กี

年目

의

iI

28
+8

$+10$

앨

$+8$

농

둥

फ

睍要

孚点 

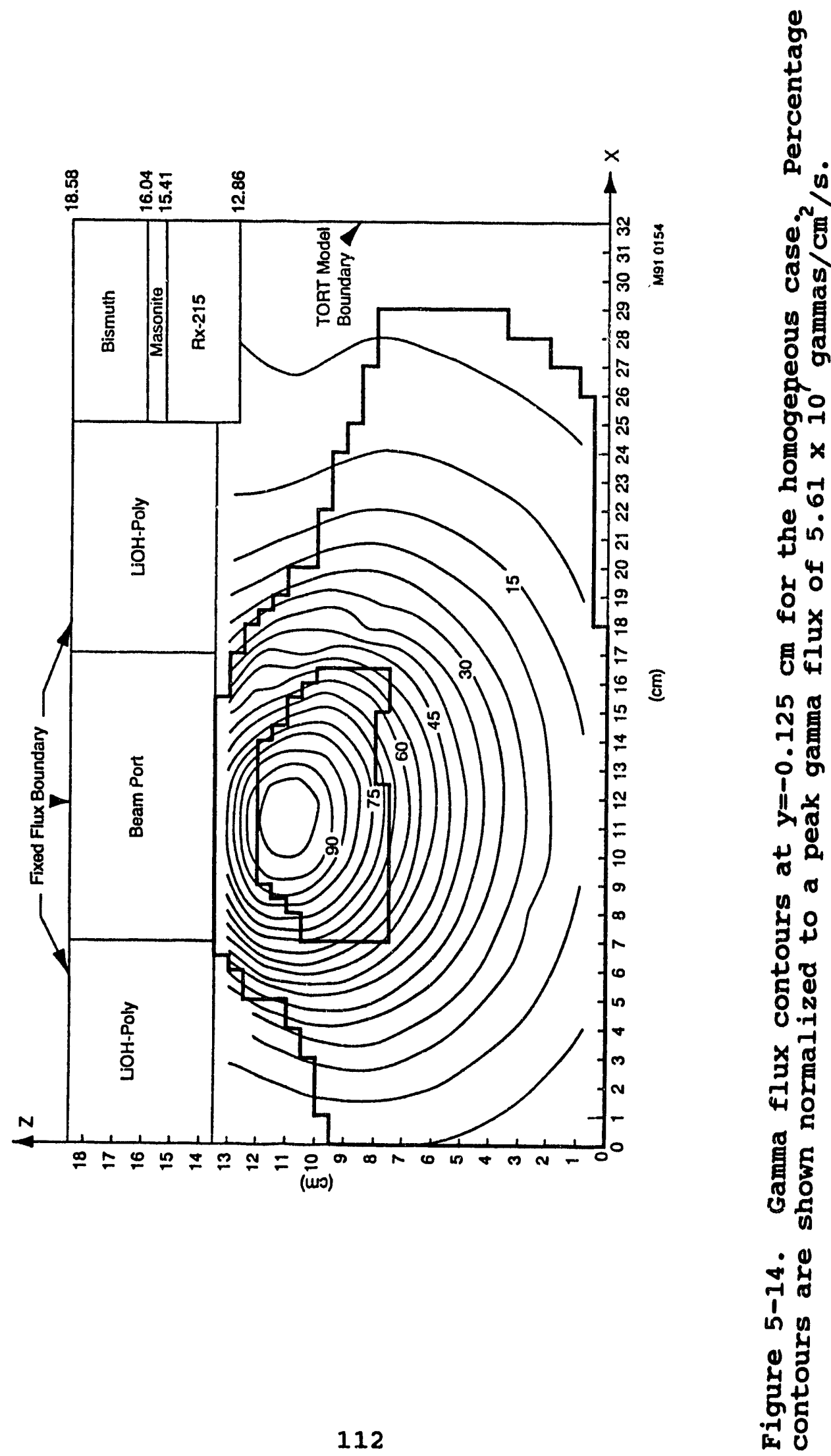
perturbed significantly by Type 3 (electron non-equilibrium) heterogeneity effects especially at surfaces or at the other side of a void.

To demonstrate the importance of considering heterogeneities when using homogeneous approximations, the contours at the $y=-0.375 \mathrm{~cm}$ plane were examined for thermal neutron, fast neutron, and gamma fluxes. The occipital crest spans from $y=-1.0 \mathrm{~cm}$ to $y=1.0 \mathrm{~cm}$. From the $y=-0.125 \mathrm{~cm}$ plane, studied above, to $y=-0.375 \mathrm{~cm}$, there is a decrease in the amount of bone on the top of the head. There is a noticeable effect between the heterogeneous cases due to this decrease in bone, as large as $10 \%$ for the fast neutron flux in some areas. This effect may be substantial in therapy.

Figures 5-15 and 5-16 depict the thermal neutron flux at $y=-0.375 \mathrm{~cm}$ for the heterogeneous and homogeneous cases. The spatial distributions are similar to each other and to the homogeneous thermal flux at $y=-0.125 \mathrm{~cm}$ (Figure 5-8). A slight decrease in flux is expected and shown in the comparison of the homogeneous contours, but this decrease does not amount to more than 1\%. Figure 5-15 is closer in spatial distribution to the homogeneous case than Figure 5-7 due to the decrease of bone (heterogeneity) upon the entrance of the beam into the dog head. The peak 


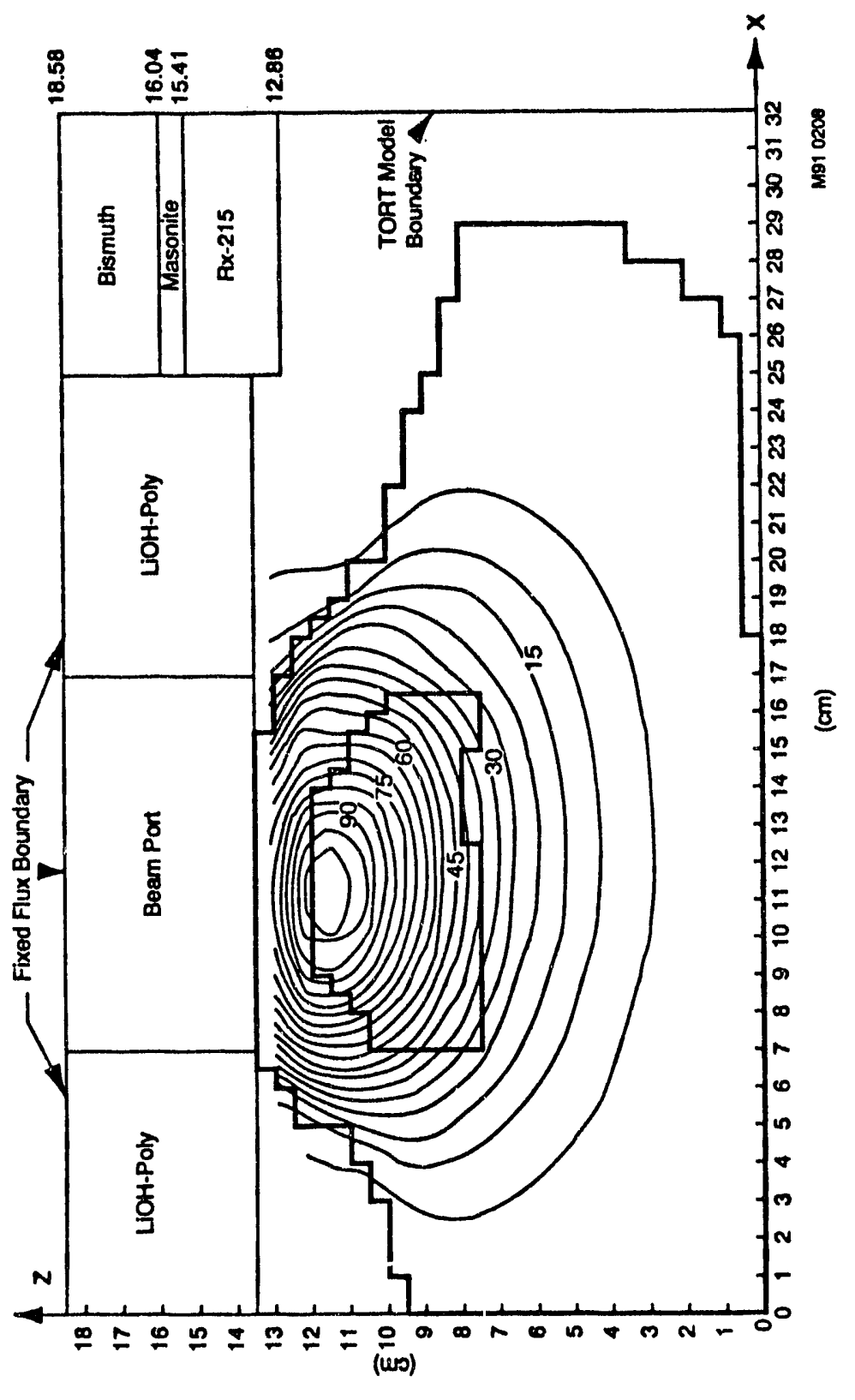

哭

马

당요

$8 x$

40

\$ٔ

an

년

幽点

당

$\leadsto$

in

m

1.

ᄀ.

to

هי

范

8 .

荥造

ᄃ

영

乌

c

兽䏛

ने

in 8

o)

मु 


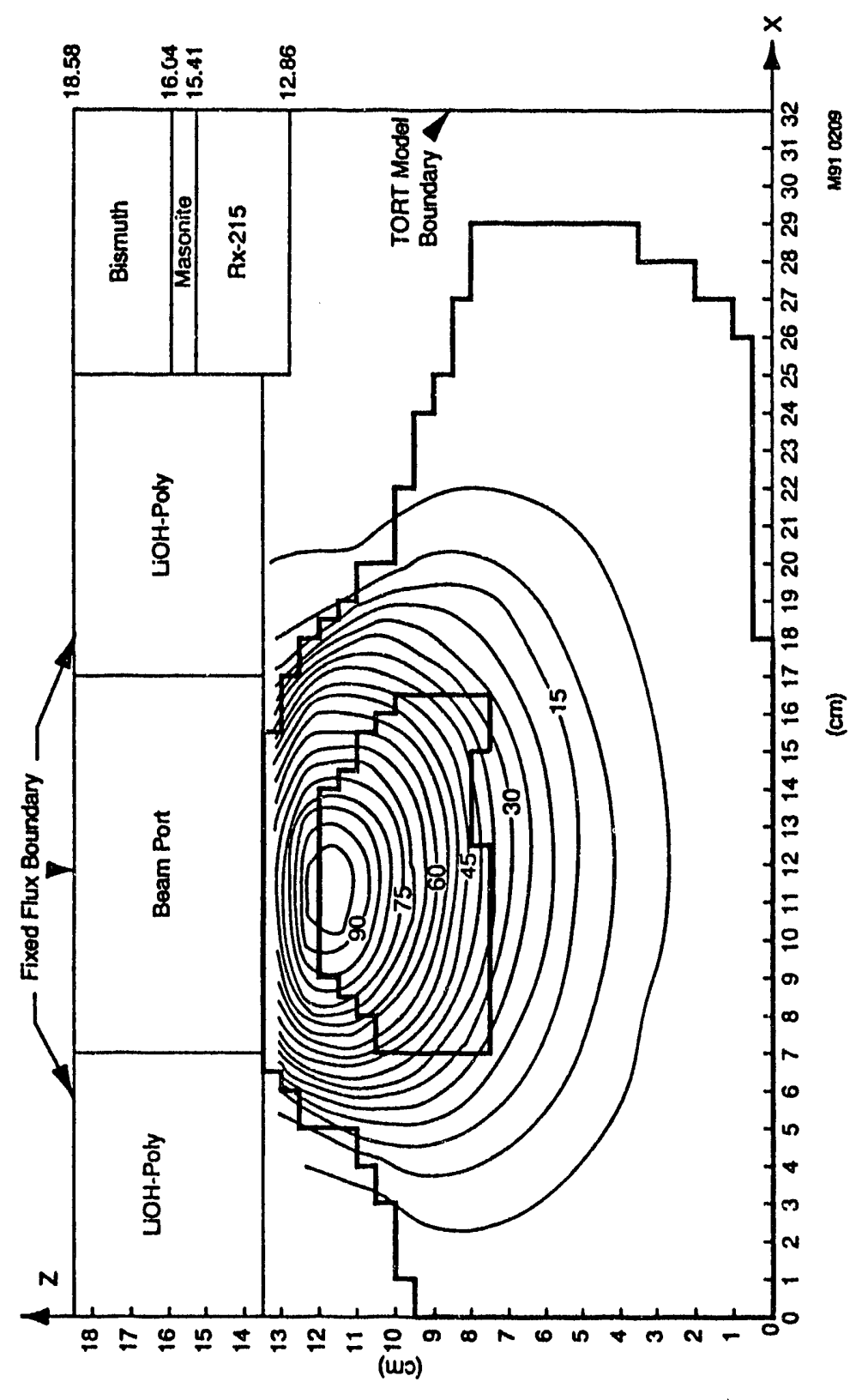

䑻

응

8

ปั

8 .

号4

过希

告罂

$+$

응

$y$

욤

政

ठ덩

点

年 
thermal flux occurs about $5 \mathrm{~mm}$ higher in the irradiation volume and in a slightly larger area in Figure 5-15 than in Figure 5-7. Also, the effect due to the nasal cavity is not as pronounced in Figure 5-15. In the upper regions of the dog head, there is as much as a $5 \%$ increase in the thermal flux in the $y=-0.375 \mathrm{~cm}$ plane. There is a decrease in the ventral direction of about $3 \%$ in some regions.

The fast fluxes can be compared in a similar manner at these two planes. Figures 5-17 and 5-18 show the fast flux at $y=-0.375 \mathrm{~cm}$. The homogeneous cases are identical at both planes (Figures 5-11 and 5-18) as expected. When Figures 5-17 and 5-18 are compared, a very slight decrease (less than 1\%) in the fast neutron flux can be seen in the heterogeneous model in the ventral direction. However, more noticeable differences, on the order of $10 \%$ in some regions, occur between the heterogeneous fast neutron fluxes at the $y$ $=-0.125 \mathrm{~cm}$ and $y=-0.375 \mathrm{~cm}$ planes. The presence of bone results in a much deeper penetration of the fast flux at the top of the dog head. The effect decreases to approximately a 48 difference between the two cases. One can observe a decreased effect due to the nasal cavity in Figure 5-17 corresponding to almost $10 \mathrm{~mm}$ in this region when compared with the flux contours in Figure 5-10. There is also a decrease of approximately $5 \%$ in the caudal direction near the entrance plane in Figure 5-17 corresponding to the 

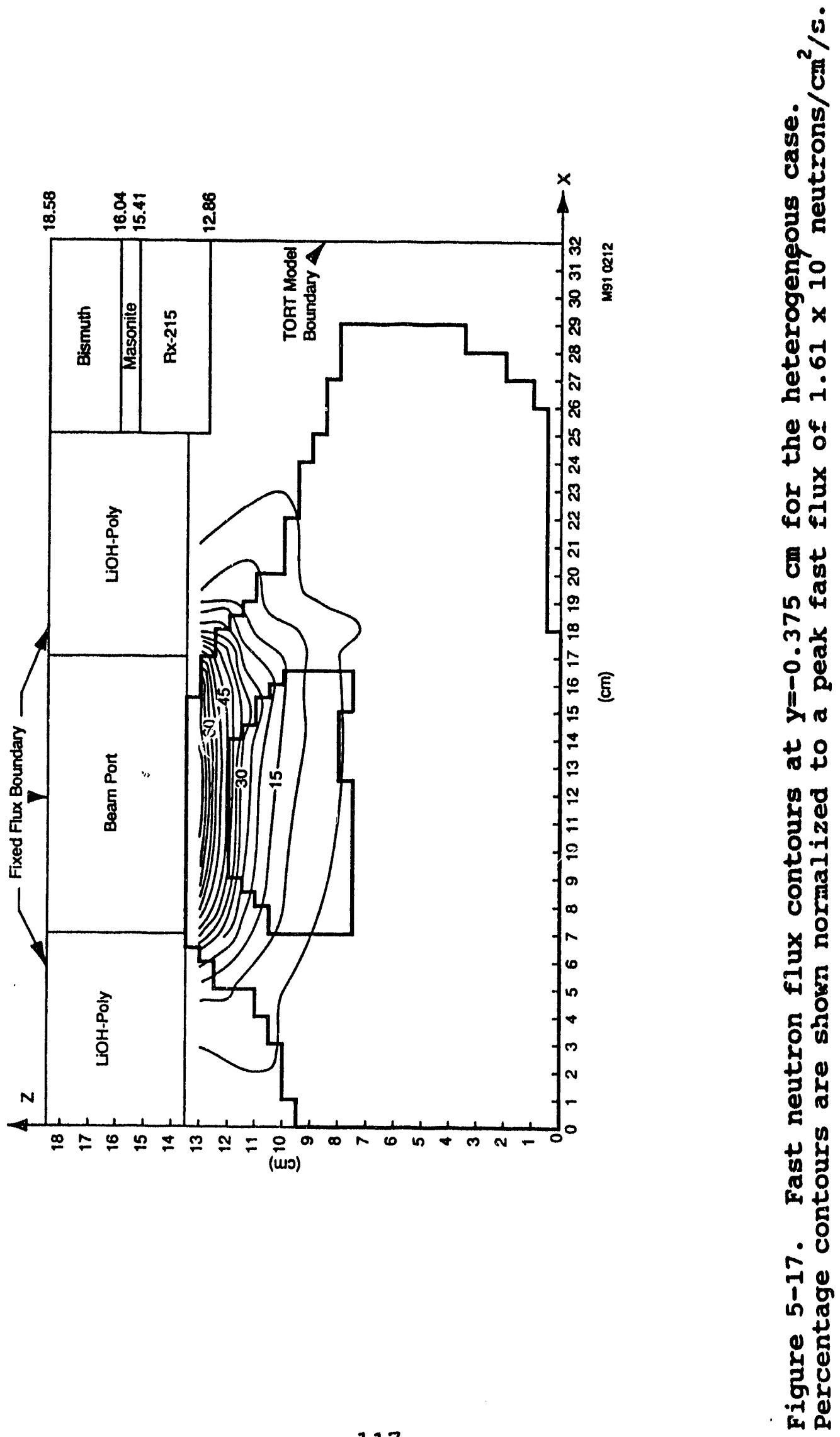

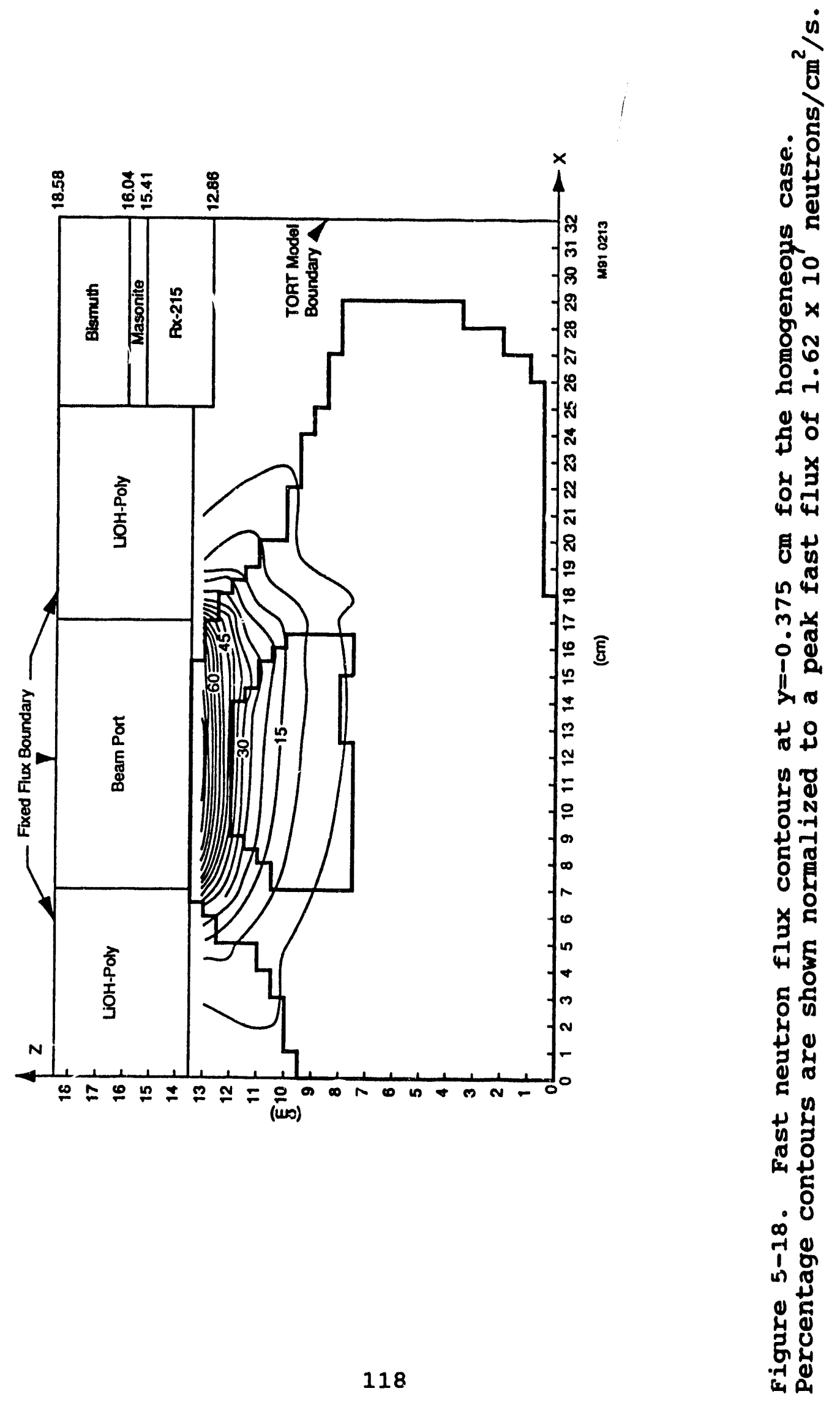
region of decreased bone. In all other regions, the spatial distributions and depths are similar. Again, this effect is due to the differing neutron transport properties in different tissues, especially bone in this case.

Finally, Figures 5-19 and 5-20 depict the gamma flux at $y=-0.375 \mathrm{~cm}$. Between the homogeneous contours shown in Figures 5-14 and 5-20, $y=-0.125 \mathrm{~cm}$ and $y=-0.375 \mathrm{~cm}$, respectively, there is only a slight decrease (approximately 18) in the gamma flux seen in the $y=-0.375 \mathrm{~cm}$ plane. The homogeneous flux in Figure 5-20 predicts a development of the flux deeper than is seen in the heterogeneous model results in Figure 5-19. The peak flux is almost identical, but one can see a $5 \%$ increase in flux in the region of the nasal cavity in the rostral direction. The spatial distributions between the homogeneous and heterogeneous cases are slightly different. In the ventral direction, the homogeneous model overestimates the gamma flux by about $5 q$ in some regions. The use of a homogeneous model in this case would lead to an underestimate of dose in the rostral direction and an overestinate of the dose in the ventral direction, including regions of the brain.

When Figures 5-13 and 5-19 are compared to see how a decrease in bone can affect the gamma flux, one can see that the peak is shifted about $3 \mathrm{~mm}$ higher in Figure 5-19 when 


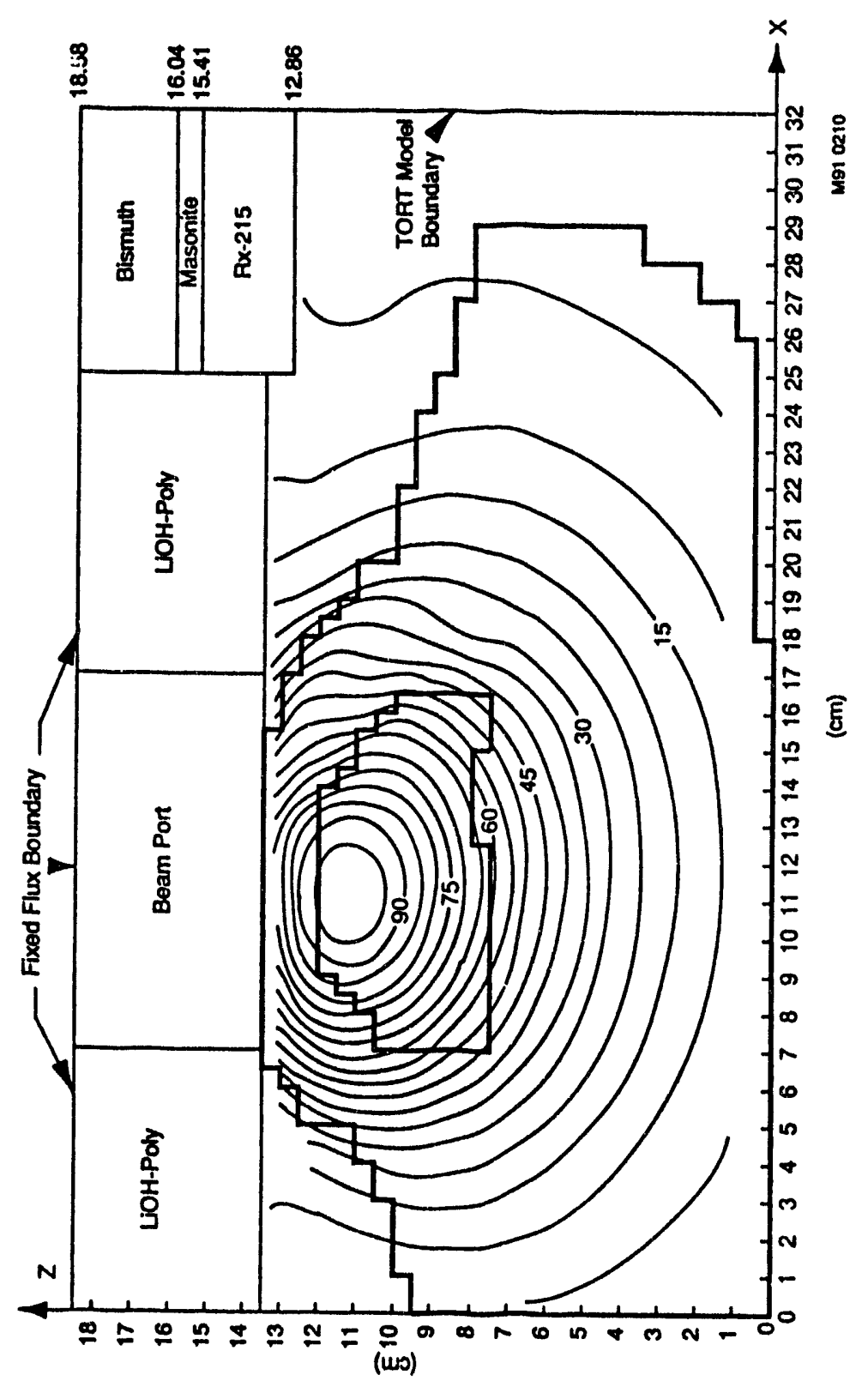

兽

造

n

m

$\circ$ G

II

$+\infty$

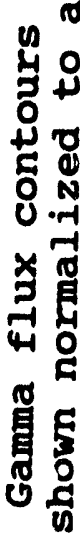

点 


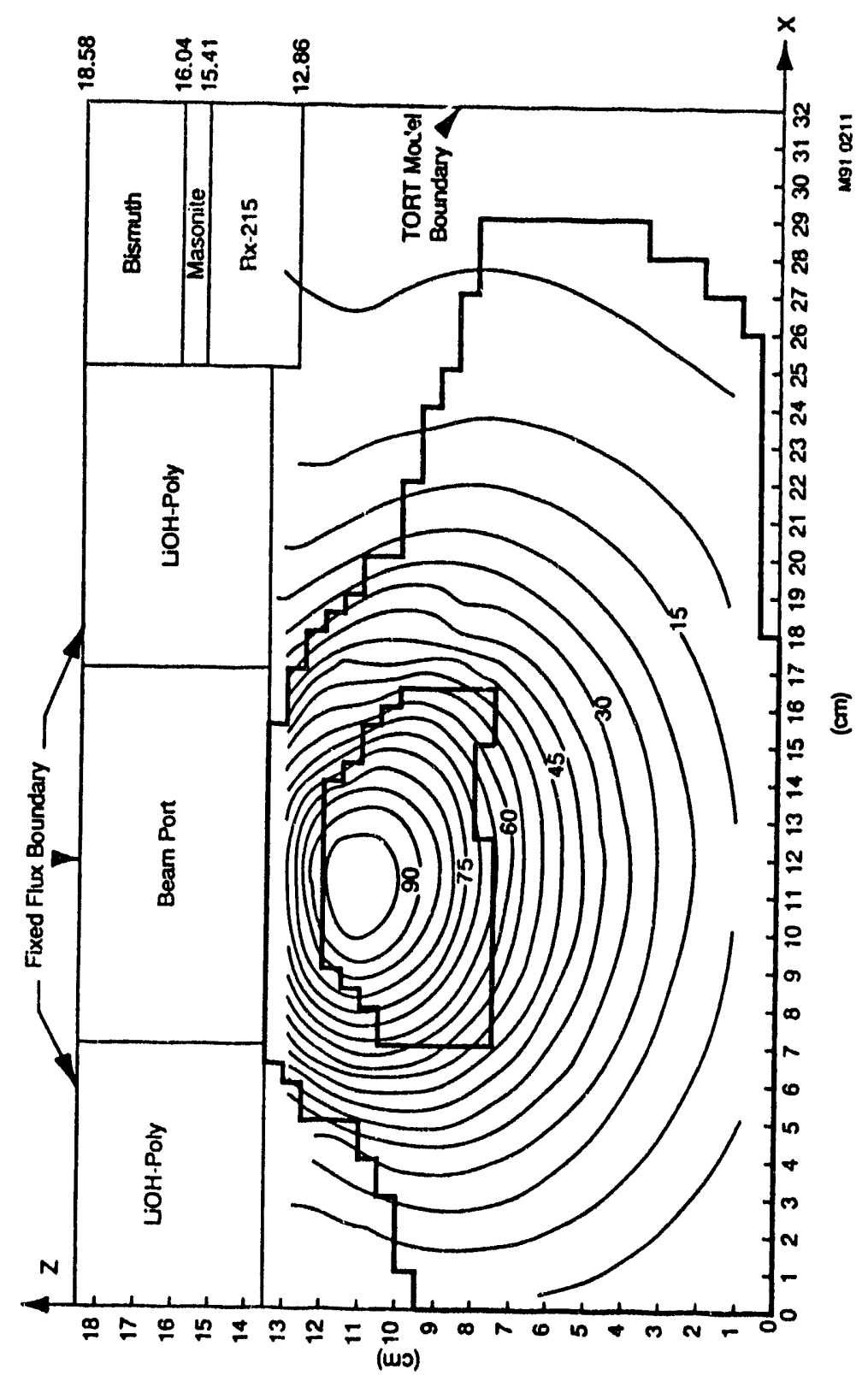

雚

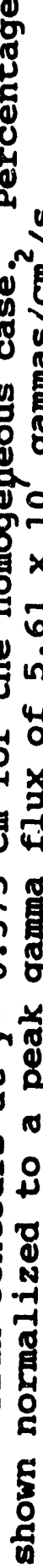

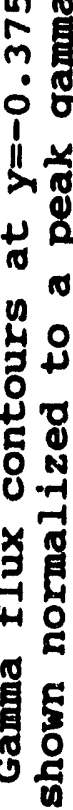

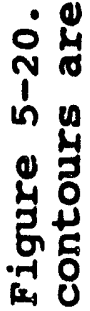


compared with the gamma flux at $y=-0.125 \mathrm{~cm}$ shown in Figure 5-13. The gamma flux is decreased by about 38 for the contours at $y=-0.375 \mathrm{~cm}$ in the regions of the nasal cavity. There is less of a decrease in flux in the ventral direction ranging from 1-2\%. In the caudal direction, there is about a $5 \%$ increase in flux due to the decreased bone at the top of the head. The changes in flux from $y=-0.125 \mathrm{~cm}$ to $y=-0.375 \mathrm{~cm}$ illustrate not only the effect of different materials such as bone, but also the necessity for examining the spatial distributions for each flux at different planes within the irradiation volume. Certain planes of interest such as ones including the tumor, brain, eyes, and the pituitary gland should be examined for treatment planning.

It is useful to examine the thermal neutron flux at sagittal planes further from the midplane. Figures 5-21 and 5-22 depict the thermal neutron flux at $y=-2.5 \mathrm{~cm}$. A small region of the brain is present in this slice as seen by the outline in both figures. The peak of the heterogeneous model is shifted in the caudal direction. In the 50-75\% flux range, there is a 3-5\% decrease in the thermal neutron flux of the heterogeneous case when compared with the contours for the homogenous case in the rostral direction. This is due to passage through bone. There is an increase in the thermal neutron flux when heterogeneities are considered in the rostral direction in the 5-15\% range 

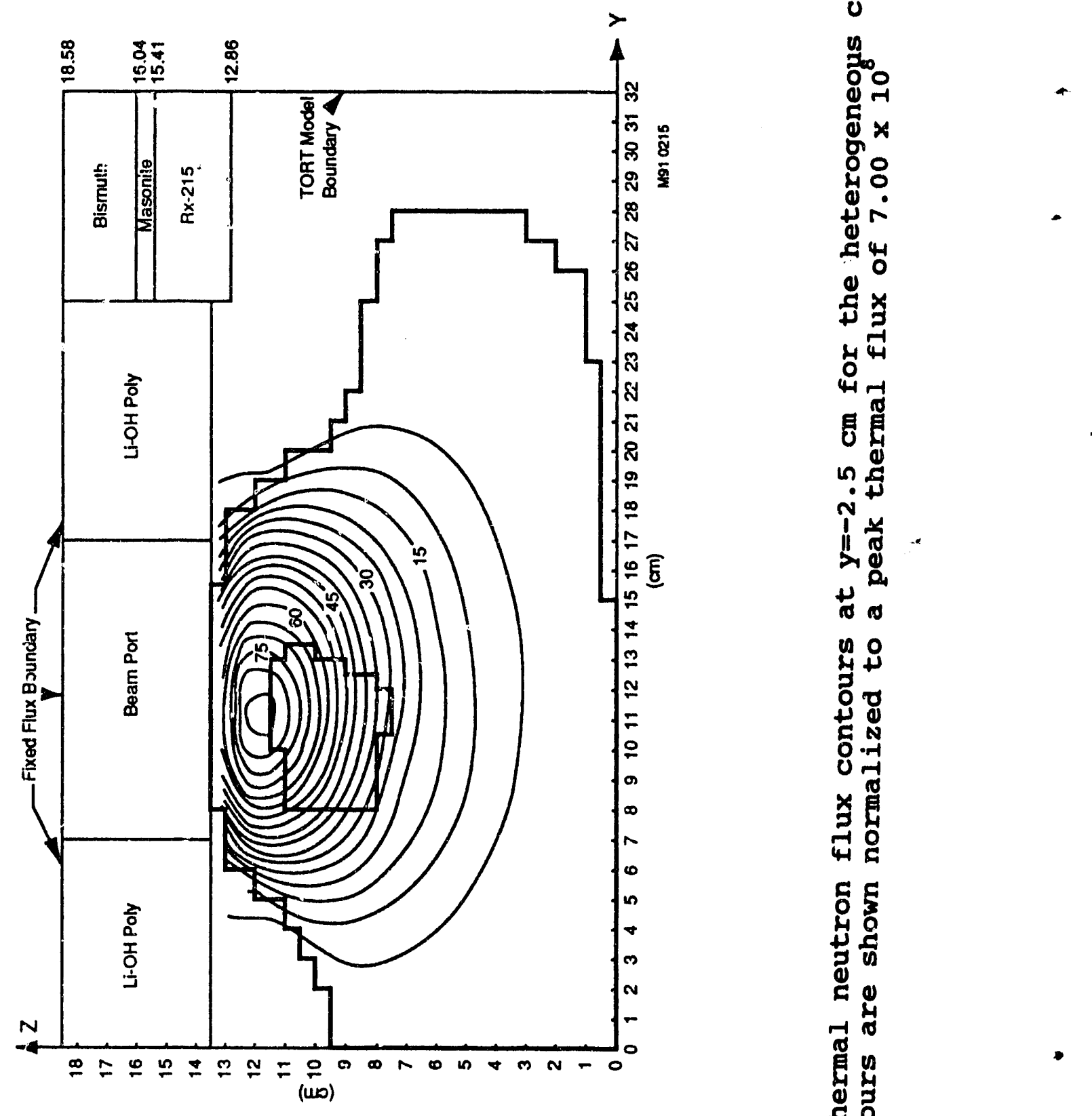

造爻

4

ชี

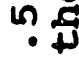

藏贶

옥

วิ

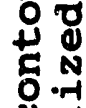

$0-1$

肴首员

ธ도

岁造

a

兽告

ن 


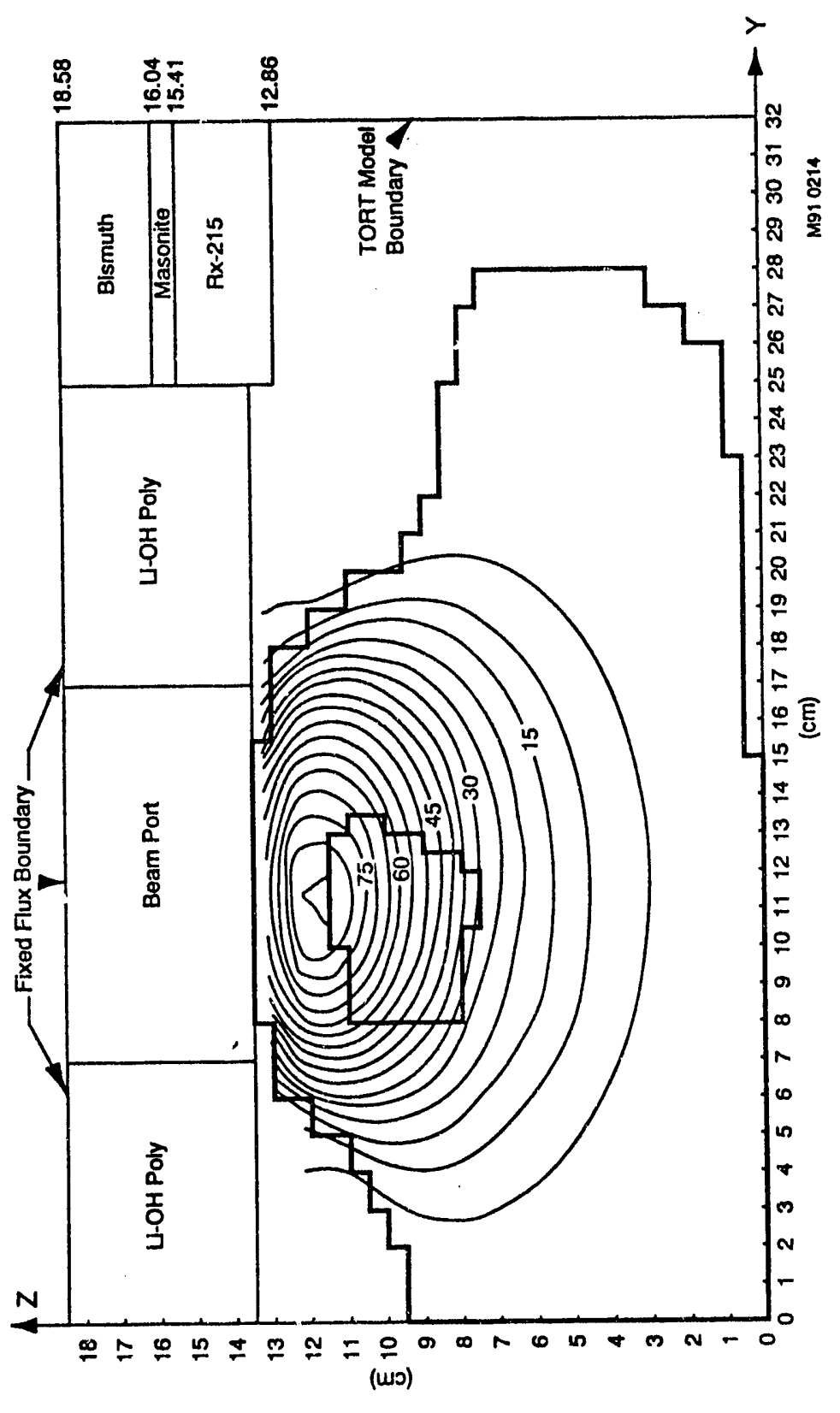

告

$n^{\infty} \circ$
0
0
0
0
0

岳齐

44

ह

in $\frac{1}{x}$

N

II

范

운

강

용

8 .

苜

건

동

언

马

\&

兽

ธn

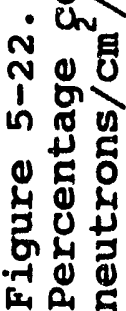


because of the neutron transport properties through the nasal cavity (a decreased density region).

In Figures 5-23 and 5-24, the thermal neutron flux contours for $y=-4.5 \mathrm{~cm}$ are shown. This is an outer section of the dog head and contains part of the eye and skull but no brain. It corresponds to sagittal slice 3 which is Figure B-3 in Appendix B for the photograph and Figure $\mathrm{C}-3$ in Appendix $\mathrm{C}$ for the detailed mesh. The peak flux in this section is only about 508 of the peak flux of the entire head for both cases. The spatial distributions are almost identical, although there is a slight decrease of the thermal neutron flux for the heterogeneous case. This is most likely due to the neutrons' passage through the skull. Contributions from effects due to the nasal cavity are less significant when examining the outer regions of the dog head.

To gain a better understanding of the spatial distribution of the fluxes over the entire head, an axial plane was examined. Figures 5-25 and 5-26 show the thermal neutron flux at $x=17.25 \mathrm{~cm}$. The detailed mesh for this slice was shown earlier in this chapter in Figure 5-3. Several different tissues were modeled in this section including the eyes, regions of the nasal cavity (including void), teeth, and bone in addition to skin and muscle. 


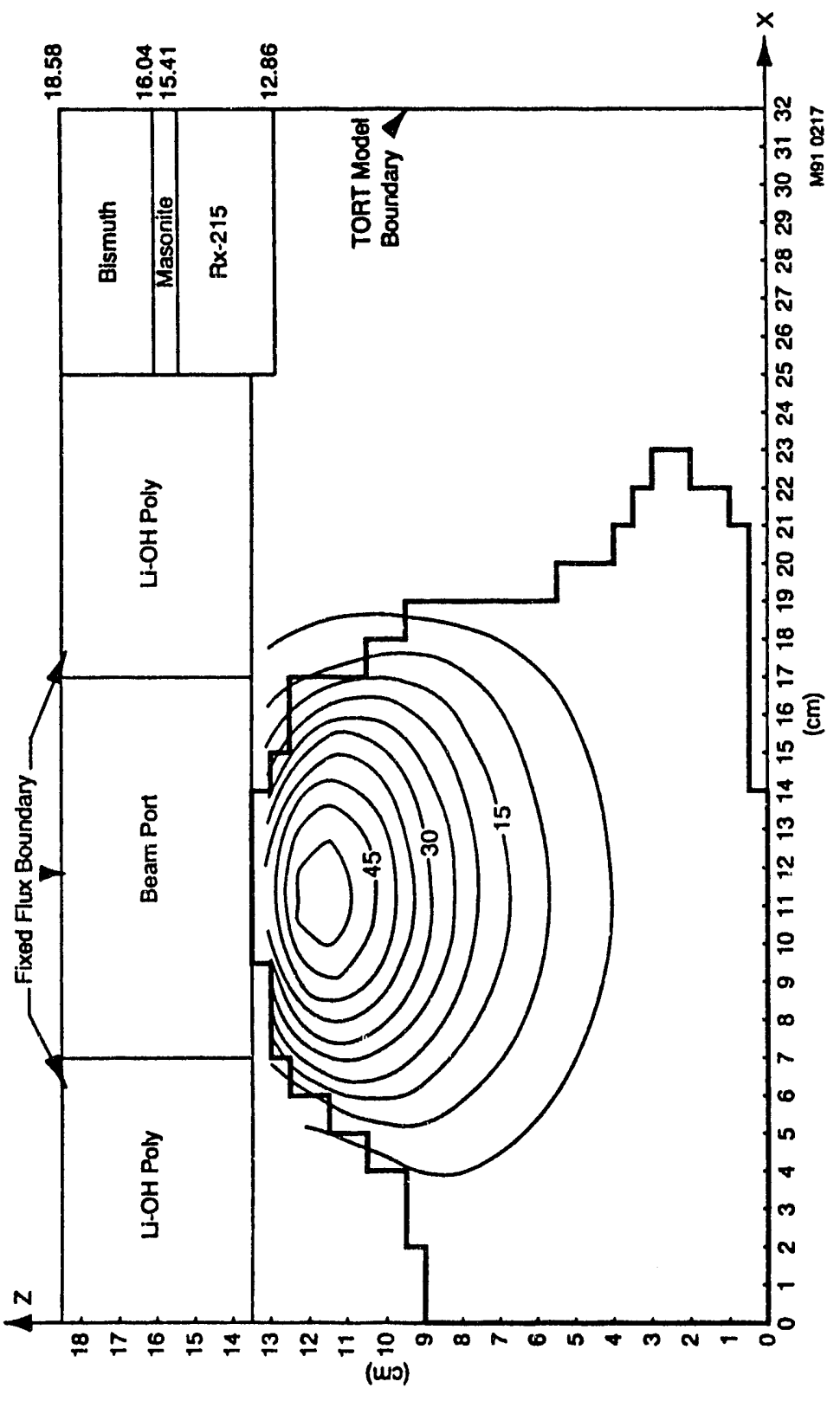

n

焉

ơ

씽

证

c. 4

题

म4

우

ํํㄹ

in

$\therefore$

î

苗

ํㅜㄴ

웡

雪

8

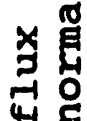

起

c

-

息

可

E्-

is

- फ

ஸ 0 ह

1 o

蛅

동

U

- $\begin{aligned} & 0 \\ & 0\end{aligned}$ 


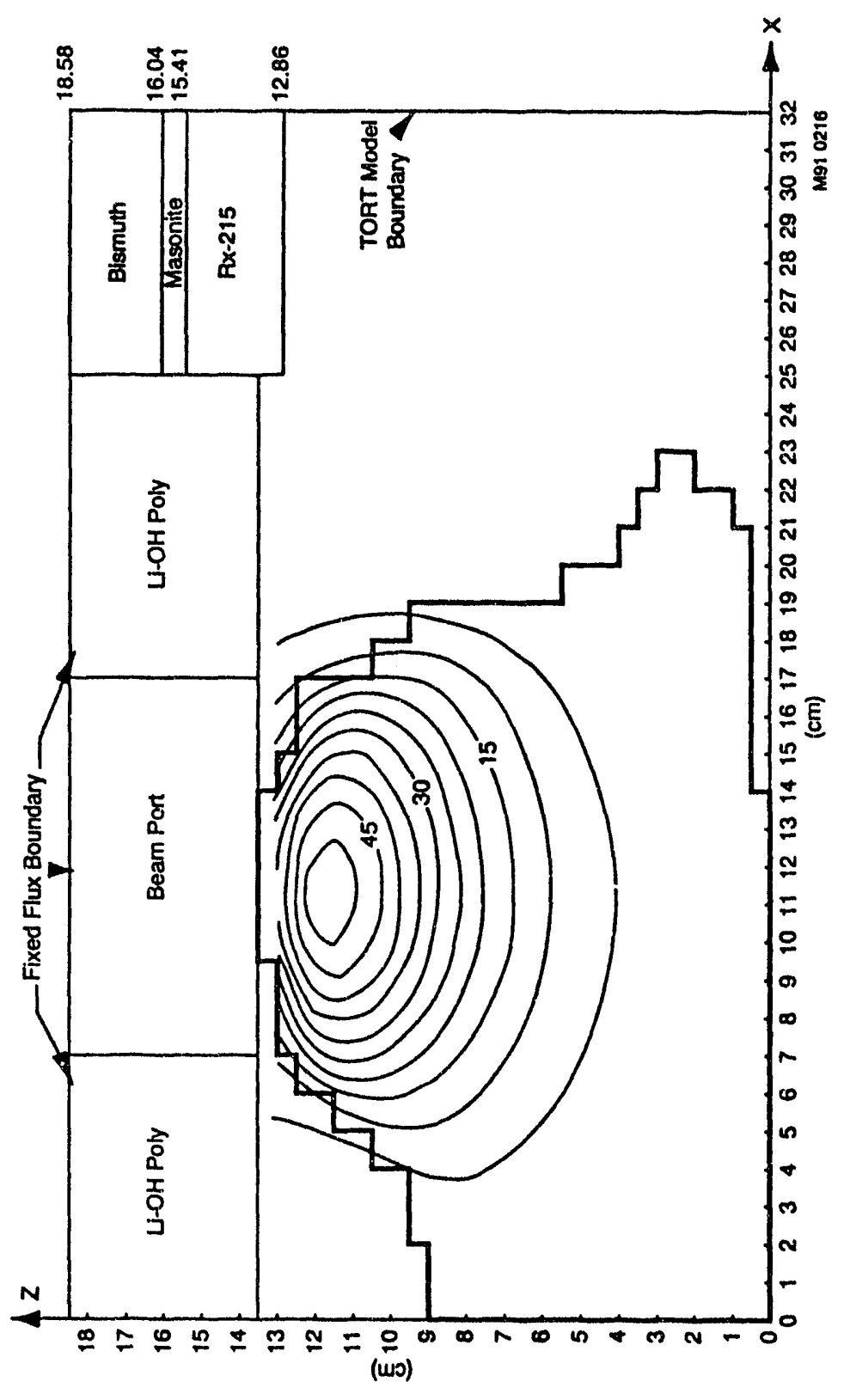

草

$n^{\infty} \circ$
0
0
0
0
0
0
0
0
0
0

욤

मै

4

罗息

뇽

i.

$\rightarrow 8$

幽

o

늘

80

은

8 .

금

때올

동

(1)

促

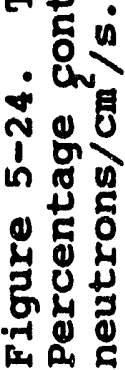




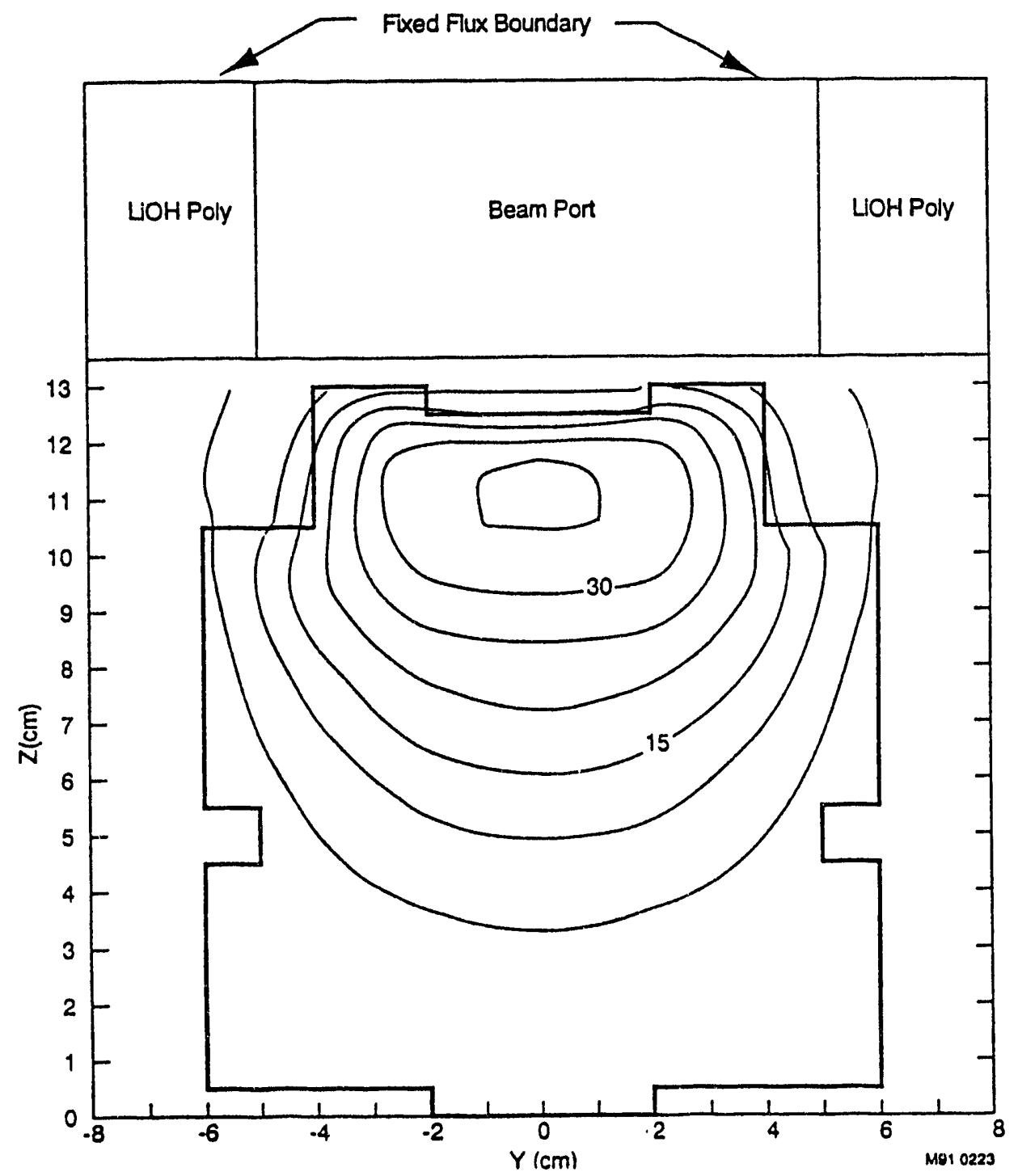

Figure 5-25. Thermal neutron flux contours at $x=17.25 \mathrm{~cm}$ for the heterogeneous case. Percentage contours are shown normalized tc a peak thermal flux of $7.00 \times 10^{8}$ neutrons $/ \mathrm{cm}^{2} / \mathrm{s}$. 


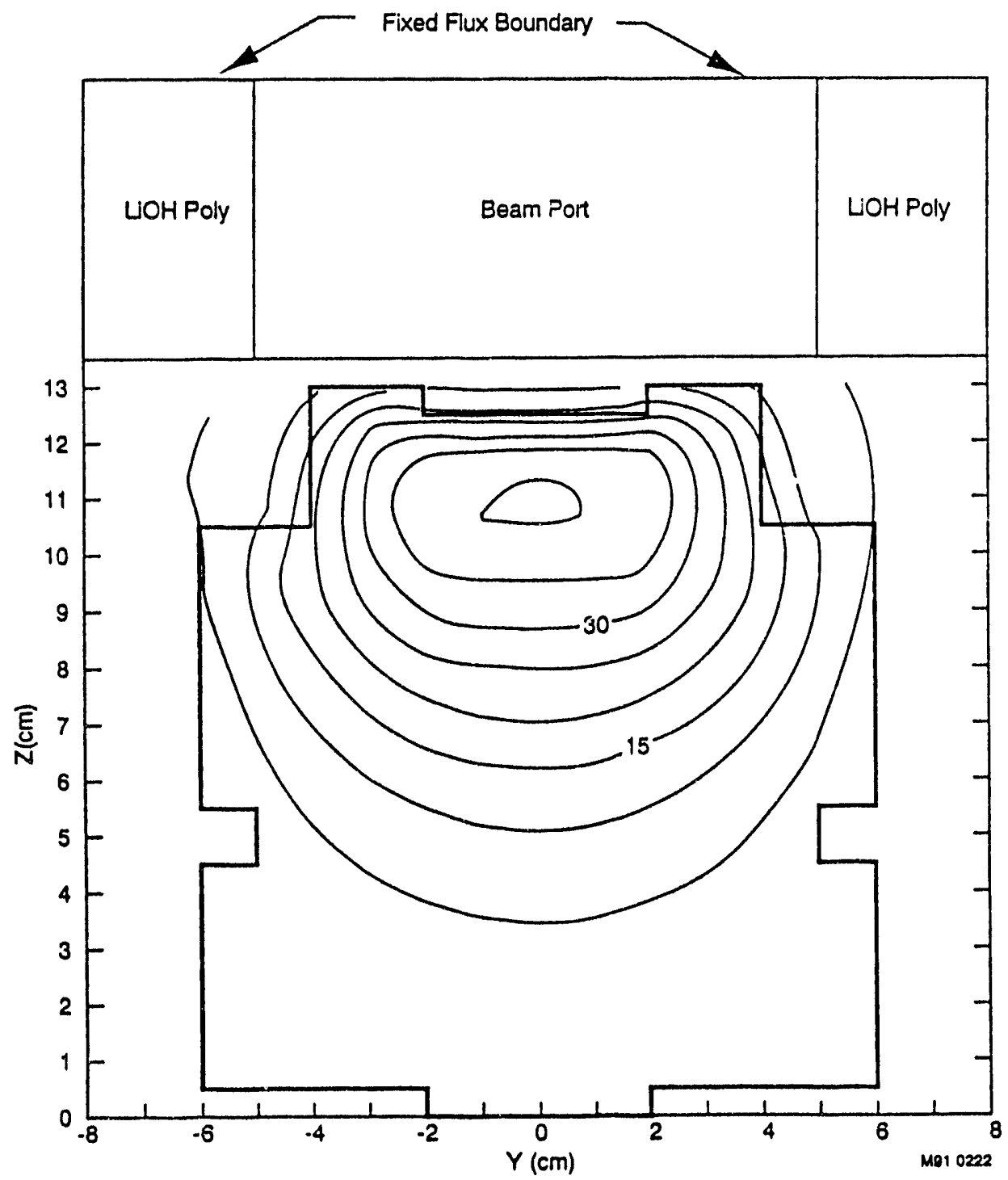

Figure 5-26. Thermal neutron flux contours at $x=17.25 \mathrm{~cm}$ for the homogeneous case. Percentage contours are shown normalized to a peak thermal flux of $6.92 \times 10^{8}$ neutrons $/ \mathrm{cm}^{2} / \mathrm{s}$. 
Neutrons interact differently in each tissue as mentioned in section 2.4. The peak thermal neutron flux is approximately $2.76 \times 10^{8} \mathrm{n} / \mathrm{cm}^{2} / \mathrm{sec}$ for the homogeneous case and approximately $2.45 \times 10^{8} \mathrm{n} / \mathrm{cm}^{2} / \mathrm{sec}$ for the heterogeneous case. Hence, overall the thermal neutron flux for the homogeneous case is greater. In terms of percentages, the flux in the homogeneous model predicts a flux that is about 5\% greater than that predicted with the heterogeneous model from 8-11 cm in the $z$ direction. The effect is more pronounced in the center of the head. At about $x=6.5 \mathrm{~cm}$, the heterogeneous flux is greater than the homogeneous flux. This trend continues as seen in the $5 \%$ and $10 \%$ contours. The homogeneous model is unable to represent these variations in the thermal neutron flux.

Figures 5-27 and 5-28 depict the spatial distribution of the gamma flux at $x=17.25 \mathrm{~cm}$. In Figure 5-27, the heterogeneous results, there is approximately a 7-8\% decrease in the flux in the region of $z=8 \mathrm{~cm}$ to $z=10 \mathrm{~cm}$ and $y=-3 \mathrm{~cm}$ to $y=3 \mathrm{~cm}$ relative to the homogeneous results. This effect is due to passage through bone and is not seen at all in the homogeneous contours. As a result, the spatial distributions of the gamma flux are different for each model. The homogeneous model overestimates the gamma flux by about 5\%. This effect is more pronounced near the peak flux and less pronounced further away from the beam 


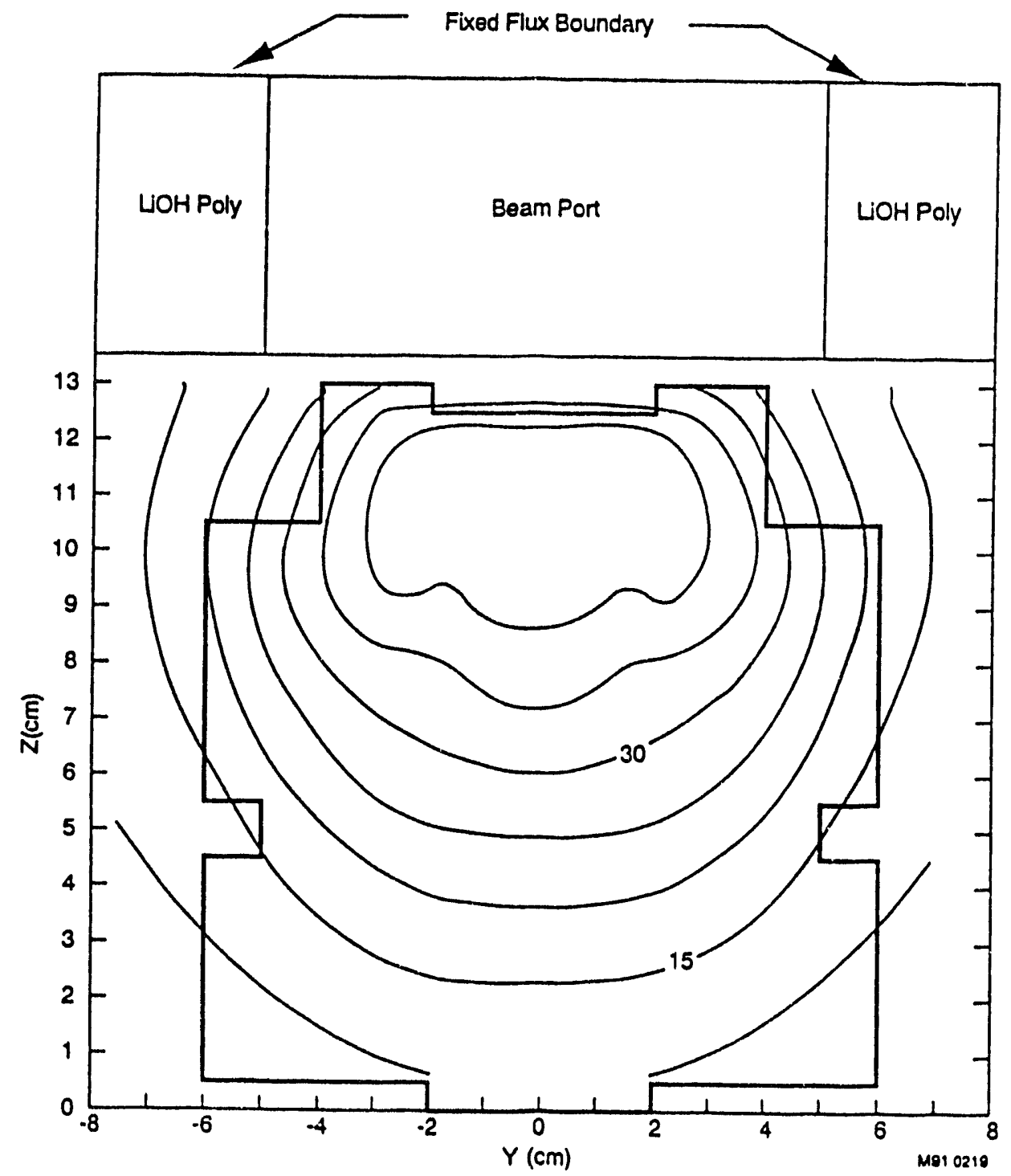

Figure 5-27. Gamma flux contours at $x=17.25 \mathrm{~cm}$ for the heterogeneous case. Percentage contours are shown normalized to a peak gamma flux of $5.63 \times 10^{7}$ gammas $/ \mathrm{cm}^{2} / \mathrm{s}$. 


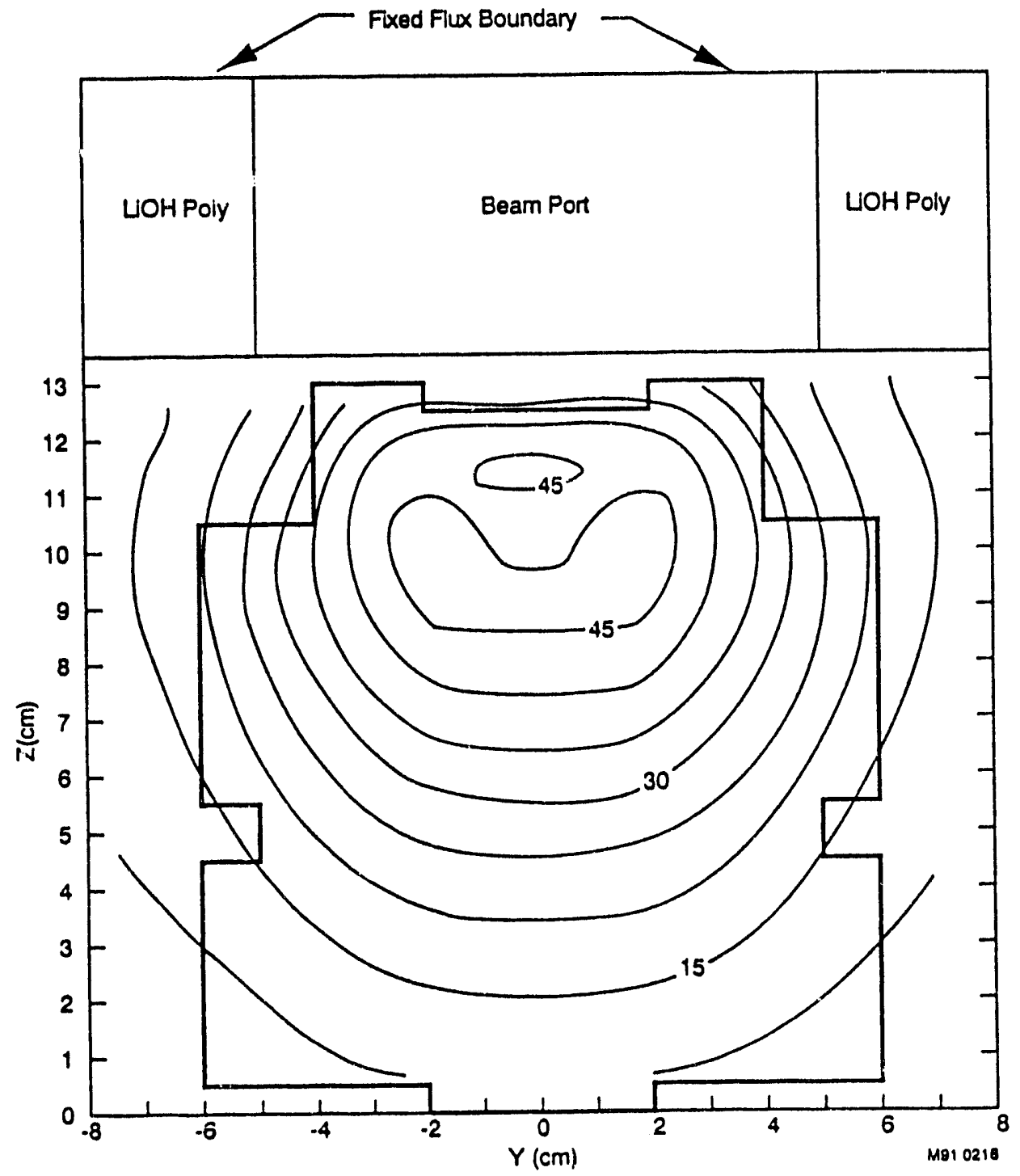

Figure 5-28. Gamma flux contours at $x=17.25 \mathrm{~cm}$ for the homogeneous case. Percentage contours are shown normalized to a peak gamma flux of $5.61 \times 10^{7}$ gammas $/ \mathrm{cm}^{2} / \mathrm{s}$. 
delimiter as expected.

As previously mentioned, calculations were performed for a $10 \mathrm{~cm} \times 10 \mathrm{~cm}$ and a $5 \mathrm{~cm} \times 10 \mathrm{~cm}$ beam delimiter for the BMRR beam. The above results are for the full beam. Additional information regarding the contours was found with the $5 \mathrm{~cm} \times 10 \mathrm{~cm}$ beam port. The beam size for this case extended from $y=-5 \mathrm{~cm}$ to $y=0 \mathrm{~cm}$. This beam has been used for canine irradiations in the INEL program.

Figures 5-29 and 5-30 depict the thermal neutron flux contours at $x=17.25 \mathrm{~cm}$ for the $5 \mathrm{~cm} \times 10 \mathrm{~cm}$ beam delimiter. As expected, the peak is shifted about $2.5 \mathrm{~cm}$ to below the center of the beam port. The spatial distributions for the two models differ. scatter contributes less in these cases when compared with the $10 \mathrm{~cm}$ $x 10 \mathrm{~cm}$ contours depicted in Figures 5-25 and 5-26. The peak flux does not develop into the brain region as with the $10 \mathrm{~cm} \times 10 \mathrm{~cm}$ beam port. However, similar heterogeneity effects are seen in Figures 5-29 and 5-30. The thermal neutron flux of the heterogeneous case is decreased by $5 \%$ when compared with the homogeneous results.

Figures 5-31 and 5-32 show the thermal neutron flux for the $5 \mathrm{~cm} \times 10 \mathrm{~cm}$ beam delimiter at $\mathrm{x}=12.25 \mathrm{~cm}$. The brain is outlined in both figures. The peak is located just above 


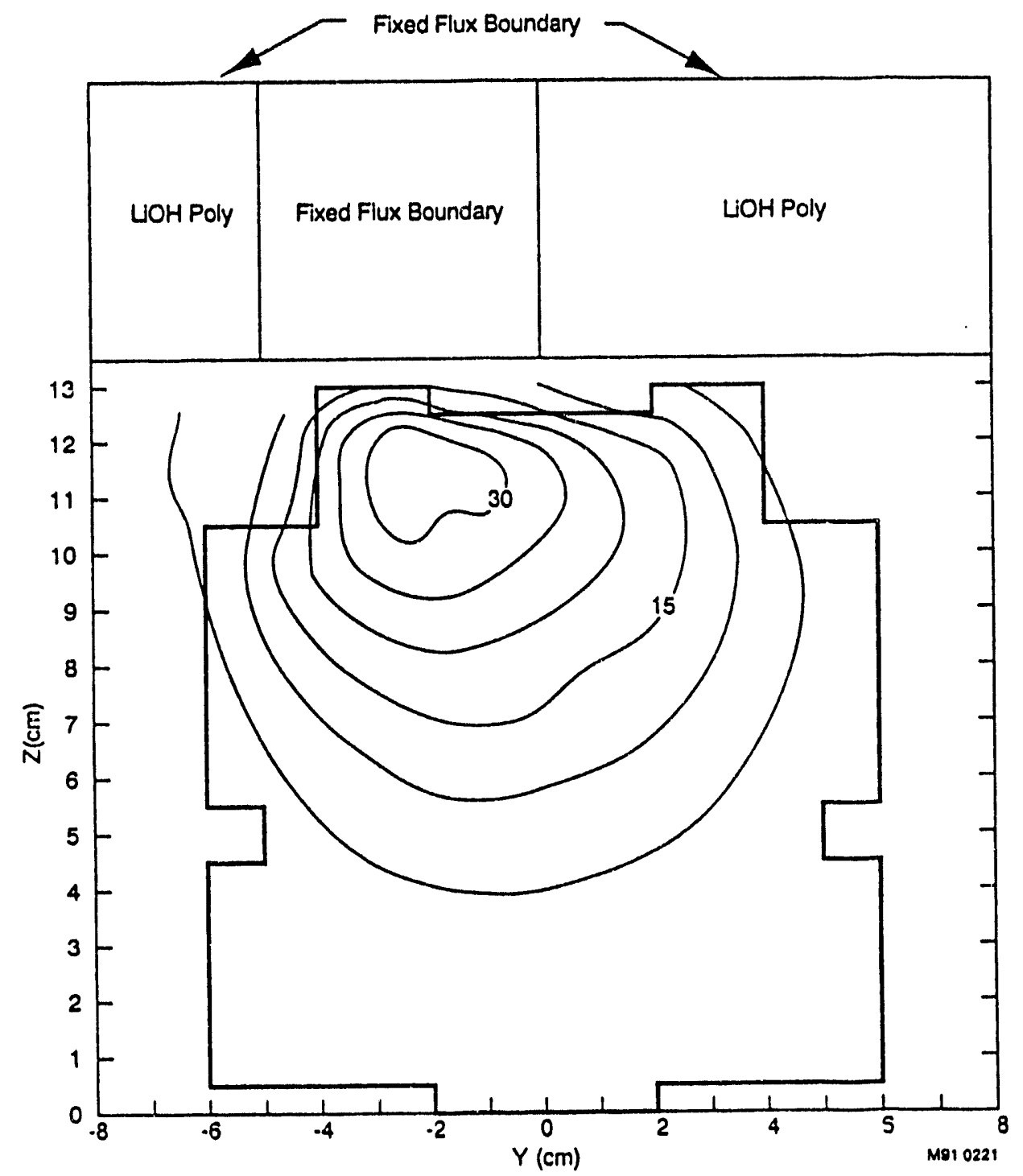

Figure 5-29. Thermal neutron flux contours at $x=17.25 \mathrm{~cm}$ for the heterogeneous case with the $5 \mathrm{~cm} \times 10 \mathrm{~cm}$ beam aperture. Percentage contours are shown normalized to a peak thermal flux of $4.14 \times 10^{8} \mathrm{n} / \mathrm{cm}^{2} / \mathrm{s}$. 


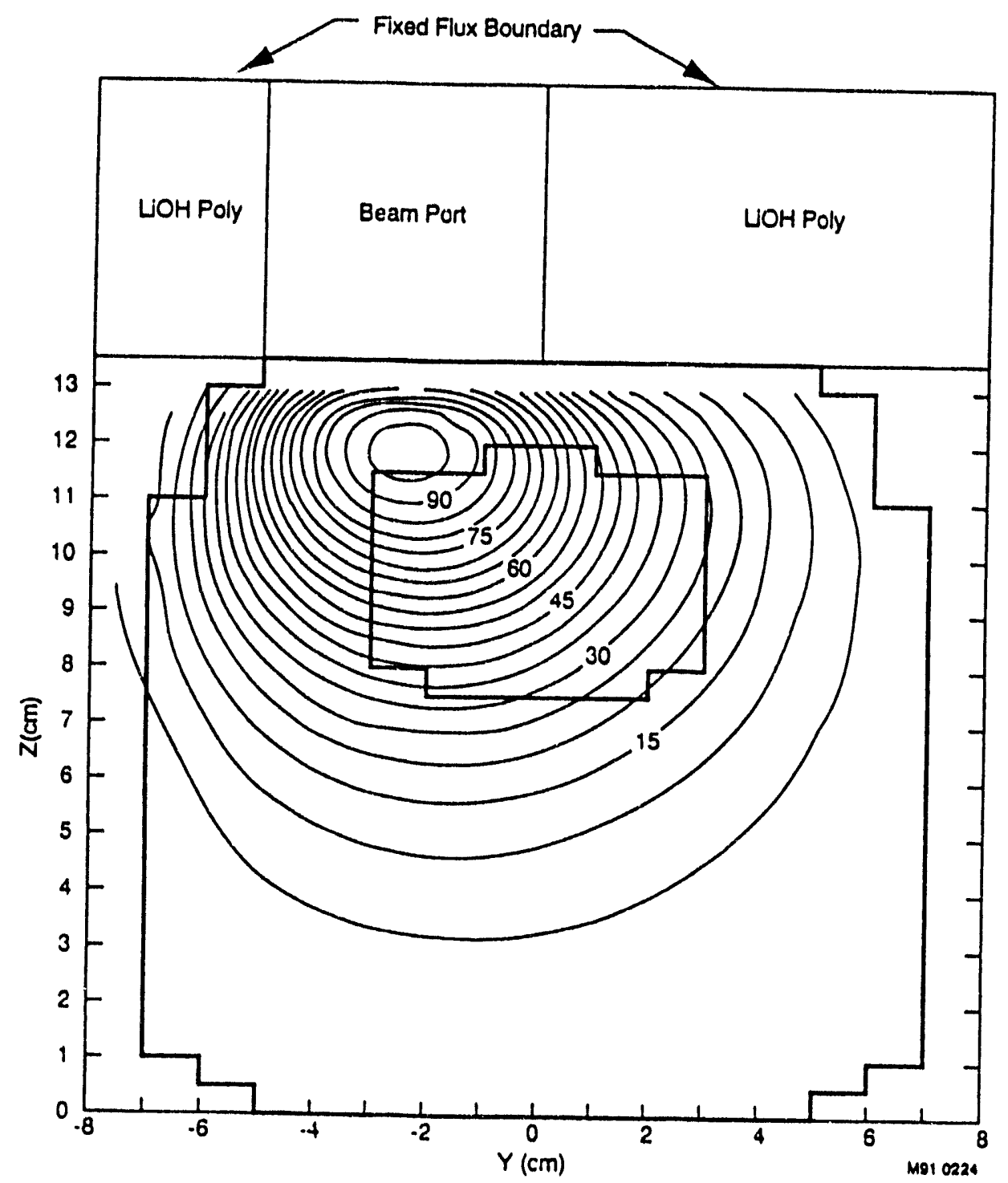

Figure 5-30. Thermal neutron flux contours at $x=17.25 \mathrm{~cm}$ for the homogeneous case with the $5 \mathrm{~cm} \times 10 \mathrm{~cm}$ beam aperture. Percentage contours are shown normalized to a peak thermal flux of $4.04 \times 10^{8} \mathrm{n} / \mathrm{cm}^{2} / \mathrm{s}$. 


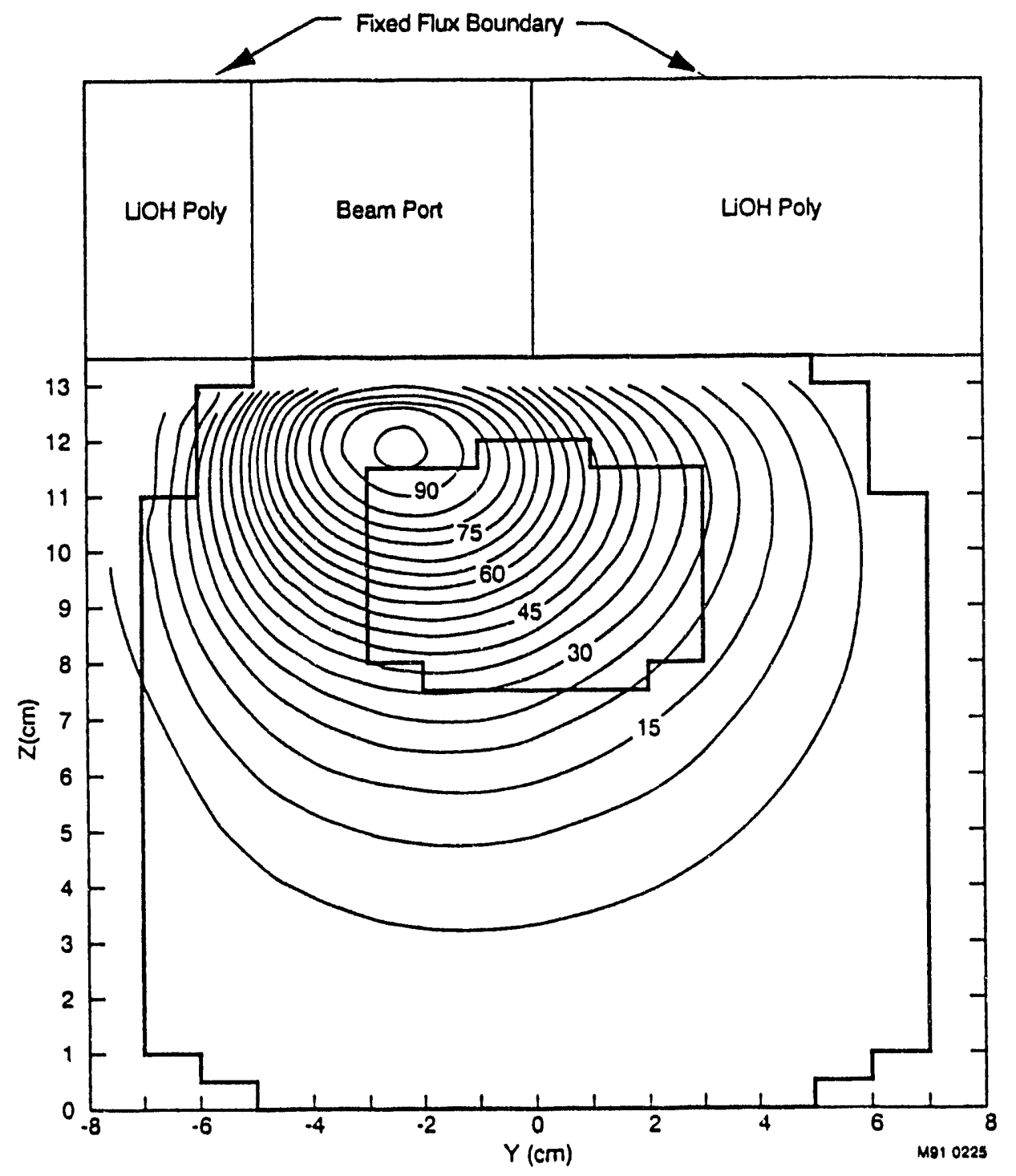

Figure 5-31. Thermal neutron flux contours at $x=12.25 \mathrm{~cm}$ for the heterogeneous case with the $5 \mathrm{~cm} \times 10 \mathrm{~cm}$ beam aperture. Percentage contours are shown normalized to a peak thermal flux of $4.14 \times 10^{8} \mathrm{n} / \mathrm{cm}^{2} / \mathrm{s}$. 


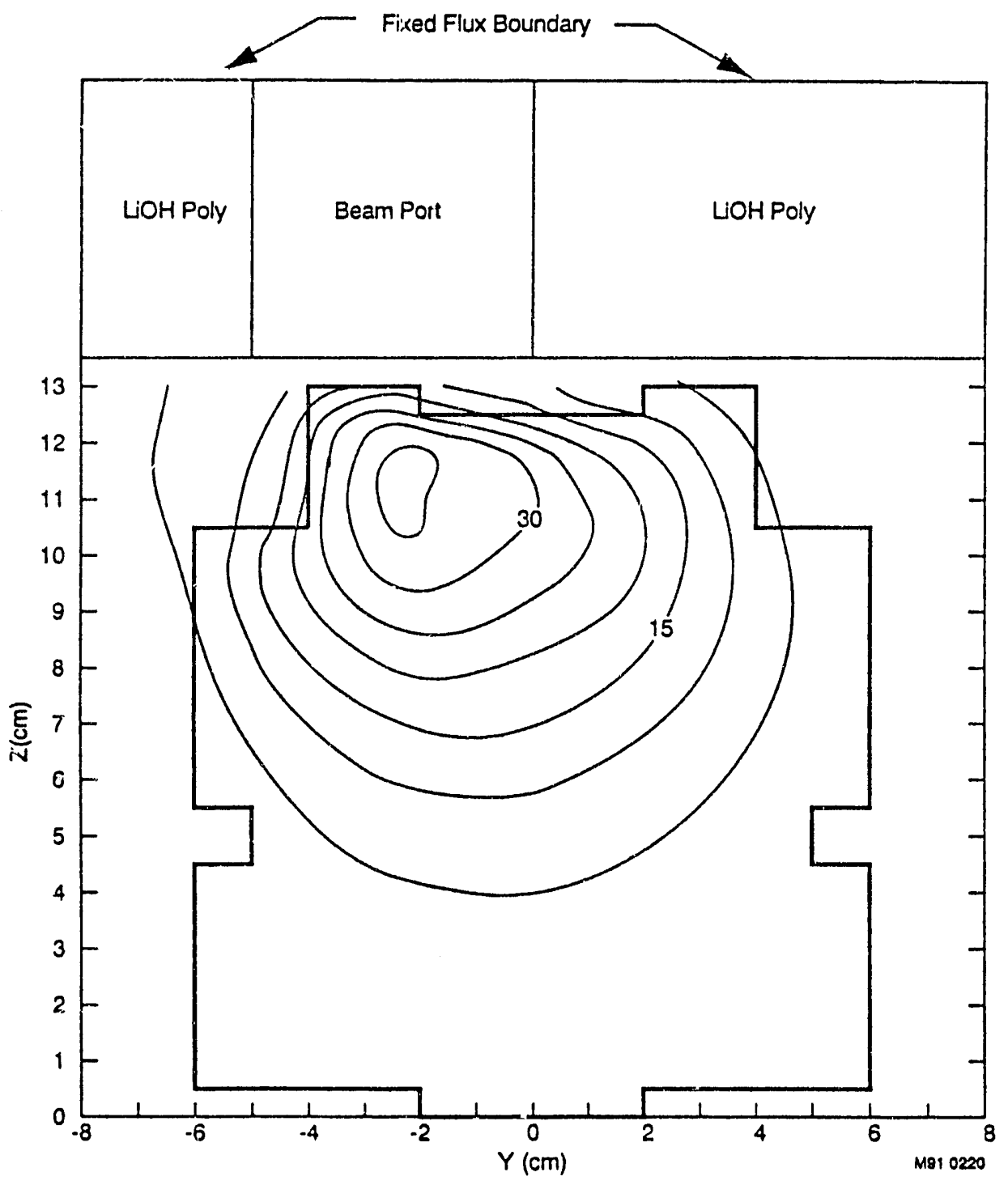

Figure 5-32. Thermal neutron flux contours at $x=12.25 \mathrm{~cm}$ for the homogeneous case with the $5 \mathrm{~cm} \times 10 \mathrm{~cm}$ beam aperture. Percentage contours are shown normalized to a peak thermal flux of $4.04 \times 1)^{8} \mathrm{n} / \mathrm{cm}^{2} / \mathrm{s}$. 
the urain region. Both peaks are $i_{\text {at }}$ the right hemisphere below the beam port. The region of peak flux is smaller in the heterogeneous case. This causes a shift in all the contours. The shift originally leads to a decrease of the thermal neutron $f$ lux in the rostral direction. At about $z=$ $8.5 \mathrm{~cm}$, there is a $2-3 \%$ decrease of flux in the ventral direction due to heterogeneities.

Finally, the fast neutron flux just below the exit of the beam port, $z=13.25 \mathrm{~cm}$, can be observed for both the 5 $\mathrm{cm} \times 10 \mathrm{~cm}$ and $10 \mathrm{~cm} \times 10 \mathrm{~cm}$ beam delimiter. The beam port is outlined over the contours. This is the fast neutron flux at the skin layer. The fast neutron fluxes for the two models with the $10 \mathrm{~cm} \times 10 \mathrm{~cm}$ beam delimiter are depicted in Figures 5-33 and 5-34. The fluxes are identical as expected silce the only materials traversed at this plane are skin and air.

Figures 5-35 and 5-36 show the fast neutron flux for the $5 \mathrm{cin} \times 10 \mathrm{~cm}$ beam delimiter at $z=13.25 \mathrm{~cm}$. These two figures are identical as expected because the neutrons are transported either through skin or air as mentioned above. When Figures 5-33 and 5-35 are compared, there is a noticeable decrease in the fast flux with the smaller beam port due to less contributions from scatter. The peak is also shifted to the right hemisphere for the $5 \mathrm{~cm} \times 10 \mathrm{~cm}$ 


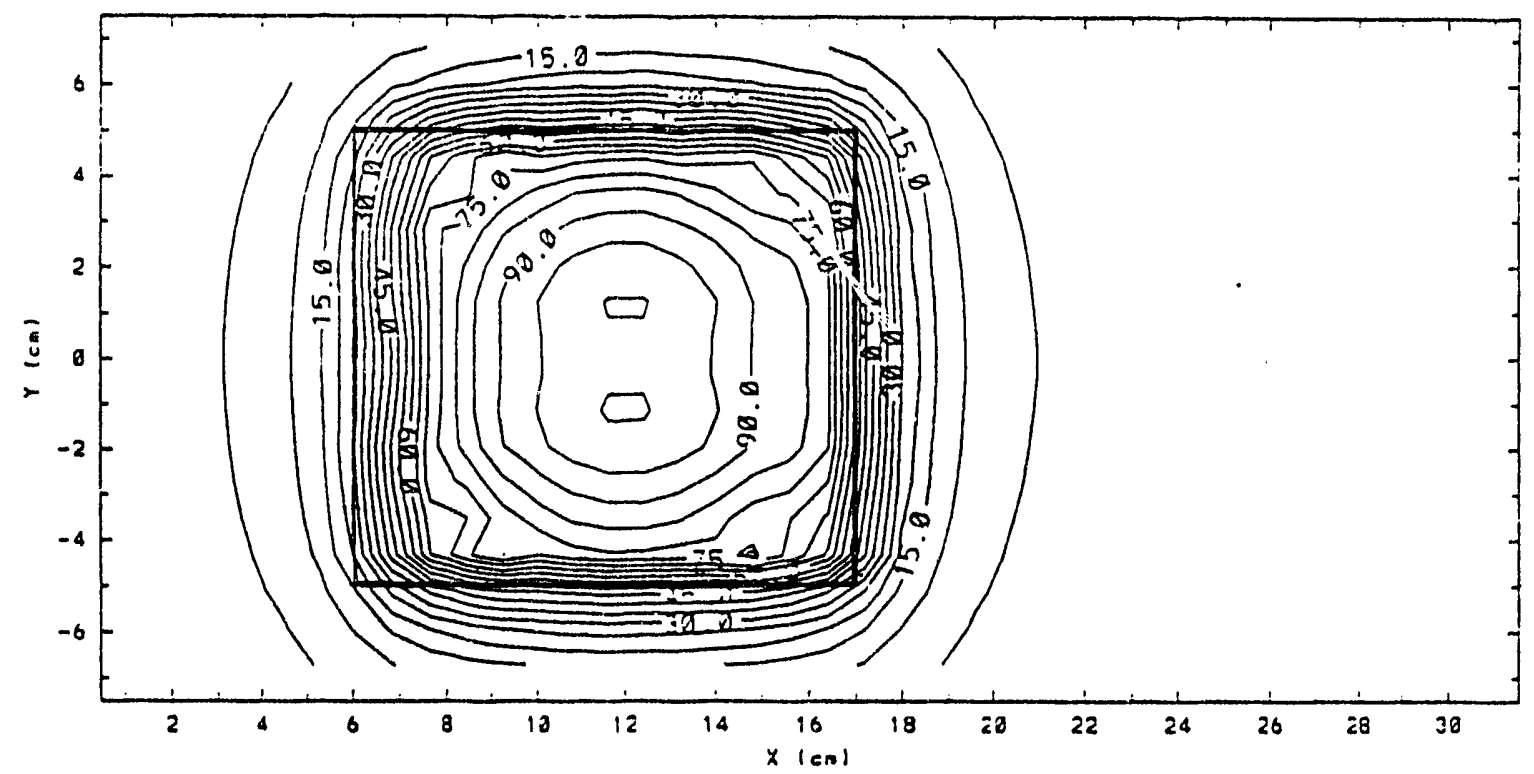

Figure 5-33. Fast neutron flux contours at $z=13.25 \mathrm{~cm}$ for the heterogeneous case with the $10 \mathrm{~cm} \times 10 \mathrm{~cm}$ beam aperture. Percentage contours are shown normalized to a peak fast flux of $1.61 \times 10^{7} \mathrm{n} / \mathrm{cm}^{2} / \mathrm{s}$. 


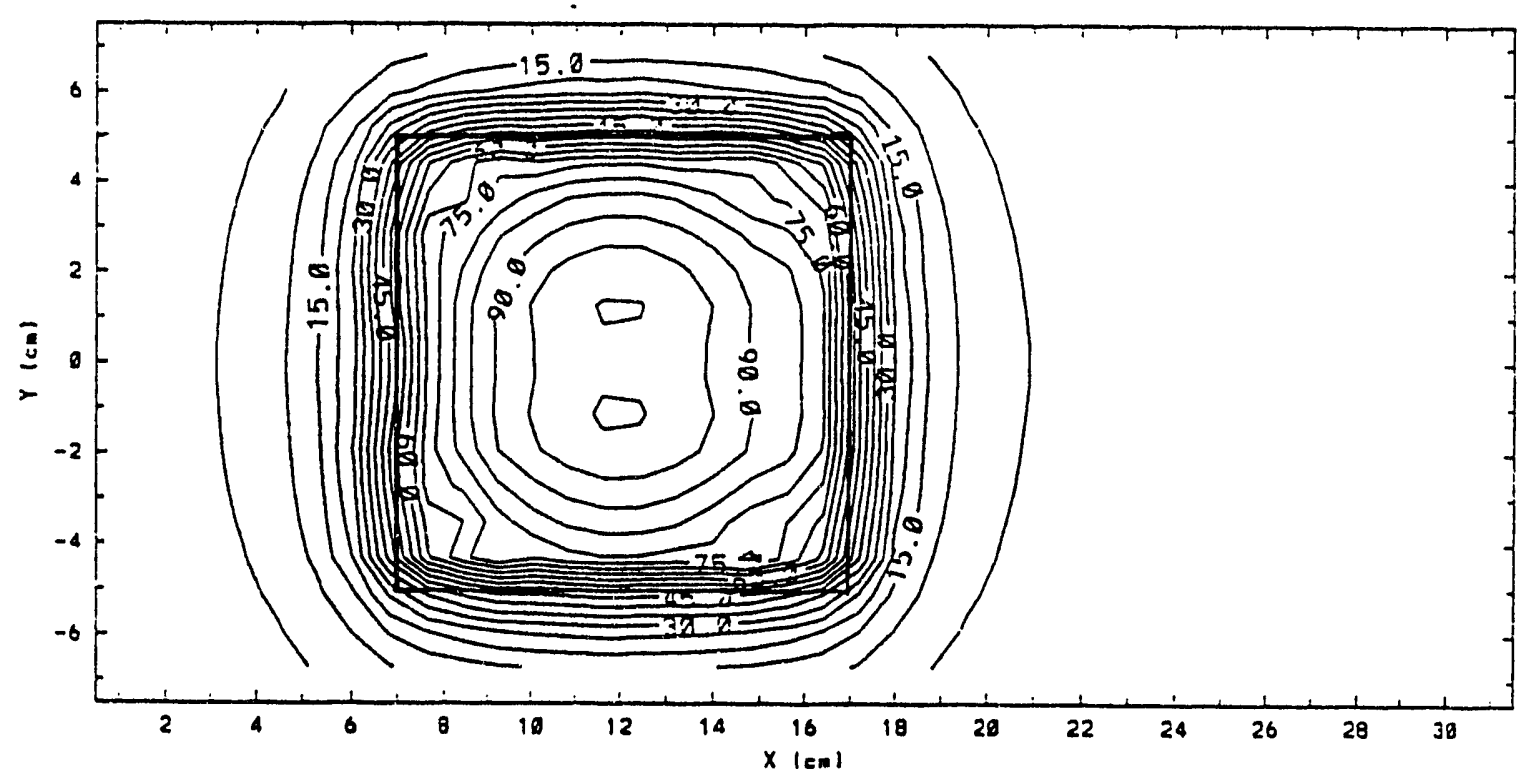

Figure 5-34. Fast neutron flux contours at $z=13.25 \mathrm{~cm}$ for the homogeneous case with the $10 \mathrm{~cm} \times 10 \mathrm{~cm}$ beam aperture. Percentage contours are shown normalized to a peak fast flux of $1.62 \times 10^{7} \mathrm{n} / \mathrm{cm}^{2} / \mathrm{s}$. 


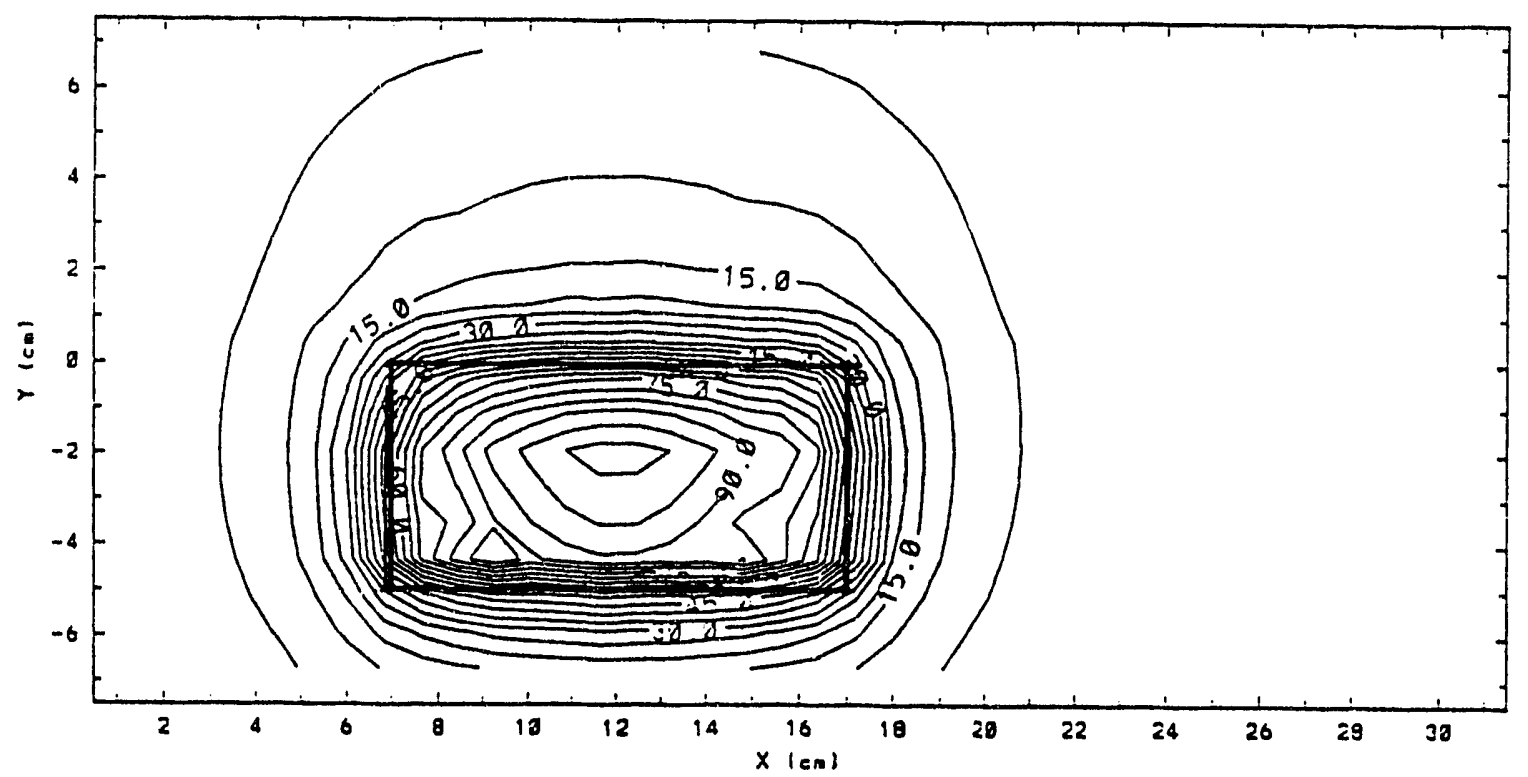

Figure 5-35. Fast neutron flux contours at $z=13.25 \mathrm{~cm}$ for the heterogeneous case with the $5 \mathrm{~cm} \times 10 \mathrm{~cm}$ beam aperture. Percentage contours are shown normalized to a peak fast flux of $1.26 \times 10^{7} \mathrm{n} / \mathrm{cm}^{2} / \mathrm{s}$. 


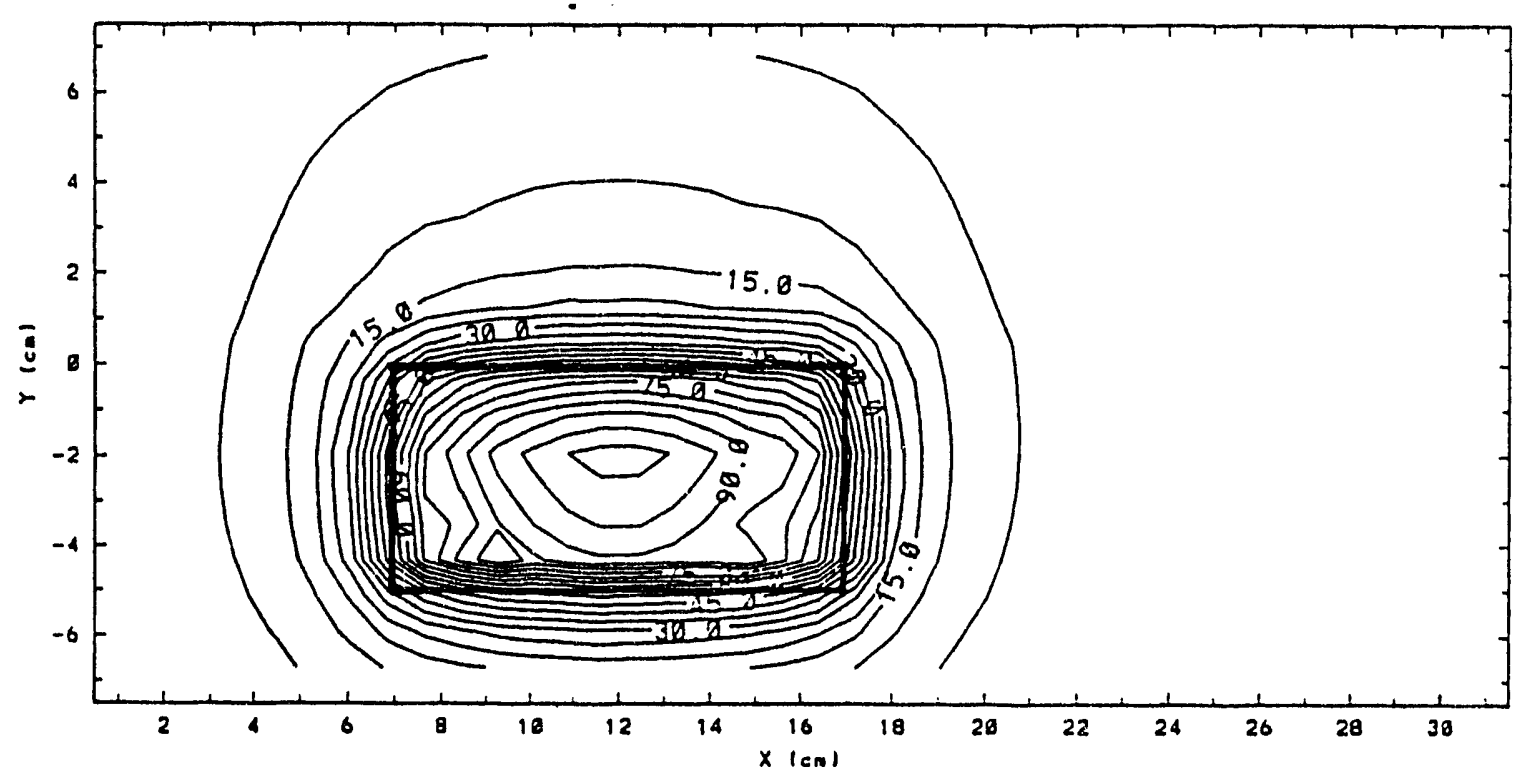

Figure 5-36. Fast neutron flux contours at $z=13.25 \mathrm{~cm}$ for the homogeneous case with the $5 \mathrm{~cm} \times 10 \mathrm{~cm}$ beam aperture. Percentage contours are shown normalized to a peak fast flux of $1.27 \times 10^{7} \mathrm{n} / \mathrm{cm}^{2} / \mathrm{s}$. 
beam delimiter as expected.

The figures presented here are meant to examine the heterogeneity effects at different planes of interest for use in the evaluation of the homogeneous approximation in modeling the Labrador Retriever head. Additional information and contour plots are presented in Appendix F.

The results obtained for these models can also be compared with the experimental results obtained for a lucite dog-head phantom at BMRR. ${ }^{4}$ The thermal neutron flux along the $10 \mathrm{~cm} \times 10 \mathrm{~cm}$ beam centerline can be examined. Figure 37 shows a comparison of the thermal flux through the entire head. Figure 38 examines the flux from $1.0 \mathrm{E}+8 \mathrm{n} / \mathrm{cm}^{2} / \mathrm{s}$ to $1.0 \mathrm{E}+9 \mathrm{n} / \mathrm{cm}^{2} / \mathrm{s}$ and $-1 \mathrm{~cm}$ to $8 \mathrm{~cm}$ for all models. The lucite dog-head phantom has a slightly larger volume than the Labrador Retriever head model used in this study. This leads to a higher development of flux in the phantom. These results are just presented to show that the fluxes obtained with the heterogeneous and homogeneous models are reasonable when compared with experimental results. 


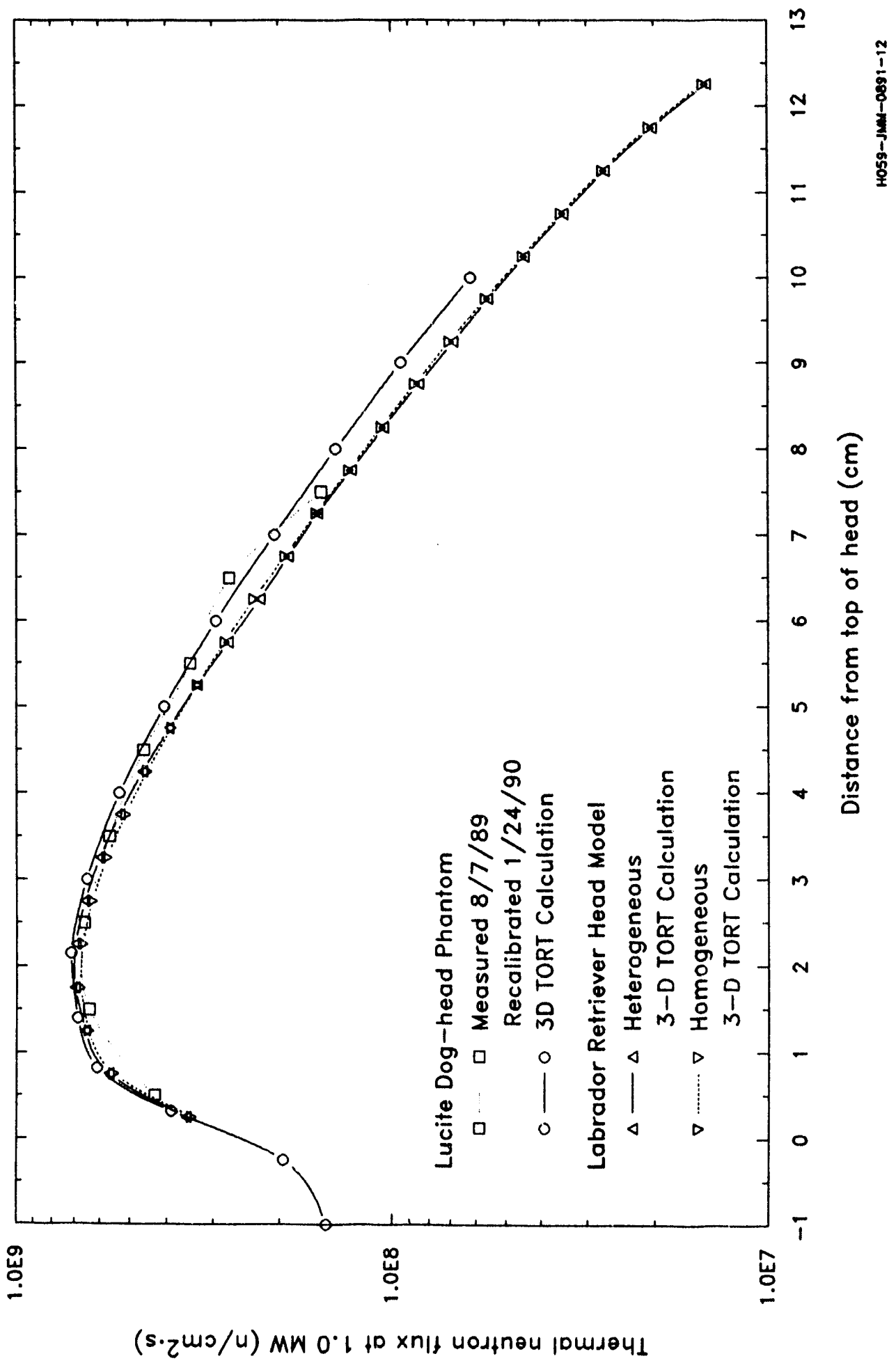

g 


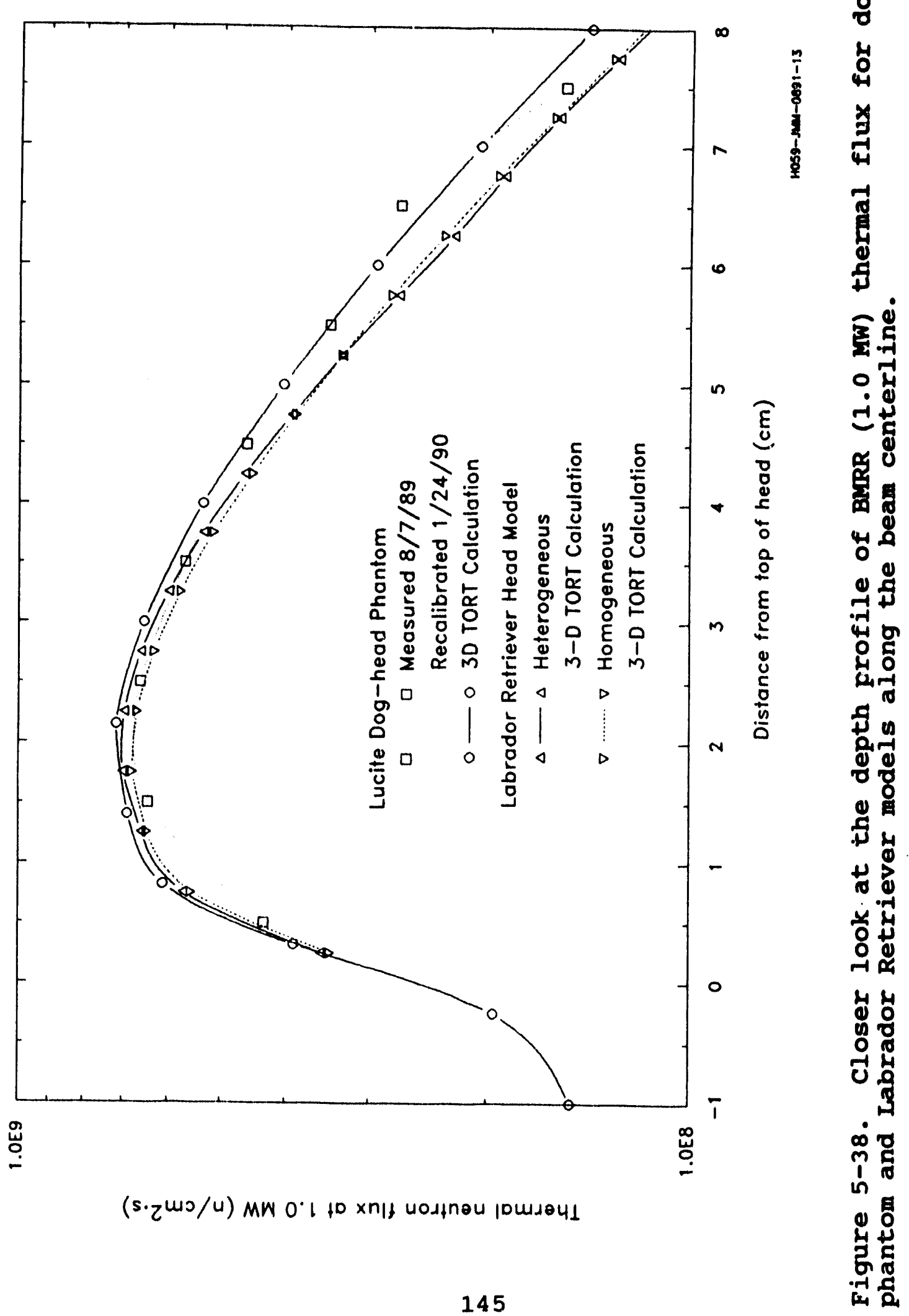


6. CONCLUSIONS AND RECOMMENDATIONS FOR FUTURE WORK

\subsection{Conclusions}

The results presented in this work indicate that, for the canine head model examined, a properly constructed homogeneous representation may be expected to provide reasonably accurate calculated neutron and gamma fluxes for use in BNCT dose distribution. The effects of macroscopic tissue composition heteroceneities and void regions are generally small, although there are some regions where the differences between the calculated heterogeneous and homogeneous results amount to 5-20\% which may be therapeutically significant. These regions include the streaming of fast neutrons behind the eyes which may be dose-limiting. There are also effects due to the nasal cavity and bone that result in 10-15\% decreases in the thermal neutron flux in certain regions of the brain when the heterogeneous case is compared with the homogeneous case. Heterogeneities result in both overestimates and underestimates of the thermal, fast, and gamma fluxes and need to be considered for each irradiation volume. Localized flux variations may be radiobiologically significant in some situations, and therefore cannot be neglected in the patient treatment planning process. 
It should be noted that this conclusion is valid only for the calculated fluxes. When converting local flux to absorbed dose for BNCT, one should always properly account for the actual local tissue composition. In addition, perturbations of the photon-induced dose profile that are caused by macroscopic heterogeneity-induced local secondary charged particle imbalance effects should also be explicitly accounted for in any calculation where the greatest achievable accuracy is required.

From the results presented in the previous chapter, one can observe that the heterogeneity effects are more pronounced at the midplane of the dog head which corresponds to the midplane of the beam. As one moves further in the $y$ direction, the peak fluxes decrease along with the heterogeneity effects. Also, the outer regions contain fewer heterogeneities leading to thermal neutron, fast neutron, and gamma fluxes that are similar to the homogeneous approximations.

The heterogeneous model presented here is an approximation in that the actual curvature and anatomy of the canine head were represented by mesh intervals of $0.5 \mathrm{~cm}$ due to the rectangular geometric structure requirements of the code. Because there were only twenty mesh intervals in the $y$ direction, materials modeled in one sagittal section 
may no longer be in the next. Also, the data available were in increments of $1 \mathrm{~cm}$ unless each axial section was used. The use of the axial sections to augment the geometric data from the sagittal sections would have made the modeling more anatomically complex, requiring finer mesh sizing, and may not have been more accurate due to the slightly different sizes of the two dogs. Additionally, the computer may not have been able to handle such an increase in the mesh structure. However, the model includes inhomogeneities that have not been addressed previously and is detailed in many respects, including the chemical compositions of canine tissues and the number of tissues modeled. In the future, software will be available to more accurately model heterogeneities using magnetic resonance images. This study will aid in the validation of such software at the INEL.

The canine tissue compositions measured in this work are, for the most part, consistent with available published data, but relatively large statistical variations are observed for some elements in some samples. The nitrogen content of various tissues is of particular importance for BNCT because of the nitrogen $(n, p)$ interaction. This contributes a fraction of the total non-selective background dose at depth which cannot be neglected. It should also be noted that the measurements presented here are for the sample-average compositions and are appropriate only for 
neutron and gamma transport analysis on a macroscopic scale. Specific substructures within a given tissue sample (e.g. capillary walls) could have quite different elemental compositions compared to the sample average. This must be recognized when performing radiation transport and dosimetry calculations on a microscopic scale.

Because canine head structures vary to some extent among different breeds, caution should be used when extrapolating the results presented here to other canine breeds. The general trends observed here should be reasonably valid for other breeds, but the detailed results will not be identical. Heterogeneity effects of the type examined here would be expected to be somewhat less significant in breeds having smaller heads than the Labrador Retriever. This is due to the fact that the physical size of structural heterogeneities in the heads of such breeds will be smaller relative to the characteristic neutron and photon relaxation lengths. For extremely large dogs, the opposite would be true.

Finally, the human cranial structure in the region of interest for BNCT of brain tumors is geometrically somewhat simpler, at least as far as macroscopic radiation transport calculations are concerned, than is the case with dogs. For most typical beam orientations, a relatively simple 
heterogeneous model consisting of skin, skull, and brain is probably sufficient for most applications, although it is useful to also explicitly represent other structures such as the tumor, eyes, and the pituitary gland for dose editing purposes.

\subsection{Recommendations for Future Work}

In the field of patient treatment planning, many areas need to be expanded upon and are currently being investigated. One of these fields is in the area of elemental tissue analysis for canines and humans. This data, along with the geometric information, is essential for a detailed heterogeneous model. In addition, base values, such as those established by the ICRU for the average man in dosimetry, are needed for the elemental tissue compositions required in the detailed modeling of heterogeneities.

In the area of patient treatment planning, further work is needed to properly individually validate homogeneous models which are used to model clinical trials. This research is being performed to evaluate boron neutron capture therapy as a potential treatment for human brain tumors. However, the study presented here only examines the heterogeneity effects in the Labrador Retriever head. The effect of inhomogeneities will differ in other breeds, and 
will also differ in humans. As a result, both macroscopic and microscopic heterogeneity effects should be examined in detail before using any data from an approximation for actual human treatment. It is also recommended that a detailed model be made of any animal that is to be treated witl. BNCT (e.g. one large canine head model may be sufficient for other large dogs, but not for rabbits or rats). If a dog is to be irradiated, then the size of this dog should be taken into consideration when utilizing the results froi: this model or another.

In the validation of homogeneous models, both Type 1 and Type 3 heterogeneity effects need to undergo further investigation. Type 1 effects, those due to spatial variations in tissue density and composition, can be examined in studies such as this one where a detailed heterogeneous model is compared with a volume-weighted homogeneous model. Type 3 effects are those heterogeneity effects that can occur on both a macroscopic and microscopic scale, induced by charged-particle imbalance. The field of microdosimetry includes examination of Type 3 effects on a microsiopic scale in BNCT.

Finally, this study only examines the effects of the inclusion of heterogeneities in modeling. It does not eximine the effect of including the proper tissue 
composition data in the model. It also does not include a tumor in the model. The elemental composition of the tumor is most likely different from other tissues and should be modeled as such as more refinements are required and made in three-dimensional modeling techniques. 


\section{REFERENCES}

1. G. Locher, "Biological effects and therapeutic possibilities of neutrons," American Journal of Roentgenology $36,1-13$ (1936).

2. L. E. Farr, W. H. Sweet, J. S. Robertson, C. G. Foster, H. B. Locksley, D. L. Sutherland, M. L. Mendelsohn, and E. E. Stickley, "Neutron capture therapy with boron in the treatment of glioblastoma multiforme," American Journal of Roentgenology 71, 279-291 (1954).

3. J. R. Choi, "Development and characterization of an epithermal beam for boron neutron capture therapy at the MITR-II Research Reactor," doctoral thesis in Nuclear Engineering, Massachusetts Institute of Technology, (April 1991).

4. H. Hatanaka, "Clinical results of boron neutron capture therapy," Neutron Beam Design, Development, and Performance for Neutron Capture Therapy, New York: Plenum Press, 15-21 (1990).

5. F. J. Wheeler, M. L. Griebenow, D. W. Wessol, and D. W. Nigg, "Analytical modeling for neutron capture therapy," Strahlentherapie und Onkologie 165, 186-188 (1989).

6. D. W. Nigg, P. D. Randolph, and F. J. Wheeler, "Demonstration of three-dimensional deterministic radiation transport theory dose distribution analysis for boron neutron capture therapy," Medical Physics 18, 43-53 (1991).

7. F. J. Wheeler and D. W. Nigg, "Three-dimensional radiation dose distribution analysis for boron neutron capture therapy," To be published, Nuclear Science and Engineering (January 1992).

8. R. G. Zamenhof, S. Clement, K. Lin, C. Lui, D. Ziegelmiller, and 0 . K. Harling, "Monte Carlo treatment planning and high-resolution alpha-track autoradiography for neutron capture therapy," strahlentherapie und onkologie 165, 188-192 (1989).

9. J. C. Yanch, X-L. Zhou, and G. L. Brownell, "A Monte Carlo investigation of the dosimetric properties of monoenergetic neutron beams for neutron capture therapy," Radiation Research 126, 1-20 (1991).

10. W. A. Rhoades and R. I. Childs, "The TORT threedimensional discrete ordinates neutron/photon transport code," Report No. ORNL-6262, Oak Ridge National Laboratory, 
Oak Ridge, TN (1987).

11. A. J. Vander, J. H. Sherman, and D. S. Luciano, Human Physiology 5th ed. New York: McGraw-Hill Publishing (1990).

12. G. J. Pilkington, "The biology, pathogenesis and spread of malignant glioma," Strahlentherapie und onkologie 165, 235-238 (1989).

13. R. V. Dorn, J. H. Spickard, and M. L. Griebenow, "A proposed protocol for clinical trials of boron neutron capture therapy," Clinical Aspects of Boron Neutron Capture Therapy, New York: Plenum Press, 135-144 (1989).

14. N. K. Wessells and J. L. Hopson, Biology 1st ed. U. S.: Random House (1988).

15. T. Kobayashi, K. Aoki, M. Ono, and K. Kanda, "Measurement of ${ }^{10} \mathrm{~B}$ concentrations and its location in phantom by prompt gamma-ray spectroscopy," Neutron Capture Therapy, Japan: Nishimura, 2-7 (1986).

16. A. H. Soloway, F. Alam, F. F. Barth, A. K. M. Anisuzzaman, and B. V. Bapat, "Tumor targeting agents for neutron capture therapy," Neutron Beam Design, Development, and Performance for Neutron Capture Therapy, New York:

Plenum Press, 37-47 (1990).

17. D. Gabel, S. Foster, and R. G. Fairchild, "The Monte Carlo simulation of the biological effect of the ${ }^{10} \mathrm{~B}(n, \gamma){ }^{7} \mathrm{Li}$ reaction in cells and tissue and its implication for boron neutron capture therapy," Radiation Research 111, 14-25 (1987).

18. E. J. Hall, Radiobjology for the Radiologist, 3rd ed., Philadelphia: J. B. Lippincott (1988).

19. D. Gabel, "Tumor-seeking compounds for boron neutron capture therapy: synthesis and biodistribution," clinical Aspects of Neutron Capture Therapy, New York: Plenum Press, 233 (1989).

20. J. M. Moran, D. W. Nigg, F. J. Wheeler, and W. F. Bauer, "Macroscopic geometric heterogeneity effects in radiation dose distribution analysis for boron neutron capture therapy," Isulcal Physics, accepted for publication (1992).

21. F. J. Wheeler, M. L. Griebenow, D. E. Wessol, D. W. Nigg, and R. A. Anderl, "A Stochastic Model for High-LET 
Response for Boron Neutron Capture Therapy," Clinical Aspects of Neutron Capture Therapy, New York: Plenum Press (1989).

22. V. P. Bond, M. N. Varma, C. A. SonDnaus, and L. E. Feinendegen, "An Alternative to Absorbed Dose, Quality, and RBE at Low Exposures," Radiation Research 104, S-52-S-57 (1985).

23. P. R. Gavin, S. L. Kraft, L. R. Wendling, and D. L. Miller, "Canine spontaneous brain tumors--a large animal model for BNCT," Strahlentherapie und onkologie 165, 225-228 (1989).

24. T. Matsumoto and O. Aizawa, "Head phantom experiment and calculation for neutron capture therapy using various neutron beams," Strahlentherapie und Onkologie 165, 98-100 (1989).

25. W. A. Rhodes and F. R. Mynatt, "The DOT III twodimensional discrete ordinates transport code," Report No. ORNL-TM-4230, Oak Ridge National Laboratory, Oak Ridge, TN (1973) .

26. O. L. Deutsch and B. W. Murray, "Monte Carlo dosimetry calculation for boron neutron capture therapy in the treatment of brain tumors," Nuclear Technology 26, 320-339 (1975).

27. O. K. Harling, S. D. Clement, J. R. Choi, J.A. Bernard, and R. G. Zamenhof, "Neutron beams for neutron capture therapy at the MIT Research Reactor," Strahlentherapie und Onkologie 165, 90-92 (1989).

28. R. G. Zamenhof, S. clement, K. Lin, C. Lui, D. Ziegelmiller, and 0 . K. Harling, "Monte carlo treatment planning and high-resolution alpha-track autoradiography for neutron capture therapy," Strahlentherapie und onkologie $165,188-192$ (1989).

29. R. G. Zamenhof, S. D. Clement, O. K. Harling, J. F. Brenner, D. E. Wazer, H. Madoc-Jones, and J. C. Yanch, "Monte Carlo based dosimetry and treatment planning for neutron capture therapy of brain tumors," Neutron Beam Design, Development, and Performance for Neutron Capture Therapy, New York: Plenum Press, 283-305 (1990).

30. J. R. Mehta, K. G. Braund, R. E. McKerrell, and M. Toivio-Kinnucan, "Analysis of muscle elements, water, and total lipids from healthy dogs and Labrador Retrievers with hereditary muscular dystrophy," American Journal of Veterinary Research 50, 640-644 (1989). 
31. J. R. Mehta, K. G. Braund, and M. Toivio-Kinnucan, "Elemental composition, water, and total lipid content in peripheral nerves, spinal cord and brain of healthy adult dogs," Research in Veterinary science 49, 250-252 (1990).

32. P. Altman and D. Dittmer, eds., Biolow Data Book, Federation of American Societies for Experimental Biology, Bethesda, MD (1972).

33. W. S. Spector, ed., Handbook of Biological Data,

Philadelphia: W. B. Saunders (1956).

34. F. A. Duck, Physical Properties of Tissue: A Comprehensive Reference Book, San Diego: Academic Press (1990).

35. S. L. Kraft, P. R. Gavin, L. R. Wendling, and V. K. Reddy, "Canine brain anatomy on magnetic resonance images," Veterinary Radiology 30, 147-158 (1989).

36. W. F. Bauer, D. A. Johnson, S. M. Steele, K. Messick, D. L. Miller and W. A. Propp, "Gross boron determination in biological samples by inductively coupled plasma-atomic emission spectroscopy," strahlentherapie und onkologie 165, 176-179 (1989).

37. ICRU Report 10b, Physical Aspects of Irradiation. National Bureau Standard Handbook 85. Washington, DC: U. s. Government Printing office (1964).

38. Y. S. Kim, "Human tissues: Chemical composition and photon dosimetry data," Radiation Research 57, 38-45 (1974).

39. M. Awschalom, I. Rosenberg, and A. Mravca, "Kermas for various substances averaged over the energy spectra of fast neutron therapy beams: A study in uncertainties," Medical Physics 10, 395-409 (1983).

40. R. A. Brooks, G. Dichiro, and M. R. Keller, "Explanation of cerebral white-gray contrast in computed tomography," Journal of Computed Assisted Tomography 4, 489491 (1980).

41. H. Q. Woodard and D. R. White, "The composition of body tissues," British Journal of Radiology 59, 1209-1219 (1986).

42. D. R. White, H. Q. Woodard, and S. M. Hammond, "Average soft-tissue and bone models for use in radiation dosimetry," British Journal of Radiology 60, 907-913 (1987).

43. F. J. Wheelex, D. K. Parsons, B. L. Rushton, and D. W. 
Nigg, "Epithermal neutron beam design for neutron capture therapy at the PBF and BMRR facilities," Nuclear Technology 92, 106-117 (1990).

44. R. W. Roussin, "BUGLE-80 coupled 47-neutron, 20-gamma ray $P 3$ cross section library," Report No. DLC-75, Radiation Shielding Information Center (1980). 
The submitted thesis publication has been authored by a contractor of the U.S. Government under DOE Contract No. DE-AC07-76ID01570. Accordingly, the U.S. Government retains a nonexclusive, royalty-free license to publish or reproduce the published form of this contribution, or allow others to do so, for U.S. Government purposes. 
APPENDIX A

TABLE OF MEASURED ELEMENTAL COMPOSITIONS 


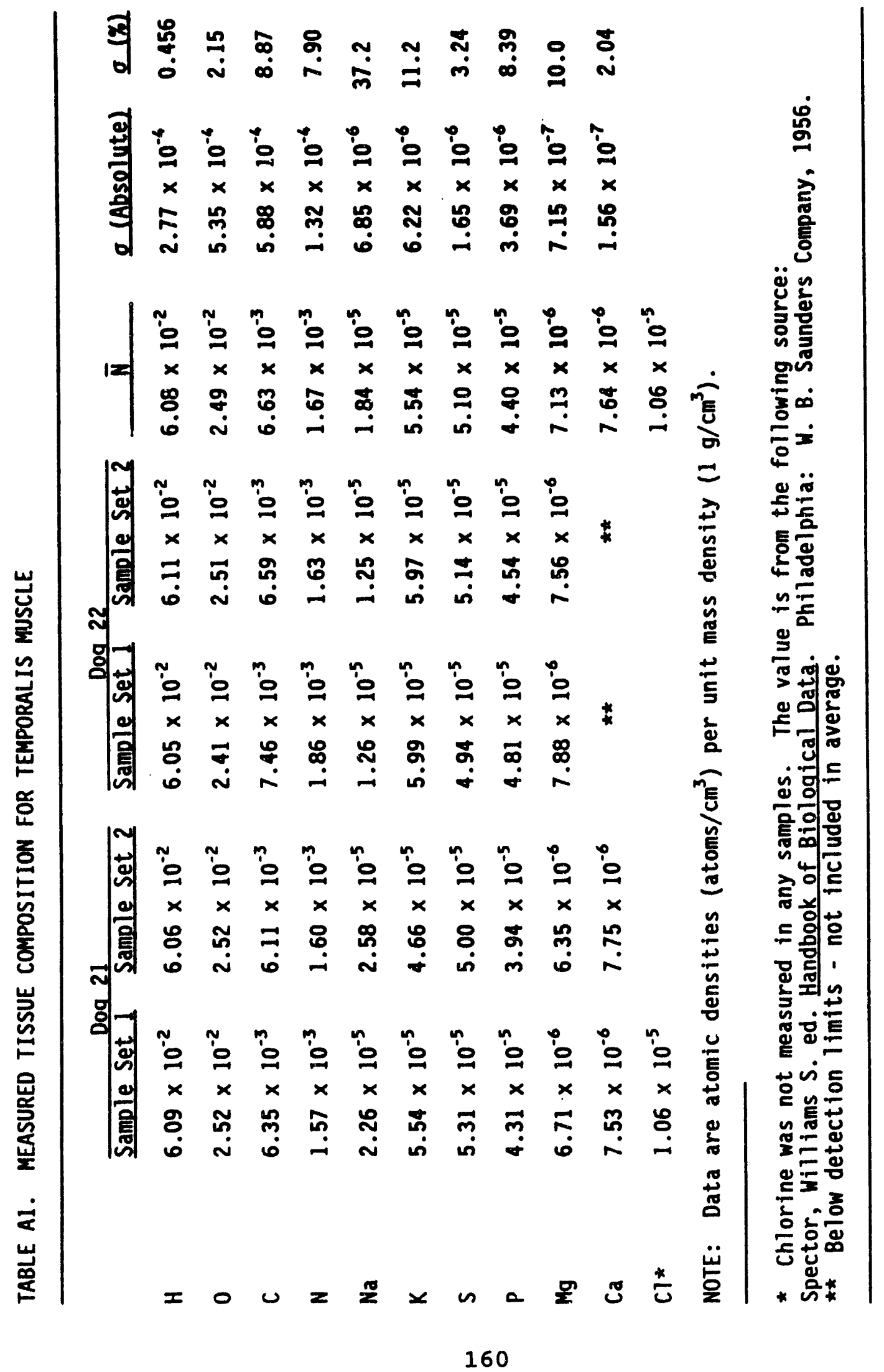




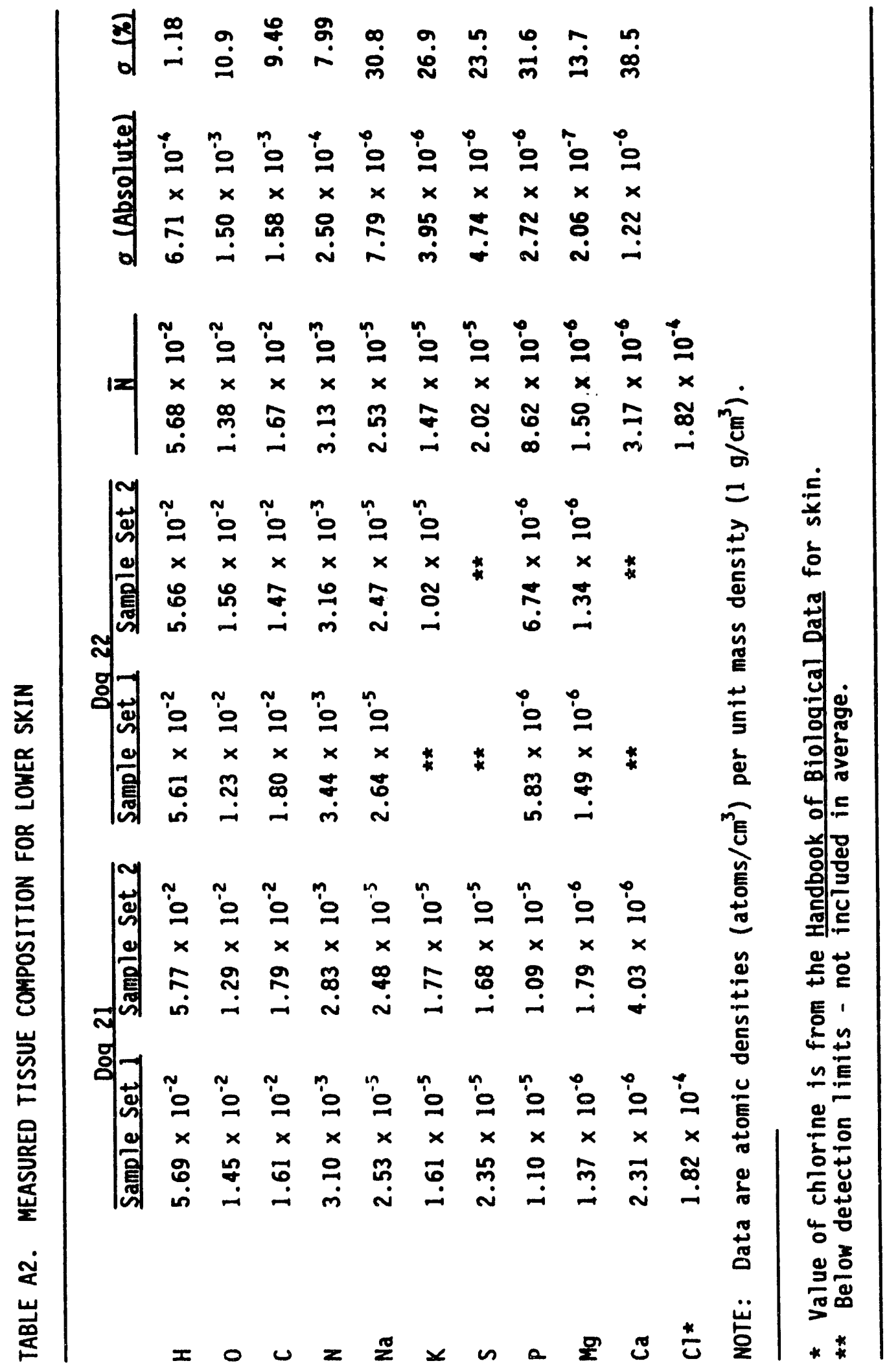




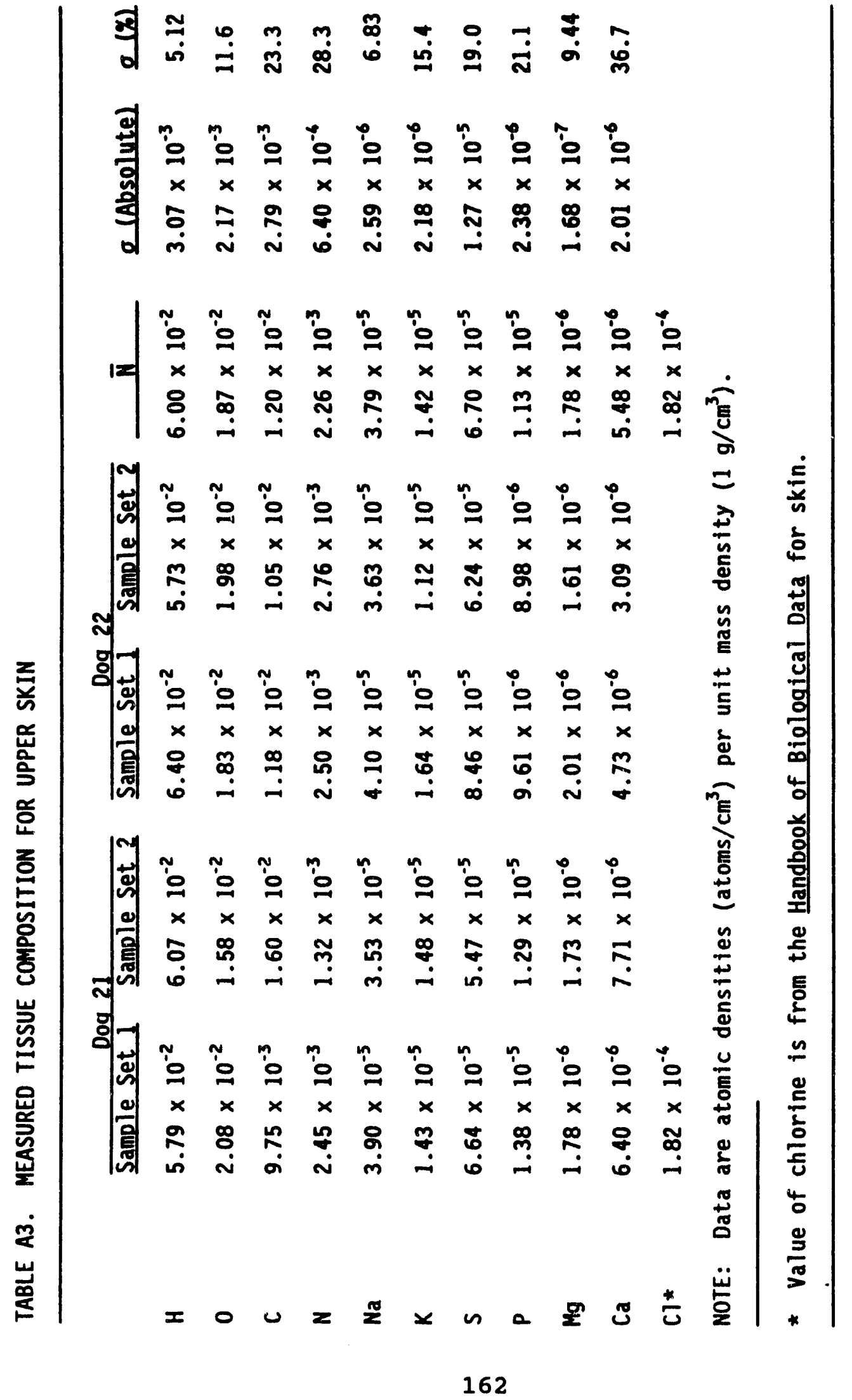




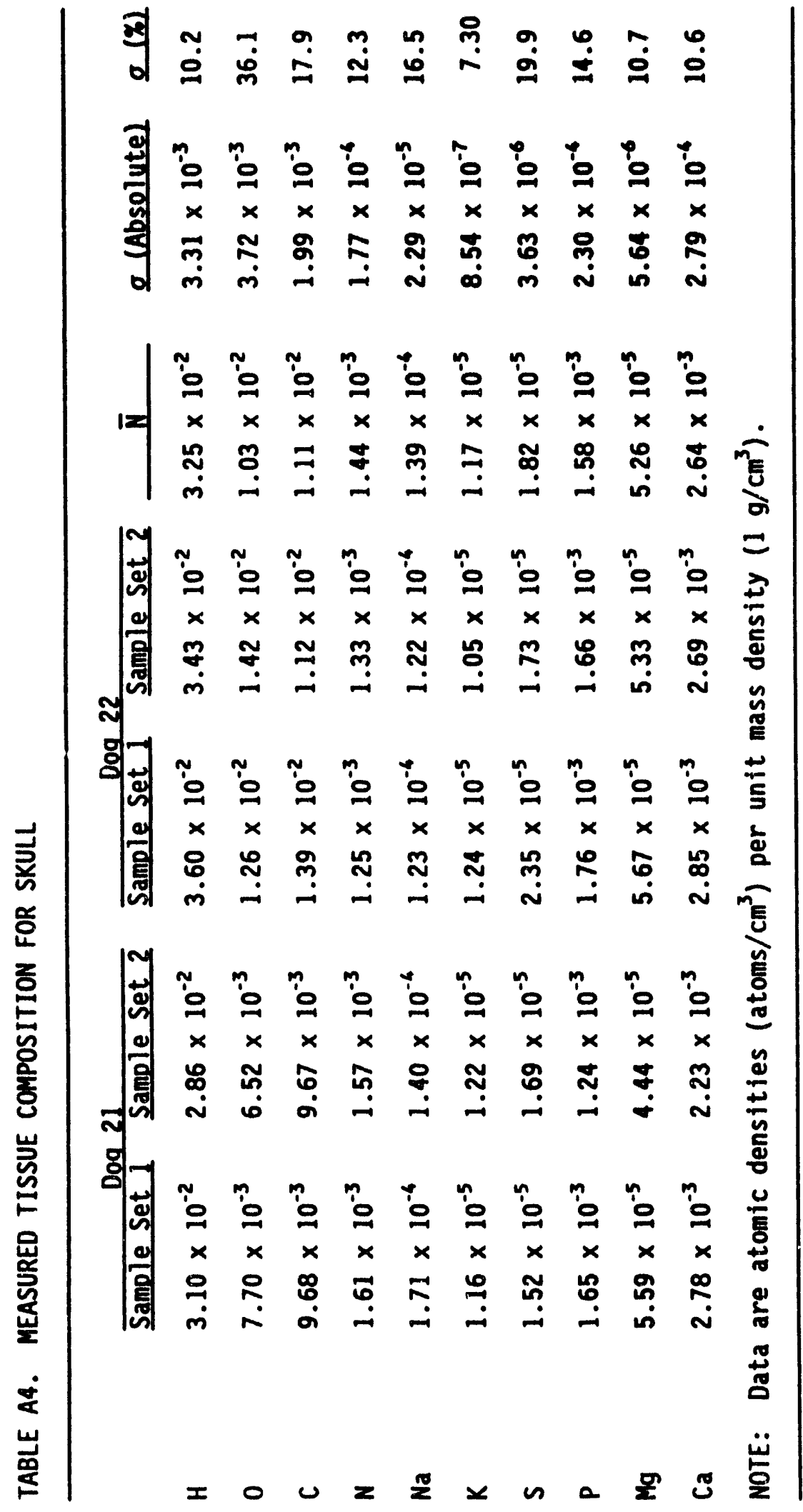




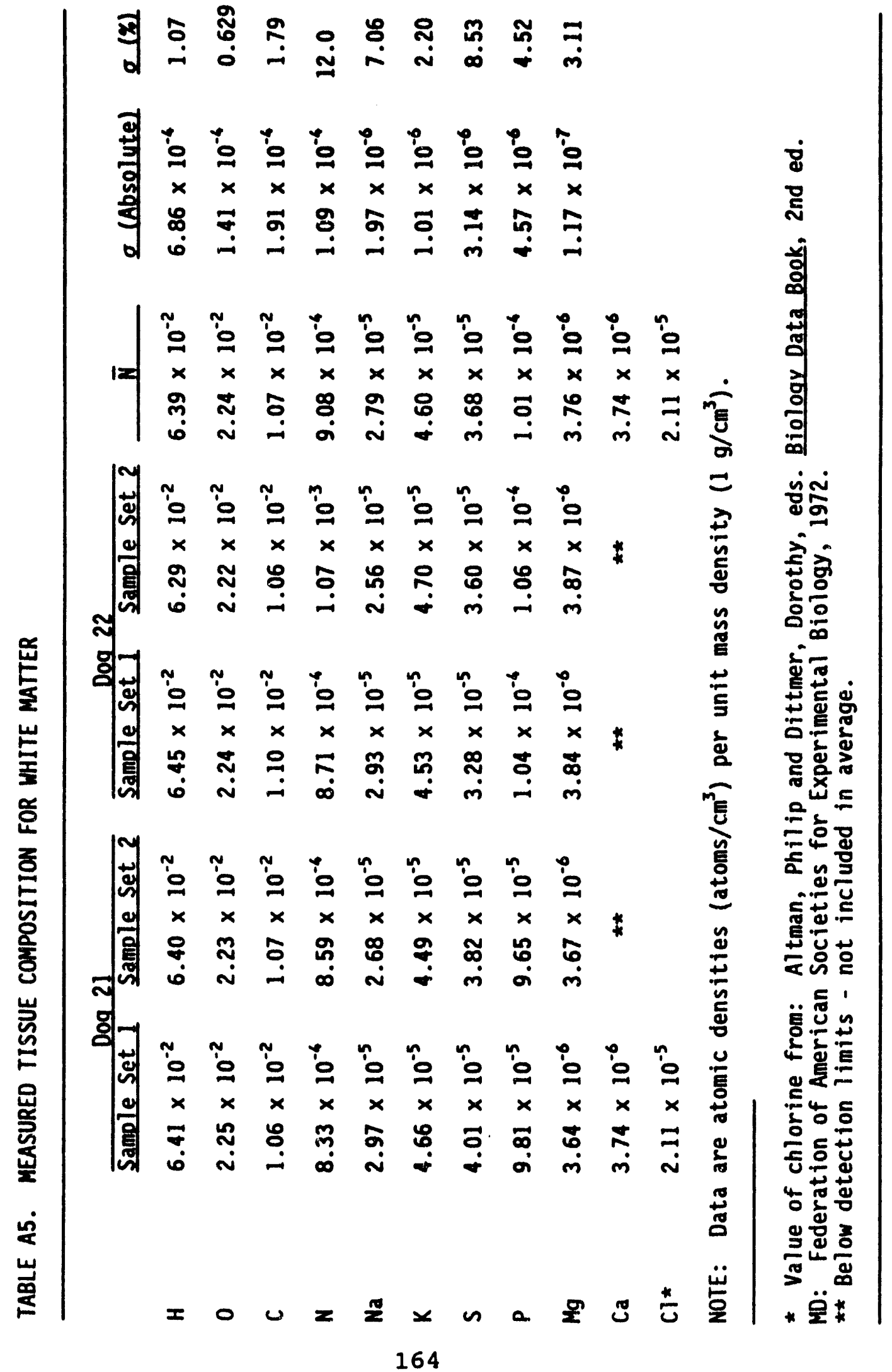




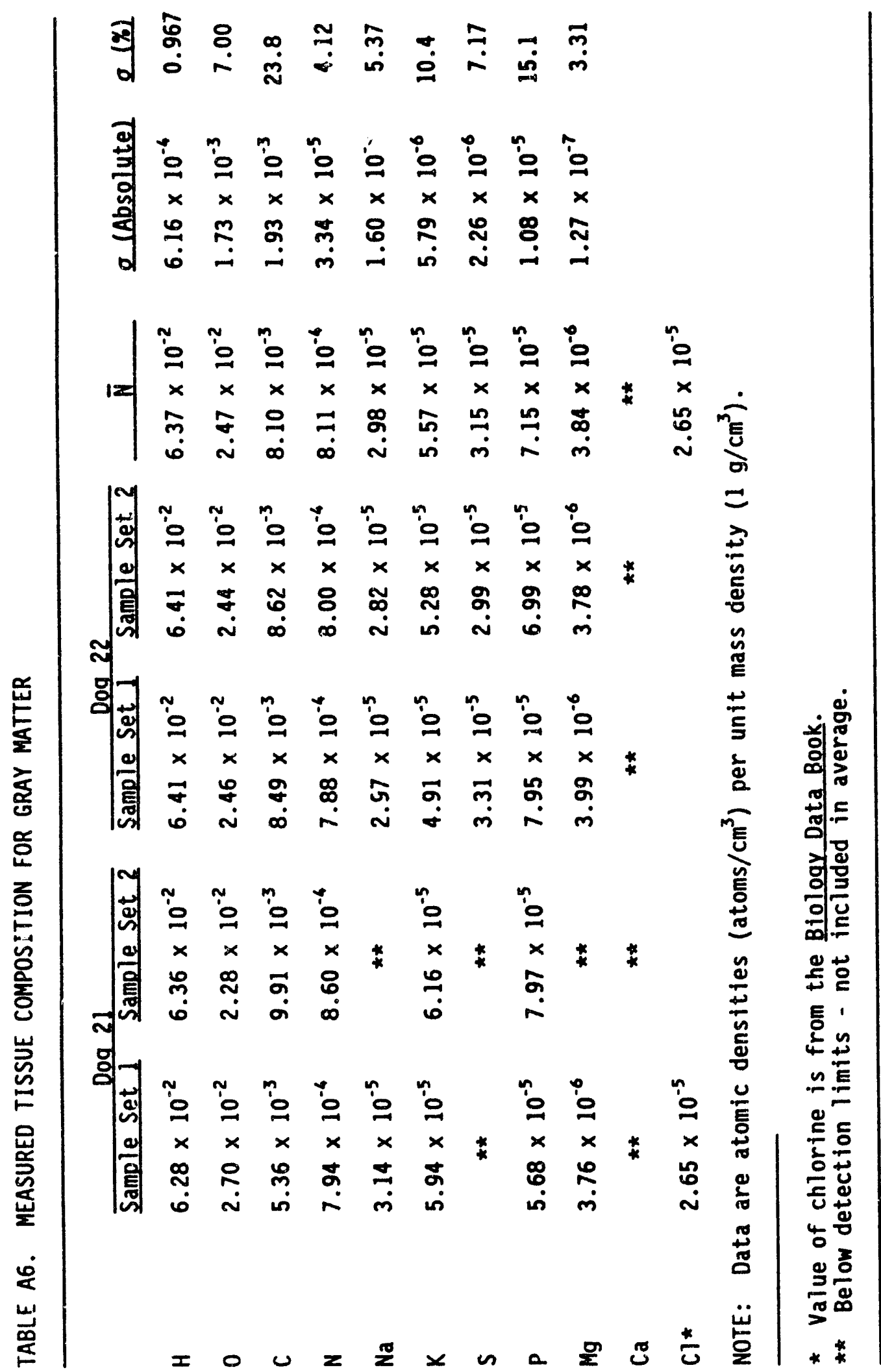




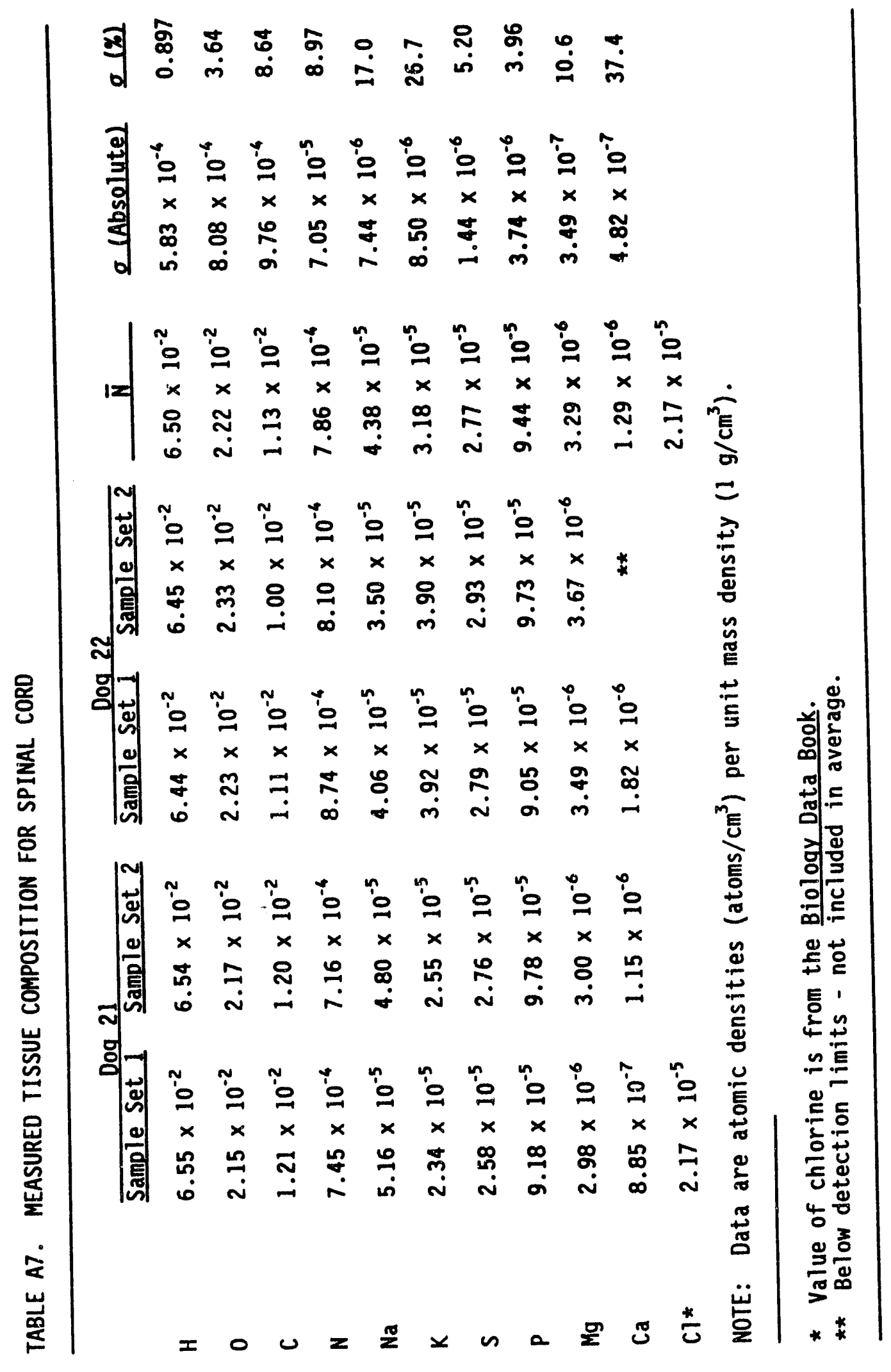




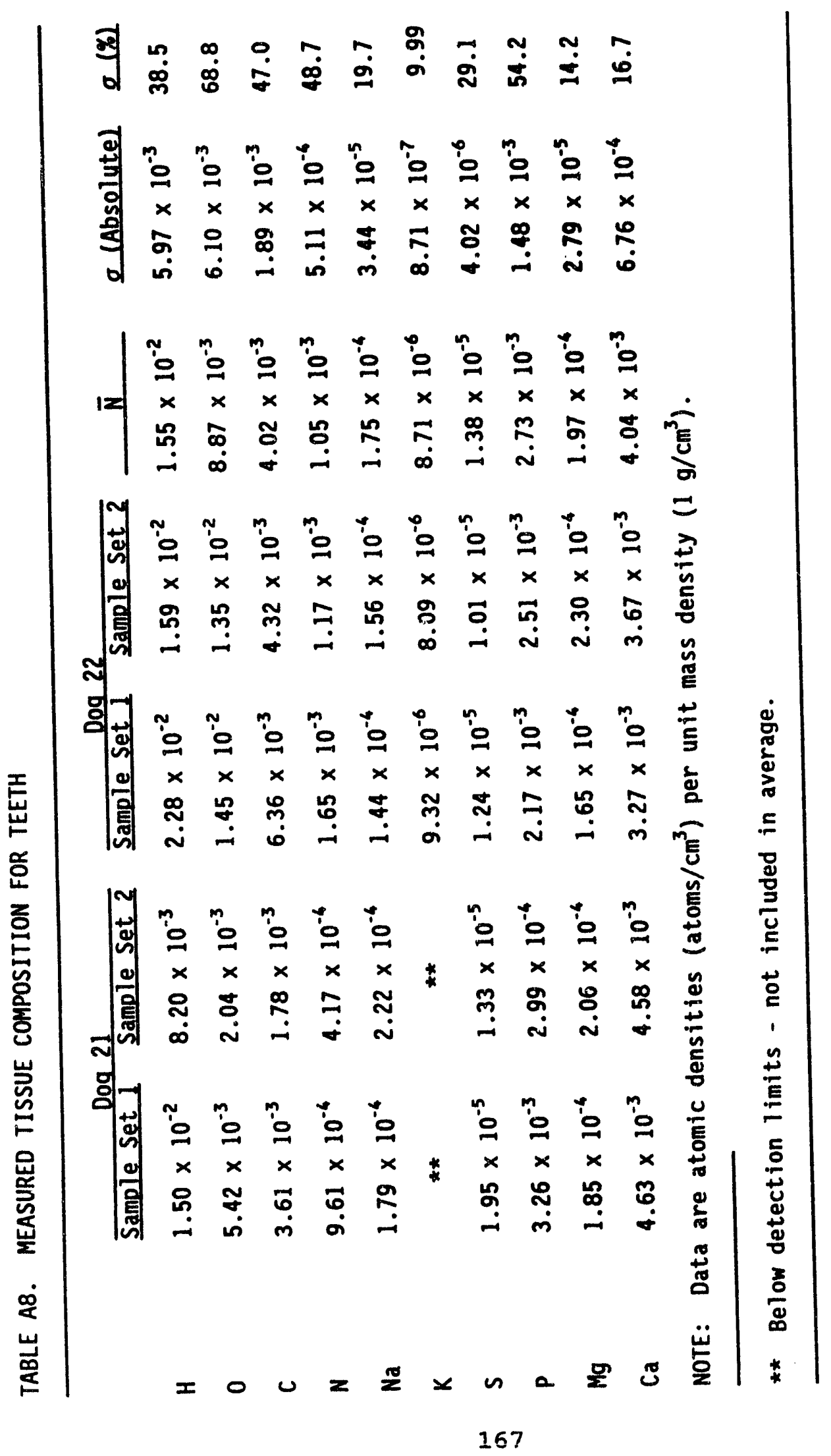




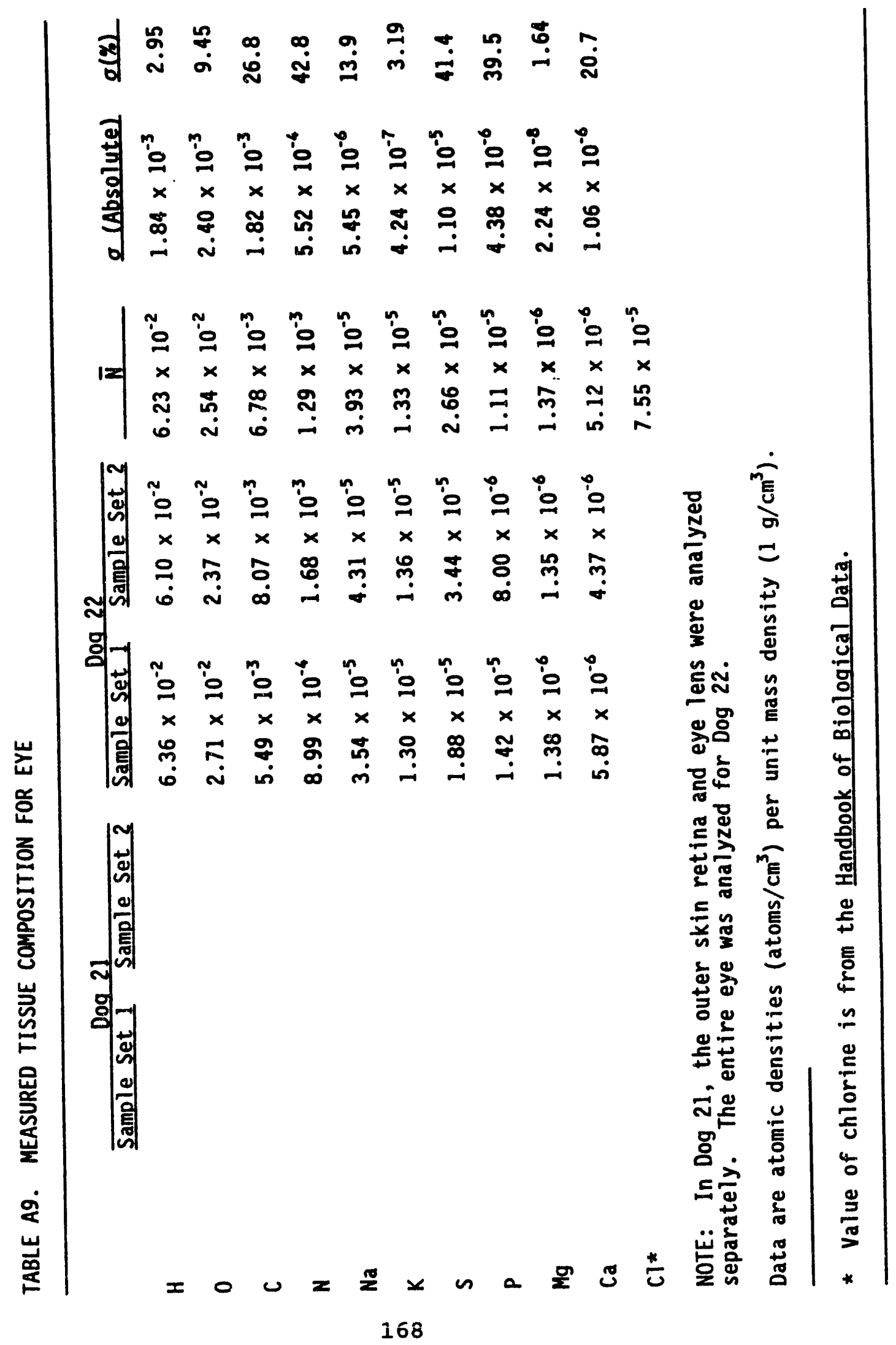




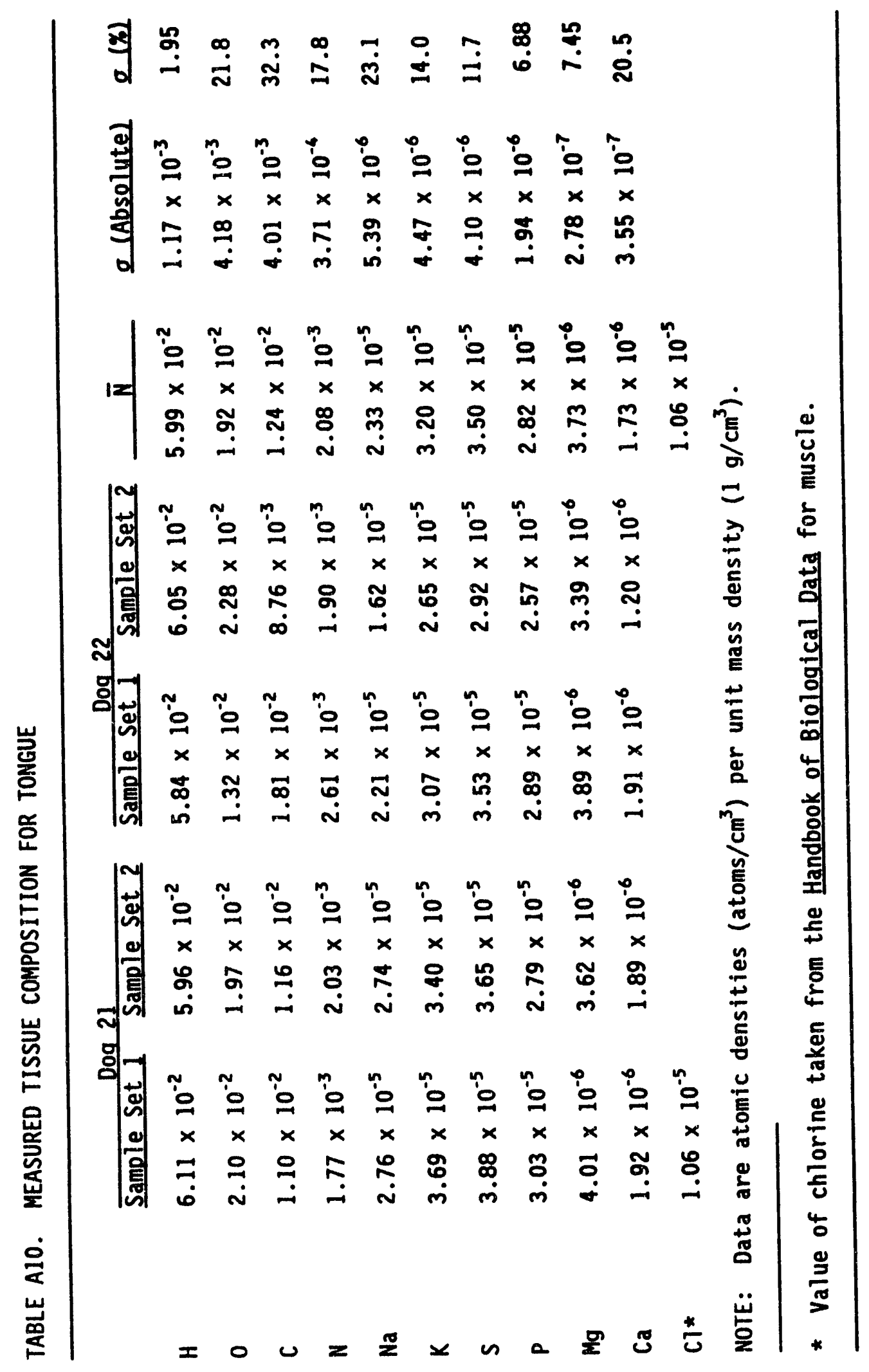




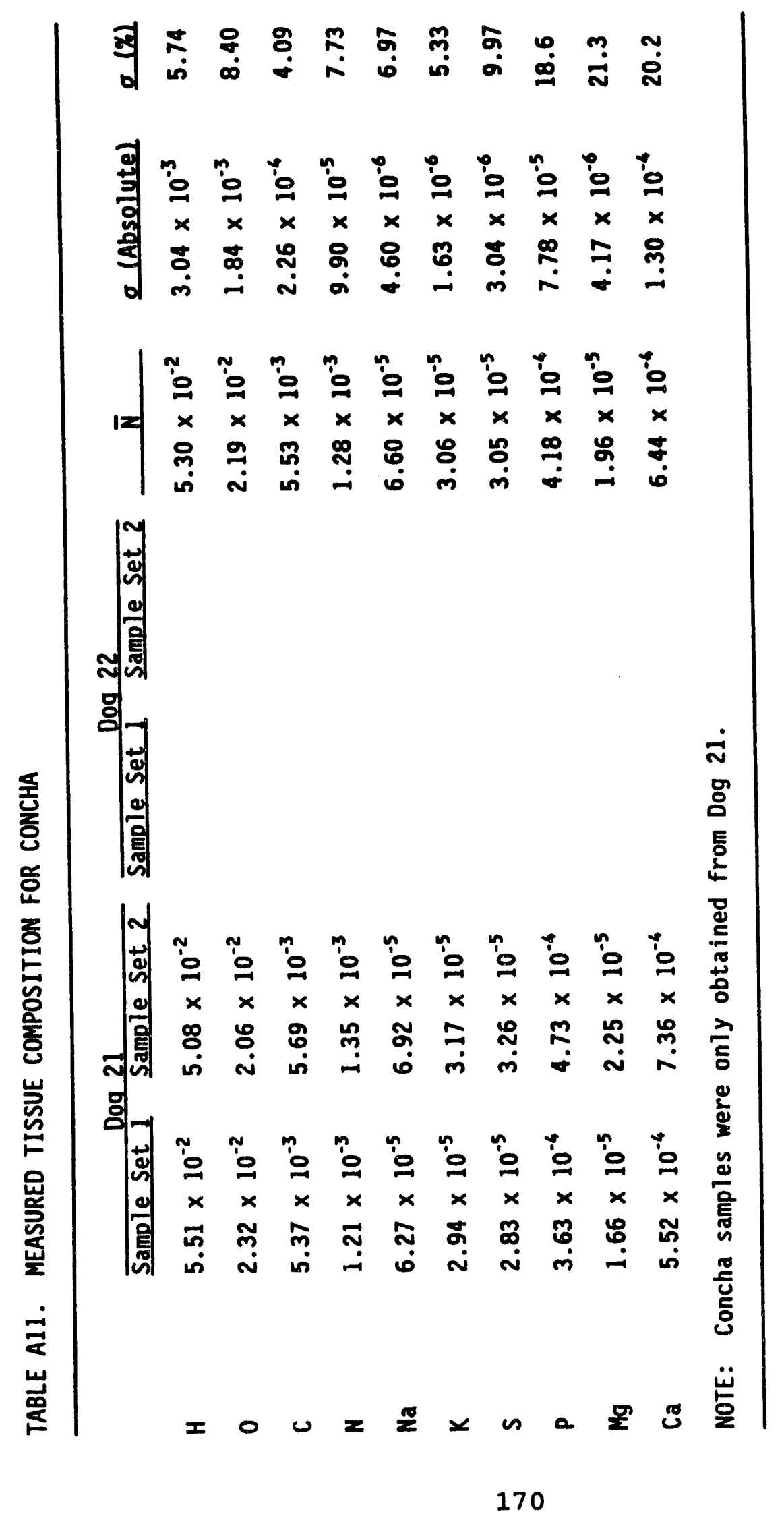




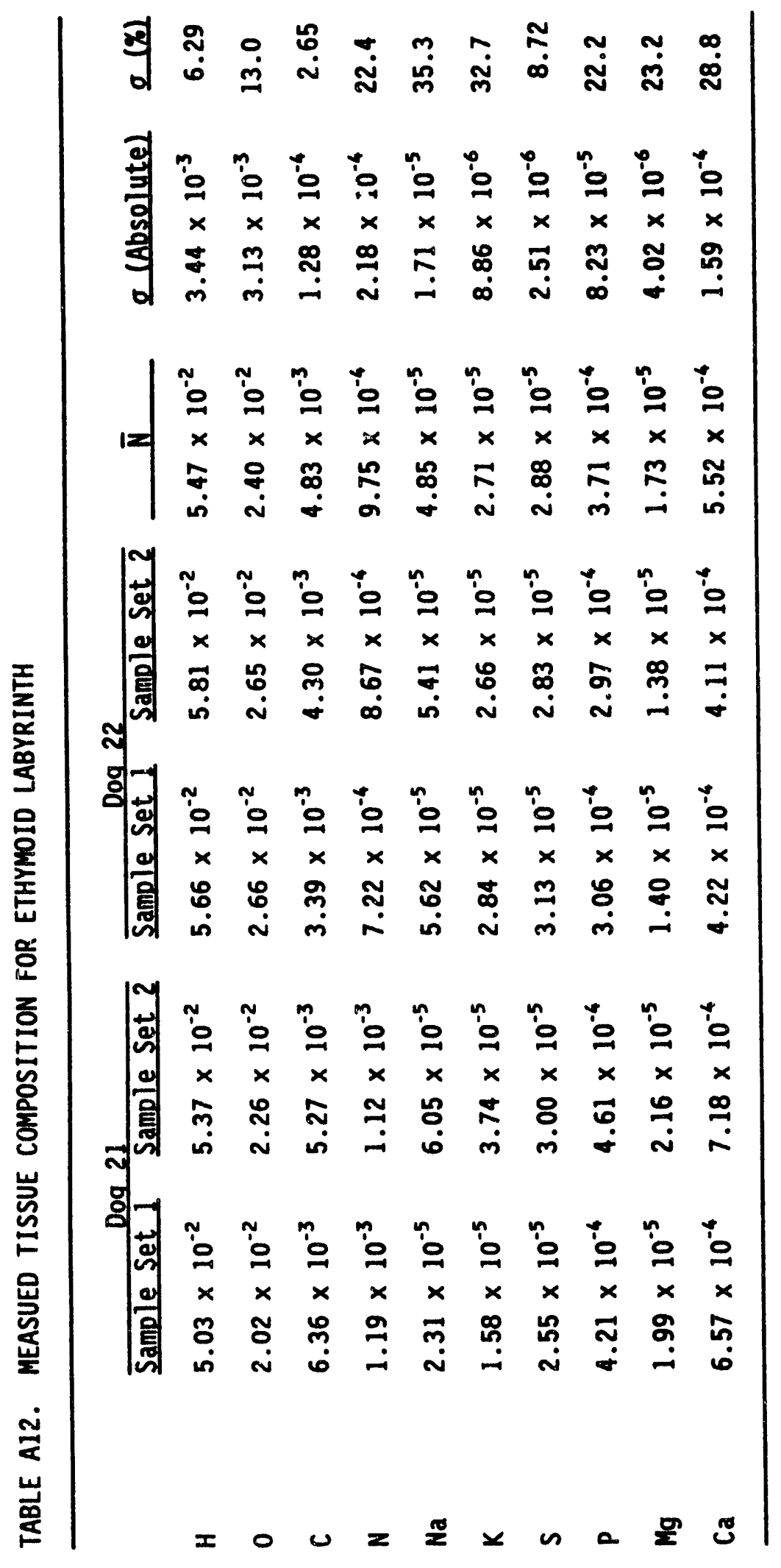




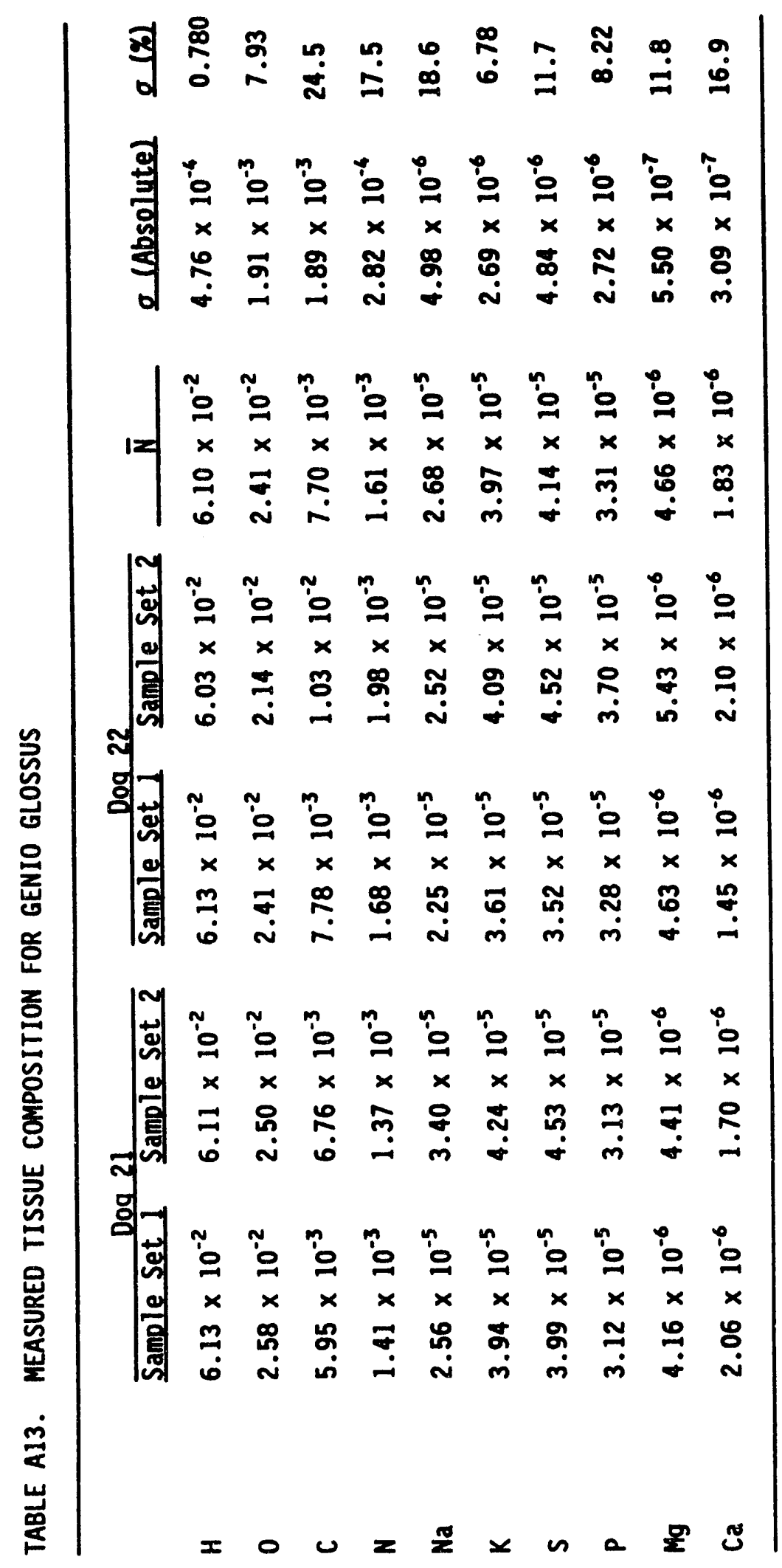




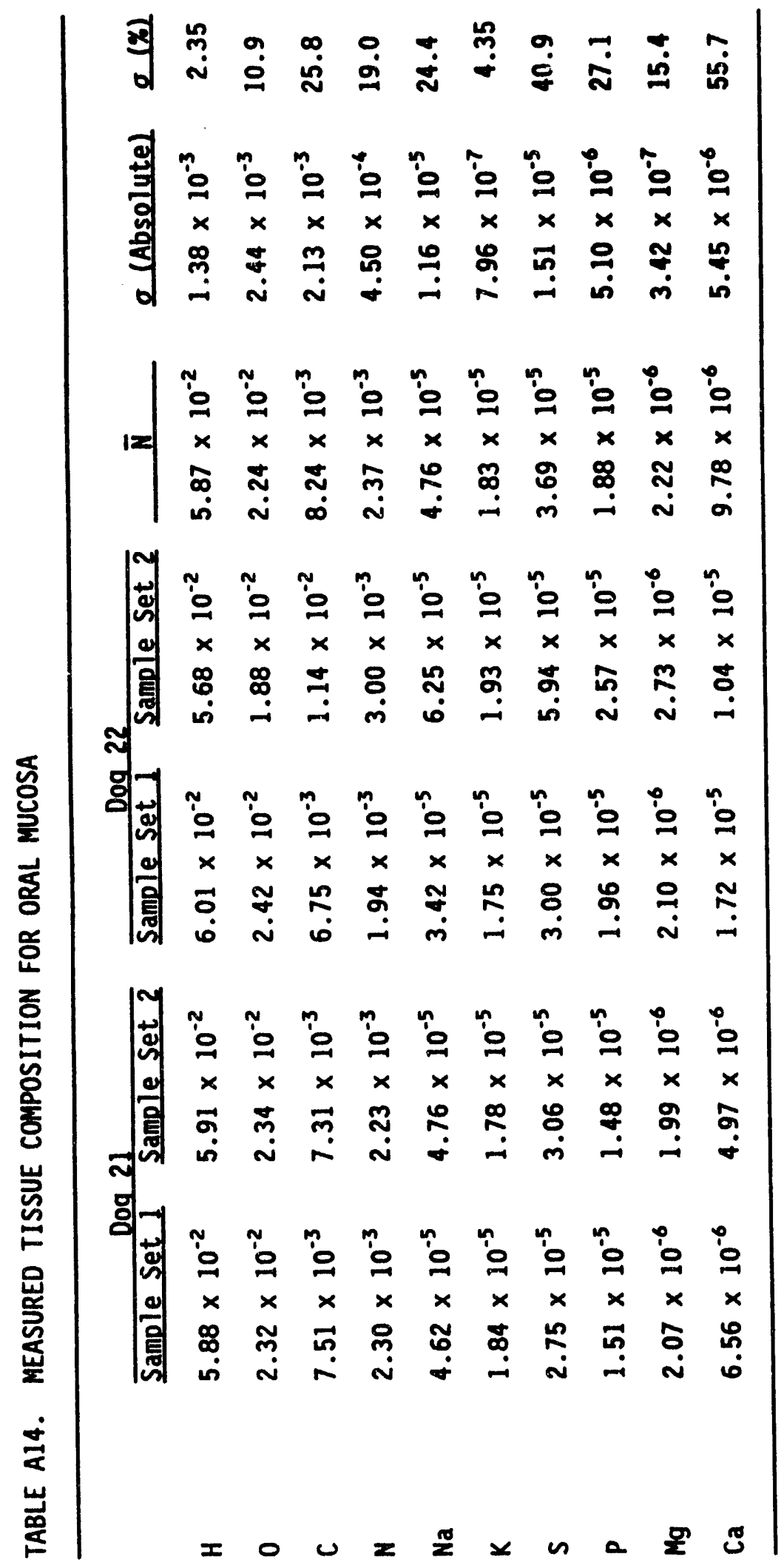




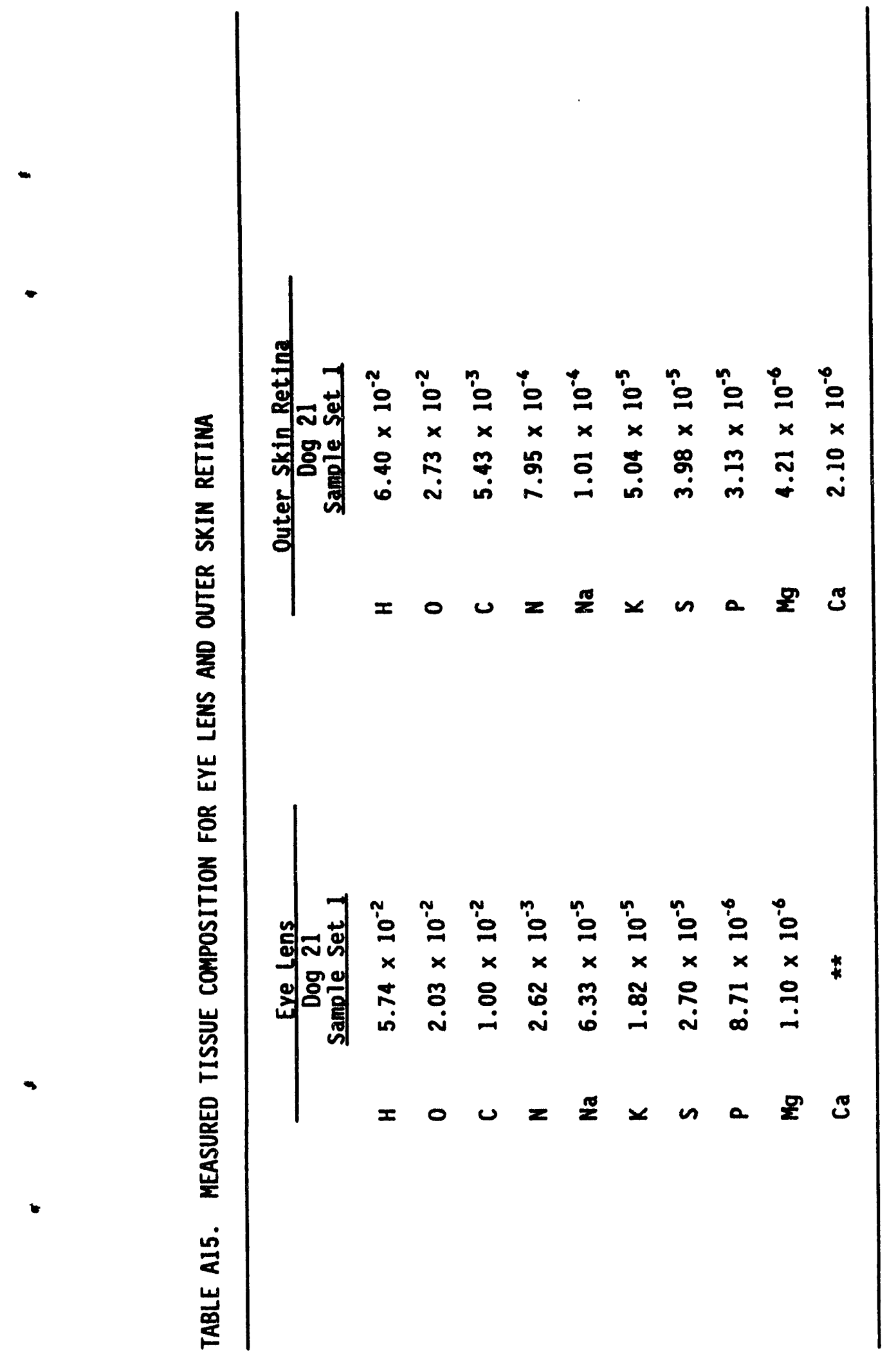


APPENDIX B

SAGITTAL SECTION PHOTOGRAPHS

175 


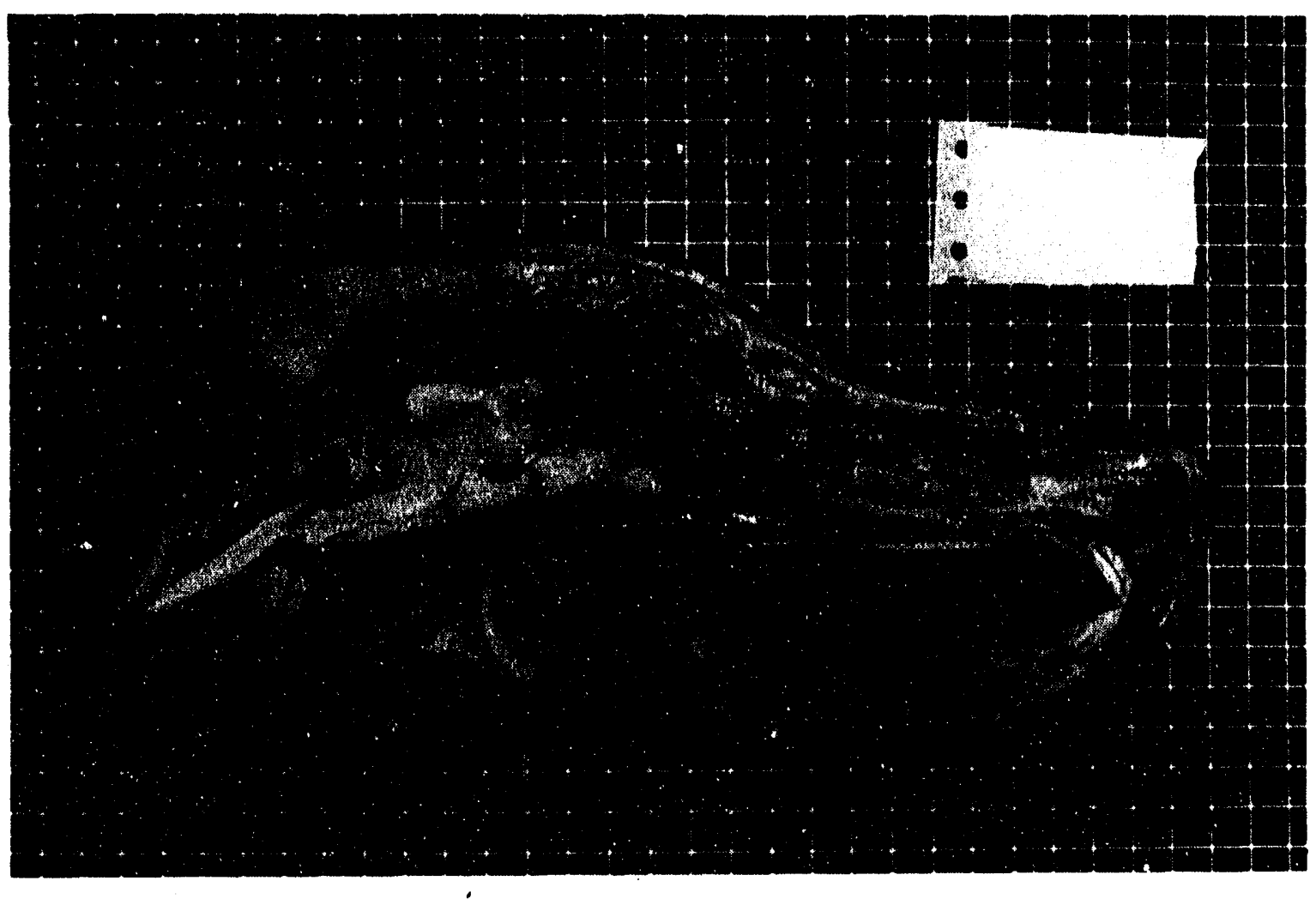

Figure B-1. Photograph of sagittal slice 7 right at $y=0 \mathrm{~cm}$. 


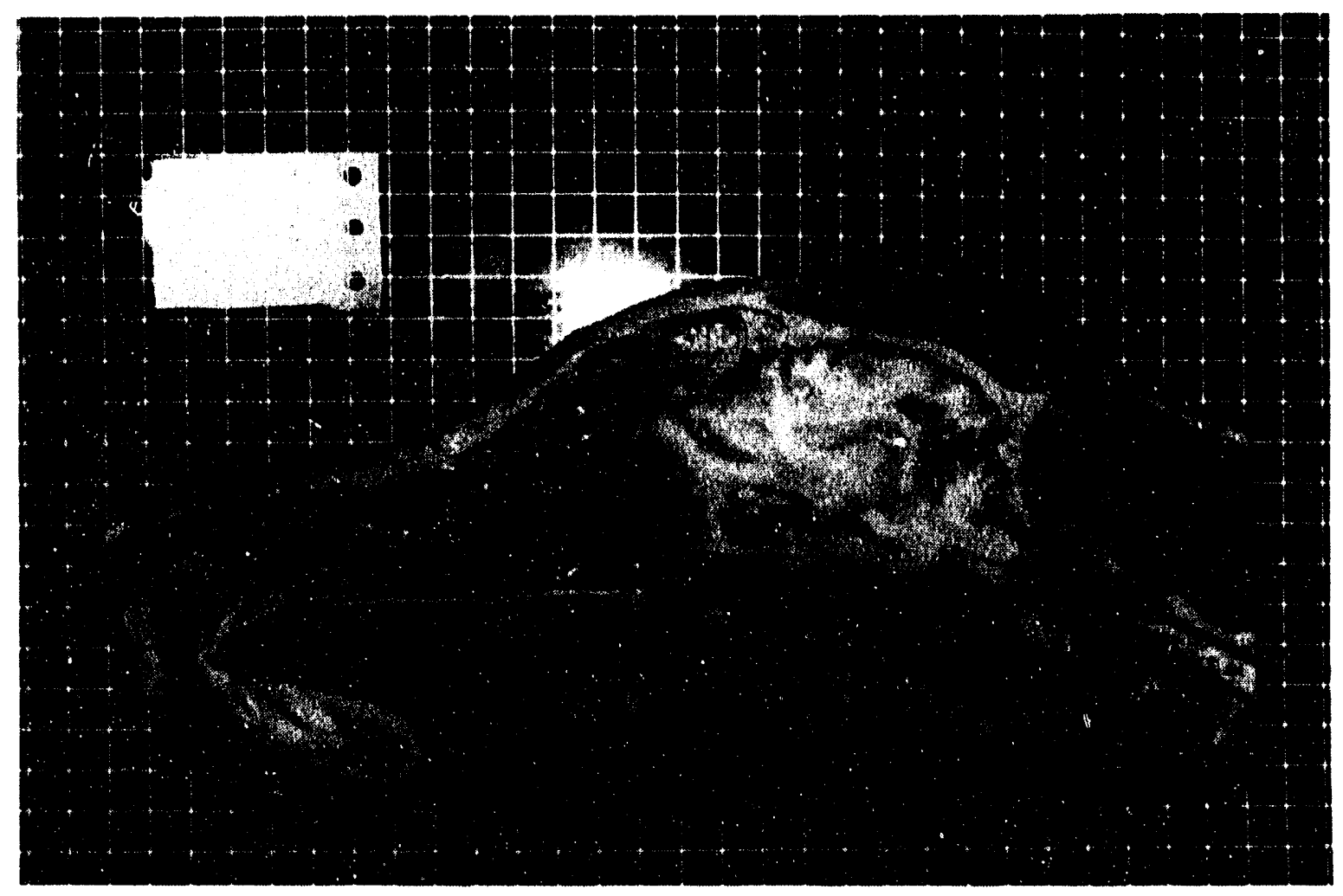

Figure B-2. Photograph of sagittal slice 7 left at $y=1 \mathrm{~cm}$. 


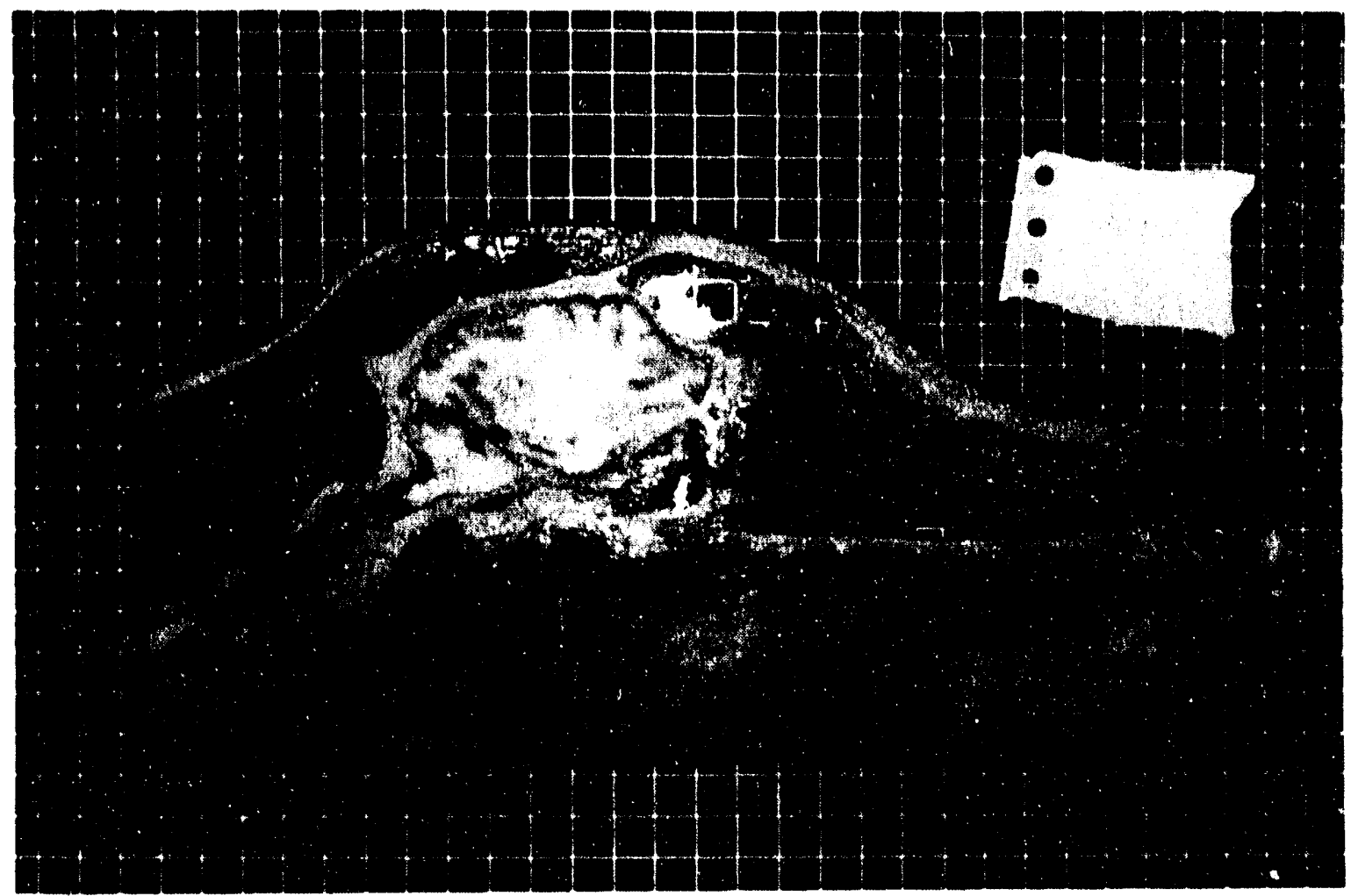

Figure B-3. Photograph of sagittal slice 6 right at $y=1 \mathrm{~cm}$. 


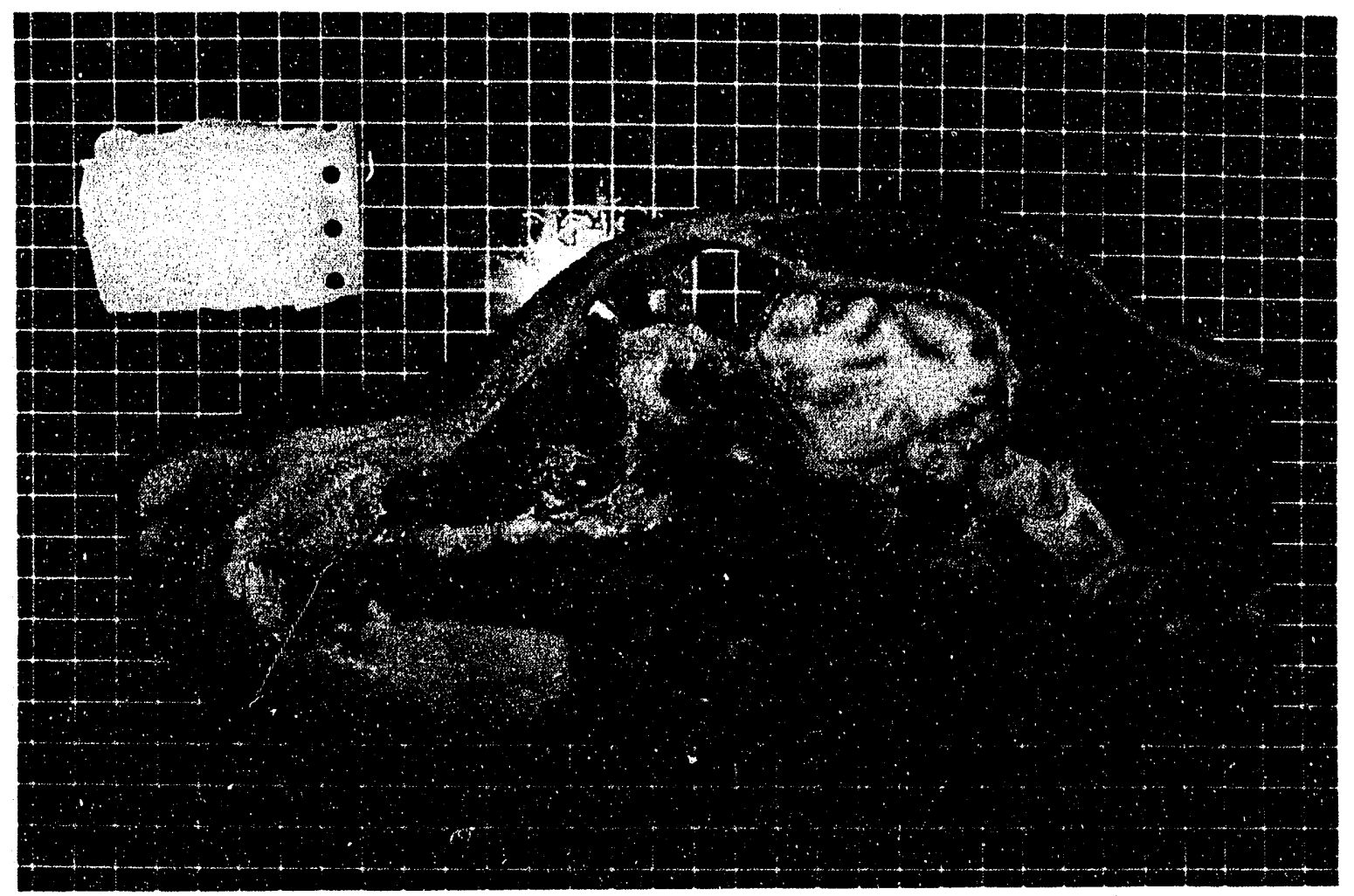

Figure B-4. Photograph of sagittal slice 6 left at $y=2 \mathrm{~cm}$. 


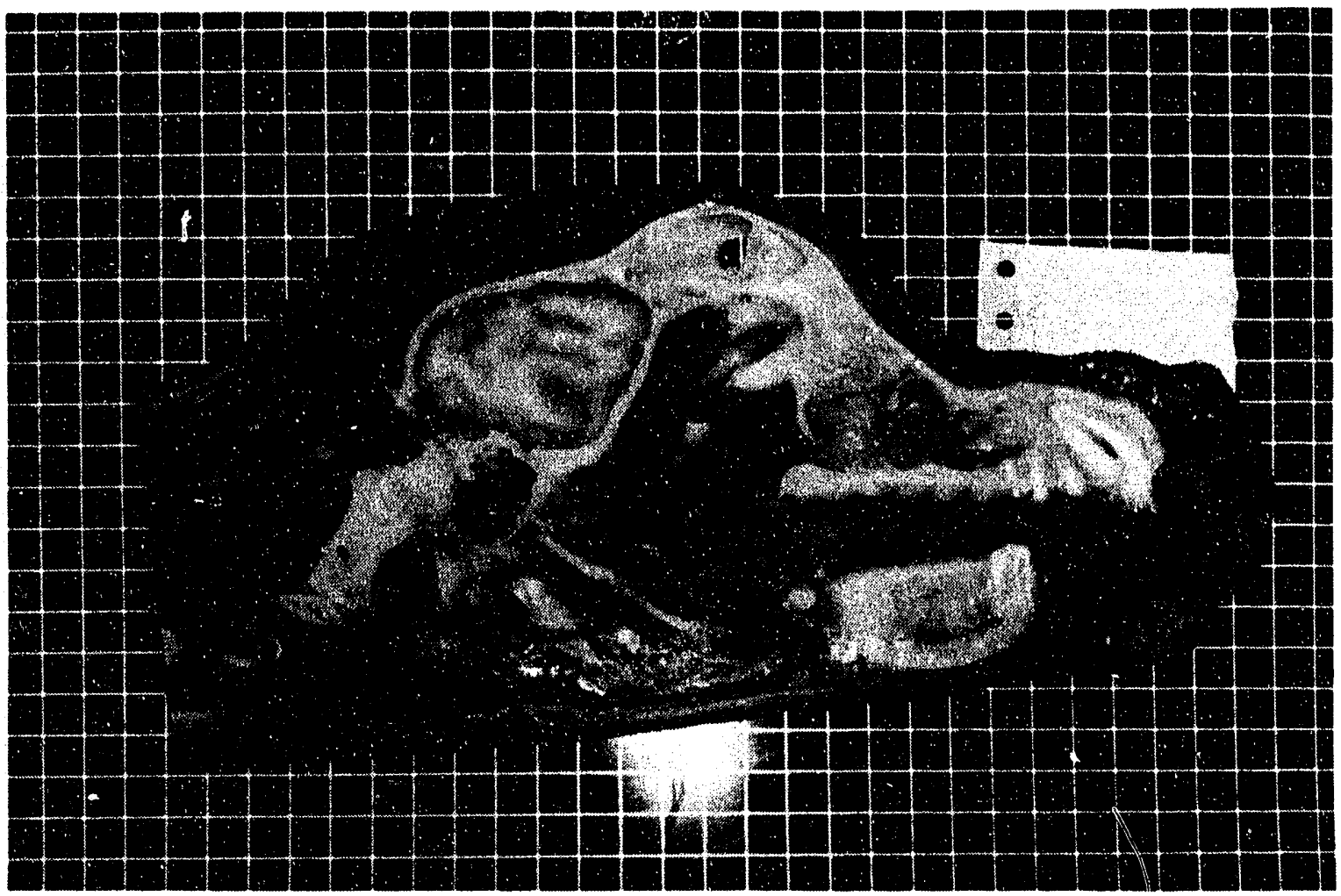

Figure B-5. Photograph of sagittal slice 5 right at $y=2 \mathrm{~cm}$. 


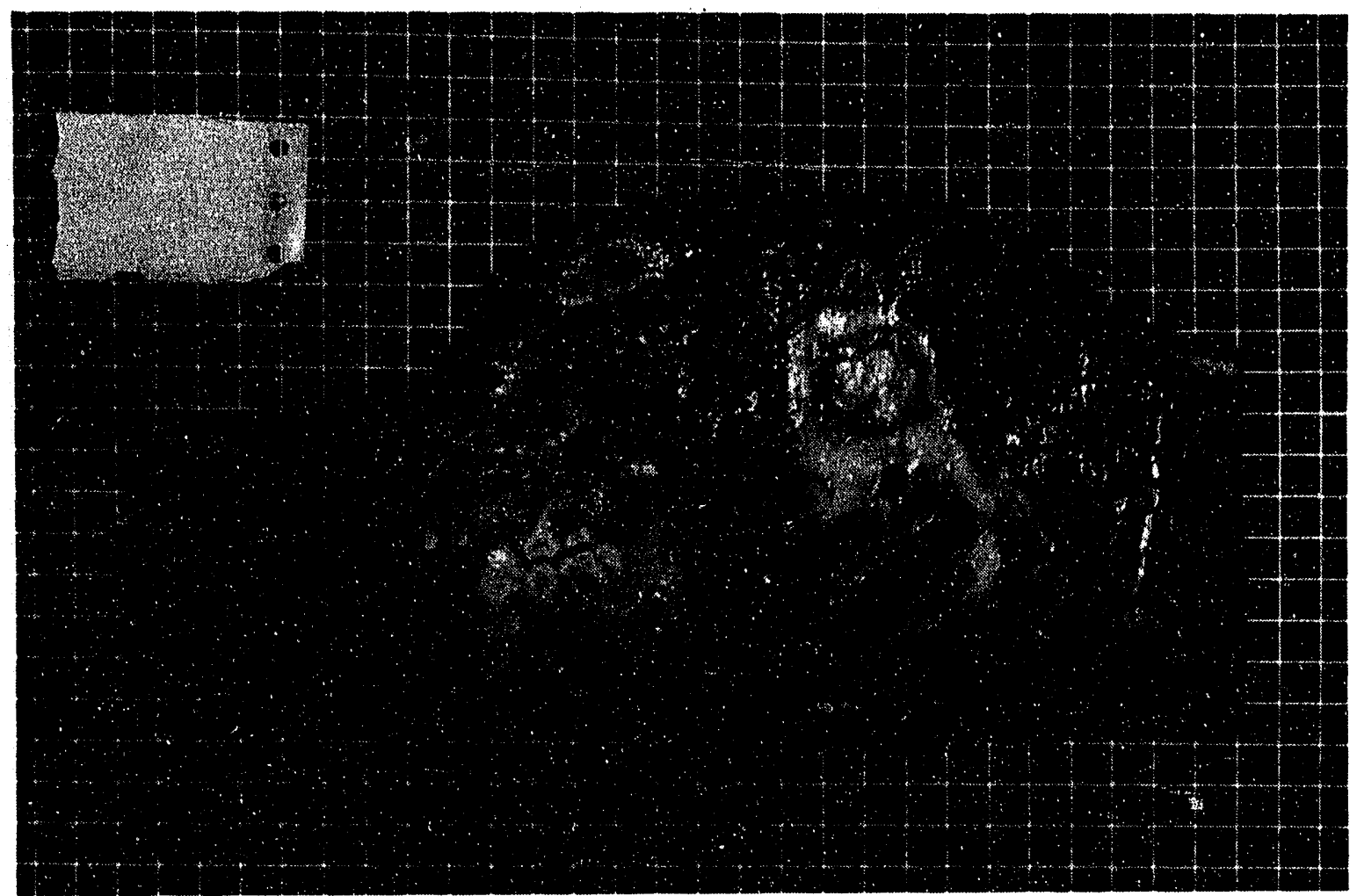

Figure B-6. Photograph of sagittal slice 5 left at $y=3 \mathrm{~cm}$. 


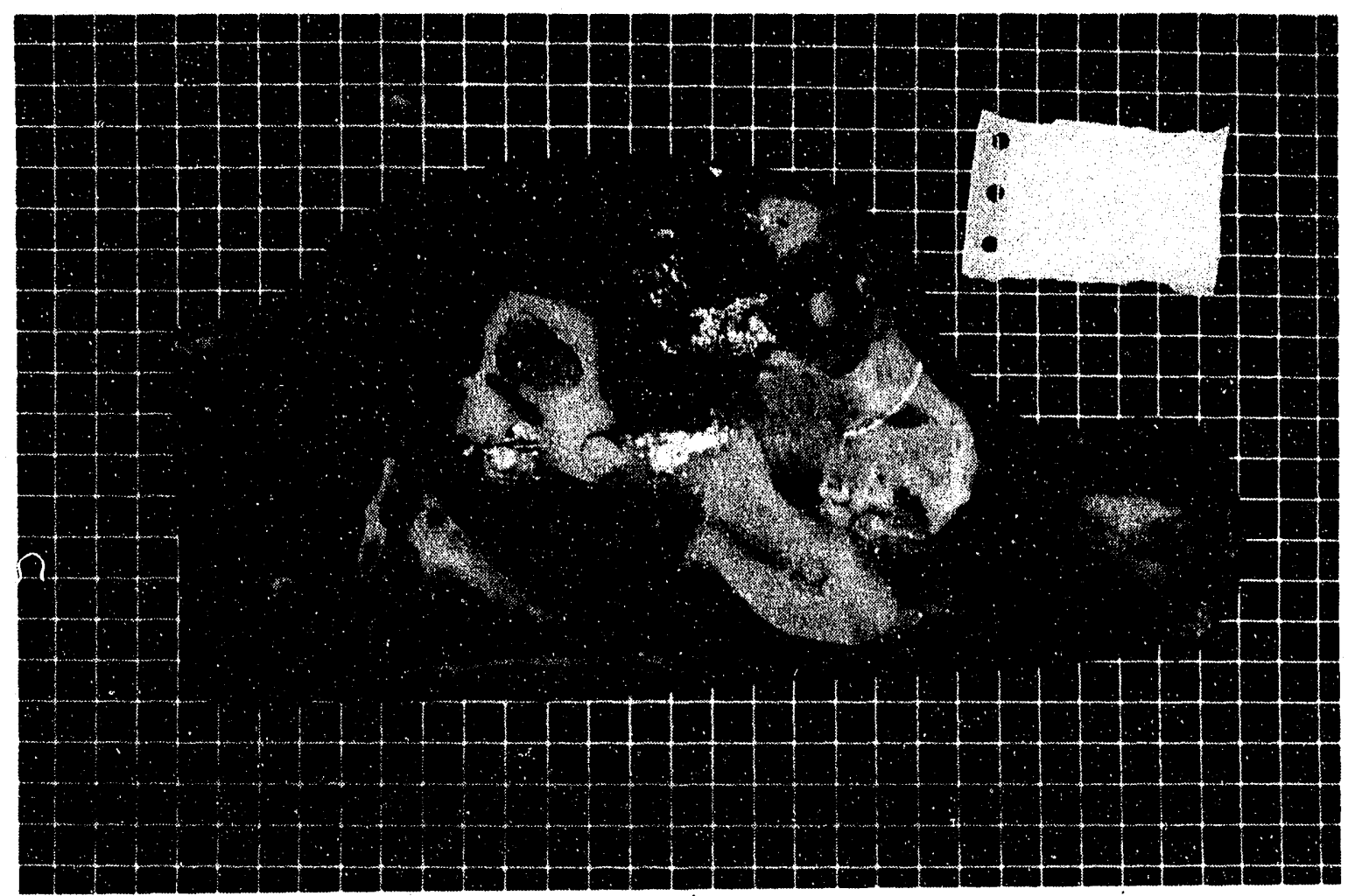

Figure B-7. photograph of sagittal slice 4 right at $y=3 \mathrm{~cm}$. 


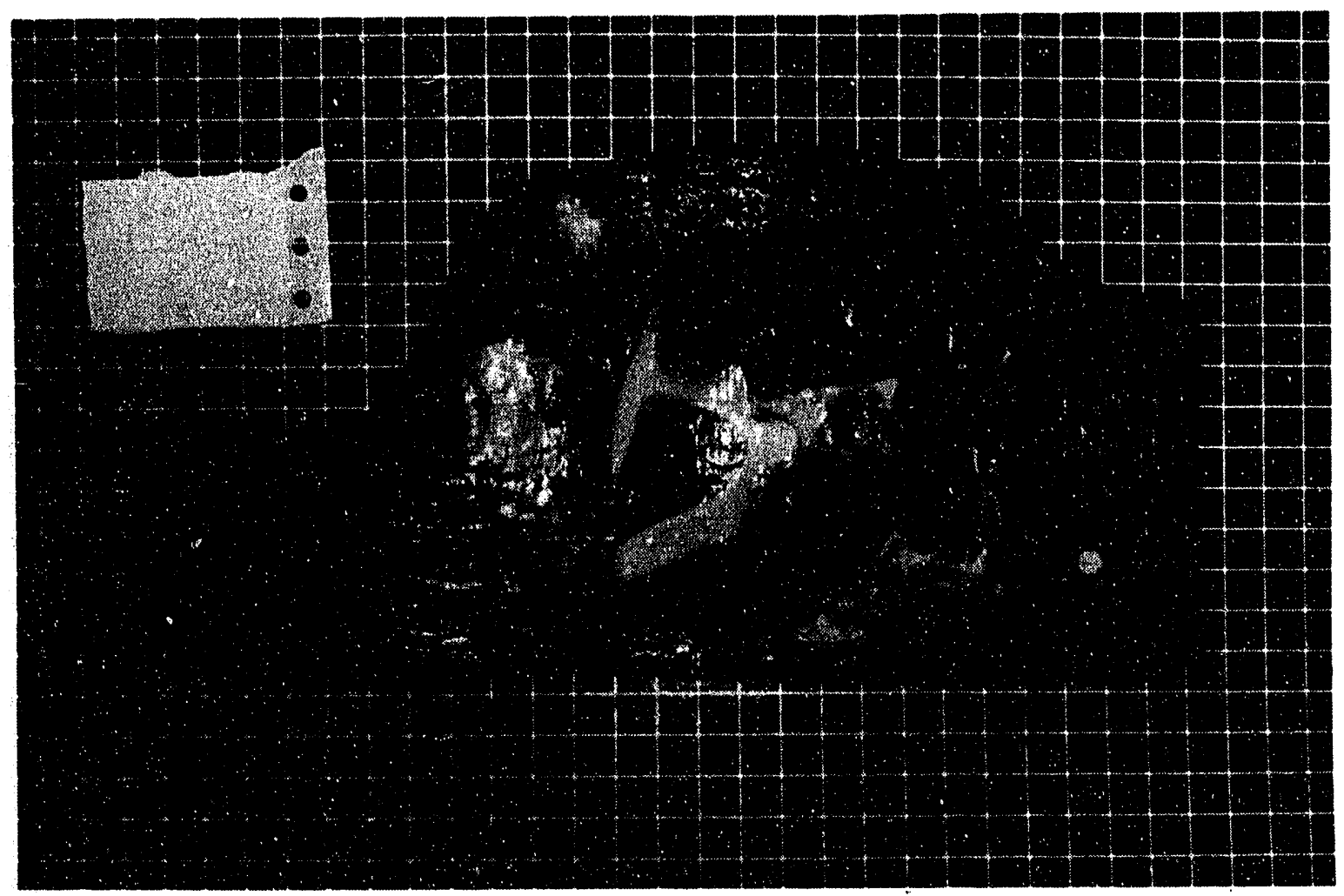

Figure B-8. Photograph of sagittal slice 4 left at $y=4 \mathrm{~cm}$. 


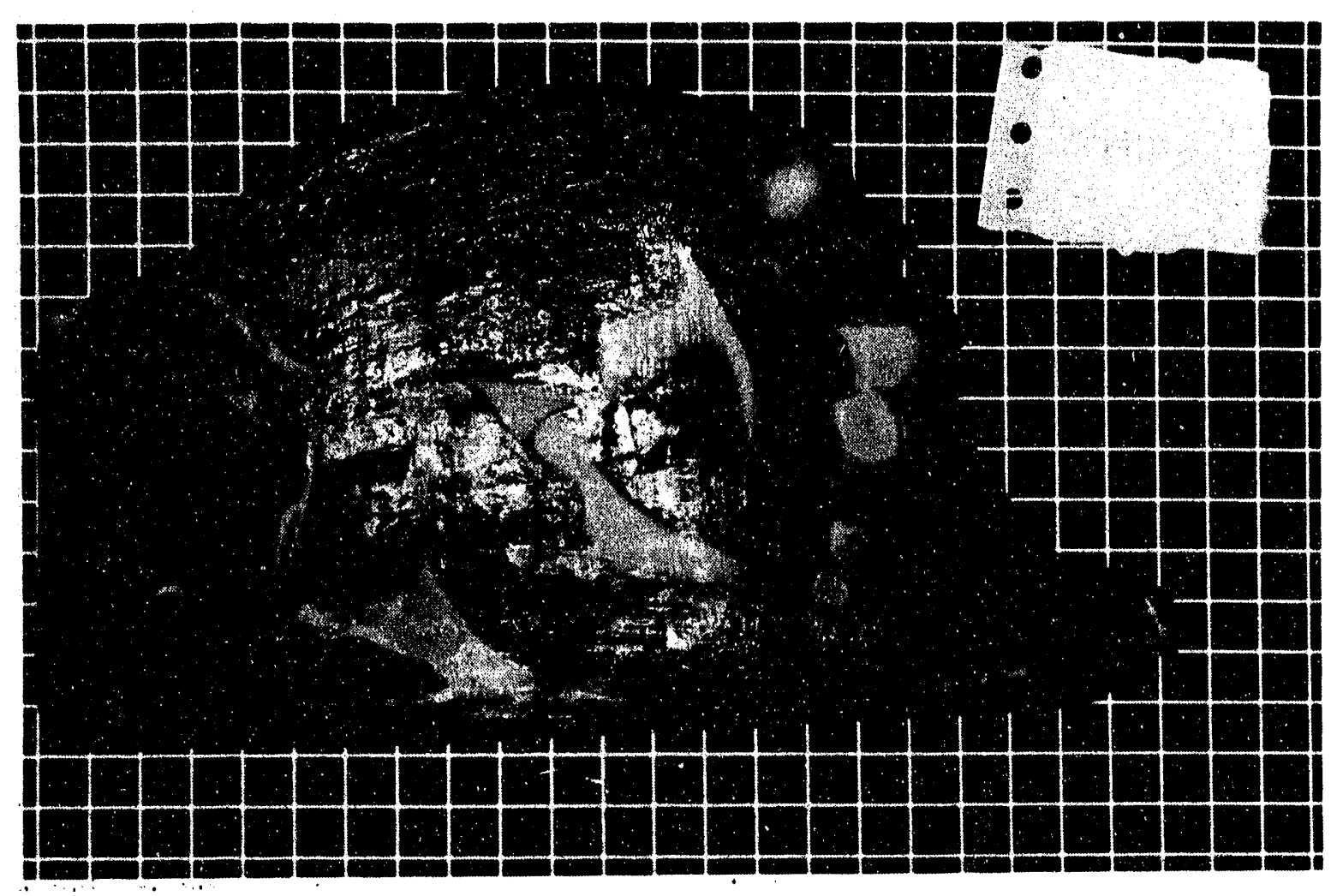

Figure B-9. Photograph of sagittal slice 3 right at $y=4 \mathrm{~cm}$. . . 


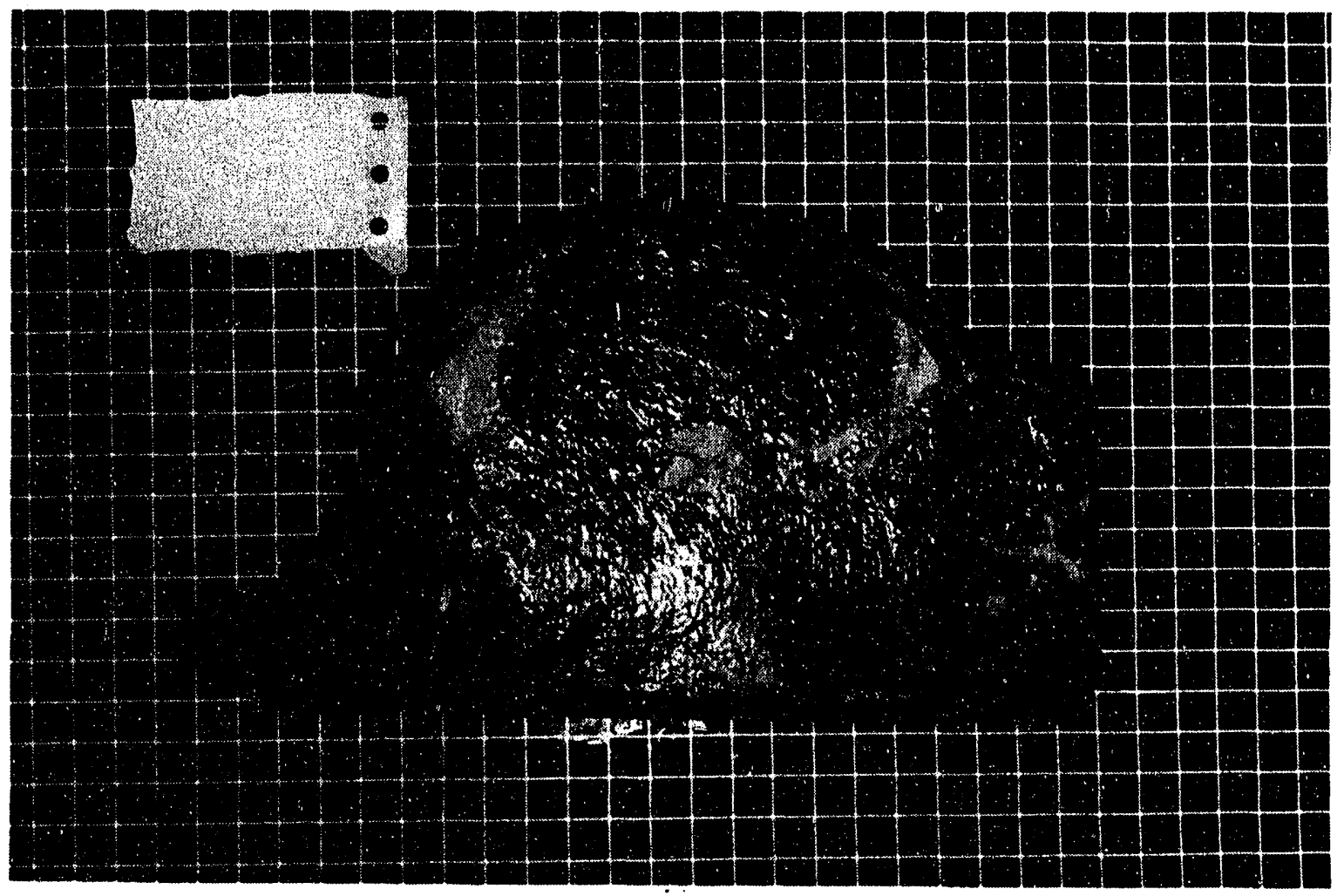

Figure B-10. Photograph of sagittal slice 3 left at $y=5 \mathrm{~cm}$. 


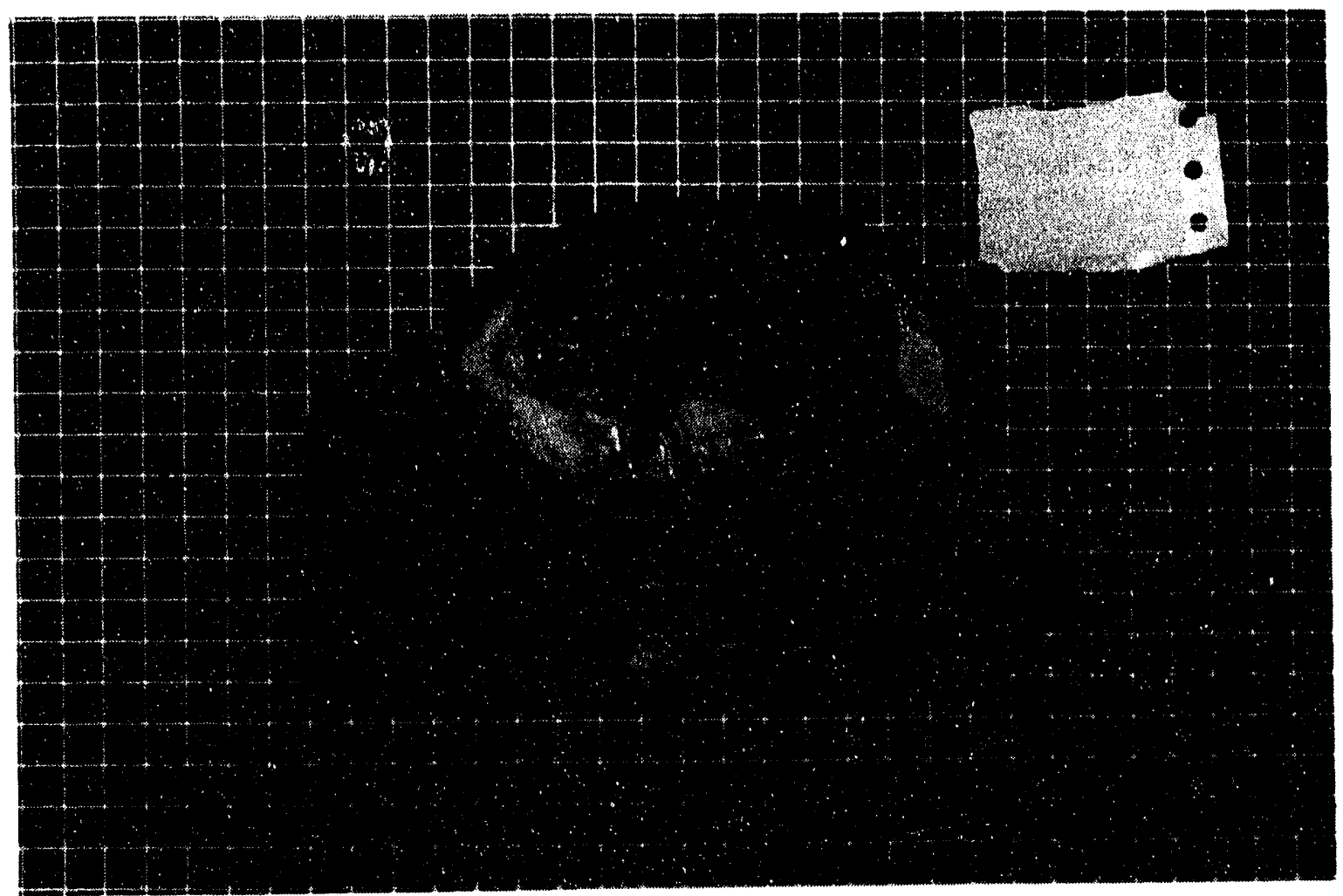

Figure B-11. Photograph of sagittal slice 2 right at $y=5$ cm. 


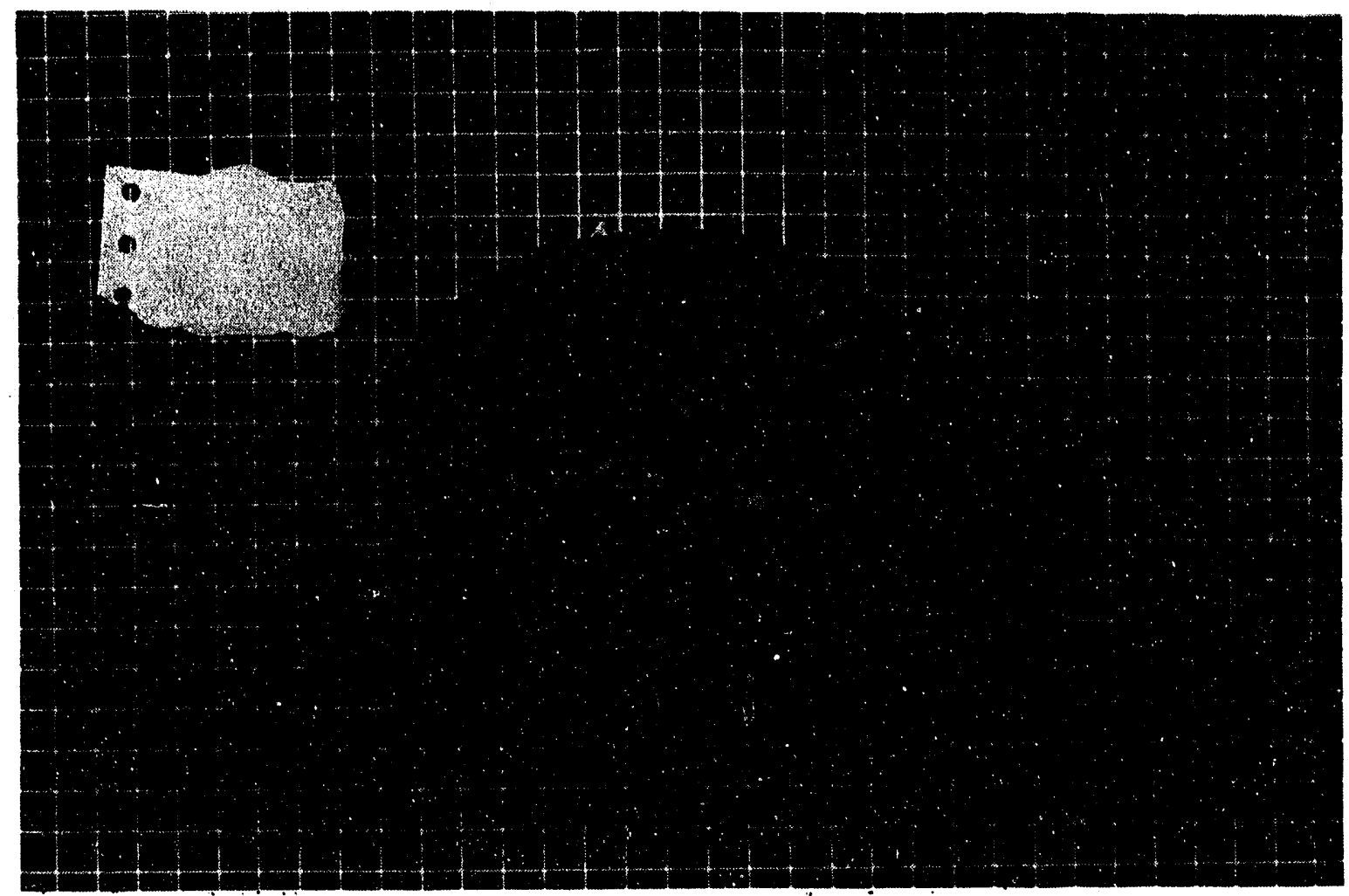

Figure B-12. Photograph of sagittal slice 2 left at $y=6 \mathrm{~cm}$. 


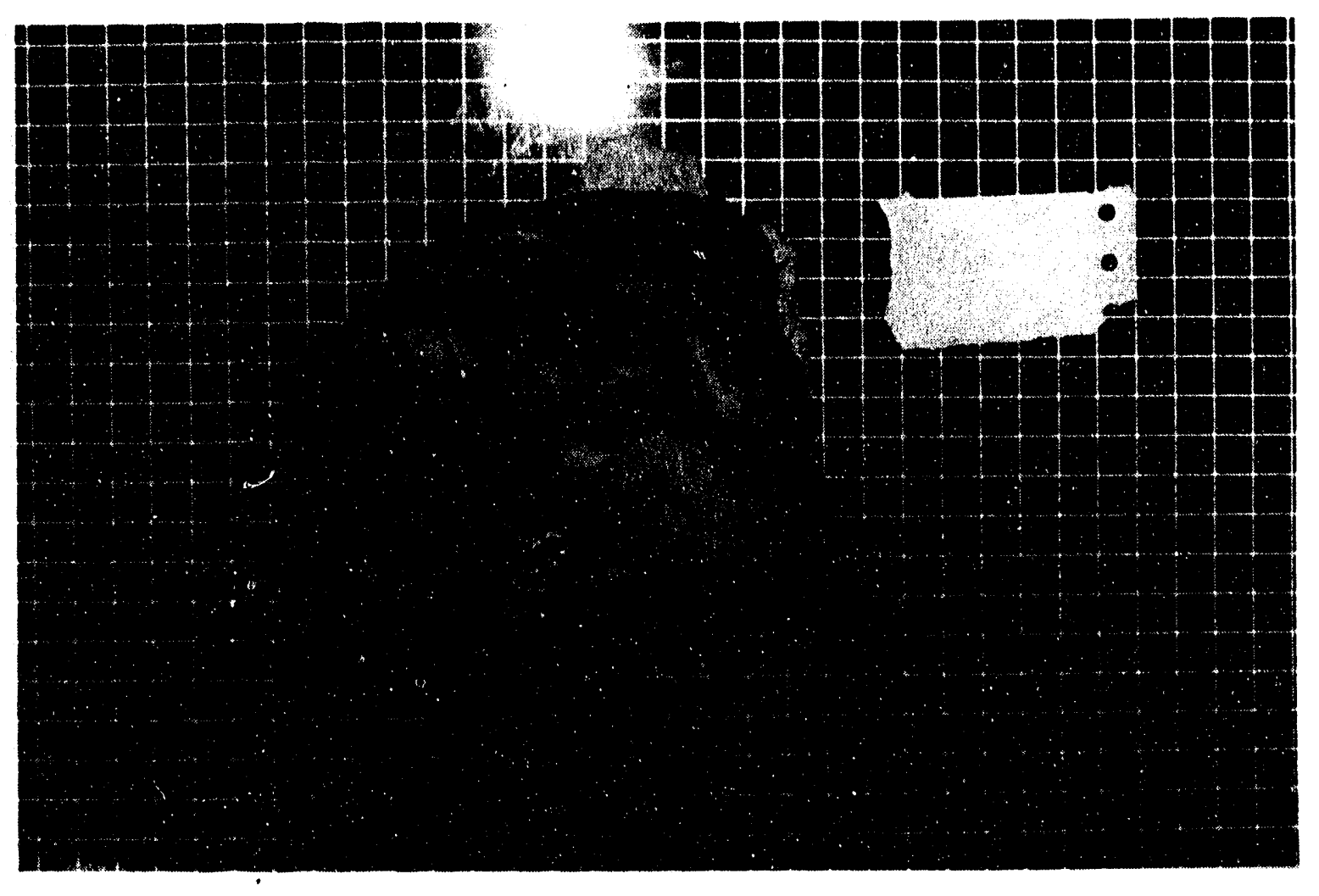

Figure B-13. Photograph of sagittal slice 1 right at $y=6$ cm. 


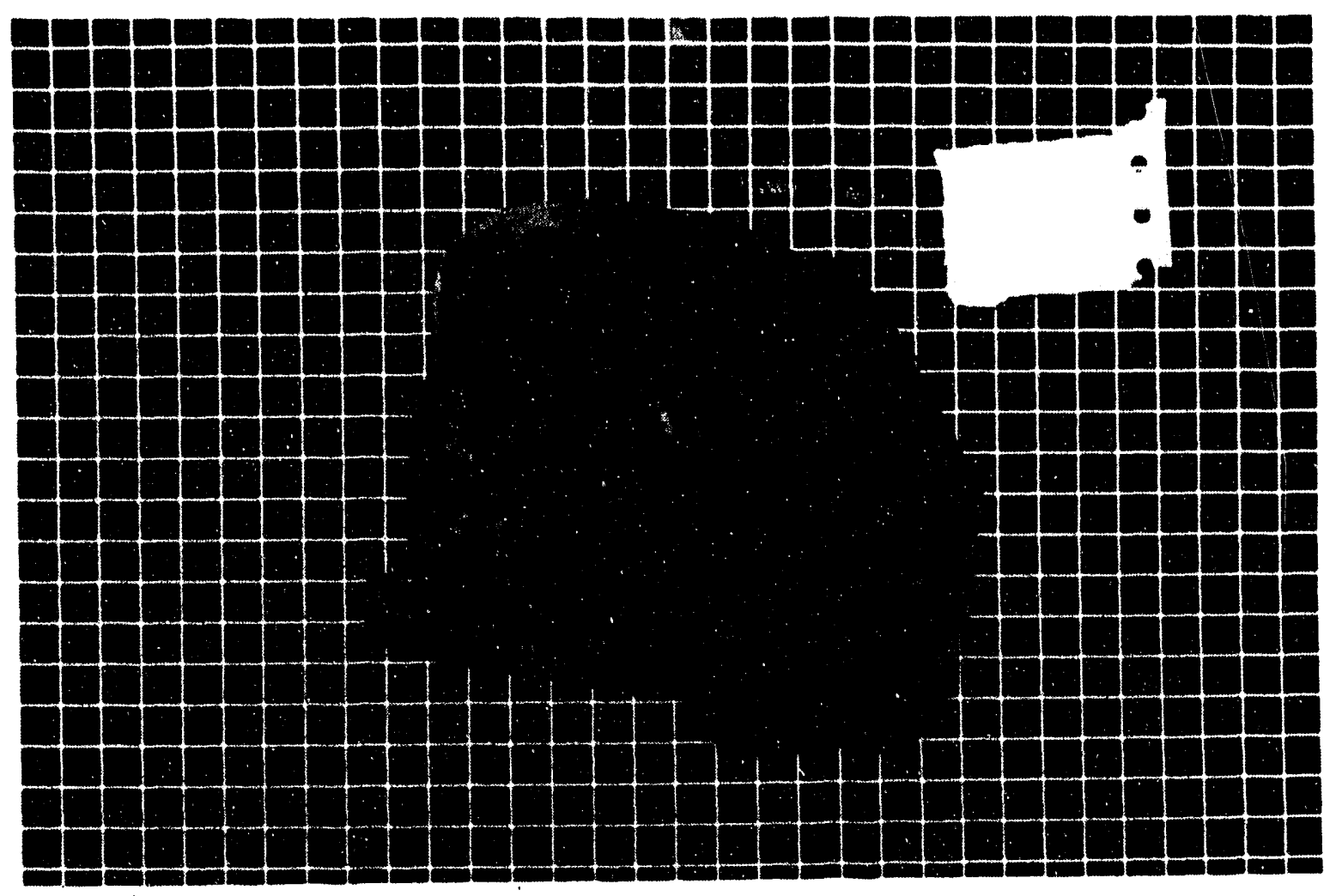

Figure B-14. Photograph of sagittal slice 1 left at $y=7 \mathrm{~cm}$. 


\section{THIS PAGE INTENTIONALLY LEFT BLANK}


APPENDIX C

DETAILED SAGITTAL MESH SECTIONS USED IN MODELING

191 


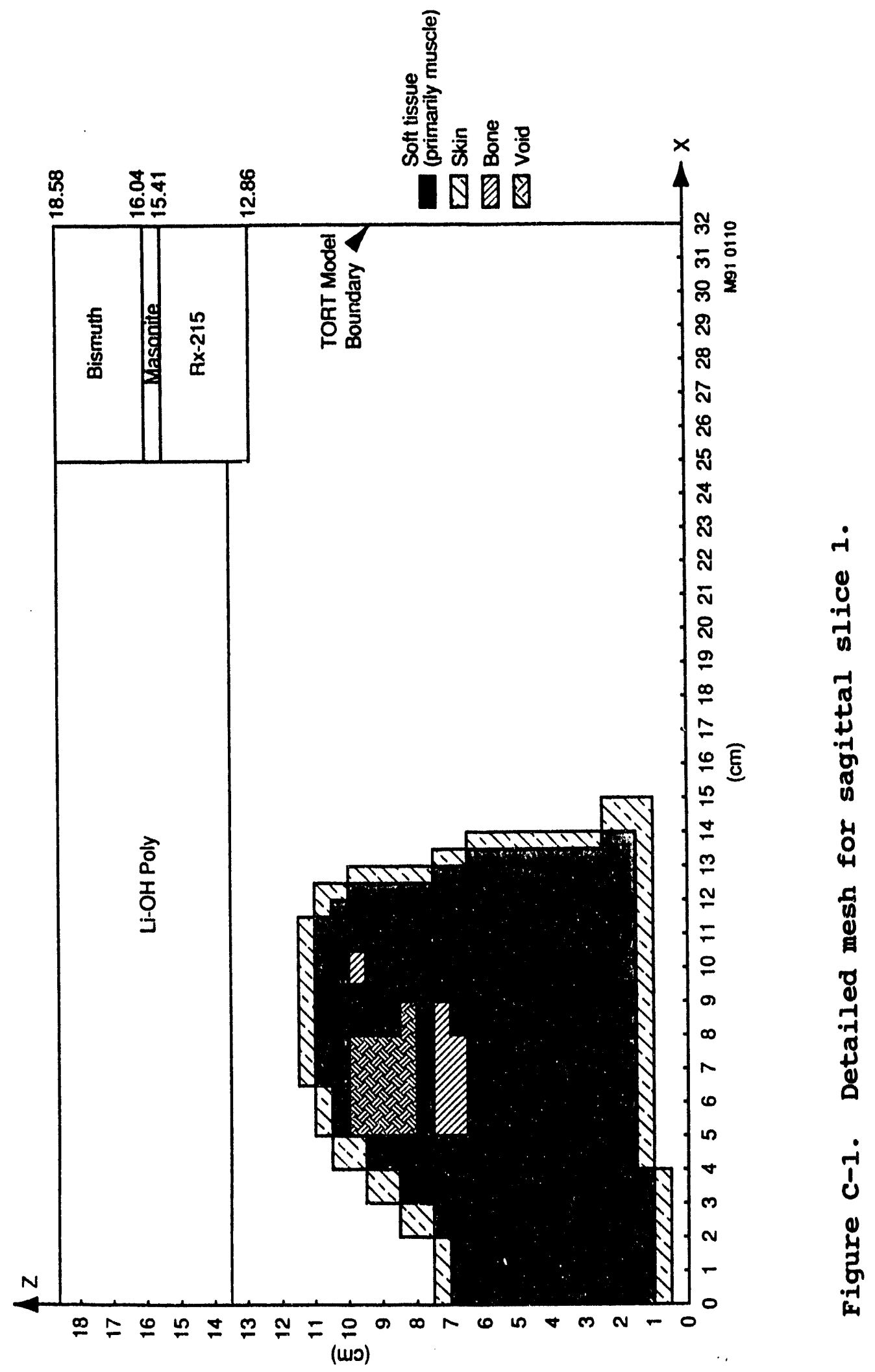




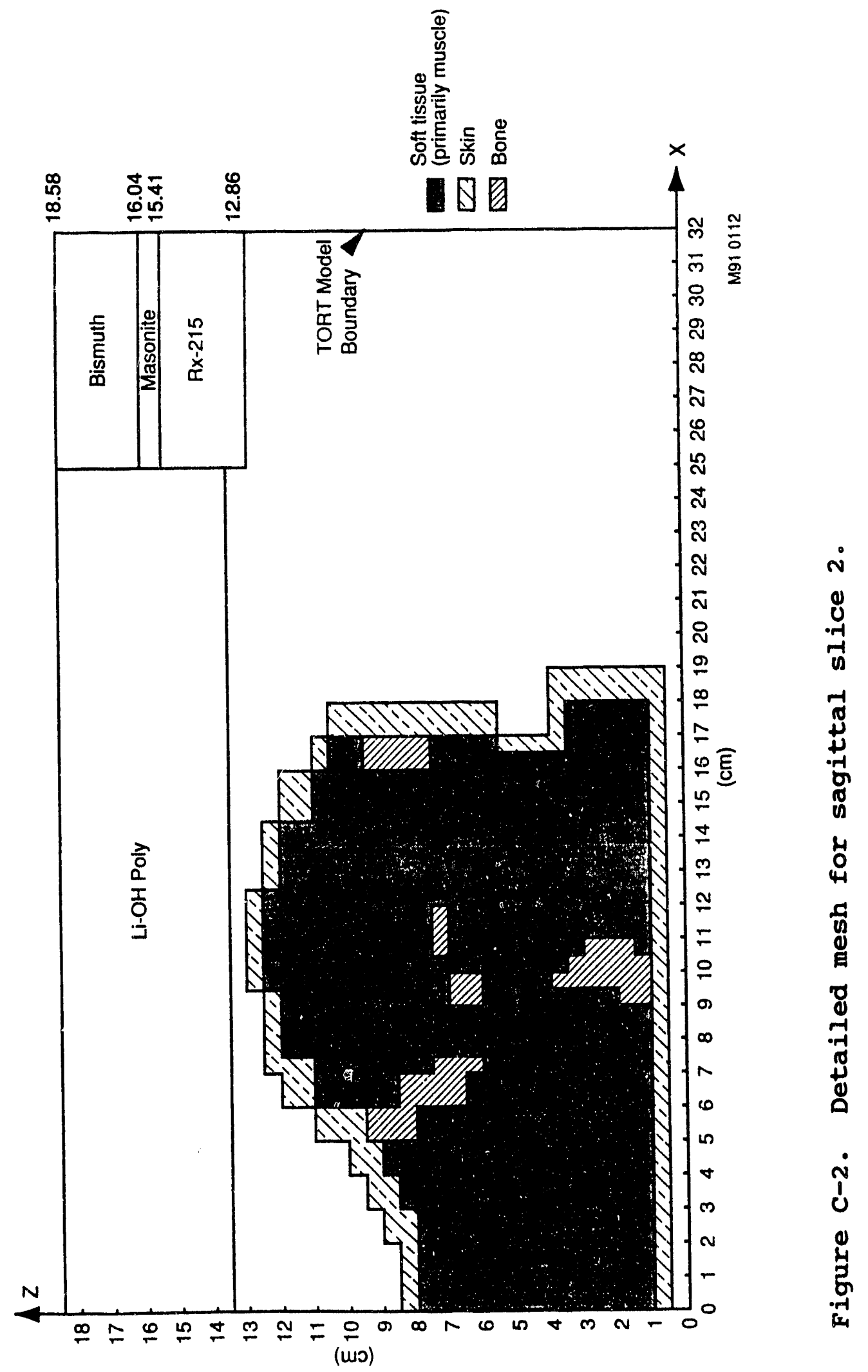




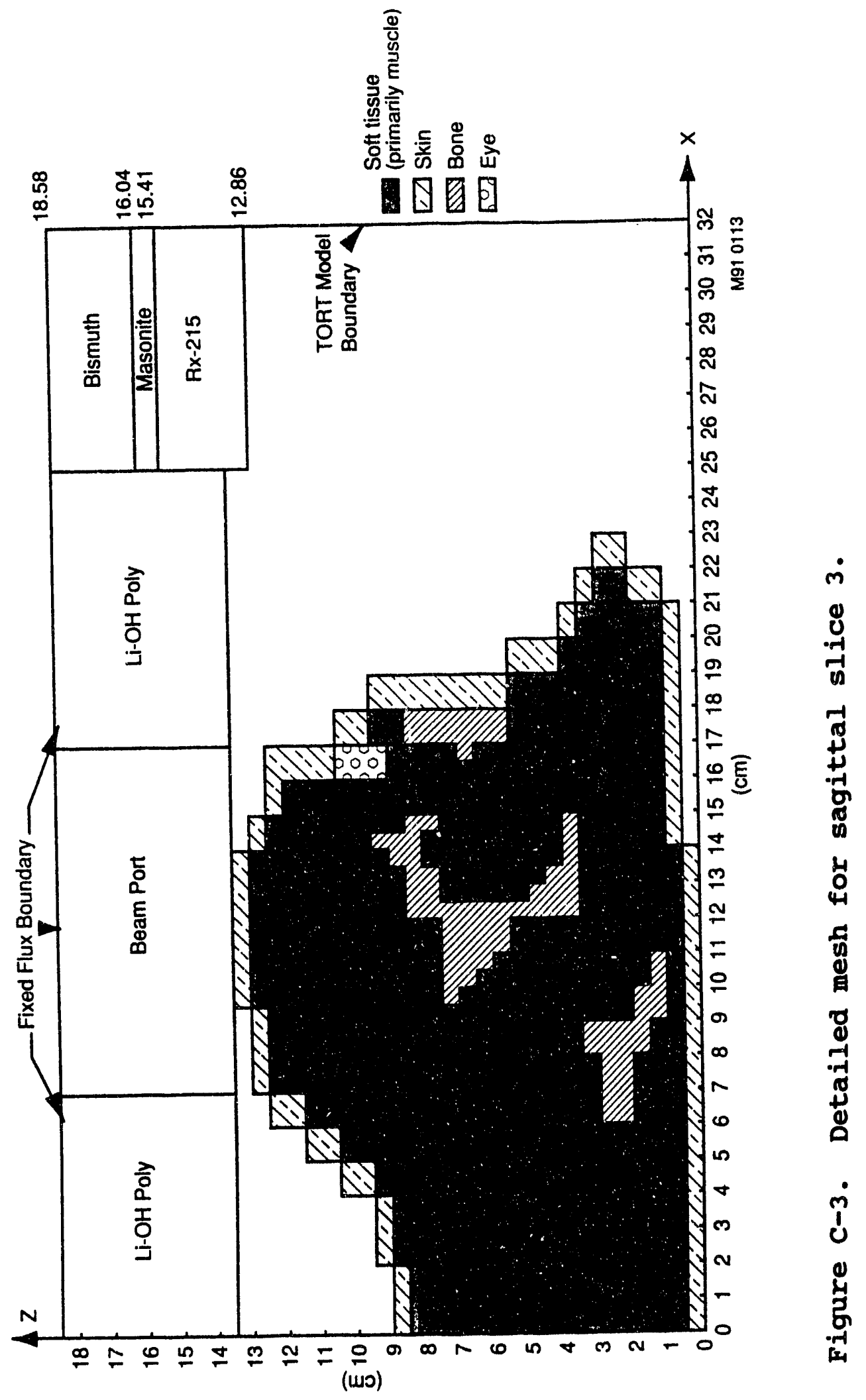




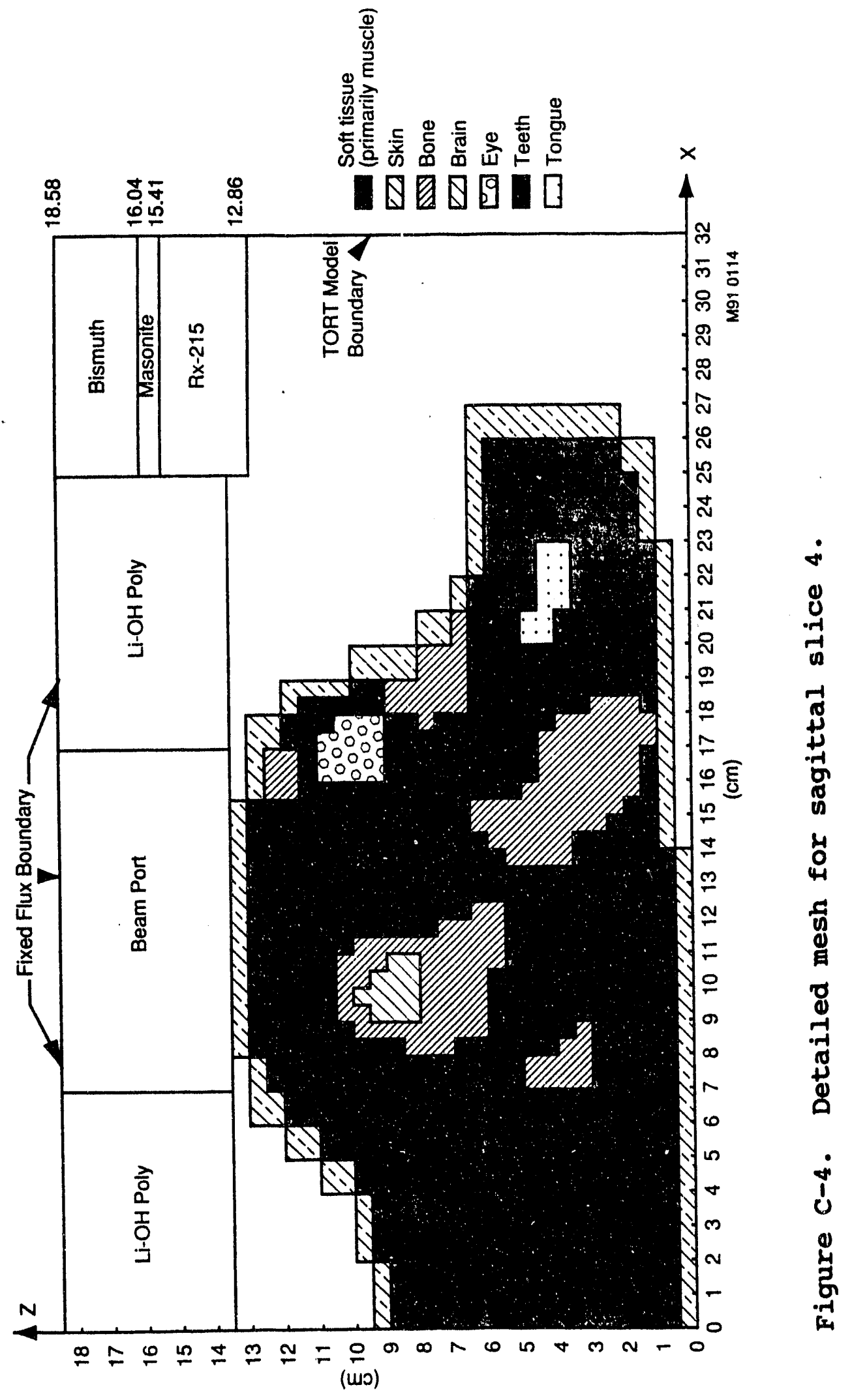




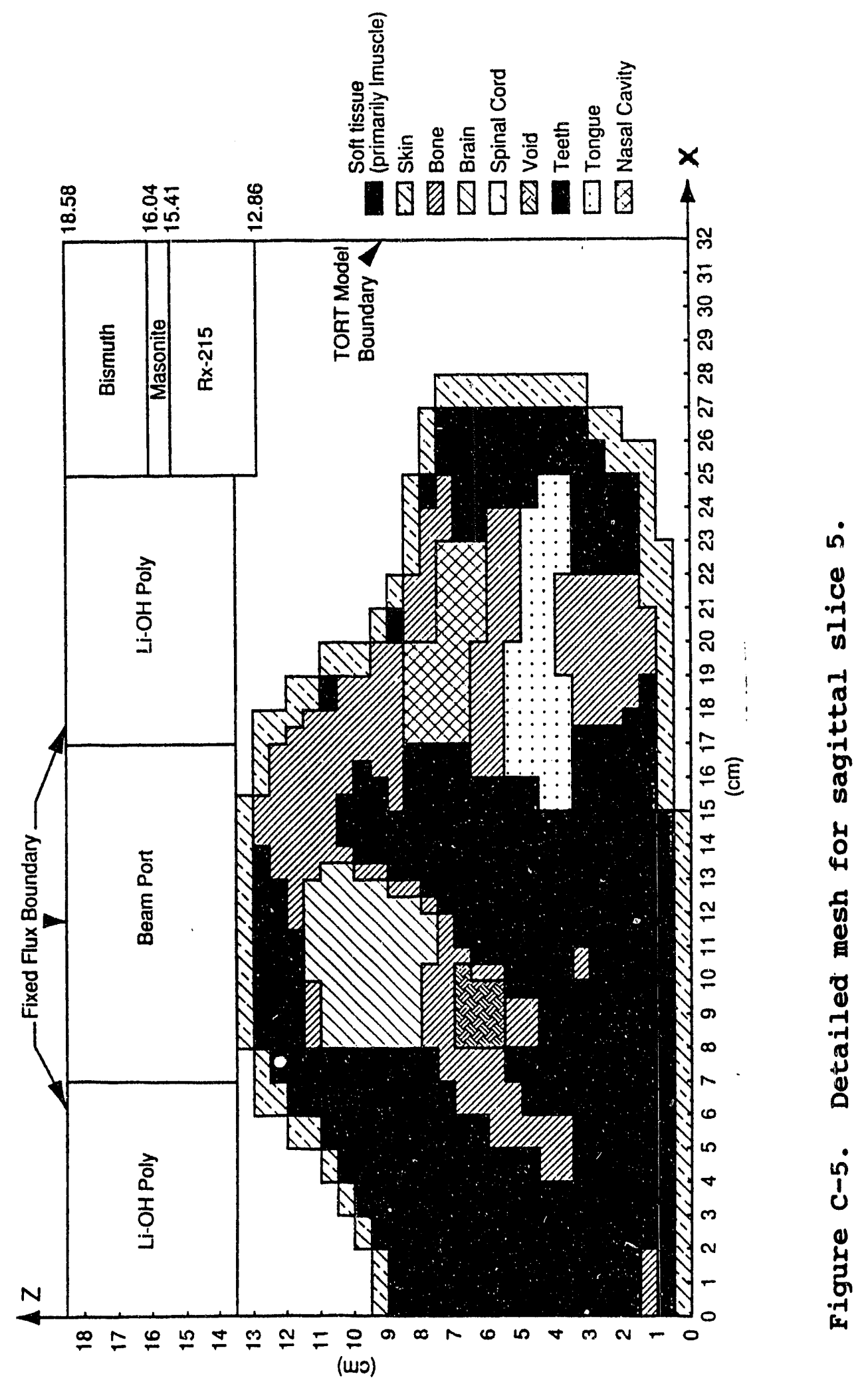




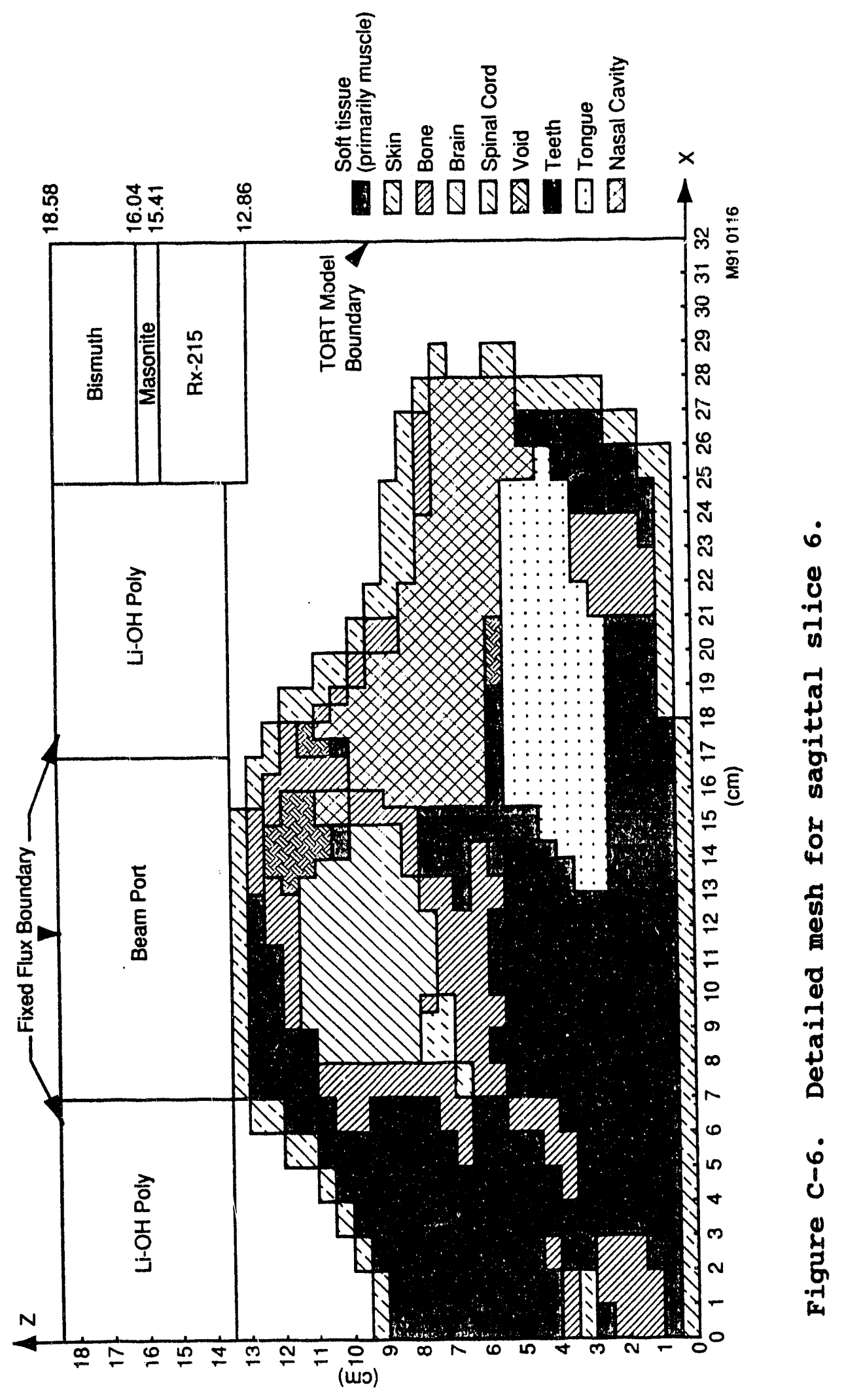




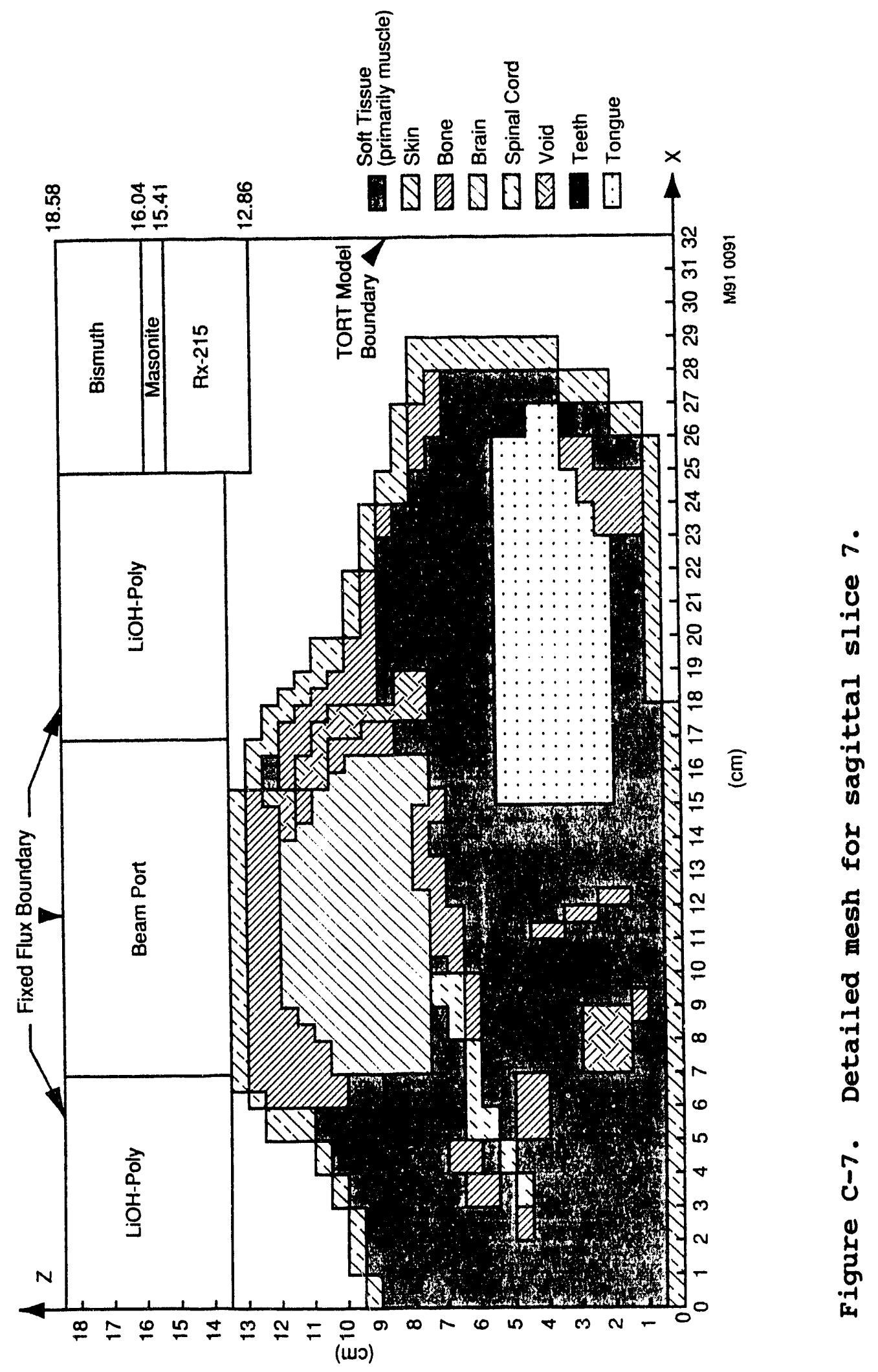


APPENDIX D

AXIAL SECTION PHOTOGRAPHS 


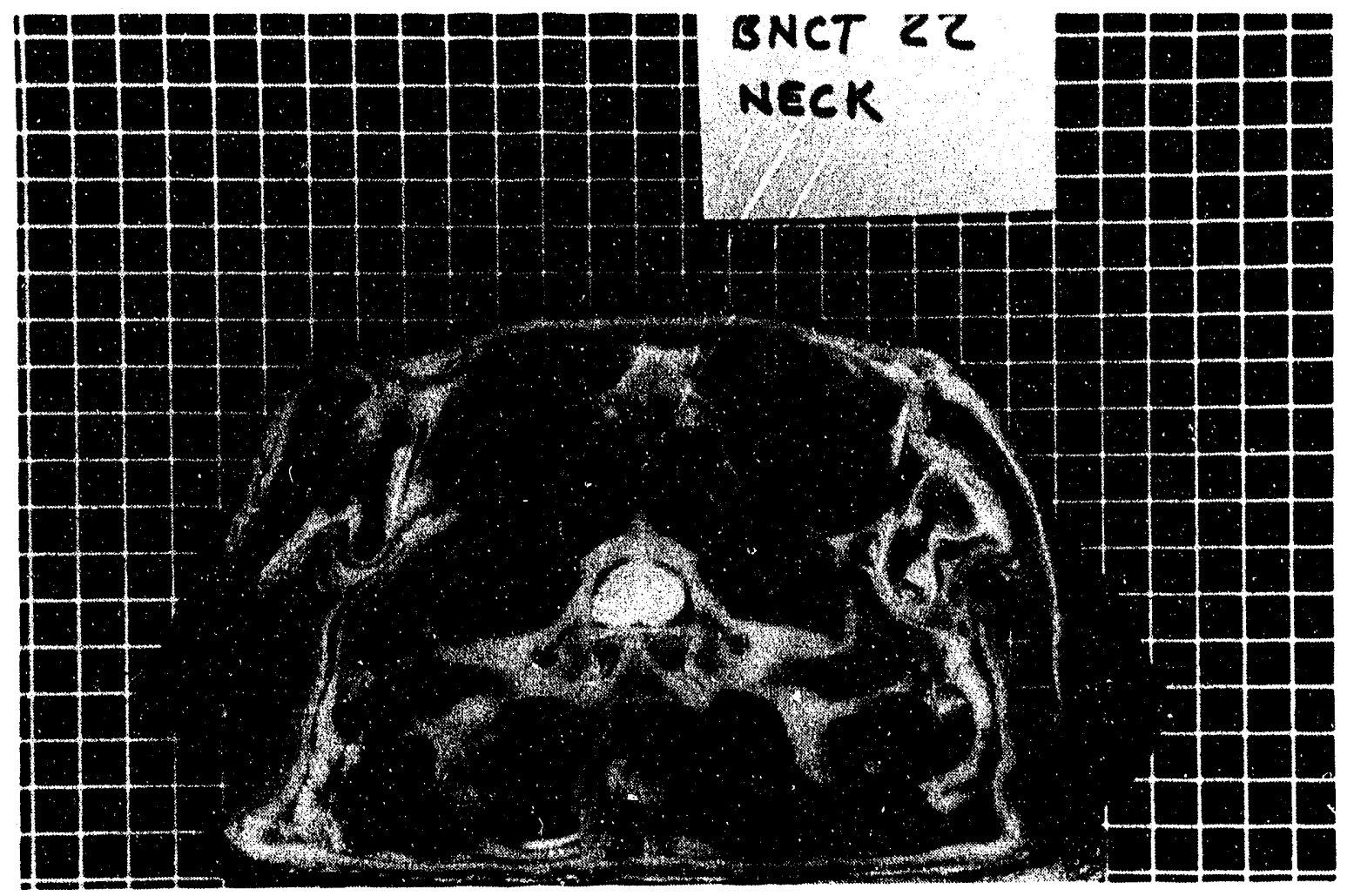

Figure D-1. Photograph of the neck at $x=7 \mathrm{~cm}$. 


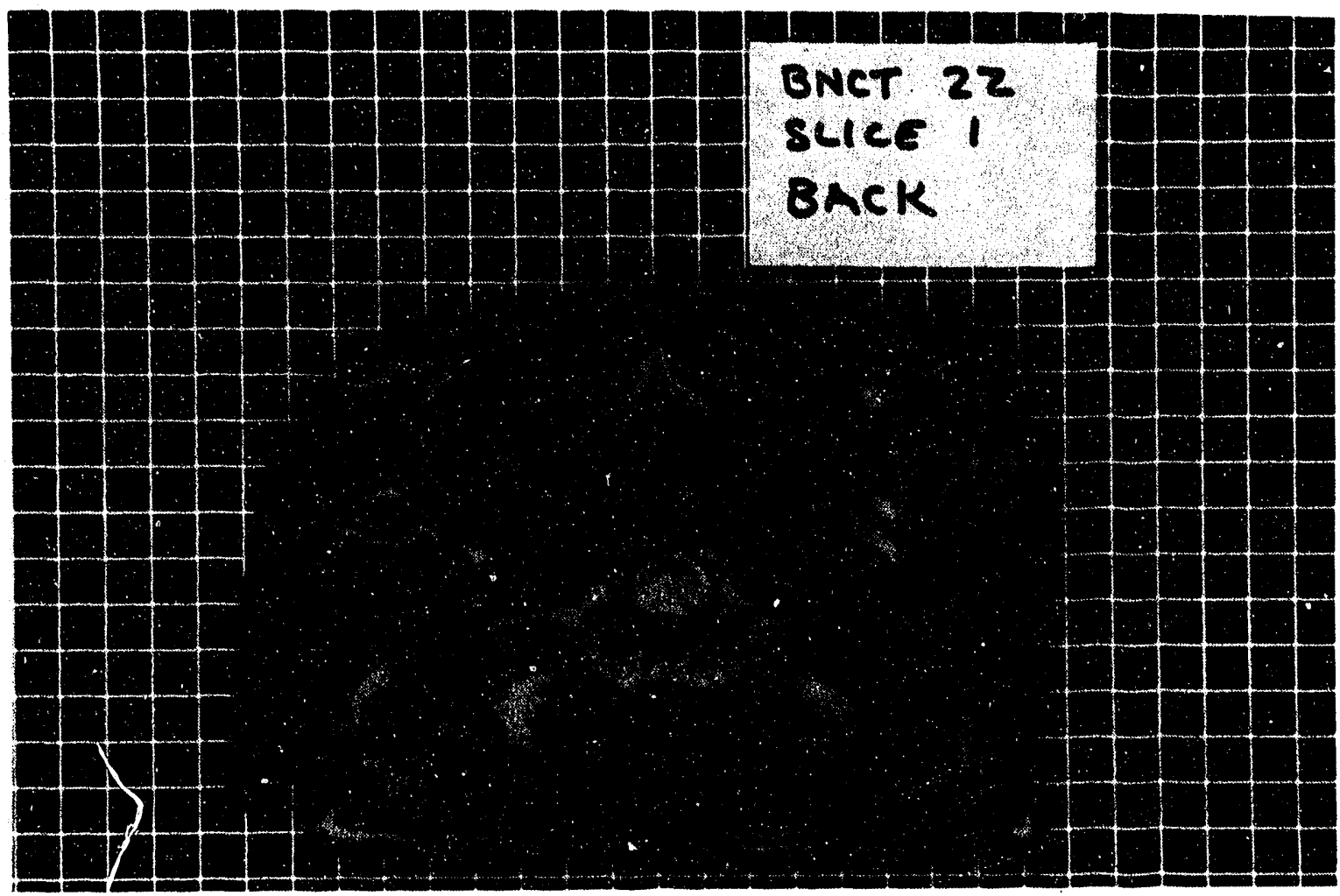

Figure D-2. Photograph of axial slice 1 back at $x=7 \mathrm{~cm}$. 


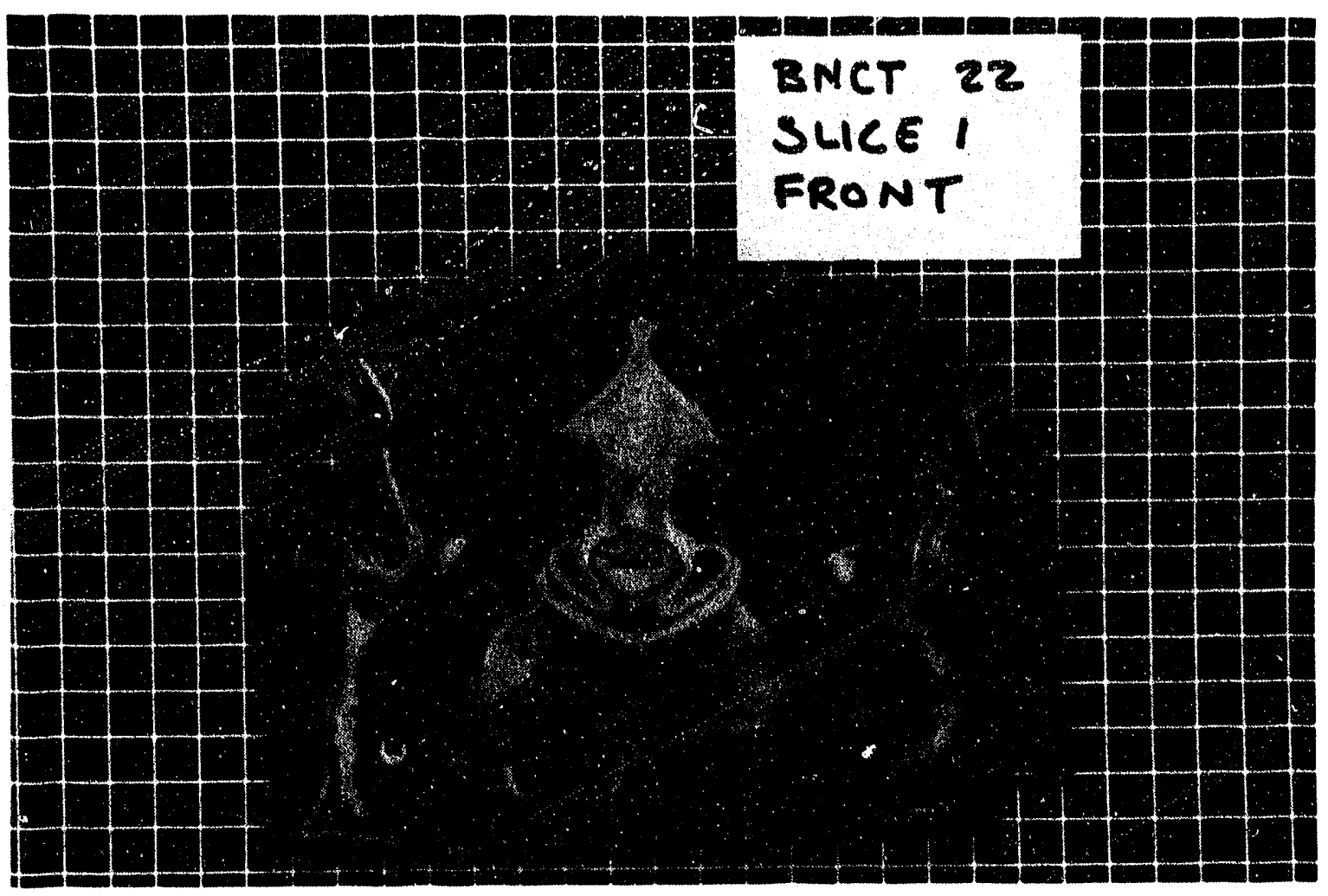

Figure D-3. Photograph of axial slice 1 front at $x=8 \mathrm{~cm}$. 


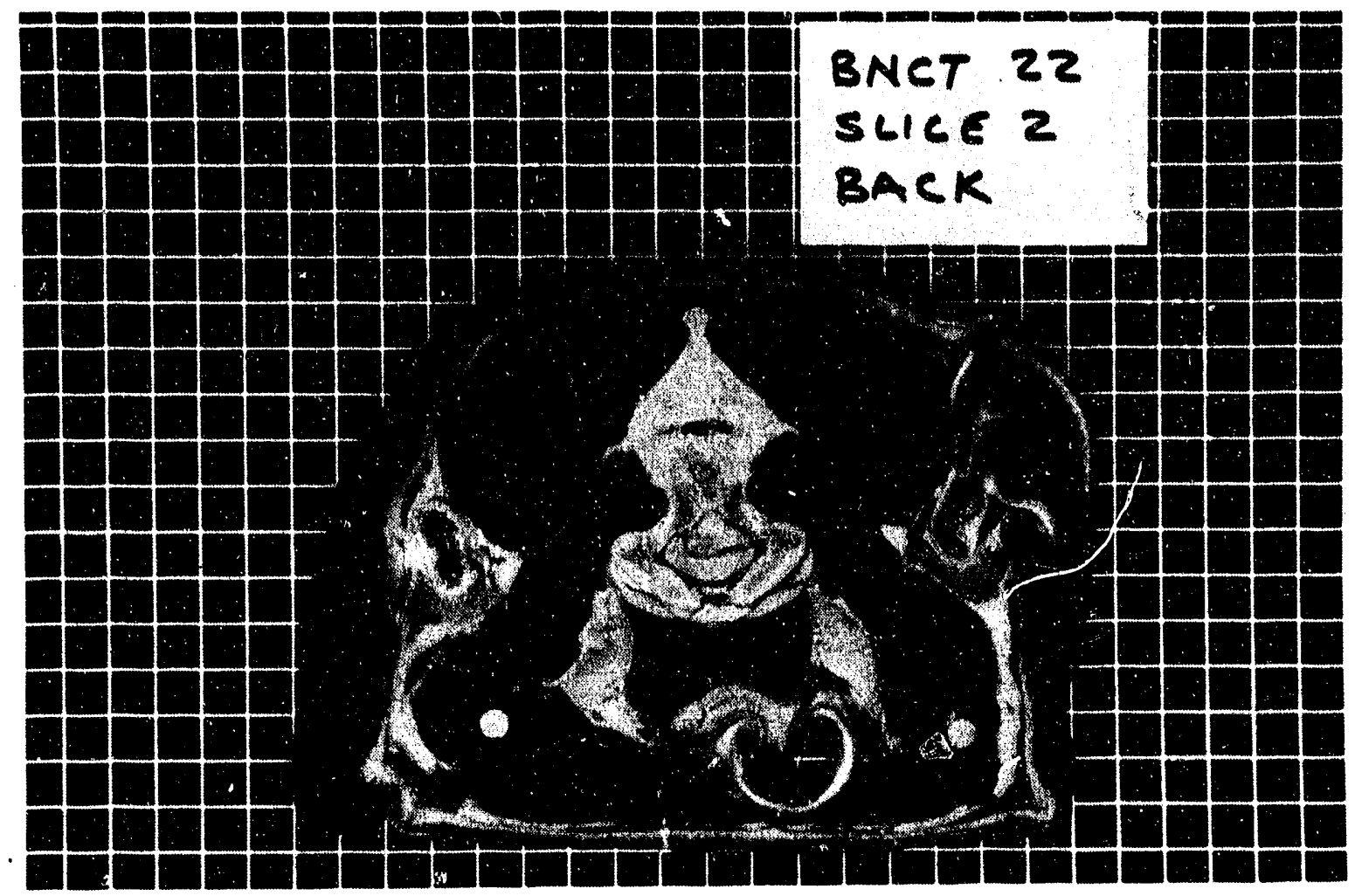

Figure D-4. Photograph of axial slice 2 back at $x=8 \mathrm{~cm}$. 


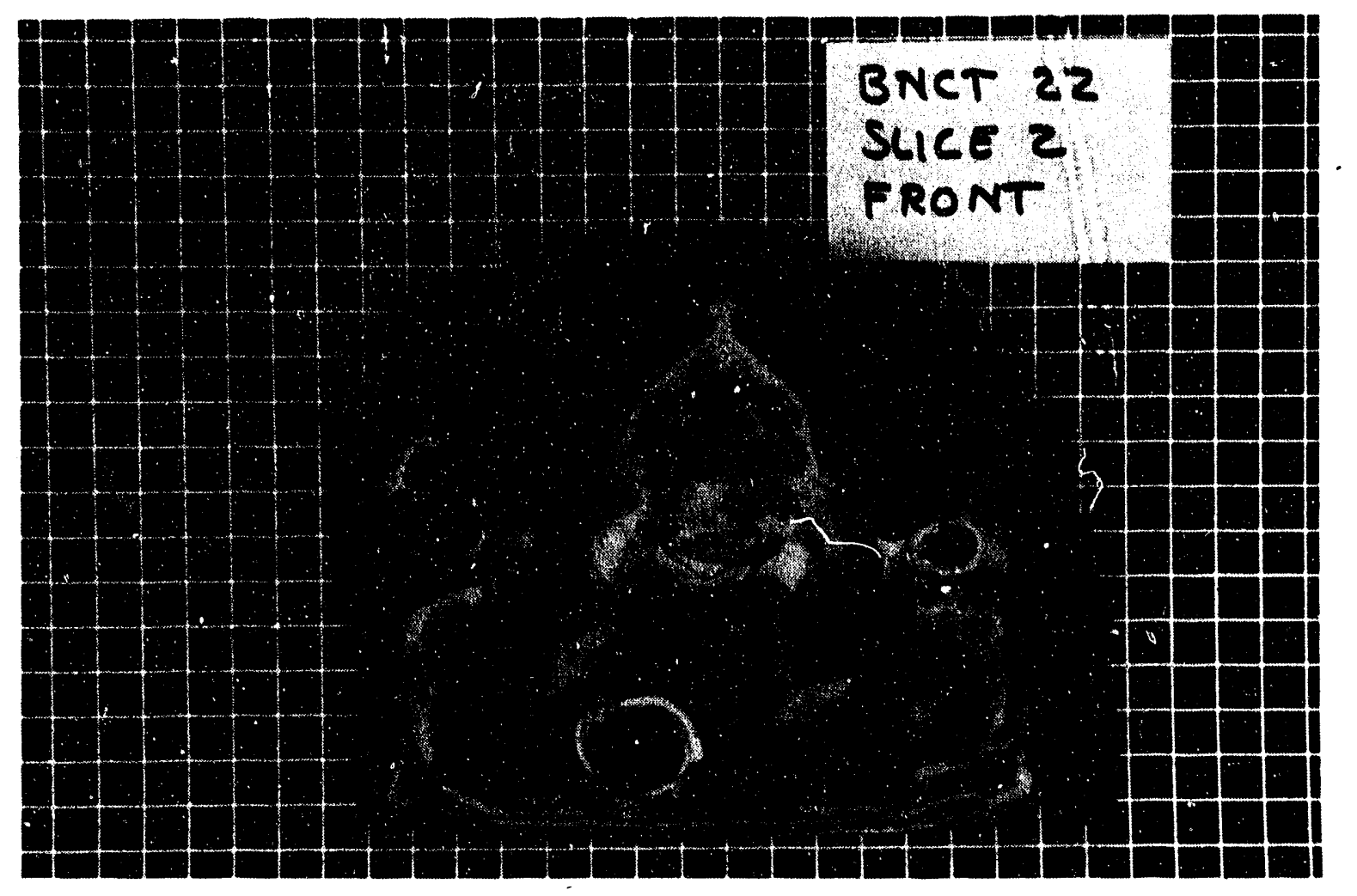

Figure D-5. Photograph of axial slice 2 front at $x=9 \mathrm{~cm}$. 


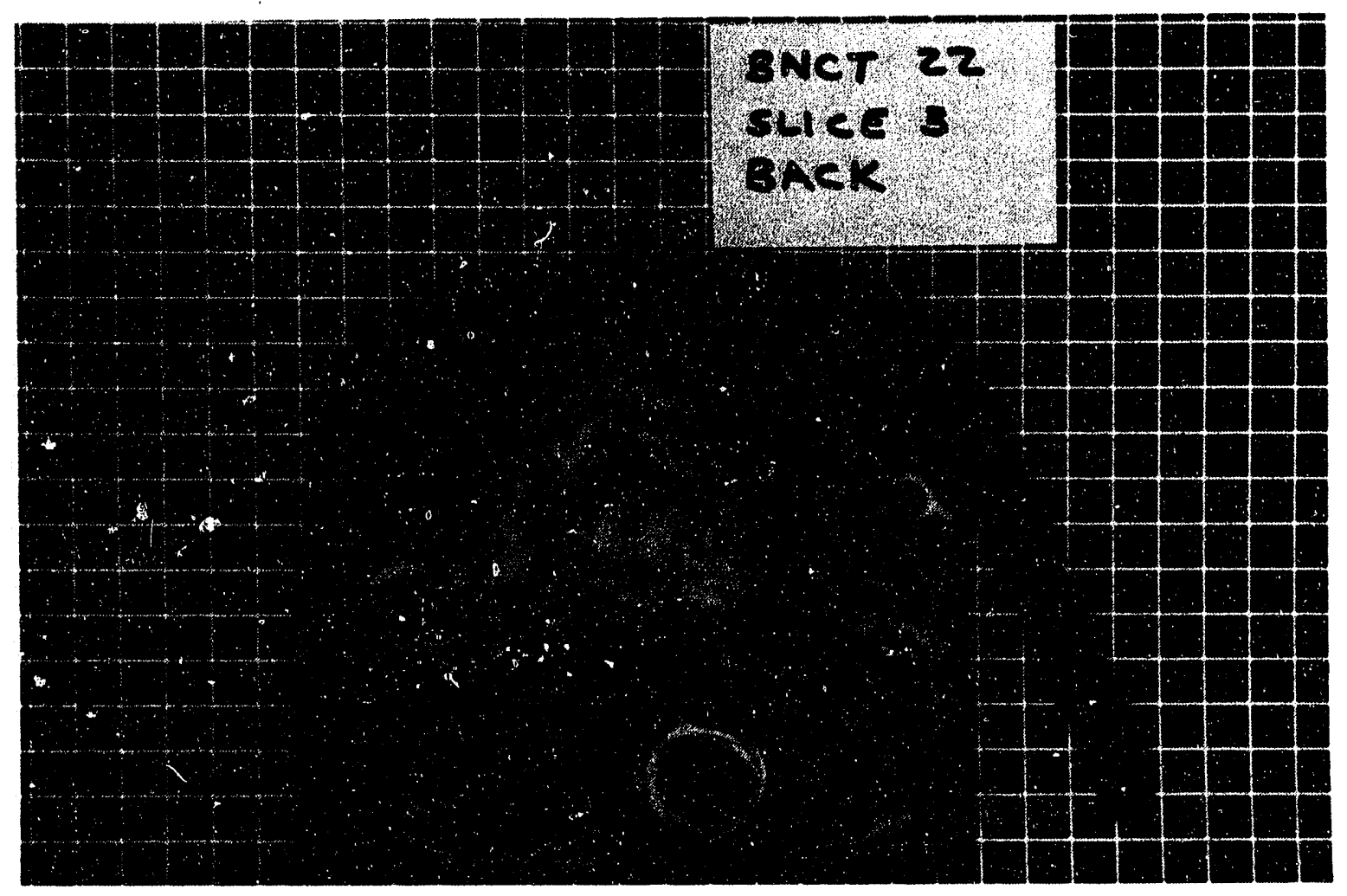

Figure D-6. Photograph of axial slice 3 back at $x=9 \mathrm{~cm}$. 


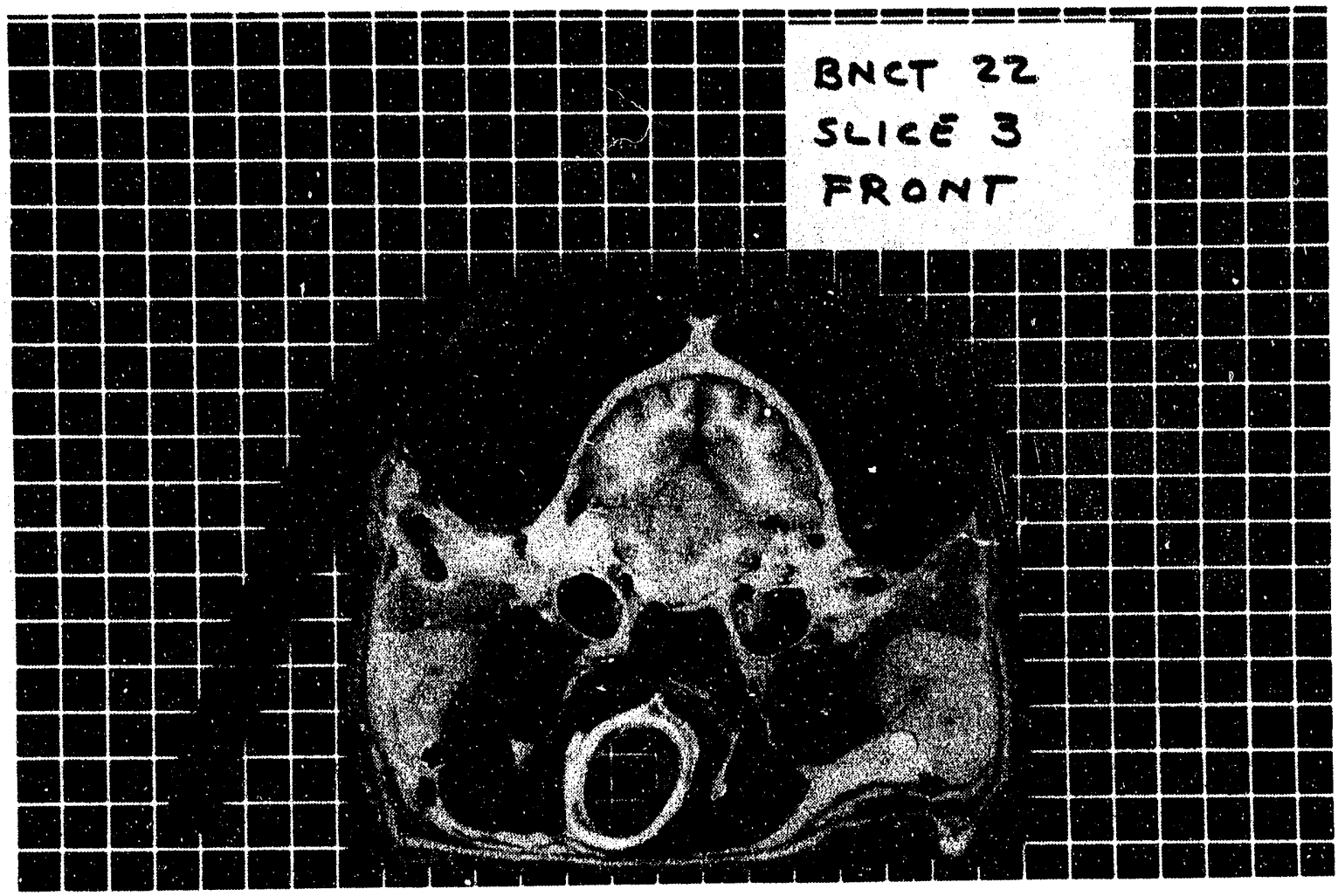

Figure D-7. Photograph of axial slice 3 front at $x=10 \mathrm{~cm}$. 


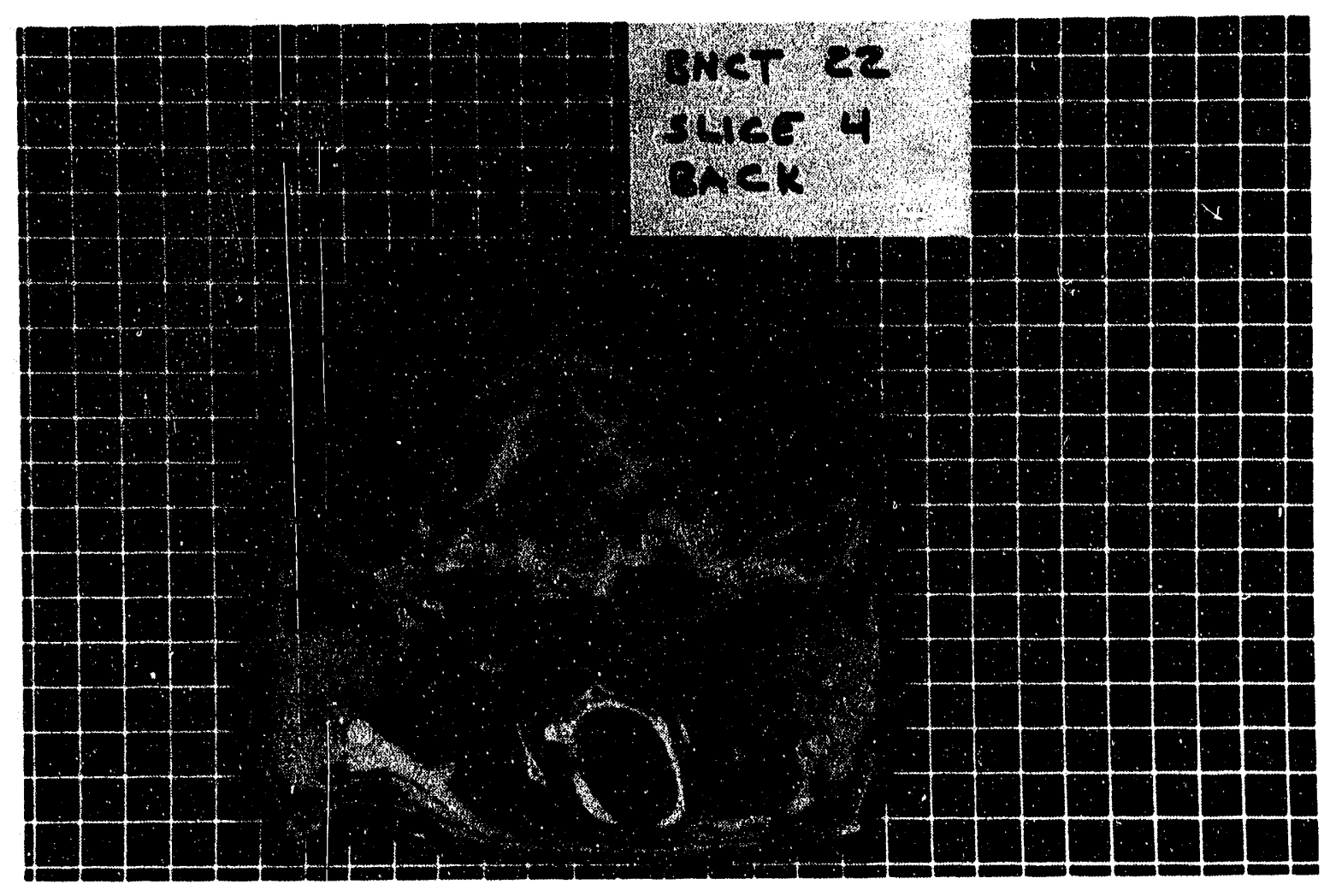

Figure D-8. Fhotograph of axial slice 4 back at $x=10 \mathrm{~cm}$. 


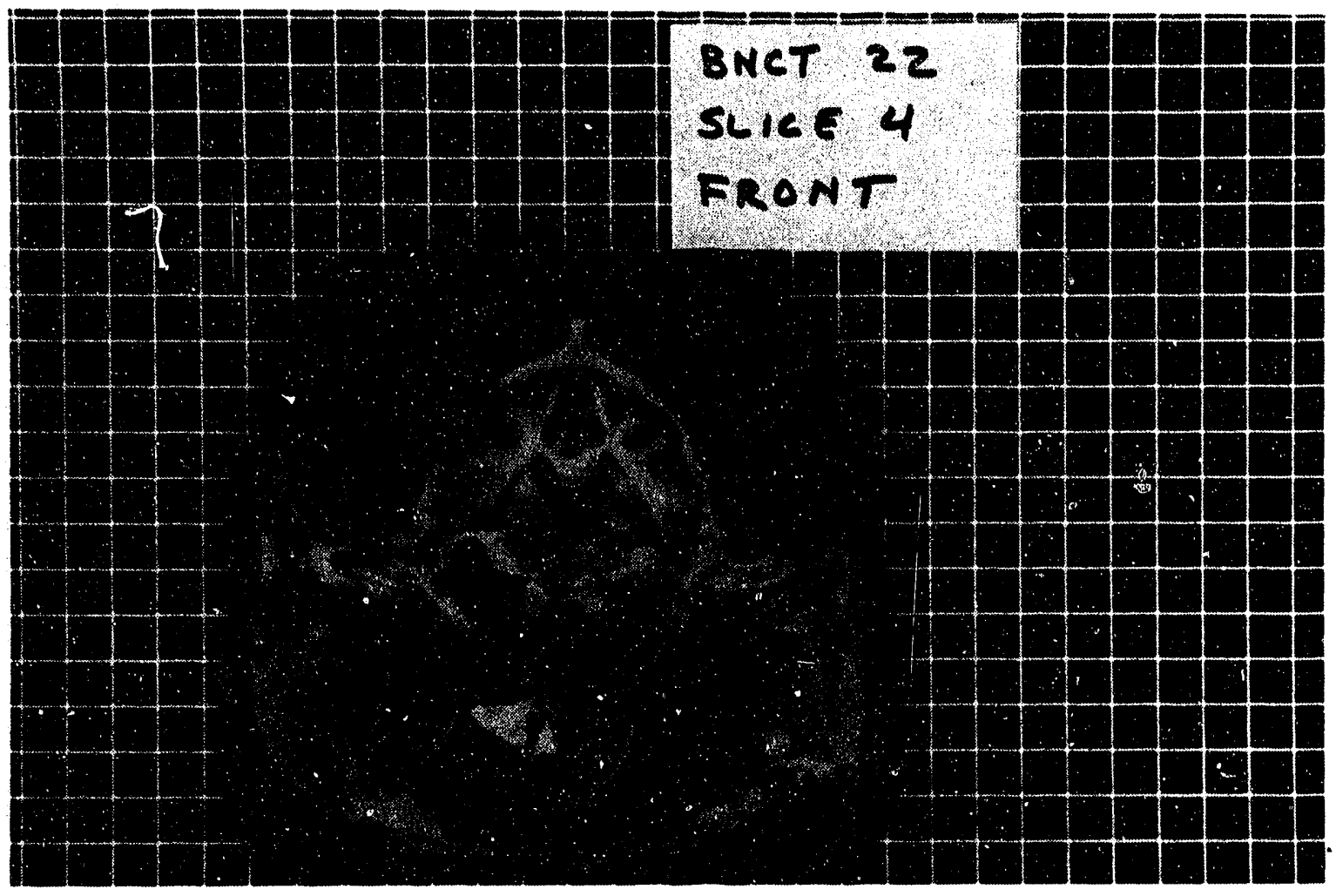

Figure D-9. Photograph of axial slice 4 front at $x=11 \mathrm{~cm}$. 


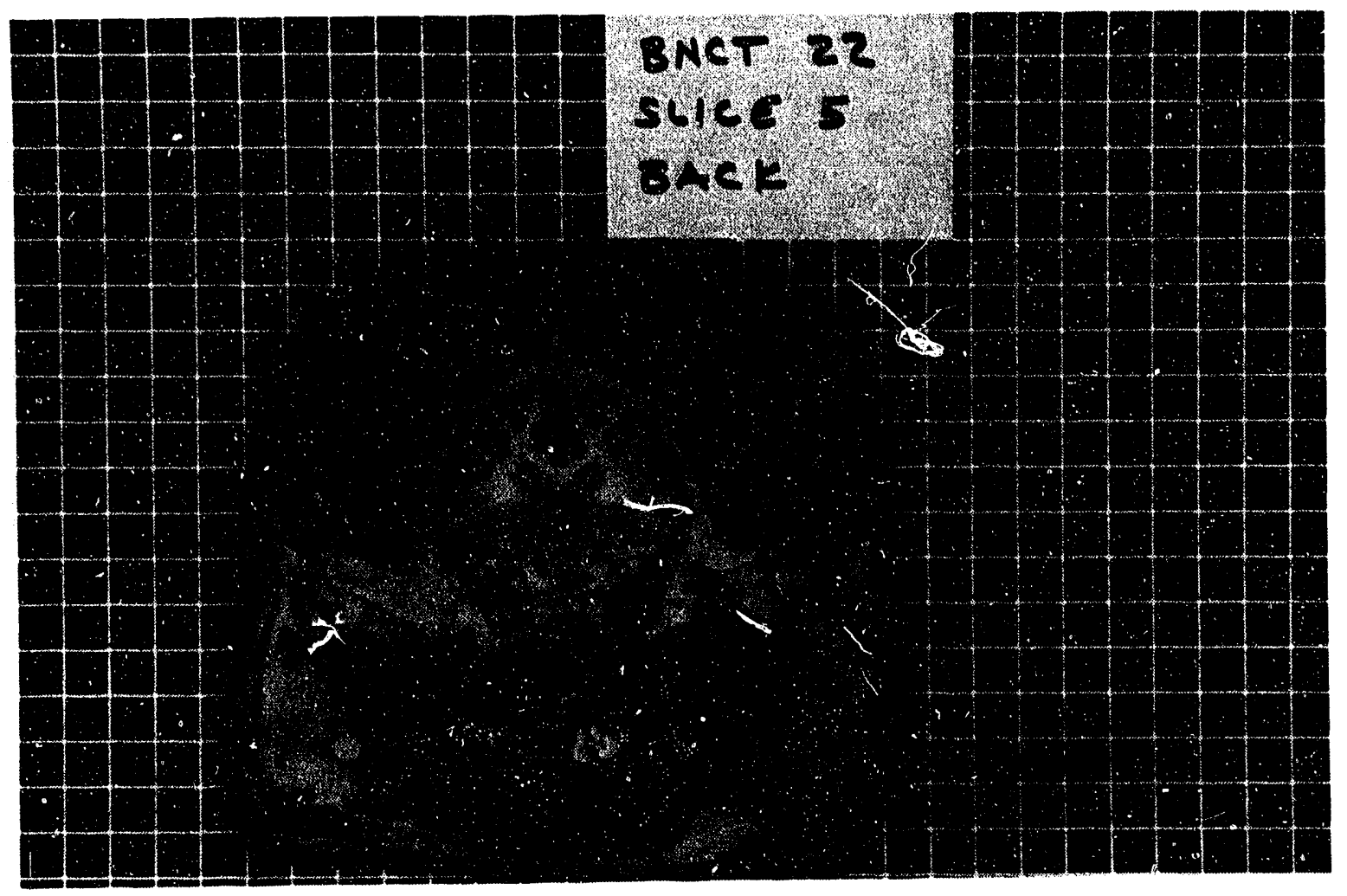

Figure D-10. Photograph of axial slice 5 back at $x=11 \mathrm{~cm}$. 


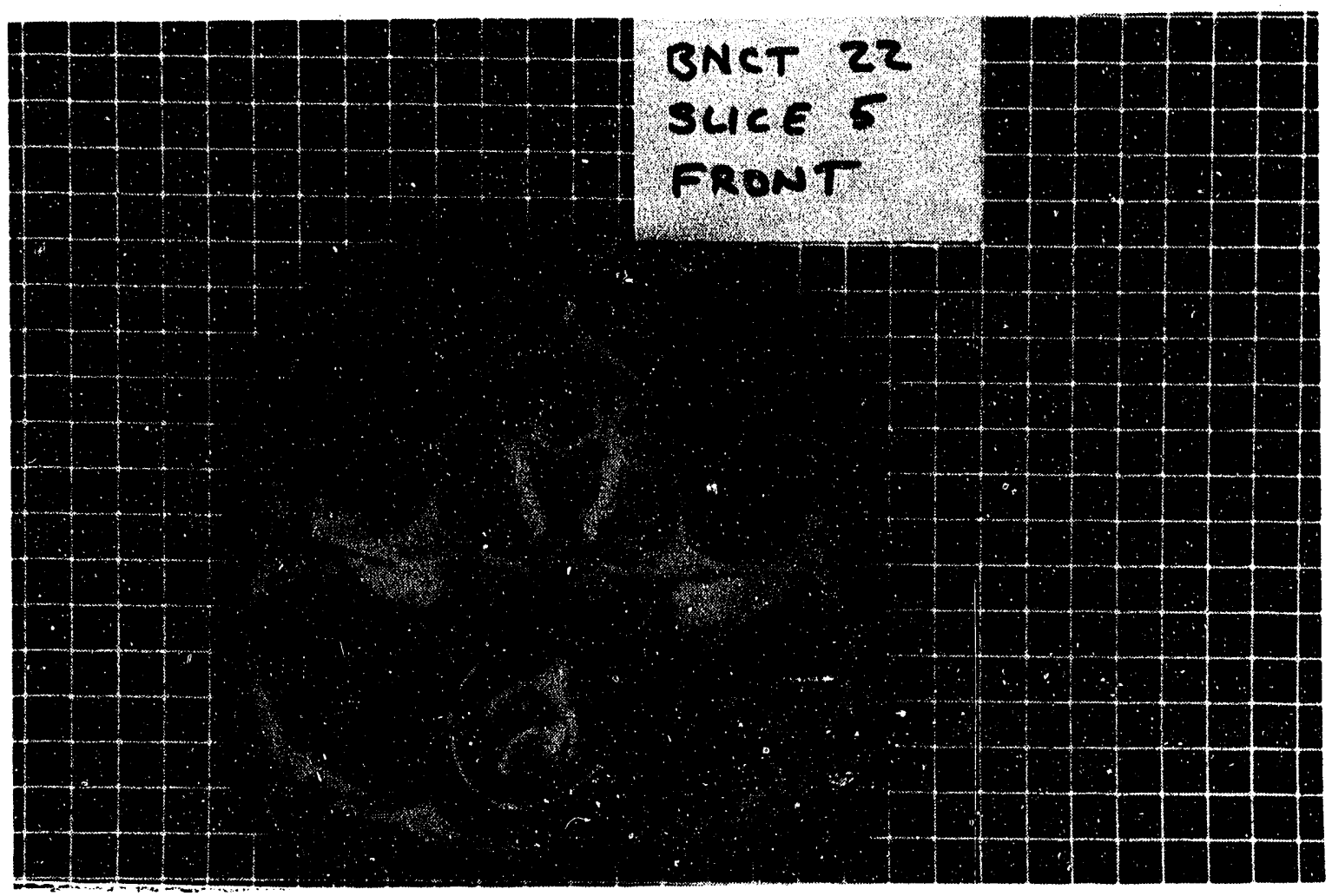

Figure D-11. Photograph of axial slice 5 front at $x=12 \mathrm{~cm}$. 


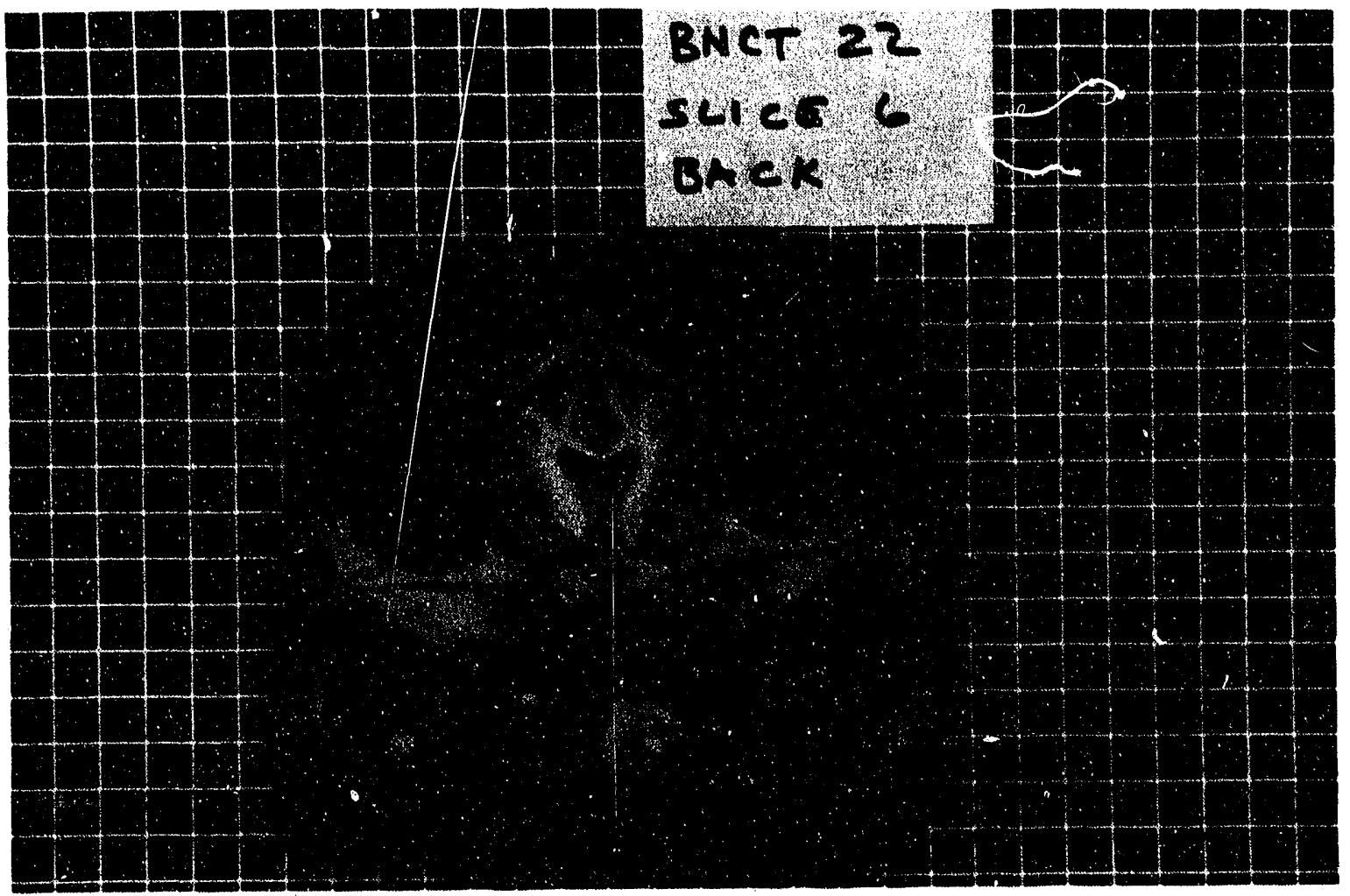

Figure D-12. Photograph of axial slice 6 back at $x=12 \mathrm{~cm}$. 


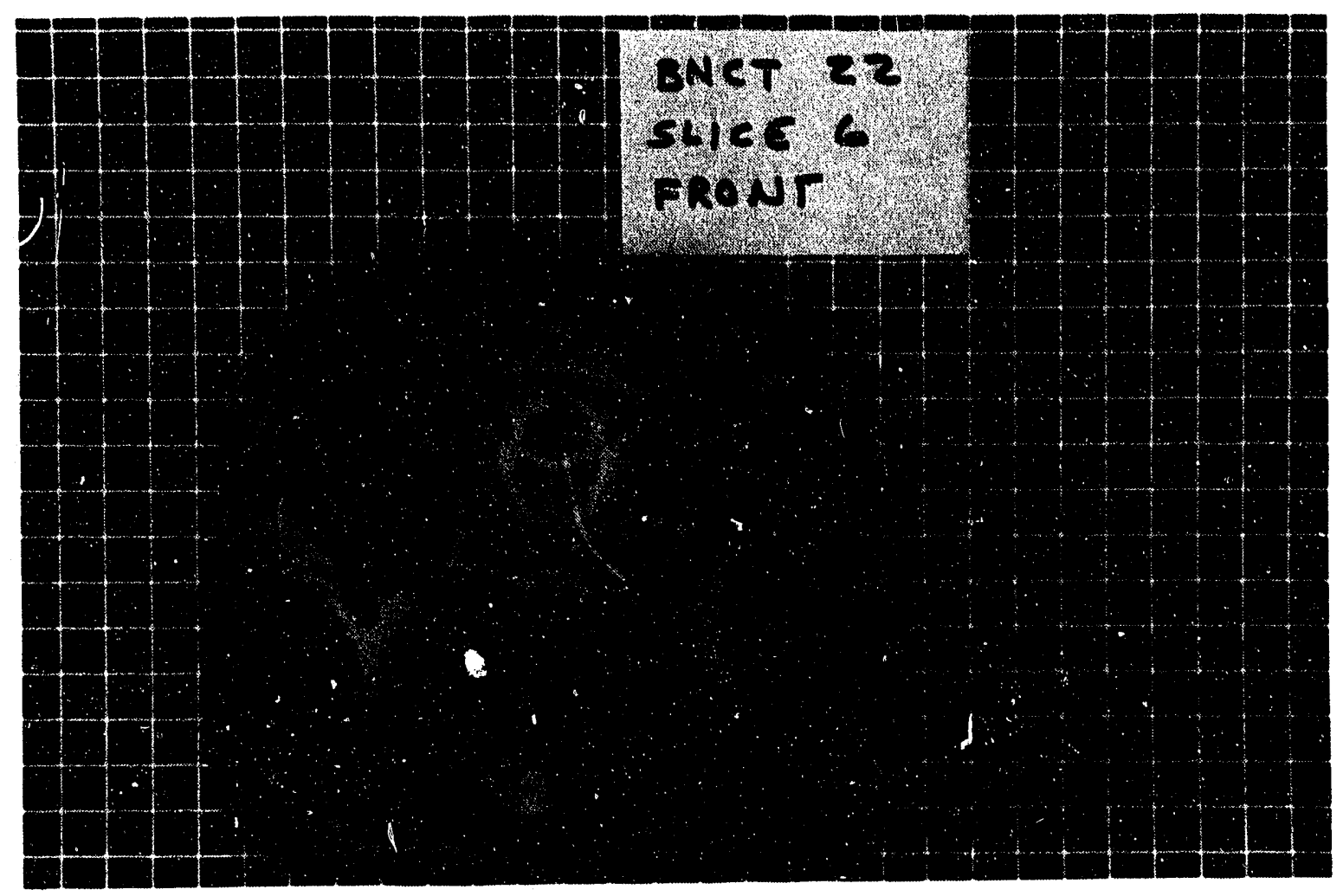

Figure D-13. Photograph of axial slice 6 front at $x=13 \mathrm{~cm}$. 


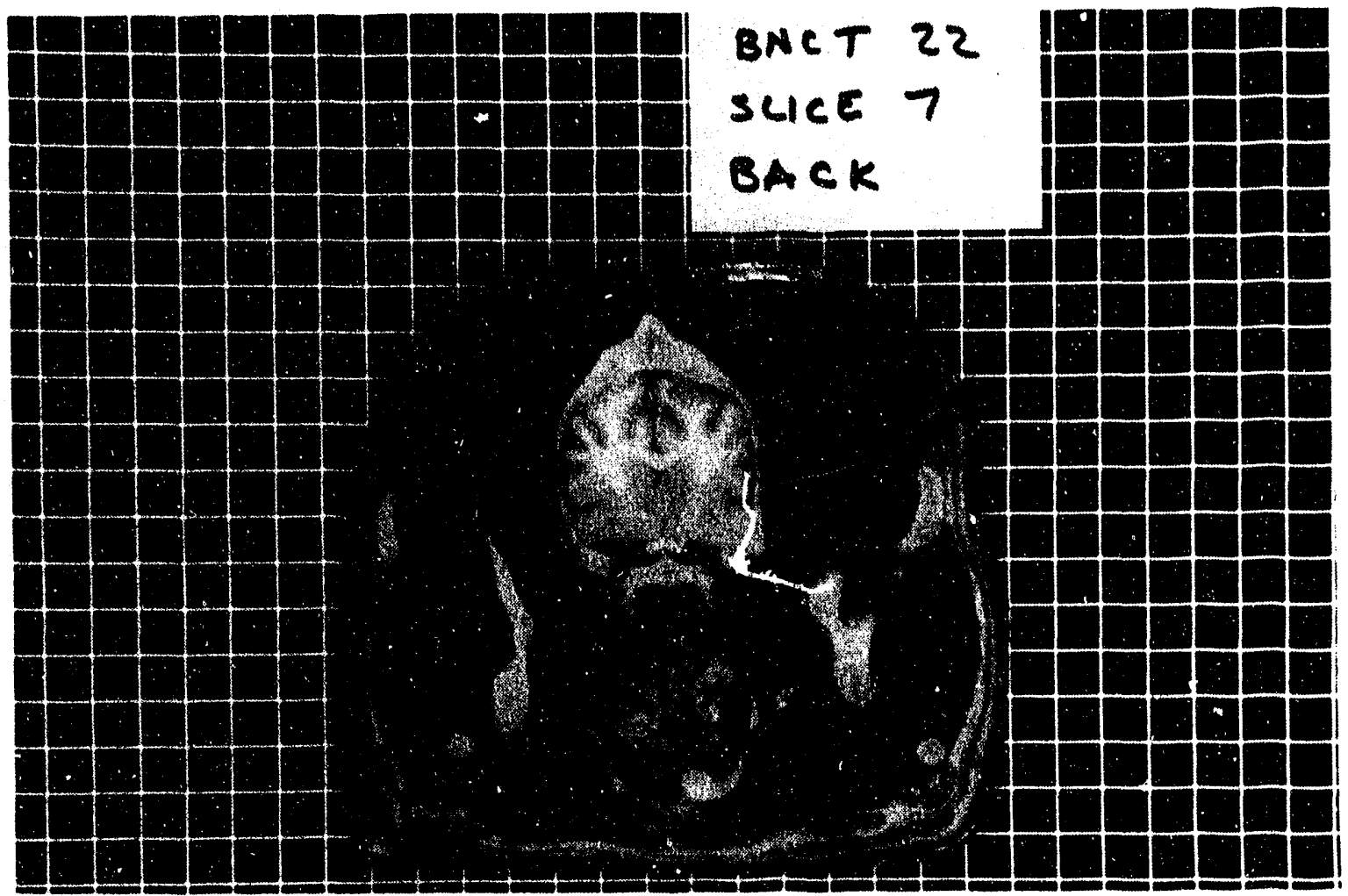

Figure D-14. Photograph of axial slice 7 back at $x=13 \mathrm{~cm}$. 


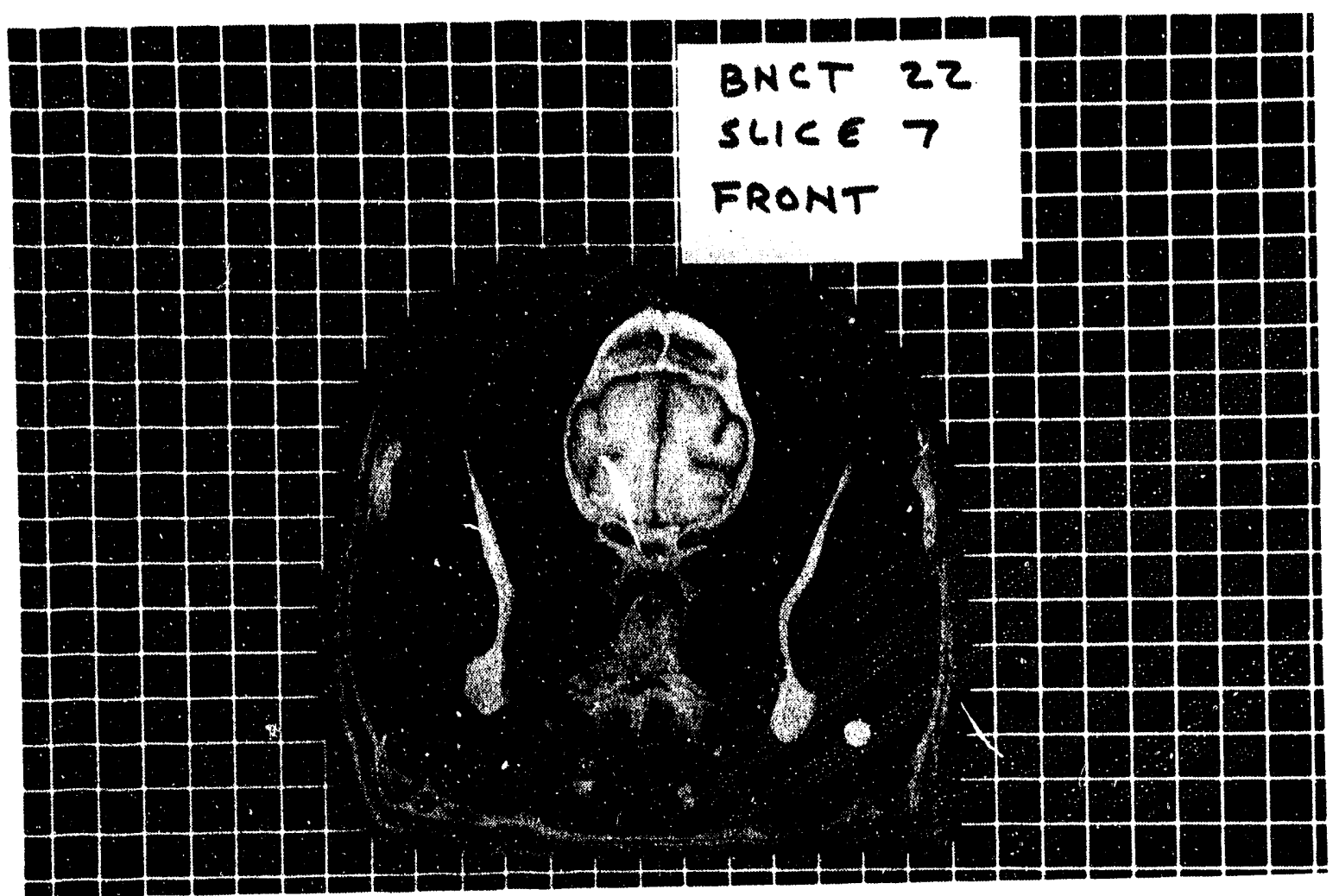

Figure D-15. Photograph of axial slice 7 front at $x=14 \mathrm{~cm}$. 


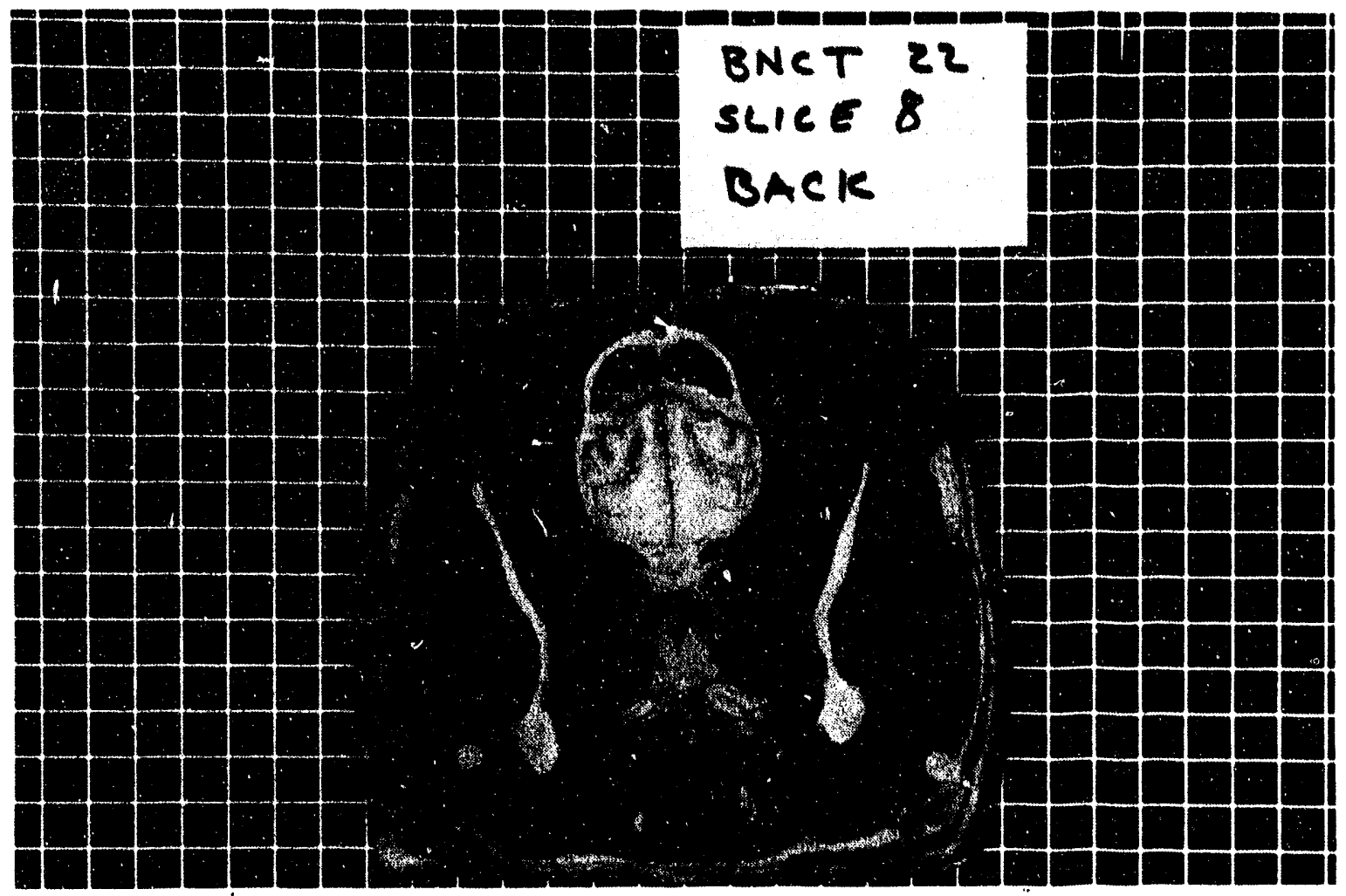

Figure D-16. Photograph of axial slice 8 back at $x=14 \mathrm{~cm}$. 


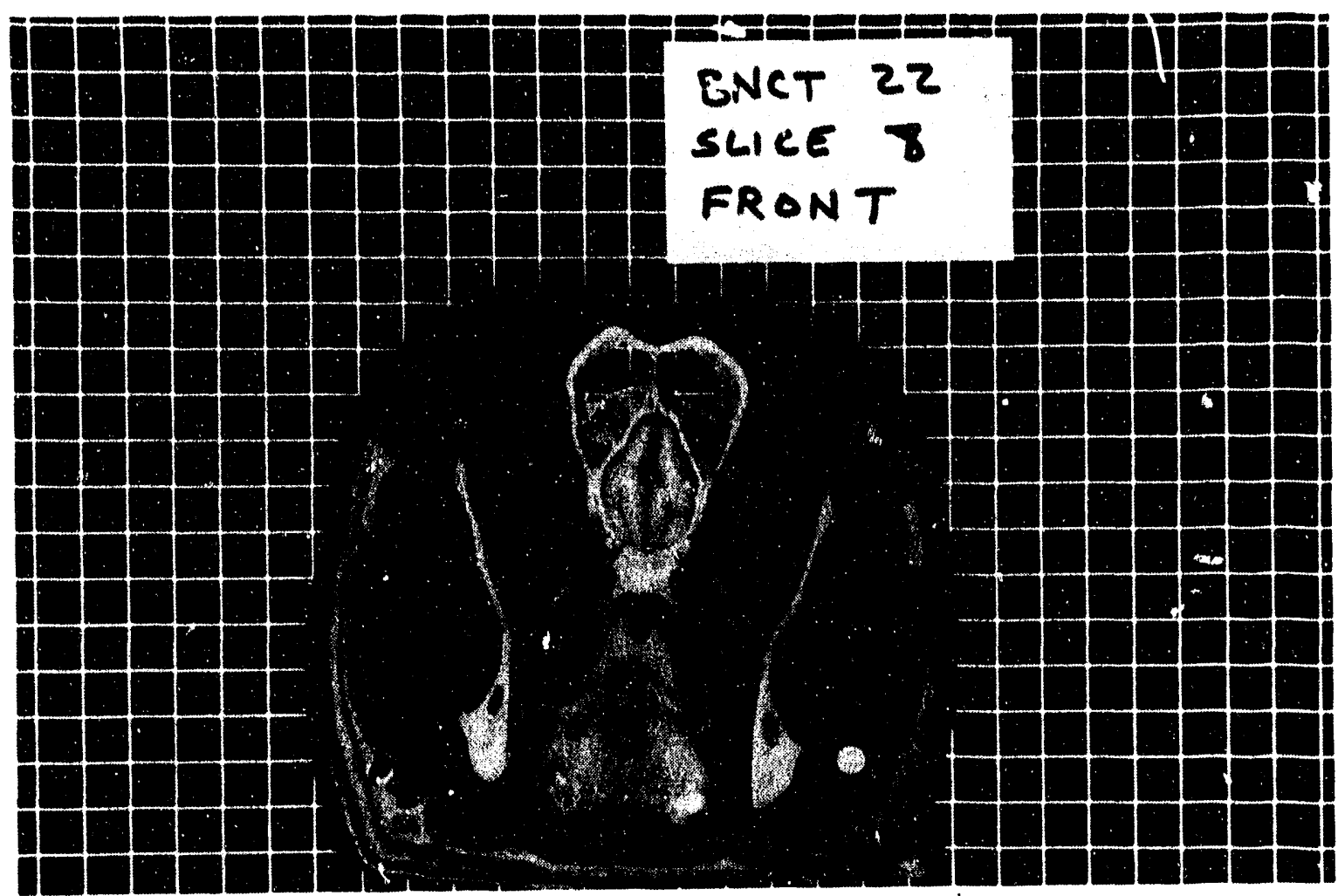

Figure D-17. Photograph of axial slice 8 front at $x=15 \mathrm{~cm}$. 


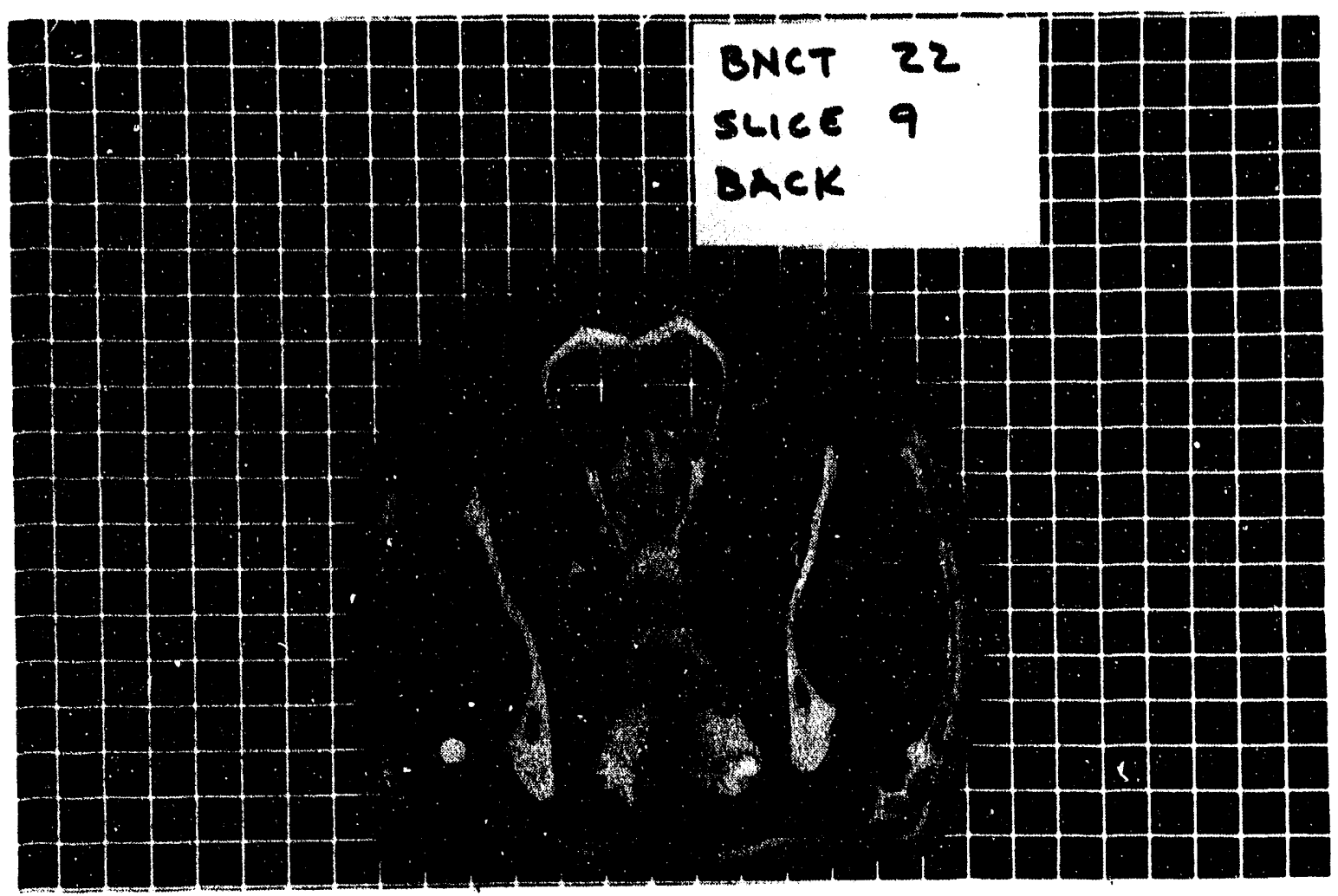

Figure D-18. Photograph of axial slice 9 back at $x=15 \mathrm{~cm}$. 


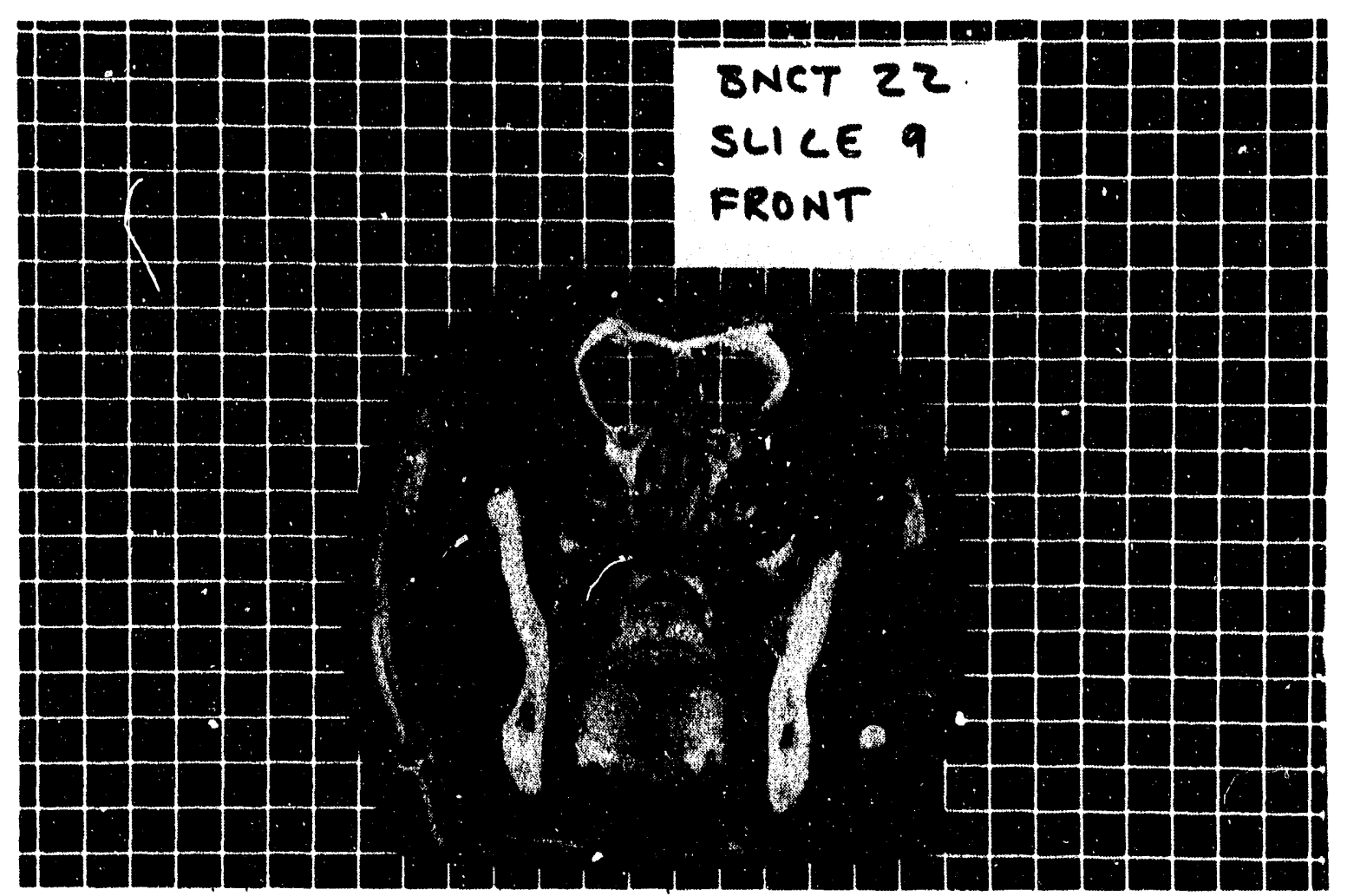

Figure D-19. Photograph of axial slice 9 front at $x=16 \mathrm{~cm}$. 


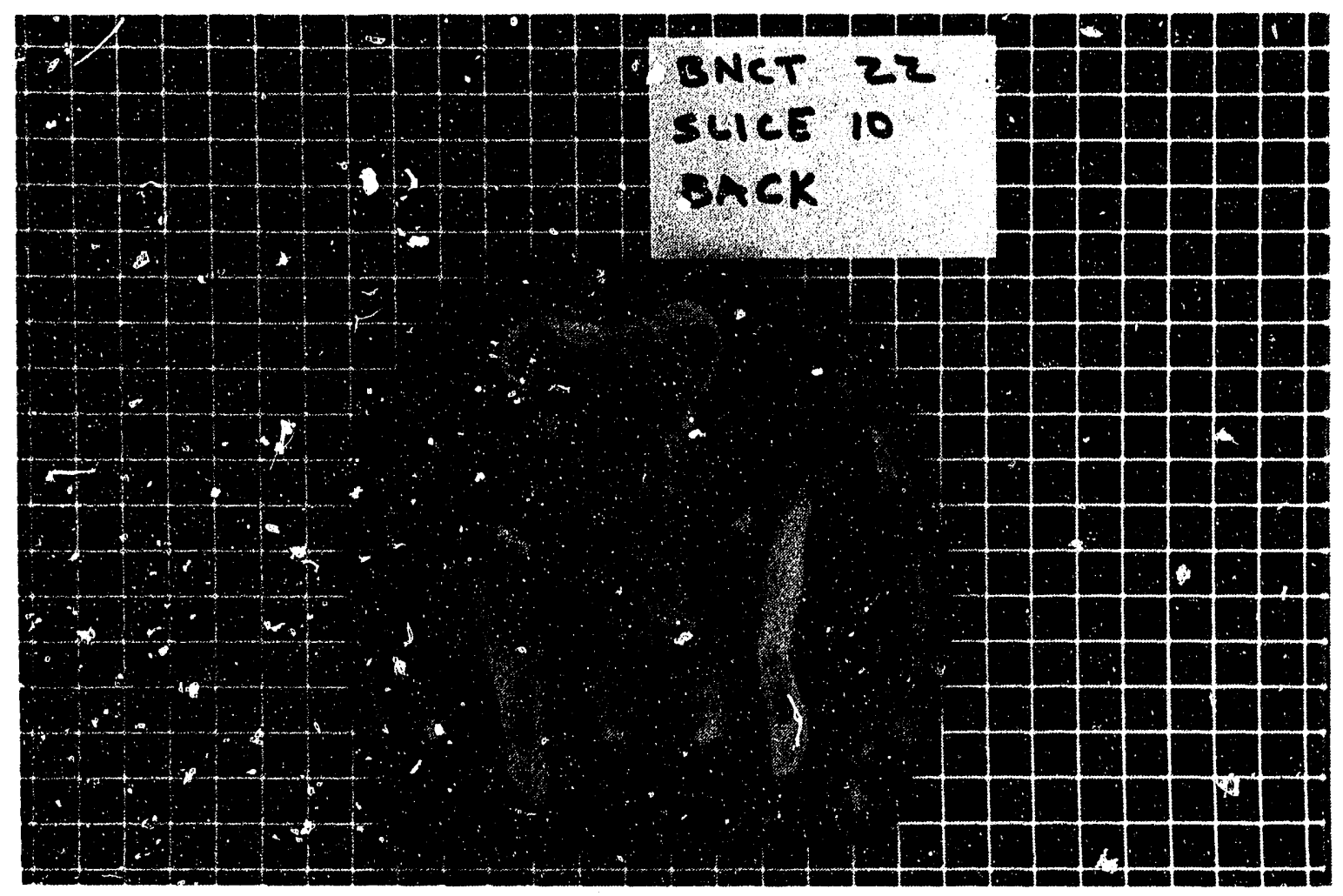

Figure D-20. Photograph of axial slice 10 back at $x=16 \mathrm{~cm}$. 


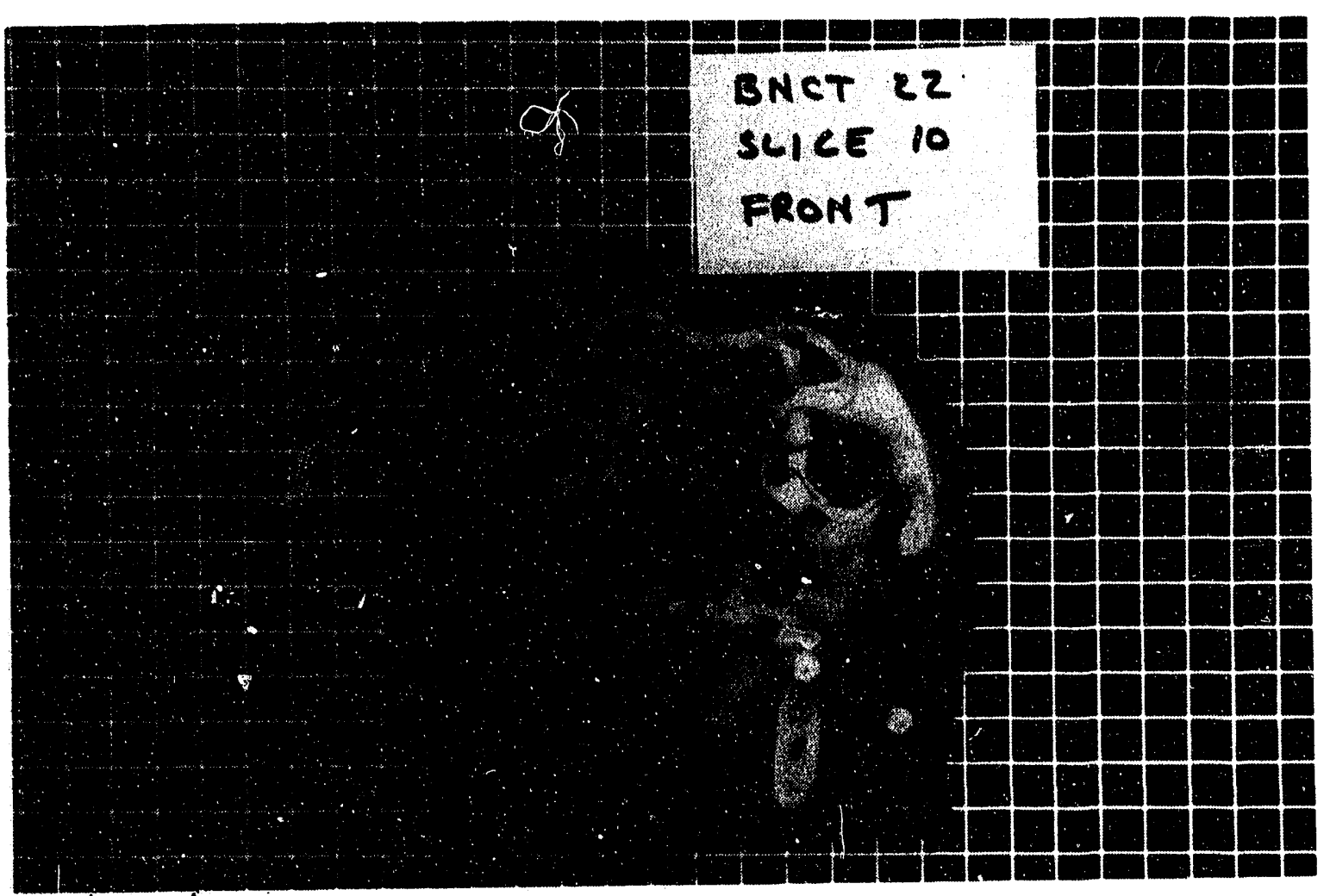

Figure D-21. Photograph of axial slice 10 front at $x=17 \mathrm{~cm}$. 


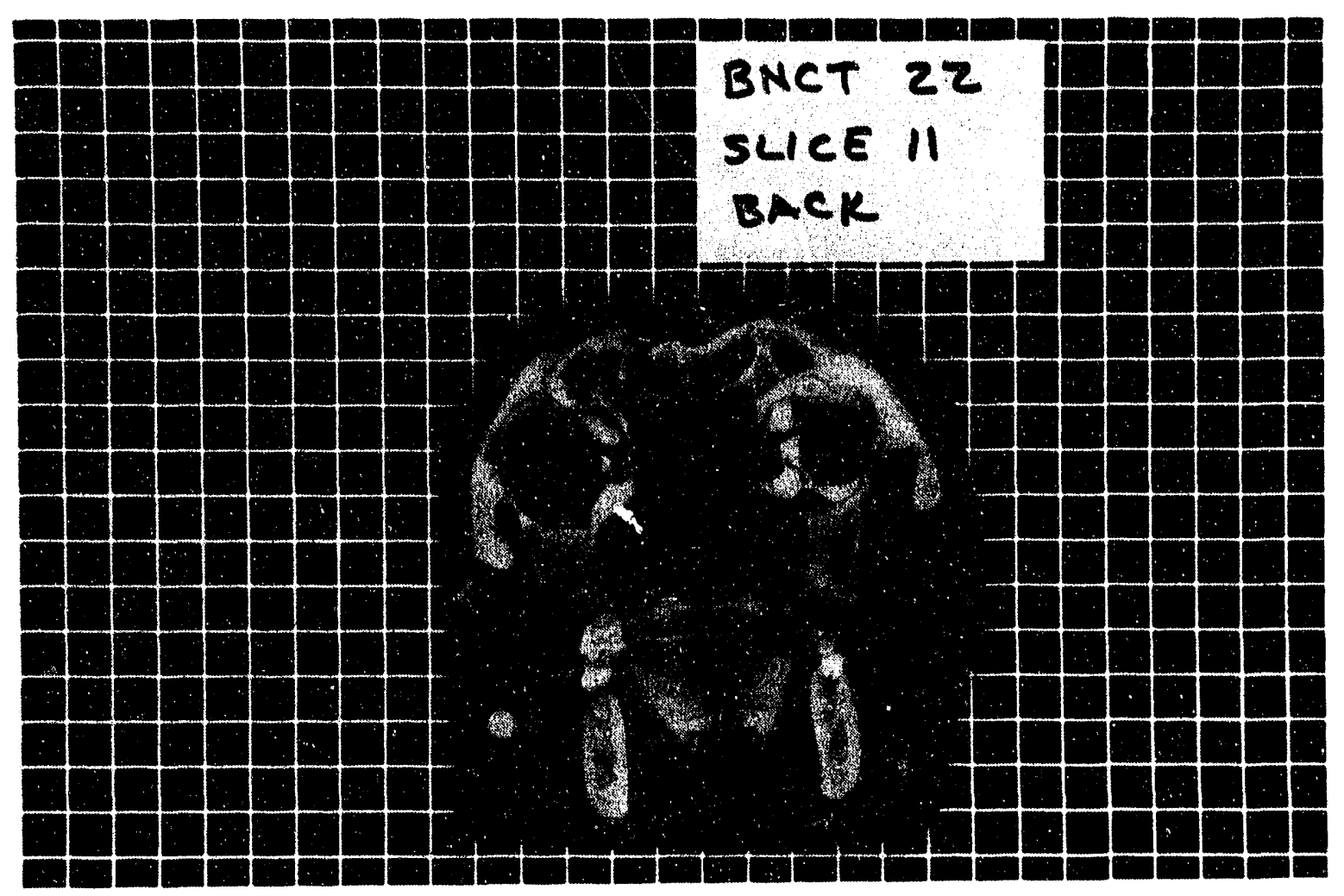

Figure D-22. Photograph of axial slice 11 back at $x=17 \mathrm{~cm}$. 


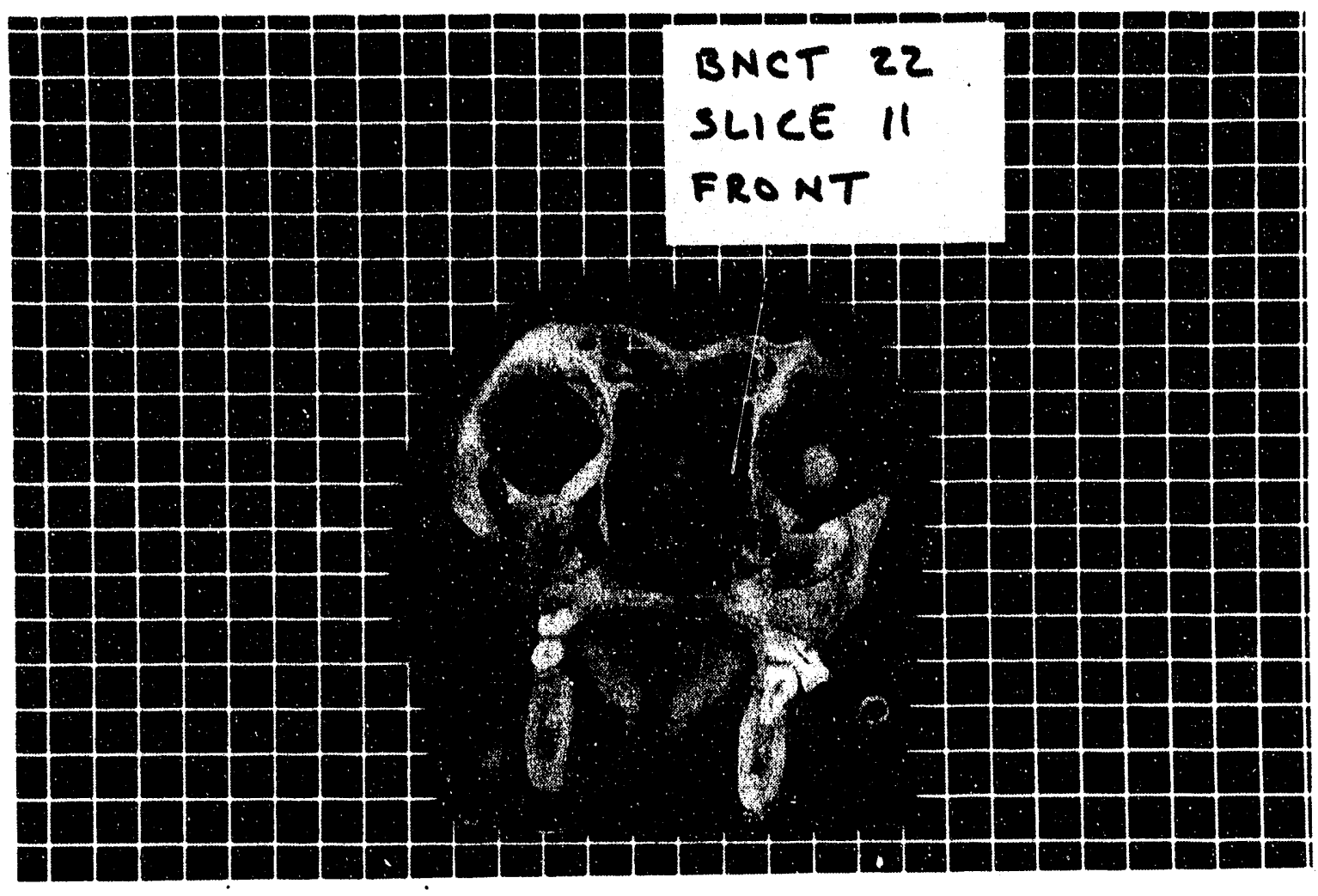

Figure D-23. Photograph of axial slice 11 front at $x=18 \mathrm{~cm}$. 


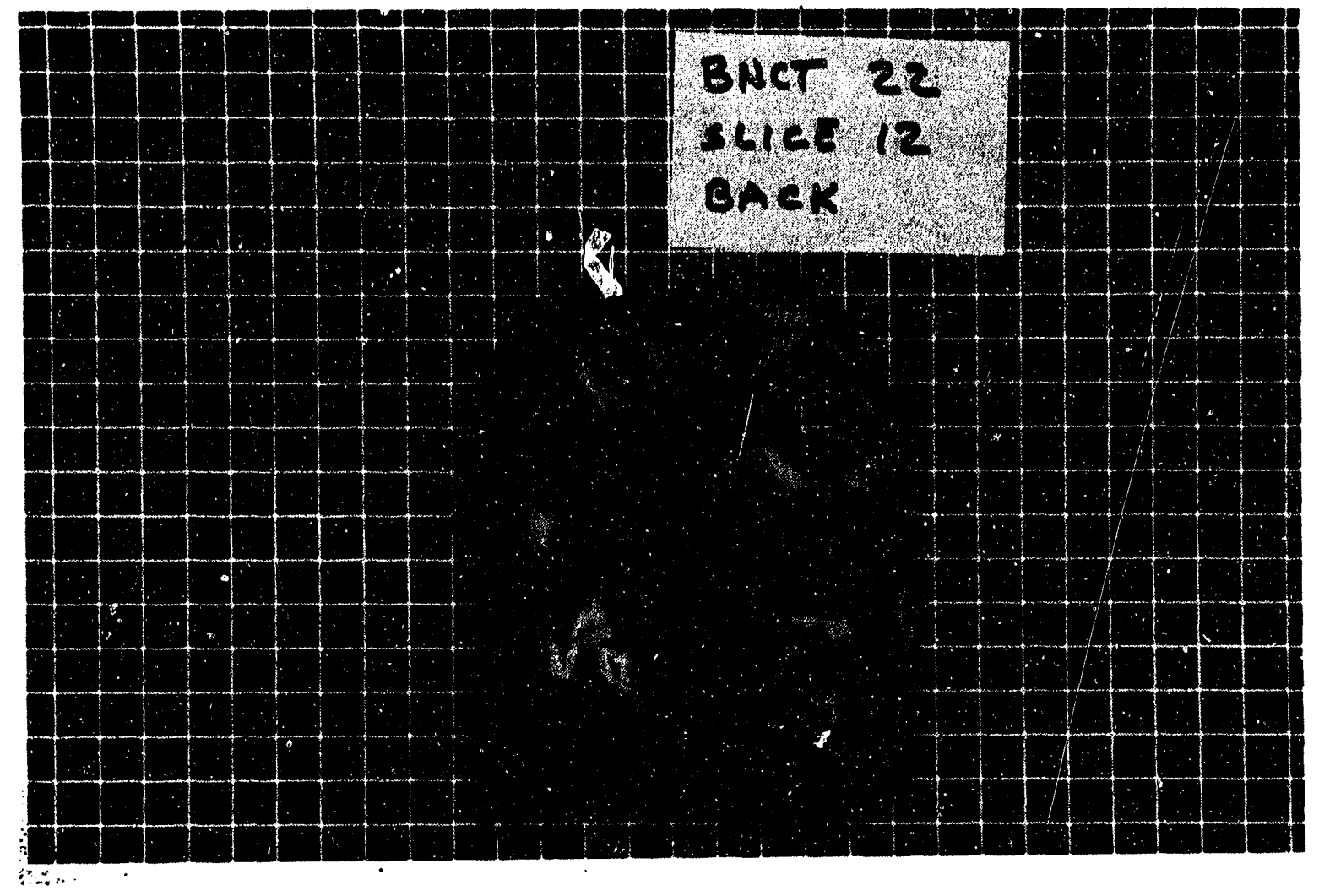

Figure D-24. Photograph of axial slice 12 back at $x=18 \mathrm{~cm}$. 


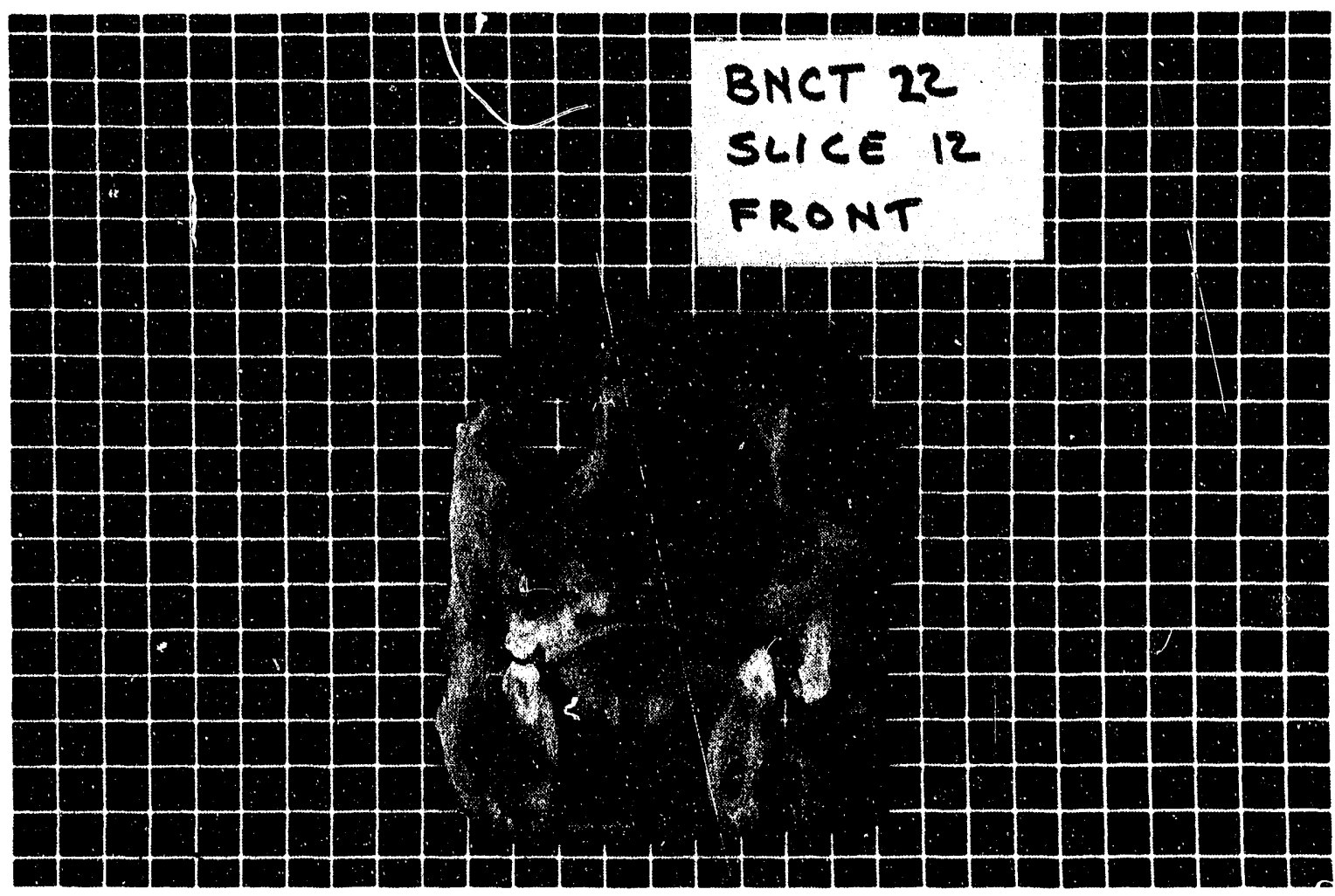

Figure D-25. Photograph of axial slice 12 front at $x=19 \mathrm{~cm}$. 


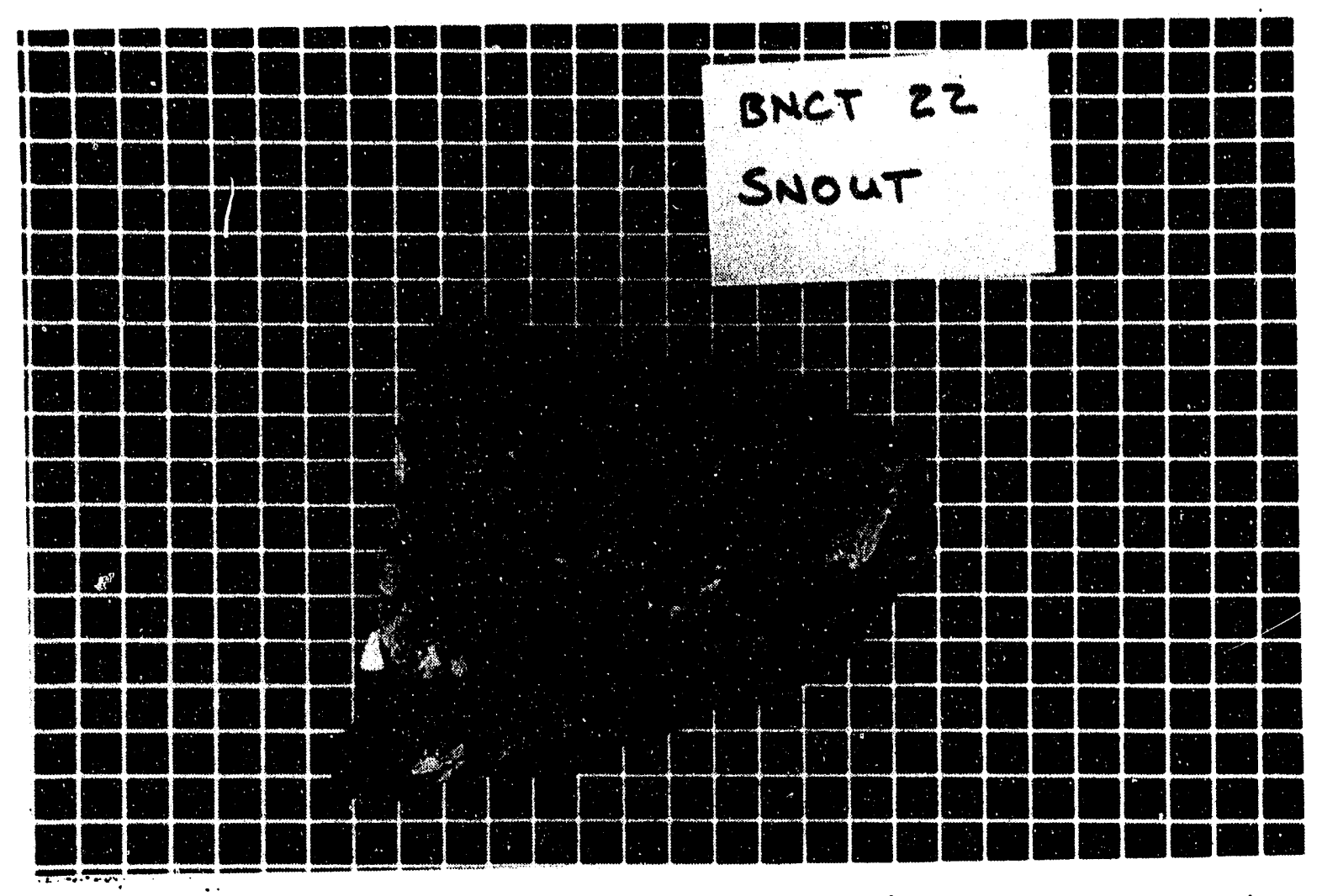

Figure D-26. Photograph of snout extending from $x=19 \mathrm{~cm}$ to approximately $30 \mathrm{~cm}$. 


\section{THIS PAGE INTENTIONALLY LEFT BLANK}




\section{APPENDIX E}

GIP AND TORT INPUT 
GIP Input for TORT Dog Head Calculations 6/05/91

- Includes Density fac:tors and Homogeneous Mix.

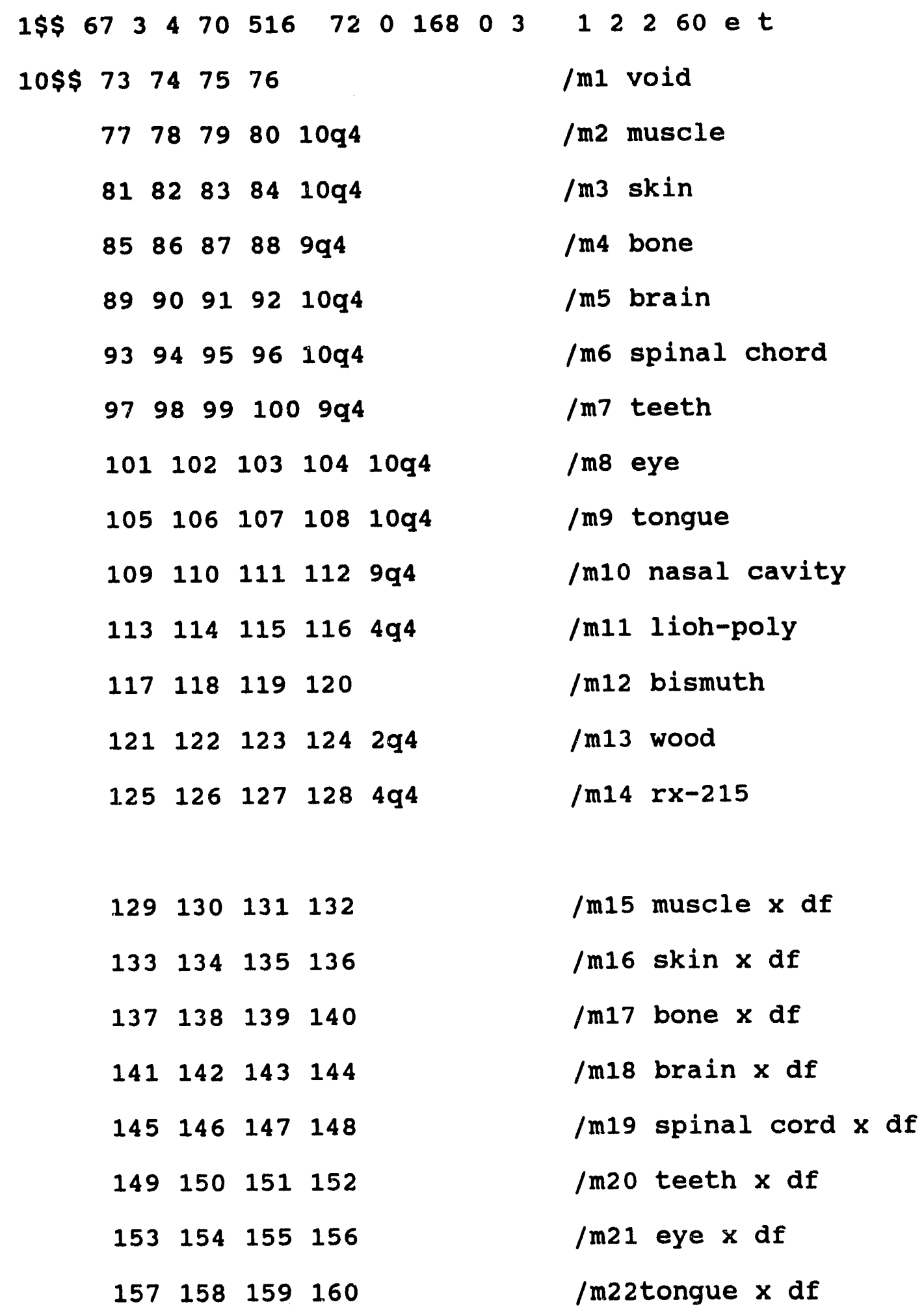




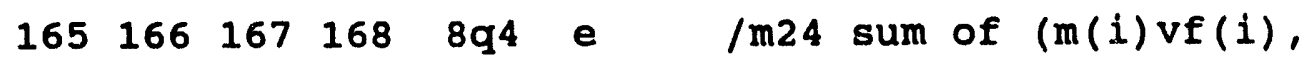
$i=15,23$ )

- Isotope List (BUGLE80):

1

- 11 PO H-1 CHECK CASE FOR COMBINED LIBRARY PROCESSI FROM CARDS (TAPE10)

- 22 P1 H-1 CHECK CASE FOR COMBINED LIBRARY PROCESSI FROM CARDS (TAPE10)

- 33 P2 H-1 CHECK CASE FOR COMBINED LIBRARY PROCESSI FROM CARDS (TAPE10)

1 44 P3 H-1 CHECK CASE FOR COMBINED LIBRARY PROCESSI FROM CARDS (TAPE10)

- 55 PO B-10 CASE FOR COMBINED CTR + CRBR PROCESSIN FROM CARDS (TAPE10)

- 66 P1 B-10 CASE FOR COMBINED CTR + CRBR PROCESSIN FROM CARDS (TAPE10)

1 77 P2 B-10 CASE FOR COMBINED CTR + CRBR PROCESSIN FROM CARDS (TAPE10)

1 88 P3 B-10 CASE FOR COMBINED CTR + CRBR PROCESSIN FROM CARDS (TAPE10)

- 99 PO 0-16 RE-RUN (FIXUP MT=102 X-SECTION)

FROM CARDS (TAPE10)

1 1010 P1 0-16 RE-RUN (FIXUP MT=102 X-SECTION) 
FROM CARDS (TAPE10)

11111 P2 0-16 RE-RUN (FIXUP MT=102 X-SECTION)

FROM CARDS (TAPE10)

- 1212 P3 0-16 RE-RUN (FIXUP MT=102 X-SECTION)

FROM CARDS (TAPE10)

- 1341 PO C-12 CASE FOR COMBINED CTR + CRBR PROCESSING FROM CARDS (TAPE10)

- 1442 P1 C-12 CASE FOR COMBINED CTR + CRBR PROCESSING FROM CARDS (TAPE10)

- 1543 P2 C-12 CASE FOR COMBINED CTR + CRBR PROCESSING FROM CARDS (TAPE10)

1 1644 P3 C-12 CASE FOR COMBINED CTR + CRBR PROCESSING FROM CARDS (TAPE10)

- 1753 PO NA CASE FOR REM SHIELDING PROBLEM FROM CARDS (TAPE10)

1 1854 P1 NA CASE FOR REM SHIELDING PROBLEM FROM CARDS (TAPE10)

- 1955 P2 NA CASE FOR REM SHIELDING PROBLEM FROM CARDS (TAPE10)

- 2056 P3 NA CASE FOR REM SHIELDING PROBLEM FROM CARDS (TAPE10)

1 2157 PO MG CASE FOR COMBINED CTR + CRBR PROCESSIN FROM CARDS (TAPE10)

1 2258 P1 MG CASE FOR COMBINED CTR + CRBR PROCESSIN FROM CARDS (TAPE10)

1 2359 P2 MG CASE FOR COMBINED CTR + CRBR PROCESSIN 
FROM CARDS (TAPE10)

- 2460 P3 MG CASE FOR COMBINED CTR + CRBR PROCESSIN FROM CARDS (TAPE10)

- 2565 PO S32 CASE FOR COMBINED CTR + CRBR PROCESSIN FROM CARDS (TAPE10)

- 2666 P1 S32 CASE FOR COMBINED CTR + CRBR PROCESSIN FROM CARDS (TAPE10)

1 2767 P2 S32 CASE FOR COMBINED CTR + CRBR PROCESSIN FROM CARDS (TAPE10)

$\begin{array}{lllll}28 & 68 & \text { P3 } & \text { S32 } & \text { CASE FOR COMBINED CTR + CRBR PROCESSIN }\end{array}$ FROM CARDS (TAPE10)

' 2969 PO K CASE FOR COMBINED CTR + CRBR PROCESSIN FROM CARDS (TAPE10)

- 3070 P1 K CASE FOR COMBINED CTR + CRBR PROCESSIN FROM CARDS (TAPE10)

' $31 \quad 71$ P2 $\mathrm{K}$ CASE FOR COMBINED CTR + CRBR PROCESSIN FROM CARDS (TAPE10)

- 3272 P3 K CASE FOR COMBINED CTR + CRBR PROCESSIN FROM CARDS (TAPE10)

- 3373 PO CA CASE FOR COMBINED CTR + CRBR PROCESSIN FROM CARDS (TAPE10)

1 3474 P1 CA CASE FOR COMBINED CTR + CRBR PROCESSIN FROM CARDS (TAPE10)

1 3575 P2 CA CASE FOR COMBINED CTR + CRBR PROCESSIN FROM CARDS (TAPE10)

- 3676 P3 CA CASE FOR COMBINED CTR + CRBR PROCESSIN 
FROM CARDS (TAPE10)

- 37101 PO LI-6 CASE FOR COMBINED CTR + CRBR PROCESSIN FROM CARDS (TAPE10)

- 38102 P1 LI-6 CASE FOR COMBINED CTR + CRBR PROCESSIN FROM CARDS (TAPE10)

- 39103 P2 LI-6 CASE FOR COMBINED CTR + CRBR PROCESSIN FROM CARDS (TAPE10)

1 40104 P3 LI-6 CASE FOR COMBINED CTR + CRBR PROCESSIN FROM CARDS (TAPE10)

1 41105 PO LI-7 CASE FOR COMBINED CTR + CRBR PROCESSIN FROM CARDS (TAPE10)

- 42106 P1 LI-7 CASE FOR COMBINED CTR + CRBR PROCESSIN FROM CARDS (TAPE10)

- 43107 P2 LI-7 CASE FOR COMBINED CTR + CRBR PROCESSIN FROM CARDS (TAPE10)

1 44108 P3 LI-7 CASE FOR COMBINED CTR + CRBR PROCESSIN FROM CARDS (TAPE10)

1 45113 PO N-14 CASE FOR COMBINED CTR + CRBR PROCESSIN FROM CARDS (TAPE10)

- 46114 P1 N-14 CASE FOR COMBINED CTR + CRBR PROCESSIN FROM CARDS (TAPE10)

1 47115 P2 N-14 CASE FOR COMBINED CTR + CRBR PROCESSIN FROM CARDS (TAPE10)

- 48116 P3 N-14 CASE FOR COMBINED CTR + CRBR PROCESSIN FROM CARDS (TAPE1O)

1 49121 PO P31 CASE FOR COMBINED CTR + CRBR PROCESSIN 
FROM CARDS (TAPE10)

- 50122 PJ. 931 CASE FOR COMBINED CTR + CRBR PRLCESSIN FROM SARDS (TAPE10)

- 51123 P2 P31 CASE FOR COMBINED CTR + CRBR PROCESSIN FROM CARDS (TAPE10)

1 52124 P3 P31 CASE FOR COMBINED CT'R + CRBR PROCESSIN FROM CARDS (TAPE10)

- 53125 PO CL CASE FOR COMBINED CTR + CRBR PROCESSIN FROM CARDS (TAPE10)

- 54126 T1 CL CASE FOR COMBINED CTR + CRBR PROCESSIN FROM CARDS (TAPE10)

1 55127 P2 CL CASE FOR COMBINED CTR + CRBR PROCESSIN FROM CARDS (TAPE10)

- 56128 P3 CL CASE FOR COMBINED CTR + CRBR PROCESSIN FROM CARDS (TAPE10)

- 57137 PO CO59 CASE FOR COMBINED CTR + CRBR PROCESSIN FROM CARDS (TAPE10)

- 58138 P1 CO59 CASE FOR COMBINED CTR + CRBR PROCESSIN FROM CARDS (TAPE10)

- 59139 P2 CO59 CASE FOR COMBINED CTR + CRBR PROCESSIN FROM CARDS (TAPE10)

- 60140 P3 CO59 CASE FOR COMBINED CTR + CRBR PROCESSIN FROM CARDS (TAPE10)

- 61141 PO CU CAEE FOR COMBINED CTR + CRBR PROCESSIN FROM CARDS (TAPE10)

- 62142 P1 CU CASE FOR COMBINED CTR + CRBR PROCESSIN 
FROM C. IRDS (TAPE10)

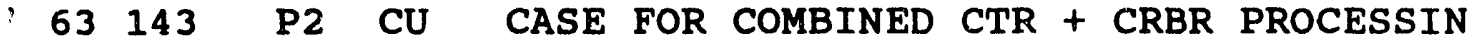
FROM CARDS (TAPE10)

- 64144 P3 CU CASE FOR COMBINED CTR + CRBR PROCESSIN FROM CARDS (TAPE10)

' 65185 PO PB CASE FOR COMBINED CTR + CRBR PROCESSIN FROM CARDS (TAPE10)

1 66186 P1 PB CASE FOR COMBINED CTR + CRBR PROCESSIN FROM CARDS (TAPE10)

1 67187 P2 PB CASE FOR COMBINED CTR + CRBR PROCESSIN FROM CARDS (TAPE10)

- 68188 P3 PB CASE FOR COMBINED CTR + CRBR PROCESSIN FROM CARDS (TAPE10)

1 69201 PO BI-209 1375/1 04-21-85 174N MINX VITAMIN-E FROM CARDS (TAPE10)

- 70202 P1 BI-209 1375/1 04-21-85 174N MINX VITAMIN-E FROM CARDS (TAPE10)

- 71203 P2 BI-209 1375/1 04-21-85 174N MINX VITAMIN-E FROM CARDS' (TAPE10)

- 72204 P3 BI-209 1375/1 04-21-85 174N MINX VITAMIN-E FROM CARDS (TAPE10)

$\begin{array}{rrrrrrl}11 \$ \$ & 1 & 2 & 3 & 4 & / \mathrm{m} 1 \text { void } \\ 1 & 2 & 3 & 4 & / \mathrm{m} 2 \text { muscle } \\ 9 & 10 & 11 & 12 & \end{array}$




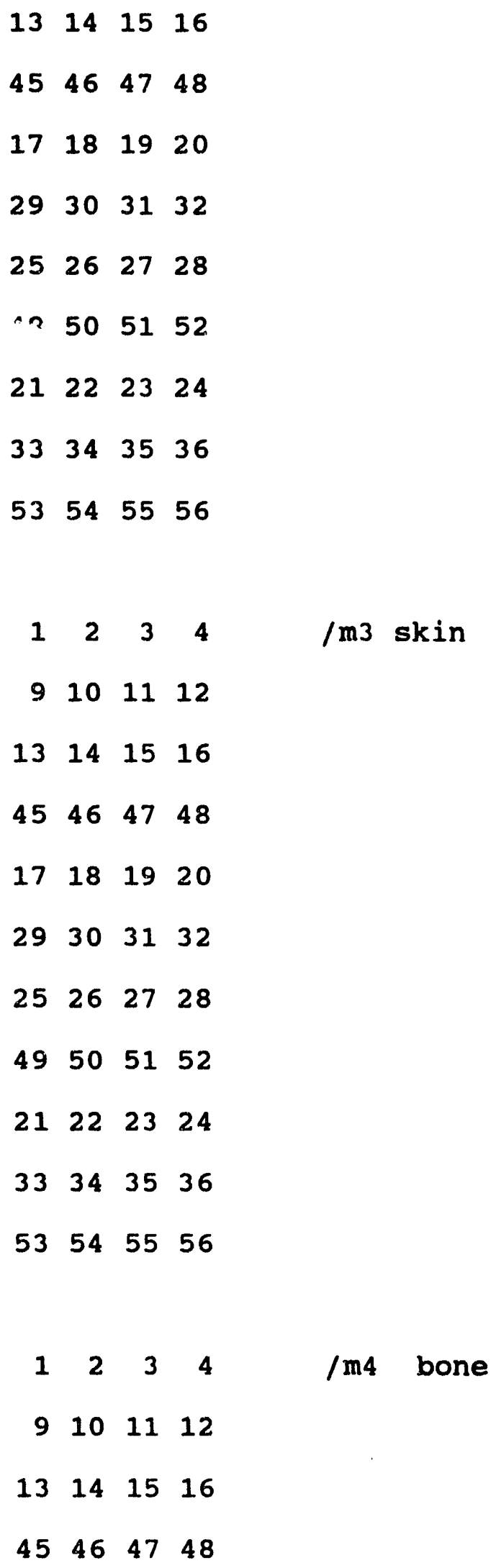




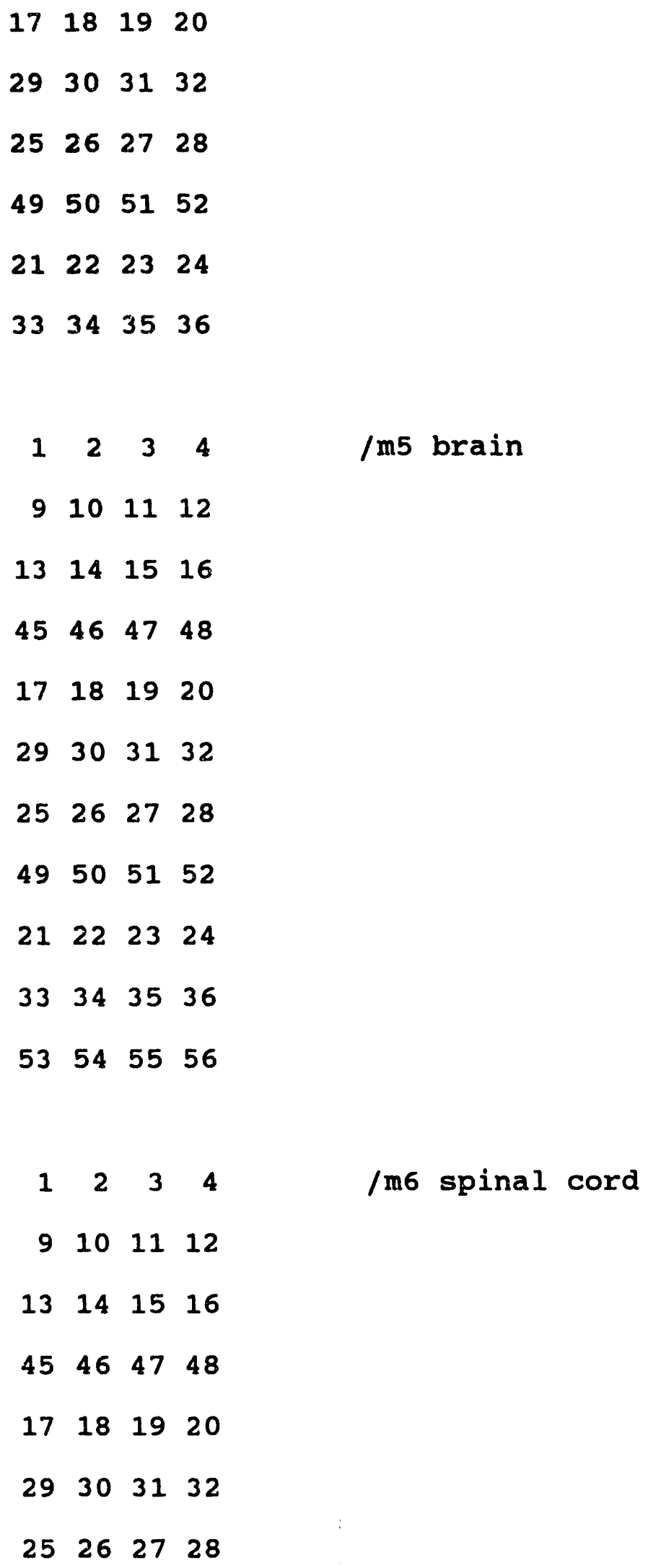




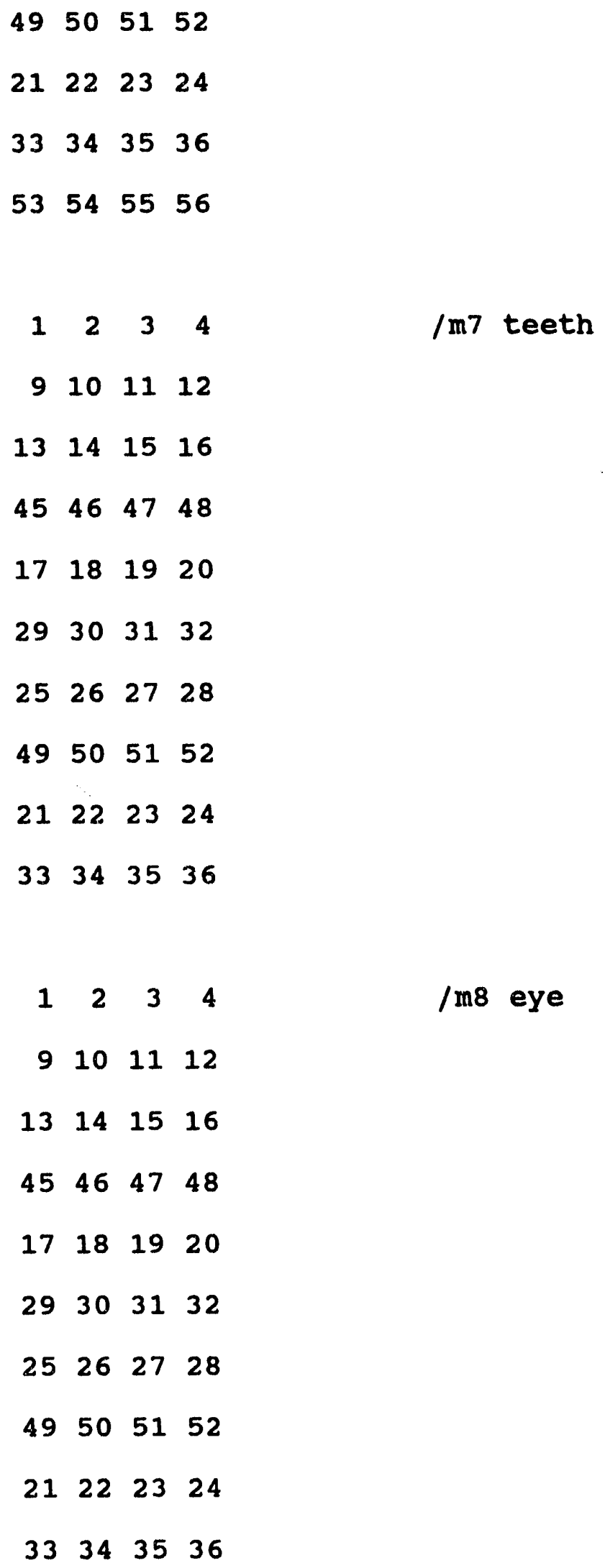


$\begin{array}{llll}53 & 54 \quad 55 & 56\end{array}$

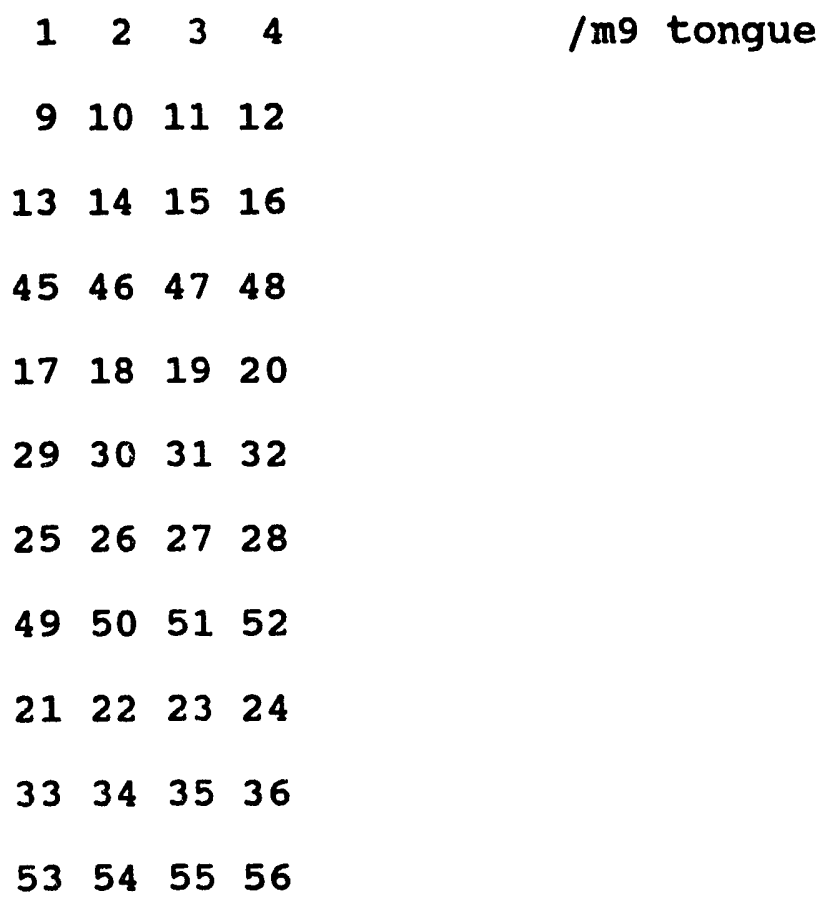




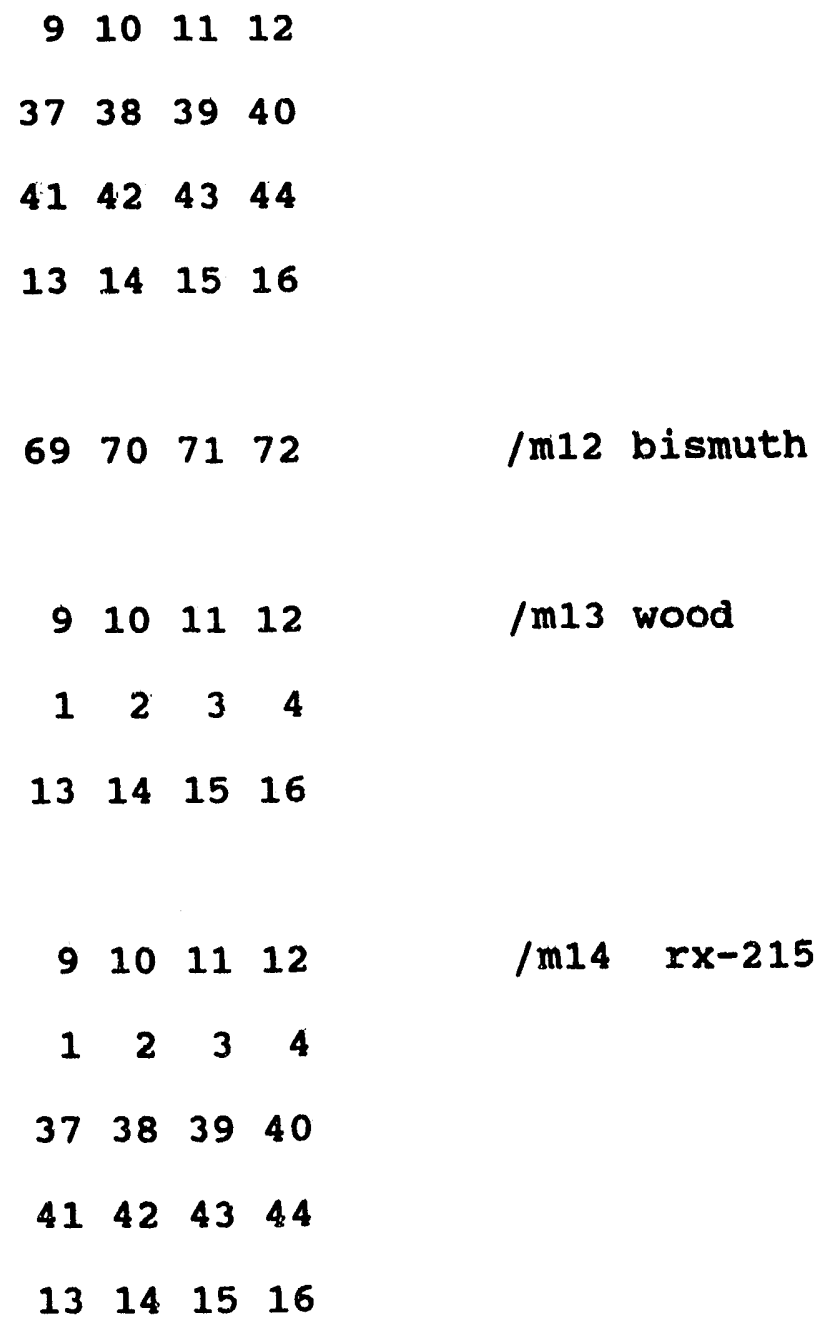

$\begin{array}{rrrr}77 & 78 & 79 & 80 \\ 81 & 82 & 83 & 84 \\ 85 & 86 & 87 & 88 \\ 89 & 90 & 91 & 92 \\ 93 & 94 & 95 & 96 \\ 97 & 98 & 99 & 100 \\ 101 & 102 & 103 & 104 \\ 105 & 106 & 107 & 108 \\ 109 & 110 & 111 & 112\end{array}$

/m15 muscle $x$ df $/ \mathrm{m} 16$ skin $\times$ df $/ \mathrm{m} 17$ bone $x \mathrm{df}$ /m18 brain $x$ df /m19 spinal chord $x$ df /m20 teeth $x$ df /m21 eye $x$ df /m22tongue $x d f$ /m23 nasal cavity $x d f$ 


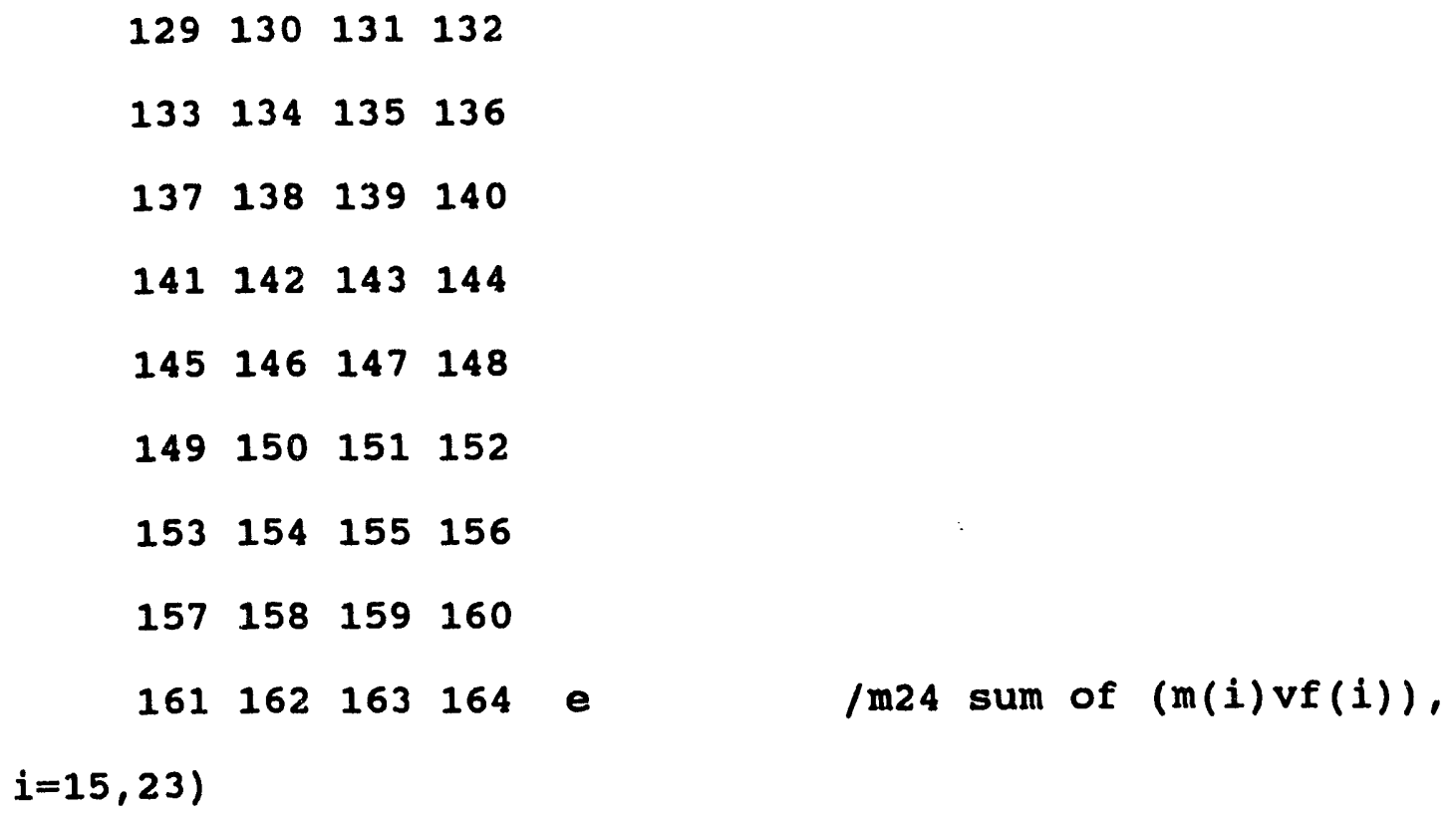


$4 r 6.50 e-2 \quad 4 r 2.22 e-24 r 1.13 e-2 \quad 4 r 7.86 e-4 \quad 4 r 4.38 e-5 \quad 4 r 3.18 e-5$ $4 \times 2.77 e-5 \quad 4 \times 9.44 e^{-5} \quad 4 \times 3.29 e-6 \quad 4 r 1.29 e-6 \quad 4 r 2.17 e-5$

$4 \times 1.55 e-2 \quad 4 \times 8.87 e-3 \quad 4 \times 4.02 e-3 \quad 4 r 1.05 e-3 \quad 4 \times 1.75 e-4 \quad 4 r 8.71 e-6$ $4 \times 1.38 e-5 \quad 4 \times 2.73 e-3 \quad 4 \times 1.97 e-4 \quad 4 \times 4.04 e-3$

$4 r 6.23 e-2 \quad 4 \times 2.54 e-2 \quad 4 \times 6.78 e-3 \quad 4 \times 1.29 e-3 \quad 4 \times 3.93 e-5 \quad 4 r 1.33 e-5$ $4 \times 2.66 e-54 r 1.11 e-5 \quad 4 \times 1.37 e-6 \quad 4 \times 5.12 e-6 \quad 4 \times 7.55 e-5$

$4 r 5.99 e-2 \quad 4 r 1.92 e-2 \quad 4 \times 1.24 e-2 \quad 4 r 2.08 e-3 \quad 4 r 2.33 e-5 \quad 4 r 3.20 e-5$ $4 \times 3.50 e-5 \quad 4 \times 2.82 e-5 \quad 4 \times 3.73 e-6 \quad 4 \times 1.73 e-6 \quad 4 \times 1.06 e-5$

$4 \times 5.30 e-24 \times 2.19 e-2 \quad 4 \times 5.53 e-3 \quad 4 r 1.28 e^{-3} \quad 4 \times 6.60 e-5 \quad 4 \times 3.06 e-5$ $4 \times 3.05 e-5 \quad 4 \times 4.18 e^{-4} 4 \times 1.96 e-5 \quad 4 \times 6.44 e^{-4}$

$4 r 0.07065 \quad 4 r 0.011830 \quad 4 r 0.006432 \quad 4 r 0.0003385 \quad 4 r 0.02656$

$4 r 0.028240$

$4 r 0.01189 \quad 4 r 0.02378,4 r 0.01426$

$4 r 0.01043 \quad 4 r 0.05445 \quad 4 r 0.0005025 \quad 4 r 0.006198 \quad 4 r 0.03073$

$4 r 1.041$

$4 \times 1.10$

$4 \times 1.61$ /m15 muscle $x$ df

$/ m 16$ skin $x$ df

$/ m 17$ bone $x d f$ 


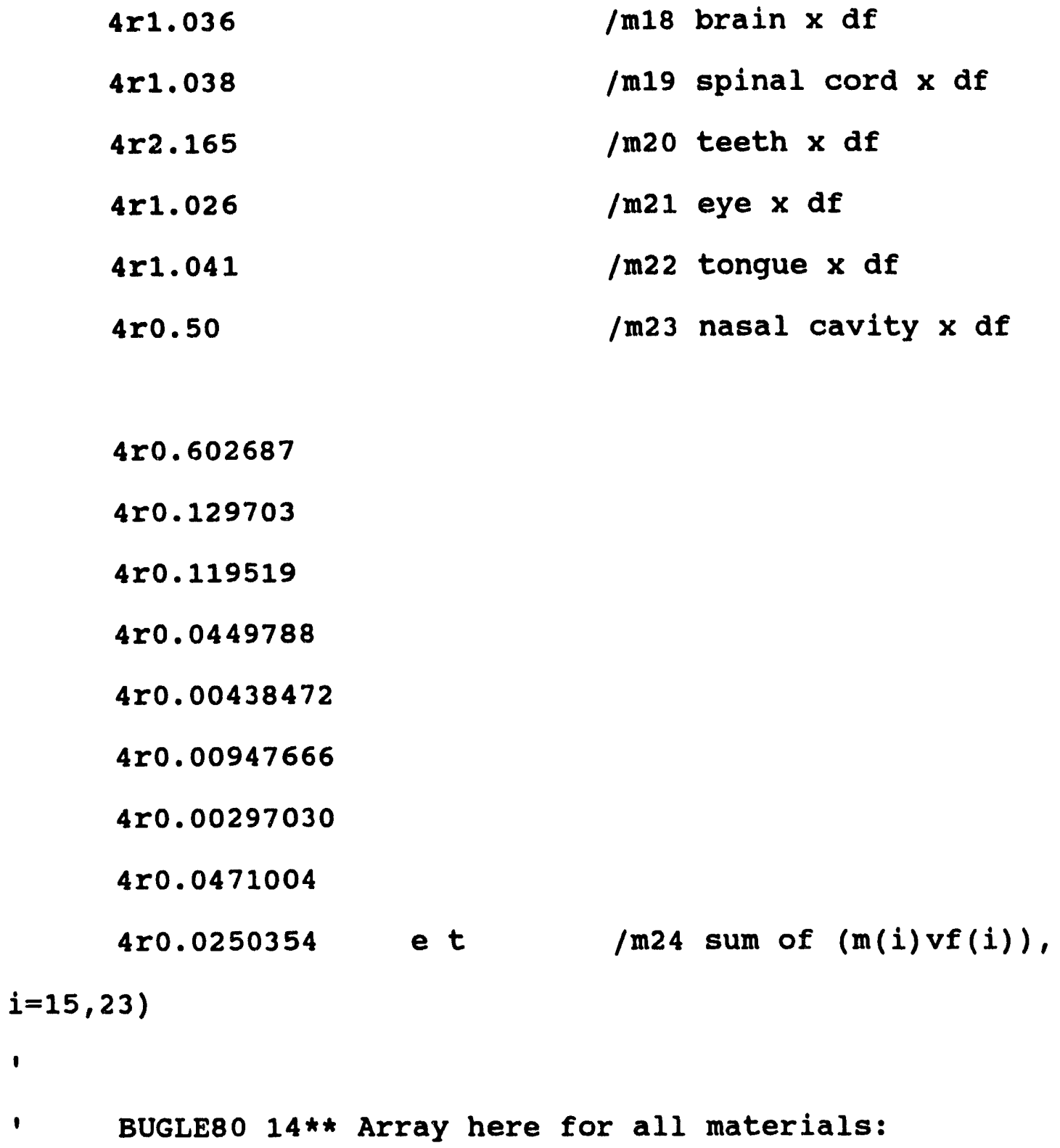

/m18 brain $x$ df

/m19 spinal cord $x$ df

/m20 teeth $x \mathrm{df}$

/m21 eye $x$ df

/m22 tongue $x$ df

/m23 nasal cavity $x$ df 
Tort Dog Benchmark 6/05/91 (s8)

- Heterogeneous model with $9 \$ \$$ change noted to

- create a homogeneous model.

- Density Factors in Library

- Manual Photographic Geometry

- $10 \times 10 \mathrm{~cm}$ delimiter. $17 \mathrm{c}$ flux inward.

- $17 *$ * change for $5 \times 10$ delimiter noted.

- $14 \times 14$ source.

- Total current in $=5.77945 \mathrm{e}-01$ (17c s8 angular.)

- Total flux inward $=1.00000$ (17c s8 angular.)

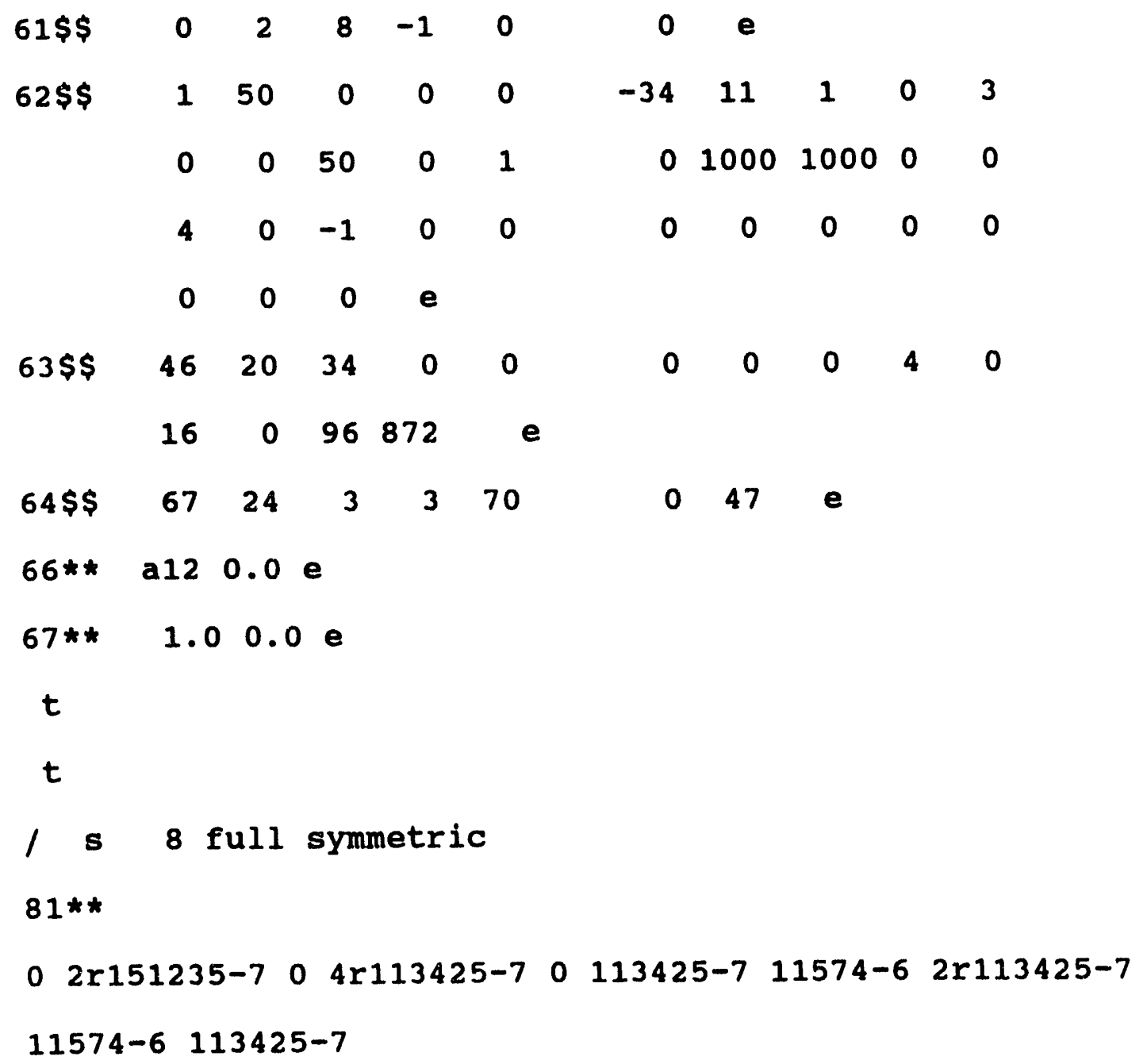


0 151235-7 2 r113425-7 2 r151235-7 2 r113425-7 $151235-7$

$3 q \quad 24$

$82 * *$

$-30861-5-21822-5 \quad 21822-5-61721-5-57735-5-21822-5 \quad 21822-5$ 57735-5

$-8165-4-7868-4-57735-5-21822-5 \quad 21822-5 \quad 57735-5 \quad 7868-4$

$-9759-4$

$-95119-5-7868-4 \quad-57735-5 \quad-21822-5 \quad 21822-5 \quad 57735-5 \quad 7868-4$

95119-5

$3 q \quad 24$

$83 * *$

$3 r-95119-5 \quad 5 r-7868-4 \quad 7 r-57735-5 \quad 9 r-21822-5$

q $\quad 249 \quad 48$

$t$

$1 * * \quad$ f0.0

$2 * * 0.0 \quad 1.00 \quad 2.00 \quad 3.00 \quad 4.00 \quad 5.00 \quad 5.50 \quad 6.00 \quad 6.50 \quad 7.00$ $\begin{array}{lllllllllll}7.50 & 8.00 & 8.50 & 9.00 & 9.50 & 10.00 & 10.50 & 11.00 & 11.50 & 12.0\end{array}$ $12.50 \quad 13.00 \quad 13.50 \quad 14.00 \quad 14.50 \quad 15.00 \quad 15.50 \quad 16.00 \quad 16.50 \quad 17.0$ $17.50 \quad 18.00 \quad 18.50 \quad 19.00$ 20.0021 .0022 .0023 .0024 .0025 .0026 .0027 .0028 .0029 .0 30.0031 .0032 .00

$$
\begin{array}{lllllllllllll}
3 * * & -8.00 & -7.00 & -6.00 & -5.00 & -4.00 & -3.00 & -2.00 & -1.00 & -0.50 \\
- & 0.25 \\
& 0.00 & 0.25 & 0.50 & 1.00 & 2.00 & 3.00 & 4.00 & 5.00 & 6.00 \\
7.00 & 8.00
\end{array}
$$




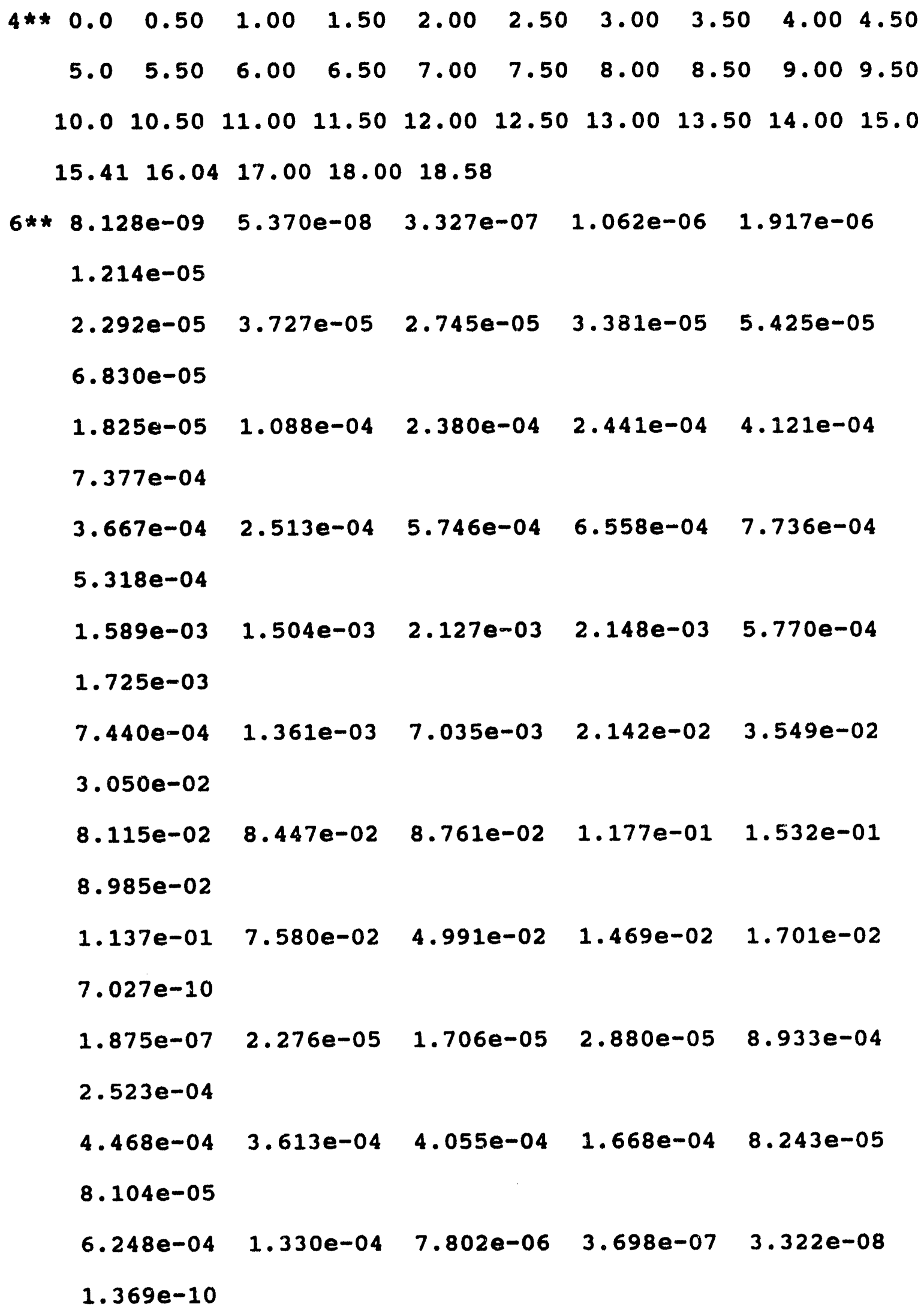




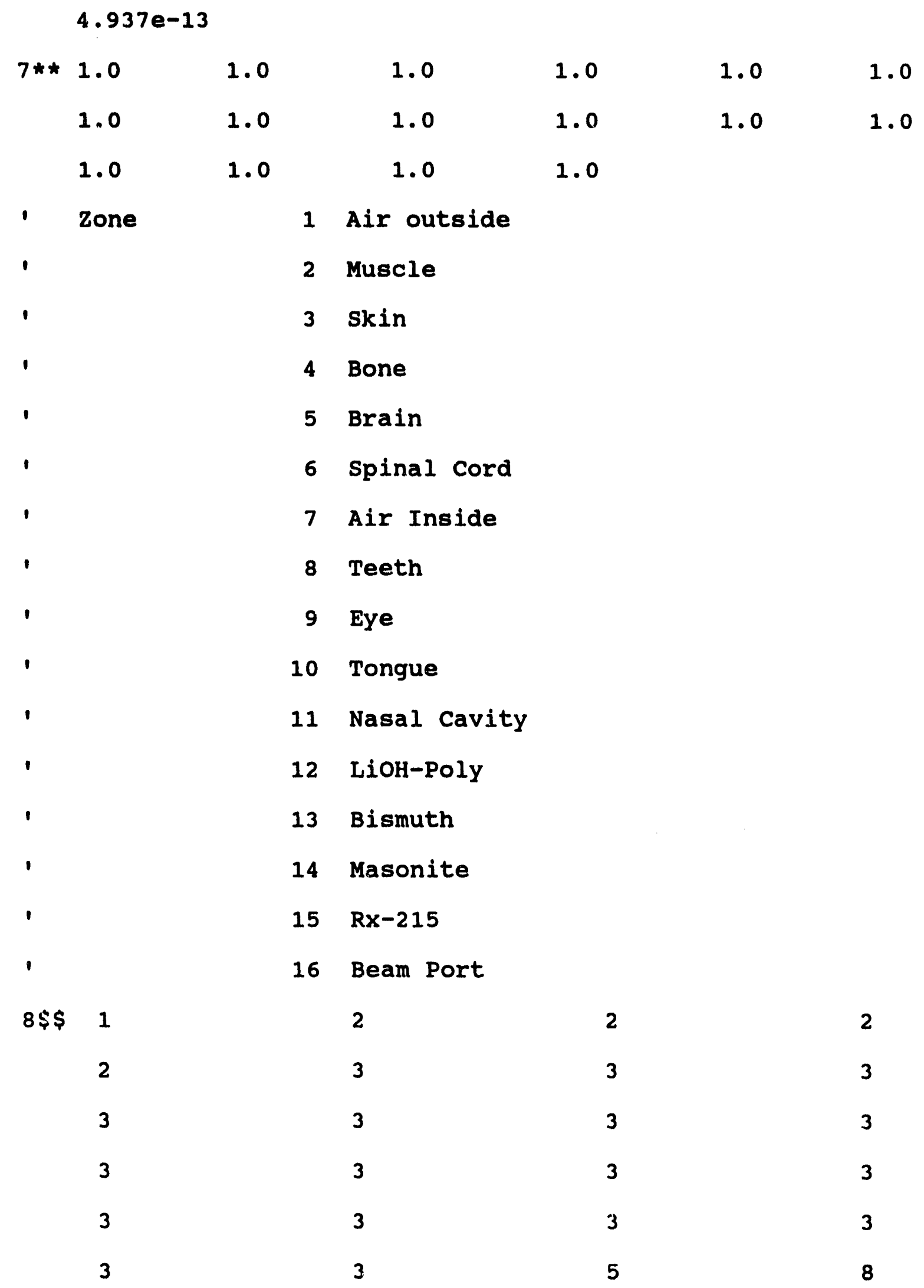




\begin{tabular}{|c|c|c|c|}
\hline 10 & 10 & 2 & 2 \\
\hline 6 & 6 & 6 & 6 \\
\hline 6 & 6 & 4 & 4 \\
\hline 4 & 4 & 4 & 4 \\
\hline 4 & 4 & 4 & 4 \\
\hline 4 & 4 & 4 & 4 \\
\hline 4 & 4 & 4 & 4 \\
\hline 4 & 2 & 2 & 4 \\
\hline 4 & 4 & 4 & 4 \\
\hline 4 & 4 & 4 & 4 \\
\hline 4 & 7 & 7 & 7 \\
\hline 7 & 7 & 7 & 7 \\
\hline 4 & 2 & 2 & 2 \\
\hline 4 & 4 & 4 & 6 \\
\hline 10 & 10 & 10 & 10 \\
\hline 4 & 4 & 4 & 8 \\
\hline 8 & 8 & 4 & 4 \\
\hline 4 & 4 & 4 & 4 \\
\hline 4 & 4 & 6 & 4 \\
\hline 4 & 6 & 6 & 5 \\
\hline 3 & 3 & 3 & 3 \\
\hline 3 & 3 & 3 & 3 \\
\hline 3 & 3 & 3 & 3 \\
\hline 3 & 3 & 3 & 3 \\
\hline 3 & 4 & 3 & 3 \\
\hline 2 & 2 & 2 & 2 \\
\hline
\end{tabular}




\begin{tabular}{|c|c|c|c|}
\hline 2 & 2 & 2 & 2 \\
\hline 2 & 4 & 5 & 5 \\
\hline 5 & 5 & 7 & 7 \\
\hline 7 & 7 & 7 & 7 \\
\hline 7 & 11 & 11 & 11 \\
\hline 11 & 11 & 11 & 4 \\
\hline 4 & 4 & 4 & 11 \\
\hline 11 & 11 & 4 & 2 \\
\hline 2 & 2 & 2 & 2 \\
\hline 2 & 3 & 3 & 3 \\
\hline 3 & 3 & 3 & 3 \\
\hline 3 & 2 & 2 & 2 \\
\hline 2 & 2 & 2 & 2 \\
\hline 2 & 5 & 5 & 5 \\
\hline 10 & 10 & 4 & 4 \\
\hline 4 & 4 & 4 & 4 \\
\hline 8 & 4 & 4 & 4 \\
\hline 4 & 11 & 11 & 4 \\
\hline 4 & 4 & 4 & 4 \\
\hline 4 & 4 & 4 & 4 \\
\hline 4 & 4 & 4 & 4 \\
\hline 4 & 4 & 4 & 4 \\
\hline 4 & 4 & 4 & 4 \\
\hline 4 & 3 & 2 & 2 \\
\hline 2 & 3 & 3 & 3 \\
\hline 3 & 3 & 3 & 2 \\
\hline
\end{tabular}




\begin{tabular}{|c|c|c|c|}
\hline 2 & 2 & 2 & 2 \\
\hline 2 & 2 & 4 & 9 \\
\hline 9 & 4 & 4 & 4 \\
\hline 8 & 10 & 10 & 4 \\
\hline 4 & 4 & 4 & 4 \\
\hline 4 & 4 & 4 & 4 \\
\hline 4 & 4 & 4 & 4 \\
\hline 4 & 4 & 4 & 4 \\
\hline 4 & 5 & 5 & 5 \\
\hline 3 & 3 & 3 & 3 \\
\hline 3 & 3 & 3 & 3 \\
\hline 2 & 2 & 3 & 3 \\
\hline 3 & 2 & 3 & 3 \\
\hline 3 & 3 & 3 & 3 \\
\hline 2 & 3 & 2 & 3 \\
\hline 2 & 3 & 2 & 2 \\
\hline 3 & 2 & 3 & 9 \\
\hline 4 & 4 & 4 & 4 \\
\hline 4 & 4 & 4 & 4 \\
\hline 4 & 4 & 4 & 4 \\
\hline 4 & 4 & 4 & 4 \\
\hline 4 & 4 & 3 & 3 \\
\hline 3 & 3 & 3 & 3 \\
\hline 3 & 3 & 3 & 3 \\
\hline 3 & 3 & 3 & 3 \\
\hline 2 & 2 & 2 & 2 \\
\hline
\end{tabular}




\begin{tabular}{|c|c|c|}
\hline 2 & 2 & 2 \\
\hline 2 & 2 & 2 \\
\hline 4 & 4 & 4 \\
\hline 4 & 4 & 4 \\
\hline 4 & 4 & 3 \\
\hline 2 & 3 & 3 \\
\hline 3 & 2 & 2 \\
\hline 2 & 3 & 3 \\
\hline 3 & 3 & 3 \\
\hline 3 & 3 & 4 \\
\hline 4 & 7 & 7 \\
\hline 3 & 3 & 3 \\
\hline 3 & 3 & 3 \\
\hline 3 & 7 & 7 \\
\hline 3 & 3 & 2 \\
\hline 4 & 4 & 4 \\
\hline 4 & 2 & 2 \\
\hline 2 & 2 & 2 \\
\hline 3 & 2 & 3 \\
\hline 2 & 4 & 2 \\
\hline 2 & 3 & 3 \\
\hline 4 & 3 & 3 \\
\hline 3 & 3 & 2 \\
\hline 3 & 2 & 3 \\
\hline 2 & 2 & 2 \\
\hline 2 & 2 & 3 \\
\hline
\end{tabular}




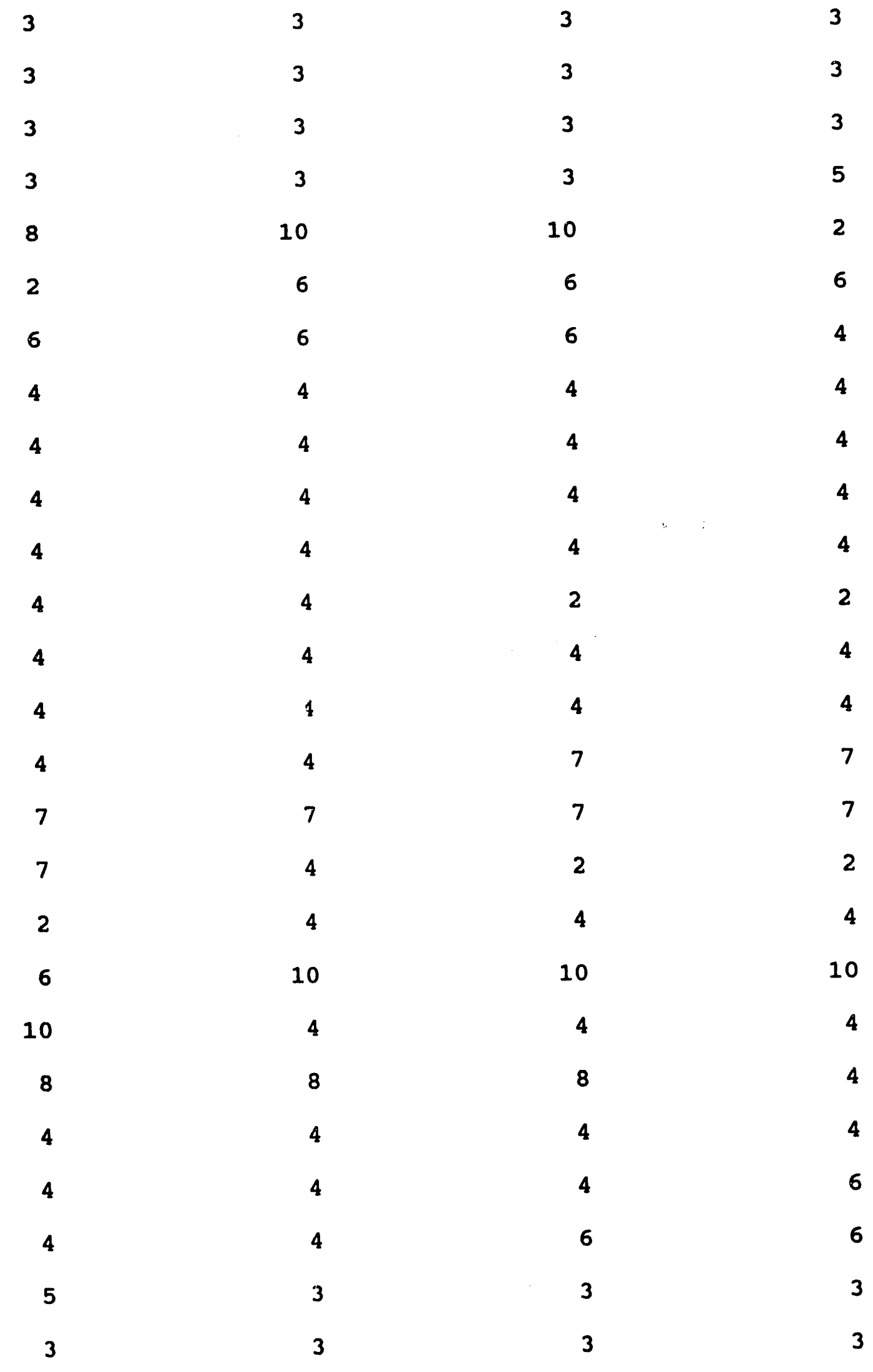




\begin{tabular}{|c|c|c|c|}
\hline 3 & 3 & 3 & 3 \\
\hline 3 & 3 & 3 & 3 \\
\hline 3 & 3 & 4 & 3 \\
\hline 3 & 2 & 2 & 2 \\
\hline 2 & 2 & 2 & 2 \\
\hline 2 & 2 & 4 & 5 \\
\hline 5 & 5 & 5 & 7 \\
\hline 7 & 7 & 7 & 7 \\
\hline 7 & 7 & 11 & 11 \\
\hline 11 & 11 & 11 & 11 \\
\hline 4 & 4 & 4 & 4 \\
\hline 11 & 11 & 11 & 4 \\
\hline 2 & 2 & 2 & 2 \\
\hline 2 & 2 & 3 & 3 \\
\hline 3 & 3 & 3 & 3 \\
\hline 3 & 3 & 2 & 2 \\
\hline 2 & 2 & 2 & 2 \\
\hline 2 & 2 & 5 & 5 \\
\hline 5 & 10 & 10 & 4 \\
\hline 4 & 4 & 4 & 4 \\
\hline 4 & 8 & 4 & 4 \\
\hline 4 & 4 & 11 & 11 \\
\hline 4 & 4 & 4 & 4 \\
\hline 4 & 4 & 4 & 4 \\
\hline 4 & 4 & 4 & 4 \\
\hline 4 & 4 & 4 & 4 \\
\hline
\end{tabular}




\begin{tabular}{|c|c|c|c|}
\hline 4 & 4 & 4 & 4 \\
\hline 4 & 4 & 3 & 2 \\
\hline 2 & 2 & 3 & 3 \\
\hline 3 & 3 & 3 & 3 \\
\hline 2 & 2 & 2 & 2 \\
\hline 2 & 2 & 2 & 4 \\
\hline 9 & 9 & 4 & 4 \\
\hline 4 & 8 & 10 & 10 \\
\hline 4 & 4 & 4 & 4 \\
\hline 4 & 4 & 4 & 4 \\
\hline 4 & 4 & 4 & 4 \\
\hline 4 & 4 & 4 & 4 \\
\hline 4 & 4 & 5 & 5 \\
\hline 5 & 3 & 3 & 3 \\
\hline 3 & 3 & 3 & 3 \\
\hline 3 & 2 & 2 & 3 \\
\hline 3 & 3 & 2 & 3 \\
\hline 3 & 3 & 3 & 3 \\
\hline 3 & 2 & 3 & 2 \\
\hline 3 & 2 & 3 & 2 \\
\hline 2 & 3 & 2 & 3 \\
\hline 9 & 4 & 4 & 4 \\
\hline 4 & 4 & 4 & 4 \\
\hline 4 & 4 & 4 & 4 \\
\hline 4 & 4 & 4 & 4 \\
\hline 4 & 4 & 4 & 3 \\
\hline
\end{tabular}




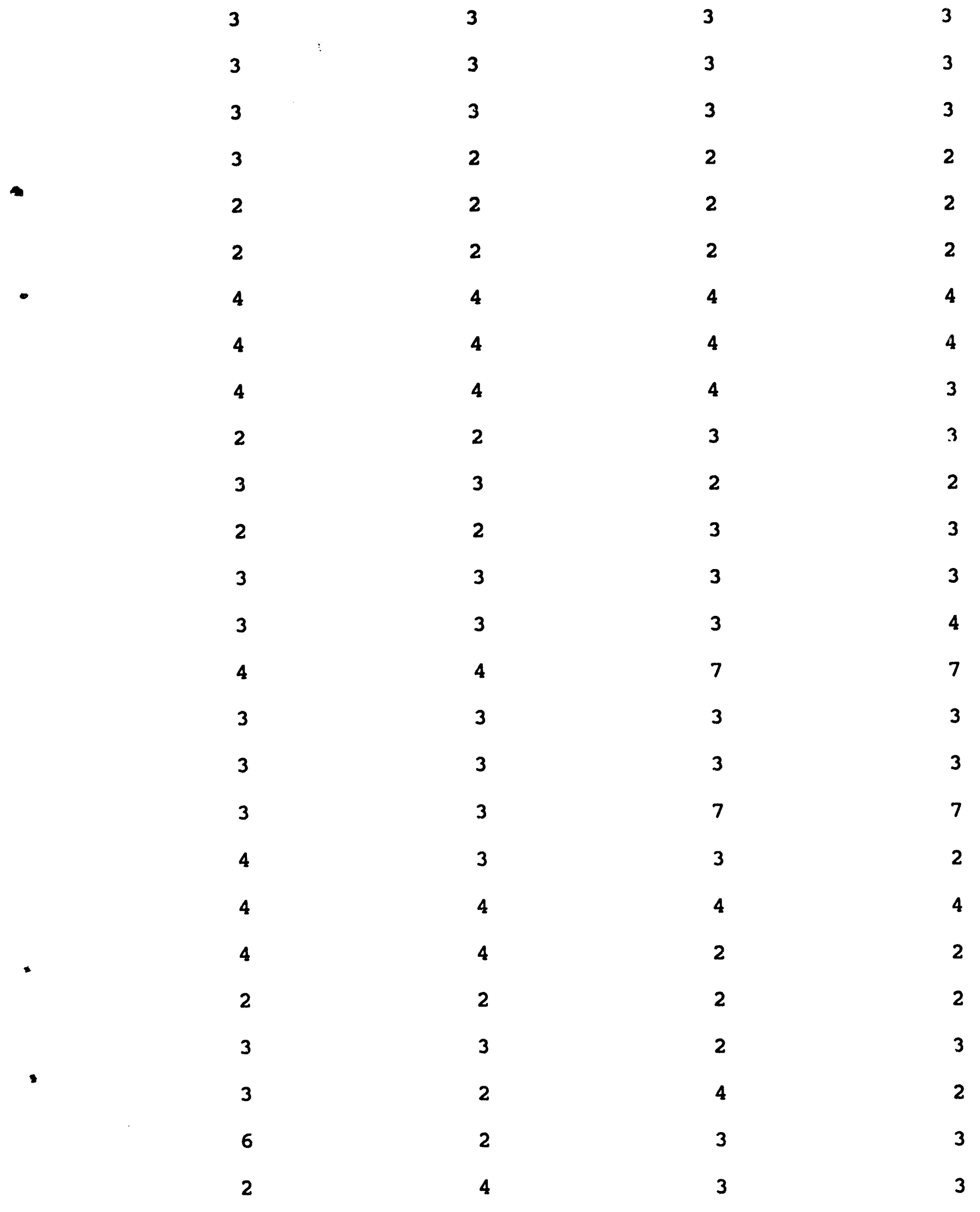




$\begin{array}{lrrr}2 & 3 & 3 & 2 \\ 3 & 3 & 2 & 3 \\ 3 & 2 & 2 & \\ 12 & 13 & 14 & 15 \\ 16 & & & \\ \text { 1 Material to region assignments: } & \end{array}$

The line below creates the heterogeneous model.

$\begin{array}{lllllllllllllllll}9 \$ \$ & 1 & 15 & 16 & 17 & 18 & 19 & 1 & 20 & 21 & 22 & 23 & 11 & 12 & 13 & 14 & 1\end{array}$

- Substitution of the line below creates the homogeneous model.

$\begin{array}{llllllllllllllll}9 \$ \$ & 1 & 24 & 24 & 24 & 24 & 24 & 24 & 24 & 24 & 24 & 24 & 11 & 12 & 13 & 14\end{array}$

1

$\begin{array}{rrrrrrrrrr}14 * \star 0.0 & 1.0 & 1.0 & 1.0 & 26.0 & 1.0 & 3.0 & 4.0 & 5.0 & 5.0 \\ 5.0 & 6.0 & 6.5 & 22.0 & 24.0 & 24.0 & 27.0 & 28.0 & 27.0 & 26.0 \\ 18.0 & 1.0 & 7.0 & 26.0 & 15.0 & 26.0 & 4.0 & 5.0 & 3.0 & 4.0 \\ 5.0 & 6.0 & 8.0 & 9.0 & 12.0 & 11.5 & 11.0 & 23.0 & 24.0 & 25.0 \\ 5.0 & 2.0 & 3.0 & 4.0 & 8.0 & 10.0 & 10.5 & 14.5 & 12.5 & 6.0 \\ 6.0 & 6.0 & 6.0 & 6.0 & 6.5 & 15.5 & 14.5 & 17.5 & 16.0 & 16.5 \\ 18.0 & 16.5 & 23.0 & 25.0 & 26.0 & 14.0 & 15.5 & 16.5 & 17.0 & 17.5\end{array}$ 
$\begin{array}{llllllllll}17.5 & 7.0 & 8.5 & 1.0 & 1.0 & 1.0 & 1.0 & 2.0 & 2.0 & 1.0\end{array}$ $\begin{array}{llllllllll}13.0 & 14.0 & 14.5 & 15.5 & 21.0 & 22.0 & 23.0 & 24.0 & 25.0 & 26.0\end{array}$ $\begin{array}{llllllllll}4.0 & 5.0 & 6.0 & 7.0 & 9.0 & 12.5 & 7.0 & 5.0 & 7.0 & 6.0\end{array}$

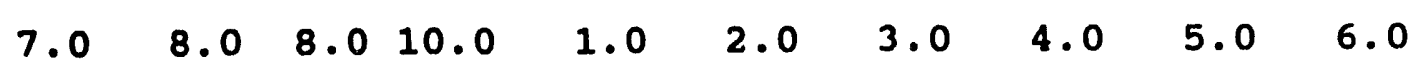
$\begin{array}{llllllllll}7.0 & 21.0 & 22.0 & 27.0 & 28.0 & 28.0 & 27.0 & 26.0 & 25.0 & 18.0\end{array}$

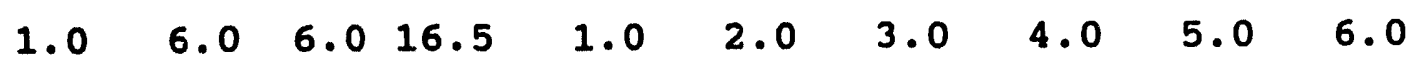
$\begin{array}{llllllllll}6.0 & 7.0 & 7.0 & 13.0 & 8.0 & 13.5 & 14.0 & 9.0 & 14.0 & 13.5\end{array}$ $\begin{array}{lllllllllll}13.0 & 13.5 & 17.0 & 17.0 & 19.0 & 15.0 & 15.5 & 16.0 & 17.0 & 17.5\end{array}$ $\begin{array}{llllllllll}18.0 & 18.0 & 18.5 & 19.0 & 20.0 & 20.0 & 22.0 & 24.0 & 24.0 & 1.0\end{array}$ $\begin{array}{llllllllll}1.0 & 2.0 & 1.0 & 1.0 & 1.0 & 1.0 & 2.0 & 3.0 & 4.0 & 5.0\end{array}$ $\begin{array}{llllllllll}6.0 & 6.0 & 8.0 & 2.0 & 3.0 & 4.0 & 5.0 & 6.0 & 6.0 & 7.0\end{array}$ $\begin{array}{llllllllll}8.0 & 8.0 & 12.5 & 13.0 & 15.0 & 16.0 & 1.0 & 10.0 & 19.0 & 18.0\end{array}$ $\begin{array}{llllllllll}17.5 & 19.0 & 23.0 & 20.0 & 16.0 & 24.0 & 20.0 & 17.0 & 20.0 & 4.0\end{array}$ $\begin{array}{llllllllll}5.0 & 6.0 & 7.0 & 8.0 & 8.0 & 8.0 & 10.0 & 12.0 & 12.5 & 13.0\end{array}$ $\begin{array}{lllllllllll}8.0 & 11.5 & 13.0 & 13.5 & 14.0 & 13.0 & 14.0 & 15.5 & 16.5 & 16.0\end{array}$

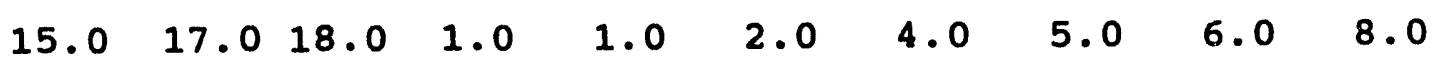
$\begin{array}{llllllllll}15.5 & 4.0 & 5.0 & 6.0 & 6.0 & 7.0 & 8.0 & 1.0 & 15.5 & 16.0\end{array}$ $\begin{array}{llllllllll}16.0 & 17.5 & 18.0 & 19.0 & 17.0 & 20.0 & 21.0 & 13.5 & 14.0 & 14.5\end{array}$ $\begin{array}{llllllllll}15.0 & 15.5 & 16.0 & 17.0 & 17.5 & 18.0 & 7.0 & 8.0 & 8.5 & 8.5\end{array}$

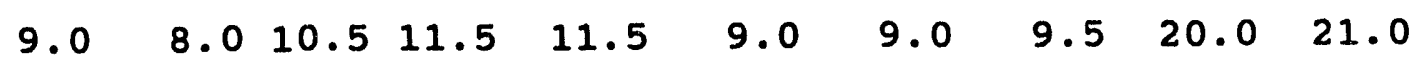

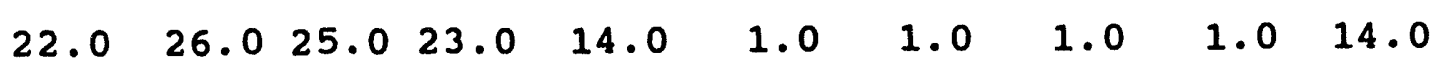
$\begin{array}{llllllllll}21.0 & 21.0 & 22.0 & 20.0 & 19.0 & 18.0 & 1.0 & 2.0 & 4.0 & 4.0\end{array}$ $\begin{array}{llllllllll}5.0 & 5.0 & 6.0 & 6.0 & 7.0 & 7.0 & 7.0 & 9.5 & 9.5 & 16.0\end{array}$ $\begin{array}{llllllllll}17.0 & 16.5 & 9.0 & 8.0 & 6.0 & 8.0 & 12.0 & 12.0 & 12.0 & 12.0\end{array}$ $\begin{array}{llllllllll}11.0 & 10.5 & 10.0 & 9.5 & 12.0 & 13.5 & 14.5 & 14.0 & 1.0 & 18.0\end{array}$

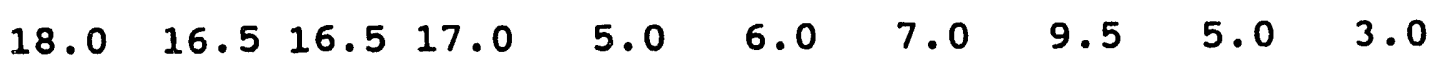


$\begin{array}{llllllllll}2.0 & 1.0 & 1.0 & 16.5 & 16.5 & 16.5 & 3.0 & 4.0 & 5.0 & 6.0\end{array}$

$\begin{array}{llllllllll}6.0 & 7.5 & 9.5 & 9.0 & 9.5 & 10.0 & 10.5 & 5.0 & 6.0 & 6.0\end{array}$ $\begin{array}{llllllllll}7.0 & 9.0 & 10.5 & 16.0 & 1.0 & 1.0 & 13.5 & 4.0 & 14.0 & 13.5\end{array}$ $\begin{array}{llllllllll}13.0 & 5.0 & 2.0 & 3.0 & 4.0 & 1.0 & 2.0 & 3.0 & 4.0 & 5.0\end{array}$

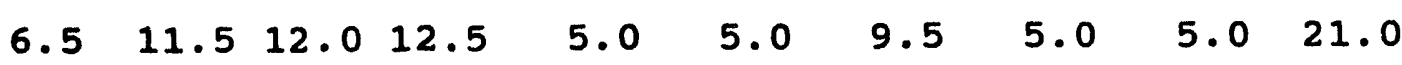
$\begin{array}{lllllllllll}22.0 & 25.0 & 27.0 & 26.0 & 25.0 & 21.0 & 15.0 & 1.0 & 17.5 & 8.0\end{array}$ $\begin{array}{llllllllll}8.0 & 10.5 & 1.0 & 19.0 & 3.0 & 6.5 & 11.0 & 12.5 & 6.0 & 11.0\end{array}$ $\begin{array}{llllllllll}12.0 & 18.0 & 18.0 & 20.0 & 14.0 & 25.0 & 27.0 & 4.0 & 0.0 & 0.0\end{array}$ $\begin{array}{llllllllll}0.0 & 0.0 & 0.0 & 0.0 & 0.0 & 0.0 & 0.0 & 0.0 & 0.0 & 0.0\end{array}$

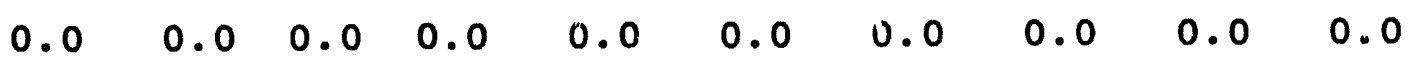
$\begin{array}{llllllllll}0.0 & 0.0 & 0.0 & 25.0 & 1.0 & 1.0 & 1.0 & 26.0 & 1.0 & 3.0\end{array}$ $\begin{array}{llllllllll}4.0 & 5.0 & 5.0 & 5.0 & 6.0 & 6.5 & 22.0 & 24.0 & 24.0 & 27.0\end{array}$ $\begin{array}{lllllllllll}28.0 & 27.0 & 26.0 & 18.0 & 1.0 & 7.0 & 26.0 & 15.0 & 26.0 & 4.0\end{array}$ $\begin{array}{llllllllll}5.0 & 3.0 & 4.0 & 5.0 & 6.0 & 8.0 & 9.0 & 12.0 & 11.5 & 11.0\end{array}$ $\begin{array}{llllllllll}23.0 & 24.0 & 25.0 & 5.0 & 2.0 & 3.0 & 4.0 & 8.0 & 10.0 & 10.5\end{array}$ $\begin{array}{llllllllll}14.5 & 12.5 & 6.0 & 6.0 & 6.0 & 6.0 & 6.0 & 6.5 & 15.5 & 14.5\end{array}$ $\begin{array}{llllllllll}17.5 & 16.0 & 16.5 & 18.0 & 16.5 & 23.0 & 25.0 & 26.0 & 14.0 & 15.5\end{array}$

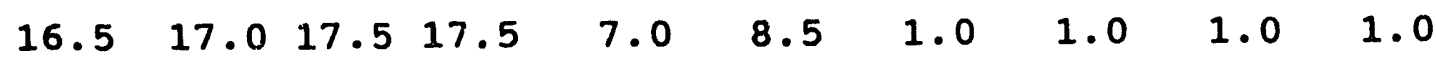
$\begin{array}{llllllllll}2.0 & 2.0 & 1.0 & 13.0 & 14.0 & 14.5 & 15.5 & 21.0 & 22.0 & 23.0\end{array}$ $\begin{array}{llllllllll}24.0 & 25.0 & 26.0 & 4.0 & 5.0 & 6.0 & 7.0 & 9.0 & 12.5 & 7.0\end{array}$ $\begin{array}{llllllllll}5.0 & 7.0 & 6.0 & 7.0 & 8.0 & 8.0 & 10.0 & 1.0 & 2.0 & 3.0\end{array}$ $\begin{array}{llllllllll}4.0 & 5.0 & 6.0 & 7.0 & 21.0 & 22.0 & 27.0 & 28.0 & 28.0 & 27.0\end{array}$ $\begin{array}{llllllllll}26.0 & 25.0 & 18.0 & 1.0 & 6.0 & 6.0 & 16.5 & 1.0 & 2.0 & 3.0\end{array}$ $\begin{array}{llllllllll}4.0 & 5.0 & 6.0 & 6.0 & 7.0 & 7.0 & 13.0 & 8.0 & 13.5 & 14.0\end{array}$ $\begin{array}{lllllllllll}9.0 & 14.0 & 13.5 & 13.0 & 13.5 & 17.0 & 17.0 & 19.0 & 15.0 & 15.5\end{array}$ $\begin{array}{llllllllll}16.0 & 17.0 & 17.5 & 18.0 & 18.0 & 18.5 & 19.0 & 20.0 & 20.0 & 22.0\end{array}$ 


\begin{tabular}{|c|c|c|c|c|c|c|c|c|c|}
\hline 24.0 & 24.0 & 1.0 & 1.0 & 2.0 & 1.0 & 1.0 & 1.0 & 1.0 & 2.0 \\
\hline 3.0 & 4.0 & 5.0 & 6.0 & 6.0 & 8.0 & 2.0 & 3.0 & 4.0 & 5.0 \\
\hline 6.0 & 6.0 & 7.0 & 8.0 & 8.0 & 12.5 & 13.0 & 15.0 & 16.0 & 1.0 \\
\hline 10.0 & 19.0 & 18.0 & 17.5 & 19.0 & 23.0 & 20.0 & 16.0 & 24.0 & 20.0 \\
\hline 17.0 & 20.0 & 4.0 & 5.0 & 6.0 & 7.0 & 8.0 & 8.0 & 8.0 & 10.0 \\
\hline 12.0 & 12.5 & 13.0 & 8.0 & 11.5 & 13.0 & 13.5 & 14.0 & 13.0 & 14.0 \\
\hline 15.5 & 16.5 & 16.0 & 15.0 & 17.0 & 18.0 & 1.0 & 1.0 & 2.0 & 4.0 \\
\hline 5.0 & 6.0 & 8.0 & 15.5 & 4.0 & 5.0 & 6.0 & 6.0 & 7.0 & 8.0 \\
\hline 1.0 & 15.5 & 16.0 & 16.0 & 17.5 & 18.0 & 19.0 & 17.0 & 20.0 & 21.0 \\
\hline 13.5 & 14.0 & 14.5 & 15.0 & 15.5 & 16.0 & 17.0 & 17.5 & 18.0 & 7.0 \\
\hline 8.0 & 8.5 & 8.5 & 9.0 & 8.0 & 10.5 & 11.5 & 11.5 & 9.0 & 9.0 \\
\hline 9.5 & 20.0 & 21.0 & 22.0 & 26.0 & 25.0 & 23.0 & 14.0 & 1.0 & 1.0 \\
\hline 1.0 & 1.0 & 14.0 & 21.0 & 21.0 & 22.0 & 20.0 & 19.0 & 18.0 & 1.0 \\
\hline 2.0 & 4.0 & 4.0 & 5.0 & 5.0 & 6.0 & 6.0 & 7.0 & 7.0 & 7.0 \\
\hline 9.5 & 9.5 & 16.0 & 17.0 & 16.5 & 9.0 & 8.0 & 6.0 & 8.0 & 12.0 \\
\hline 12.0 & 12.0 & 12.0 & 11.0 & 10.5 & 10.0 & 9.5 & 12.0 & 13.5 & 14.5 \\
\hline 14.0 & 1.0 & 18.0 & 18.0 & 16.5 & 16.5 & 17.0 & 5.0 & 6.0 & 7.0 \\
\hline 9.5 & 5.0 & 3.0 & 2.0 & 1.0 & 1.0 & 16.5 & 16.5 & 16.5 & 3.0 \\
\hline 4.0 & 5.0 & 6.0 & 6.0 & 7.5 & 9.5 & 9.0 & 9.5 & 10.0 & 10.5 \\
\hline 5.0 & 6.0 & 6.0 & 7.0 & 9.0 & 10.5 & 16.0 & 1.0 & 1.0 & 13.5 \\
\hline 4.0 & 14.0 & 13.5 & 13.0 & 5.0 & 2.0 & 3.0 & 4.0 & 1.0 & 2.0 \\
\hline 3.0 & 4.0 & 5.0 & 6.5 & 11.5 & 12.0 & 12.5 & 5.0 & 5.0 & 9.5 \\
\hline 5.0 & 5.0 & 21.0 & 22.0 & 25.0 & 27.0 & 26.0 & 25.0 & 21.0 & 15.0 \\
\hline 1.0 & 17.5 & 8.0 & 8.0 & 10.5 & 1.0 & 19.0 & 3.0 & 6.5 & 11.0 \\
\hline 2.5 & 6.0 & 11.0 & 12.0 & 18.0 & 18.0 & 20.0 & 14.0 & 25.0 & 27.0 \\
\hline 4.0 & 0.0 & 0.0 & 0.0 & 0.0 & 0.0 & 0.0 & 0.0 & 0.0 & 0.0 \\
\hline
\end{tabular}


$\begin{array}{llllllllll}0.0 & 0.0 & 0.0 & 0.0 & 0.0 & 0.0 & 0.0 & 0.0 & 0.0 & 0.0\end{array}$

$\begin{array}{lllllll}0.0 & 0.0 & 0.0 & 0.0 & 0.0 & 0.0 & 25.0\end{array}$

$0.0 \quad 25.025 .025 .0 \quad 7.0$

$\begin{array}{lllllllllll}15 * * 32.0 & 26.0 & 18.0 & 7.0 & 28.0 & 22.0 & 20.0 & 20.0 & 19.0 & 18.5\end{array}$ $\begin{array}{llllllllll}18.0 & 17.0 & 15.5 & 24.0 & 25.0 & 27.0 & 29.0 & 29.0 & 28.0 & 27.0\end{array}$ $\begin{array}{llllllllll}26.0 & 18.0 & 16.5 & 27.0 & 26.0 & 27.0 & 6.0 & 6.0 & 4.0 & 5.0\end{array}$ $\begin{array}{lllllllllll}6.0 & 8.0 & 10.0 & 10.0 & 12.5 & 12.0 & 11.5 & 24.0 & 25.0 & 26.0\end{array}$ $\begin{array}{lllllllllll}7.0 & 3.0 & 4.0 & 5.0 & 10.0 & 12.0 & 13.5 & 15.5 & 15.0 & 7.0\end{array}$ $\begin{array}{llllllllll}8.0 & 8.5 & 9.0 & 16.5 & 15.5 & 17.5 & 18.0 & 18.5 & 19.0 & 20.0\end{array}$ $\begin{array}{lllllllllll}22.0 & 17.5 & 24.0 & 27.0 & 28.0 & 15.5 & 16.5 & 17.5 & 18.0 & 18.0\end{array}$ $\begin{array}{llllllllll}19.0 & 9.0 & 9.5 & 27.0 & 25.0 & 18.0 & 2.0 & 3.0 & 3.0 & 2.0\end{array}$ $\begin{array}{llllllllll}22.0 & 25.0 & 26.0 & 25.0 & 22.0 & 23.0 & 24.0 & 25.0 & 26.0 & 27.0\end{array}$ $\begin{array}{llllllllll}6.0 & 7.0 & 7.0 & 8.0 & 10.0 & 13.5 & 14.5 & 12.5 & 8.0 & 13.5\end{array}$ $\begin{array}{llllllllll}13.5 & 10.0 & 9.5 & 12.5 & 22.0 & 21.0 & 20.0 & 20.0 & 19.0 & 17.0\end{array}$ $\begin{array}{lllllllllll}15.5 & 25.0 & 27.0 & 28.0 & 29.0 & 29.0 & 28.0 & 27.0 & 26.0 & 26.0\end{array}$ $\begin{array}{llllllllll}18.0 & 18.0 & 7.0 & 18.0 & 7.0 & 7.0 & 6.0 & 6.0 & 7.0 & 8.0\end{array}$ $\begin{array}{llllllllll}9.0 & 11.5 & 13.0 & 15.5 & 13.5 & 14.0 & 15.0 & 13.5 & 15.0 & 16.0\end{array}$ $\begin{array}{llllllllll}16.0 & 15.0 & 17.5 & 18.0 & 21.0 & 16.0 & 16.0 & 18.0 & 18.5 & 18.0\end{array}$ $\begin{array}{llllllllll}20.0 & 18.5 & 19.0 & 20.0 & 21.0 & 22.0 & 24.0 & 28.0 & 27.0 & 27.0\end{array}$

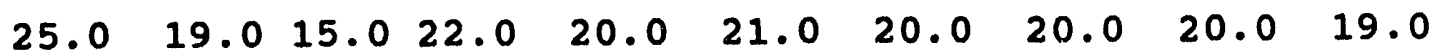
$\begin{array}{llllllllll}18.0 & 18.0 & 15.5 & 1.6 .0 & 16.5 & 8.0 & 8.0 & 8.0 & 11.5 & 13.0\end{array}$ $\begin{array}{llllllllll}14.0 & 12.5 & 13.0 & 13.5 & 25.0 & 24.0 & 2.0 & 11.0 & 21.0 & 22.0\end{array}$ $\begin{array}{llllllllll}22.0 & 22.0 & 25.0 & 24.0 & 20.0 & 25.0 & 24.0 & 20.0 & 23.0 & 6.0\end{array}$ $\begin{array}{llllllllll}6.0 & 7.0 & 8.0 & 9.5 & 12.0 & 10.0 & 10.5 & 12.5 & 13.0 & 13.5\end{array}$ $\begin{array}{llllllllll}10.0 & 13.0 & 13.5 & 14.0 & 15.5 & 17.0 & 18.0 & 19.0 & 19.0 & 20.0\end{array}$ $\begin{array}{llllllllll}20.0 & 18.0 & 19.0 & 26.0 & 19.0 & 20.0 & 19.0 & 19.0 & 18.0 & 15.5\end{array}$ 
$\begin{array}{llllllllll}18.0 & 19.0 & 18.5 & 18.5 & 18.0 & 15.5 & 17.0 & 14.0 & 17.0 & 17.5\end{array}$

$\begin{array}{llllllllll}18.0 & 18.0 & 19.0 & 21.0 & 21.0 & 21.0 & 23.0 & 14.0 & 14.5 & 15.0\end{array}$ $\begin{array}{llllllllll}15.5 & 16.0 & 17.0 & 17.5 & 18.0 & 18.5 & 8.0 & 8.5 & 9.0 & 11.5\end{array}$

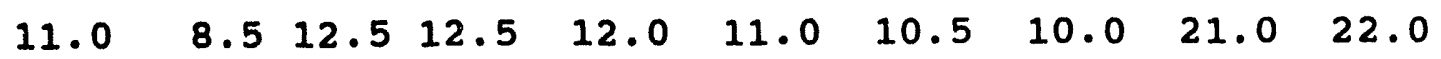
$\begin{array}{llllllllll}27.0 & 27.0 & 26.0 & 26.0 & 23.0 & 14.0 & 21.0 & 19.0 & 14.0 & 21.0\end{array}$ $\begin{array}{llllllllll}22.0 & 22.0 & 23.0 & 21.0 & 20.0 & 19.0 & 2.0 & 4.0 & 18.0 & 18.0\end{array}$ $\begin{array}{llllllllll}17.0 & 17.0 & 16.0 & 17.0 & 16.0 & 15.0 & 15.0 & 14.0 & 14.0 & 17.0\end{array}$ $\begin{array}{llllllllll}18.0 & 17.0 & 11.0 & 10.0 & 9.0 & 9.0 & 15.0 & 13.5 & 13.0 & 12.5\end{array}$ $\begin{array}{llllllllll}12.5 & 12.5 & 12.5 & 12.5 & 13.5 & 14.5 & 15.0 & 14.5 & 18.0 & 19.0\end{array}$ $\begin{array}{llllllllll}19.0 & 19.0 & 17.0 & 18.0 & 17.0 & 16.0 & 14.5 & 12.5 & 6.0 & 5.0\end{array}$ $\begin{array}{lllllllllll}4.0 & 3.0 & 16.5 & 18.0 & 18.0 & 17.0 & 16.0 & 16.0 & 16.0 & 17.0\end{array}$

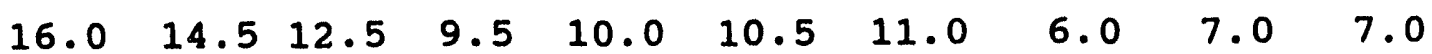
$\begin{array}{lllllllllll}7.5 & 10.0 & 12.0 & 17.0 & 4.0 & 13.5 & 14.0 & 15.0 & 15.0 & 14.0\end{array}$ $\begin{array}{llllllllll}13.5 & 12.5 & 5.0 & 5.0 & 5.0 & 2.0 & 3.0 & 4.0 & 5.0 & 6.5\end{array}$

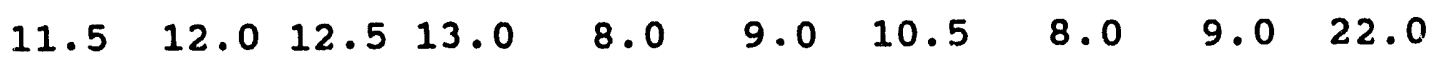
$\begin{array}{lllllllllll}25.0 & 27.0 & 28.0 & 27.0 & 26.0 & 26.0 & 23.0 & 15.0 & 18.0 & 10.0\end{array}$ $\begin{array}{llllllllll}10.5 & 11.0 & 2.0 & 20.0 & 7.0 & 15.5 & 15.0 & 14.5 & 15.0 & 15.0\end{array}$ $\begin{array}{llllllllll}15.0 & 18.5 & 23.0 & 21.0 & 15.5 & 26.0 & 28.0 & 5.0 & 1.0 & 1.0\end{array}$ $\begin{array}{llllllllll}1.0 & 1.0 & 1.0 & 1.0 & 1.0 & 1.0 & 1.0 & 1.0 & 1.0 & 1.0\end{array}$

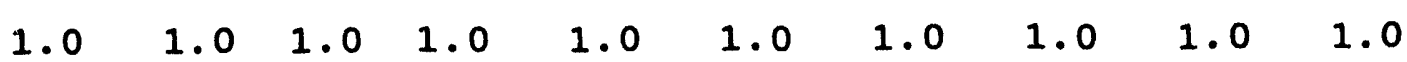
$\begin{array}{llllllllll}1.0 & 1.0 & 1.0 & 26.0 & 26.0 & 18.0 & 7.0 & 28.0 & 22.0 & 20.0\end{array}$ $\begin{array}{llllllllll}20.0 & 19.0 & 18.5 & 18.0 & 17.0 & 15.5 & 24.0 & 25.0 & 27.0 & 29.0\end{array}$ $\begin{array}{lllllllllll}29.0 & 28.0 & 27.0 & 26.0 & 18.0 & 16.5 & 27.0 & 26.0 & 27.0 & 6.0\end{array}$ $\begin{array}{llllllllll}6.0 & 4.0 & 5.0 & 6.0 & 8.0 & 10.0 & 10.0 & 12.5 & 12.0 & 11.5\end{array}$

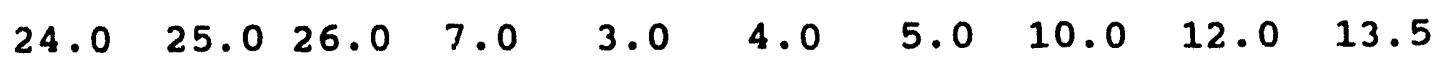
$\begin{array}{llllllllll}15.5 & 15.0 & 7.0 & 8.0 & 8.5 & 9.0 & 16.5 & 15.5 & 17.5 & 18.0\end{array}$ 
$\begin{array}{llllllllll}18.5 & 19.0 & 20.0 & 22.0 & 17.5 & 24.0 & 27.0 & 28.0 & 15.5 & 16.5\end{array}$

$\begin{array}{llllllllll}17.5 & 18.0 & 18.0 & 19.0 & 9.0 & 9.5 & 27.0 & 25.0 & 18.0 & 2.0\end{array}$ $\begin{array}{lllllllllll}3.0 & 3.0 & 2.0 & 22.0 & 25.0 & 26.0 & 25.0 & 22.0 & 23.0 & 24.0\end{array}$ $\begin{array}{llllllllll}25.0 & 26.0 & 27.0 & 6.0 & 7.0 & 7.0 & 8.0 & 10.0 & 13.5 & 14.5\end{array}$

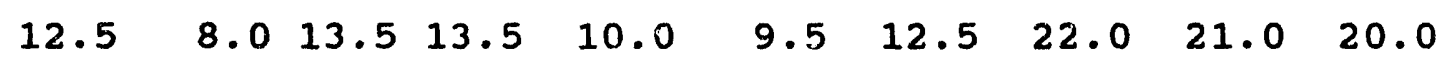
$\begin{array}{llllllllll}20.0 & 19.0 & 17.0 & 15.5 & 25.0 & 27.0 & 28.0 & 29.0 & 29.0 & 28.0\end{array}$ $\begin{array}{llllllllll}27.0 & 26.0 & 26.0 & 18.0 & 18.0 & 7.0 & 18.0 & 7.0 & 7.0 & 6.0\end{array}$ $\begin{array}{llllllllll}6.0 & 7.0 & 8.0 & 9.0 & 11.5 & 13.0 & 15.5 & 13.5 & 14.0 & 15.0\end{array}$ $\begin{array}{llllllllll}13.5 & 15.0 & 16.0 & 16.0 & 15.0 & 17.5 & 1.8 .0 & 21.0 & 16.0 & 1.6 .0\end{array}$ $\begin{array}{llllllllll}18.0 & 18.5 & 18.0 & 20.0 & 18.5 & 19.0 & 20.0 & 21.0 & 22.0 & 24.0\end{array}$ $\begin{array}{llllllllll}28.0 & 27.0 & 27.0 & 25.0 & 19.0 & 15.0 & 22.0 & 20.0 & 21.0 & 20.0\end{array}$ $\begin{array}{llllllllll}20.0 & 20.0 & 19.0 & 18.0 & 18.0 & 15.5 & 16.0 & \mp 5.5 & 8.0 & 8.0\end{array}$ $\begin{array}{lllllllllll}8.0 & 11.5 & 13.0 & 14.0 & 12.5 & 13.0 & 13.5 & 25.0 & 24.0 & 2.0\end{array}$ $\begin{array}{llllllllll}11.0 & 21.0 & 22.0 & 22.0 & 22.0 & 25.0 & 24.0 & 20.0 & 25.0 & 24.0\end{array}$ $\begin{array}{llllllllll}20.0 & 23.0 & 6.0 & 6.0 & 7.0 & 8.0 & 9.5 & 12.0 & 10.0 & 10.5\end{array}$ $\begin{array}{llllllllll}12.5 & 13.0 & 13.5 & 10.0 & 13.0 & 13.5 & 14.0 & 15.5 & 17.0 & 18.0\end{array}$ $\begin{array}{llllllllll}19.0 & 19.0 & 20.0 & 20.0 & 18.0 & 19.0 & 26.0 & 19.0 & 20.0 & 19.0\end{array}$ $\begin{array}{llllllllll}19.0 & 18.0 & 15.5 & 18.0 & 19.0 & 18.5 & 18.5 & 18.0 & 15.5 & 17.0\end{array}$ $\begin{array}{llllllllll}14.0 & 17.0 & 17.5 & 18.0 & 18.0 & 19.0 & 21.0 & 21.0 & 21.0 & 23.0\end{array}$ $\begin{array}{llllllllll}14.0 & 14.5 & 15.0 & 15.5 & 16.0 & 17.0 & 17.5 & 18.0 & 18.5 & 8.0\end{array}$ $\begin{array}{lllllllllll}3.5 & 9.0 & 11.5 & 11.0 & 8.5 & 12.5 & 12.5 & 12.0 & 11.0 & 10.5\end{array}$ $\begin{array}{lllllllllll}10.0 & 21.0 & 22.0 & 27.0 & 27.0 & 26.0 & 26.0 & 23.0 & 14.0 & 21.0\end{array}$ $\begin{array}{llllllllll}19.0 & 14.0 & 21.0 & 22.0 & 22.0 & 23.0 & 21.0 & 20.0 & 19.0 & 2.0\end{array}$ $\begin{array}{llllllllllll}4.0 & 18.0 & 18.0 & 17.0 & 17.0 & 16.0 & 17.0 & 16.0 & 15.0 & 15.0\end{array}$ $\begin{array}{llllllllll}14.0 & 14.0 & 17.0 & 18.0 & 17.0 & 11.0 & 10.0 & 9.0 & 9.0 & 15.0\end{array}$

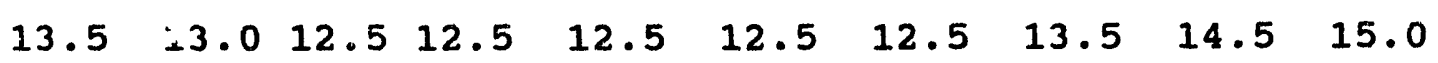


$\begin{array}{llllllllll}14.5 & 18.0 & 19.0 & 19.0 & 19.0 & 17.0 & 18.0 & 17.0 & 16.0 & 14.5\end{array}$ $\begin{array}{llllllllll}12.5 & 6.0 & 5.0 & 4.0 & 3.0 & 16.5 & 18.0 & 18.0 & 17.0 & 16.0\end{array}$

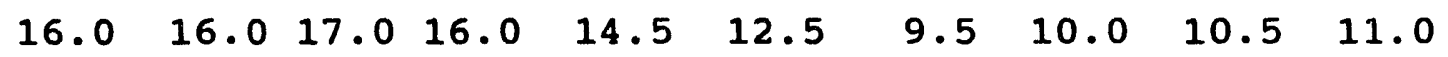
$\begin{array}{llllllllll}6.0 & 7.0 & 7.0 & 7.5 & 10.0 & 12.0 & 17.0 & 4.0 & 13.5 & 14.0\end{array}$

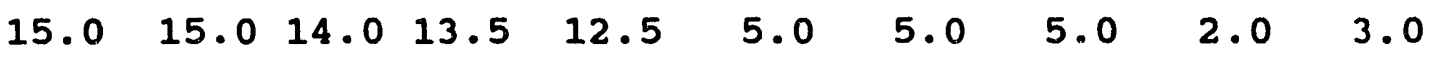
$\begin{array}{llllllllll}4.0 & 5.0 & 6.5 & 11.5 & 12.0 & 12.5 & 13.0 & 8.0 & 9.0 & 10.5\end{array}$ $\begin{array}{llllllllll}8.0 & 9.0 & 22.0 & 25.0 & 27.0 & 28.0 & 27.0 & 26.0 & 26.0 & 23.0\end{array}$ $\begin{array}{llllllllll}15.0 & 18.0 & 10.0 & 10.5 & 11.0 & 2.0 & 20.0 & 7.0 & 15.5 & 15.0\end{array}$ $\begin{array}{llllllllll}14.5 & 15.0 & 15.0 & 15.0 & 18.5 & 23.0 & 21.0 & 15.5 & 26.0 & 28.0\end{array}$ $\begin{array}{llllllllll}5.0 & 1.0 & 1.0 & 1.0 & 1.0 & 1.0 & 1.0 & 1.0 & 1.0 & 1.0\end{array}$

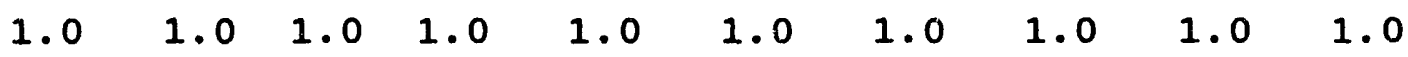
$\begin{array}{lllllll}1.0 & 1.0 & 1.0 & 1.0 & 1.0 & 1.0 & 26.0\end{array}$ $\begin{array}{llllll}25.0 & 32.0 & 32.0 & 32.0 & 17.0\end{array}$

$$
\begin{array}{rrrrrrrrrrr}
16 * * & -8.0 & -1.0 & -1.0 & -1.0 & -1.0 & -1.0 & -1.0 & -1.0 & -1.0 & -1.0 \\
-1.0 & -1.0 & -1.0 & -1.0 & -1.0 & -1.0 & -1.0 & -1.0 & -1.0 & -1.0 \\
-1.0 & -1.0 & -1.0 & -1.0 & -1.0 & -1.0 & -1.0 & -1.0 & -1.0 & -1.0 \\
-1.0 & -1.0 & -1.0 & -1.0 & -1.0 & -1.0 & -2.0 & -1.0 & -1.0 & -1.0 \\
-1.0 & -1.0 & -1.0 & -1.0 & -1.0 & -1.0 & -1.0 & -1.0 & -1.0 & -1.0 \\
-1.0 & -1.0 & -1.0 & -1.0 & -1.0 & -1.0 & -1.0 & -1.0 & -1.0 & -1.0 \\
-1.0 & -1.0 & -1.0 & -1.0 & -1.0 & -1.0 & -1.0 & -1.0 & -1.0 & -1.0 \\
-1.0 & -1.0 & -1.0 & -2.0 & -2.0 & -2.0 & -2.0 & -2.0 & -2.0 & -2.0 \\
-2.0 & -2.0 & -2.0 & -2.0 & -2.0 & -2.0 & -2.0 & -2.0 & -2.0 & -2.0 \\
-2.0 & -2.0 & -2.0 & -2.0 & -2.0 & -2.0 & -2.0 & -2.0 & -2.0 & -2.0 \\
-2.0 & -2.0 & -2.0 & -2.0 & -2.0 & -2.0 & -2.0 & -2.0 & -2.0 & -2.0 \\
-2.0 & -2.0 & -2.0 & -2.0 & -2.0 & -2.0 & -2.0 & -2.0 & -2.0 & -2.0 \\
-2.0 & -2.0 & -2.0 & -2.0 & -2.0 & -2.0 & -2.0 & -2.0 & -2.0 & -2.0
\end{array}
$$




$$
\begin{aligned}
& \begin{array}{llllllllllll}
-2.0 & -2.0 & -2.0 & -2.0 & -2.0 & -2.0 & -2.0 & -2.0 & -2.0 & -2.0
\end{array} \\
& \begin{array}{lllllllllll}
-2.0 & -2.0 & -2.0 & -2.0 & -2.0 & -2.0 & -2.0 & -2.0 & -2.0 & -2.0
\end{array} \\
& \begin{array}{lllllllllll}
-2.0 & -2.0 & -2.0 & -2.0 & -2.0 & -2.0 & -2.0 & -2.0 & -2.0 & -3.0
\end{array} \\
& \begin{array}{lllllllllll}
-3.0 & -3.0 & -3.0 & -3.0 & -3.0 & -3.0 & -3.0 & -3.0 & -3.0 & -3.0
\end{array} \\
& \begin{array}{llllllllllll}
-3.0 & -3.0 & -3.0 & -3.0 & -3.0 & -3.0 & -3.0 & -3.0 & -3.0 & -3.0
\end{array} \\
& \begin{array}{lllllllllll}
-3.0 & -3.0 & -3.0 & -3.0 & -3.0 & -3.0 & -3.0 & -3.0 & -3.0 & -3.0
\end{array} \\
& \begin{array}{lllllllllll}
-3.0 & -3.0 & -3.0 & -3.0 & -3.0 & -3.0 & -3.0 & -3.0 & -3.0 & -3.0
\end{array} \\
& \begin{array}{llllllllllll}
-3.0 & -3.0 & -3.0 & -3.0 & -3.0 & -3.0 & -3.0 & -3.0 & -3.0 & -3.0
\end{array} \\
& \begin{array}{lllllllllll}
-3.0 & -3.0 & -3.0 & -3.0 & -3.0 & -3.0 & -3.0 & -3.0 & -3.0 & -3.0
\end{array} \\
& \begin{array}{lllllllllll}
-3.0 & -3.0 & -3.0 & -4.0 & -4.0 & -4.0 & -4.0 & -4.0 & -4.0 & -4.0
\end{array} \\
& \begin{array}{lllllllllll}
-4.0 & -4.0 & -4.0 & -4.0 & -4.0 & -4.0 & -4.0 & -4.0 & -4.0 & -4.0
\end{array} \\
& \begin{array}{lllllllllll}
-4.0 & -4.0 & -4.0 & -4.0 & -4.0 & -4.0 & -4.0 & -4.0 & -4.0 & -4.0
\end{array} \\
& \begin{array}{llllllllllll}
-4.0 & -4.0 & -4.0 & -4.0 & -4.0 & -4.0 & -4.0 & -4.0 & -4.0 & -4.0
\end{array} \\
& \begin{array}{llllllllllll}
-4.0 & -4.0 & -4.0 & -4.0 & -4.0 & -4.0 & -4.0 & -4.0 & -4.0 & -4.0
\end{array} \\
& \begin{array}{lllllllllll}
-4.0 & -4.0 & -4.0 & -4.0 & -4.0 & -4.0 & -5.0 & -5.0 & -5.0 & -5.0
\end{array} \\
& \begin{array}{lllllllllll}
-5.0 & -5.0 & -5.0 & -5.0 & -5.0 & -5.0 & -5.0 & -5.0 & -5.0 & -5.0
\end{array} \\
& \begin{array}{lllllllllll}
-5.0 & -5.0 & -5.0 & -5.0 & -5.0 & -5.0 & -5.0 & -5.0 & -5.0 & -5.0
\end{array} \\
& \begin{array}{lllllllllll}
-5.0 & -5.0 & -5.0 & -5.0 & -5.0 & -5.0 & -5.0 & -5.0 & -5.0 & -5.0
\end{array} \\
& \begin{array}{lllllllllll}
-5.0 & -5.0 & -5.0 & -5.0 & -5.0 & -5.0 & -5.0 & -5.0 & -6.0 & -6.0
\end{array} \\
& \begin{array}{llllllllll}
-6.0 & -6.0 & -6.0 & -6.0 & -6.0 & -6.0 & -6.0 & -6.0 & -6.0 & -6.0
\end{array} \\
& \begin{array}{llllllllll}
-6.0 & -6.0 & -6.0 & -6.0 & -6.0 & -6.0 & -6.0 & -6.0 & -6.0 & -6.0
\end{array} \\
& \begin{array}{llllllllll}
-6.0 & -6.0 & -6.0 & -6.0 & -6.0 & -6.0 & -6.0 & -6.0 & -6.0 & -6.0
\end{array} \\
& \begin{array}{lllllllllll}
-6.0 & -6.0 & -6.0 & -6.0 & -7.0 & -7.0 & -7.0 & -7.0 & -7.0 & -7.0
\end{array} \\
& \begin{array}{llllllllllll}
-7.0 & -7.0 & -7.0 & -7.0 & -7.0 & -7.0 & -7.0 & -7.0 & -7.0 & -7.0
\end{array} \\
& \begin{array}{llllllllllll}
-7.0 & -7.0 & -7.0 & -7.0 & -7.0 & -7.0 & -7.0 & -7.0 & -7.0 & -3.0
\end{array} \\
& \begin{array}{llllllllllll}
-3.0 & -3.0 & -3.0 & -3.0 & -3.0 & -3.0 & -3.0 & -3.0 & -3.0 & -3.0
\end{array}
\end{aligned}
$$




\begin{tabular}{|c|c|c|c|c|c|c|c|c|c|}
\hline-3.0 & -3.0 & -4.0 & $-4 \cdot 0$ & -1.0 & -0.3 & -0.5 & -1.0 & -0.3 & -0.5 \\
\hline-1.0 & -2.0 & -1.0 & -3.0 & -3.0 & -1.0 & -2.0 & -6.0 & -1.0 & -1.0 \\
\hline-1.0 & -2.0 & -2.0 & -2.0 & -2.0 & -2.0 & -2.0 & -2.0 & -3.0 & -3.0 \\
\hline-3.0 & -3.0 & -4.0 & -4.0 & -4.0 & -5.0 & -5.0 & -5.0 & -6.0 & -6.0 \\
\hline-6.0 & -7.0 & -7.0 & -1.0 & 0.0 & 0.0 & 0.0 & 0.0 & 0.0 & 0.0 \\
\hline 0.0 & 0.0 & 0.0 & 0.0 & 0.0 & 0.0 & 0.0 & 0.0 & 0.0 & 0.0 \\
\hline 0.0 & 0.0 & 0.0 & 0.0 & 0.0 & 0.0 & 0.0 & 0.0 & 0.0 & 0.0 \\
\hline 0.0 & 0.0 & 0.0 & 0.0 & 0.0 & 0.0 & 0.0 & 0.0 & 0.0 & 0.0 \\
\hline 0.0 & 0.0 & 0.0 & 0.0 & 0.0 & 0.0 & 0.0 & 0.0 & 0.0 & 0.0 \\
\hline 0.0 & 0.0 & 0.0 & 0.0 & 0.0 & 0.0 & 0.0 & 0.0 & 0.0 & 0.0 \\
\hline 0.0 & 0.0 & 0.0 & 0.0 & 0.0 & 0.0 & 0.0 & 0.0 & 0.0 & 0.0 \\
\hline 0.0 & 0.0 & 0.0 & 0.0 & 0.0 & 0.0 & 1.0 & 1.0 & 1.0 & 1.0 \\
\hline 1.0 & 1.0 & 1.0 & 1.0 & 1.0 & 1.0 & 1.0 & 1.0 & 1.0 & 1.0 \\
\hline 1.0 & 1.0 & 1.0 & 1.0 & 1.0 & 1.0 & 1.0 & 1.0 & 1.0 & 1.0 \\
\hline 1.0 & 1.0 & 1.0 & 1.0 & 1.0 & 1.0 & 1.0 & 1.0 & 1.0 & 1.0 \\
\hline 1.0 & 1.0 & 1.0 & 1.0 & 1.0 & 1.0 & 1.0 & 1.0 & 1.0 & 1.0 \\
\hline 1.0 & 1.0 & 1.0 & 1.0 & 1.0 & 1.0 & 1.0 & 1.0 & 1.0 & 1.0 \\
\hline 1.0 & 1.0 & 1.0 & 1.0 & 1.0 & 1.0 & 1.0 & 1.0 & 1.0 & 1.0 \\
\hline 1.0 & 1.0 & 1.0 & 1.0 & 1.0 & 1.0 & 1.0 & 1.0 & 1.0 & 1.0 \\
\hline 1.0 & 1.0 & 1.0 & 1.0 & 1.0 & 1.0 & 1.0 & 1.0 & 1.0 & 1.0 \\
\hline 1.0 & 1.0 & 2.0 & 2.0 & 2.0 & 2.0 & 2.0 & 2.0 & 2.0 & 2.0 \\
\hline 2.0 & 2.0 & 2.0 & 2.0 & 2.0 & 2.0 & 2.0 & 2.0 & 2.0 & 2.0 \\
\hline 2.0 & 2.0 & 2.0 & 2.0 & 2.0 & 2.0 & 2.0 & 2.0 & 2.0 & 2.0 \\
\hline 2.0 & 2.0 & 2.0 & 2.0 & 2.0 & 2.0 & 2.0 & 2.0 & 2.0 & 2.0 \\
\hline 2.0 & 2.0 & 2.0 & 2.0 & 2.0 & 2.0 & 2.0 & 2.0 & 2.0 & 2.0 \\
\hline 2.0 & 2.0 & 2.0 & 2.0 & 2.0 & 2.0 & 2.0 & 2.0 & 2.0 & 2.0 \\
\hline
\end{tabular}


$\begin{array}{llllllllll}2.0 & 2.0 & 2.0 & 2.0 & 2.0 & 2.0 & 3.0 & 3.0 & 3.0 & 3.0\end{array}$

$\begin{array}{llllllllll}3.0 & 3.0 & 3.0 & 3.0 & 3.0 & 3.0 & 3.0 & 3.0 & 3.0 & 3.0\end{array}$

$\begin{array}{llllllllll}3.0 & 3.0 & 3.0 & 3.0 & 3.0 & 3.0 & 3.0 & 3.0 & 3.0 & 3.0\end{array}$

$\begin{array}{llllllllll}3.0 & 3.0 & 3.0 & 3.0 & 3.0 & 3.0 & 3.0 & 3.0 & 3.0 & 3.0\end{array}$

$\begin{array}{llllllllll}3.0 & 3.0 & 3.0 & 3.0 & 3.0 & 3.0 & 3.0 & 3.0 & 3.0 & 3.0\end{array}$

$\begin{array}{llllllllll}3.0 & 3.0 & 3.0 & 3.0 & 3.0 & 3.0 & 3.0 & 3.0 & 3.0 & 4.0\end{array}$

$\begin{array}{llllllllll}4.0 & 4.0 & 4.0 & 4.0 & 4.0 & 4.0 & 4.0 & 4.0 & 4.0 & 4.0\end{array}$

$\begin{array}{llllllllll}4.0 & 4.0 & 4.0 & 4.0 & 4.0 & 4.0 & 4.0 & 4.0 & 4.0 & 4.0\end{array}$

$\begin{array}{llllllllll}4.0 & 4.0 & 4.0 & 4.0 & 4.0 & 4.0 & 4.0 & 4.0 & 4.0 & 4.0\end{array}$

$\begin{array}{llllllllll}4.0 & 4.0 & 4.0 & 4.0 & 4.0 & 4.0 & 4.0 & 4.0 & 4.0 & 4.0\end{array}$

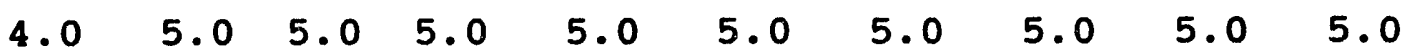

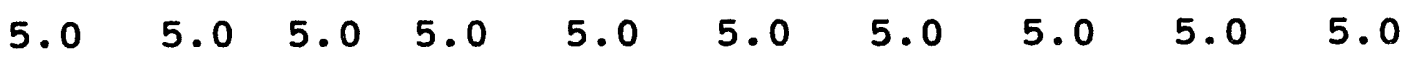

$\begin{array}{llllllllll}5.0 & 5.0 & 5.0 & 5.0 & 5.0 & 5.0 & 5.0 & 5.0 & 5.0 & 5.0\end{array}$

$\begin{array}{llllllllll}5.0 & 5.0 & 5.0 & 5.0 & 5.0 & 5.0 & 5.0 & 6.0 & 6.0 & 6.0\end{array}$

$\begin{array}{llllllllll}6.0 & 6.0 & 6.0 & 6.0 & 6.0 & 6.0 & 6.0 & 6.0 & 6.0 & 6.0\end{array}$

$\begin{array}{llllllllll}6.0 & 6.0 & 6.0 & 6.0 & 6.0 & 6.0 & 6.0 & 6.0 & 6.0 & 6.0\end{array}$

$\begin{array}{llllllllll}6.0 & 6.0 & 2.0 & 2.0 & 2.0 & 2.0 & 2.0 & 2.0 & 2.0 & 2.0\end{array}$

$\begin{array}{llllllllll}2.0 & 2.0 & 2.0 & 2.0 & 2.0 & 3.0 & 3.0 & 0.0 & 0.0 & 0.3\end{array}$

$\begin{array}{llllllllll}0.5 & 0.0 & 0.3 & 0.5 & 1.0 & 0.0 & 2.0 & 2.0 & 0.0 & 1.0\end{array}$

$\begin{array}{llllllllll}5.0 & 0.0 & 0.0 & 0.0 & 1.0 & 1.0 & 1.0 & 1.0 & 1.0 & 1.0\end{array}$

$\begin{array}{llllllllll}1.0 & 2.0 & 2.0 & 2.0 & 2.0 & 3.0 & 3.0 & 3.0 & 4.0 & 4.0\end{array}$

$\begin{array}{lllllll}4.0 & 5.0 & 5.0 & 5.0 & 6.0 & 6.0 & 0.0\end{array}$

$\begin{array}{lllll}-8.0 & -8.0 & -8.0 & -8.0 & -5.0\end{array}$

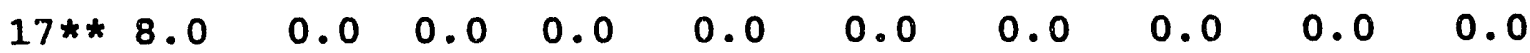

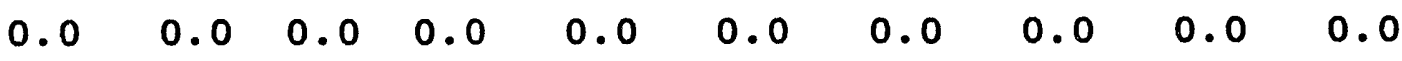
$\begin{array}{llllllllll}0.0 & 0.0 & 0.0 & 0.0 & 0.0 & 0.0 & 0.0 & 0.0 & 0.0 & 0.0\end{array}$ 


\begin{tabular}{|c|c|c|c|c|c|c|c|c|c|}
\hline 0.0 & 0.0 & 0.0 & 0.0 & 0.0 & 0.0 & 0.0 & 0.0 & 0.0 & 0.0 \\
\hline 0.0 & 0.0 & 0.0 & 0.0 & 0.0 & 0.0 & 0.0 & 0.0 & 0.0 & 0.0 \\
\hline 0.0 & 0.0 & 0.0 & 0.0 & 0.0 & 0.0 & 0.0 & 0.0 & 0.0 & 0.0 \\
\hline 0.0 & 0.0 & 0.0 & 0.0 & 0.0 & 0.0 & 0.0 & 0.0 & 0.0 & 0.0 \\
\hline 0.0 & 0.0 & 0.0 & -1.0 & -1.0 & -1.0 & -1.0 & -1.0 & -1.0 & -1.0 \\
\hline .0 & -1.0 & -1.0 & -1.0 & -1.0 & -1.0 & -1.0 & -1.0 & -1.0 & -1.0 \\
\hline 1.0 & -1.0 & -1.0 & -1.0 & -1.0 & -1.0 & -1.0 & -1.0 & -1.0 & -1.0 \\
\hline 1.0 & -1.0 & -1.0 & -1.0 & -1.0 & -1.0 & -1.0 & -1.0 & -1.0 & -1.0 \\
\hline 1.0 & -1.0 & -1.0 & -1.0 & -1.0 & -1.0 & -1.0 & -1.0 & -1.0 & -1.0 \\
\hline 1.0 & -1.0 & -1.0 & -1.0 & -1.0 & -1.0 & -1.0 & -1.0 & -1.0 & -1.0 \\
\hline-1.0 & -1.0 & -1.0 & -1.0 & -1.0 & -1.0 & -1.0 & -1.0 & -1.00 & -1.0 \\
\hline-1.0 & -1.0 & -1.0 & -1.0 & -1.0 & -1.0 & -1.0 & -1.0 & -1.0 & -1.0 \\
\hline-1.0 & -1.0 & -1.0 & -1.0 & -1.0 & -1.0 & -1.0 & -1.0 & -1.0 & -2.0 \\
\hline-2.0 & -2.0 & -2.0 & -2.0 & -2.0 & -2.0 & $-2 \cdot 0$ & -2.0 & -2.0 & -2.0 \\
\hline-2.0 & -2.0 & -2.0 & -2.0 & $-2 \cdot 0$ & -2.0 & -2.0 & -2.0 & -2.0 & -2.0 \\
\hline-2.0 & -2.0 & -2.0 & -2.0 & -2.0 & -2.0 & -2.0 & -2.0 & -2.0 & -2.0 \\
\hline-2.0 & -2.0 & -2.0 & -2.0 & -2.0 & -2.0 & -2.0 & $-2 \cdot 0$ & -2.0 & -2.0 \\
\hline-2.0 & -2.0 & -2.0 & -2.0 & -2.0 & -2.0 & $-2 \cdot 0$ & -2.0 & -2.0 & $-2 \cdot 0$ \\
\hline-2.0 & -2.0 & -2.0 & -2.0 & -2.0 & -2.0 & -2.0 & -2.0 & -2.0 & -2.0 \\
\hline-2.0 & -2.0 & -2.0 & -3.0 & -3.0 & -3.0 & -3.0 & -3.0 & -3.0 & -3.0 \\
\hline-3.0 & -3.0 & -3.0 & -3.0 & -3.0 & $-3 \cdot 0$ & -3.0 & -3.0 & -3.0 & -3.0 \\
\hline-3.0 & -3.0 & $-3 \cdot 0$ & -3.0 & -3.0 & -3.0 & -3.0 & -3.0 & -3.0 & -3.0 \\
\hline-3.0 & -3.0 & -3.0 & -3.0 & -3.0 & -3.0 & -3.0 & -3.0 & -3.0 & -3.0 \\
\hline-3.0 & -3.0 & -3.0 & -3.0 & -3.0 & -3.0 & -3.0 & -3.0 & -3.0 & -3.0 \\
\hline-3.0 & -3.0 & -3.0 & -3.0 & -3.0 & -3.0 & -4.0 & -4.0 & -4.0 & -4.0 \\
\hline-4.0 & -4.0 & -4.0 & $-4 \cdot 0$ & -4.0 & -4.0 & -4.0 & -4.0 & -4.0 & $-4 \cdot 0$ \\
\hline
\end{tabular}




\begin{tabular}{|c|c|c|c|c|c|c|c|c|c|}
\hline-4.0 & -4.0 & -4.0 & -4.0 & -4.0 & -4.0 & -4.0 & -4.0 & -4.0 & -4.0 \\
\hline-4.0 & -4.0 & -4.0 & -4.0 & -4.0 & -4.0 & -4.0 & -4.0 & -4.0 & -4.0 \\
\hline-4.0 & -4.0 & -4.0 & -4.0 & -4.0 & -4.0 & -4.0 & -4.0 & -5.0 & -5.0 \\
\hline-5.0 & -5.0 & -5.0 & -5.0 & -5.0 & -5.0 & -5.0 & -5.0 & -5.0 & -5.0 \\
\hline-5.0 & -5.0 & -5.0 & -5.0 & -5.0 & -5.0 & -5.0 & -5.0 & -5.0 & -5.0 \\
\hline-5.0 & -5.0 & -5.0 & -5.0 & -5.0 & -5.0 & -5.0 & -5.0 & -5.0 & -5.0 \\
\hline-5.0 & -5.0 & -5.0 & -5.0 & -6.0 & -6.0 & -6.0 & -6.0 & -6.0 & -6.0 \\
\hline-6.0 & -6.0 & -6.0 & -6.0 & -6.0 & -6.0 & -6.0 & -6.0 & -6.0 & -6.0 \\
\hline-6.0 & -6.0 & -6.0 & -6.0 & -6.0 & -6.0 & -6.0 & -6.0 & -6.0 & -2.0 \\
\hline-2.0 & -2.0 & -2.0 & -2.0 & -2.0 & -2.0 & -2.0 & -2.0 & -2.0 & -2.0 \\
\hline-2.0 & -2.0 & -3.0 & -3.0 & 0.0 & 0.0 & -0.3 & -0.5 & 0.0 & -0.3 \\
\hline 0.5 & -1.0 & 0.0 & -2.0 & -2.0 & 0.0 & -1.0 & -5.0 & 0.0 & 0.0 \\
\hline 0.0 & -1.0 & -1.0 & -1.0 & -1.0 & -1.0 & -1.0 & -1.0 & -2.0 & -2.0 \\
\hline-2.0 & -2.0 & -3.0 & -3.0 & -3.0 & -4.0 & -4.0 & $-4 \cdot 0$ & -5.0 & -5.0 \\
\hline-5.0 & -6.0 & -6.0 & 0.0 & 1.0 & 1.0 & 1.0 & 1.0 & 1.0 & 1.0 \\
\hline 1.0 & 1.0 & 1.0 & 1.0 & 1.0 & 1.0 & 1.0 & 1.0 & 1.0 & 1.0 \\
\hline 1.0 & 1.0 & 1.0 & 1.0 & 1.0 & 1.0 & 1.0 & 1.0 & 1.0 & 1.0 \\
\hline 1.0 & 1.0 & 1.0 & 1.0 & 1.0 & 1.0 & 1.0 & 1.0 & 1.0 & 1.0 \\
\hline 1.0 & 1.0 & 1.0 & 1.0 & 1.0 & 1.0 & 1.0 & 1.0 & 1.0 & 1.0 \\
\hline 1.0 & 1.0 & 1.0 & 1.0 & 1.0 & 1.0 & 1.0 & 1.0 & 1.0 & 1.0 \\
\hline 1.0 & 1.0 & 1.0 & 1.0 & 1.0 & 1.0 & 1.0 & 1.0 & 1.0 & 1.0 \\
\hline 1.0 & 1.0 & 1.0 & 1.0 & 1.0 & 1.0 & 2.0 & 2.0 & 2.0 & 2.0 \\
\hline 2.0 & 2.0 & 2.0 & 2.0 & 2.0 & 2.0 & 2.0 & 2.0 & 2.0 & 2.0 \\
\hline 2.0 & 2.0 & 2.0 & 2.0 & 2.0 & 2.0 & 2.0 & 2.0 & 2.0 & 2.0 \\
\hline 2.0 & 2.0 & 2.0 & 2.0 & 2.0 & 2.0 & 2.0 & 2.0 & 2.0 & 2.0 \\
\hline 2.0 & 2.0 & 2.0 & 2.0 & 2.0 & 2.0 & 2.0 & 2.0 & 2.0 & 2.0 \\
\hline
\end{tabular}


$\begin{array}{llllllllll}2.0 & 2.0 & 2.0 & 2.0 & 2.0 & 2.0 & 2.0 & 2.0 & 2.0 & 2.0\end{array}$

$\begin{array}{llllllllll}2.0 & 2.0 & 2.0 & 2.0 & 2.0 & 2.0 & 2.0 & 2.0 & 2.0 & 2.0\end{array}$

$\begin{array}{llllllllll}2.0 & 2.0 & 2.0 & 2.0 & 2.0 & 2.0 & 2.0 & 2.0 & 2.0 & 2.0\end{array}$

$\begin{array}{llllllllll}2.0 & 2.0 & 2.0 & 2.0 & 2.0 & 2.0 & 2.0 & 2.0 & 2.0 & 2.0\end{array}$

$\begin{array}{llllllllll}2.0 & 2.0 & 3.0 & 3.0 & 3.0 & 3.0 & 3.0 & 3.0 & 3.0 & 3.0\end{array}$

$\begin{array}{llllllllll}3.0 & 3.0 & 3.0 & 3.0 & 3.0 & 3.0 & 3.0 & 3.0 & 3.0 & 3.0\end{array}$

$\begin{array}{llllllllll}3.0 & 3.0 & 3.0 & 3.0 & 3.0 & 3.0 & 3.0 & 3.0 & 3.0 & 3.0\end{array}$

$\begin{array}{llllllllll}3.0 & 3.0 & 3.0 & 3.0 & 3.0 & 3.0 & 3.0 & 3.0 & 3.0 & 3.0\end{array}$

$\begin{array}{llllllllll}3.0 & 3.0 & 3.0 & 3.0 & 3.0 & 3.0 & 3.0 & 3.0 & 3.0 & 3.0\end{array}$

$\begin{array}{llllllllll}3.0 & 3.0 & 3.0 & 3.0 & 3.0 & 3.0 & 3.0 & 3.0 & 3.0 & 3.0\end{array}$

$\begin{array}{llllllllll}3.0 & 3.0 & 3.0 & 3.0 & 3.0 & 3.0 & 4.0 & 4.0 & 4.0 & 4.0\end{array}$

$\begin{array}{llllllllll}4.0 & 4.0 & 4.0 & 4.0 & 4.0 & 4.0 & 4.0 & 4.0 & 4.0 & 4.0\end{array}$

$\begin{array}{llllllllll}4.0 & 4.0 & 4.0 & 4.0 & 4.0 & 4.0 & 4.0 & 4.0 & 4.0 & 4.0\end{array}$

$\begin{array}{llllllllll}4.0 & 4.0 & 4.0 & 4.0 & 4.0 & 4.0 & 4.0 & 4.0 & 4.0 & 4.0\end{array}$

$\begin{array}{llllllllll}4.0 & 4.0 & 4.0 & 4.0 & 4.0 & 4.0 & 4.0 & 4.0 & 4.0 & 4.0\end{array}$

$\begin{array}{llllllllll}4.0 & 4.0 & 4.0 & 4.0 & 4.0 & 4.0 & 4.0 & 4.0 & 4.0 & 5.0\end{array}$

$\begin{array}{llllllllll}5.0 & 5.0 & 5.0 & 5.0 & 5.0 & 5.0 & 5.0 & 5.0 & 5.0 & 5.0\end{array}$

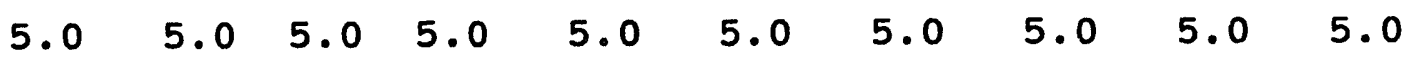

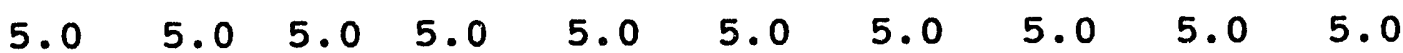

$\begin{array}{llllllllll}5.0 & 5.0 & 5.0 & 5.0 & 5.0 & 5.0 & 5.0 & 5.0 & 5.0 & 5.0\end{array}$

$\begin{array}{llllllllll}5.0 & 6.0 & 6.0 & 6.0 & 6.0 & 6.0 & 6.0 & 6.0 & 6.0 & 6.0\end{array}$

$\begin{array}{llllllllll}6.0 & 6.0 & 6.0 & 6.0 & 6.0 & 6.0 & 6.0 & 6.0 & 6.0 & 6.0\end{array}$

$\begin{array}{llllllllll}6.0 & 6.0 & 6.0 & 6.0 & 6.0 & 6.0 & 6.0 & 6.0 & 6.0 & 6.0\end{array}$

$\begin{array}{llllllllll}6.0 & 6.0 & 6.0 & 6.0 & 6.0 & 6.0 & 6.0 & 7.0 & 7.0 & 7.0\end{array}$

$\begin{array}{llllllllll}7.0 & 7.0 & 7.0 & 7.0 & 7.0 & 7.0 & 7.0 & 7.0 & 7.0 & 7.0\end{array}$

$\begin{array}{llllllllll}7.0 & 7.0 & 7.0 & 7.0 & 7.0 & 7.0 & 7.0 & 7.0 & 7.0 & 7.0\end{array}$ 
$\begin{array}{llllllllll}7.0 & 7.0 & 3.0 & 3.0 & 3.0 & 3.0 & 3.0 & 3.0 & 3.0 & 3.0\end{array}$

$\begin{array}{llllllllll}3.0 & 3.0 & 3.0 & 3.0 & 3.0 & 4.0 & 4.0 & 1.0 & 0.3 & 0.5\end{array}$

$\begin{array}{llllllllll}1.0 & 0.3 & 0.5 & 1.0 & 2.0 & 1.0 & 3.0 & 3.0 & 1.0 & 2.0\end{array}$

$\begin{array}{llllllllll}6.0 & 1.0 & 1.0 & 1.0 & 2.0 & 2.0 & 2.0 & 2.0 & 2.0 & 2.0\end{array}$

$\begin{array}{llllllllll}2.0 & 3.0 & 3.0 & 3.0 & 3.0 & 4.0 & 4.0 & 4.0 & 5.0 & 5.0\end{array}$

$\begin{array}{lllllll}5.0 & 6.0 & 6.0 & 6.0 & 7.0 & 7.0 & 1.0\end{array}$

Line below creates $10 \times 10$ delimiter from $\mathrm{Y}=-5$ to

$Y=+5$

$8.0 \quad 8.0 \quad 8.0 \quad 8.0 \quad 5.0$

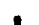

Line below creates $5 \times 10$ delimiter from $Y=-5$ to $Y=0$

$\begin{array}{lllll}8.0 & 8.0 & 8.0 & 8.0 & 0.0\end{array}$

1

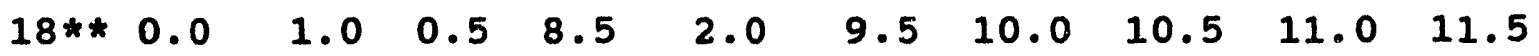
$\begin{array}{llllllllll}12.0 & 12.5 & 13.0 & 9.0 & 8.5 & 8.0 & 7.5 & 3.5 & 2.0 & 1.0\end{array}$ $\begin{array}{llllllllll}0.5 & 0.0 & 7.5 & 3.0 & 2.0 & 3.5 & 10.0 & 10.5 & 4.5 & 5.0\end{array}$ $\begin{array}{llllllllll}5.5 & 6.0 & 6.5 & 7.0 & 1.5 & 2.5 & 3.5 & 1.0 & 1.0 & 2.5\end{array}$ $\begin{array}{llllllllll}4.0 & 4.5 & 5.5 & 6.0 & 6.0 & 6.5 & 7.0 & 7.0 & 7.5 & 10.0\end{array}$ $\begin{array}{llllllllll}10.5 & 11.0 & 11.5 & 12.0 & 12.5 & 11.5 & 11.0 & 10.5 & 10.0 & 9.5\end{array}$ $\begin{array}{llllllllll}9.0 & 8.5 & 8.5 & 7.5 & 7.0 & 11.5 & 10.5 & 10.5 & 9.5 & 8.5\end{array}$ $\begin{array}{llllllllll}7.5 & 1.5 & 1.0 & 1.5 & 1.0 & 0.5 & 1.0 & 1.5 & 4.0 & 3.0\end{array}$ $\begin{array}{llllllllll}2.5 & 3.5 & 4.0 & 4.5 & 1.0 & 1.0 & 1.5 & 2.0 & 3.5 & 4.0\end{array}$ $\begin{array}{llllllllll}3.5 & 4.0 & 4.5 & 5.5 & 5.5 & 5.5 & 6.0 & 6.5 & 6.5 & 7.0\end{array}$ 
$\begin{array}{llllllllll}7.5 & 7.0 & 7.5 & 7.5 & 9.0 & 9.5 & 10.0 & 10.5 & 11.0 & 12.5\end{array}$

$\begin{array}{llllllllll}13.0 & 8.5 & 8.0 & 7.5 & 7.0 & 5.0 & 2.5 & 1.5 & 1.0 & 0.5\end{array}$ $\begin{array}{llllllllll}0.0 & 8.0 & 12.0 & 12.0 & 8.0 & 9.0 & 9.5 & 10.0 & 10.5 & 11.0\end{array}$

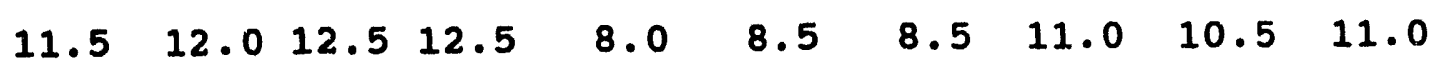

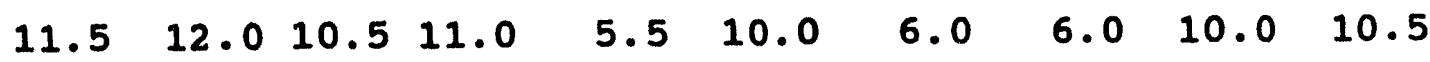
$\begin{array}{llllllllll}6.0 & 10.5 & 10.0 & 9.5 & 8.5 & 6.0 & 6.0 & 6.0 & 7.5 & 2.5\end{array}$ $\begin{array}{llllllllll}1.5 & 1.0 & 0.5 & 8.0 & 8.5 & 9.0 & 9.5 & 10.0 & 10.5 & 11.0\end{array}$ $\begin{array}{llllllllll}12.0 & 12.5 & 13.0 & 9.0 & 9.5 & 10.0 & 10.5 & 11.0 & 11.5 & 12.0\end{array}$ $\begin{array}{llllllllll}1.2 .5 & 7.5 & 9.0 & 9.5 & 3.5 & 4.5 & 1.0 & 3.0 & 1.0 & 1.5\end{array}$ $\begin{array}{llllllllll}2.0 & 3.5 & 5.0 & 5.0 & 5.5 & 7.0 & 7.5 & 6.5 & 6.0 & 3.5\end{array}$ $\begin{array}{llllllllll}3.5 & 5.0 & 5.5 & 4.5 & 7.0 & 7.5 & 5.5 & 7.5 & 8.0 & 9.0\end{array}$ $\begin{array}{llllllllll}11.0 & 11.5 & 11.0 & 10.0 & 12.5 & 12.0 & 10.5 & 10.0 & 9.5 & 9.0\end{array}$ $\begin{array}{llllllllll}8.5 & 12.0 & 10.5 & 1.0 & 6.5 & 9.5 & 10.0 & 11.0 & 12.0 & 13.0\end{array}$ $\begin{array}{llllllllll}12.5 & 9.5 & 10.0 & 11.0 & 11.5 & 12.0 & 12.5 & 0.5 & 11.5 & 10.5\end{array}$ $\begin{array}{llllllllll}9.0 & 7.5 & 6.5 & 6.5 & 4.0 & 4.0 & 3.5 & 3.5 & 3.5 & 2.5\end{array}$ $\begin{array}{llllllllll}2.0 & 1.5 & 1.5 & 1.0 & 1.0 & 1.5 & 3.0 & 3.0 & 3.0 & 6.0\end{array}$ $\begin{array}{llllllllll}10.0 & 7.0 & 5.5 & 6.0 & 6.5 & 8.0 & 9.0 & 9.5 & 7.0 & 6.5\end{array}$ $\begin{array}{llllllllll}6.0 & 2.0 & 1.5 & 1.0 & 0.5 & 0.0 & 0.5 & 4.0 & 0.0 & 0.5\end{array}$ $\begin{array}{llllllllll}1.0 & 2.0 & 2.0 & 3.5 & 4.0 & 5.5 & 8.5 & 9.0 & 9.0 & 9.5\end{array}$

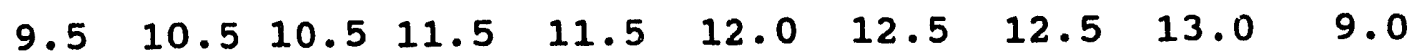
$\begin{array}{llllllllll}5.5 & 6.5 & 1.0 & 1.5 & 2.0 & 3.0 & 3.5 & 4.0 & 4.5 & 5.0\end{array}$ $\begin{array}{llllllllll}5.5 & 6.0 & 6.5 & 7.0 & 7.5 & 8.0 & 7.5 & 9.0 & 0.5 & 1.0\end{array}$ $\begin{array}{llllllllll}2.0 & 3.5 & 4.0 & 5.5 & 10.5 & 11.0 & 12.0 & 12.5 & 9.5 & 9.0\end{array}$ $\begin{array}{llllllllll}8.5 & 8.0 & 1.0 & 1.0 & 2.0 & 5.5 & 8.0 & 8.5 & 9.0 & 9.5\end{array}$

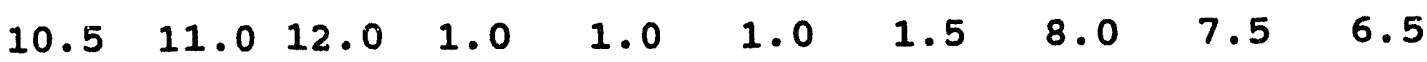
$\begin{array}{llllllllll}6.0 & 6.0 & 7.0 & 7.5 & 0.5 & 1.0 & 1.5 & 1.0 & 1.5 & 2.5\end{array}$ 
$\begin{array}{llllllllll}6.5 & 7.5 & 7.5 & 8.0 & 8.5 & 7.0 & 7.5 & 8.5 & 9.5 & 10.5\end{array}$ $\begin{array}{llllllllll}11.0 & 10.5 & 10.0 & 7.5 & 6.5 & 7.0 & 9.5 & 8.0 & 8.5 & 8.5\end{array}$ $\begin{array}{llllllllll}8.0 & 7.5 & 3.0 & 2.0 & 1.5 & 1.0 & 0.5 & 0.0 & 11.5 & 5.5\end{array}$ $\begin{array}{llllllllll}6.5 & 6.5 & 9.0 & 8.0 & 9.5 & 12.5 & 12.5 & 12.5 & 12.0 & 12.0\end{array}$ $\begin{array}{llllllllll}12.0 & 10.0 & 8.5 & 8.5 & 10.0 & 1.0 & 5.0 & 9.5 & 0.0 & 0.5\end{array}$ $\begin{array}{llllllllll}9.0 & 0.0 & 0.5 & 1.0 & 2.5 & 3.0 & 4.0 & 9.0 & 0.0 & 0.5\end{array}$ $\begin{array}{llllllllll}1.0 & 9.0 & 0.0 & 0.5 & 9.0 & 0.0 & 0.5 & 8.5 & 0.5 & 1.0\end{array}$ $\begin{array}{llllllllll}8.0 & 0.5 & 1.0 & 2.0 & 1.0 & 0.5 & 8.5 & 2.0 & 9.5 & 10.0\end{array}$

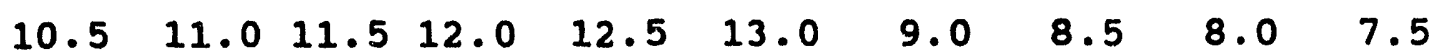
$\begin{array}{llllllllll}3.5 & 2.0 & 1.0 & 0.5 & 0.0 & 7.5 & 3.0 & 2.0 & 3.5 & 10.0\end{array}$ $\begin{array}{llllllllll}10.5 & 4.5 & 5.0 & 5.5 & 6.0 & 6.5 & 7.0 & 1.5 & 2.5 & 3.5\end{array}$ $\begin{array}{llllllllll}1.0 & 1.0 & 2.5 & 4.0 & 4.5 & 5.5 & 6.0 & 6.0 & 6.5 & 7.0\end{array}$ $\begin{array}{llllllllll}7.0 & 7.5 & 10.0 & 10.5 & 11.0 & 11.5 & 12.0 & 12.5 & 11.5 & 11.0\end{array}$ $\begin{array}{llllllllll}10.5 & 10.0 & 9.5 & 9.0 & 8.5 & 8.5 & 7.5 & 7.0 & 11.5 & 10.5\end{array}$ $\begin{array}{llllllllll}10.5 & 9.5 & 8.5 & 7.5 & 1.5 & 1.0 & 1.5 & 1.0 & 0.5 & 1.0\end{array}$ $\begin{array}{llllllllll}1.5 & 4.0 & 3.0 & 2.5 & 3.5 & 4.0 & 4.5 & 1.0 & 1.0 & 1.5\end{array}$ $\begin{array}{llllllllll}2.0 & 3.5 & 4.0 & 3.5 & 4.0 & 4.5 & 5.5 & 5.5 & 5.5 & 6.0\end{array}$ $\begin{array}{llllllllll}6.5 & 6.5 & 7.0 & 7.5 & 7.0 & 7.5 & 7.5 & 9.0 & 9.5 & 10.0\end{array}$ $\begin{array}{llllllllll}10.5 & 11.0 & 12.5 & 13.0 & 8.5 & 8.0 & 7.5 & 7.0 & 5.0 & 2.5\end{array}$ $\begin{array}{llllllllll}1.5 & 1.0 & 0.5 & 0.0 & 8.0 & 12.0 & 12.0 & 8.0 & 9.0 & 9.5\end{array}$ $\begin{array}{llllllllll}10.0 & 10.5 & 11.0 & 11.5 & 12.0 & 12.5 & 12.5 & 8.0 & 8.5 & 8.5\end{array}$

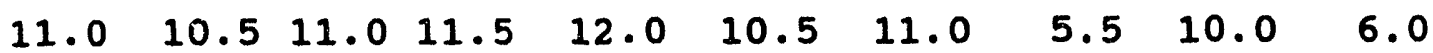

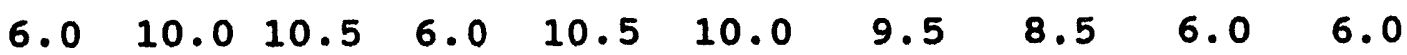
$\begin{array}{llllllllll}6.0 & 7.5 & 2.5 & 1.5 & 1.0 & 0.5 & 8.0 & 8.5 & 9.0 & 9.5\end{array}$ $\begin{array}{llllllllll}10.0 & 10.5 & 11.0 & 12.0 & 12.5 & 13.0 & 9.0 & 9.5 & 10.0 & 10.5\end{array}$

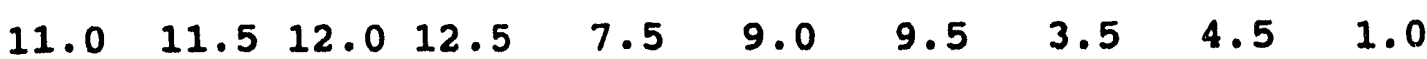


$\begin{array}{llllllllll}3.0 & 1.0 & 1.5 & 2.0 & 3.5 & 5.0 & 5.0 & 5.5 & 7.0 & 7.5\end{array}$

$\begin{array}{llllllllll}6.5 & 6.0 & 3.5 & 3.5 & 5.0 & 5.5 & 4.5 & 7.0 & 7.5 & 5.5\end{array}$

$\begin{array}{llllllllll}7.5 & 8.0 & 9.0 & 11.0 & 11.5 & 11.0 & 10.0 & 12.5 & 12.0 & 10.5\end{array}$

$\begin{array}{llllllllll}10.0 & 9.5 & 9.0 & 8.5 & 12.0 & 10.5 & 1.0 & 6.5 & 9.5 & 10.0\end{array}$

$\begin{array}{llllllllll}11.0 & 12.0 & 13.0 & 12.5 & 9.5 & 10.0 & 11.0 & 11.5 & 12.0 & 12.5\end{array}$

$\begin{array}{llllllllll}0.5 & 11.5 & 10.5 & 9.0 & 7.5 & 6.5 & 6.5 & 4.0 & 4.0 & 3.5\end{array}$

$\begin{array}{llllllllll}3.5 & 3.5 & 2.5 & 2.0 & 1.5 & 1.5 & 1.0 & 1.0 & 1.5 & 3.0\end{array}$

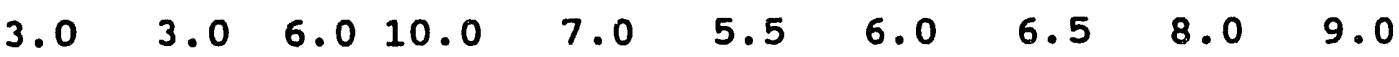

$\begin{array}{llllllllll}9.5 & 7.0 & 6.5 & 6.0 & 2.0 & 1.5 & 1.0 & 0.5 & 0.0 & 0.5\end{array}$

$\begin{array}{llllllllll}4.0 & 0.0 & 0.5 & 1.0 & 2.0 & 2.0 & 3.5 & 4.0 & 5.5 & 8.5\end{array}$

$\begin{array}{llllllllll}9.0 & 9.0 & 9.5 & 9.5 & 10.5 & 10.5 & 11.5 & 11.5 & 12.0 & 12.5\end{array}$ $\begin{array}{llllllllll}12.5 & 13.0 & 9.0 & 5.5 & 6.5 & 1.0 & 1.5 & 2.0 & 3.0 & 3.5\end{array}$ $\begin{array}{llllllllll}4.0 & 4.5 & 5.0 & 5.5 & 6.0 & 6.5 & 7.0 & 7.5 & 8.0 & 7.5\end{array}$ $\begin{array}{llllllllll}9.0 & 0.5 & 1.0 & 2.0 & 3.5 & 4.0 & 5.5 & 10.5 & 11.0 & 12.0\end{array}$ $\begin{array}{llllllllll}12.5 & 9.5 & 9.0 & 8.5 & 8.0 & 1.0 & 1.0 & 2.0 & 5.5 & 8.0\end{array}$ $\begin{array}{llllllllll}8.5 & 9.0 & 9.5 & 10.5 & 11.0 & 12.0 & 1.0 & 1.0 & 1.0 & 1.5\end{array}$ $\begin{array}{llllllllll}8.0 & 7.5 & 6.5 & 6.0 & 6.0 & 7.0 & 7.5 & 0.5 & 1.0 & 1.5\end{array}$ $\begin{array}{llllllllll}1.0 & 1.5 & 2.5 & 6.5 & 7.5 & 7.5 & 8.0 & 8.5 & 7.0 & 7.5\end{array}$ $\begin{array}{llllllllll}8.5 & 9.5 & 10.5 & 11.0 & 10.5 & 10.0 & 7.5 & 6.5 & 7.0 & 9.5\end{array}$ $\begin{array}{llllllllll}8.0 & 8.5 & 8.5 & 8.0 & 7.5 & 3.0 & 2.0 & 1.5 & 1.0 & 0.5\end{array}$ $\begin{array}{llllllllll}0.0 & 11.5 & 5.5 & 6.5 & 6.5 & 9.0 & 8.0 & 9.5 & 12.5 & 12.5\end{array}$

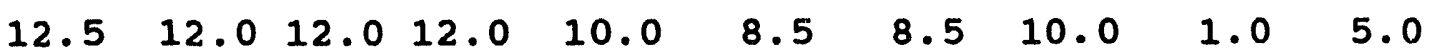

$\begin{array}{llllllllll}9.5 & 0.0 & 0.5 & 9.0 & 0.0 & 0.5 & 1.0 & 2.5 & 3.0 & 4.0\end{array}$ $\begin{array}{llllllllll}9.0 & 0.0 & 0.5 & 1.0 & 9.0 & 0.0 & 0.5 & 9.0 & 0.0 & 0.5\end{array}$ $\begin{array}{lllllll}8.5 & 0.5 & 1.0 & 8.0 & 0.5 & 1.0 & 2.0\end{array}$ $\begin{array}{lllll}13.5 & 16.04 & 15.41 & 12.86 & 13.5\end{array}$ 


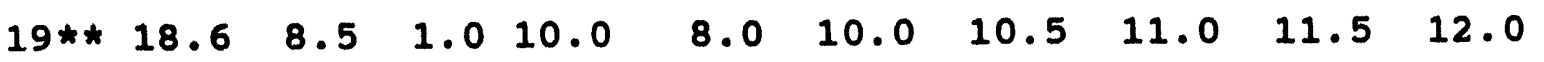
$\begin{array}{llllllllll}12.5 & 13.0 & 13.5 & 9.5 & 9.0 & 8.5 & 8.0 & 7.5 & 3.5 & 2.0\end{array}$ $\begin{array}{llllllllll}1.0 & 0.5 & 12.0 & 5.5 & 5.5 & 4.5 & 10.5 & 11.0 & 5.0 & 5.5\end{array}$ $\begin{array}{llllllllll}6.5 & 6.5 & 7.0 & 7.5 & 2.5 & 3.5 & 4.5 & 2.5 & 3.0 & 3.5\end{array}$ $\begin{array}{llllllllll}5.0 & 5.0 & 6.5 & 7.0 & 6.5 & 7.0 & 7.5 & 7.5 & 8.0 & 10.5\end{array}$ $\begin{array}{llllllllll}11.0 & 11.5 & 12.0 & 12.5 & 13.0 & 12.0 & 11.5 & 11.0 & 10.5 & 10.0\end{array}$ $\begin{array}{llllllllll}9.5 & 9.5 & 9.0 & 8.0 & 7.5 & 12.5 & 11.5 & 11.0 & 10.5 & 9.5\end{array}$ $\begin{array}{llllllllll}8.5 & 3.0 & 1.5 & 8.0 & 1.5 & 1.0 & 4.0 & 3.0 & 4.5 & 3.5\end{array}$ $\begin{array}{llllllllll}3.5 & 4.0 & 4.5 & 5.5 & 3.0 & 3.5 & 3.5 & 3.5 & 4.0 & 5.0\end{array}$ $\begin{array}{llllllllll}4.0 & 4.5 & 5.5 & 6.0 & 6.0 & 6.0 & 6.5 & 7.0 & 7.0 & 7.5\end{array}$ $\begin{array}{llllllllll}8.0 & 7.5 & 8.0 & 8.0 & 9.5 & 10.0 & 10.5 & 11.0 & 12.0 & 13.0\end{array}$ $\begin{array}{llllllllll}13.5 & 9.0 & 8.5 & 8.0 & 7.5 & 6.0 & 5.0 & 2.5 & 1.5 & 1.0\end{array}$

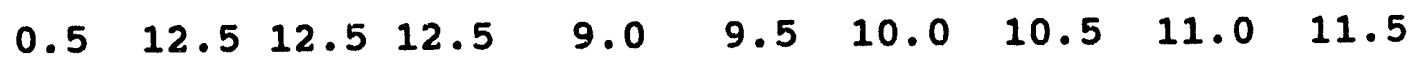
$\begin{array}{llllllllll}12.0 & 12.5 & 13.0 & 13.0 & 11.0 & 11.0 & 10.0 & 11.5 & 11.0 & 11.5\end{array}$ $\begin{array}{llllllllll}12.0 & 12.5 & 11.0 & 11.5 & 6.0 & 11.0 & 9.0 & 10.0 & 10.5 & 11.0\end{array}$

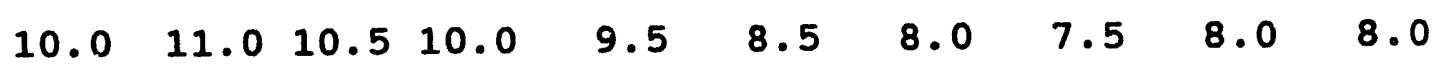
$\begin{array}{llllllllll}2.5 & 1.5 & 1.0 & 8.5 & 9.0 & 9.5 & 10.0 & 10.5 & 11.0 & 12.0\end{array}$

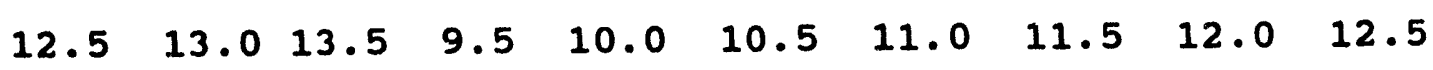

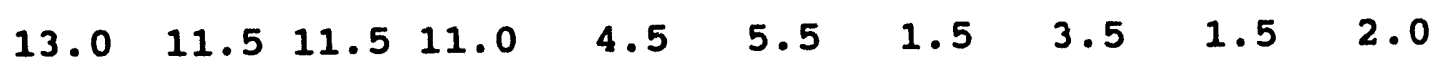
$\begin{array}{llllllllll}3.5 & 4.0 & 7.5 & 6.0 & 6.5 & 7.5 & 8.0 & 8.5 & 7.5 & 4.5\end{array}$ $\begin{array}{llllllllll}6.0 & 7.0 & 7.5 & 5.5 & 7.5 & 8.0 & 6.5 & 8.0 & 9.0 & 10.0\end{array}$ $\begin{array}{llllllllll}11.5 & 12.0 & 12.5 & 12.5 & 13.0 & 12.5 & 12.0 & 10.5 & 10.0 & 9.5\end{array}$

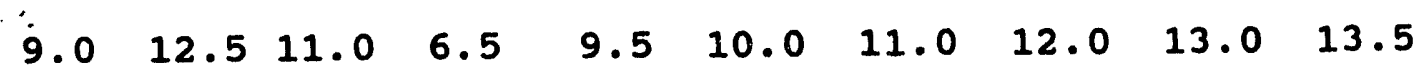
$\begin{array}{llllllllll}13.0 & 10.0 & 11.0 & 11.5 & 12.0 & 12.5 & 13.0 & 1.0 & 12.5 & 11.0\end{array}$ $\begin{array}{llllllllll}10.5 & 8.0 & 9.0 & 8.0 & 6.5 & 5.0 & 4.5 & 5.5 & 6.0 & 6.5\end{array}$ $\begin{array}{llllllllll}6.5 & 5.0 & 4.5 & 4.5 & 4.0 & 3.0 & 5.0 & 4.0 & 3.5 & 10.0\end{array}$ 
$\begin{array}{llllllllll}10.5 & 8.5 & 6.0 & 6.5 & 7.5 & 9.0 & 9.5 & 10.0 & 8.0 & 7.0\end{array}$ $\begin{array}{llllllllll}6.5 & 6.5 & 2.0 & 1.5 & 1.0 & 0.5 & 4.0 & 9.0 & 0.5 & 1.0\end{array}$

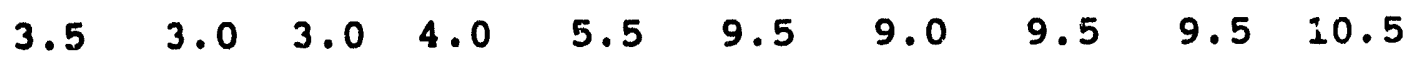
$\begin{array}{llllllllll}10.5 & 11.5 & 11.5 & 12.5 & 12.0 & 12.5 & 13.0 & 13.0 & 13.5 & 10.5\end{array}$ $\begin{array}{llllllllll}8.5 & 7.0 & 1.5 & 2.0 & 3.0 & 3.5 & 4.0 & 4.5 & 5.0 & 5.5\end{array}$ $\begin{array}{llllllllll}6.0 & 6.5 & 7.0 & 7.5 & 8.5 & 9.0 & 8.5 & 9.5 & 1.0 & 2.0\end{array}$ $\begin{array}{llllllllll}3.5 & 4.0 & 5.5 & 10.5 & 11.0 & 12.0 & 12.5 & 13.0 & 10.5 & 9.5\end{array}$ $\begin{array}{llllllllll}9.0 & 8.5 & 8.0 & 2.0 & 3.5 & 8.0 & 8.5 & 9.0 & 9.5 & 10.5\end{array}$ $\begin{array}{llllllllll}11.0 & 12.0 & 12.5 & 2.0 & 4.0 & 3.5 & 3.0 & 9.5 & 8.5 & 7.5\end{array}$ $\begin{array}{llllllllll}7.5 & 7.0 & 7.5 & 9.5 & 1.0 & 7.5 & 2.5 & 1.5 & 2.5 & 6.5\end{array}$ $\begin{array}{llllllllll}7.5 & 11.0 & 8.0 & 8.5 & 9.5 & 7.5 & 8.5 & 9.5 & 10.5 & 11.0\end{array}$ $\begin{array}{llllllllll}11.5 & 11.0 & 11.0 & 10.0 & 7.0 & 7.5 & 10.0 & 8.5 & 10.0 & 9.0\end{array}$ $\begin{array}{llllllllll}8.5 & 8.0 & 7.5 & 3.0 & 2.5 & 1.5 & 1.0 & 0.5 & 12.0 & 6.5\end{array}$ $\begin{array}{llllllllll}7.0 & 7.0 & 9.5 & 9.5 & 10.0 & 13.0 & 13.0 & 13.0 & 12.5 & 12.5\end{array}$ $\begin{array}{llllllllll}12.5 & 10.5 & 9.0 & 9.0 & 10.5 & 2.5 & 6.0 & 10.0 & 0.5 & 9.0\end{array}$ $\begin{array}{llllllllll}9.5 & 0.5 & 1.0 & 4.0 & 3.0 & 3.5 & 9.0 & 9.5 & 0.5 & 9.0\end{array}$ $\begin{array}{llllllllll}1.5 & 9.5 & 0.5 & 9.0 & 9.5 & 0.5 & 8.5 & 9.0 & 1.0 & 8.0\end{array}$ $\begin{array}{llllllllll}8.5 & 7.5 & 7.0 & 2.5 & 8.5 & 1.0 & 10.0 & 8.0 & 10.0 & 10.5\end{array}$

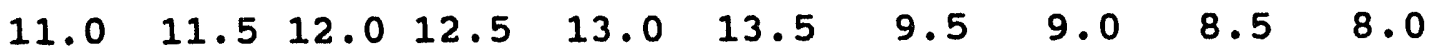
$\begin{array}{llllllllll}7.5 & 3.5 & 2.0 & 1.0 & 0.5 & 12.0 & 5.5 & 5.5 & 4.5 & 10.5\end{array}$ $\begin{array}{llllllllll}11.0 & 5.0 & 5.5 & 6.5 & 6.5 & 7.0 & 7.5 & 2.5 & 3.5 & 4.5\end{array}$ $\begin{array}{llllllllll}2.5 & 3.0 & 3.5 & 5.0 & 5.0 & 6.5 & 7.0 & 6.5 & 7.0 & 7.5\end{array}$ $\begin{array}{lllllllllll}7.5 & 8.0 & 10.5 & 11.0 & 11.5 & 12.0 & 12.5 & 13.0 & 12.0 & 11.5\end{array}$ $\begin{array}{llllllllll}11.0 & 10.5 & 10.0 & 9.5 & 9.5 & 9.0 & 8.0 & 7.5 & 12.5 & 1.1 .5\end{array}$ $\begin{array}{llllllllll}11.0 & 10.5 & 9.5 & 8.5 & 3.0 & 1.5 & 8.0 & 1.5 & 1.0 & 4.0\end{array}$ $\begin{array}{llllllllll}3.0 & 4.5 & 3.5 & 3.5 & 4.0 & 4.5 & 5.5 & 3.0 & 3.5 & 3.5\end{array}$ 
$\begin{array}{llllllllll}3.5 & 4.0 & 5.0 & 4.0 & 4.5 & 5.5 & 6.0 & 6.0 & 6.0 & 6.5\end{array}$

$\begin{array}{llllllllll}7.0 & 7.0 & 7.5 & 8.0 & 7.5 & 8.0 & 8.0 & 9.5 & 10.0 & 10.5\end{array}$ $\begin{array}{lllllllllll}11.0 & 1 \text { ? } 0 & 13.0 & 13.5 & 9.0 & 8.5 & 8.0 & 7.5 & 6.0 & 5.0\end{array}$ $\begin{array}{llllllllll}2.5 & 1.5 & 1.0 & 0.5 & 12.5 & 12.5 & 12.5 & 9.0 & 9.5 & 10.0\end{array}$ $\begin{array}{llllllllll}10.5 & 11.0 & 11.5 & 12.0 & 12.5 & 13.0 & 13.0 & 11.0 & 11.0 & 10.0\end{array}$

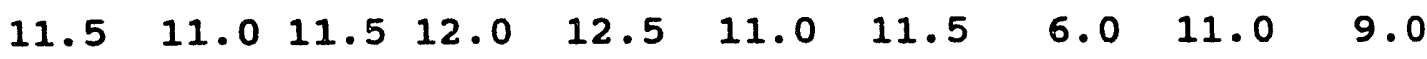
$\begin{array}{llllllllll}10.0 & 10.5 & 11.0 & 10.0 & 11.0 & 10.5 & 10.0 & 9.5 & 8.5 & 8.0\end{array}$ $\begin{array}{llllllllll}7.5 & 8.0 & 8.0 & 2.5 & 1.5 & 1.0 & 8.5 & 9.0 & 9.5 & 10.0\end{array}$

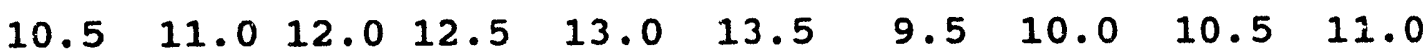
$\begin{array}{llllllllll}11.5 & 12.0 & 12.5 & 13.0 & 11.5 & 11.5 & 11.0 & 4.5 & 5.5 & 1.5\end{array}$ $\begin{array}{llllllllll}3.5 & 1.5 & 2.0 & 3.5 & 4.0 & 7.5 & 6.0 & 6.5 & 7.5 & 8.0\end{array}$ $\begin{array}{llllllllll}8.5 & 7.5 & 4.5 & 6.0 & 7.0 & 7.5 & 5.5 & 7.5 & 8.0 & 6.5\end{array}$ $\begin{array}{llllllllll}8.0 & 9.0 & 10.0 & 11.5 & 12.0 & 12.5 & 12.5 & 13.0 & 12.5 & 12.0\end{array}$ $\begin{array}{llllllllll}10.5 & 10.0 & 9.5 & 9.0 & 12.5 & 11.0 & 6.5 & 9.5 & 10.0 & 11.0\end{array}$ $\begin{array}{llllllllll}12.0 & 13.0 & 13.5 & 13.0 & 10.0 & 11.0 & 11.5 & 12.0 & 12.5 & 13.0\end{array}$

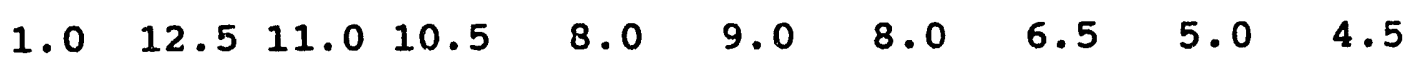
$\begin{array}{llllllllll}5.5 & 6.0 & 6.5 & 6.5 & 5.0 & 4.5 & 4.5 & 4.0 & 3.0 & 5.0\end{array}$

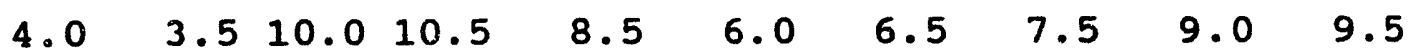
$\begin{array}{llllllllll}10.0 & 8.0 & 7.0 & 6.5 & 6.5 & 2.0 & 1.5 & 1.0 & 0.5 & 4.0\end{array}$ $\begin{array}{llllllllll}9.0 & 0.5 & 1.0 & 3.5 & 3.0 & 3.0 & 4.0 & 5.5 & 9.5 & 9.0\end{array}$

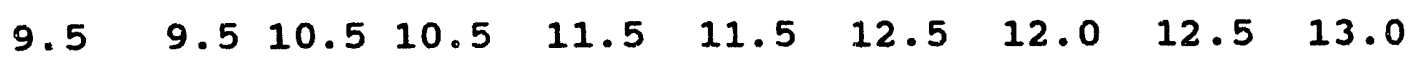

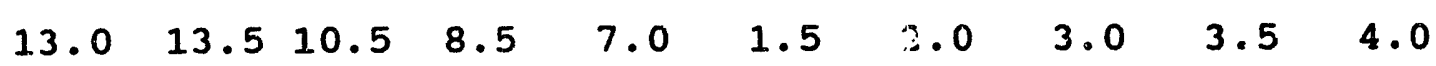
$\begin{array}{llllllllll}4.5 & 5.0 & 5.5 & 6.0 & 6.5 & 7.0 & 7.5 & 8.5 & 9.0 & 8.5\end{array}$ $\begin{array}{llllllllll}9.5 & 1.0 & 2.0 & 3.5 & 4.0 & 5.5 & 10.5 & 11.0 & 12.0 & 12.5\end{array}$ $\begin{array}{llllllllll}13.0 & 10.5 & 9.5 & 9.0 & 8.5 & 8.0 & 2.0 & 3.5 & 8.0 & 8.5\end{array}$ $\begin{array}{llllllllll}9.0 & 9.5 & 10.5 & 11.0 & 12.0 & 12.5 & 2.0 & 4.0 & 3.5 & 3.0\end{array}$ 


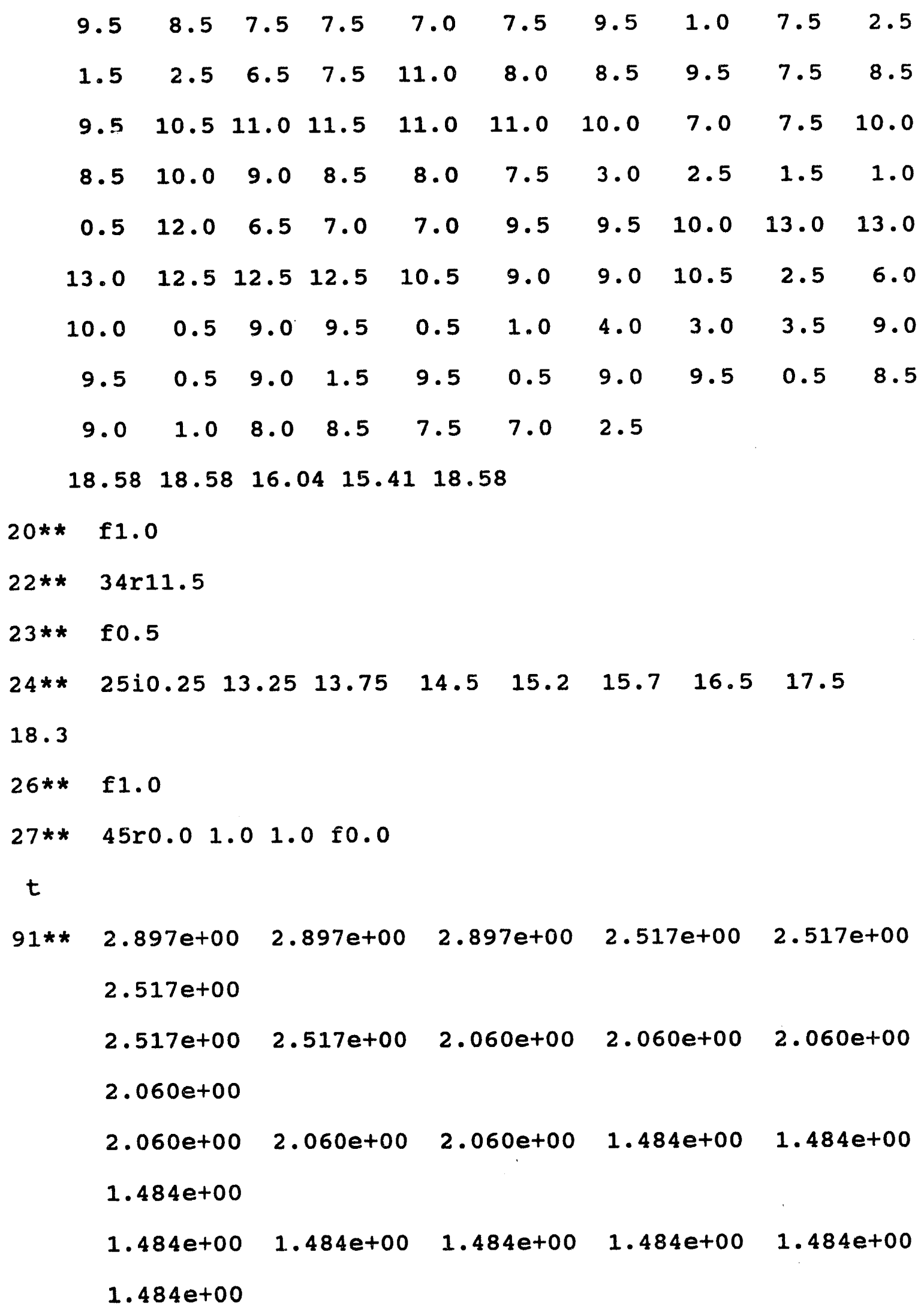




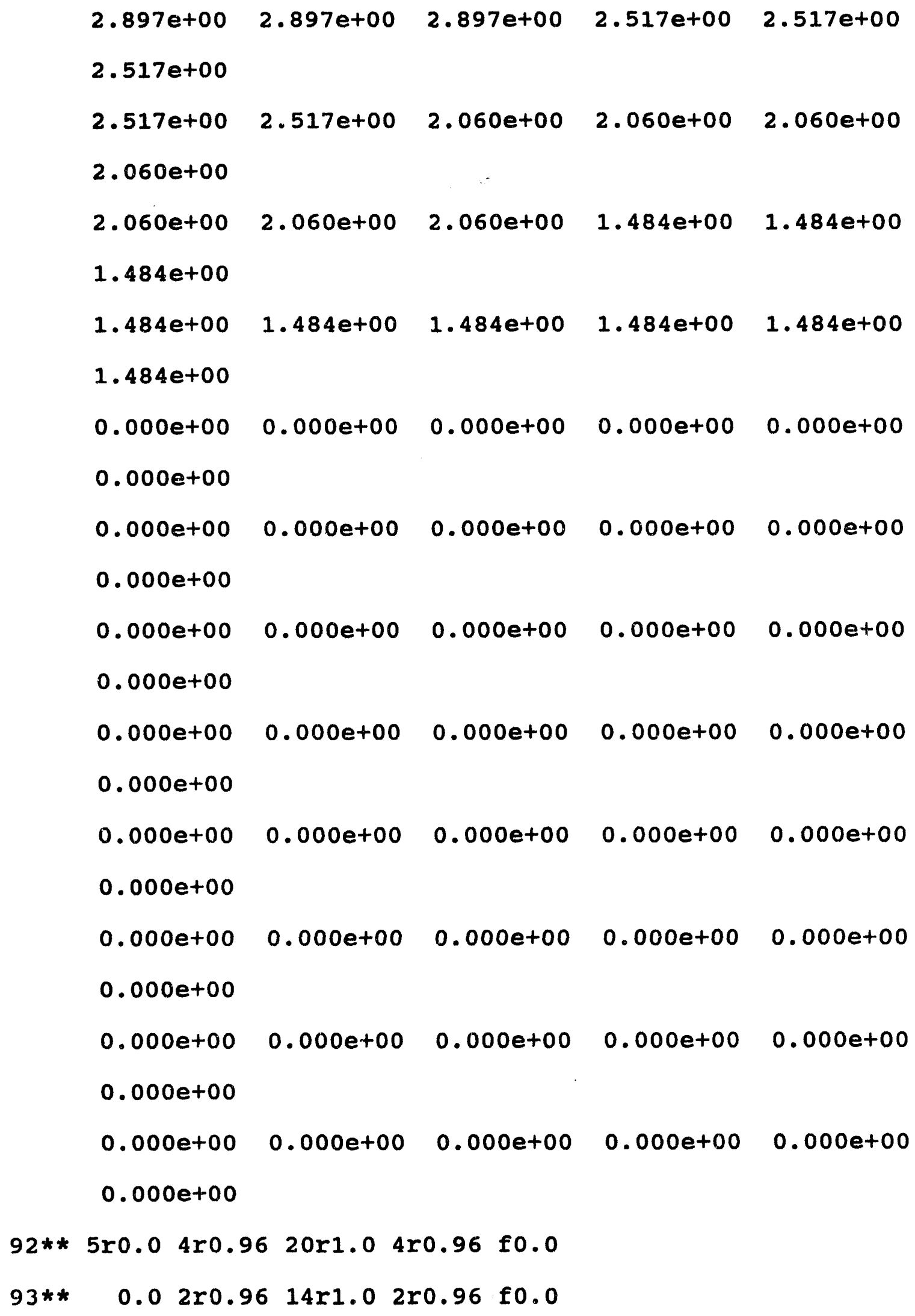




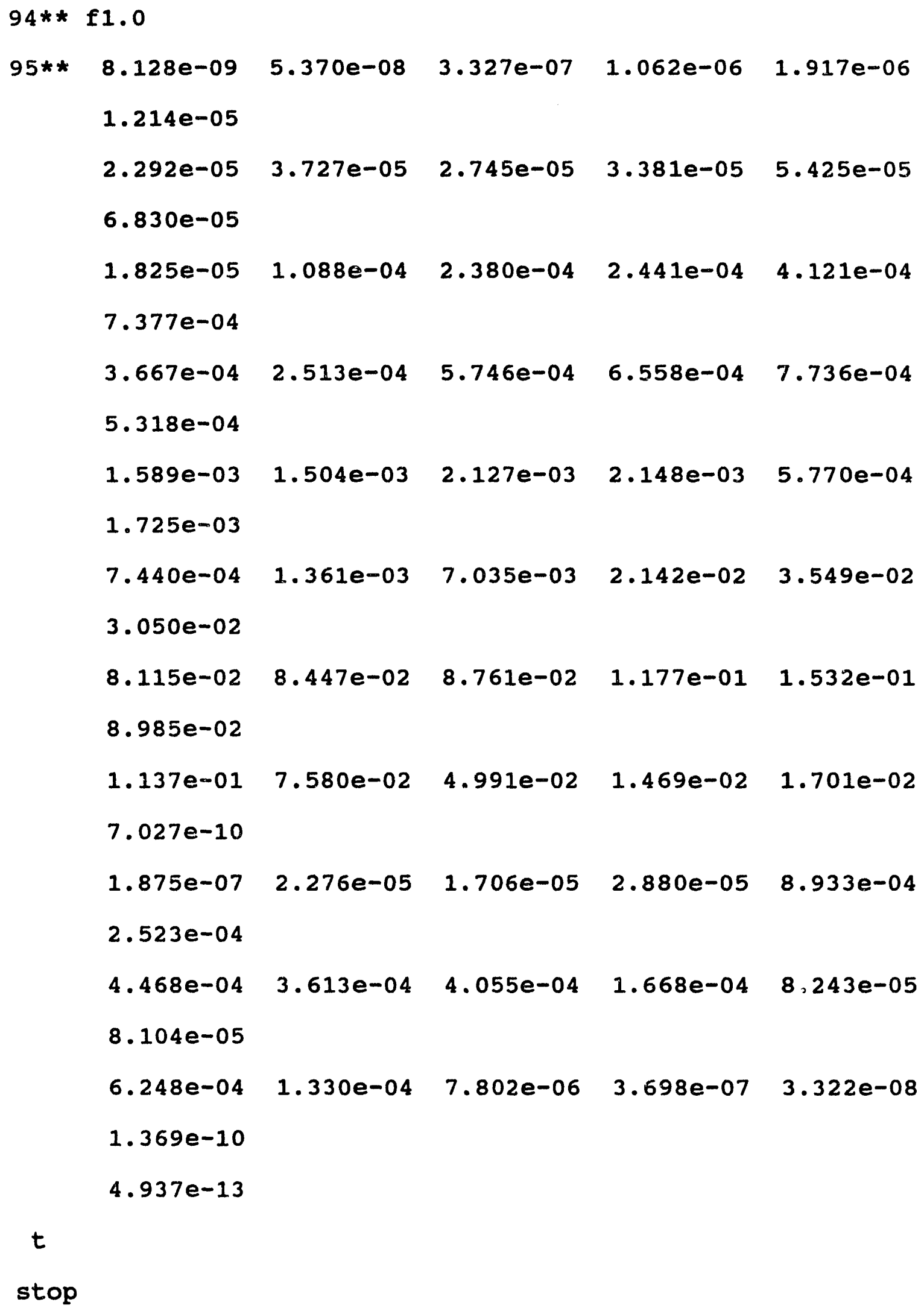




\section{APPENDIX F}

ADDITIONAL CONTOUR PLOTS 


\section{APPENDIX F. ADDITIONAL CONTOUR PLOTS}

This appendix includes additional contour plots to further examine Type 1 heterogeneity effects. Only the contour plots are shown here. A number of sagittal, axial, and dorsal planes will be examined for both the $5 \mathrm{~cm} \times 10 \mathrm{~cm}$ and $10 \mathrm{~cm} \times 10 \mathrm{~cm}$ beams at the Brookhaven Medical Research Reactor.

Figures F-1 and F-2 depict the heterogeneous and homogeneous fast neutron flux at $y=-2.5 \mathrm{~cm}$ which is sagittal slice 5 (see Figures $B-5$ and $C-5$ for the photograph and modeling of this slice). The shapes of the fluxes are essentially identical, although the flux does penetrate about $1 \%$ deeper due to the presence of bone. (Note that the effect is not as great as that seen at the midline of the beam.) Figures $F-3$ and $F-4$ show the fast neutron flux at $y$ $=-4.5 \mathrm{~cm}$, equivalent to sagittal slice 3 (Figures $\mathrm{B}-3$ and C-3) for the heterogeneous and homogeneous cases, respectively. These fluxes are also identical. This is expected since there are fewer heterogeneities in the outer regions of the head.

The gamma fluxes can also be examined at these sagittal planes. The gamma $f l u x$ at $y=-2.5 \mathrm{~cm}$ for the heterogeneous and homogeneous models, respectively, are shown in Figures 


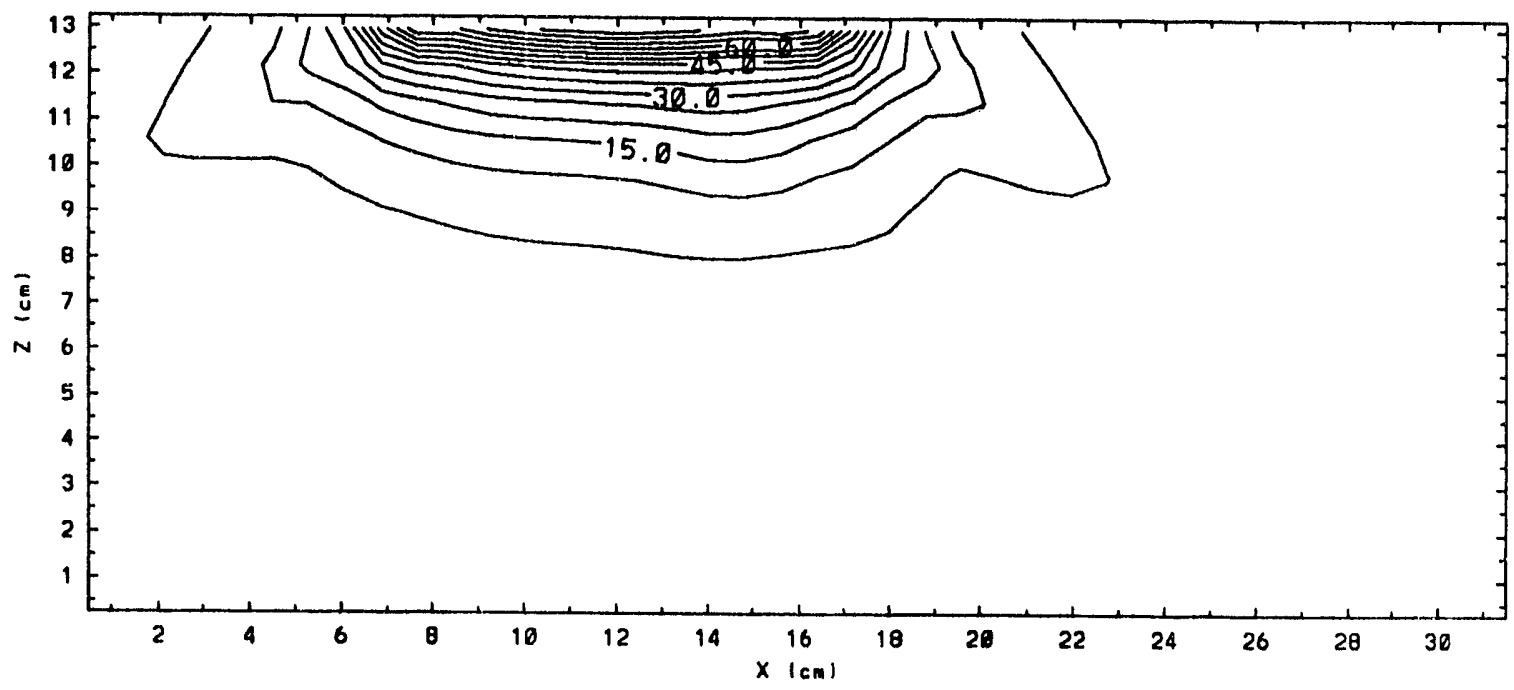

Figure F-1. Fast neutron flux contours at $y=-2.5 \mathrm{~cm}$ for the heterogeneous case. Percentage contours are shown normalized to a peak fast flux of $1.61 \times 10^{7} \mathrm{n} / \mathrm{cm}^{2} / \mathrm{s}$. 


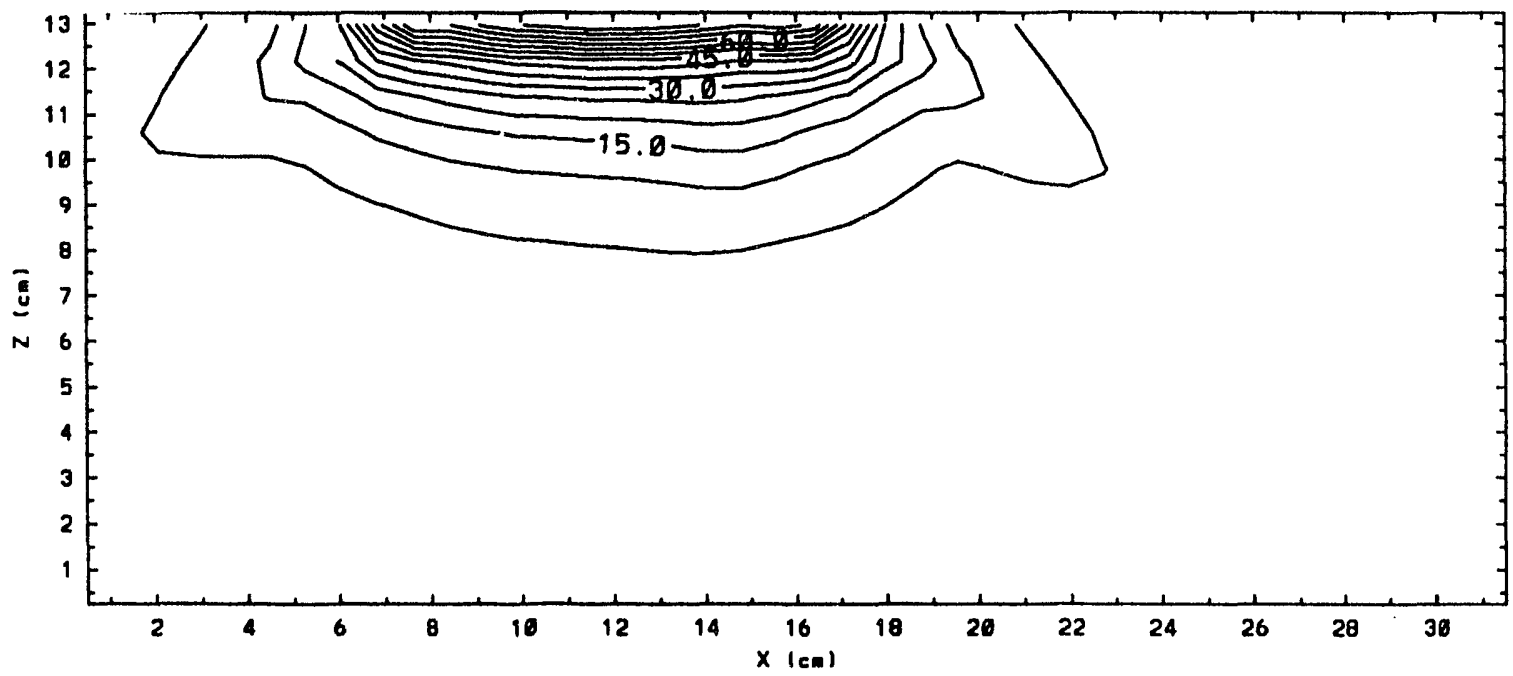

Figure F-2. Fast neutron flux contours at $y=-2.5 \mathrm{~cm}$ for the homogeneous case. Percentage contours are shown normalized to a peak fast flux of $1.62 \times 10^{7} \mathrm{n} / \mathrm{cm}^{2} / \mathrm{s}$. 


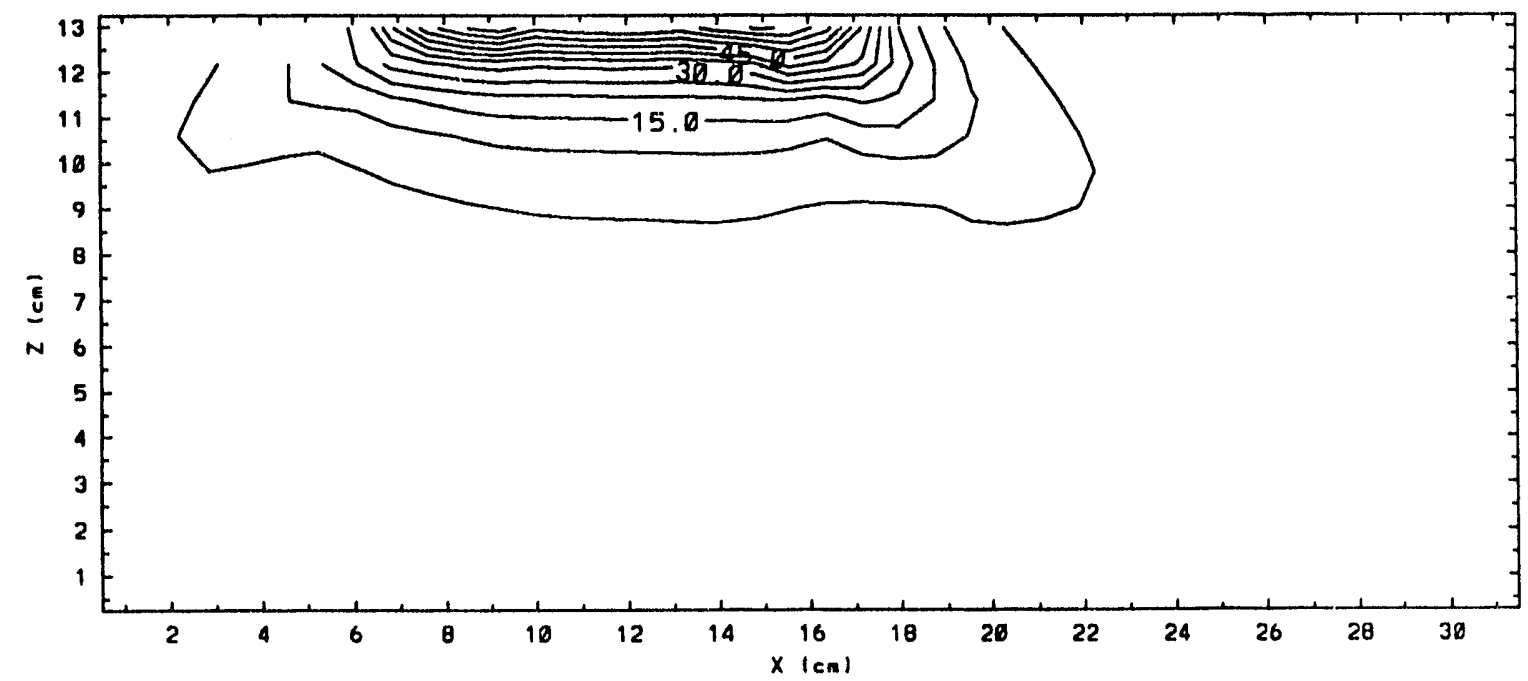

Figure F-3. Fast neutron flux contours at $y=-4.5 \mathrm{~cm}$ for the heterogeneous case. Percentage contours are shown normalized to a peak fast flux of $1.61 \times 10^{7} \mathrm{n} / \mathrm{cm}^{2} / \mathrm{s}$. 


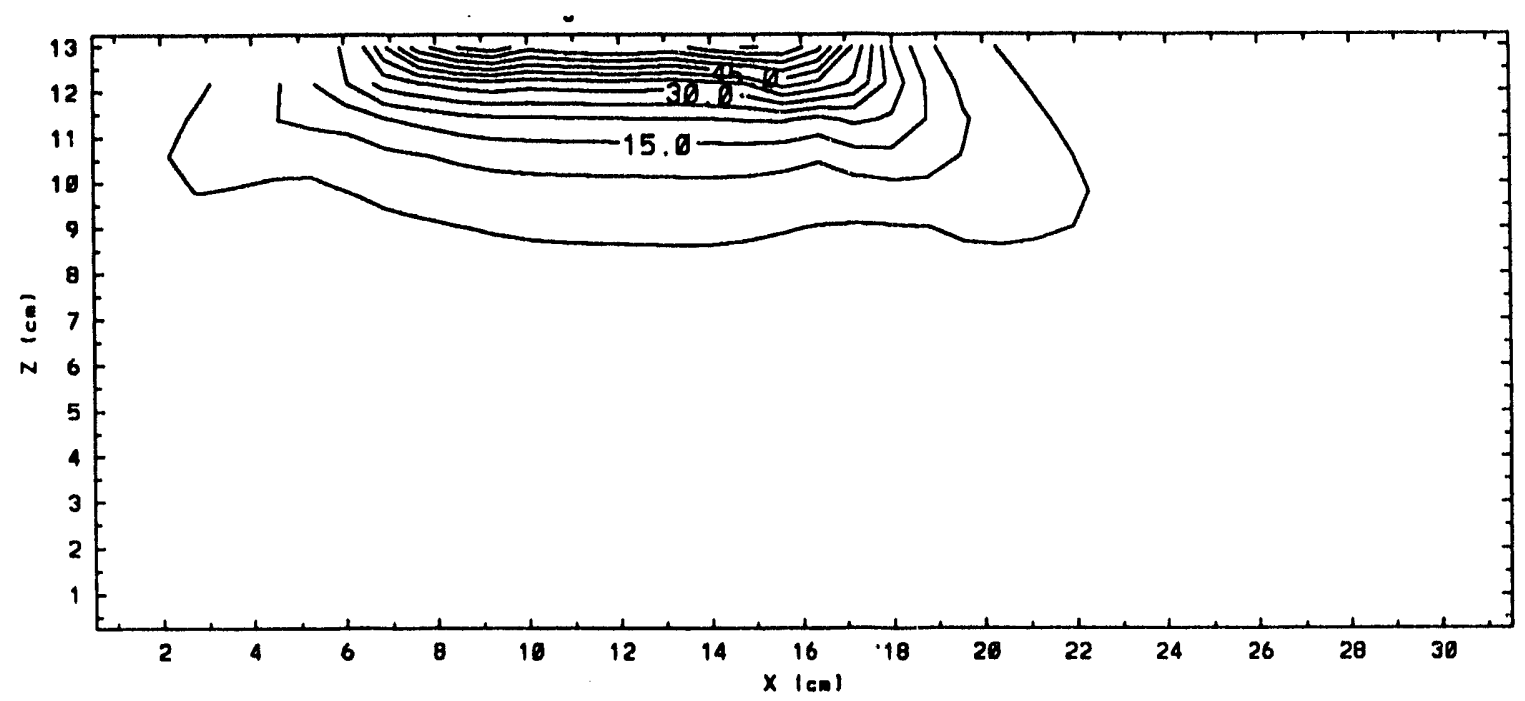

Figure F-4. Fast neutron flux contours at $y=-4.5 \mathrm{~cm}$ for the homogeneous case. Percentage contours are shown normalized to a peak fast flux of $1.62 \times 10^{7} \mathrm{n} / \mathrm{cm}^{2} / \mathrm{s}$. 
F-5 and F-6. A decrease of the flux can be seen due to inhomogeneities in the ventral and rostral directions. The decrease ranges from $3-5 \%$ and is a result of passage through bone. From these results, the homogeneous case overestimates the dose to the eye from gamma rays. It should be noted again that the photon-induced absorbed dose distribution can be perturbed significantly by Type 3 (electron non-equilibrium) heterogeneity effects.

Figures $F-7$ and $F-8$ depict the gamma flux at $Y=-4.5$ cm. Again, a decrease in the heterogeneous flux is seen in the ventral direction when compared with the homogeneous results. There is less contributions to the flux from the sides. This is reasonable because the flux was decreasing at $y=-2.5 \mathrm{~cm}$ as seen above. The peak flux of this region is only about 608 of the total peak flux.

Several axial planes can be examined as well to better understand the distribution of the fluxes throughout the canine head. The thermal neutron fluxes at $x=7.25 \mathrm{~cm}$ are shown in Figures $F-9$ and $F-10$. The heterogeneous and homogeneous cases have the same spatial distributions. A $2 \%$ decrease is seen in the flux of the heterogeneous case in the ventral direction. Figures $F-11$ and $F-12$ show the thermal neutron $f l u x$ at $x=12.25 \mathrm{~cm}$. This is a plane through the brain. The peak flux is smaller in the 


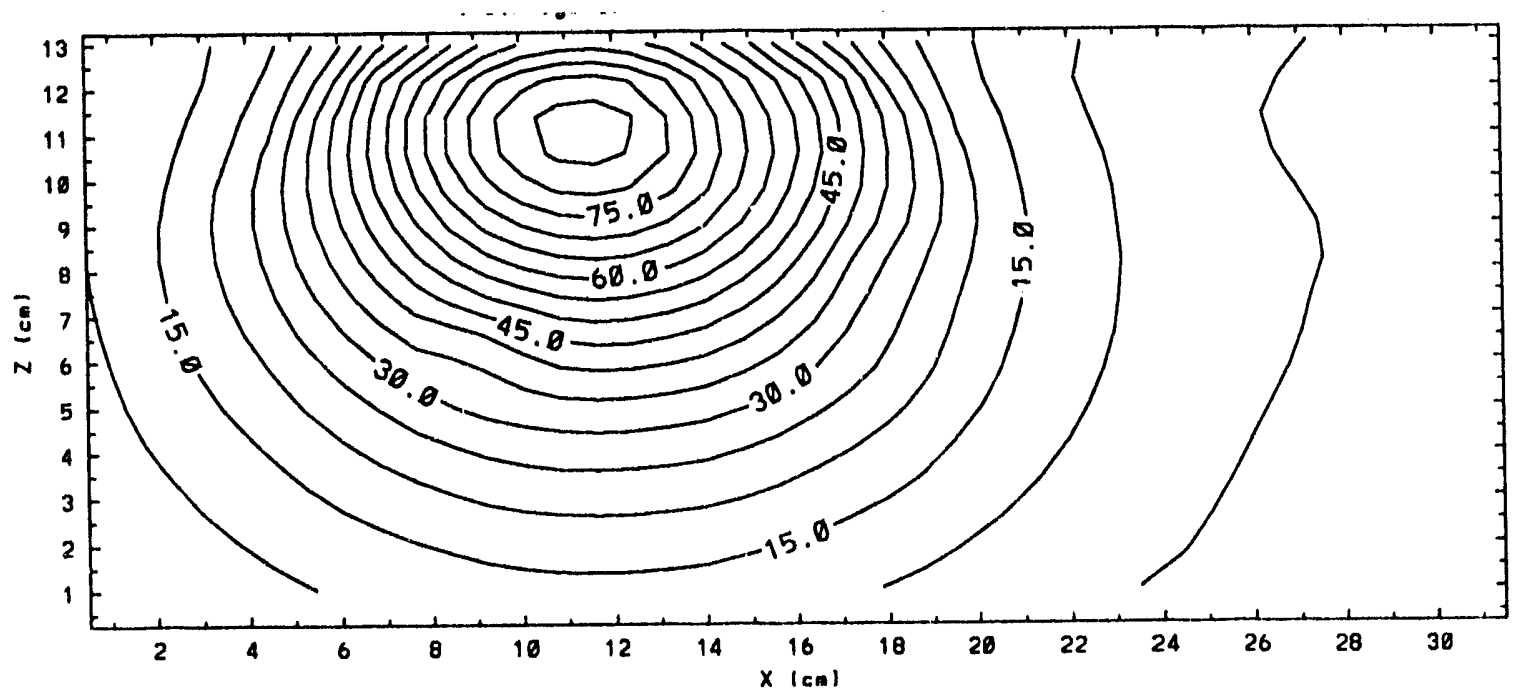

Figure F-5. Gamma flux contours at $y=-2.5 \mathrm{~cm}$ for the heterogeneous case. Percentage contours are shown normalized to a peak gamma flux of $5.63 \times 10^{7} \mathrm{gammas} / \mathrm{cm}^{2} / \mathrm{s}$. 


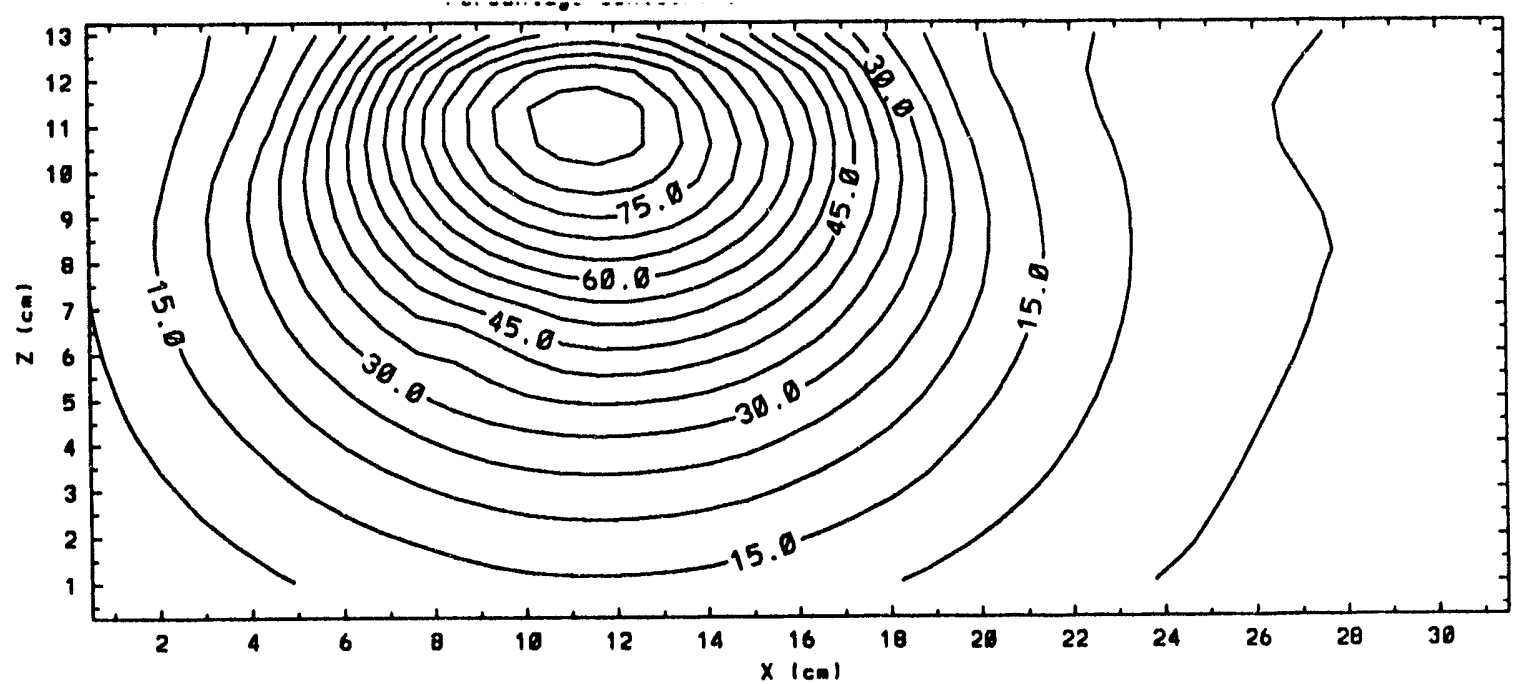

Figure F-6. Gamma flux contours at $y=-2.5 \mathrm{~cm}$ for the homogeneous case. Percentage contours are shown normalized to a peak gamma flux of $5.61 \times 10^{7}$ gammas $/ \mathrm{cm}^{2} / \mathrm{s}$. 


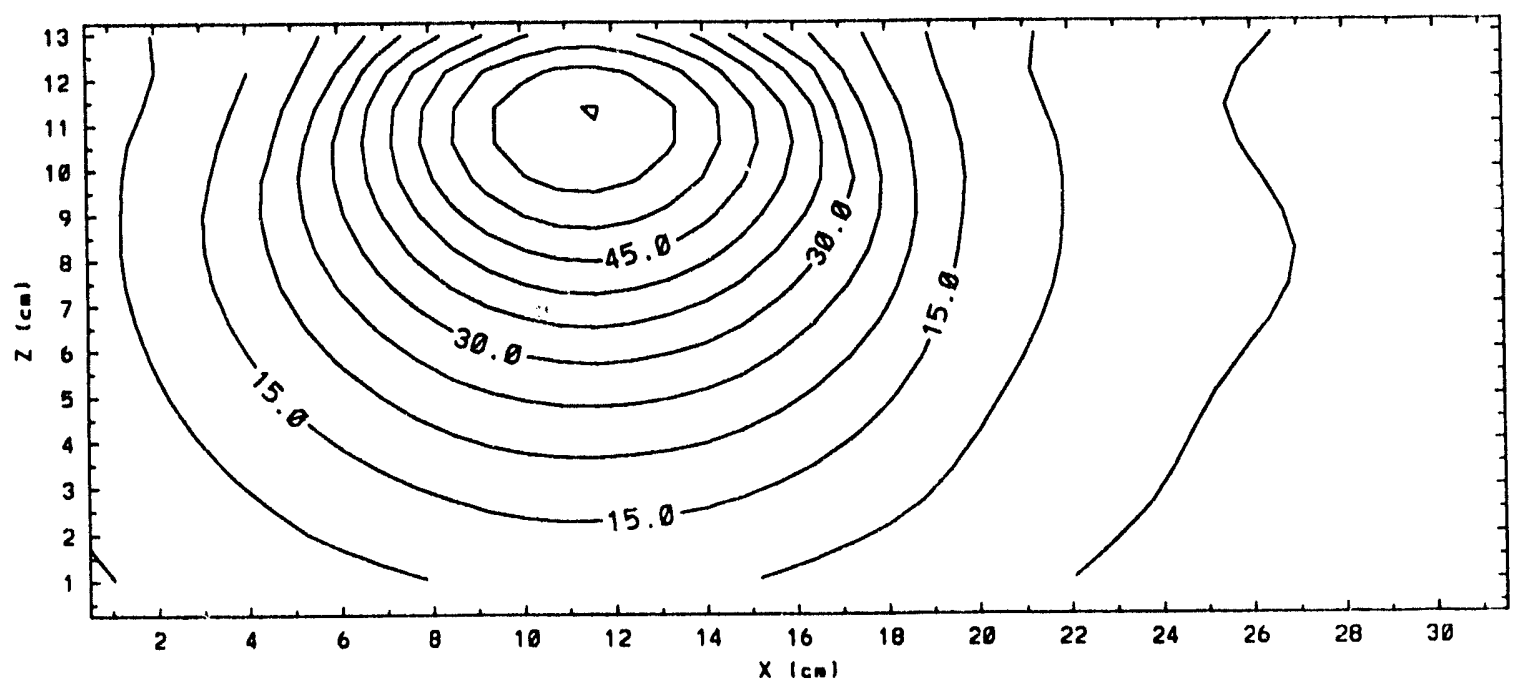

Figure F-7. Gamma flux contours at $y=-4.5 \mathrm{~cm}$ for the heterogeneous case. Percentage contours are shown normalized to a peak gamma flux of $5.63 \times 10^{7}$ gammas $/ \mathrm{cm}^{2} / \mathrm{s}$. 


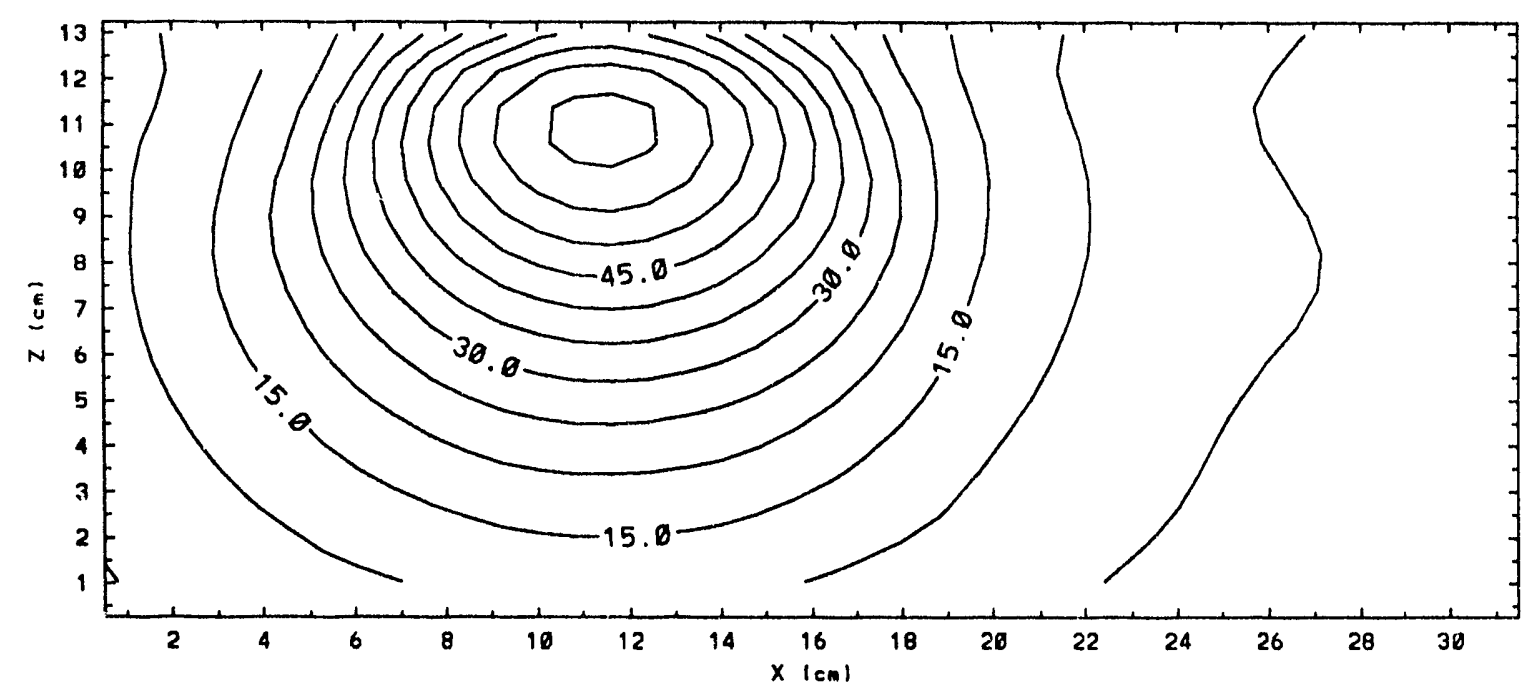

Figure F-8. Gamma flux contours at $y=-4.5 \mathrm{~cm}$ for the homogeneous case. Percentage contours are shown normalized to a peak gamma flux of $5.61 \times 10^{7} \mathrm{gammas} / \mathrm{cm}^{2} / \mathrm{s}$. 


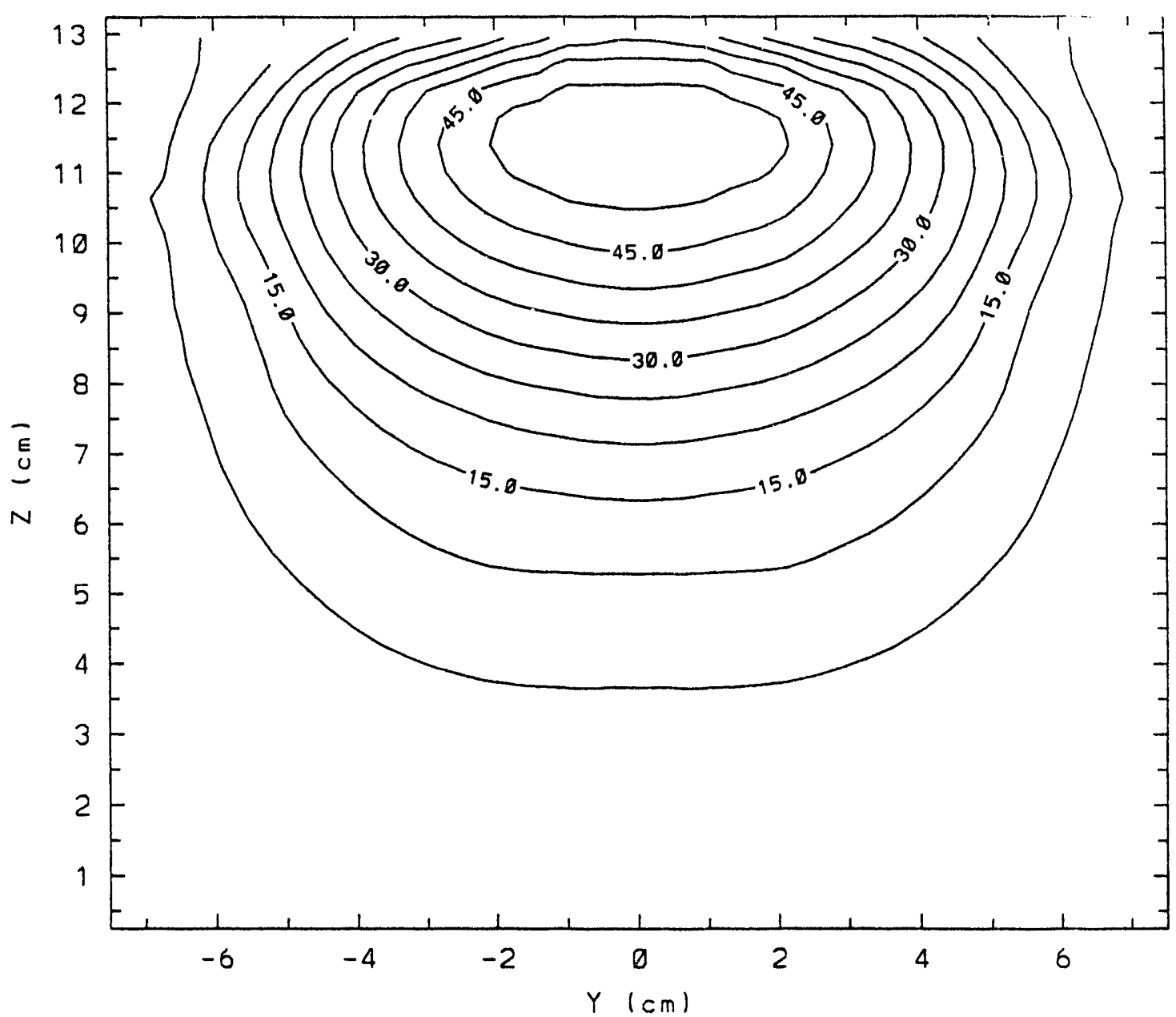

Figure F-9. Thermal neutron flux contours at $x=7.25 \mathrm{~cm}$ for the heterogeneous case. Percentage contours are shown normalized to a peak thermal flux of $7.00 \times 10^{8} \mathrm{n} / \mathrm{cm}^{2} / \mathrm{s}$. 


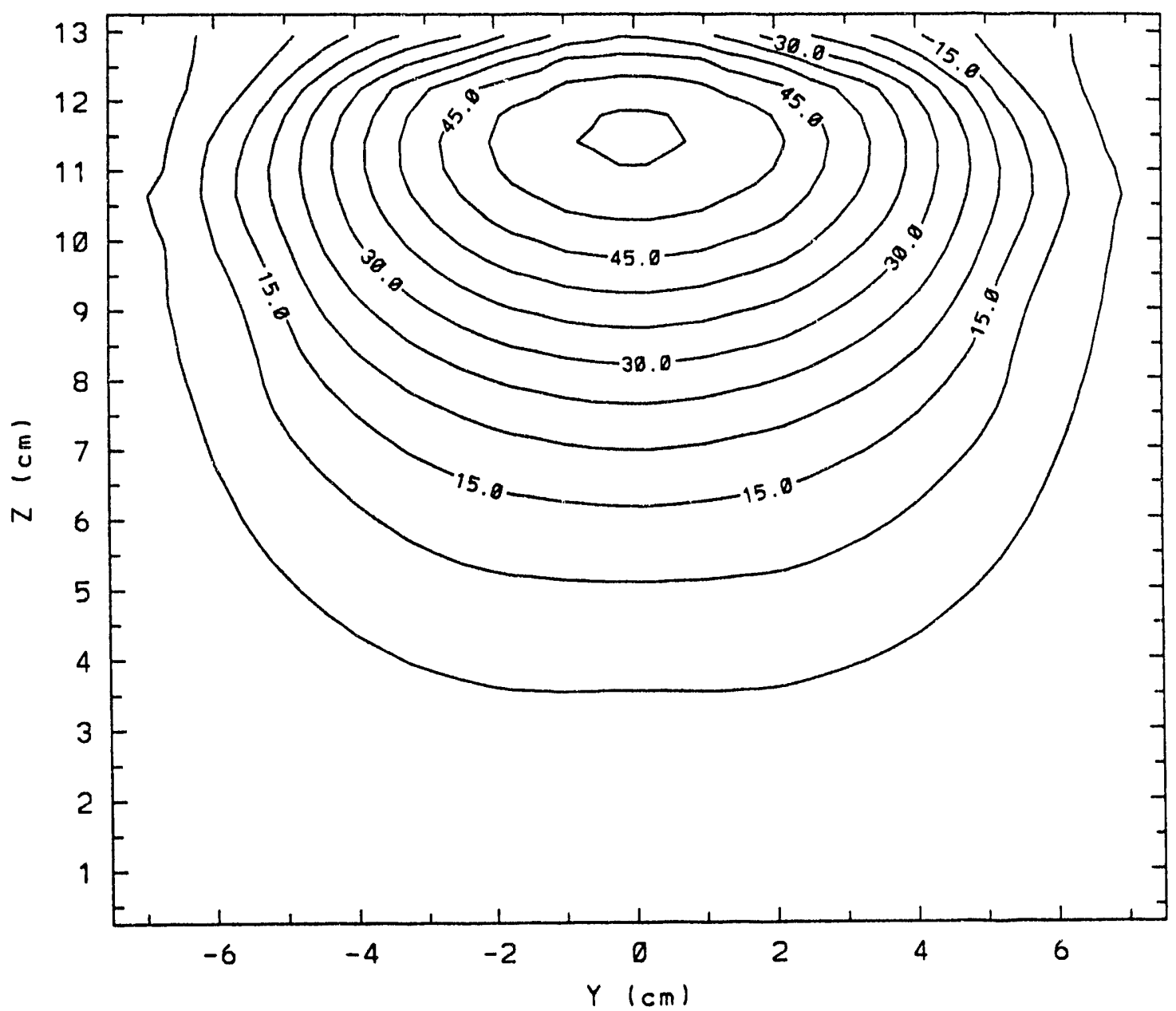

Figure F-10. Thermal neutron flux contours at $x=7.25 \mathrm{~cm}$ for the homogeneous case. Percentage contours are shown normalized to a peak thermal flux of $6.92 \times 10^{8} \mathrm{n} / \mathrm{cm}^{2} / \mathrm{s}$. 


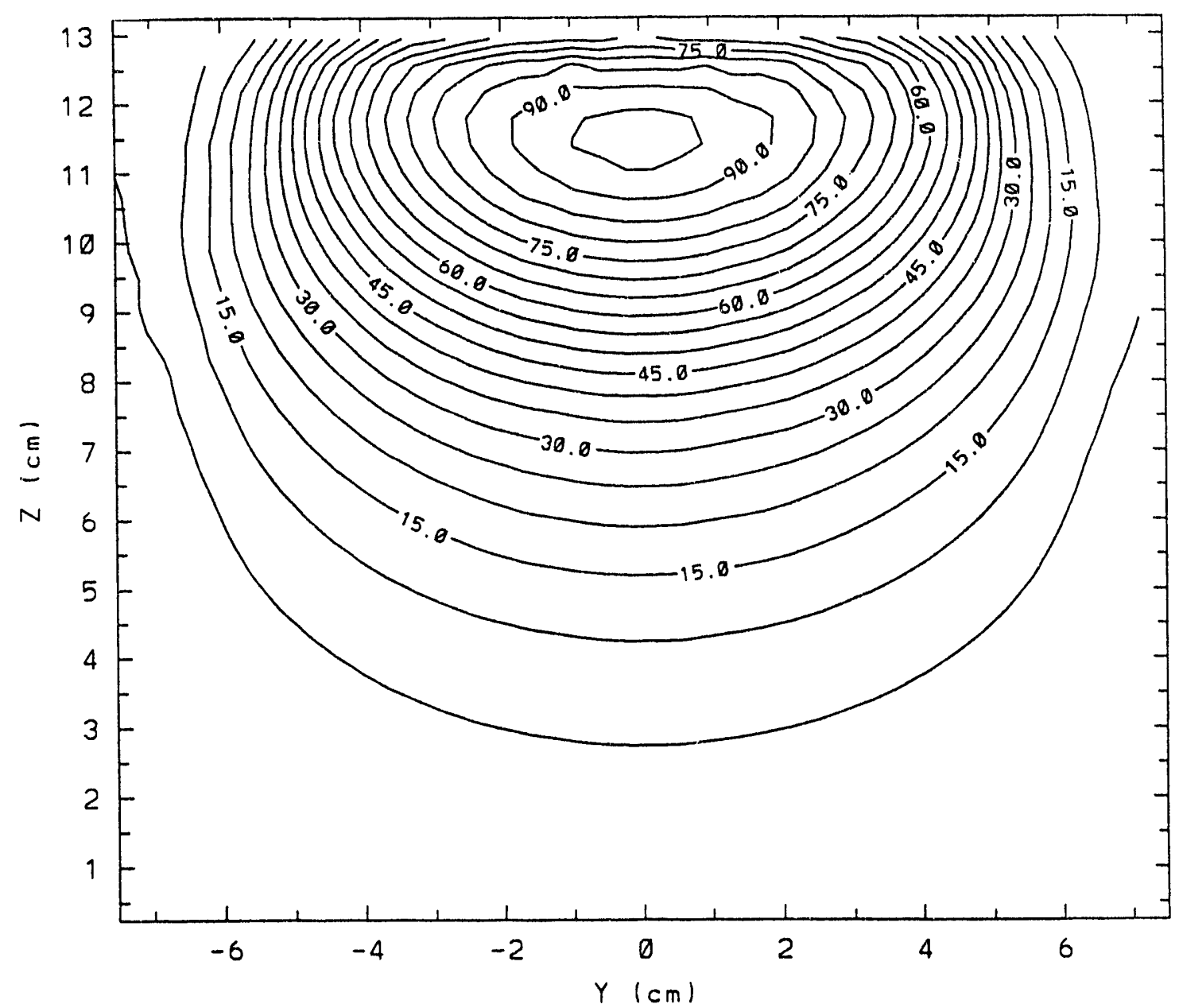

Figure F-11. Thermal neutron flux contours at $x=12.25 \mathrm{~cm}$ for the heterogeneous case. Percentage contours are shown normalized to a peak thermal flux of $7.00 \times 10^{8} \mathrm{n} / \mathrm{cm}^{2} / \mathrm{s}$. 


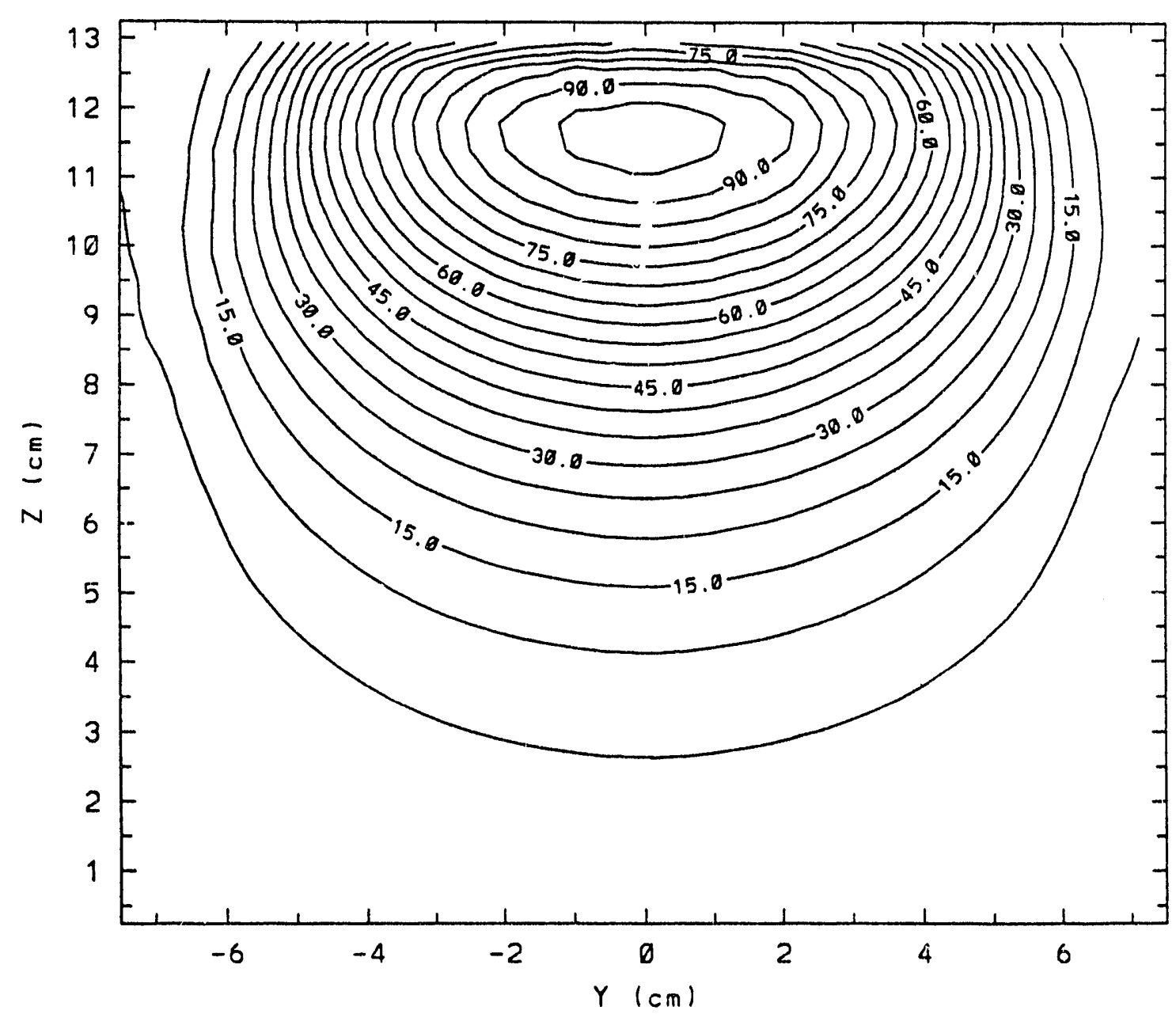

Figure F-12. Thermal neutron flux contours at $x=12.25 \mathrm{~cm}$ for the homogeneous case. Percentage contours are shown normalized to a peak thermal flux of $6.92 \times 10^{8} \mathrm{n} / \mathrm{cm}^{2} / \mathrm{s}$. 
heterogeneous case (Figure $F-11$ ) relative to the flux in the homogeneous case (Figure $\mathrm{F}-12$ ). There is also a decrease in the ventral directions. This decrease becomes larger after the $55 \%$ contour. The decrease is only approximately $3 \%$. In both axial planes examined, the observed decrease corresponds to the presence of bone and other inhomogeneities in these regions.

The fast neutron fluxes at $x=7.25 \mathrm{~cm}$ are presented in Figures $\mathrm{F}-13$ and $\mathrm{F}-14$. The spatial distributions are essentially identical, as expected. Figures F-15 and F-16 show the fast flux at $x=12.25 \mathrm{~cm}$. The fluxes between the two cases are similar. A slight decrease (less than 1\%) is seen in the 5-15\% contours of the heterogeneous results relative to the homogeneous results in the ventral direction. More inhomogeneities and bone are present in the $x=12.25 \mathrm{~cm}$ plane when compared with the $\mathrm{x}=7.25 \mathrm{~cm}$ plane which explains why a slight effect was seen in one but not the other.

The results of the fast neutron flux at $x=17.25 \mathrm{~cm}$ (shown in Figures $F-17$ and $F-18$ ) have a different spatial distribution than the fast neutron fluxes at the planes examined above. One reason is that in the model this slice only extends from $y=-6 \mathrm{~cm}$ to $y=6 \mathrm{~cm}$ whereas it extended from $y=-7 \mathrm{~cm}$ to $y=7 \mathrm{~cm}$ in the other planes examined. 


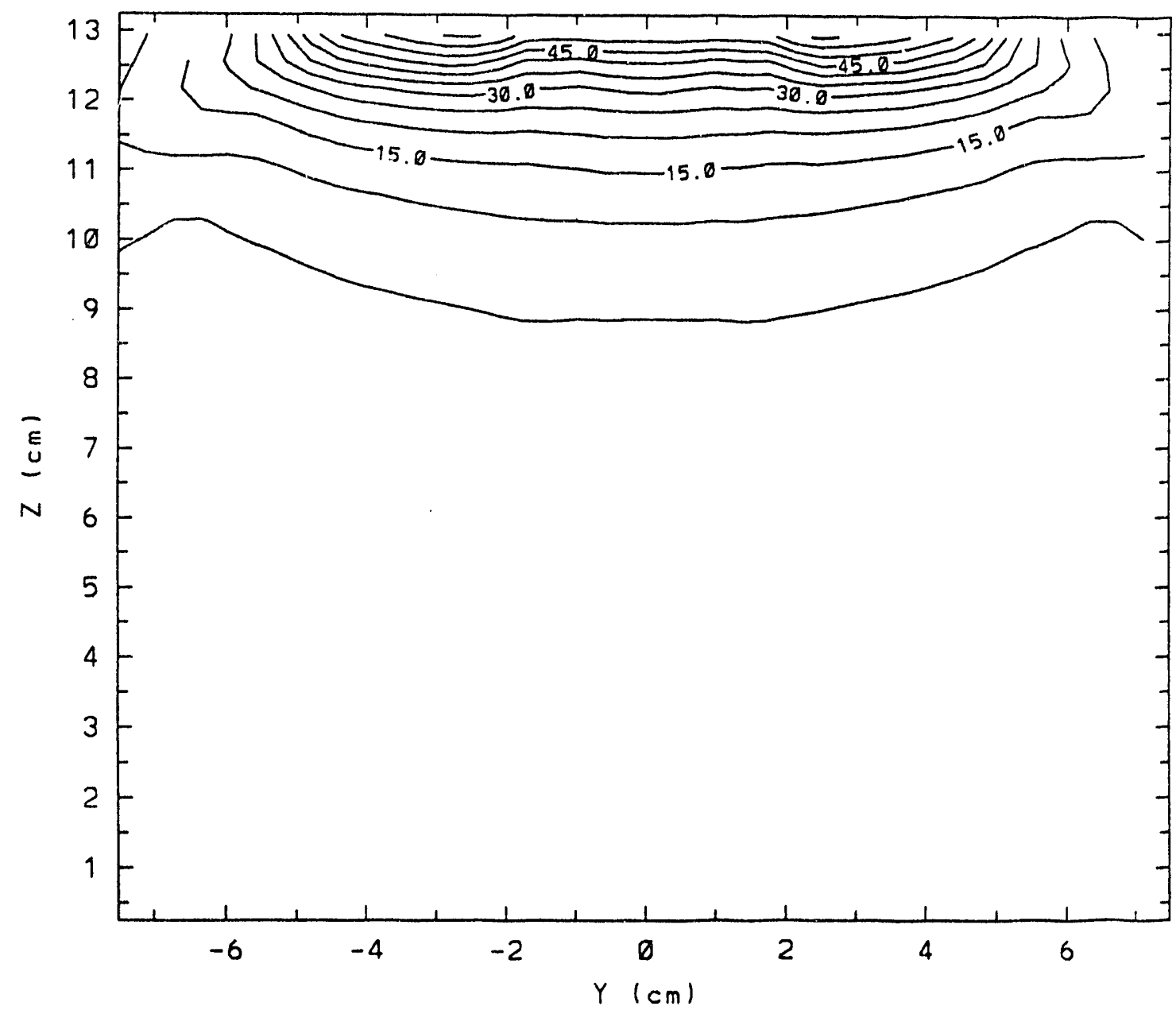

Figure F-13. Fast neutron flux contours at $x=7.25 \mathrm{~cm}$ for the heterogeneous case. Percentage contours are shown normalized to a peak fast flux of $1.61 \times 10^{7} \mathrm{n} / \mathrm{cm}^{2} / \mathrm{s}$. 


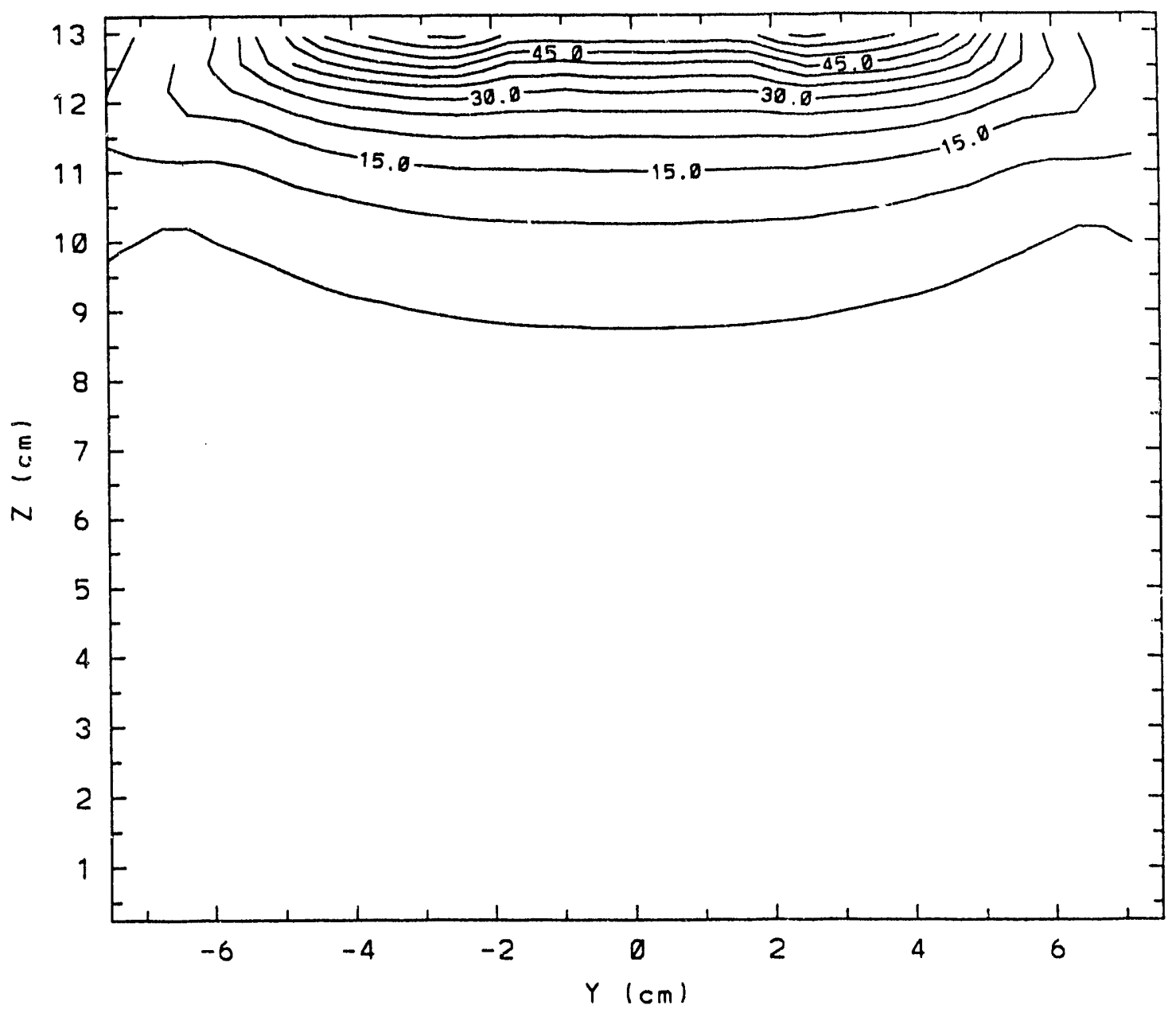

Figure F-14. Fast neutron flux contours at $x=7.25 \mathrm{~cm}$ for the homogeneous case. Percentage contours are shown normalized to a peak fast flux of $1.62 \times 10^{7} \mathrm{n} / \mathrm{cm}^{2} / \mathrm{s}$. 


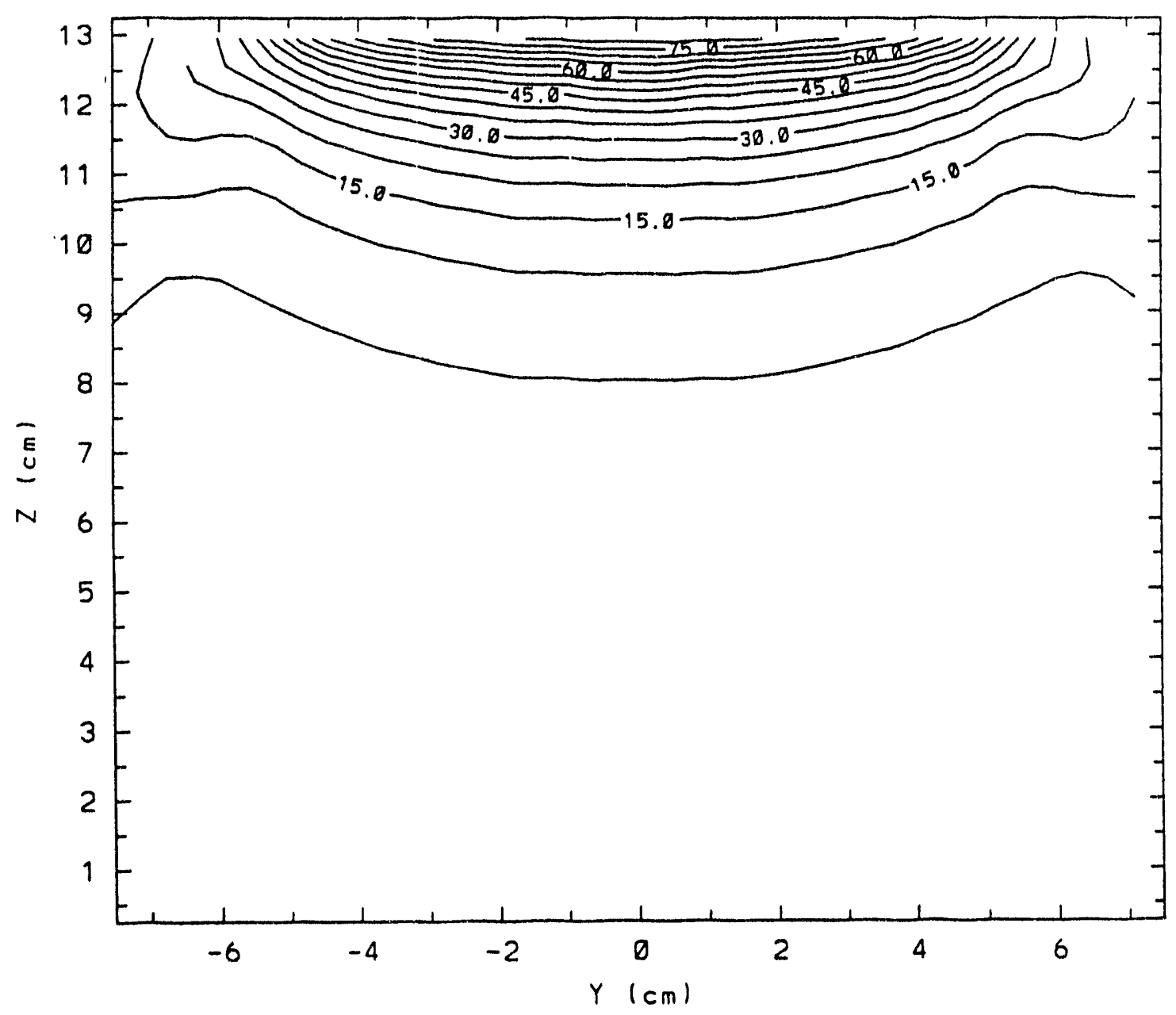

Figure F-15. Fast neutron flux contours at $x=12.25 \mathrm{~cm}$ for the heterogeneous case. Percentage contours are shown normalized to a peak fast flux of $1.61 \times 10^{7} \mathrm{n} / \mathrm{cm}^{2} / \mathrm{s}$. 


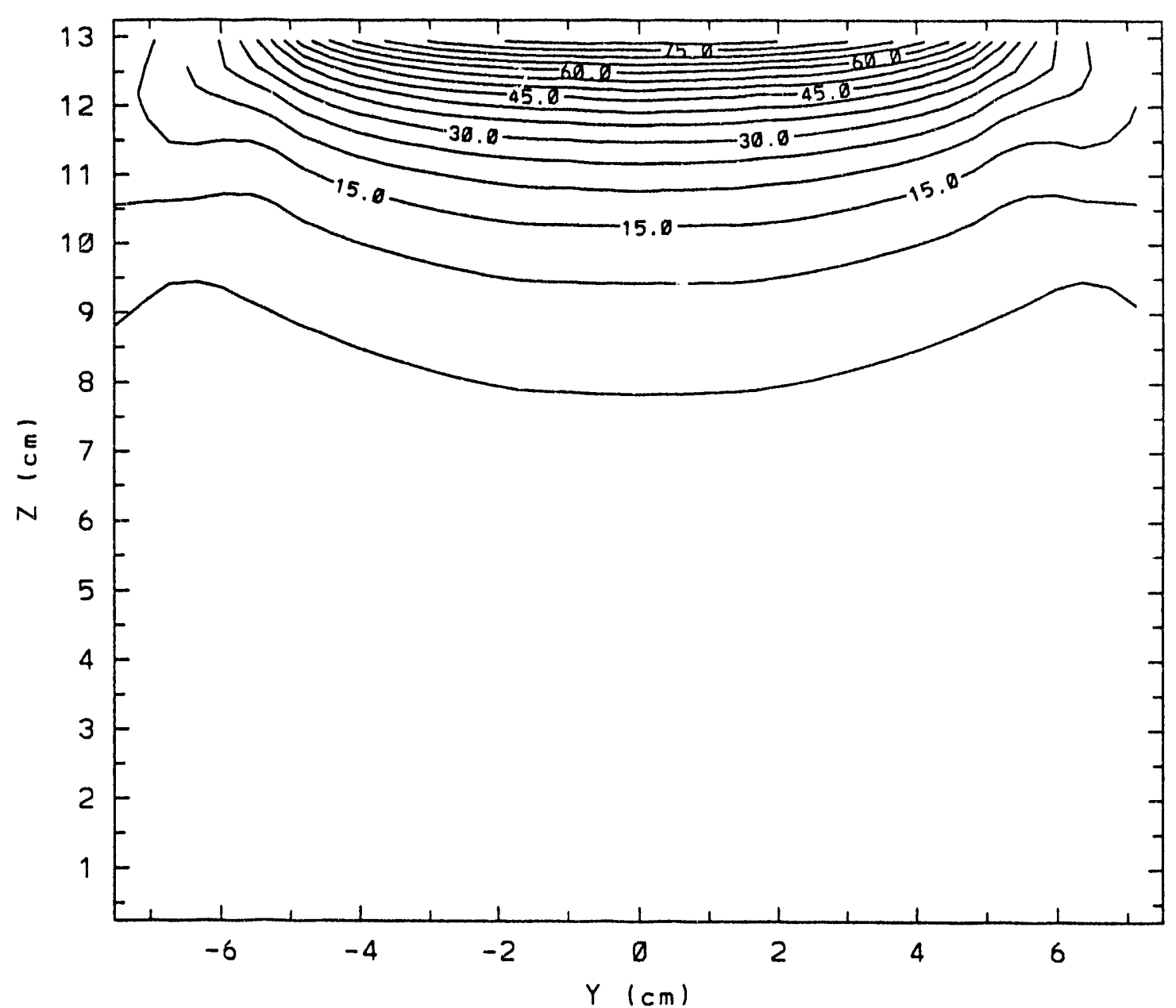

Figure F-16. Fast neutron flux contours at $x=12.25 \mathrm{~cm}$ for the homogeneous case. Percentage contours are shown normalized to a peak fast flux of $1.62 \times 10^{7} \mathrm{n} / \mathrm{cm}^{2} / \mathrm{s}$. 


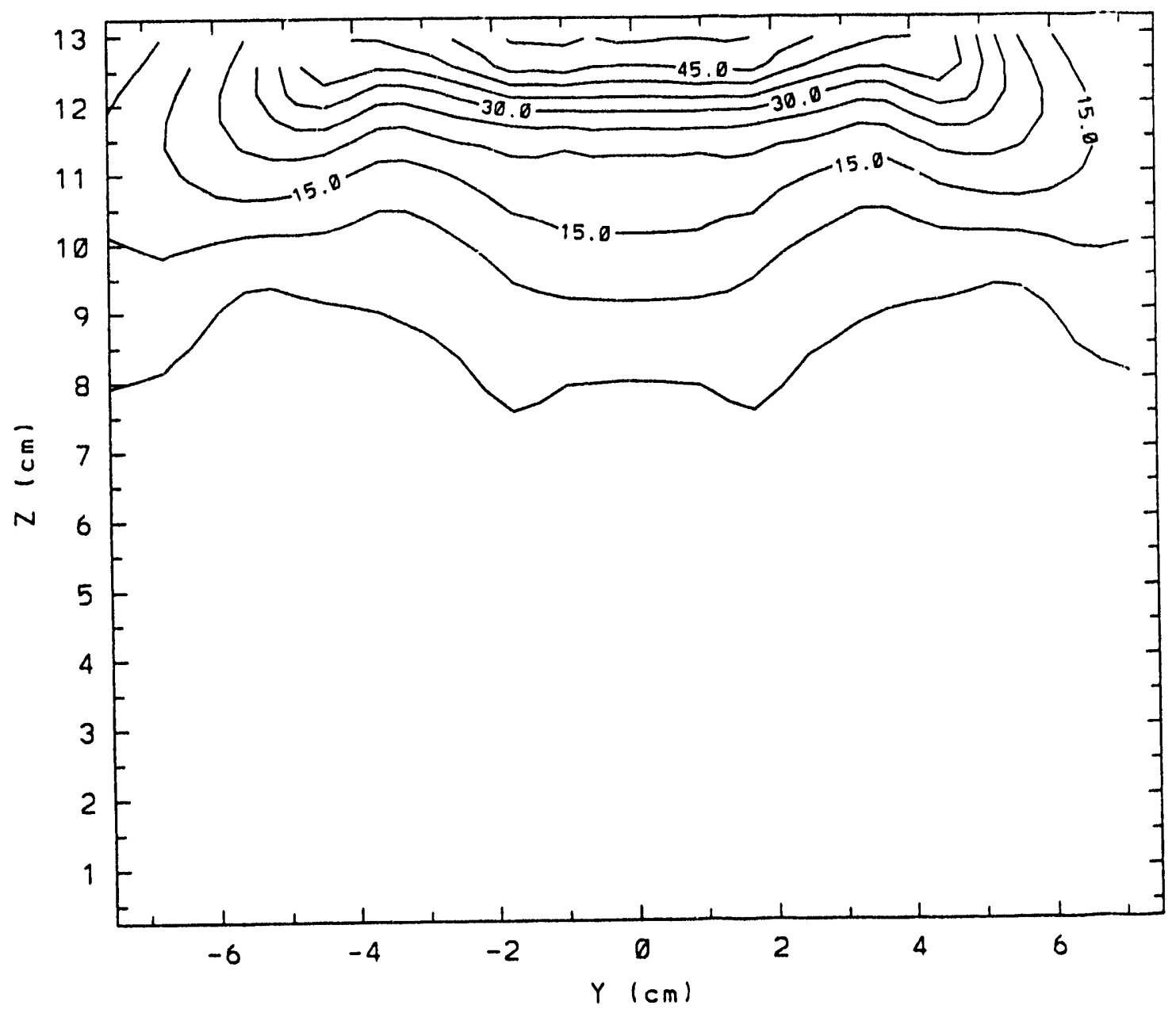

Figure F-17. Fast neutron flux contours at $x=17.25 \mathrm{~cm}$ for the heterogeneous case. Percentage contours are shown normalized to a peak fast flux of $1.61 \times 10^{7} \mathrm{n} / \mathrm{cm}^{2} / \mathrm{s}$. 


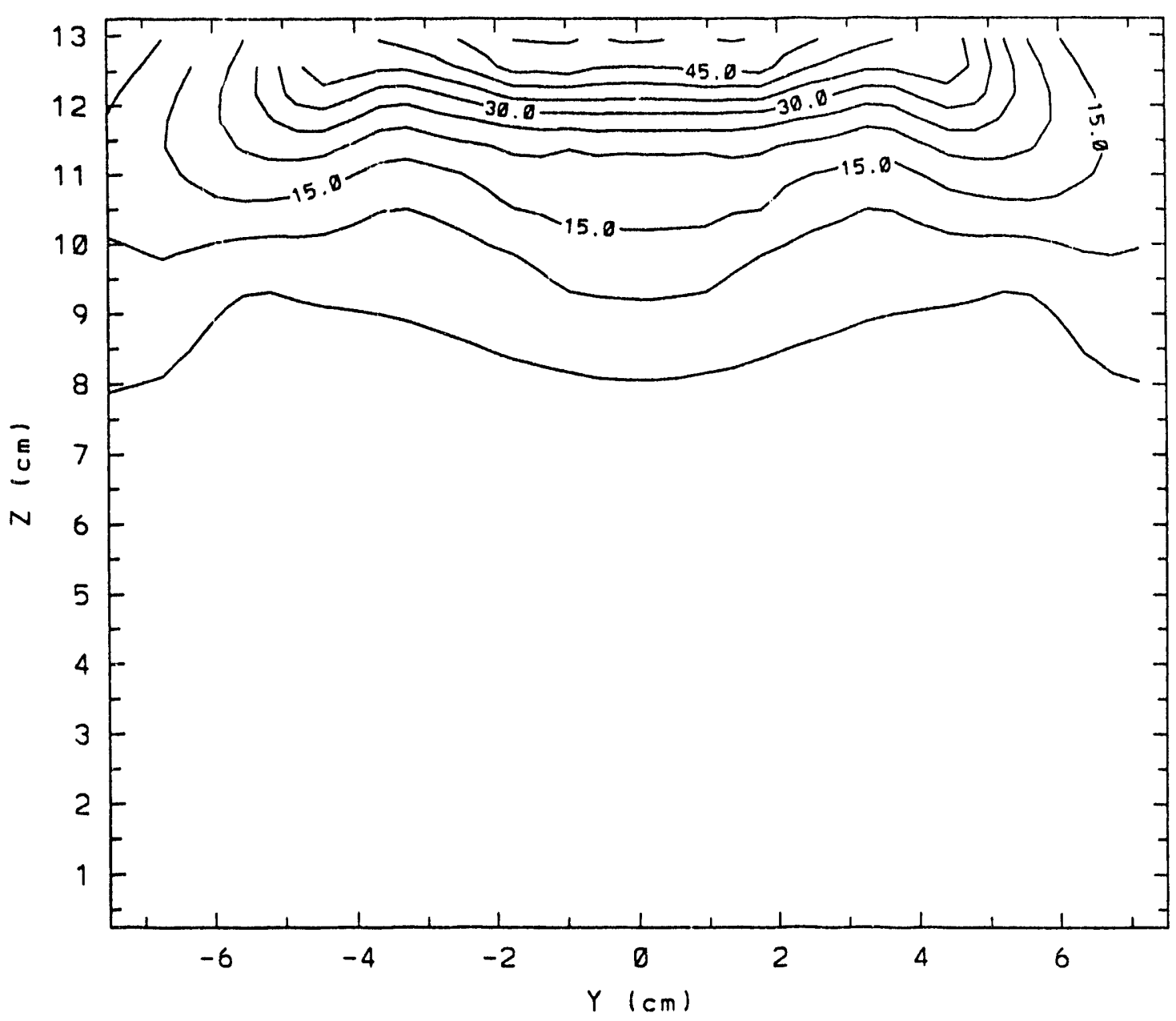

Figure $F-18$. Fast neutron flux contours at $x=17.25 \mathrm{~cm}$ for the homogeneous case. Percentage contours are shown normalized to a peak fast flux of $1.62 \times 10^{7} \mathrm{n} / \mathrm{cm}^{2} / \mathrm{s}$. 
There is an increase of the flux in the heterogeneous case due to void and nasal cavity regions. Streaming of the flux occurs at approximately $y=-2 \mathrm{~cm}$ due to the passage of neutrons through the nasal cavity. This streaming results in the penetration of the flux about $1 \mathrm{~cm}$ deeper than predicted with the homogeneous model. Although this specific increase may not be significant in therapy because of the magnitude of the fluxes involved, it is significant to note that because of the heterogeneities of an irradiation volume, a homogeneous approximation may not be sufficient for patient treatment planning.

The gamma flux can be examined at the same planes as the fast neutron flux. Figures $F-19$ and $F-20$ present the gamma flux at $x=7.25 \mathrm{~cm}$. The peak flux of the heterogeneous case (Figure F-19) is located slightly higher and is smaller than that of the homogeneous case. This results in approximately a $2 \%$ (upward) shift of all the contours in the heterogeneous results. The spatial distributions between the two cases are similar however. This is tne same effect that was seen in the thermal neutron flux at this plane. Finally, the gamma fluxes at $x=12.25$ $\mathrm{cm}$ are shown in Figures $\mathrm{F}-21$ and $\mathrm{F}-22$ for the heterogeneous and homogeneous cases, respectively. Again, there is approximately a 2-3z decrease in the gamma flux of the heterogeneous case in the ventral direction as seen in the 


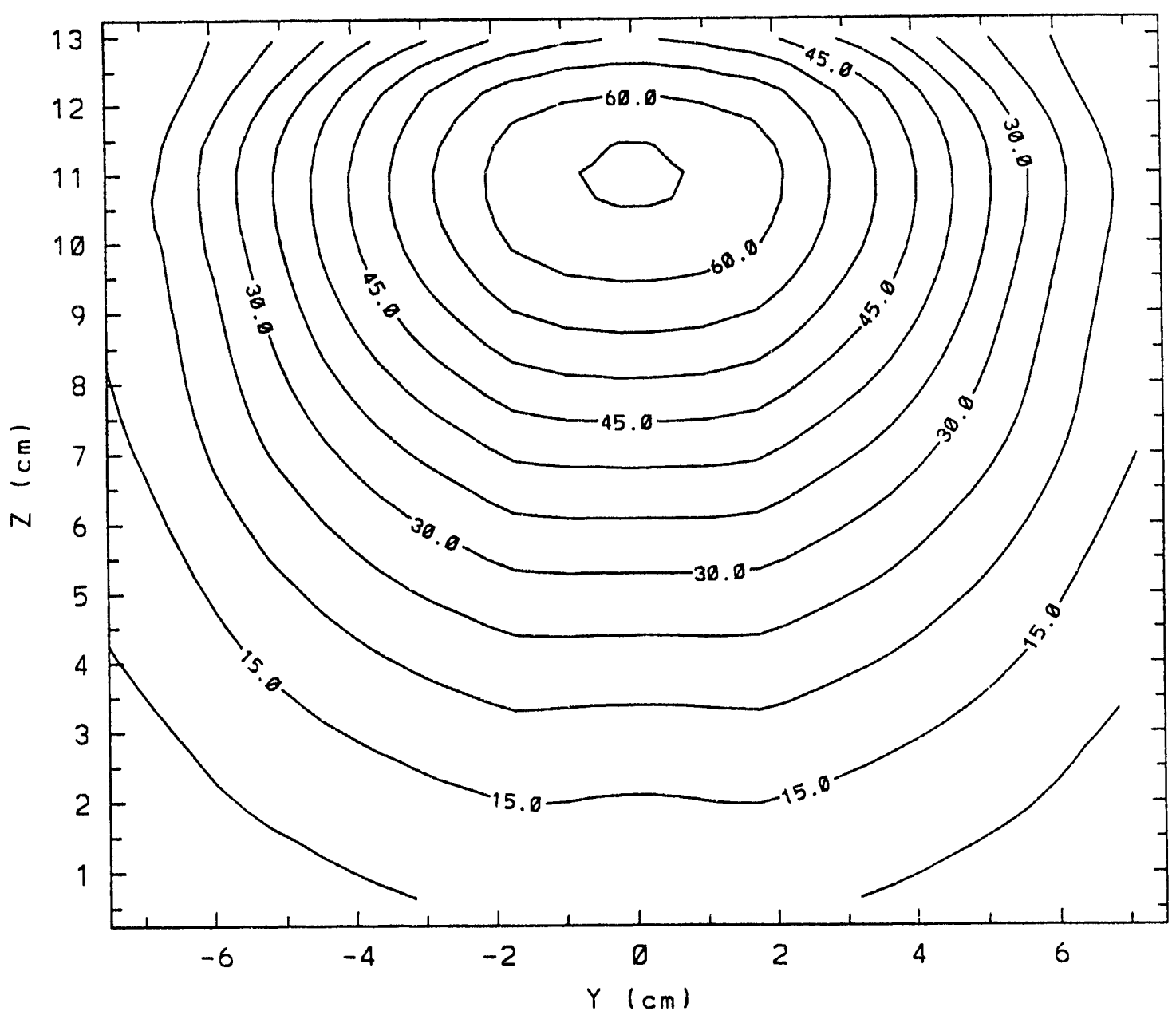

Figure F-19. Gamma flux contours at $x=7.25 \mathrm{~cm}$ for the heterogeneous case. Percentage contours are shown normalized to a peak gamma flux of $5.63 \times 10^{7}$ gammas $/ \mathrm{cm}^{2} / \mathrm{s}$. 


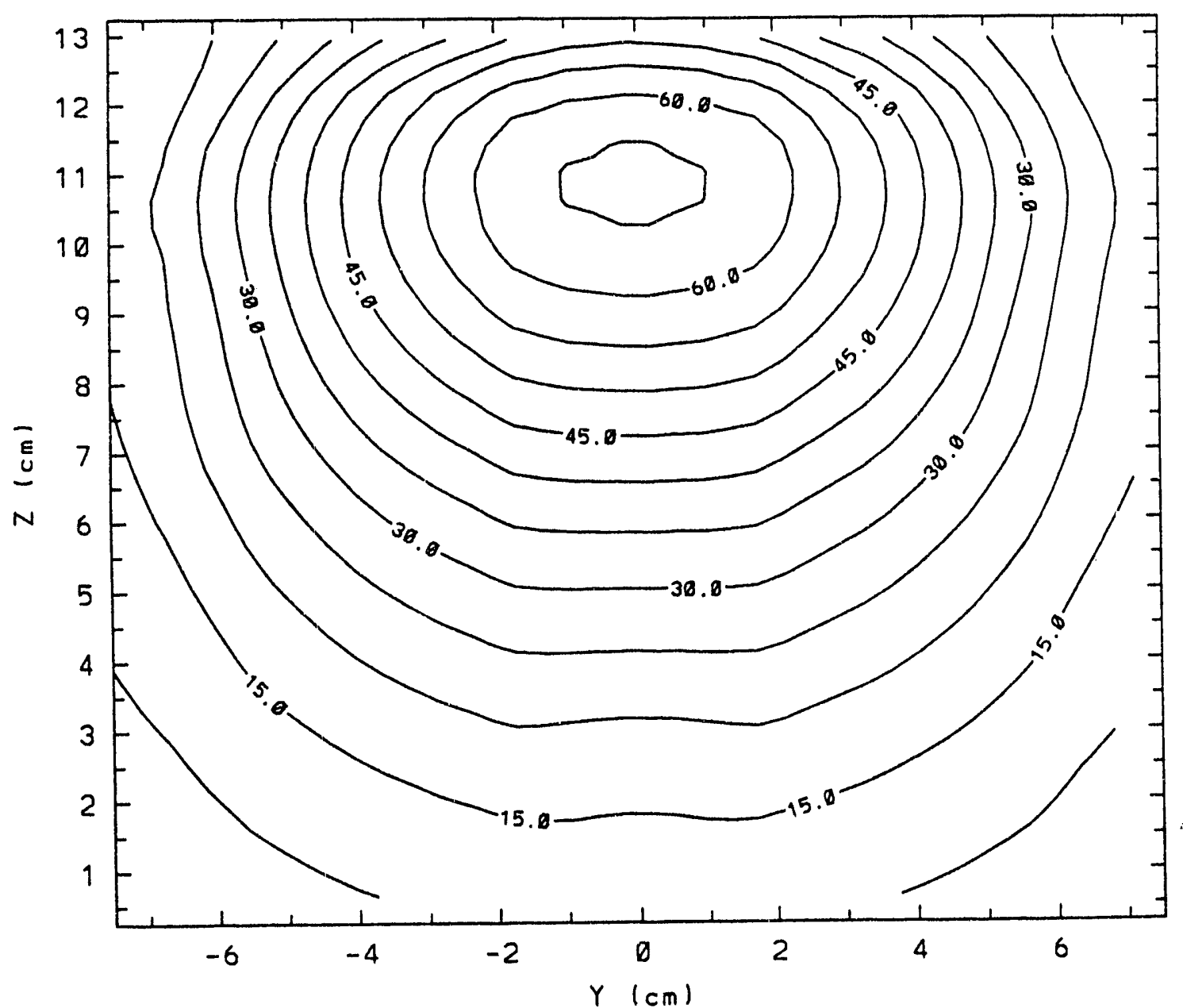

Figure F-20. Gamma flux contours at $x=7.25 \mathrm{~cm}$ for the homogeneous case. Percentage contours are shown normalized to a peak gamma flux of $5.61 \times 10^{7}$ gammas $/ \mathrm{cm}^{2} / \mathrm{s}$. 


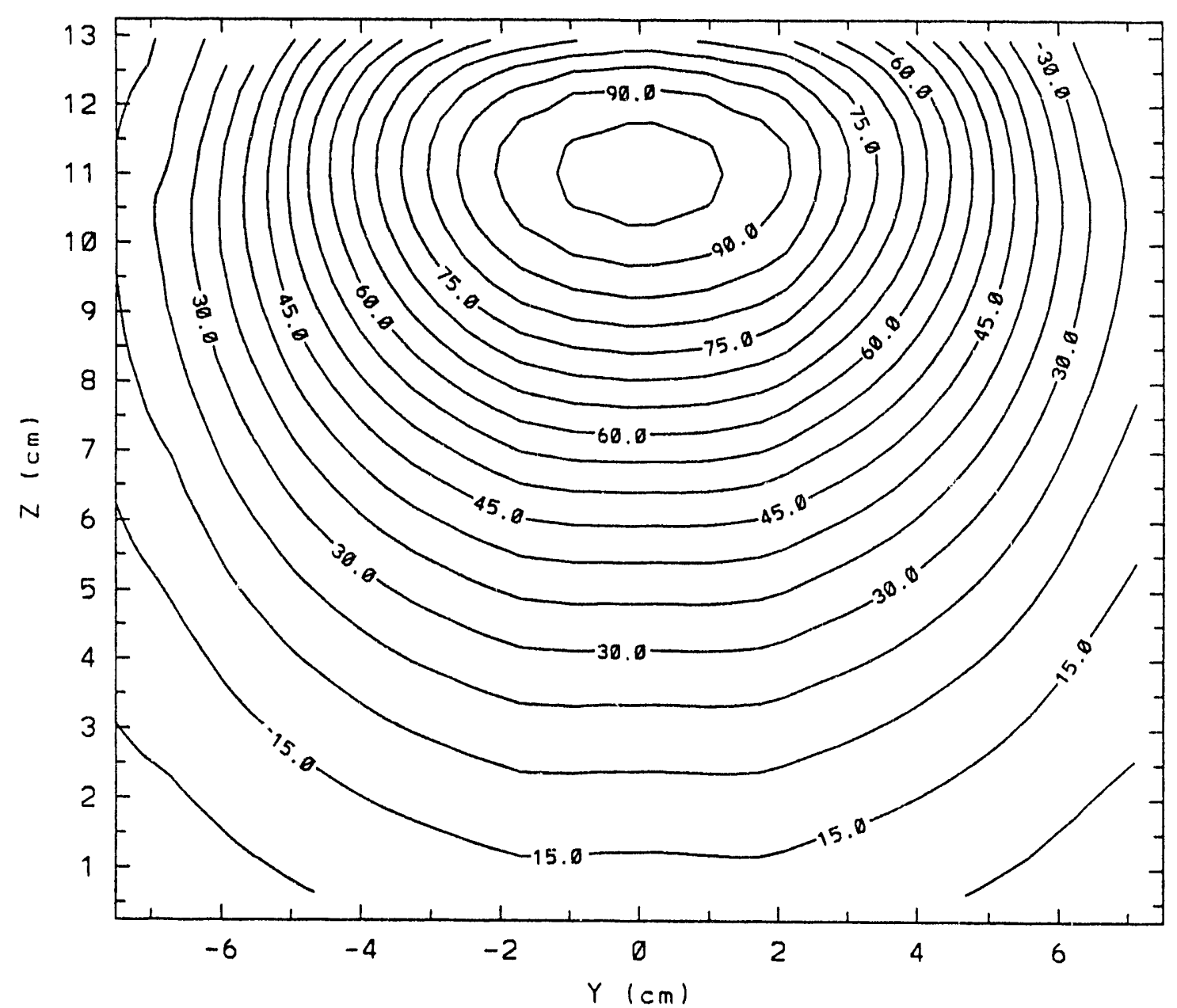

Figure F-21. Gamma flux contours at $x=12.25 \mathrm{~cm}$ for the heterogeneous case. Percentage contours are shown normalized to a peak gamma flux of $5.63 \times 10^{7} \mathrm{gammas} / \mathrm{cm}^{2} / \mathrm{s}$. 


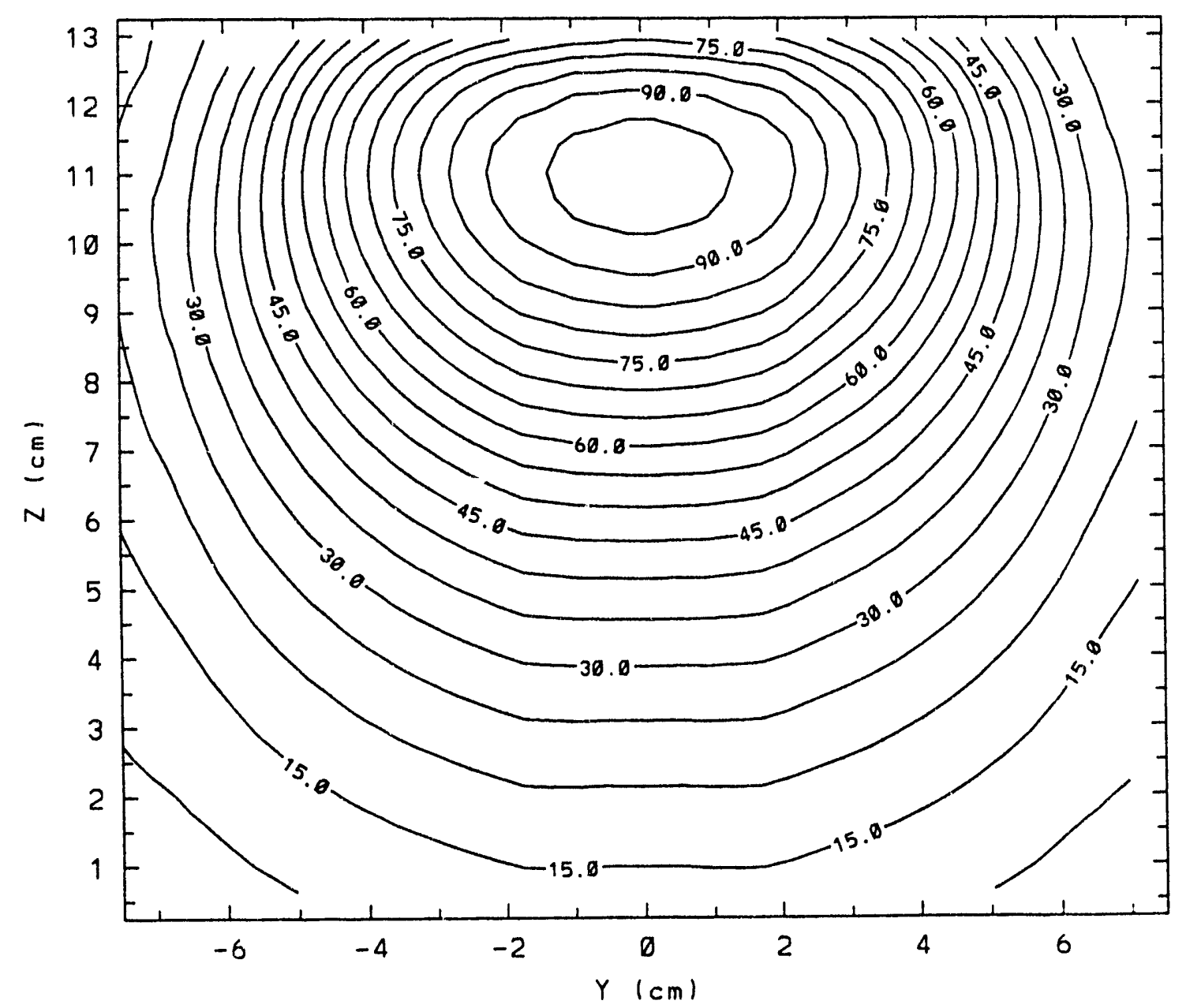

Figure F-22. Gamma flux contours at $x=12.25 \mathrm{~cm}$ for the homogeneous case. Percentage contours are shown normalized to a peak gamma flux of $5.61 \times 10^{7}$ gammas $/ \mathrm{cm}^{2} / \mathrm{s}$. 
thermal neutron flux at this plane.

The thermal neutron and gamma fluxes for the dorsal plane at $z=13.25 \mathrm{~cm}$, which is the top of the dog head and just after the entrance of the beam, are presented here. The results of the fast flux at this plane were presented in section 5.3. Figures $F-23$ and F-24 show the thermal neutron flux at this plane. The results are identical as expected. The gamma fluxes at the same plane are presented in Figures F-25 and F-26. From a comparison of the two results, the homogeneous case underestimates the flux by about $3-5 \%$ in the 55-65\% contours at the center of the top of the dog head. This is a contributing factor to the skin dose which would be underestimated if this homogeneous model was used for therapy planning.

The heterogeneity effects for the $5 \mathrm{~cm} \times 10 \mathrm{~cm}$ beam aperture will be similar to those presented for the $10 \mathrm{~cm} \times$ $10 \mathrm{~cm}$ beam in section 5.3 and in the first part of this appendix. These results are presented here for those interested in the spatial distributions for the smaller beam aperture.

Figures F-27 and F-28 show the thermal neutron flux at $y=-0.125 \mathrm{~cm}$. The spatial distributions differ slightly. There is approximately a $5 \%$ decrease in the thermal neutron 


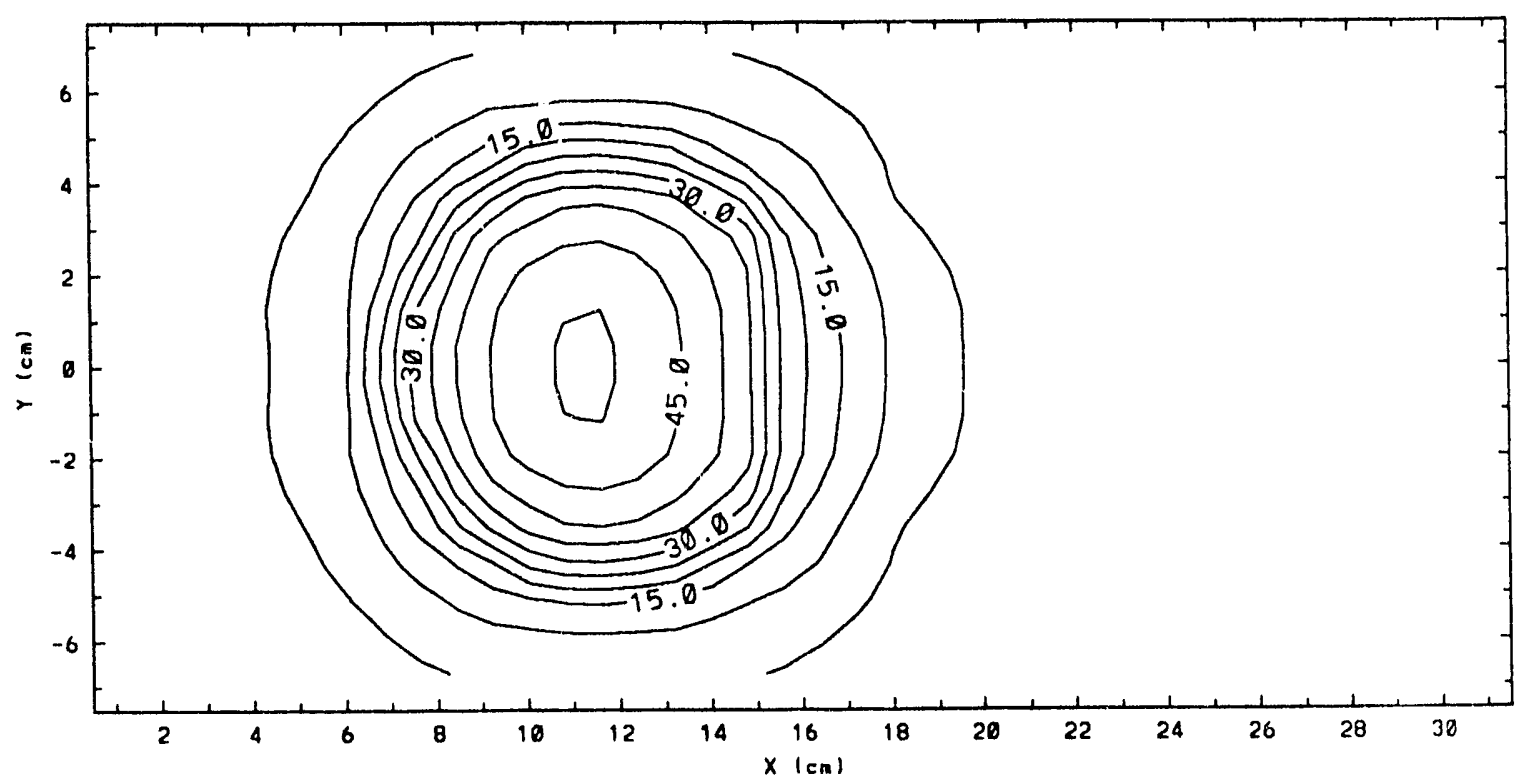

Figure F-23. Thermal neutron flux contours at $z=13.25 \mathrm{~cm}$ for the heterogeneous case with the $10 \mathrm{~cm} \times 10 \mathrm{~cm}$ beam aperture. Percentage contours are shown normalized to a peak thermal flux of $7.00 \times 10^{8} \mathrm{n} / \mathrm{cm}^{2} / \mathrm{s}$. 


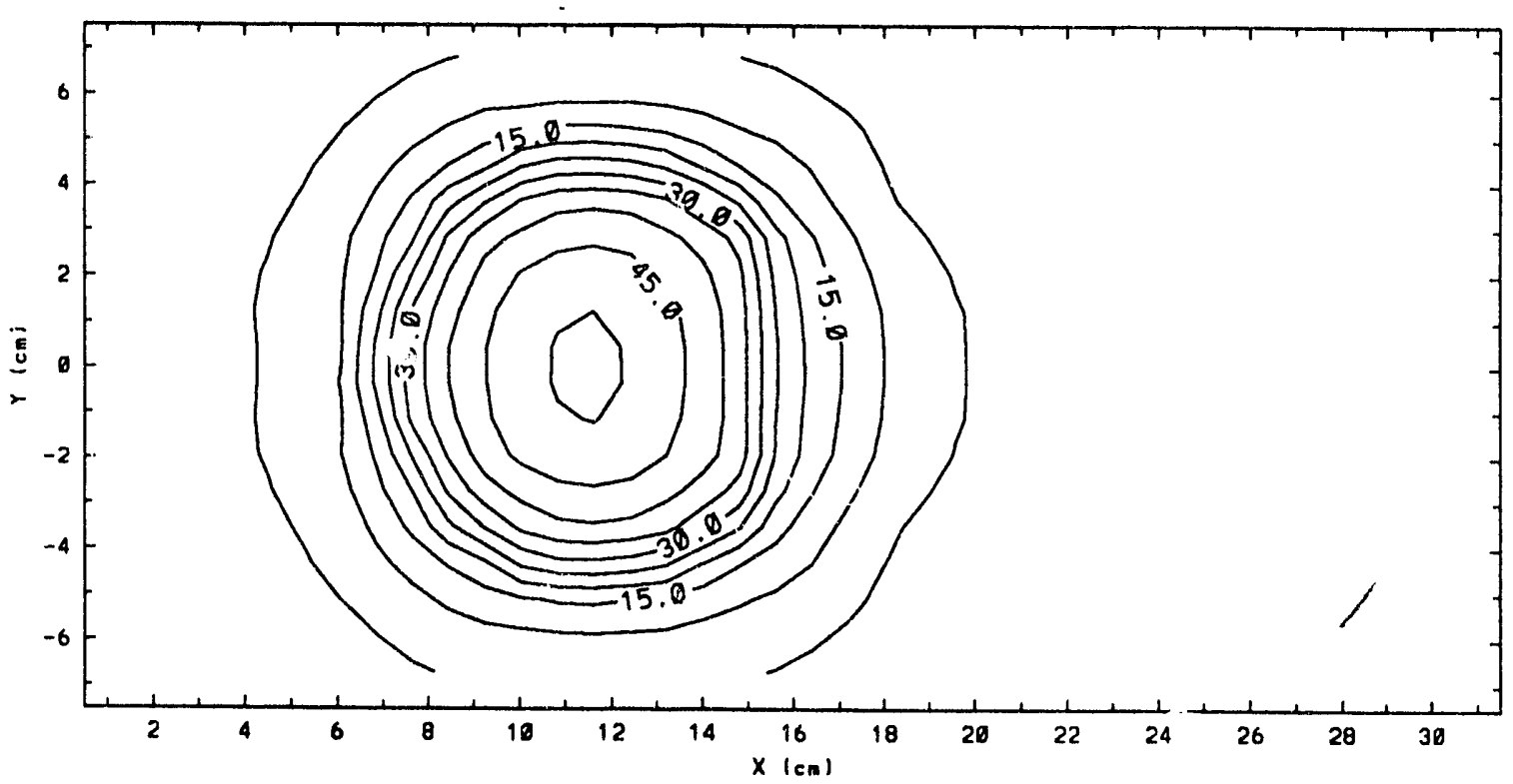

Figure F-24. Thermal neutron flux contours at $z=13.25 \mathrm{~cm}$ for the homogeneous case with the $10 \mathrm{~cm} \times 10 \mathrm{~cm}$ beam aperture. Percentage contours are shown normalized to a peak thermal flux of $6.92 \times 10^{8} \mathrm{n} / \mathrm{cm}^{2} / \mathrm{s}$. 


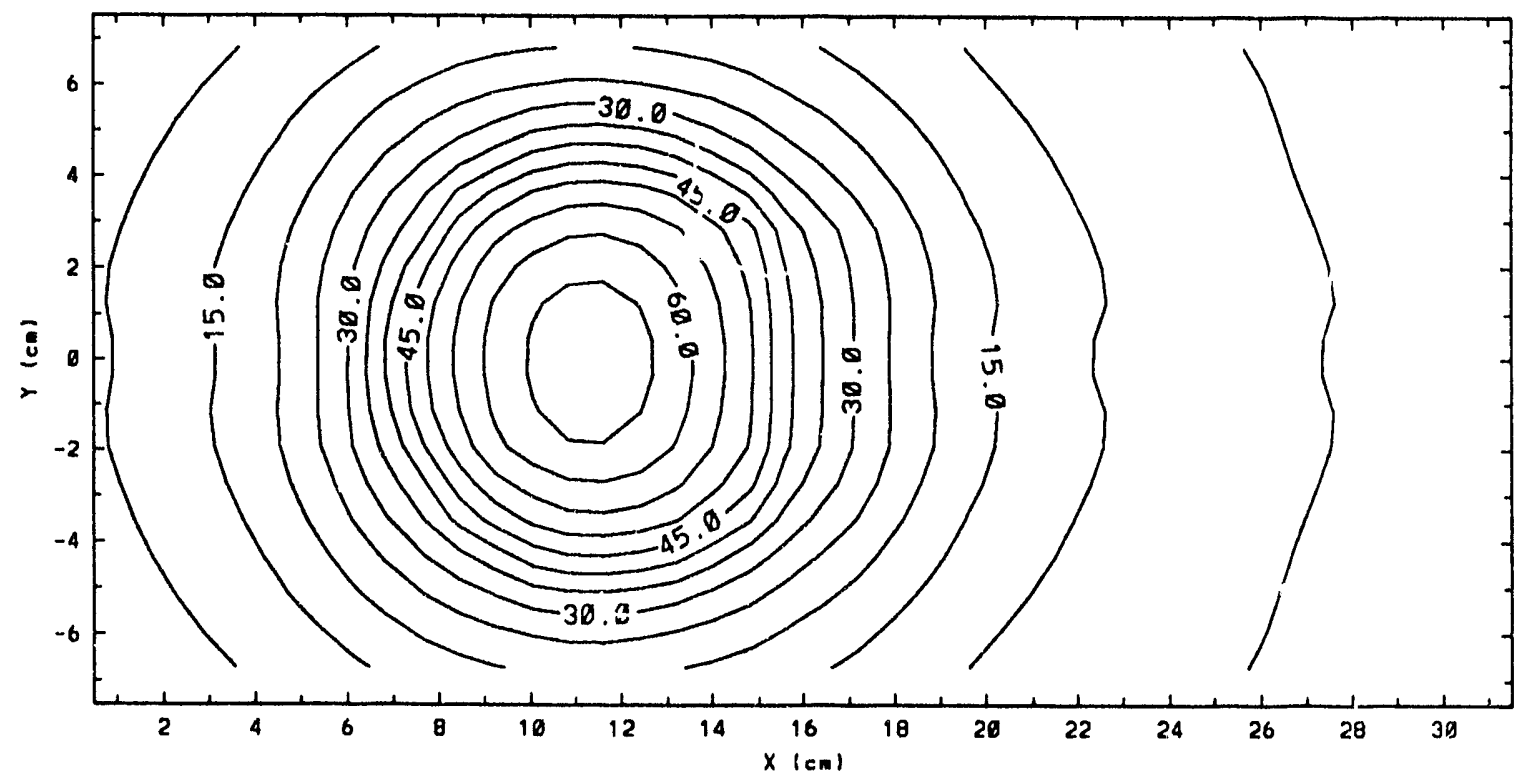

Figure F-25. Gamma flux contours at $z=13.25 \mathrm{~cm}$ for the heterogeneous case with the $10 \mathrm{~cm} \times 10 \mathrm{~cm}$ beam aperture. Percentage contours are shown normalized to a peak gamma flux of $5.63 \times 10^{7}$ gammas $/ \mathrm{cm}^{2} / \mathrm{s}$. 


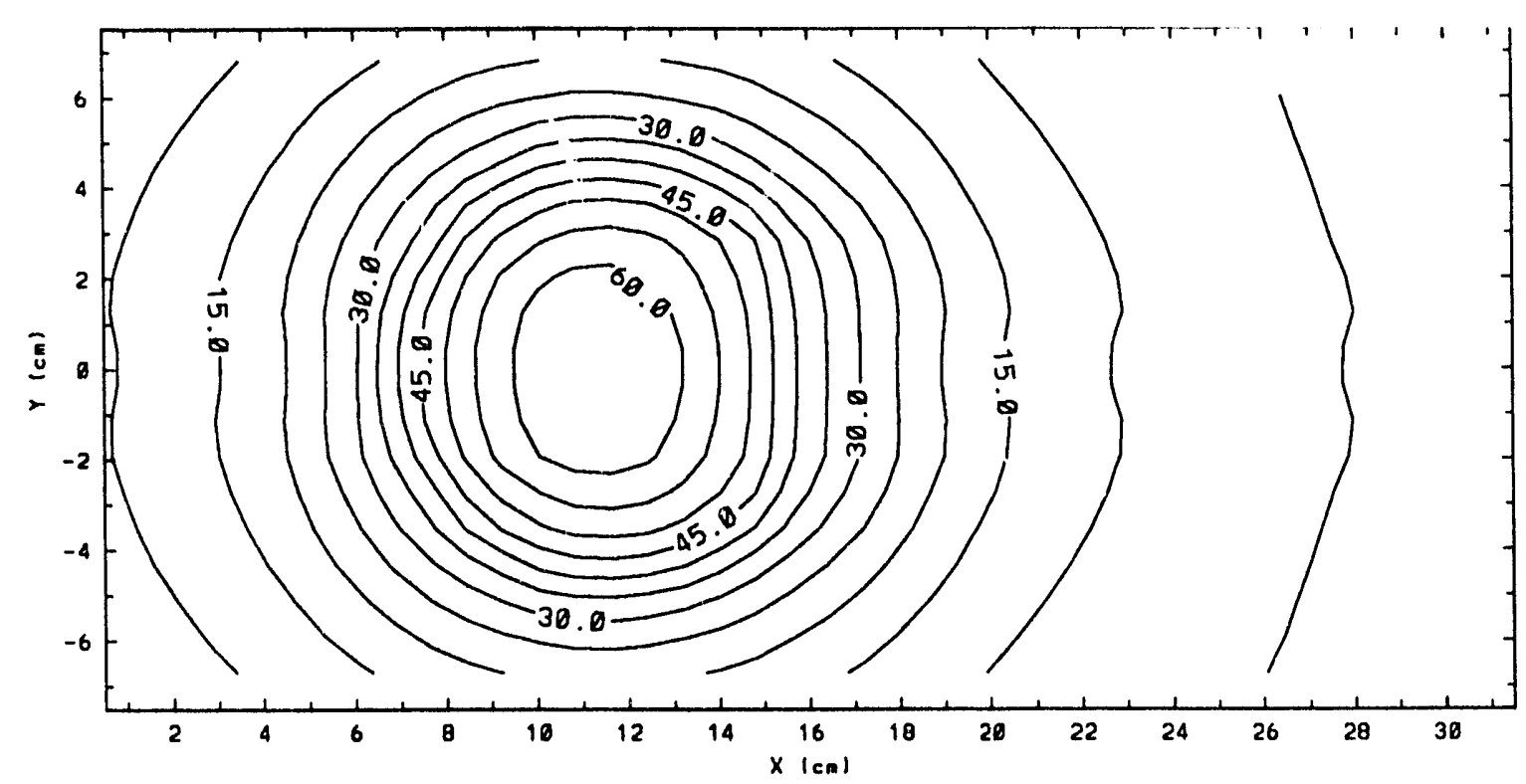

Figure F-26. Gamma flux contours at $z=13.25 \mathrm{~cm}$ for the homogeneous case with the $10 \mathrm{~cm} \times 10 \mathrm{~cm}$ beam aperture. Percentage contours are shown no:malized to a peak gamma flux of $5.61 \times 10^{7}$ gammas $/ \mathrm{cm}^{2} / \mathrm{s}$. 


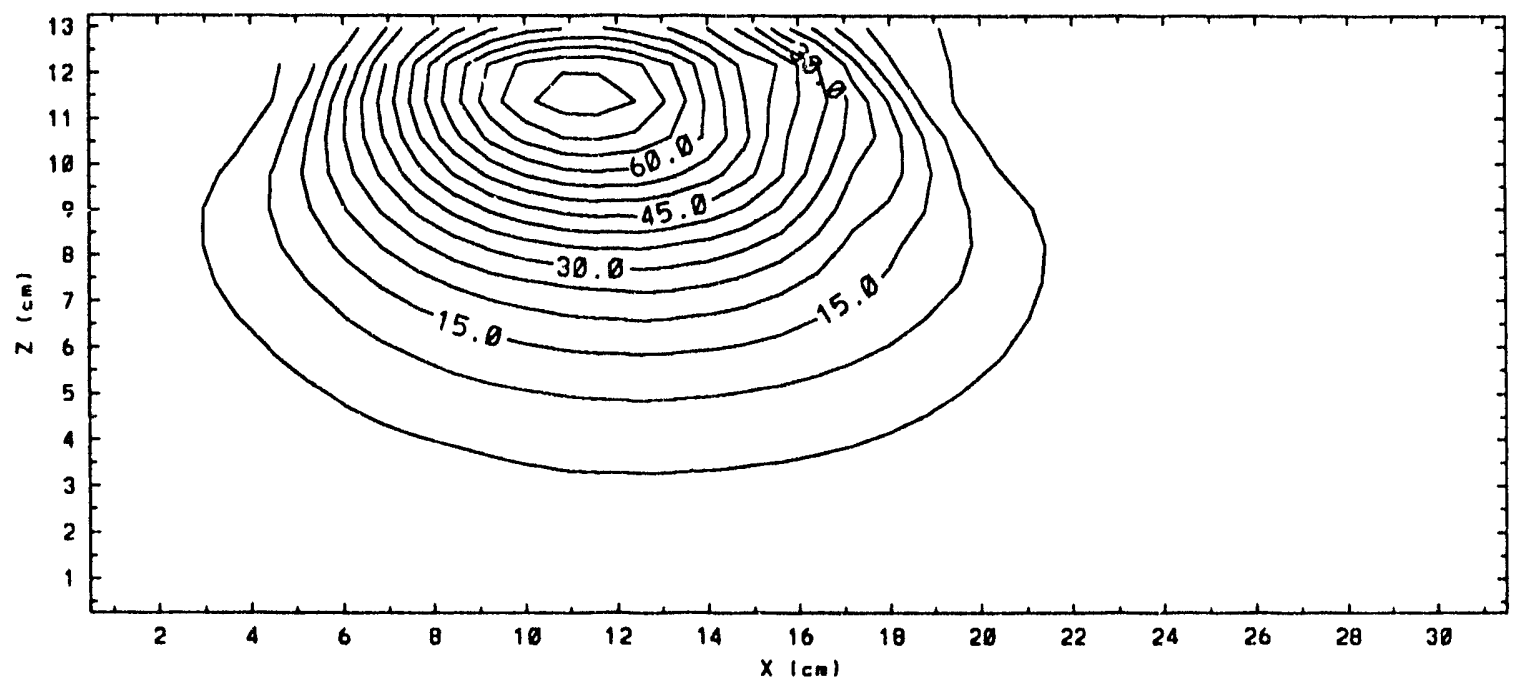

Figure F-27. Thermal neutron flux contours at $y=-0.125 \mathrm{~cm}$ for the heterogeneous case with the $5 \mathrm{~cm} \times 10 \mathrm{~cm}$ beam aperture. Percentage contours are shown normalized to a peak thermal flux of $4.14 \times 10^{8} \mathrm{n} / \mathrm{cm}^{2} / \mathrm{s}$. 


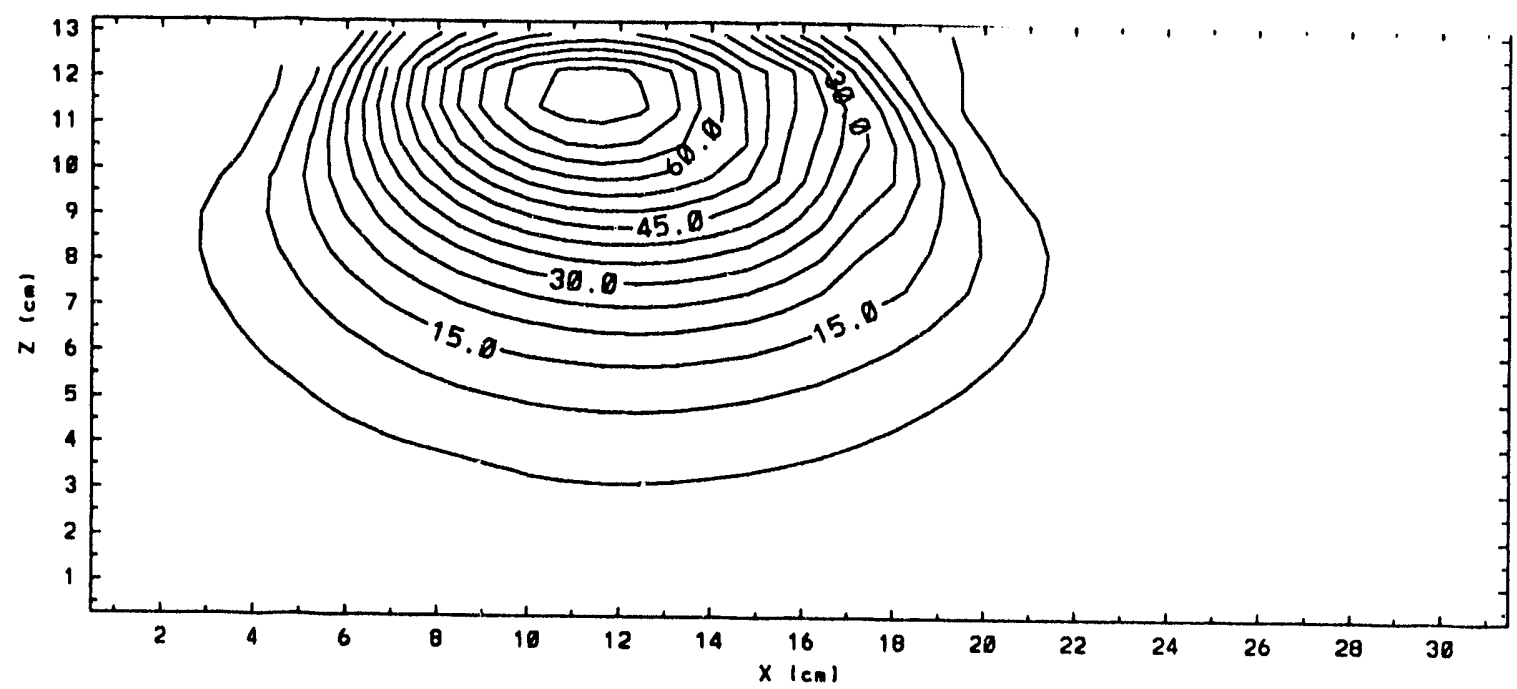

Figure F-28. Thermal neutron flux contours at $y=-0.125 \mathrm{~cm}$ for the homogeneous case with the $5 \mathrm{~cm} \times 10 \mathrm{~cm}$ beam aperture. Percentage contours are shown normalized to a peak thermal flux of $4.04 \times 10^{8} \mathrm{n} / \mathrm{cm}^{2} / \mathrm{s}$. 
flux of the heterogeneous case relative to the homogeneous one in the rostral direction. The hetelogeneous peak is smaller and is shifted in the ventral direction. These results are those seen in the same plane for the $10 \mathrm{~cm} \times 10$ cm case. As noted in section 5.3, the difference in flux is due to the presence of bone and the sinuses in these regions. There is a difference in the magnitude of the thermal neutron fluxes for the different size beams. For example, the thermal neutron fluxes in the heterogeneous cases are $7.00 \times 10^{8} \mathrm{n} / \mathrm{cm}^{2} / \mathrm{s}$ and $4.14 \times 10^{8} \mathrm{n} / \mathrm{cm}^{2} / \mathrm{s}$ for the 10 $\mathrm{cm} \times 10 \mathrm{~cm}$ beam and $5 \mathrm{~cm} \times 10 \mathrm{~cm}$ beam, respectively.

The fast neutron flux contours at the $y=-0.125 \mathrm{~cm}$ plane are shown in Figures F-29 and F-30. The results are essentially identical.

The calculated gamma fluxes for the heterogeneous and homogeneous cases at $y=-0.125 \mathrm{~cm}$ are shown in Figures $\mathrm{F}-31$ and $\mathrm{F}-32$, respectively. There is approximately a 58 decrease in the gamma flux when heterogeneities are included. There is also a small difference between the spatial distributions of the two cases. The peak gamma fluxes are $2.98 \times 10^{7} \mathrm{n} / \mathrm{cm}^{2} / \mathrm{s}$ and $2.97 \times 10^{7} \mathrm{n} / \mathrm{cm}^{2} / \mathrm{s}$ for the heterogeneous and homogeneous cases, respectively.

The thermal, fast, and gamma fluxes can be examined at 


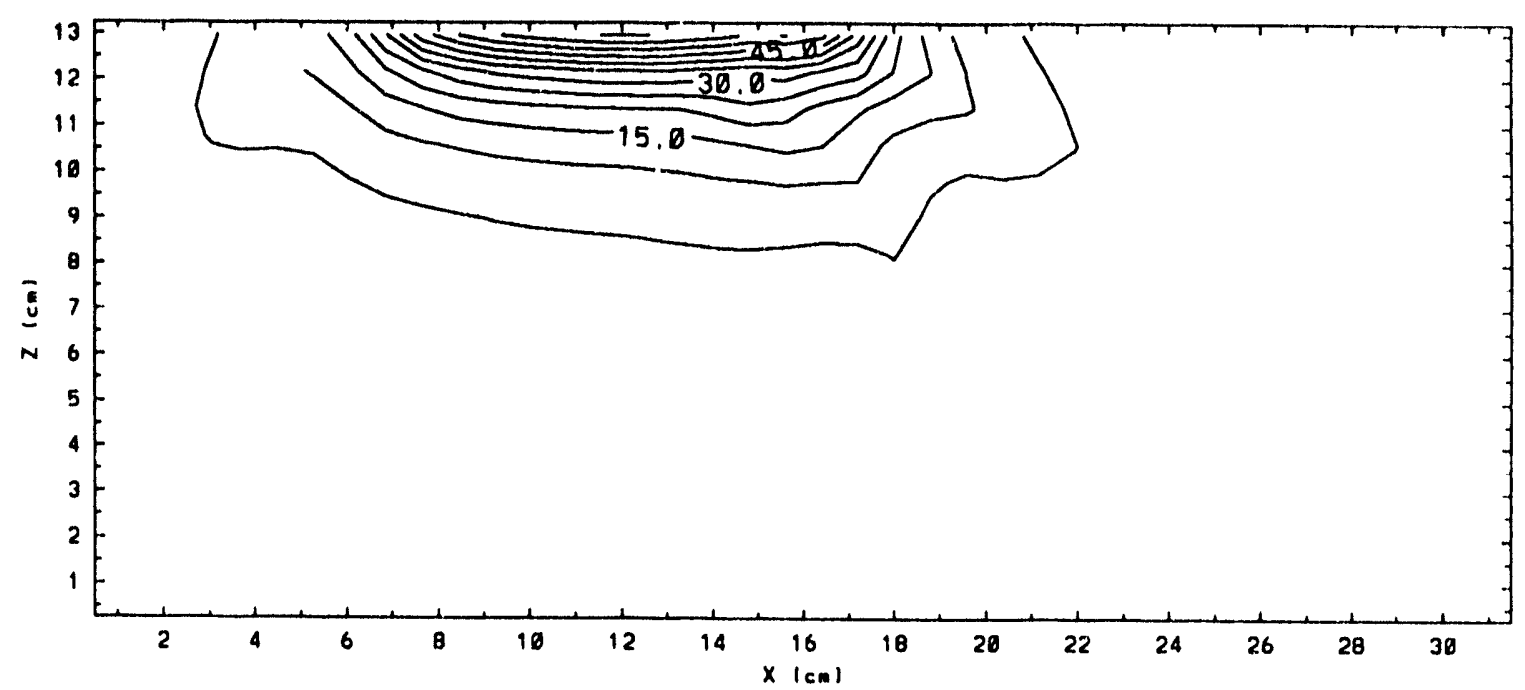

Figure F-29. Fast neutron flux contours at $y=-0.125 \mathrm{~cm}$ for the heterogeneous case with the $5 \mathrm{~cm} \times 10 \mathrm{~cm}$ beam aperture. Percentage contours are shown normalized to a peak fast flux of $1.26 \times 10^{7} \mathrm{n} / \mathrm{cm}^{2} / \mathrm{s}$. 


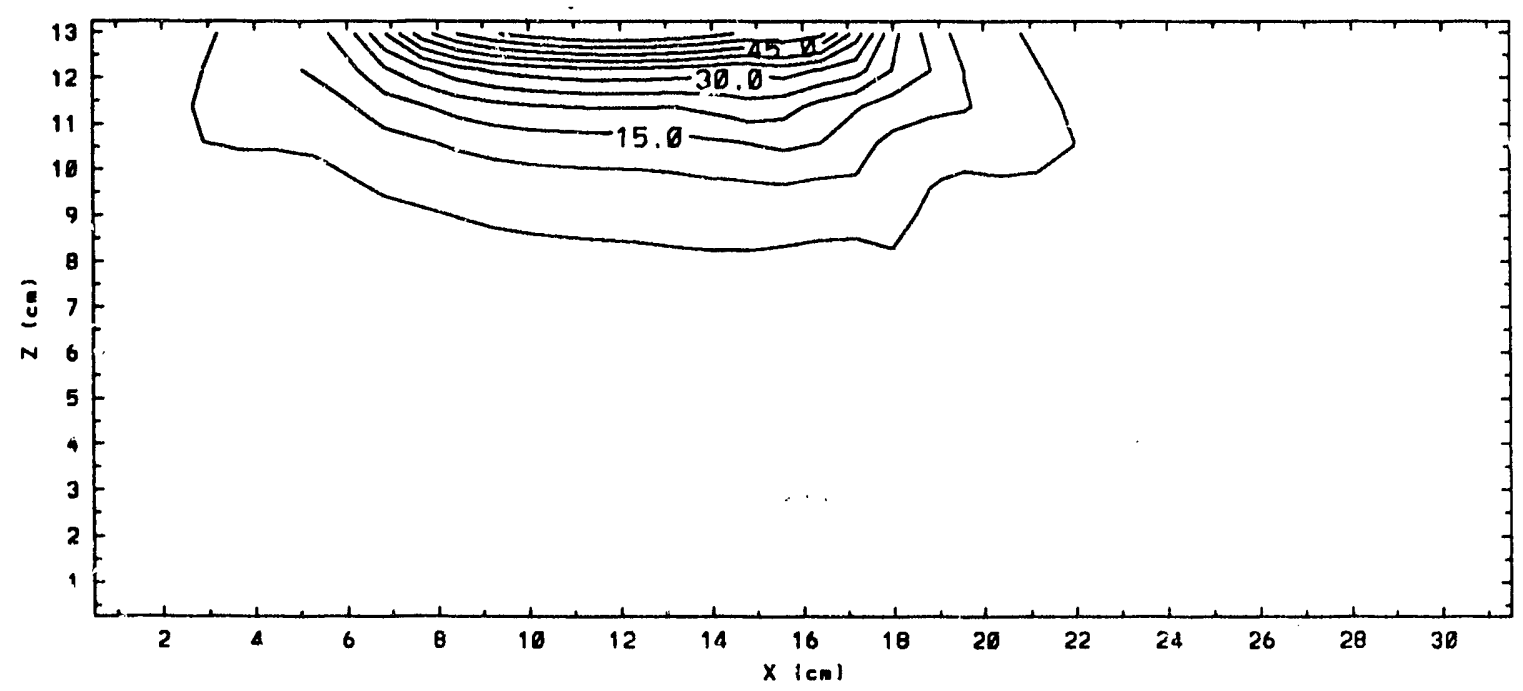

Figure F-30. Fast neutron flux contours at $y=-0.125 \mathrm{~cm}$ for the homogeneous case with the $5 \mathrm{~cm} \times 10 \mathrm{~cm}$ beam aperture. Percentage contours are shown normalized to a peak fast flux of $1.27 \times 10^{7} \mathrm{n} / \mathrm{cm}^{2} / \mathrm{s}$. 


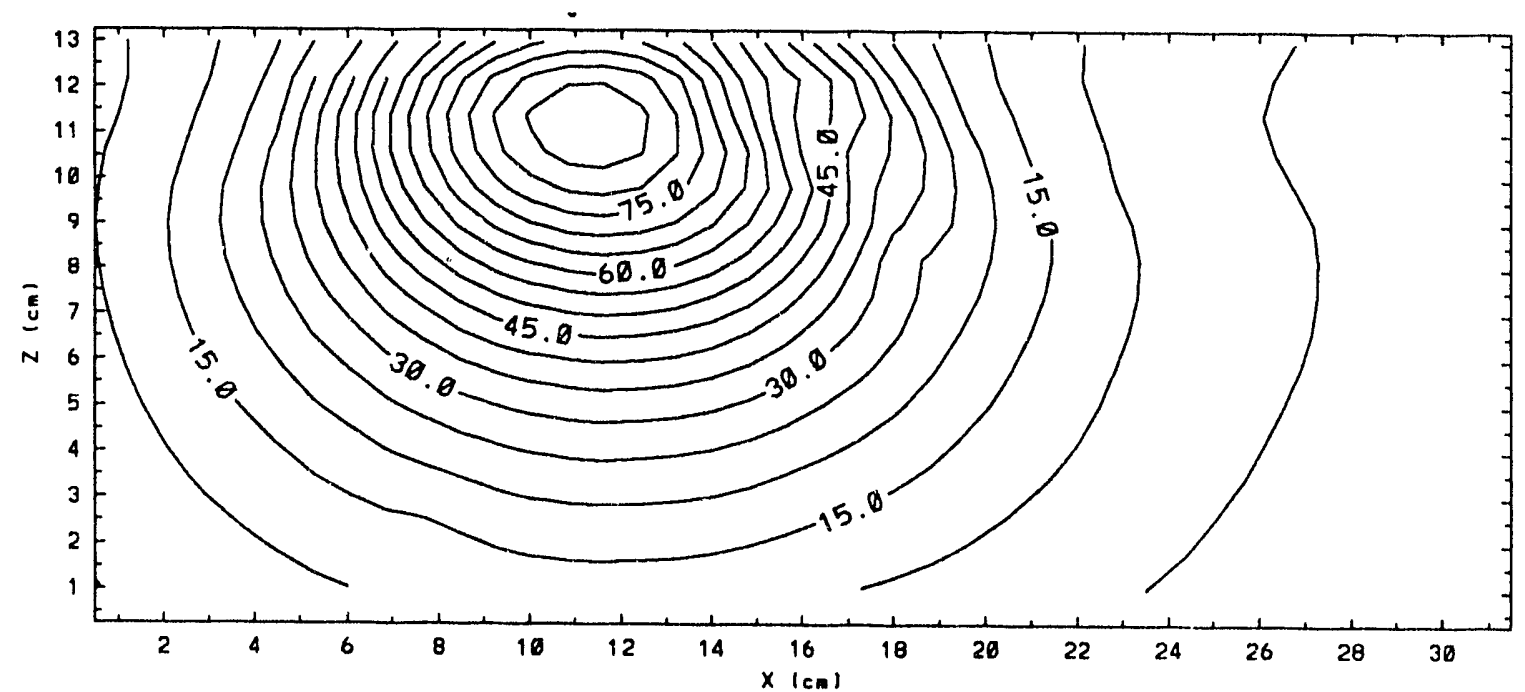

Figure F-31. Gamma flux contours at $y=-0.125 \mathrm{~cm}$ for the heterogeneous case with the $5 \mathrm{~cm} \times 10 \mathrm{~cm}$ beam aperture. Percentage contours are shown normalized to a peak gamma flux of $2.98 \times 10^{7}$ gammas $/ \mathrm{cm}^{2} / \mathrm{s}$. 


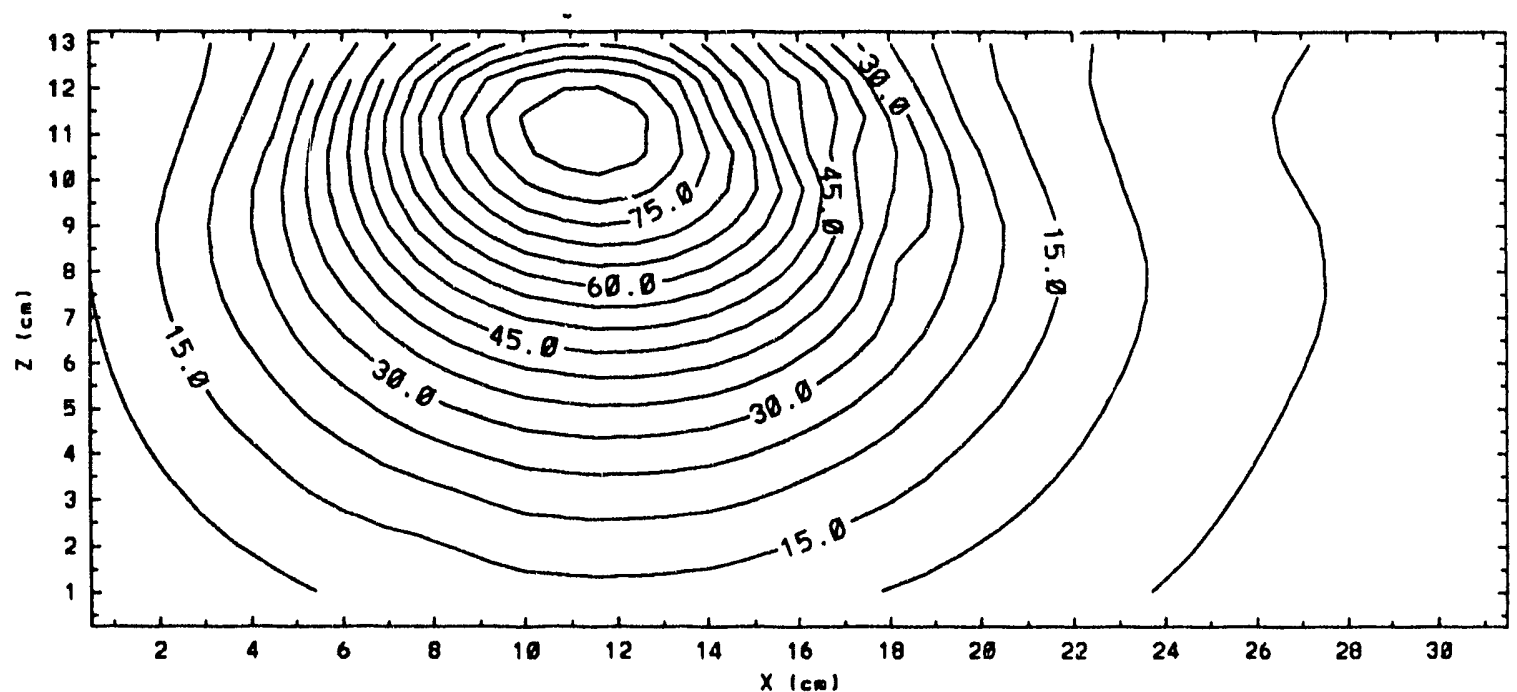

Figure F-32. Gamma flux contours at $y=-0.125 \mathrm{~cm}$ for the homogeneous case with the $5 \mathrm{~cm} \times 10 \mathrm{~cm}$ beam aperture. Percentage contsurs are shown normalized to a peak gamma flux of $2.97 \times 10^{i}$ gammas $/ \mathrm{cm}^{2} / \mathrm{s}$. 
$y=-2.5 \mathrm{~cm}$. This plane corresponds to sagittal slice 5 which is shown in Figures B-5-(photograph) and C-5 (detailed mesh). This section contains a large section of bone from $x$ $=13 \mathrm{~cm}$ to $\mathrm{x}=19 \mathrm{~cm}$ in the region of $\mathrm{z}=9 \mathrm{~cm}$ to $\mathrm{z}=13 \mathrm{~cm}$. It also contains the nasal cavity. A $5 \%$ decrease in the thermal flux in the rostral direction is seen due to the bone in this slice when Figures F-33 (heterogeneous case) and F-34 (homogeneous case) are compared.

Figures F-35 and F-36 show the calculated fast flux contours at $\mathrm{y}=-2.5 \mathrm{~cm}$ for the two models. The contours are essentially identical, following the same trend as the results for the $10 \mathrm{~cm} \times 10 \mathrm{~cm}$ oeam at this plane. The fast neutron flux is not as sensitive to the bone region in this slice as the thermal and gamma fluxes.

The contours of the spatial distributions of the gamma flux are depicted in Figures F-37 and F-38. A slight decrease of the flux is seen in Figure F-37, the heterogenous case. The peak flux is slightly smaller (less than 18 ) due to the inclusion of inhomogeneities in modeling. There is a $3-5 \%$ decrease in the flux in the rostral direction due to passage through bone.

As mentioned in section 6.1 , heterogeneity effects are not as pronounced in the outer regions of the dog head. The 


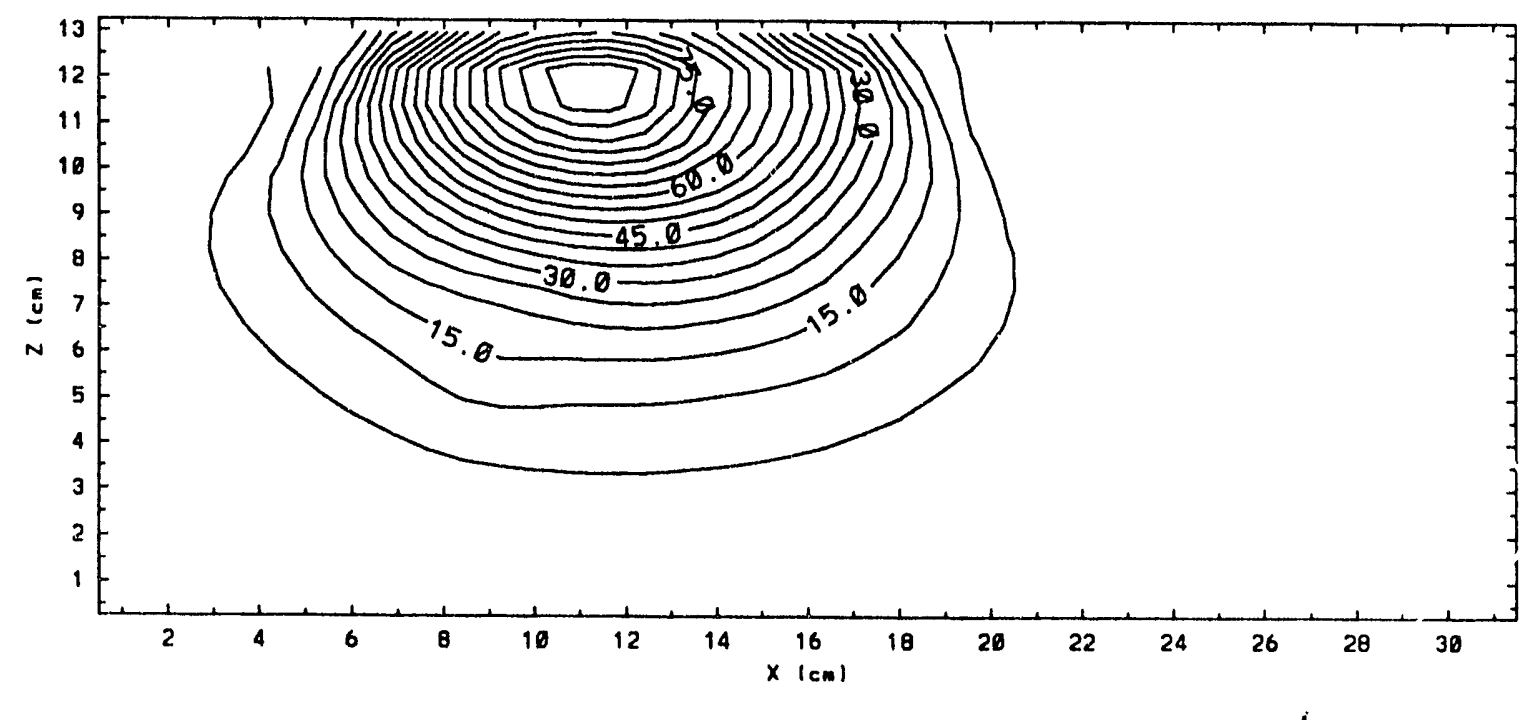

Figure F-33. Thermal neutron flux contours at $y=-2.5 \mathrm{~cm}$ for the heterogeneous case with the $5 \mathrm{~cm} \times 10 \mathrm{~cm}$ beam aperture. Percentage contours are shown normalized to a peak thermal flux of $4.14 \times 10^{8} \mathrm{n} / \mathrm{cm}^{2} / \mathrm{s}$. 


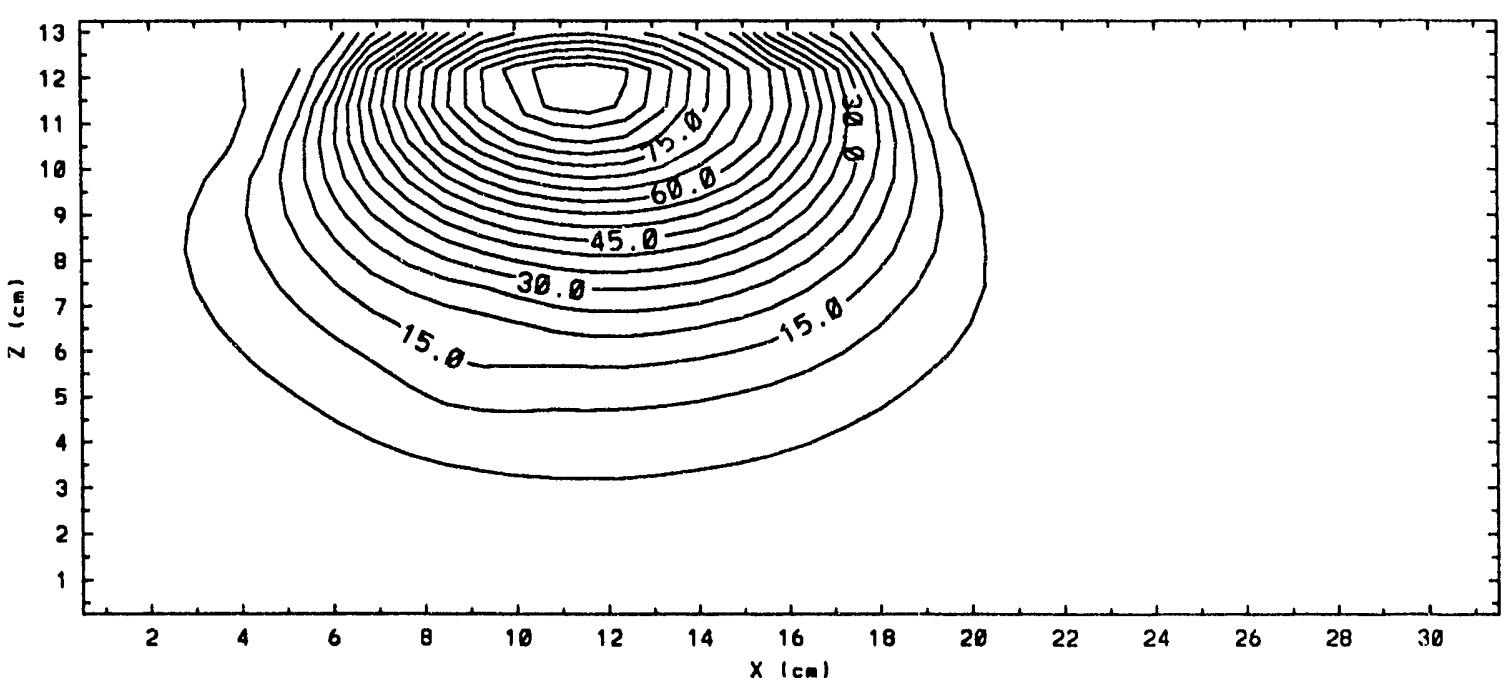

Figure F-34. Thermal neutron flux contours at $y=-2.5 \mathrm{~cm}$ for the homogeneous case with the $5 \mathrm{~cm} \times 10 \mathrm{~cm}$ beam aperture. Percentage contours are shown normalized to a peak thermal flux of $4.04 \times 10^{8} \mathrm{n} / \mathrm{cm}^{2} / \mathrm{s}$. 


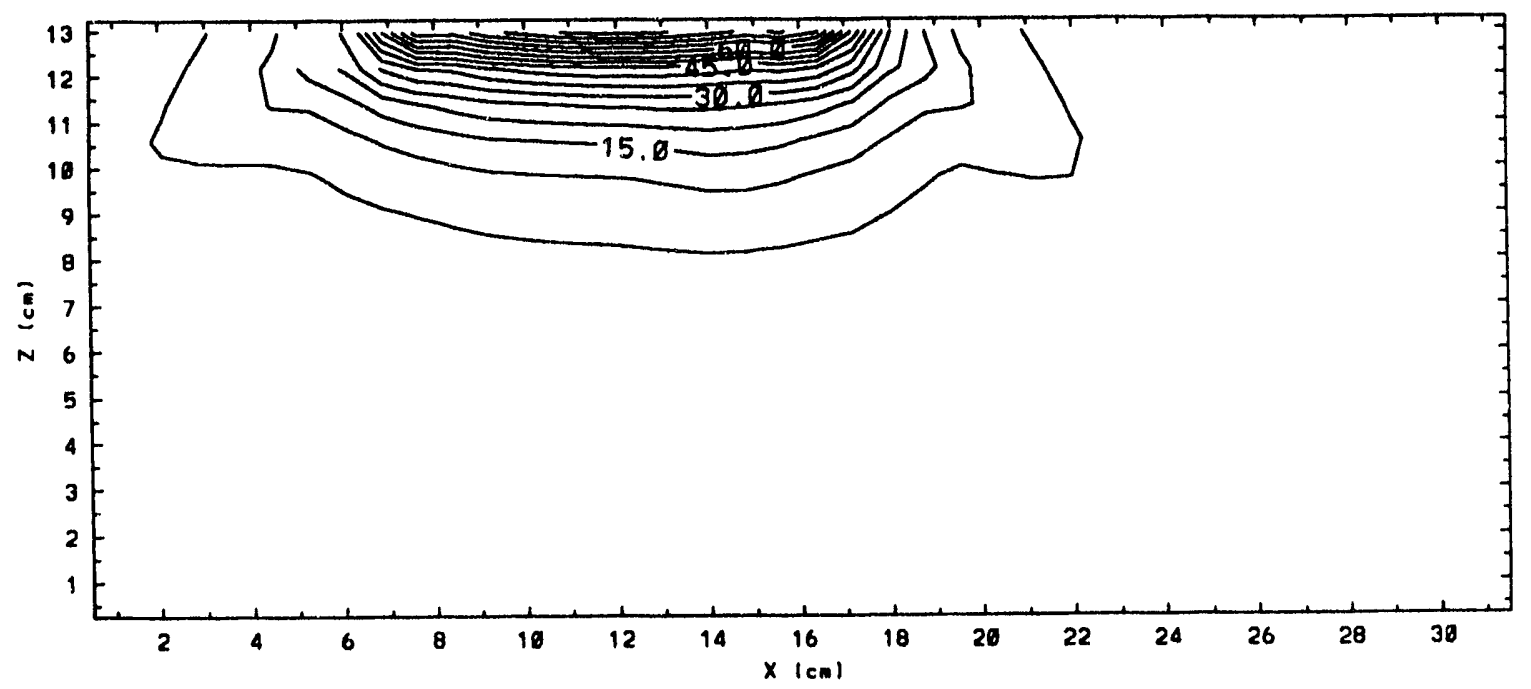

Figure F-35. Fast neutron flux contours at $y=-2.5 \mathrm{~cm}$ for the heterogeneous case with the $5 \mathrm{~cm} \times 10 \mathrm{~cm}$ beam aperture. Percentage contours are shown normalized to a peak fast flux of $1.26 \times 10^{7} \mathrm{n} / \mathrm{cm}^{2} / \mathrm{s}$. 


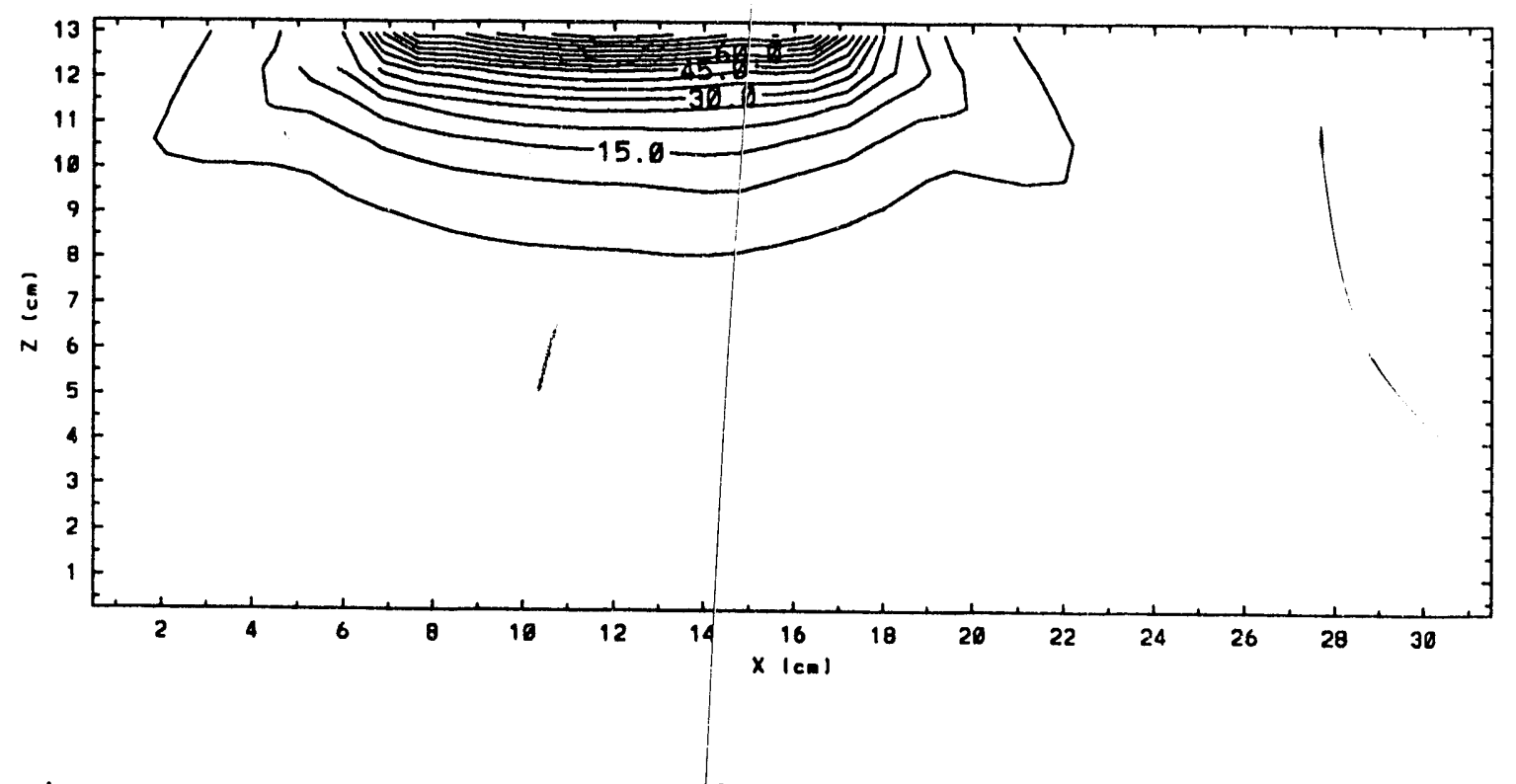

Figure F-36. Fast neutron flux contours at $y=-2.5 \mathrm{~cm}$ for the homogeneous case with the $5 \mathrm{~cm} \times 10 \mathrm{~cm}$ beam aperture. Percentage contours are shown normalized to a peak fast flux of $1.27 \times 10^{7} \mathrm{n} / \mathrm{cm}^{2} / \mathrm{s}$. 


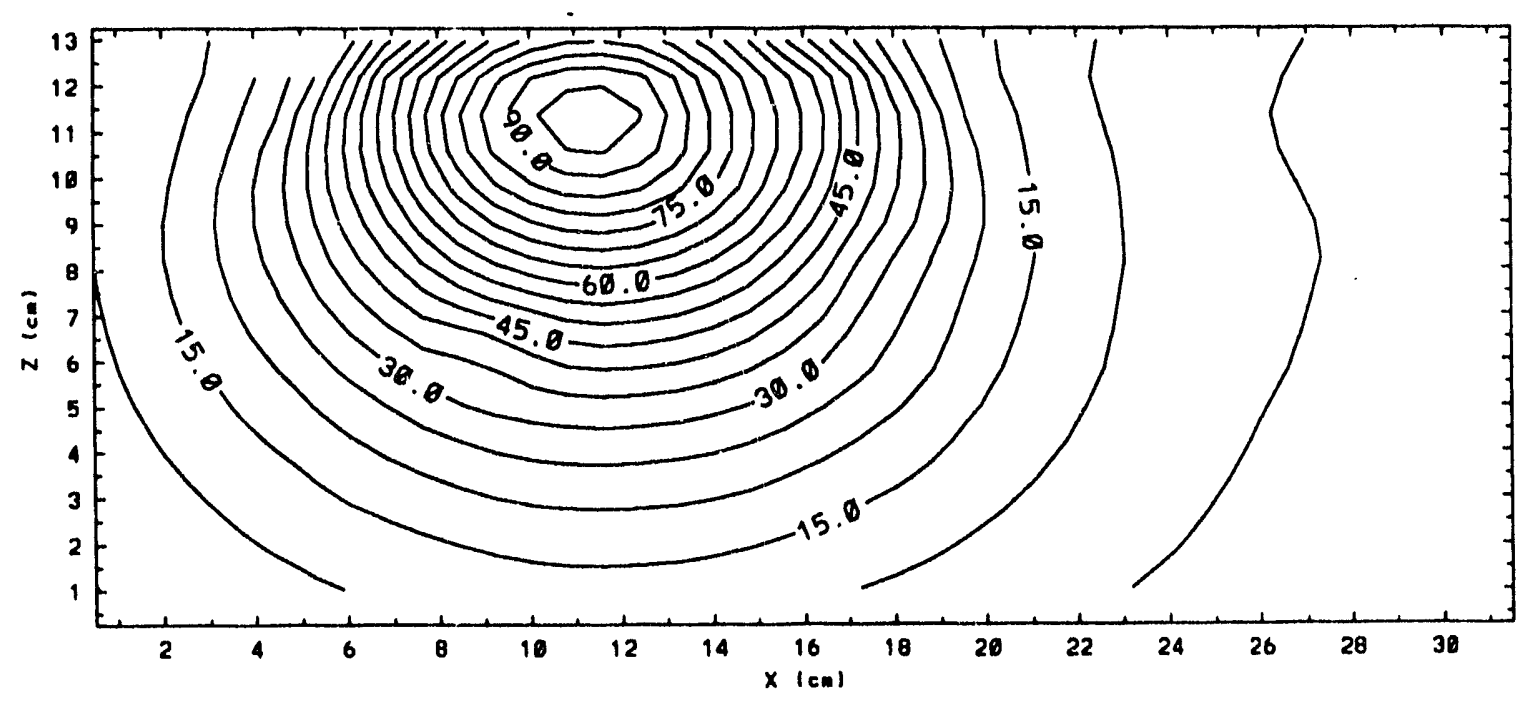

Figure F-37. Gamma flux contours at $y=-2.5 \mathrm{~cm}$ for the heterogeneous case with the $5 \mathrm{~cm} \times 10 \mathrm{~cm}$ beam aperture. Percentage contours are shown normalized to a peak gamma flux of $2.98 \times 10^{7}$ gammas $/ \mathrm{cm}^{2} / \mathrm{s}$. 


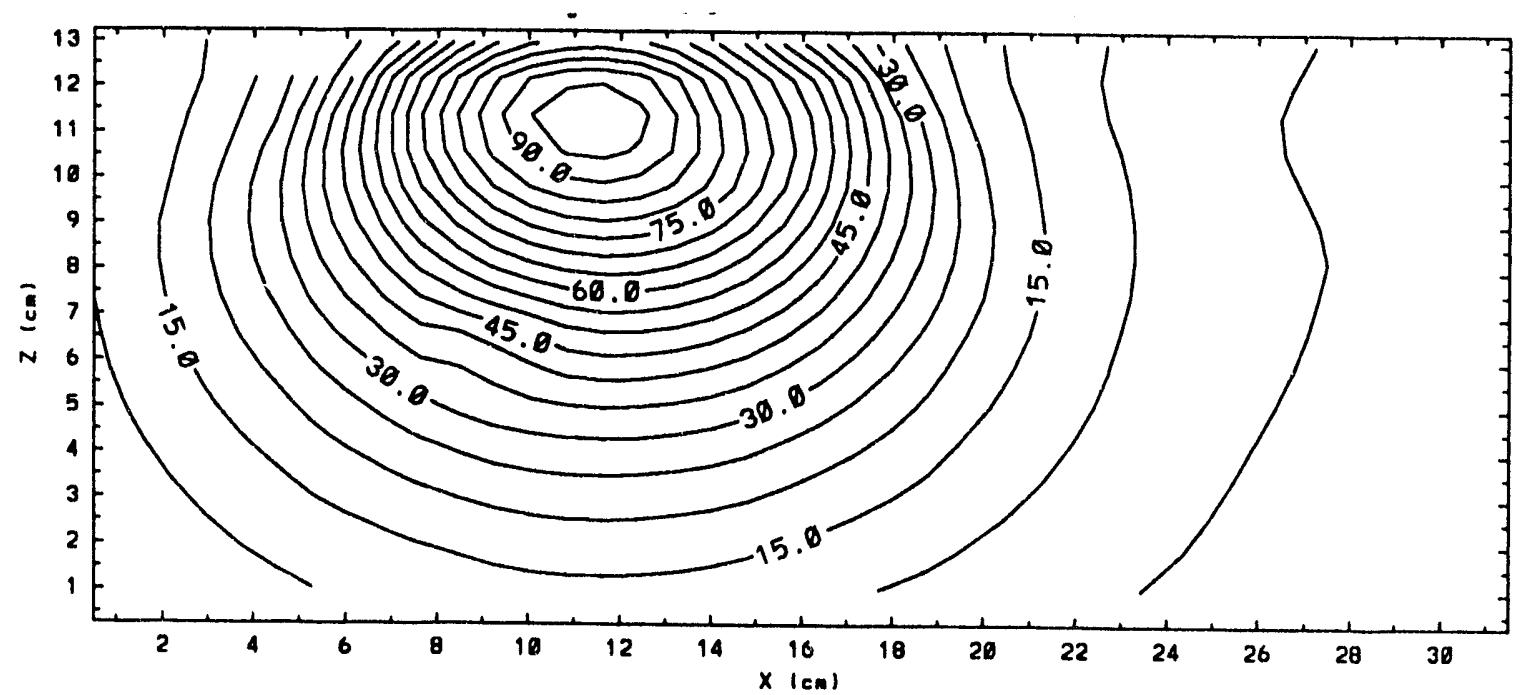

Figure F-38. Gamma flux contours at $y=-2.5 \mathrm{~cm}$ for the homogeneous case with the $5 \mathrm{~cm} \times 10 \mathrm{~cm}$ beam aperture. Percentage contours are shown normalized to a peak gamma flux of $2.97 \times 10^{7}$ gammas $/ \mathrm{cm}^{2} / \mathrm{s}$. 
thermal fluxes at $y=-4.5 \mathrm{~cm}$, corresponding to sagittal slice 3, Figure B-3 (photograph) and Figure C-3 (detailed mesh), are shown in Figures F-39 and F-40. The thermal fluxes for the two cases are essentially identical. Figures F-41 and F-42 depict the fast neutron flux at $y=-4.5 \mathrm{~cm}$. There are no differences between the heterogeneous and homogeneous cases as expected because of fewer heterogeneities in this plane.

An effect is seen, however, in the gamma flux. Relative to the total peak flux, the peak gamma flux at the $y=-4.5 \mathrm{~cm}$ plane is 708 of the total. The gamma fluxes are shown in Figures F-43 and F-44 for the two models. The peak flux for the heterogeneous case is smaller than that of the homogeneous case. Also, a slight decrease $(1-2 \%)$ is seen in the ventral direction for the heterogeneous case.

The thermal, fast, and gamma fluxes can also be examined at a plane not under the beam delimiter. The following contour plots depict the fluxes at the $y=2.5 \mathrm{~cm}$ plane (sagittal slice 5). These may not be necessary for therapy since the dose to tissues not underneath the beam port should not affect the therapy, but it is interesting to observe the spatial distribution of the fluxes in these regions along with any heterogeneity effects that might be observed. 


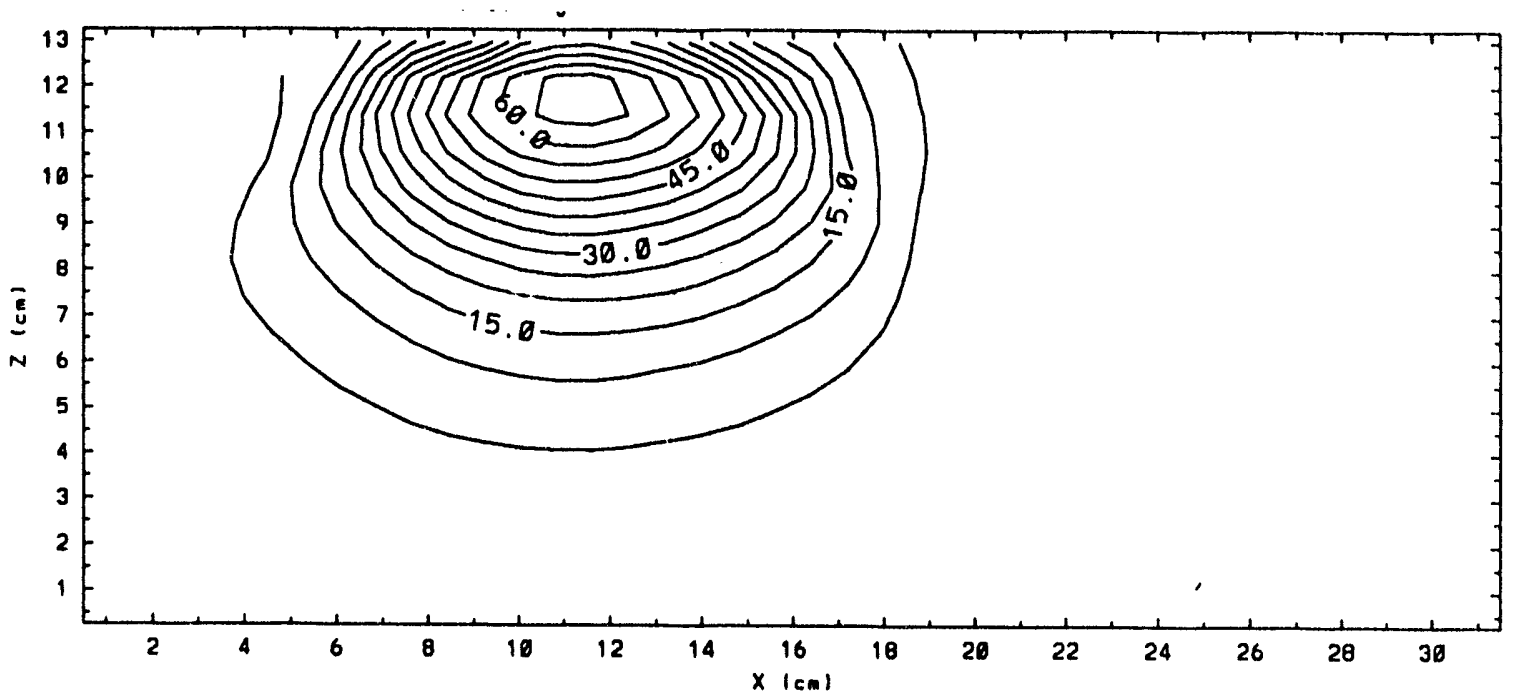

Figure F-39. Thermal neutron flux contours at $y=-4.5 \mathrm{~cm}$ for the heterogeneous case with the $5 \mathrm{~cm} \times 10 \mathrm{~cm}$ beam aperture. Percentage contours are shown normalized to a peak thermal flux of $4.14 \times 10^{8} \mathrm{n} / \mathrm{cm}^{2} / \mathrm{s}$. 


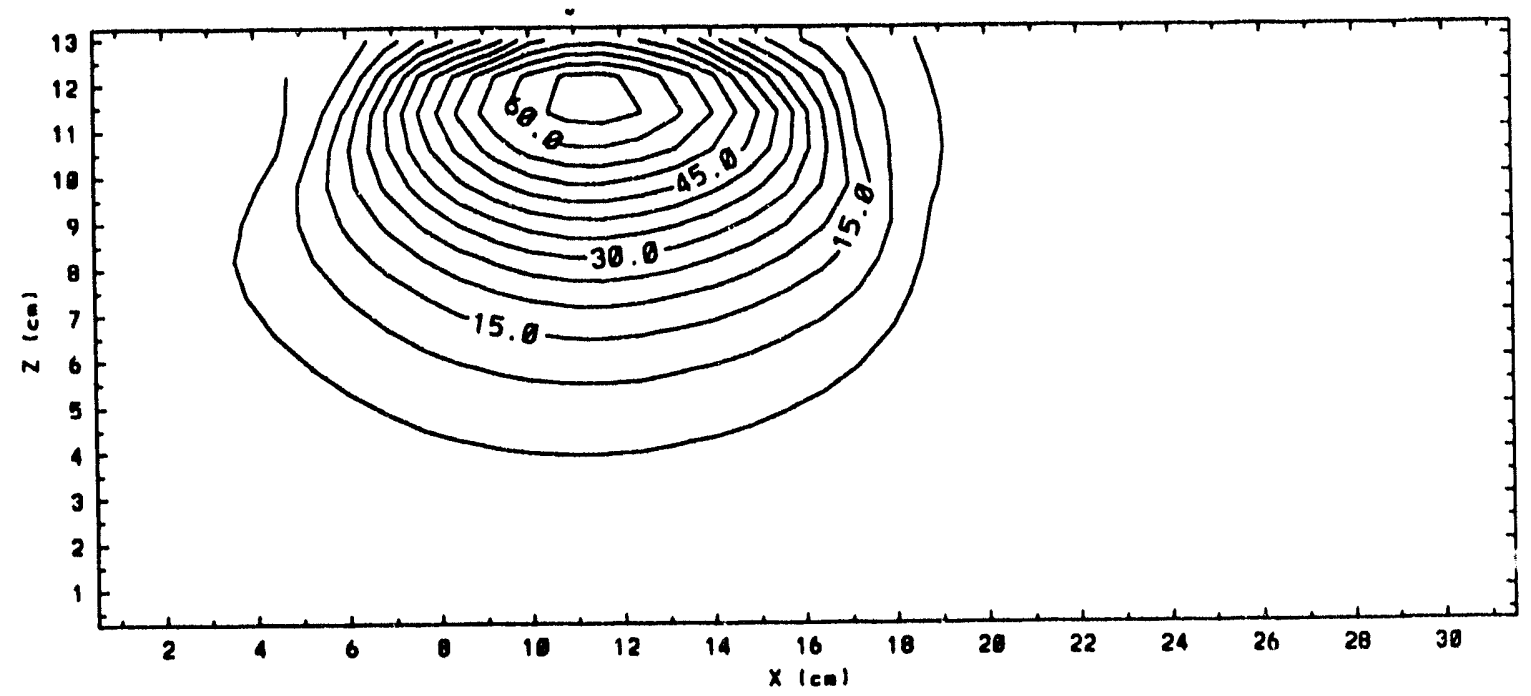

Figure F-40. Thermal neutron flux contours at $y=-4.5 \mathrm{~cm}$ for the homogeneous case with the $5 \mathrm{~cm} \times 10 \mathrm{~cm}$ beam aperture. Percentage contours are shown normalized to a peak thermal flux of $4.04 \times 10^{8} \mathrm{n} / \mathrm{cm}^{2} / \mathrm{s}$. 


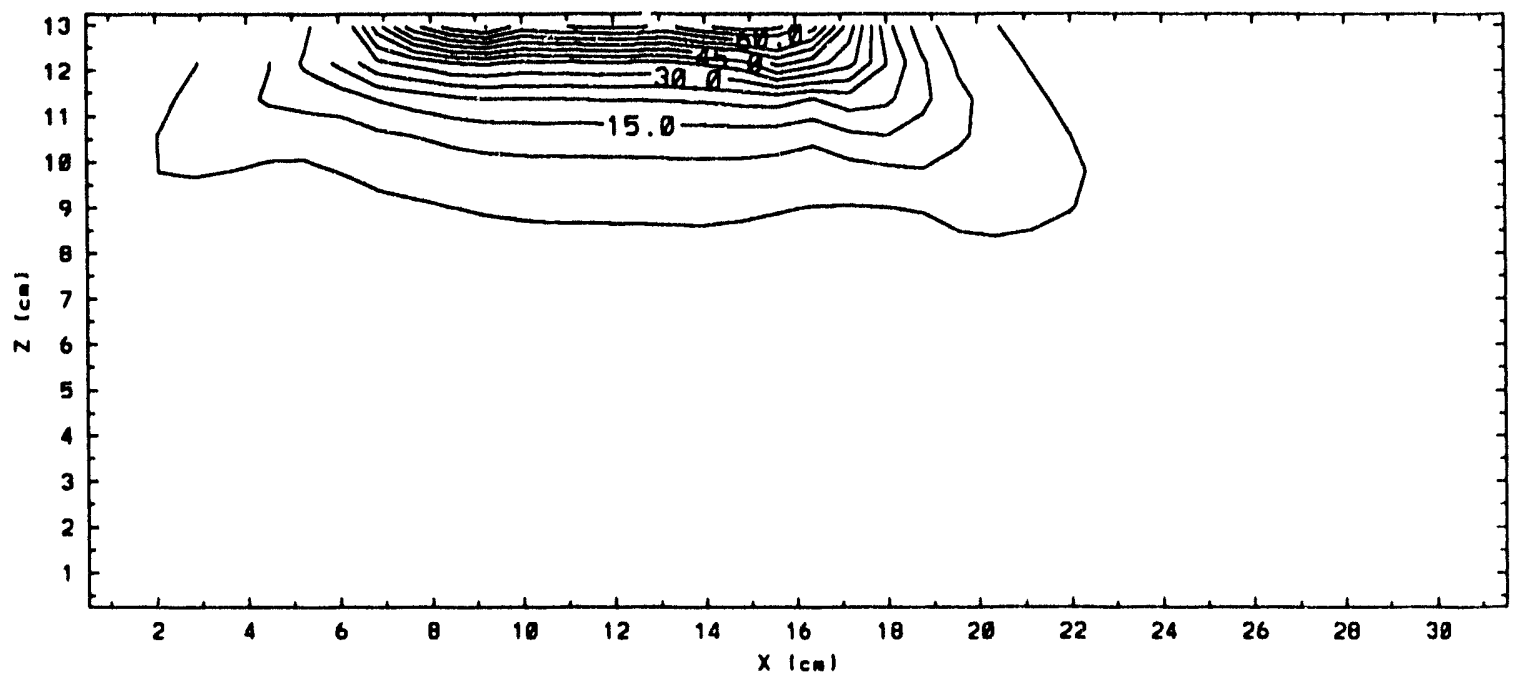

Figure F-41. Fast neutron flux contours at $y=-4.5 \mathrm{~cm}$ for the heterogeneous case with the $5 \mathrm{~cm} \times 10 \mathrm{~cm}$ beam aperture. Percentage contours are shown normalized to a peak fast flux of $1.26 \times 10^{7} \mathrm{n} / \mathrm{cm}^{2} / \mathrm{s}$. 


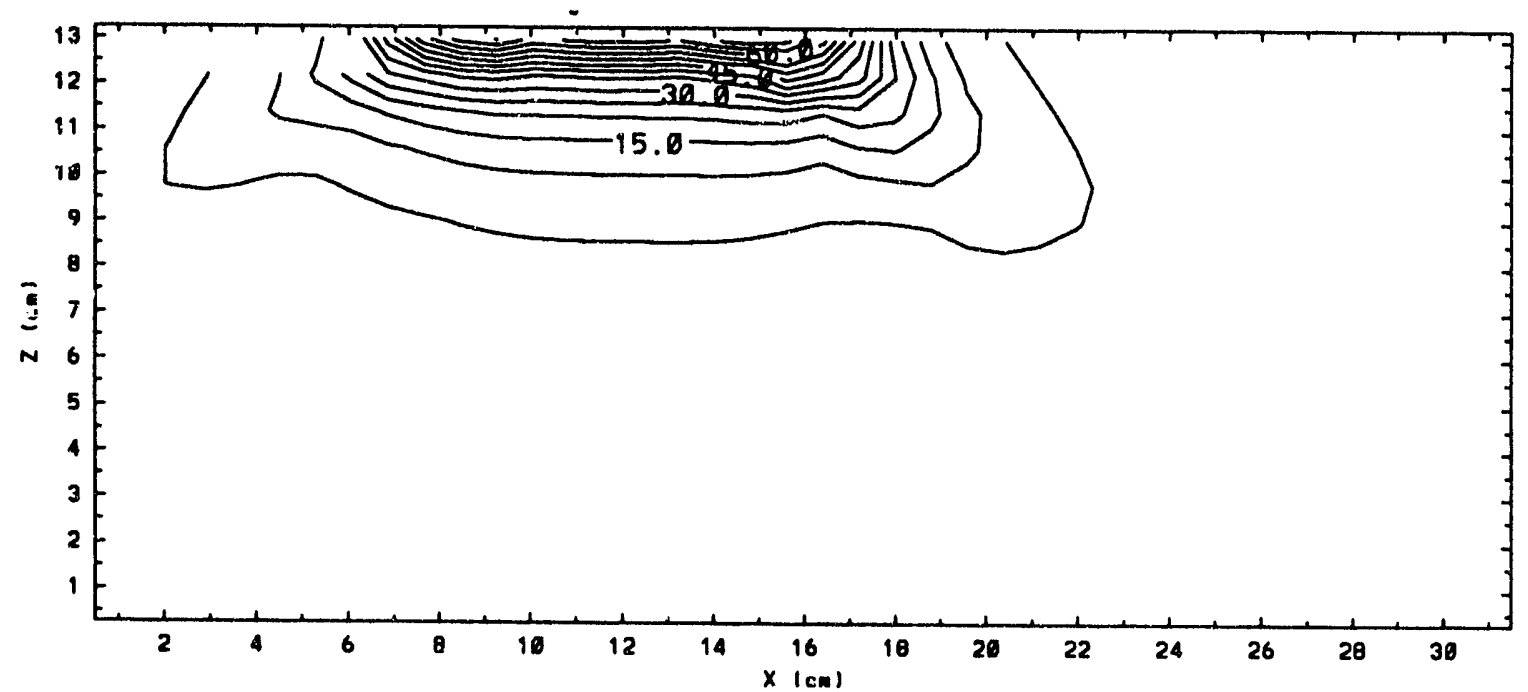

Figure F-42. Fast neutron flux contours at $y=-4.5 \mathrm{~cm}$ for the homogeneous case with the $5 \mathrm{~cm} \times 10 \mathrm{~cm}$ beam aperture. Percentage contours are shown normalized to a peak fast flux of $1.27 \times 10^{7} \mathrm{n} / \mathrm{cm}^{2} / \mathrm{s}$. 


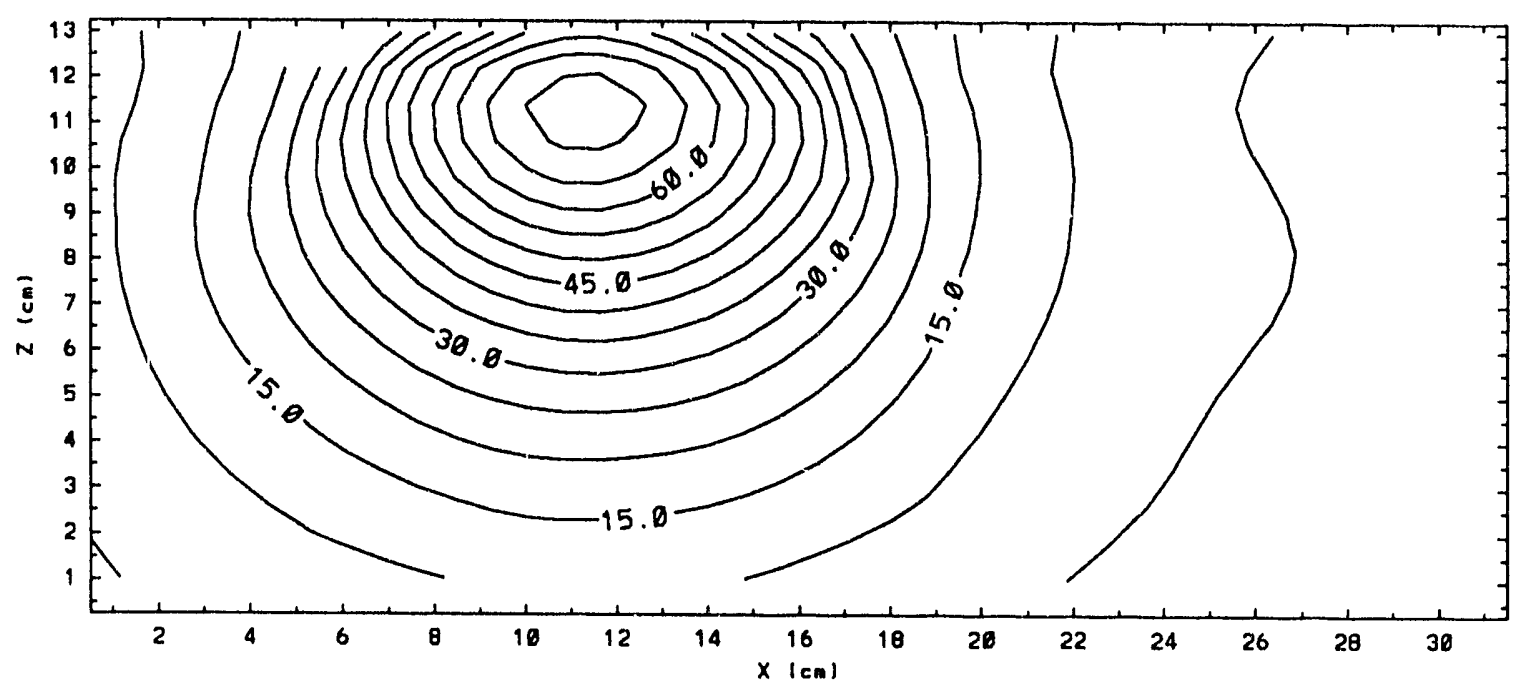

Figu-e F-43. Gamma flux contours at $y=-4.5 \mathrm{~cm}$ for the heterogeneous case with the $5 \mathrm{~cm} \times 10 \mathrm{~cm}$ beam aperture. Percentage contours are shown normalized to a peak gamma flux of $2.98 \times 10^{7}$ gammas $/ \mathrm{cm}^{2} / \mathrm{s}$. 


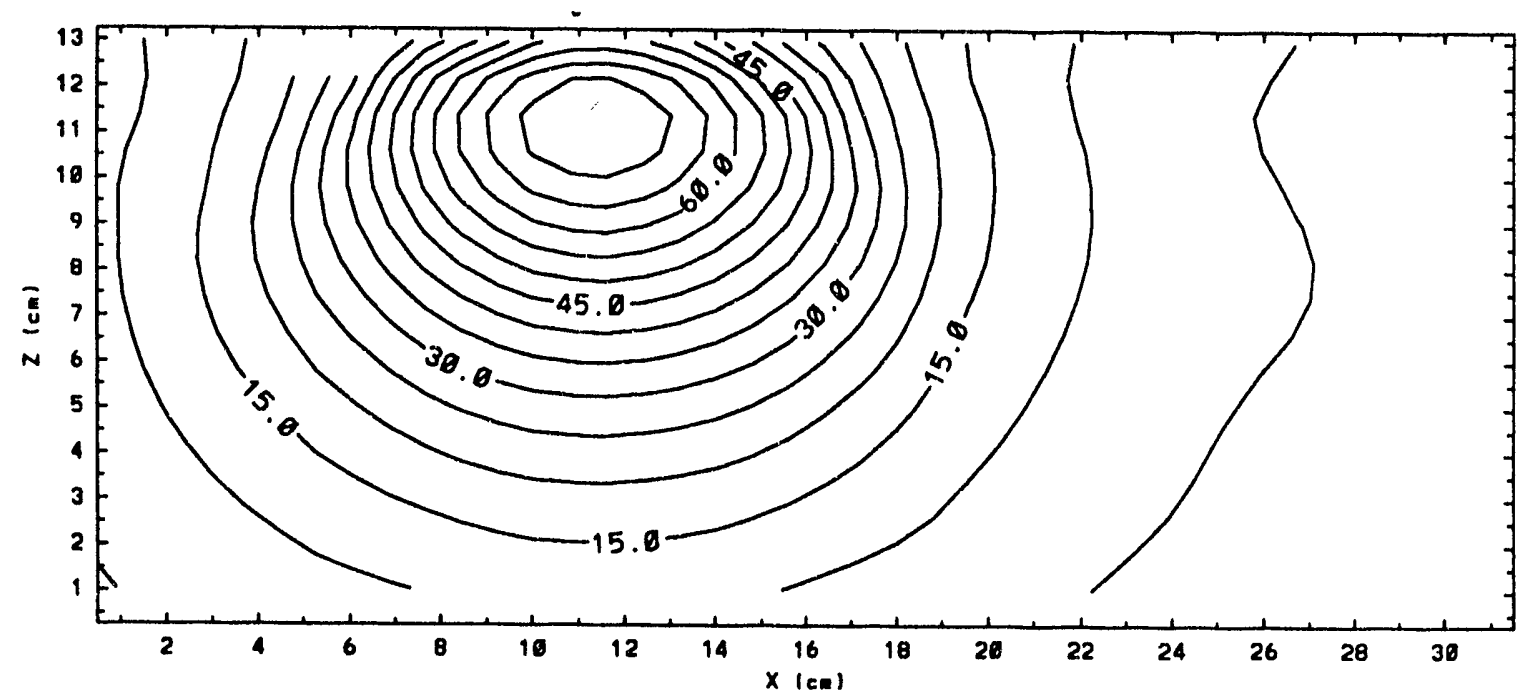

Figure F-44. Gamma flux contours at $y=-4.5 \mathrm{~cm}$ for the homogeneous case with the $5 \mathrm{~cm} \times 10 \mathrm{~cm}$ beam aperture. Percentage contours are shown normalized to a peak gamma flux of $2.97 \times 10^{7}$ gammas $/ \mathrm{cm}^{2} / \mathrm{s}$. 
Figures F-45 and F-46 show the thermal neutron flux at $y=2.5 \mathrm{~cm}$. The peak flux for both cases is only $45 \%$ of the total peak flux. The peak is slightly smaller in the heterogeneous case. Differences can also be seen between the spatial distributions. In the rostral direction, where the neutrons are traveling through bone, a 3-4\% decrease in flux is noted because of inhomogeneities. The spatial distributions are similar in all other regions (ventral and caudal directions).

The fast neutron fluxes at $y=2.5 \mathrm{~cm}$ are shown in Figures $F-47$ and F-48. These contours are only $15 \%$ of the total peak fast flux. An effect is seen due to heterogeneities, although the difference in dose is not significant, especially when compared with the heterogeneity effects at the midplane. There is streaming of neutrons due to bone which results in a penetration of the $5 \%$ contour 5 $\mathrm{mm}$ deeper than that seen in the homogeneous case.

The spatial distributions of the gamma flux for both cases are depicted in Figures F-49 and F-50. The peak fluxes at the $y=2.5 \mathrm{~cm}$ plane are $65 \%$ of the total peak flux. The contours differ by approximately 3-5\% in the rostral direction due to bone and the nasal cavity as seen in the thermal flux distributions. 


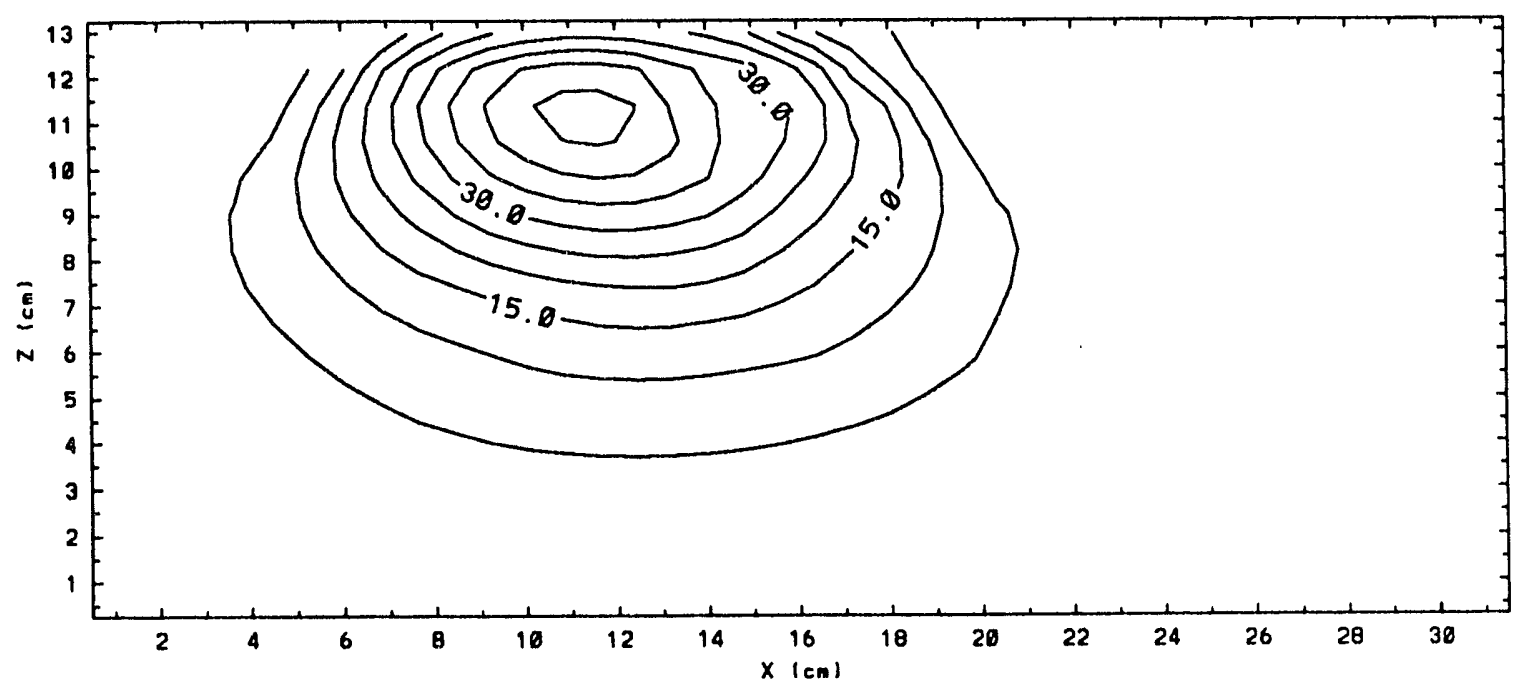

Figure F-45. Thermal neutron flux contours at $y=2.5 \mathrm{~cm}$ for the heterogeneous case with the $5 \mathrm{~cm} \times 10 \mathrm{~cm}$ beam aperture. Percentage contours are shown normalized to a peak thermal flux of $4.14 \times 10^{8} \mathrm{n} / \mathrm{cm}^{2} / \mathrm{s}$. 
\

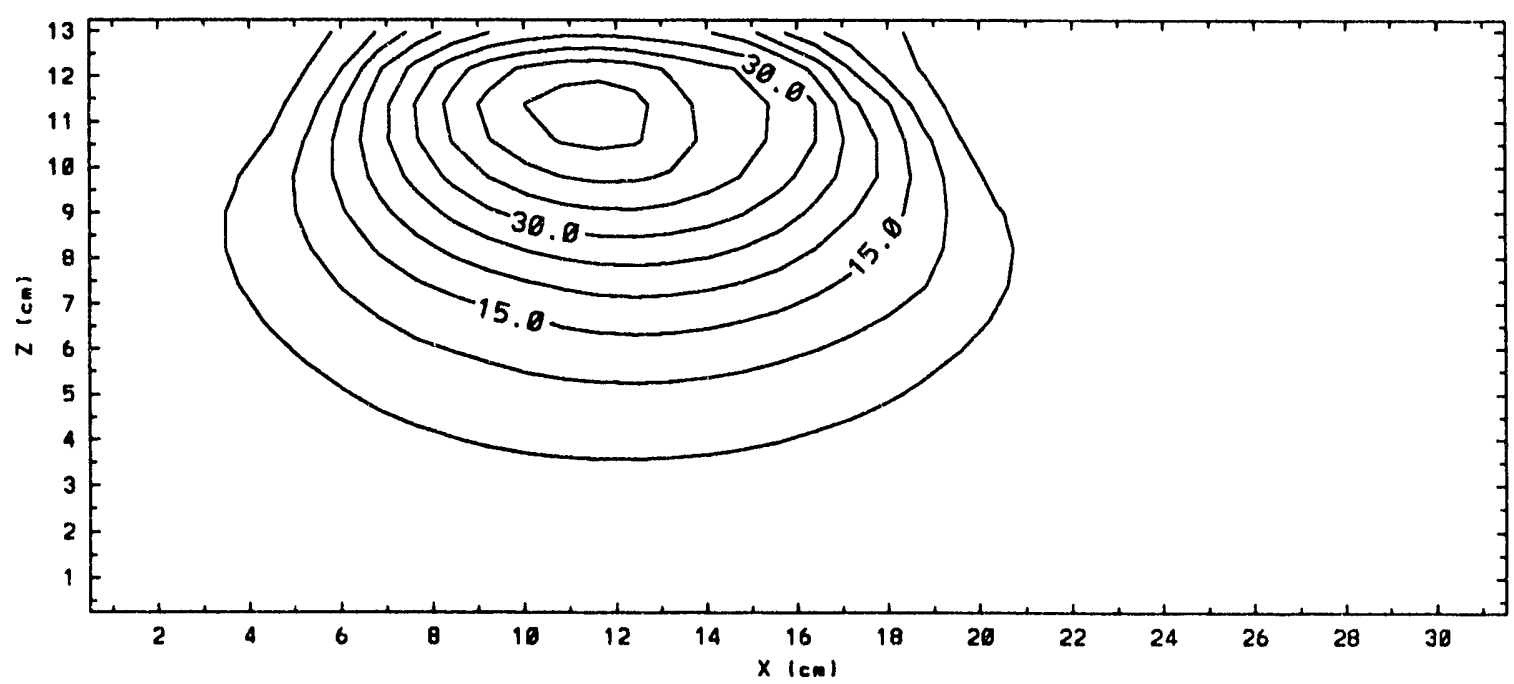

Figure F-46. Thermal neutron flux contours at $y=2.5 \mathrm{~cm}$ for the homogeneous case with the $5 \mathrm{~cm} \times 10 \mathrm{~cm}$ beam aperture. Percentage contours are shown normalized to a peak thermal flux of $4.04 \times 10^{8} \mathrm{n} / \mathrm{cm}^{2} / \mathrm{s}$. 


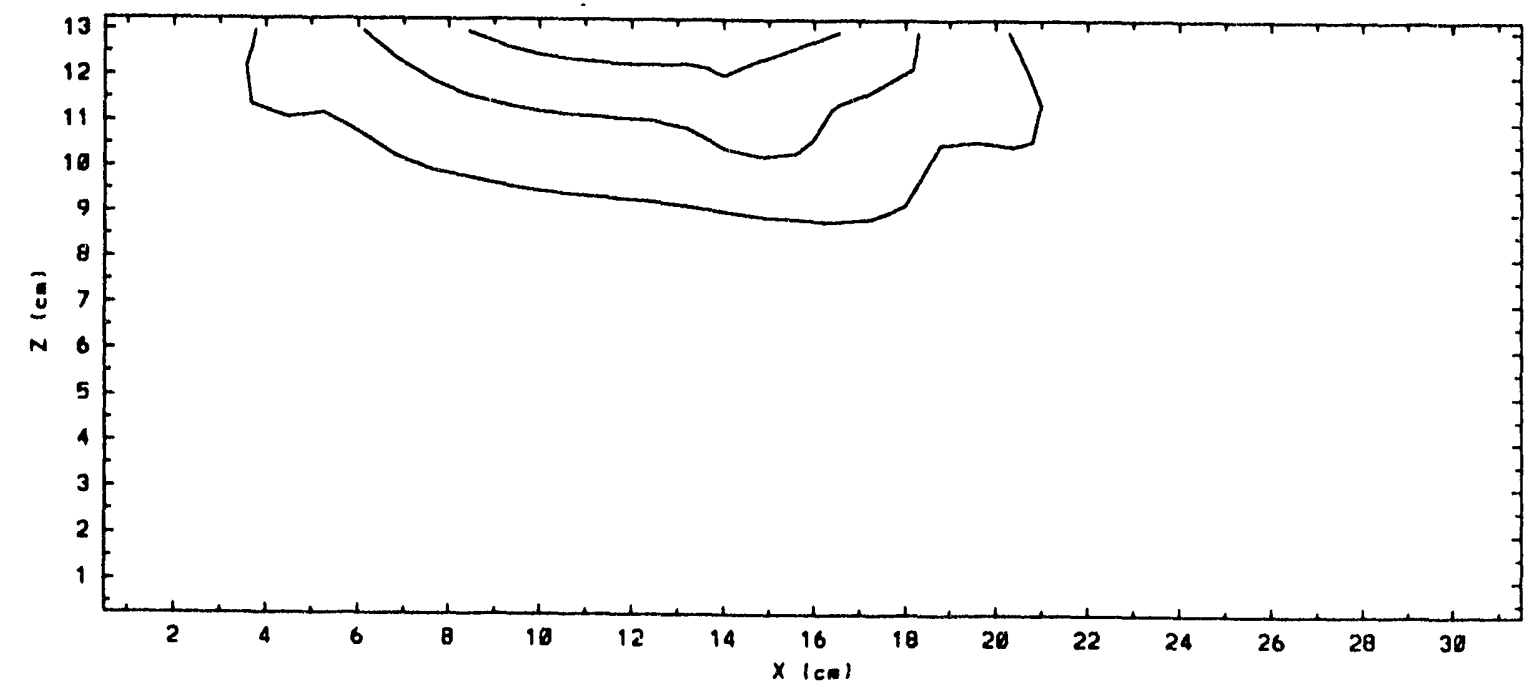

Figure $\mathbf{p - 4 7}$. Fast neutron flux contours at $y=2.5 \mathrm{~cm}$ for the heterogeneous case with the $5 \mathrm{~cm} \times 10 \mathrm{~cm}$ beam aperture. Percentage contours are shown normalized to a peak fast flux of $1.26 \times 10^{7} \mathrm{n} / \mathrm{cm}^{2} / \mathrm{s}$. 
$\checkmark$

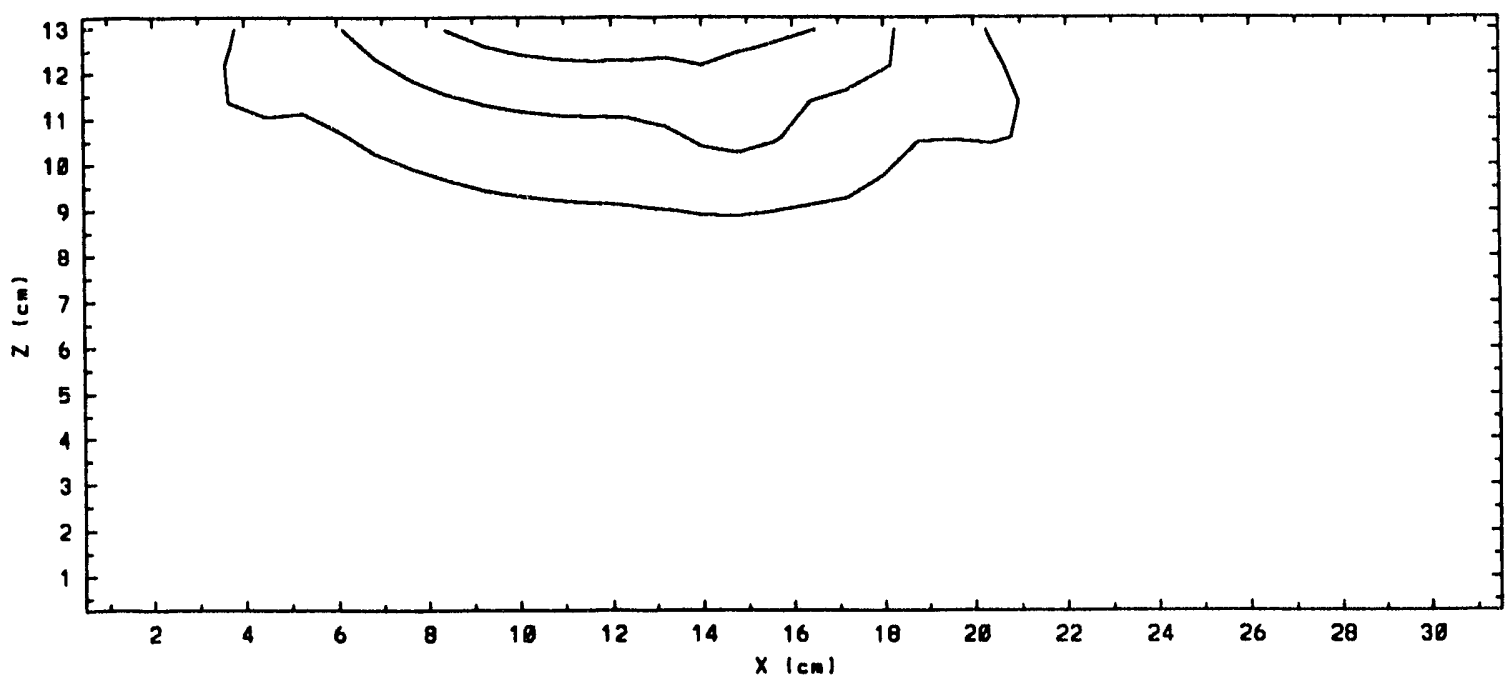

Figure F-48. Fast neutron flux contours at $y=2.5 \mathrm{~cm}$ for the homogeneous case with the $5 \mathrm{~cm} \times 10 \mathrm{~cm}$ beam aperture. Percentage contours are shown normalized to a peak fast flux of $1.27 \times 10^{7} \mathrm{n} / \mathrm{cm}^{2} / \mathrm{s}$. 


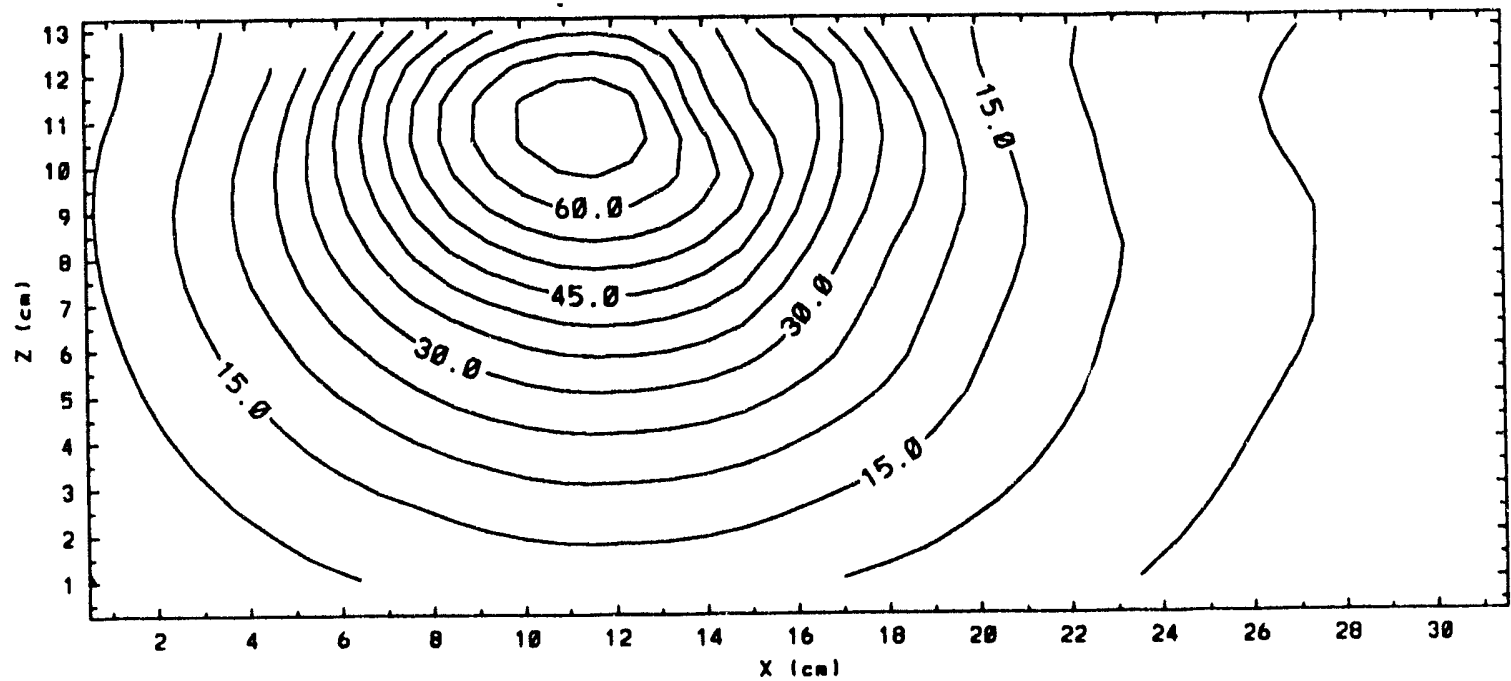

Figure F-49. Gamma flux contours at $y=2.5 \mathrm{~cm}$ for the heterogeneous case with the $5 \mathrm{~cm} \times 10 \mathrm{~cm}$ beam aperture. Percentage contours are shown normalized to a peak gamma flux of $2.98 \times 10^{7}$ gammas $/ \mathrm{cm}^{2} / \mathrm{s}$. 
-

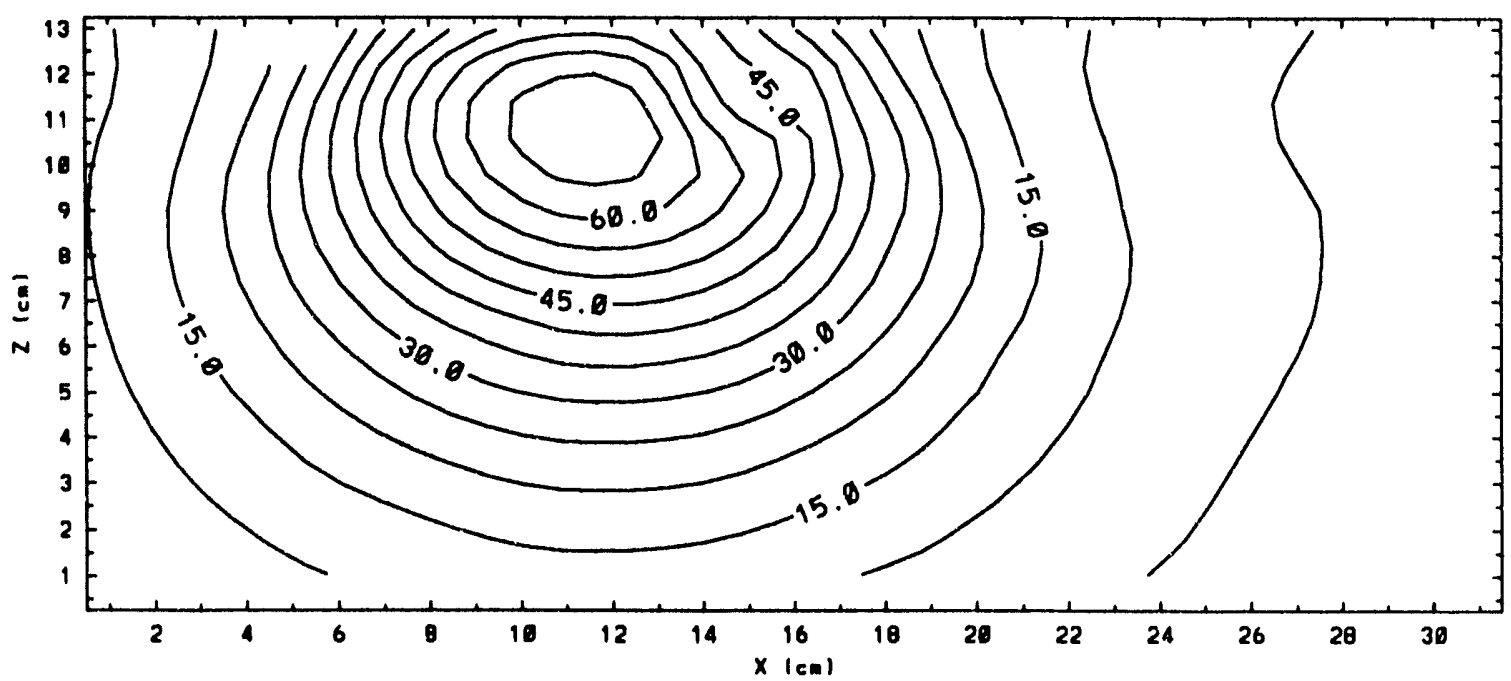

Figure F-50. Gamma flux contours at $y=2.5 \mathrm{~cm}$ for the homogeneous case with the $5 \mathrm{~cm} \times 10 \mathrm{~cm}$ beam aperture. Percentage contours are shown normalized to a peak gamma flux of $2.97 \times 10^{7}$ gammas $/ \mathrm{cm}^{2} / \mathrm{s}$. 
The flux contours through various axial planes offer a different way to look at the data. Planes through $x=7.25$ $\mathrm{cm}, \mathrm{x}=12.25 \mathrm{~cm}$, and $\mathrm{x}=17.25 \mathrm{~cm}$ are examined here. All of the peak fluxes are in the left half of the head (remember that the beam extends from $y=-5 \mathrm{~cm}$ to $y=0 \mathrm{~cm}$ ).

Figures F-51 and F-52 contain the thermal flux distributions at $x=7.25 \mathrm{~cm}$. The peak is only $50 z$ of the total peak. The peak of the heterogeneous case is smaller relative to the homogeneous peak. There is approximately a 28 decrease in flux to the right side (positive $y$ ) of the dog head. The fast neutron fluxes are presented in Figures F-53 and F-54. The spatial distributions are similar as expected. There is a slight decrease (less than 18) in the flux in the left hemisphere due to inhomogeneities. The gamma flux contours are shown in Figures F-55 and F-56. similar to the thermal flux trends, a 1-28 decrease in the gamma flux is seen in the heterogeneous case.

The axial plane at $x=12.25 \mathrm{~cm}$ consists mainly of muscle and the brain surrounded by some parts of the skull. The fast neutron fluxes at this plane are shown in Figures F-57 and F-58. The spatial distributions are similar for both cases. Figures F-59 and F-60 depict the gamma fluxes at $\mathrm{x}=12.25 \mathrm{~cm}$. The spatial distributions are similar, although there is a $1-28$ decrease in the gamma flux for the 


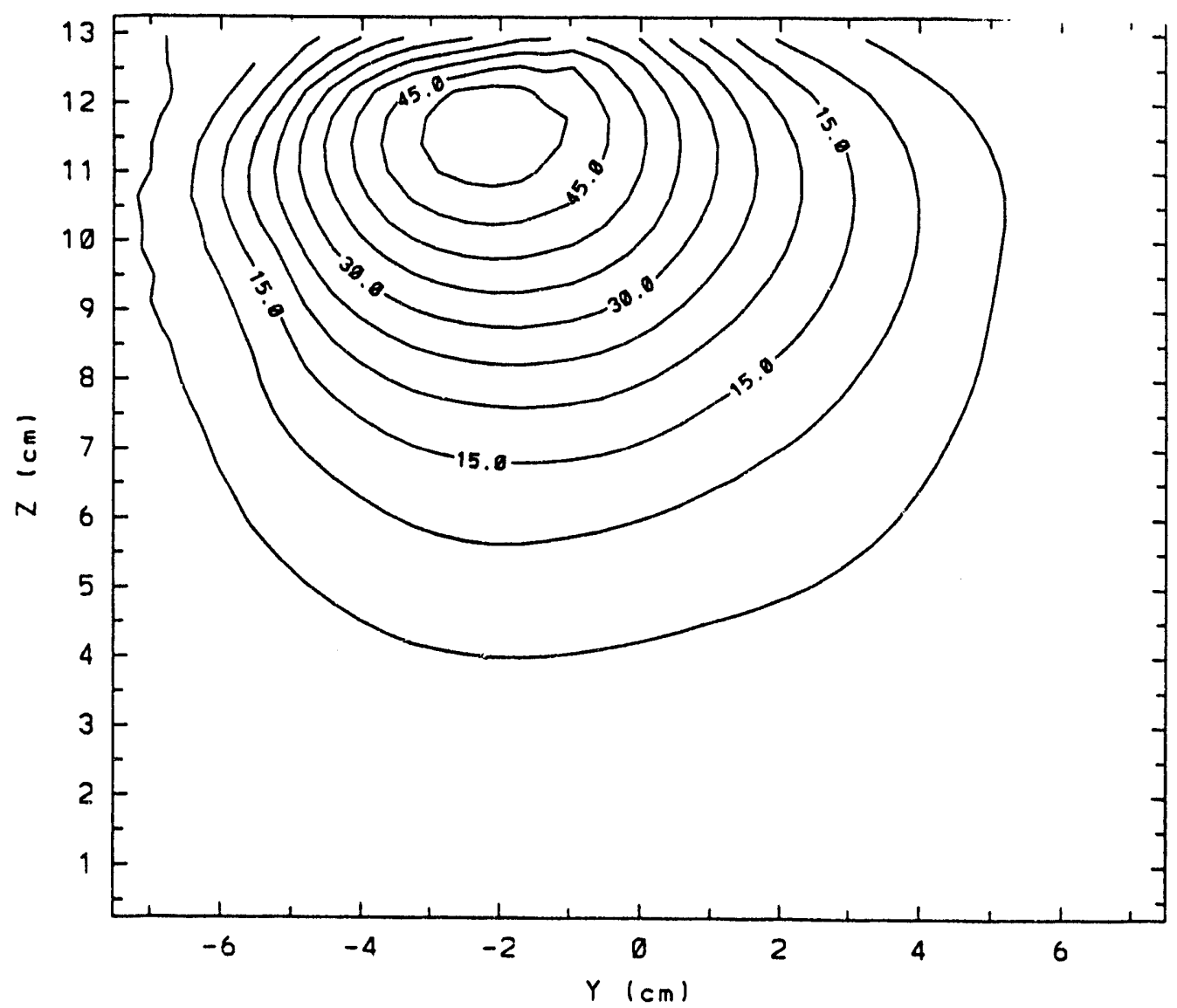

Figure F-51. Thermal neutron flux contours at $x=7.25 \mathrm{~cm}$ for the heterogeneous case with the $5 \mathrm{~cm} \times 10 \mathrm{~cm}$ beam aperture. Percentage contours are shown normalized to a peak thermal flux of $4.14 \times 10^{8} \mathrm{n} / \mathrm{cm}^{2} / \mathrm{s}$. 


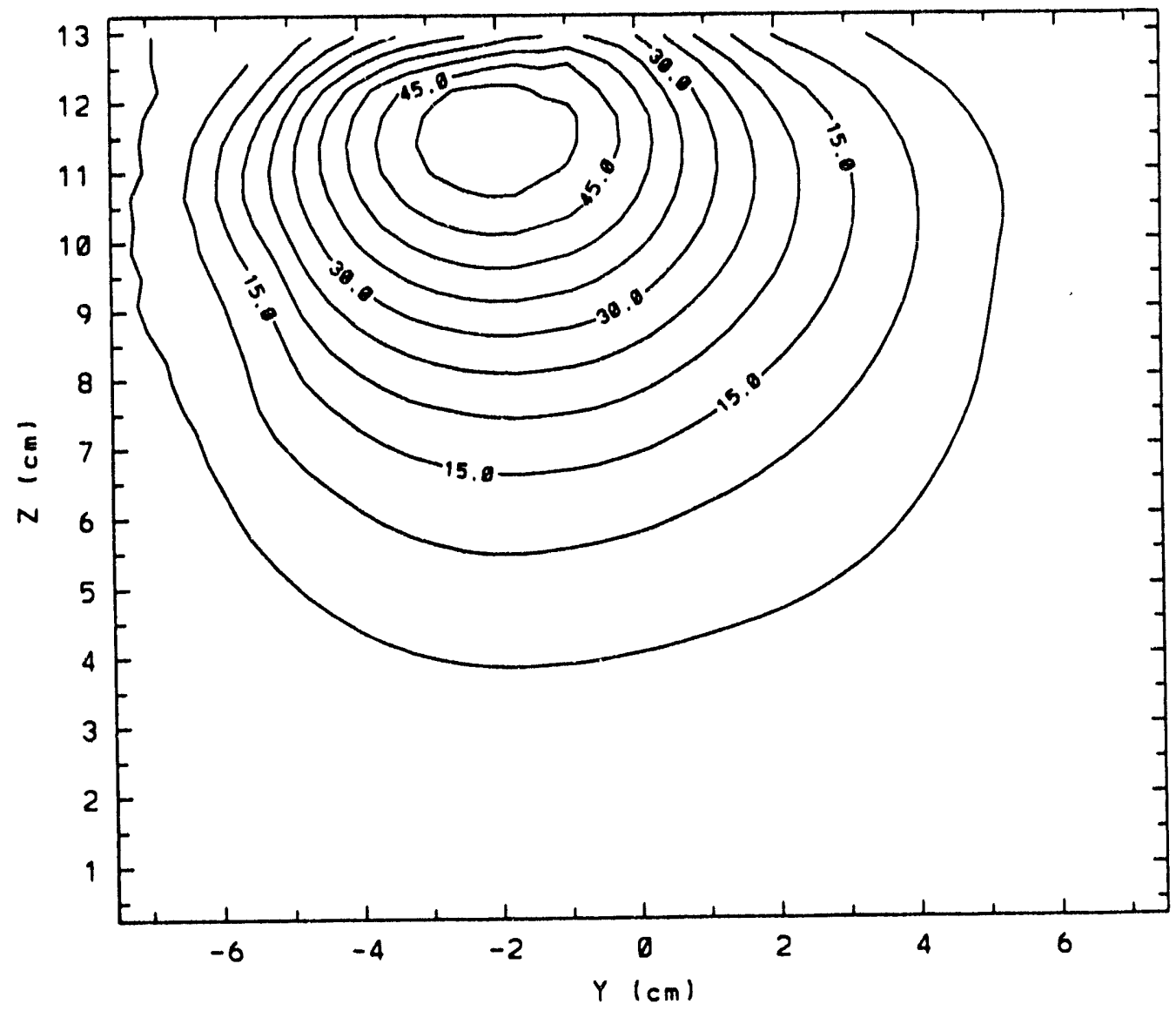

$\rightarrow$

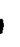

Figure F-52. Thermal neutron flux contours at $x=7.25 \mathrm{~cm}$ for the homogeneous case with the $5 \mathrm{~cm} \times 10 \mathrm{~cm}$ beam aperture. Percentage contours are shown normalized to a peak thermal flux of $4.04 \times 10^{8} \mathrm{n} / \mathrm{cm}^{2} / \mathrm{s}$. 


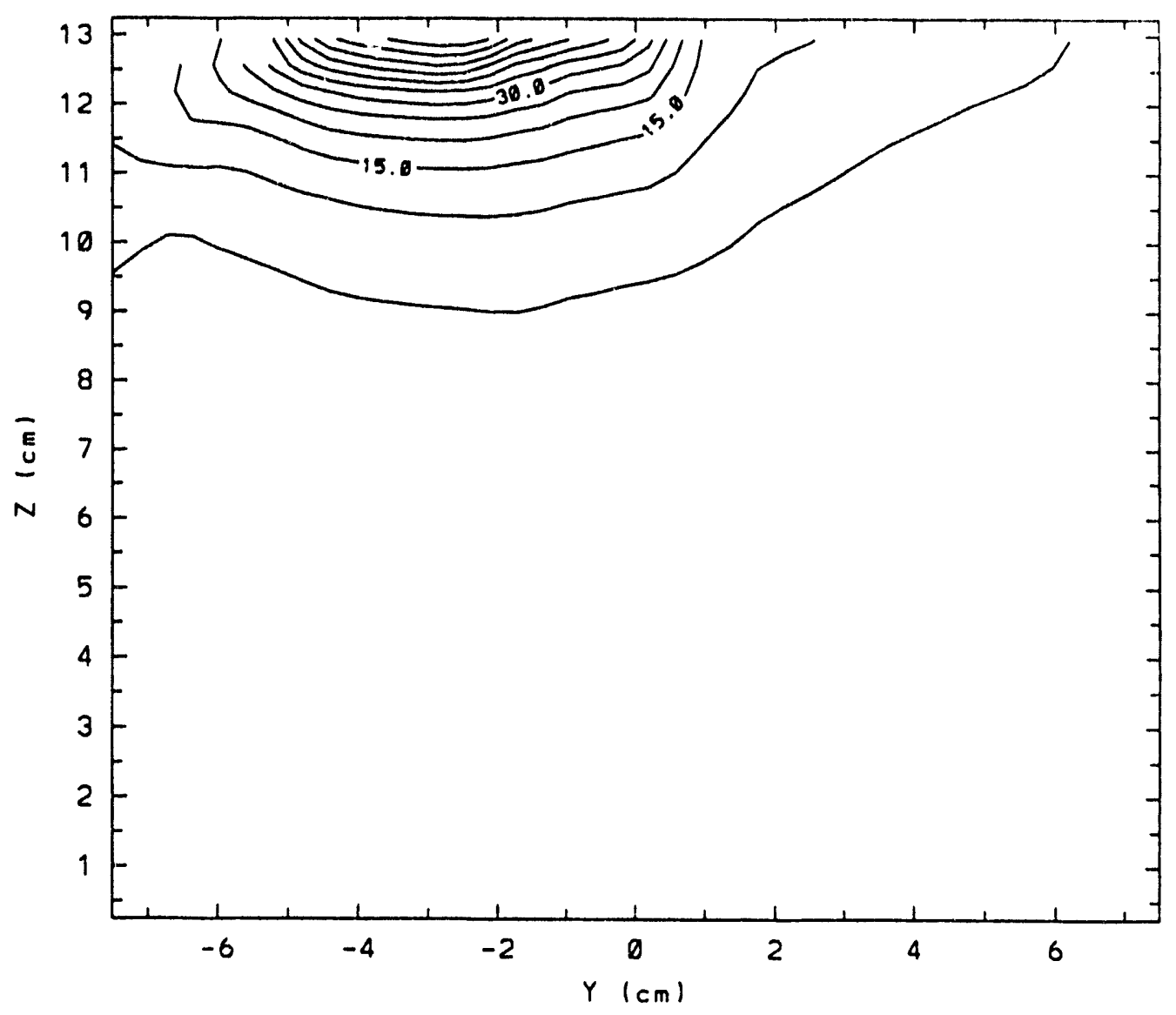

Figure F-53. Fast neutron flux contours at $x=7.25 \mathrm{~cm}$ for the heterogeneous case with the $5 \mathrm{~cm} \times 10 \mathrm{~cm}$ beam aperture. Percentage contours are shown normalized to a peak fast flux of $1.26 \times 10^{7} \mathrm{n} / \mathrm{cm}^{2} / \mathrm{s}$. 


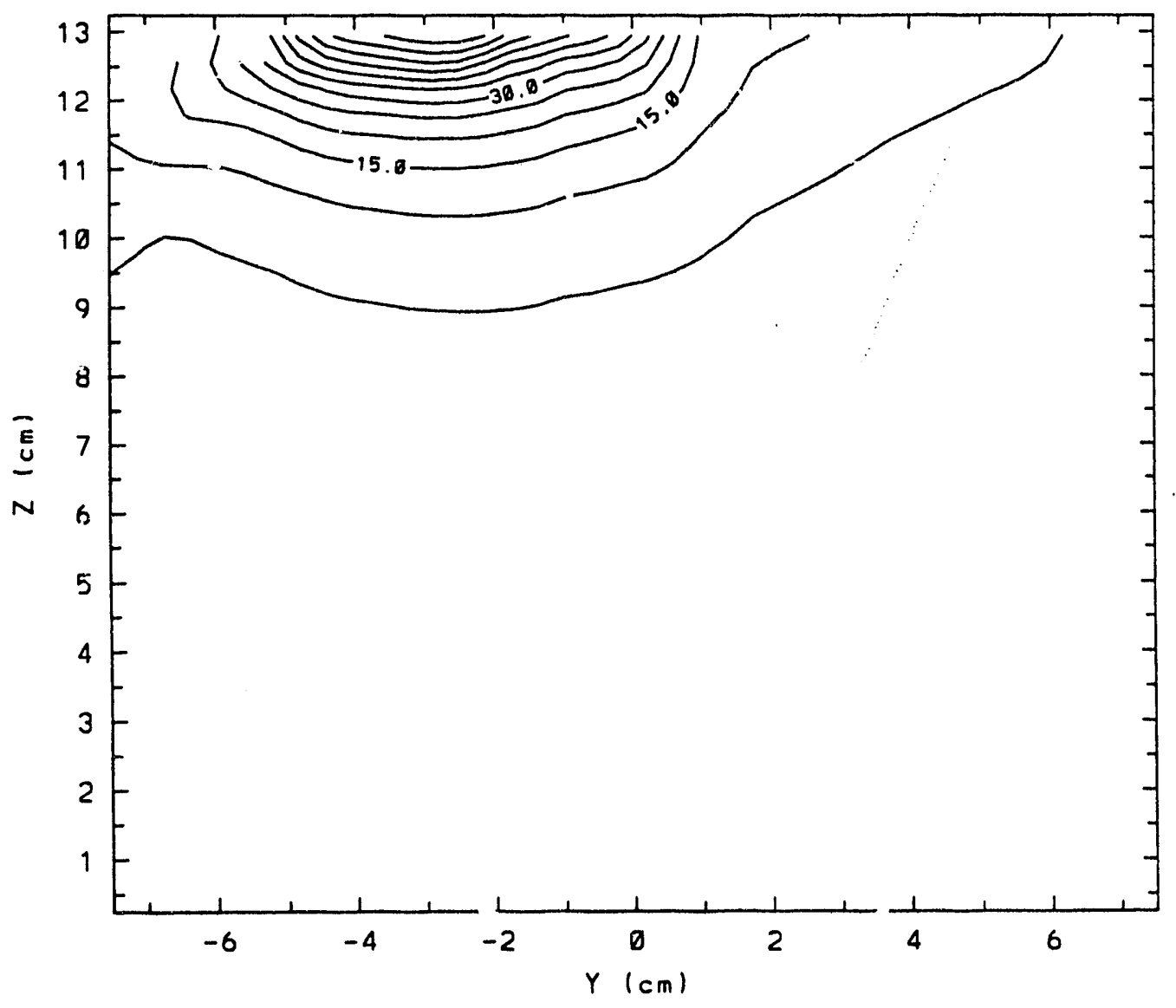

Figure F-54. Fast neutron flux contours at $x=7.25 \mathrm{~cm}$ for the homogeneous case with the $5 \mathrm{~cm} \times 10 \mathrm{~cm}$ beam aperture. Percentage contours are shown normalized to a peak fast flux of $2.27 \times 10^{7} \mathrm{n} / \mathrm{cm}^{2} / \mathrm{s}$. 


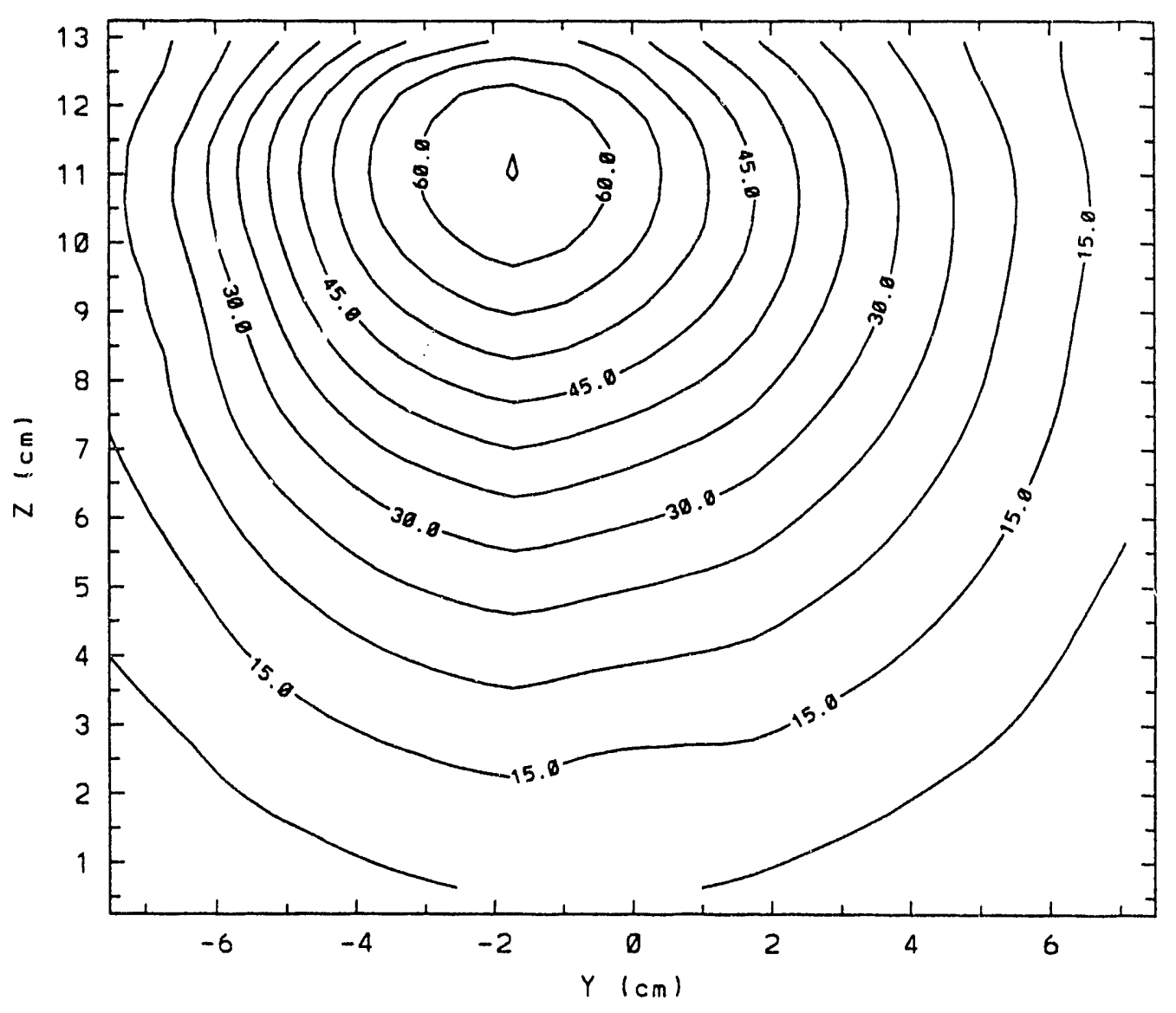

Figure F-55. Gamma flux contours at $x=7.25 \mathrm{~cm}$ for the heterogeneous case with the $5 \mathrm{~cm} \times 10 \mathrm{~cm}$ beam aperture. Percentage contours are shown normalized to a peak gamma flux of $2.98 \times 10^{7}$ gammas $/ \mathrm{cm}^{2} / \mathrm{s}$. 


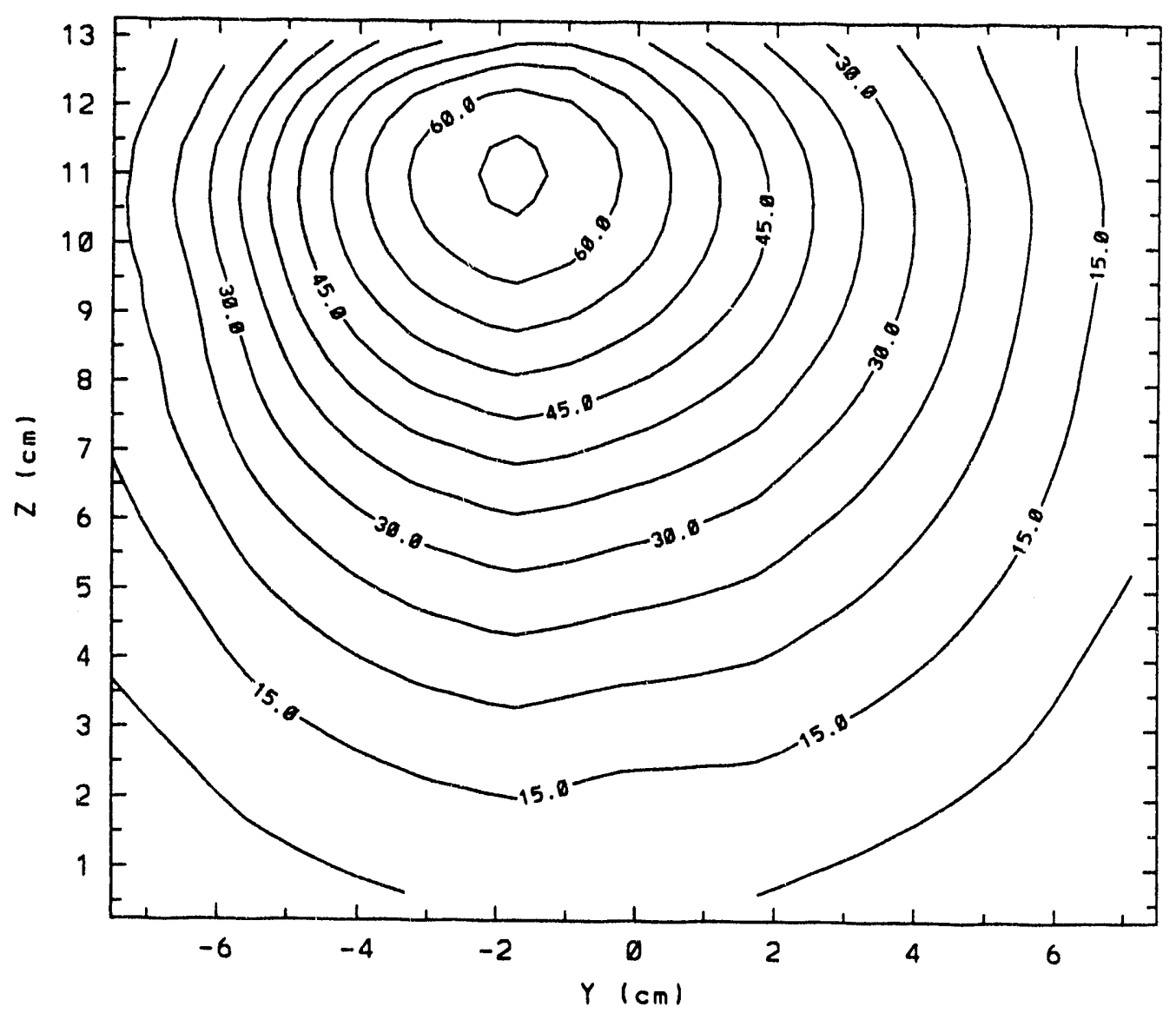

Figure F-56. Gamma flux contours at $x=7.25 \mathrm{~cm}$ for the homogeneous case with the $5 \mathrm{~cm} \times 10 \mathrm{~cm}$ beam aperture. Percentage contours are shown normalized to a peak gamma flux of $2.97 \times 10^{7}$ gammas $/ \mathrm{cm}^{2} / \mathrm{s}$. 


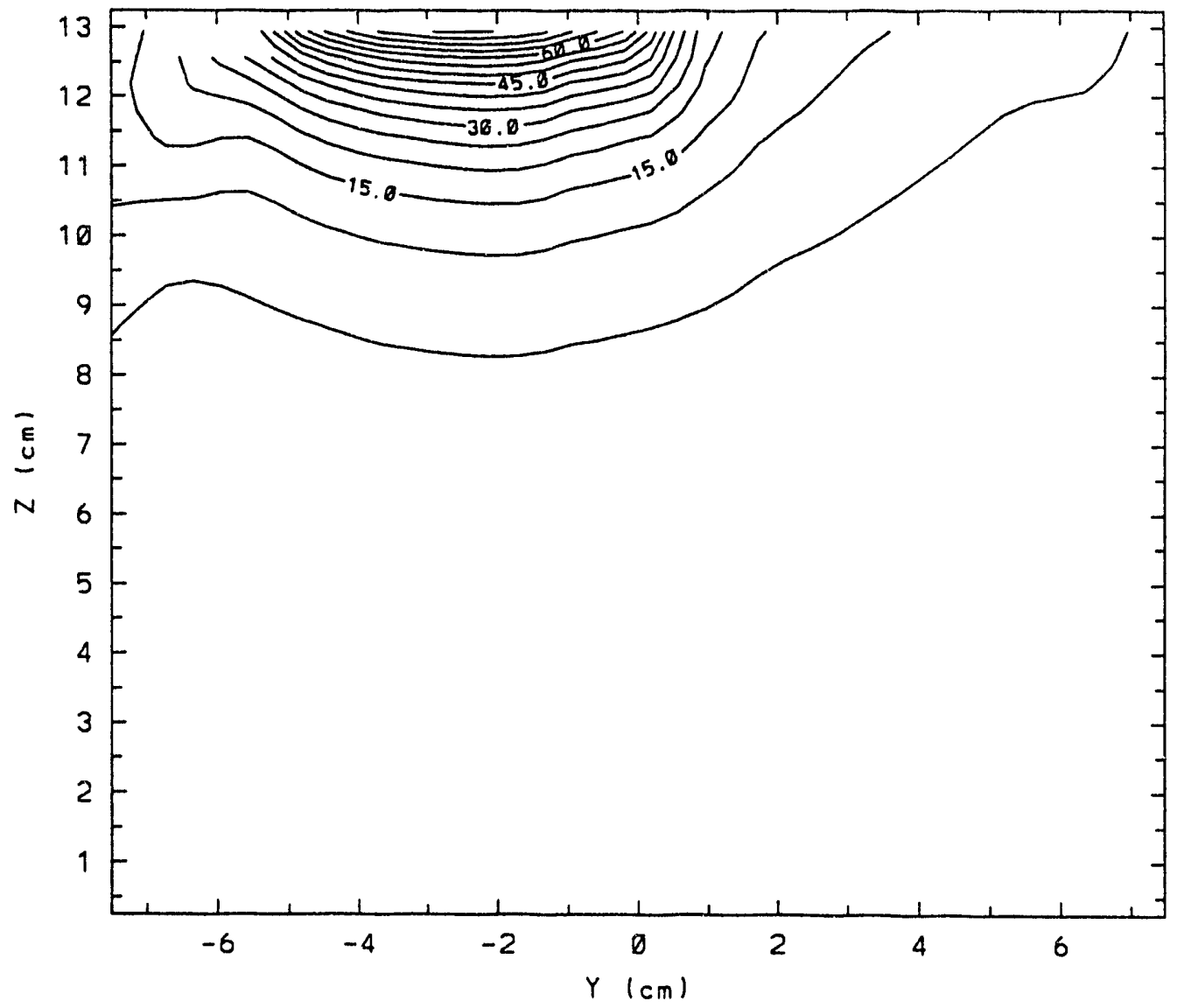

Figure F-57. Fast neutron flux contours at $x=12.25 \mathrm{~cm}$ for the heterogerieous case with the $5 \mathrm{~cm} \times 10 \mathrm{~cm}$ beam aperture. Percentage contours are shown normalized to a peak fast flux of $1.26 \times 10^{7} \mathrm{n} / \mathrm{cm}^{2} / \mathrm{s}$. 


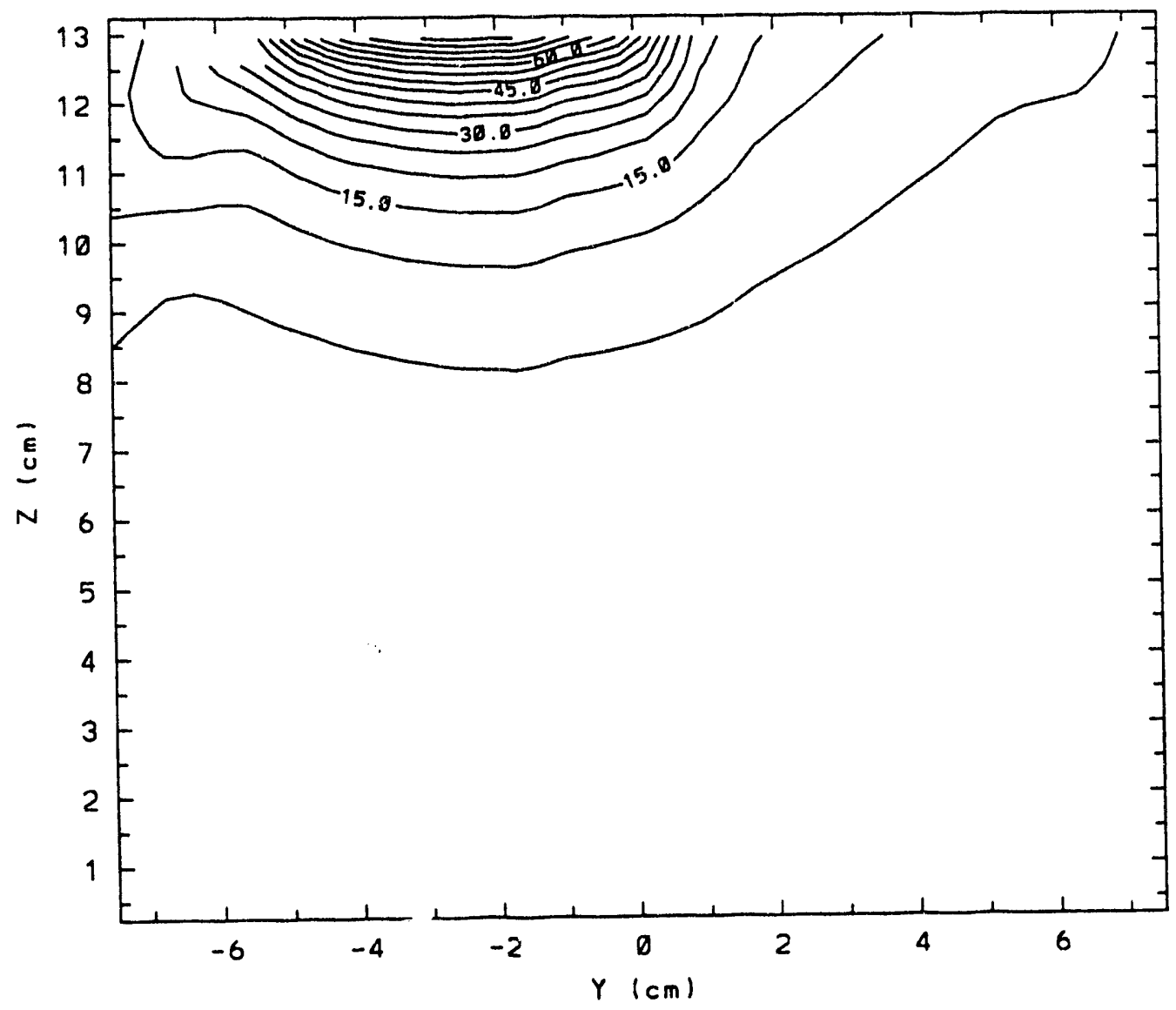

7

Figure F-58. Fast neutron flux contours at $x=12.25 \mathrm{~cm}$ for the homogeneous case with the $5 \mathrm{~cm} \times 10 \mathrm{~cm}$ beam aperture. percentage contours are shown normalized to a peak fast flux of $1.27 \times 10^{7} \mathrm{n} / \mathrm{cm}^{2} / \mathrm{s}$. 


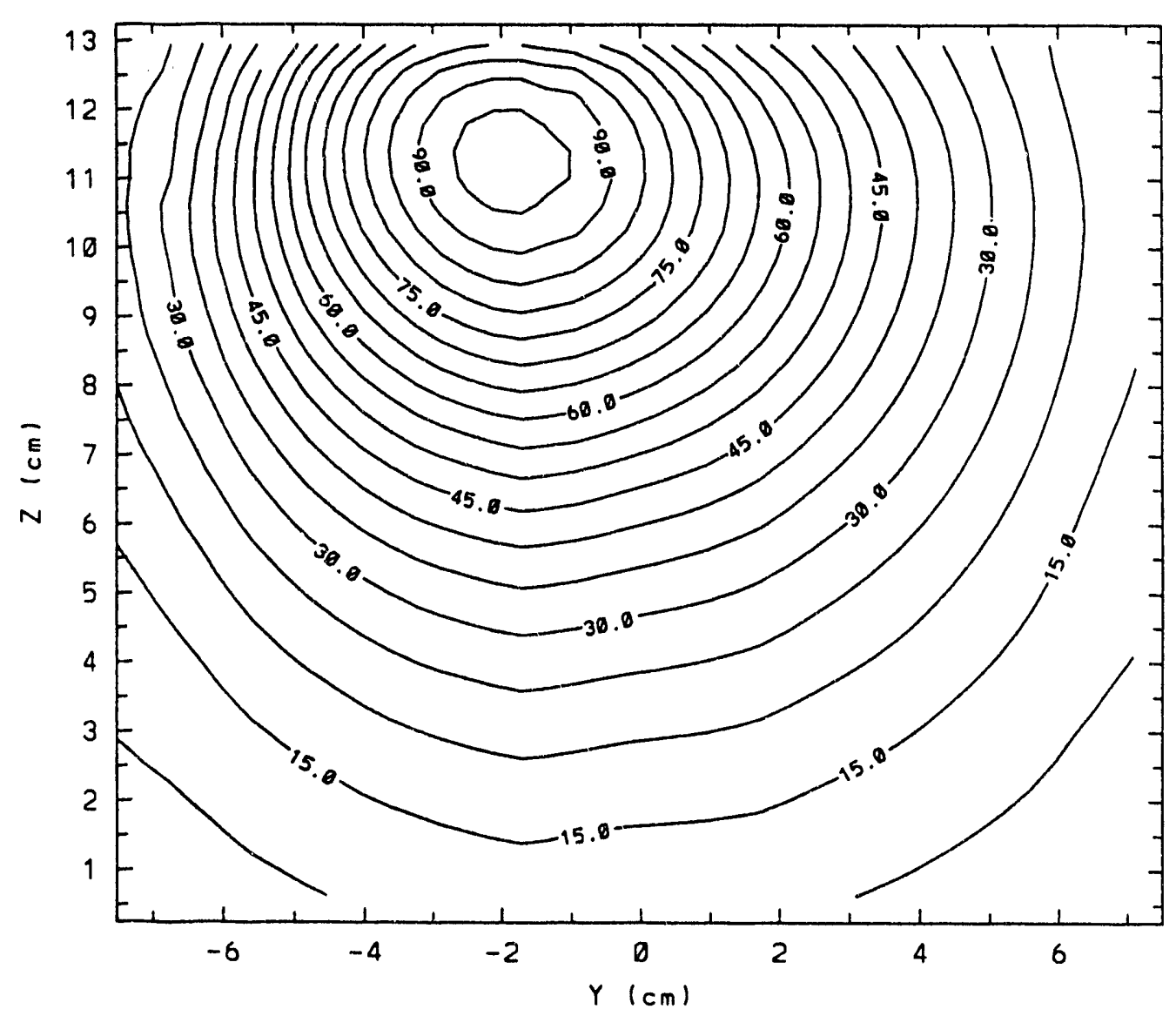

Figure F-59. Gamma flux contours at $x=12.25 \mathrm{~cm}$ for the heterogeneous case with the $5 \mathrm{~cm} \times 10 \mathrm{~cm}$ beam aperture. Percentage contours are shown normalized to a peak gamma flux of $2.98 \times 10^{7}$ gammas $/ \mathrm{cm}^{2} / \mathrm{s}$. 


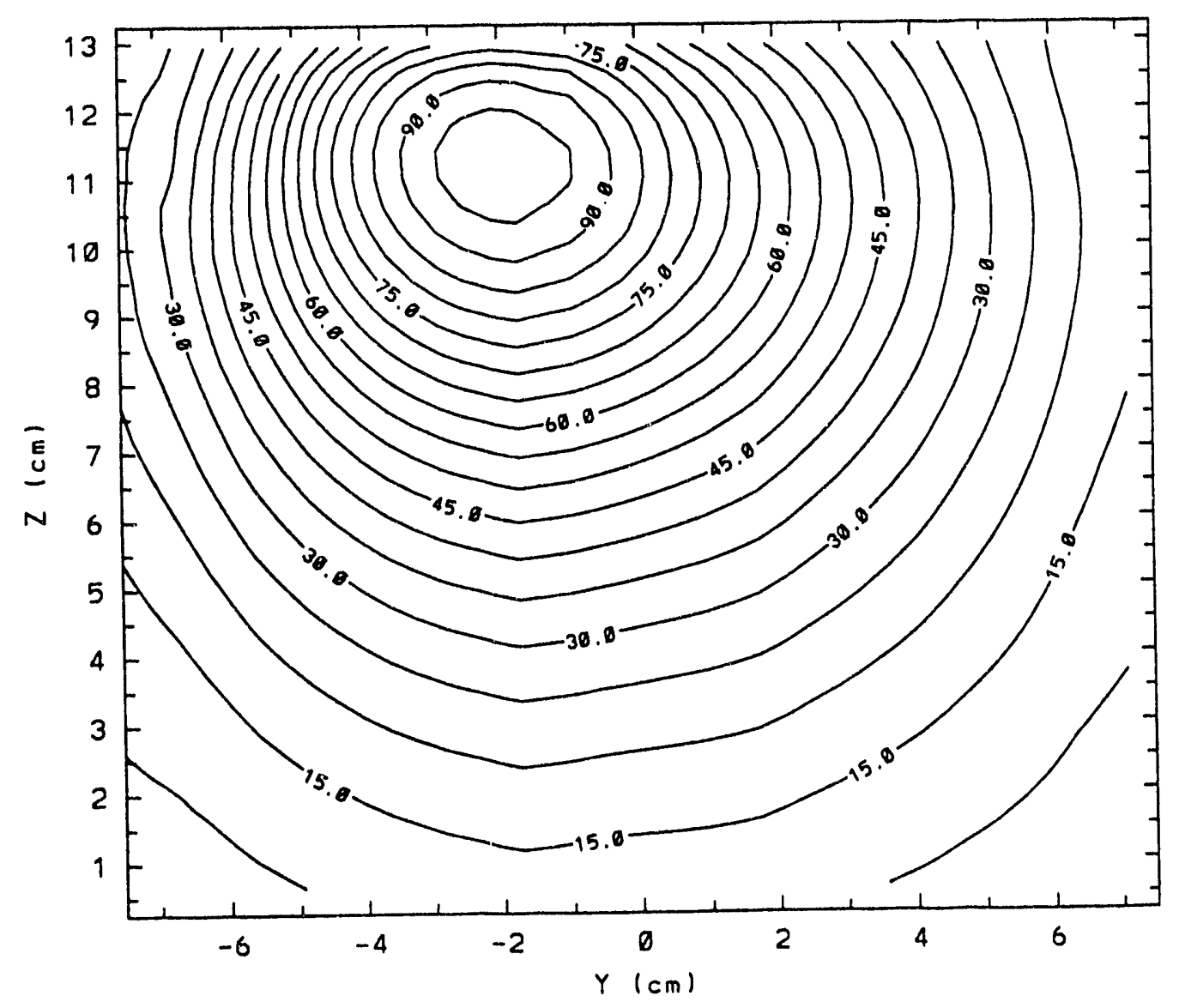

Figure F-60. Gamma flux contours at $x=12.25 \mathrm{~cm}$ for the homogeneous case with the $5 \mathrm{~cm} \times 10 \mathrm{~cm}$ beam aperture. Percentage contours are shown normalized to a peak gamma flux of $2.97 \times 10^{7}$ gammas $/ \mathrm{cm}^{2} / \mathrm{s}$. 
heterogeneous case with all contours shifted due to the effects of bone.

The fast neutron and gamma fluxes can be examined at $x$ $=17.25 \mathrm{~cm}$. The regions in this axial plane include the eyes, bone, nasal cavity, and void. Figures F-61 and F-62 show the spatial distributions of the fast neutron flux. streaming of the $5 \%$ contour amounting to deeper penetration of $1 \mathrm{~cm}$ through the nasal cavity is seen underneath the beam port in the heterogeneous case. Also due to inhomogeneities, the $10 \%$ contour penetrates a little deeper. The spatial distributions for the remaining contours are similar for both cases. Figures F-63 and F-64 present the gamma fluxes at $x=17.25 \mathrm{~cm}$. The shapes of the contours differ significantly, although this will not have an effect on therapy. The contours for the homogeneous cases are larger than those for the heterogeneous case. In some areas, the flux decreases 5-8\% due to heterogeneities such as bone and the sinuses.

Finally, the dorsal plane at $z=13.25 \mathrm{~cm}$ can be examined for the thermal and gamma fluxes. The fast neutron fluxes at this plane were presented in section 5.3. Figures F-65 and F-66 show the thermal neutron flux in the skin layer. The flux of the homogeneous case is slightly larger (less than 1\%). As expected, the flux is not as large as 


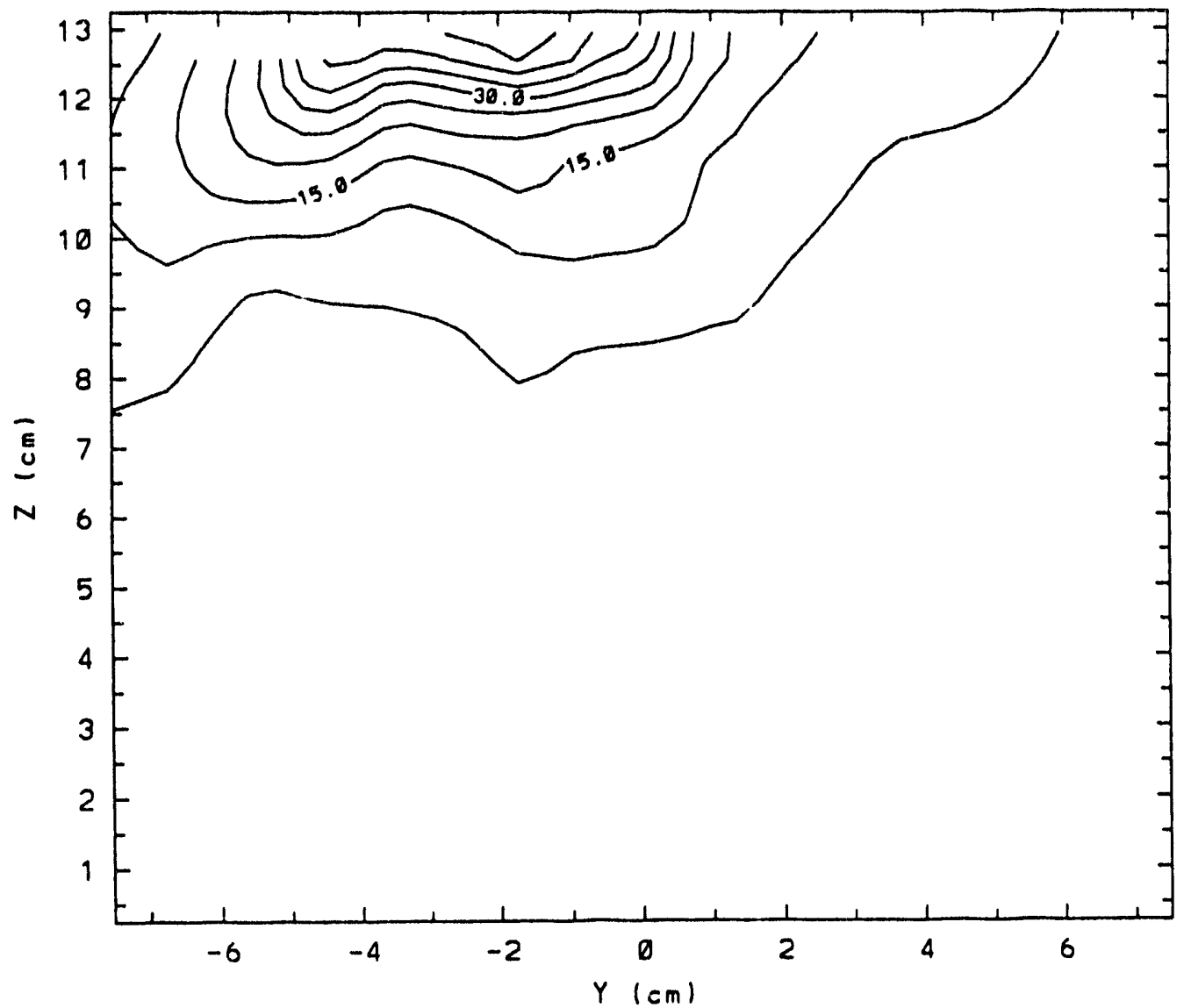

Figure F-61. Fast neutron flux contours at $x=17.25 \mathrm{~cm}$ for the heterogeneous case with the $5 \mathrm{~cm} \times 10 \mathrm{~cm}$ beam aperture. Percentage contours are shown normalized to a peak fast flux of $1.26 \times 10^{7} \mathrm{n} / \mathrm{cm}^{2} / \mathrm{s}$. 


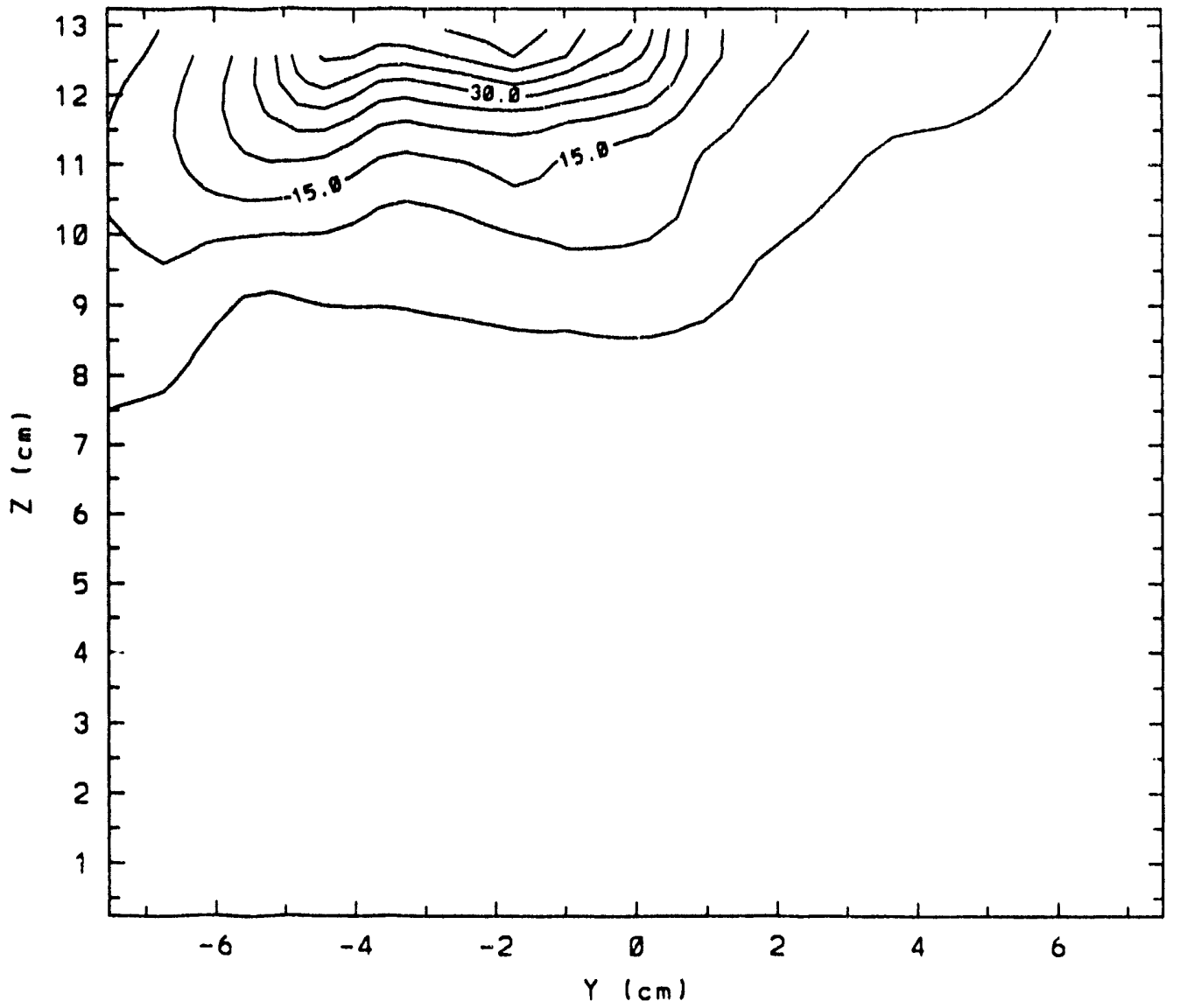

Figure F-62. Fast neutron flux contours at $x=17.25 \mathrm{~cm}$ for the homogeneous case with the $5 \mathrm{~cm} \times 10 \mathrm{~cm}$ beam aperture. Percentage contours are shown normalized to a peak fast flux of $1.27 \times 10^{7} \mathrm{n} / \mathrm{cm}^{2} / \mathrm{s}$. 


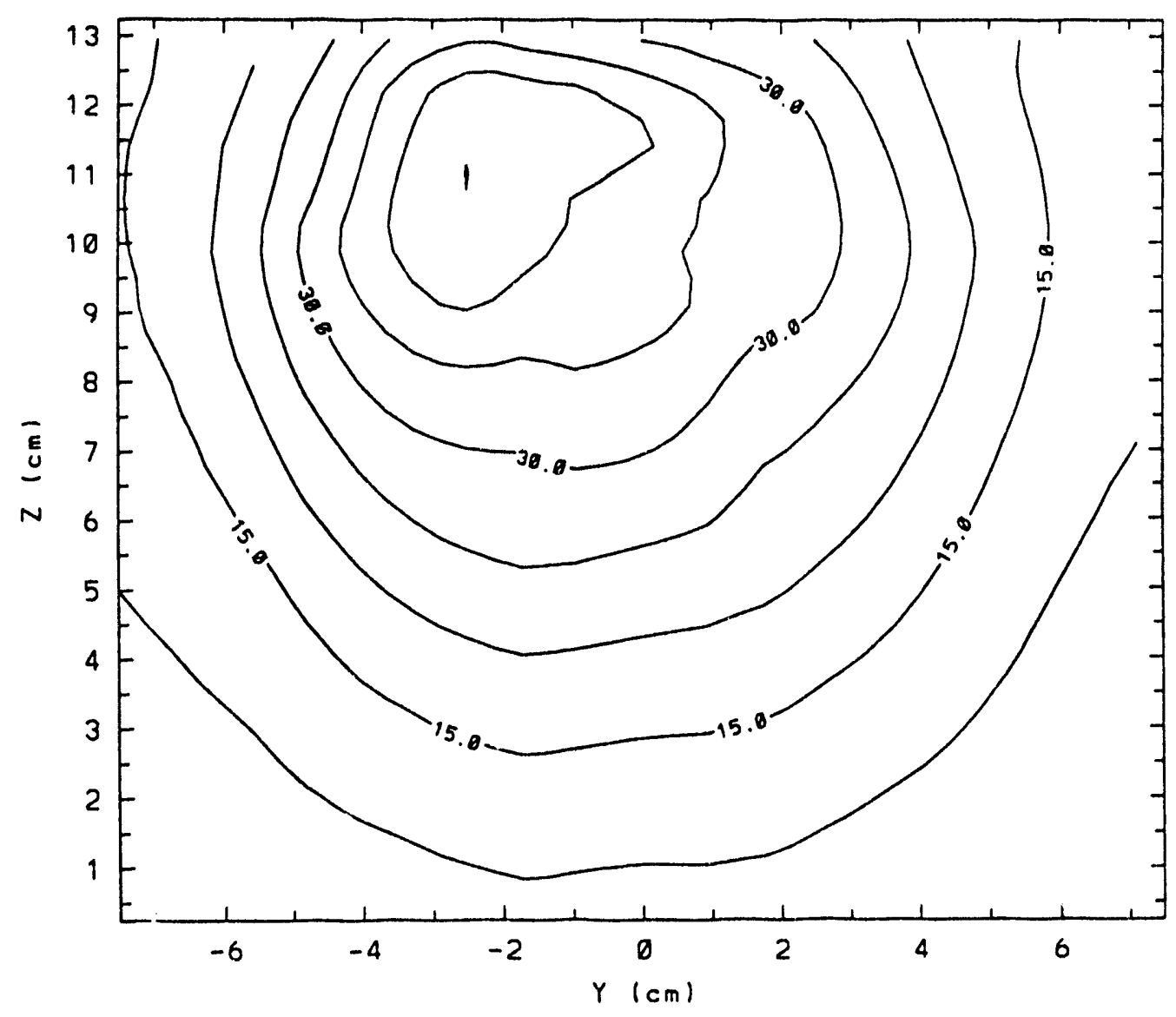

Figure F-63. Gamma flux contours at $x=17.25 \mathrm{~cm}$ for the heterogeneous case with the $5 \mathrm{~cm} \times 10 \mathrm{~cm}$ beam aperture. Percentage contours are shown normalized to a peak gamma flux of $2.98 \times 10^{7}$ gammas $/ \mathrm{cm}^{2} / \mathrm{s}$. 


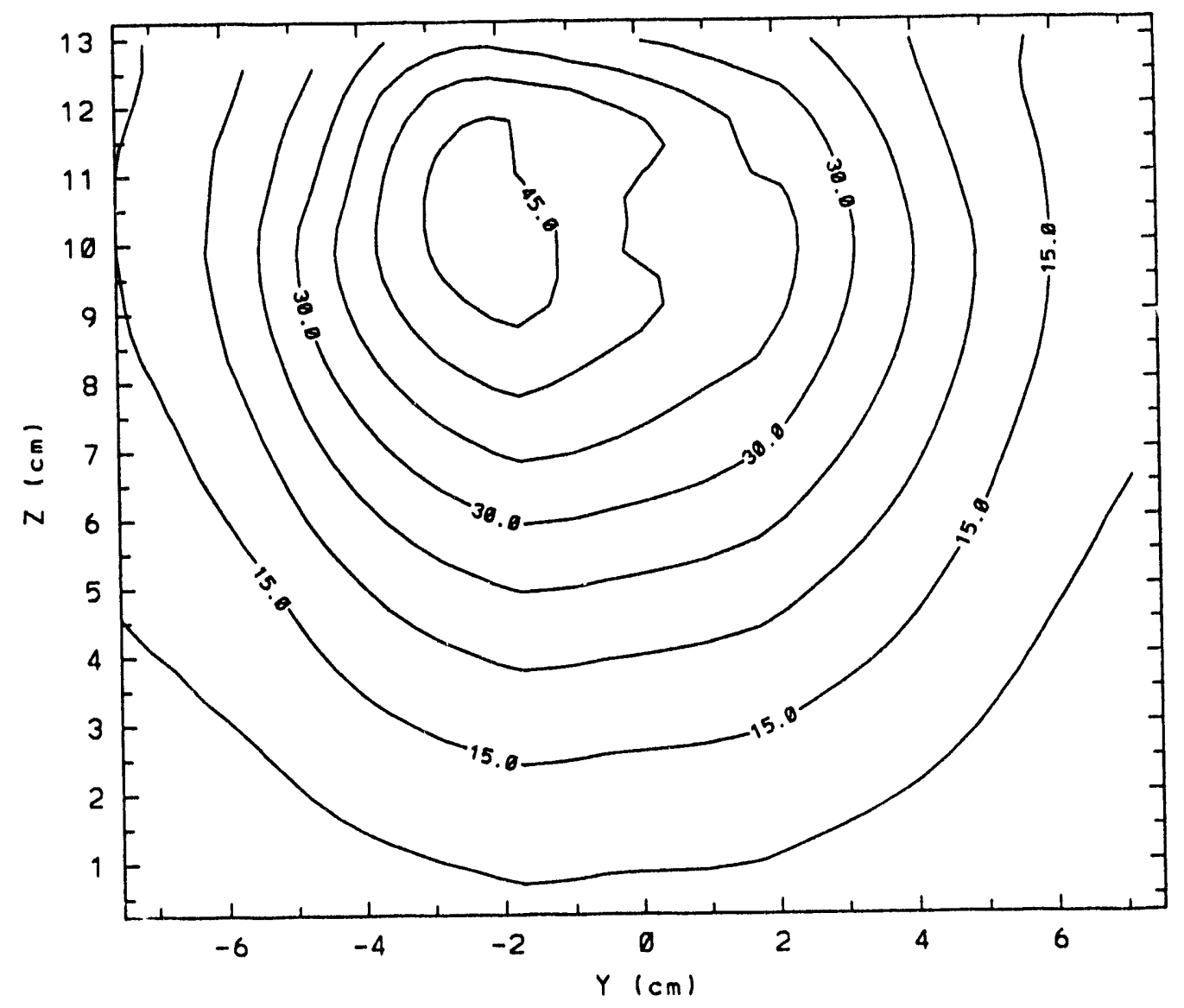

Figure F-64. Gamma flux at $x=17.25 \mathrm{~cm}$ for the homogeneous case with the $5 \mathrm{~cm} \times 10 \mathrm{~cm}$ beam aperture. Percentage contours are shown normalized to a peak gamma flux of $2.97 \mathrm{x}$ $10^{7}$ gammas $/ \mathrm{cm}^{2} / \mathrm{s}$. 


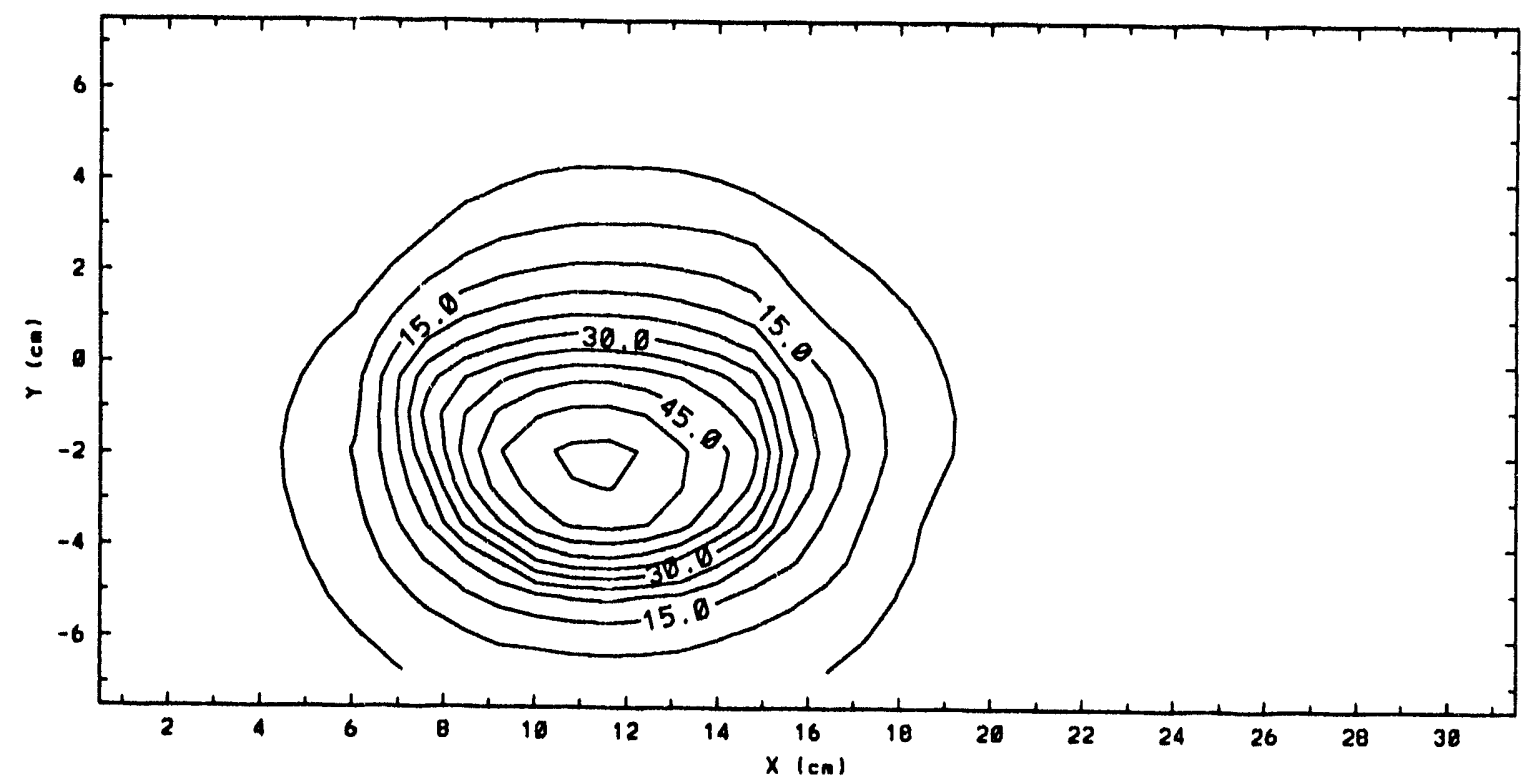

Figure F-65. Thermal neutron flux contours at $z=13.25 \mathrm{~cm}$ for the heterogeneous case with the $5 \mathrm{~cm} \times 10 \mathrm{~cm}$ beam aperture. Percentage contours are shown normalized to a peak thermal flux of $4.14 \times 10^{8} \mathrm{n} / \mathrm{cm}^{2} / \mathrm{s}$. 


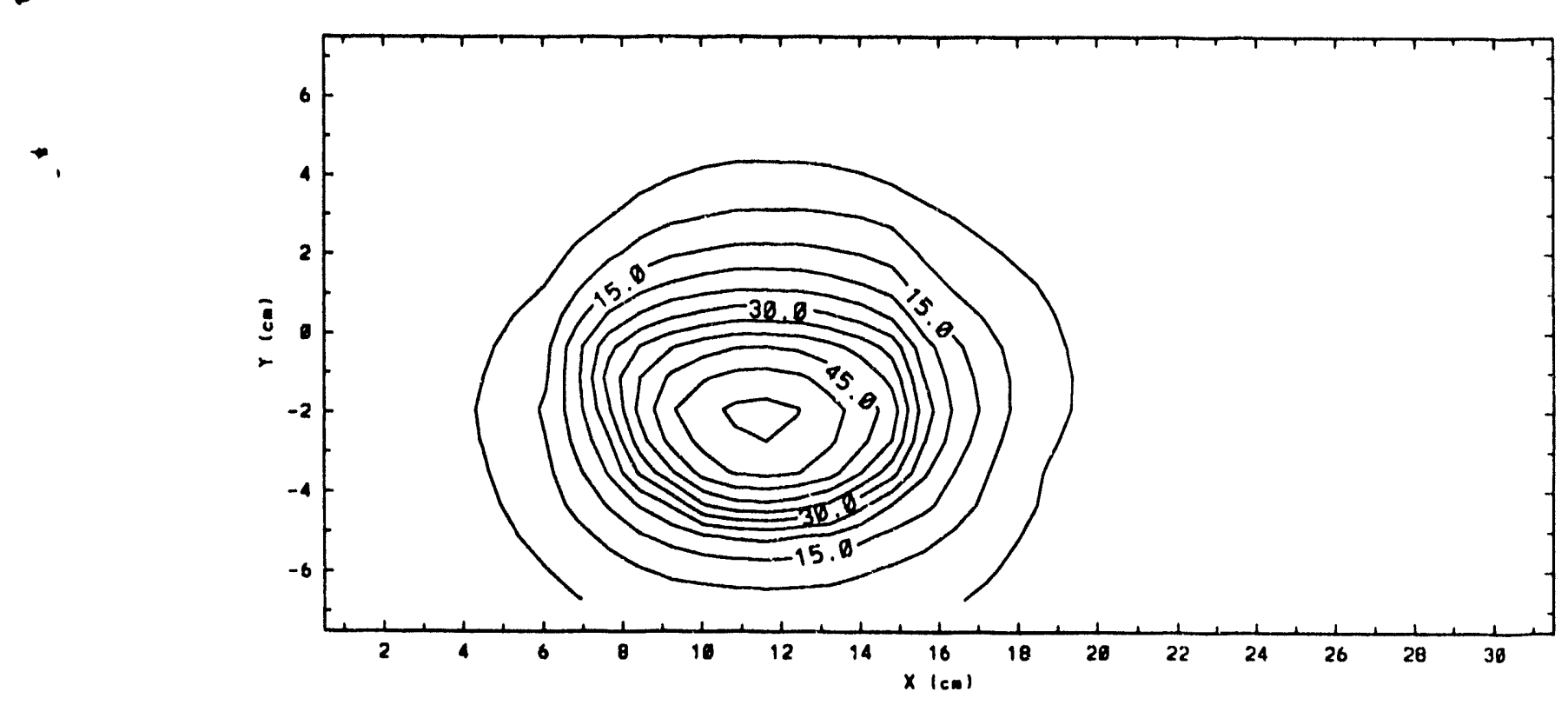

Figure F-66. Thermal neutron flux contours at $z=13.25 \mathrm{~cm}$ for the homogeneous case with the $5 \mathrm{~cm} \times 10 \mathrm{~cm}$ beam aperture. Percentage contours are shown normalized to a peak thermal flux of $4.04 \times 10^{8} \mathrm{n} / \mathrm{cm}^{2} / \mathrm{s}$. 
that for the $10 \mathrm{~cm} \times 10 \mathrm{~cm}$ beam port (shown in Figures F-23 and $(\mathrm{F}-24)$. The gamma fluxes at $z=13.25 \mathrm{~cm}$ are depicted in Figures F-67 and F-68. The effect is similar to that seen for the $10 \mathrm{~cm} \times 10 \mathrm{~cm} \mathrm{cdse}$. For the $55 \%$ to $70 \%$ contours, the heterogenecus fluxes are greater $(1-3 \%)$ for the portion of the contour that is not underneath the beam port. The spatial distributions are similar for all other contours.

The results presented in this appendix are to supplement those presented in section 5.3. While the planes examined here may not be those most relevant to therapy, they further explore Type 1 heterogeneity effects in selected sagittal, axial, and dorsal planes to provide an analysis of 1luxes throughout the canine head. This further analysis was performed to stress the importance of not only making calculations for heterogenous models but of examining the results of such calculations over the entire head volume to further understand the flux distributions. 


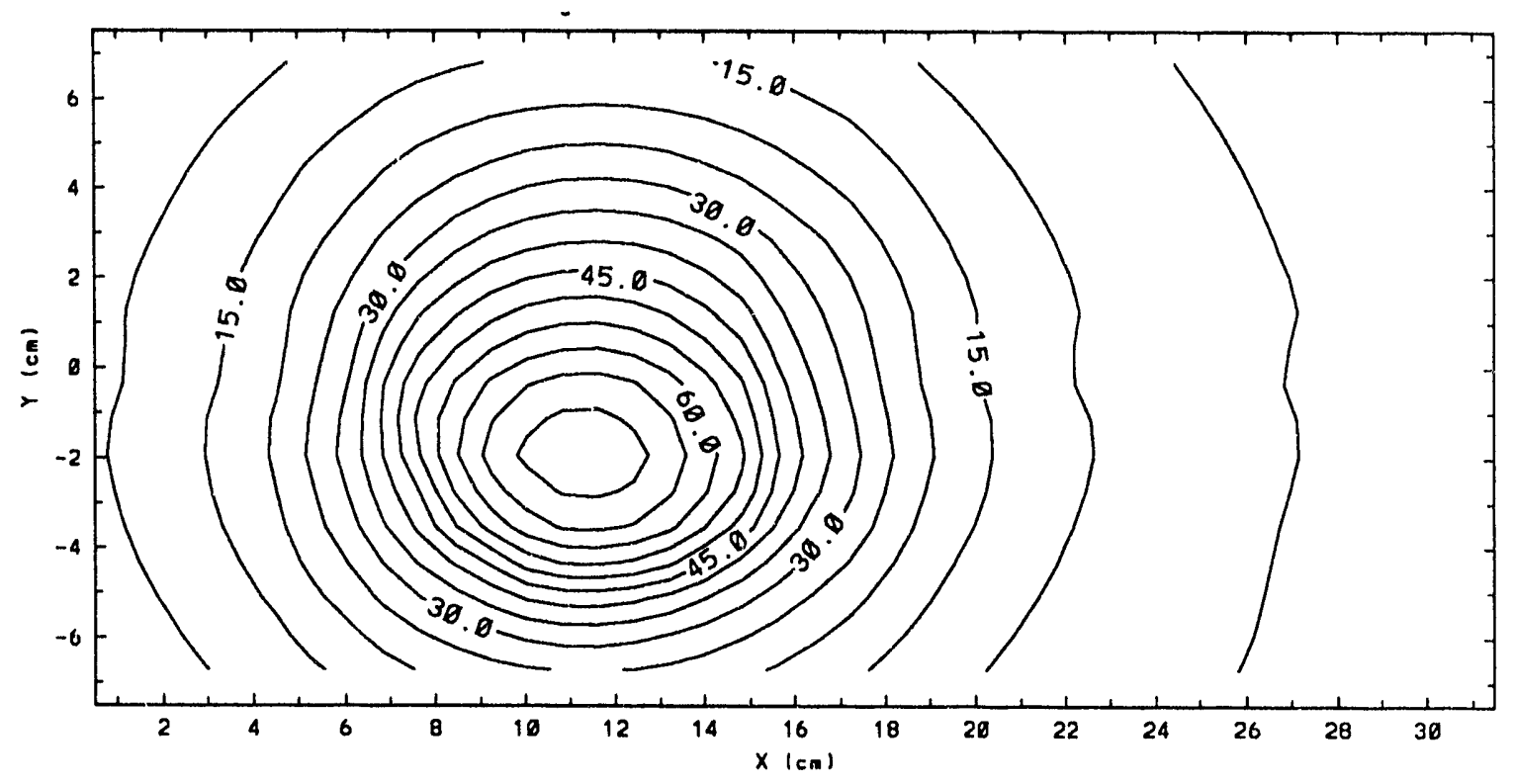

Figure F-66. Gamma flux contours at $z=13.25 \mathrm{~cm}$ for the heterogeneous case with the $5 \mathrm{~cm} \times 10 \mathrm{~cm}$ beam aperture. Percentage contours are shown normalized to a peak gamma flux of $2.98 \times 10^{7}$ gammas $/ \mathrm{cm}^{2} / \mathrm{s}$. 


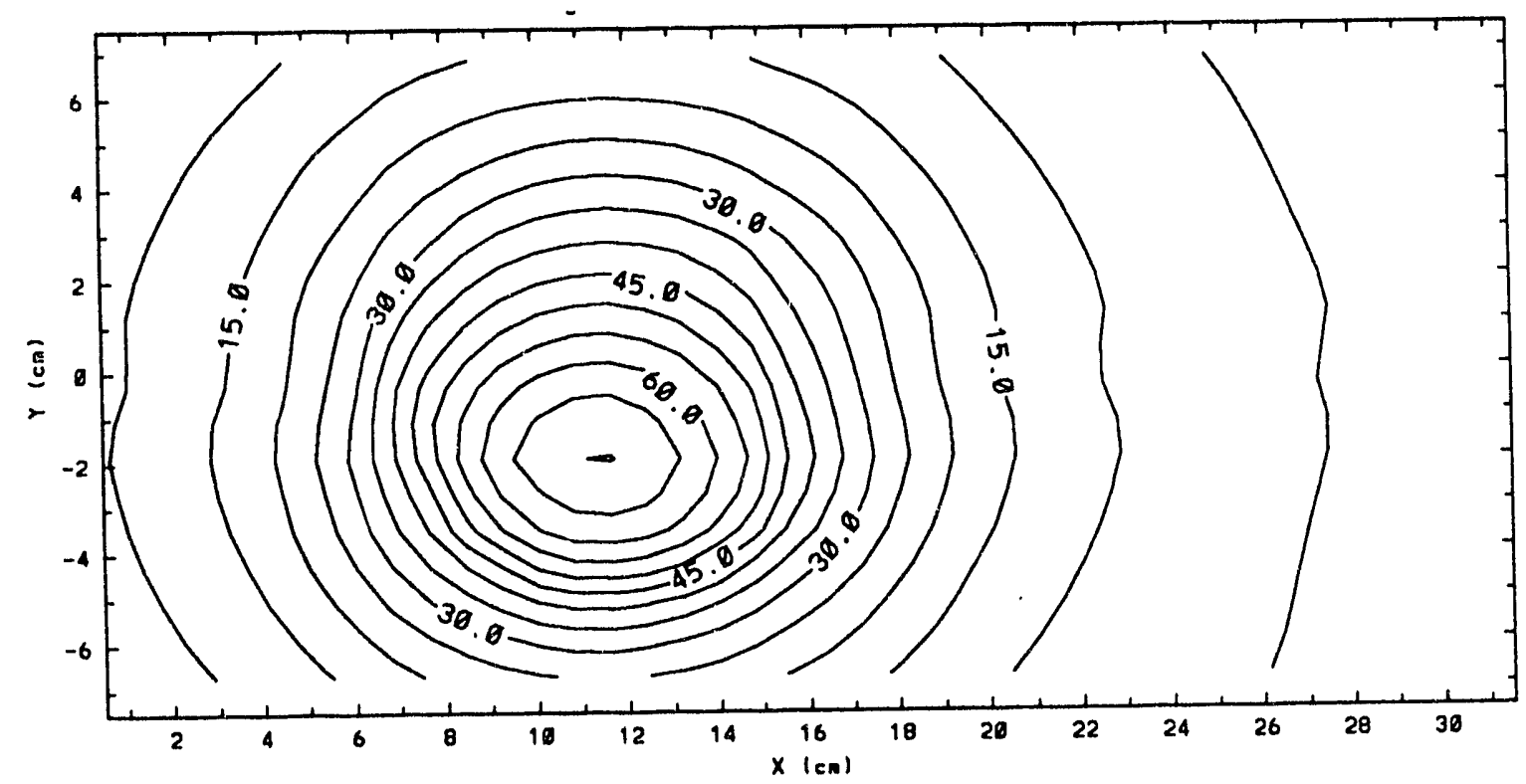

Figure F-67. Gamma flux contours at $z=13.25 \mathrm{~cm}$ for the homogeneous case with the $5 \mathrm{~cm} \times 10 \mathrm{~cm}$ beam aperture. Percentage contours are shown normalized to a peak gamma flux of $2.97 \times 10^{7}$ gammas $/ \mathrm{cm}^{2} / \mathrm{s}$. 


\section{THIS PAGE INTENTIONALLY LEFT BLANK}


APPENDIX G

RAW DATA-CHEMICAL ANALYSES

361 
This appendix contains the unreduced data obtained by the chemical analysis procedures described in section 4-3. In Table G-1, the results obtained for the first dog (sagittal sections, Dog 21) for carbon, hydrogen, nitrogen, and oxygen are presented. Table G-2 contains the measurements obtained by ICP-AES for the trace elements in these samples. The carbon, hydrogen, nitrogen, and oxygen data for the second dog (axial sections, Dog 22) are presented in Table G-3. The data obtained for the trace elements from these samples are shown in Table G-4. It is from the data contained in these four tables that Appendix $A$ was constructed.

As noted in section 4.3 , all number density calculations were made assuming unit mass density. The following is a sample calculation to convert from $\mu \mathrm{g} / \mathrm{g}$ into atoms $/ \mathrm{cm}^{3}$ for the \#1 sample of white matter for carbon (Dog 21):

$$
\begin{gathered}
\rho_{\mathrm{C}}=(212270 \mathrm{\mu g} / \mathrm{g})\left(1 \mathrm{~g} / \mathrm{cm}^{3}\right) /\left(1 \times 10^{6}\right)=0.21227 \mathrm{~g} / \mathrm{cm}^{3} \\
\mathrm{~N}_{\mathrm{C}}=\left(0.21227 \mathrm{~g} / \mathrm{cm}^{3}\right)(0.6022 \text { atoms } / \mathrm{mole}) /(12.0 \mathrm{~g} / \mathrm{mole}) \\
\mathrm{N}_{\mathrm{C}}=1.06 \times 10^{.2} \text { atoms } / \mathrm{g}
\end{gathered}
$$

The values are expressed in terms of atoms $/ \mathrm{cm}^{3} \times 10^{-24}$ or atoms $/ 9 \times 10^{-24}$ as shown in Appendix $A$. 
TABLE G-1. SUMMARY OF CARBON, HYDROGEN, NITROGEN, AND OXYGEN CONTENT IN DOG 21 SAMPLES

\begin{tabular}{|c|c|c|c|c|c|c|}
\hline$\#$ & Sample & $8 \mathrm{C}$ & $\frac{8 H}{6}$ & $8 \mathrm{~N}$ & 80 & Tota ${ }^{a}$ \\
\hline$\# 1$ & $\begin{array}{l}\text { White Matter } \\
\text { CHN (dry wt.) } \\
\text { SD } \\
\% \text { (wet wt.) } \\
\% \text { from water } \\
\text { \& from } \mathrm{PO}_{4} \\
\text { Total (wet wt.) } \\
\mu g / g\end{array}$ & $\begin{array}{r}64.130 \\
0.330 \\
21.227 \\
\\
21.227 \\
212270\end{array}$ & $\begin{array}{r}9.960 \\
0.180 \\
3.297 \\
7.433 \\
10.730 \\
107301\end{array}$ & $\begin{array}{l}5.850 \\
0.070 \\
1.936\end{array}$ & $\begin{array}{c}59.467 \\
0.010 \\
59.467 \\
594667\end{array}$ & 96 \\
\hline$\# 2$ & $\begin{array}{l}\text { White matter } \\
\text { CHN (dry wt.) } \\
\text { SD (wet wt.) } \\
\text { \& (wet water } \\
\text { \& from wat } \\
\text { \& from } \mathrm{PO}_{4} \\
\text { Total (wet wt.) } \\
\mu \mathrm{g} / \mathrm{g}\end{array}$ & $\begin{array}{r}63.520 \\
0.930 \\
21.406\end{array}$ & $\begin{array}{l}9.910 \\
0.120 \\
3.340 \\
7.367 \\
10.706 \\
107063\end{array}$ & $\begin{array}{l}5.930 \\
0.180 \\
1.998\end{array}$ & $\begin{array}{c}58.933 \\
0.011 \\
58.933 \\
589333\end{array}$ & 95 \\
\hline$\# 3$ & $\begin{array}{l}\text { Gray matter } \\
\text { CHN (dry wt.) } \\
\text { SD } \\
\% \text { (wet wt.) } \\
\& \text { from water } \\
\& \text { from } \mathrm{PO}_{4} \\
\text { Total (wet wt.) } \\
\mu \mathrm{g} / \mathrm{g}\end{array}$ & $\begin{array}{r}54.500 \\
0.410 \\
10.682\end{array}$ & $\begin{array}{r}8.050 \\
0.070 \\
1.578 \\
8.933 \\
10.511 \\
105111\end{array}$ & $\begin{array}{l}9.420 \\
0.070 \\
1.846\end{array}$ & $\begin{array}{c}71.467 \\
0.010 \\
71.467 \\
714667\end{array}$ & 96 \\
\hline$\# 4$ & $\begin{array}{l}\text { Gray matter } \\
\text { CHN (dry wt.) } \\
\text { SD } \\
q \text { (wet wt.) } \\
\% \text { from water } \\
q \text { from } \mathrm{PO}_{4} \\
\text { Total (wet wt.) } \\
\mu g / g\end{array}$ & $\begin{array}{r}61.410 \\
0.850 \\
19.774\end{array}$ & $\begin{array}{l}9.670 \\
0.170 \\
3.114 \\
7.533 \\
10.647 \\
106471\end{array}$ & $\begin{array}{l}6.210 \\
0.170 \\
2.000\end{array}$ & $\begin{array}{c}60.267 \\
0.011 \\
60.267 \\
602667\end{array}$ & 94 \\
\hline$\# 5$ & $\begin{array}{l}\text { Skull } \\
\text { CHN (dry wt.) } \\
\text { SD } \\
q \text { (wet wt.) } \\
q \text { from water } \\
q \text { from } \mathrm{PO}_{4} \\
\text { Total (wet wt.) } \\
\mu \mathrm{g} / \mathrm{g}\end{array}$ & $\begin{array}{r}23.980 \\
0.170 \\
19.304\end{array}$ & $\begin{array}{l}3.750 \\
0.070 \\
3.019 \\
2.167 \\
5.185 \\
51854\end{array}$ & $\begin{array}{l}4.640 \\
0.230 \\
3.735\end{array}$ & $\begin{array}{c}17.333 \\
0.187 \\
17.333 \\
173333\end{array}$ & 91 \\
\hline
\end{tabular}


TABLE G-1. CONTINUED

\begin{tabular}{|c|c|c|c|c|c|c|}
\hline$\#$ & Sample & $8 \mathrm{C}$ & $8 \mathrm{H}$ & $8 \mathrm{~N}$ & 80 & Total \\
\hline$\# 6$ & $\begin{array}{l}\text { Skull } \\
\text { CHN (dry wt.) } \\
\text { SD } \\
\text { \& (wet wt.) } \\
\text { \& from water } \\
\text { \& from } \mathrm{PO}_{4} \\
\text { Total (wet wt.) } \\
\mu \mathrm{g} / \mathrm{g}\end{array}$ & $\begin{array}{r}23.050 \\
1.470 \\
19.293 \\
\\
19.293 \\
192929\end{array}$ & $\begin{array}{l}3.550 \\
0.160 \\
2.971 \\
1.811 \\
4.782 \\
47825\end{array}$ & $\begin{array}{l}4.350 \\
0.120 \\
3.641 \\
3.641 \\
36410\end{array}$ & $\begin{array}{c}14.489 \\
0.169 \\
14.489 \\
144889\end{array}$ & 77 \\
\hline$\# 7$ & $\begin{array}{l}\text { Dermis } \\
\text { CHN (dry wt.) } \\
\text { SD } \\
\& \text { (wet wt.) } \\
\text { \& from water } \\
\& \text { from } \mathrm{PO}_{4} \\
\text { Total (wet wt.) } \\
\mu g / g\end{array}$ & $\begin{array}{r}56.210 \\
1.900 \\
32.040 \\
\\
32.040 \\
320397\end{array}$ & $\begin{array}{l}8.340 \\
0.240 \\
4.754 \\
4.778 \\
9.532 \\
95316\end{array}$ & $\begin{array}{r}12.640 \\
0.870 \\
7.205\end{array}$ & $\begin{array}{c}38.222 \\
0.011 \\
38.222 \\
382222\end{array}$ & 88 \\
\hline$\# 8$ & $\begin{array}{l}\text { Dermis } \\
\text { CHN (dry wt.) } \\
\text { SD } \\
\text { \& (wet wt.) } \\
\text { \& from water } \\
\text { \& from } \mathrm{PO}_{4} \\
\text { Total (wet wt.) } \\
\mu \mathrm{g} / \mathrm{g}\end{array}$ & $\begin{array}{r}57.940 \\
2.330 \\
35.691\end{array}$ & $\begin{array}{l}8.760 \\
0.320 \\
5.396 \\
4.267 \\
9.663 \\
96628\end{array}$ & $\begin{array}{r}10.690 \\
1.620 \\
6.585\end{array}$ & $\begin{array}{c}34.133 \\
0.012 \\
34.133 \\
341333\end{array}$ & 87 \\
\hline$\# 9$ & $\begin{array}{l}\text { Temporalis Muscl } \\
\text { CHN (dry wt.) } \\
\text { SD } \\
\text { \& (wet wt.) } \\
\text { \& from water } \\
\text { \& from } \mathrm{PO}_{4} \\
\text { Total (wet wt.) } \\
\mu \mathrm{g} / \mathrm{g}\end{array}$ & $\begin{array}{r}\text { e } \\
51.490 \\
0.100 \\
12.667\end{array}$ & $\begin{array}{r}7.410 \\
0.120 \\
1.823 \\
8.378 \\
10.201 \\
102006\end{array}$ & $\begin{array}{r}14.870 \\
0.180 \\
3.658\end{array}$ & $\begin{array}{l}67.022 \\
0.0 \\
67.022 \\
670222\end{array}$ & 95 \\
\hline$\# 10$ & $\begin{array}{l}\text { Temporalis Muscle } \\
\text { CHN (dry wt.) } \\
\text { SD } \\
\text { \& (wet wt.) } \\
\text { \& from water } \\
\text { \& from } \mathrm{PO}_{4} \\
\text { Total (wet wt.) } \\
\mu \mathrm{g} / \mathrm{g}\end{array}$ & $\begin{array}{r}49.130 \\
0.190 \\
12.184 \\
\\
12.184 \\
121842\end{array}$ & $\begin{array}{r}7.210 \\
0.040 \\
1.788 \\
8.356 \\
10.144 \\
101436\end{array}$ & $\begin{array}{r}15.020 \\
0.120 \\
3.725\end{array}$ & $\begin{array}{l}66.844 \\
0.0 \\
66.844 \\
668444\end{array}$ & 94 \\
\hline
\end{tabular}




\begin{tabular}{|c|c|c|c|c|c|c|}
\hline$\#$ & Sample & $8 \mathrm{C}$ & $8 \mathrm{H}$ & $\frac{8}{8} \mathrm{~N}$ & 80 & Total" \\
\hline$\# 11$ & $\begin{array}{l}\text { Epidermis } \\
\text { CHN (dry wt.) } \\
\text { SD } \\
\text { \& (wet wt.) } \\
\text { \& from water } \\
\text { \& from } \mathrm{PO}_{4} \\
\text { Total (wet wt.) } \\
\mu \mathrm{g} / \mathrm{g}\end{array}$ & $\begin{array}{r}51.460 \\
0.390 \\
19.452 \\
\\
19.452 \\
194519\end{array}$ & $\begin{array}{l}7.340 \\
0.140 \\
2.775 \\
6.911 \\
9.686 \\
96856\end{array}$ & $\begin{array}{r}15.100 \\
0.410 \\
5.708\end{array}$ & $\begin{array}{l}55.289 \\
0.0 \\
55.289 \\
552889\end{array}$ & 91 \\
\hline$\# 12$ & $\begin{array}{l}\text { Epidermis } \\
\text { CHN (dry wt.) } \\
\text { SD (wet wt.) } \\
\text { \% (wrom water } \\
\text { \& from } \\
\text { \% from } \mathrm{PO}_{4} \\
\text { Total (wet wt.) } \\
\mu g / g\end{array}$ & $\begin{array}{r}60.680 \\
1.850 \\
31.978 \\
\\
31.978 \\
319784\end{array}$ & $\begin{array}{l}9.310 \\
0.310 \\
4.906 \\
5.256 \\
10.162 \\
101619\end{array}$ & $\begin{array}{l}5.840 \\
0.180 \\
3.078 \\
3.078 \\
30777\end{array}$ & $\begin{array}{c}42.044 \\
0.0 \\
42.044 \\
420444\end{array}$ & 88 \\
\hline$\# 13$ & $\begin{array}{l}\text { Ethmoid Labyrint } \\
\text { CHN (dry wt.) } \\
\text { SD } \\
\% \text { (wet wt.) } \\
\& \text { from water } \\
\% \text { from PO } \\
\text { Total (wet wt.) } \\
\mu g / g\end{array}$ & $\begin{array}{r}31.030 \\
9.470 \\
12.691 \\
12.691 \\
126913\end{array}$ & $\begin{array}{l}4.540 \\
1.240 \\
1.857 \\
6.567 \\
8.424 \\
84235\end{array}$ & $\begin{array}{l}6.750 \\
2.240 \\
2.761\end{array}$ & $\begin{array}{c}52.533 \\
0.057 \\
52.533 \\
525333\end{array}$ & 88 \\
\hline$\# 14$ & $\begin{array}{l}\text { Ethmoid Labyrint } \\
\text { CHN (dry wt.) } \\
\text { SD } \\
\text { \& (wet wt.) } \\
\text { \& from water } \\
\text { \& from } \mathrm{PO}_{4} \\
\text { Total (wet wt.) } \\
\mu \mathrm{g} / \mathrm{g}\end{array}$ & $\begin{array}{r}31.490 \\
2.690 \\
10.518 \\
10.518 \\
105177\end{array}$ & $\begin{array}{l}4.730 \\
0.290 \\
1.580 \\
7.400 \\
8.980 \\
89798\end{array}$ & $\begin{array}{l}7.800 \\
0.440 \\
2.605 \\
2.605 \\
26052\end{array}$ & $\begin{array}{c}59.200 \\
0.053 \\
59.200 \\
592000\end{array}$ & 94 \\
\hline$\# 15$ & $\begin{array}{l}\text { Concha } \\
\text { CHN (dry wt.) } \\
\text { SD } \\
\& \text { (wet wt.) } \\
: \text { from water } \\
\% \text { from } \mathrm{PO}_{4} \\
\text { Total (wet wt.) } \\
\mu \mathrm{g} / \mathrm{g}\end{array}$ & $\begin{array}{r}33.990 \\
0.990 \\
10.707 \\
10.707 \\
107069\end{array}$ & $\begin{array}{l}5.090 \\
0.110 \\
1.603 \\
7.611 \\
9.214 \\
92145\end{array}$ & $\begin{array}{l}8.960 \\
0.400 \\
2.822 \\
\\
2.822 \\
28224\end{array}$ & $\begin{array}{c}60.889 \\
0.043 \\
60.889 \\
608889\end{array}$ & 94 \\
\hline
\end{tabular}

a. Total includes trace elements (see TABLE G-2). 
TABLE G-1. CONTINUED

\begin{tabular}{|c|c|c|c|c|c|c|}
\hline \pm & Sample & $\frac{8}{8}$ & $8 \mathrm{H}$ & $\frac{8 N}{N}$ & 80 & Total \\
\hline$\# 16$ & $\begin{array}{l}\text { Concha } \\
\text { CHN (dry wt.) } \\
\text { SD (wet wt.) } \\
\& \text { (wet water } \\
\text { \& from wat } \\
\text { \& from } \mathrm{PO}_{4} \\
\text { Total (wet wt.) } \\
\mu g / g\end{array}$ & $\begin{array}{r}28.850 \\
0.360 \\
11.338 \\
11.338 \\
113380\end{array}$ & $\begin{array}{l}4.480 \\
0.100 \\
1.761 \\
6.744 \\
8.505 \\
85051\end{array}$ & $\begin{array}{l}7.970 \\
0.110 \\
3.132 \\
3.132 \\
31322\end{array}$ & $\begin{array}{c}53.956 \\
0.056 \\
53.956 \\
539556\end{array}$ & 90 \\
\hline$\# 17$ & $\begin{array}{l}\text { Genio Glossus } \\
\text { CHN (dry wt.) } \\
\text { SD } \\
\text { \& (wet wt.) } \\
\text { \& from water } \\
\text { \& from } \mathrm{PO}_{4} \\
\text { Total (wet wt.) } \\
\mu \mathrm{g} / \mathrm{g}\end{array}$ & $\begin{array}{r}51.620 \\
1.140 \\
11.873 \\
11.873 \\
118726\end{array}$ & $\begin{array}{r}7.430 \\
0.200 \\
1.709 \\
8.556 \\
10.264 \\
102645\end{array}$ & $\begin{array}{r}14.270 \\
0.280 \\
3.282 \\
\\
3.282 \\
32821\end{array}$ & $\begin{array}{l}68.444 \\
0.0 \\
68.444 \\
684444\end{array}$ & 95 \\
\hline \#18 & $\begin{array}{l}\text { Genio Glossus } \\
\text { CHN (dry wt.) } \\
\text { SD } \\
\text { \& (wet wt.) } \\
\text { \& from water } \\
\text { \& from } \mathrm{PO}_{4} \\
\text { Total (wet wt.) } \\
\mu \mathrm{g} / \mathrm{g}\end{array}$ & $\begin{array}{r}53.070 \\
0.630 \\
13.480 \\
\\
13.480 \\
134798\end{array}$ & $\begin{array}{r}7.630 \\
0.130 \\
1.938 \\
8.289 \\
10.227 \\
102269\end{array}$ & $\begin{array}{r}12.590 \\
0.240 \\
3.198\end{array}$ & $\begin{array}{l}66.311 \\
0.0 \\
66.311 \\
663111\end{array}$ & 94 \\
\hline \#19 & $\begin{array}{l}\text { Tongue } \\
\text { CHN (dry wt.) } \\
\text { SD } \\
\text { \& (wet wt.) } \\
\text { \& from water } \\
\text { \& from } \mathrm{PO}_{4} \\
\text { Total (wet wt.) } \\
\mu \mathrm{g} / \mathrm{g}\end{array}$ & $\begin{array}{r}58.960 \\
0.920 \\
21.992\end{array}$ & $\begin{array}{r}8.720 \\
0.100 \\
3.253 \\
6.967 \\
10.219 \\
102192\end{array}$ & $\begin{array}{r}11.060 \\
0.850 \\
4.125\end{array}$ & $\begin{array}{c}55.733 \\
0.0 \\
55.733 \\
557333\end{array}$ & 93 \\
\hline$\# 20$ & $\begin{array}{l}\text { Tongue } \\
\text { CHN (dry wt.) } \\
\text { SD } \\
\& \text { (wet wt.) } \\
\& \text { from water } \\
\& \text { from } \mathrm{PO}_{4} \\
\text { Total (wet wt.) } \\
\mu g / g\end{array}$ & $\begin{array}{r}56.380 \\
0.850 \\
23.116 \\
\\
23.116 \\
231158\end{array}$ & $\begin{array}{l}8.360 \\
0.330 \\
3.428 \\
6.556 \\
9.983 \\
99832\end{array}$ & $\begin{array}{r}11.500 \\
0.750 \\
4.715\end{array}$ & $\begin{array}{c}52.444 \\
0.0 \\
52.444 \\
524444\end{array}$ & 91 \\
\hline
\end{tabular}


TABLE G-1. CONTINUED

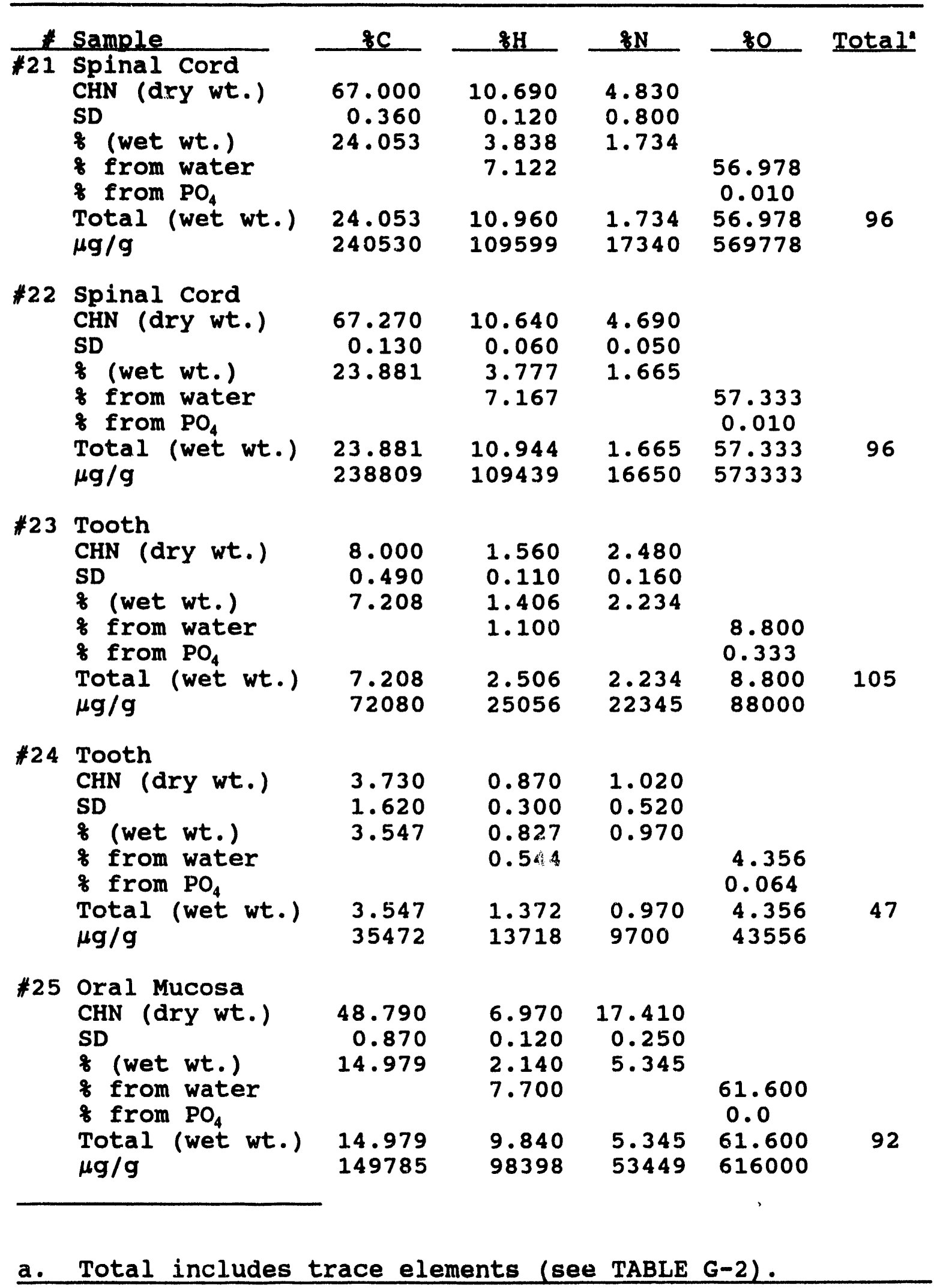


TABLE G-1. CONTINUED

\begin{tabular}{|c|c|c|c|c|c|c|}
\hline$\#$ & Sample & $8 \mathrm{C}$ & $8 \mathrm{H}$ & $\frac{8 N}{N}$ & 80 & Total" \\
\hline$\# 26$ & $\begin{array}{l}\text { Oral Mucosa } \\
\text { CHN (dry wt.) } \\
\text { SD } \\
\& \text { (wet wt.) } \\
\& \text { from water } \\
\& \text { from } \mathrm{PO}_{4} \\
\text { Total (wet wt.) } \\
\mu \mathrm{g} / \mathrm{g}\end{array}$ & $\begin{array}{r}48.250 \\
0.440 \\
14.572 \\
\\
14.572 \\
145715\end{array}$ & $\begin{array}{l}7.060 \\
0.060 \\
2.132 \\
7.756 \\
9.888 \\
98877\end{array}$ & $\begin{array}{r}17.140 \\
0.390 \\
5.176\end{array}$ & $\begin{array}{l}62.044 \\
0.0 \\
62.044 \\
620444\end{array}$ & 92 \\
\hline$\# 27$ & no sample & & & & & \\
\hline$\# 28$ & $\begin{array}{l}\text { Eye Lens } \\
\text { CHN (dry wt.) } \\
\text { SD } \\
\text { \& (wet wt.) } \\
\text { \& from water } \\
\text { of from } \mathrm{PO}_{4} \\
\text { Total (wet wt.) } \\
\mu \mathrm{g} / \mathrm{g}\end{array}$ & $\begin{array}{r}50.950 \\
0.160 \\
20.023 \\
20.023 \\
200234\end{array}$ & $\begin{array}{l}7.270 \\
0.030 \\
2.857 \\
6.744 \\
9.602 \\
96016\end{array}$ & $\begin{array}{r}15.490 \\
0.040 \\
6.088 \\
\\
6.088 \\
60876\end{array}$ & $\begin{array}{l}53.956 \\
0.0 \\
53.956 \\
539556\end{array}$ & 90 \\
\hline$\# 29$ & $\begin{array}{l}\text { Outer Skin Retina } \\
\text { CHN (dry wt.) } \\
\text { SD } \\
\& \text { (wet wt.) } \\
\text { \& from water } \\
\text { \& from } \mathrm{PO}_{4} \\
\text { Total (wet wt.) } \\
\mu \mathrm{g} / \mathrm{g}\end{array}$ & $\begin{array}{r}58.880 \\
0.760 \\
10.834\end{array}$ & $\begin{array}{l}8.950 \\
0.220 \\
1.647 \\
9.067 \\
10.713 \\
107135\end{array}$ & $\begin{array}{r}10.050 \\
0.630 \\
1.849\end{array}$ & $\begin{array}{c}72.533 \\
0.0 \\
72.533 \\
725333\end{array}$ & 97 \\
\hline
\end{tabular}

a. Total includes trace elements (see TABLE G-2). 
TABLE G-2. SUMMARY OF TRACE ELEMENT CONTENT IN DOG 21 SAMPLES

\begin{tabular}{|c|c|c|c|c|c|c|c|}
\hline \multirow{8}{*}{ 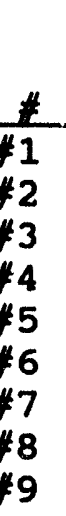 } & \multirow[b]{2}{*}{ Sample } & \multicolumn{6}{|c|}{ TOTAL (WET WEIGHT) } \\
\hline & & \&Na & $8 \mathrm{~g}$ & 85 & $\& \mathrm{P}$ & \&.Mg & $\& \mathrm{Ca}$ \\
\hline & White Matter & $\overline{0.113}$ & $\overline{0.302}$ & $\overline{0.213}$ & $\overline{0.505}$ & 0.015 & 0.025 \\
\hline & White matter & 0.102 & 0.292 & 0.203 & 0.496 & 0.015 & $\star *$ \\
\hline & Gray matter & 0.120 & 0.386 & * & 0.292 & 0.015 & $\star \star$ \\
\hline & Gray matter & $0^{* *}$ & 0.400 & $\star \star \star$ & 0.410 & $\star \star$ & $\star *$ \\
\hline & $\begin{array}{l}\text { Skull } \\
\text { skull }\end{array}$ & $\begin{array}{l}0.654 \\
0.534\end{array}$ & $\begin{array}{l}0.075 \\
0.079\end{array}$ & $\begin{array}{l}0.081 \\
0.090\end{array}$ & $\begin{array}{l}8.476 \\
6.377\end{array}$ & $\begin{array}{l}0.226 \\
0.179\end{array}$ & $\begin{array}{l}18.513 \\
14.836\end{array}$ \\
\hline & Dermis & $\begin{array}{l}0.097 \\
0.095\end{array}$ & $\begin{array}{l}0.104 \\
0.115\end{array}$ & $\begin{array}{l}0.125 \\
0.090\end{array}$ & $\begin{array}{l}0.057 \\
0.056\end{array}$ & $\begin{array}{l}0.006 \\
0.007\end{array}$ & $\begin{array}{r}0.015 \\
0.027\end{array}$ \\
\hline$\# 10$ & $\begin{array}{l}\text { Muscle } \\
\text { Temporalis }\end{array}$ & 0.086 & 0.360 & 0.283 & 0.222 & 0.027 & 0.050 \\
\hline $\begin{array}{l}\# 11 \\
\# 12 \\
\# 13\end{array}$ & $\begin{array}{l}\text { Muscle } \\
\text { Epidermis } \\
\text { Epidermis } \\
\text { Ethmoid }\end{array}$ & $\begin{array}{l}0.099 \\
0.149 \\
0.135\end{array}$ & $\begin{array}{l}0.302 \\
0.093 \\
0.096\end{array}$ & $\begin{array}{l}0.266 \\
0.353 \\
0.291\end{array}$ & $\begin{array}{l}0.203 \\
0.071 \\
0.066\end{array}$ & $\begin{array}{l}0.026 \\
0.007 \\
0.007\end{array}$ & $\begin{array}{l}0.052 \\
0.043 \\
0.051\end{array}$ \\
\hline$\# 14$ & $\begin{array}{l}\text { Labyrinth } \\
\text { Ethmoid }\end{array}$ & .088 & 0.102 & 0.136 & 2.167 & 0.080 & 4.373 \\
\hline $\begin{array}{l}\# 15 \\
\# 16 \\
\# 17\end{array}$ & $\begin{array}{l}\text { Labyrinth } \\
\text { Concha } \\
\text { Concha } \\
\text { Genio }\end{array}$ & $\begin{array}{l}0.231 \\
0.239 \\
0.264\end{array}$ & $\begin{array}{l}0.243 \\
0.191 \\
0.206\end{array}$ & $\begin{array}{l}0.160 \\
0.150 \\
0.173\end{array}$ & $\begin{array}{l}2.371 \\
1.866 \\
2.434\end{array}$ & $\begin{array}{l}0.087 \\
0.067 \\
0.091\end{array}$ & $\begin{array}{l}4.778 \\
3.673 \\
4.901\end{array}$ \\
\hline$\# 18$ & $\begin{array}{l}\text { Glossus } \\
\text { Genio }\end{array}$ & 0.098 & 0.256 & 0.213 & 0.161 & 0.017 & 0.014 \\
\hline & Glossus & 0.130 & 0.275 & 0.241 & 0.161 & 0.018 & 0.011 \\
\hline $\begin{array}{l}\# 19 \\
\# 20\end{array}$ & $\begin{array}{l}\text { Tongue } \\
\text { Tongue }\end{array}$ & $\begin{array}{l}0.105 \\
0.105\end{array}$ & $\begin{array}{l}0.239 \\
0.221\end{array}$ & $\begin{array}{l}0.207 \\
0.194\end{array}$ & $\begin{array}{l}0.156 \\
0.144\end{array}$ & $\begin{array}{l}0.016 \\
0.015\end{array}$ & $\begin{array}{l}0.013 \\
0.013\end{array}$ \\
\hline$\# 21$ & Spinal Cord & 0.197 & 0.152 & 0.137 & 0.472 & 0.012 & 0.006 \\
\hline $\begin{array}{l}\# 22 \\
\# 23\end{array}$ & $\begin{array}{l}\text { Spinal Cord } \\
\text { Tooth }\end{array}$ & $\begin{array}{l}0.183 \\
0.683\end{array}$ & $\begin{array}{c}0.166 \\
* \star\end{array}$ & $\begin{array}{l}0.147 \\
0.104\end{array}$ & $\begin{array}{r}0.503 \\
16.788\end{array}$ & $\begin{array}{l}0.012 \\
0.745\end{array}$ & $\begin{array}{r}0.008 \\
30.782\end{array}$ \\
\hline $\begin{array}{l}\# 24 \\
\# 25\end{array}$ & Tooth & 0.847 & ** & 0.071 & 1.539 & 0.830 & 30.516 \\
\hline $\begin{array}{l}\# 25 \\
\# 26 \\
\# 27\end{array}$ & $\begin{array}{l}\text { Oral Mucosa } \\
\text { Oral Mucosa } \\
\text { no sample }\end{array}$ & $\begin{array}{l}0.177 \\
0.182\end{array}$ & $\begin{array}{l}0.120 \\
0.115\end{array}$ & $\begin{array}{l}0.147 \\
0.163\end{array}$ & $\begin{array}{l}0.078 \\
0.076\end{array}$ & $\begin{array}{l}0.008 \\
0.008\end{array}$ & $\begin{array}{l}0.044 \\
0.033\end{array}$ \\
\hline$\# 28$ & $\begin{array}{l}\text { Eye Lens } \\
\text { outer skin }\end{array}$ & 0.242 & 0.118 & 0.144 & 0.045 & 0.004 & ** \\
\hline & Retina & 0.385 & 0.327 & 0.212 & 0.161 & 0.017 & 0.014 \\
\hline
\end{tabular}

** Sample was below detection limits. 
TABLE G-3. SUMMARY OF CARBON, HYDROGEN, NITROGEN, AND OXYGEN CONTENT IN DOG 22 SAMPLES

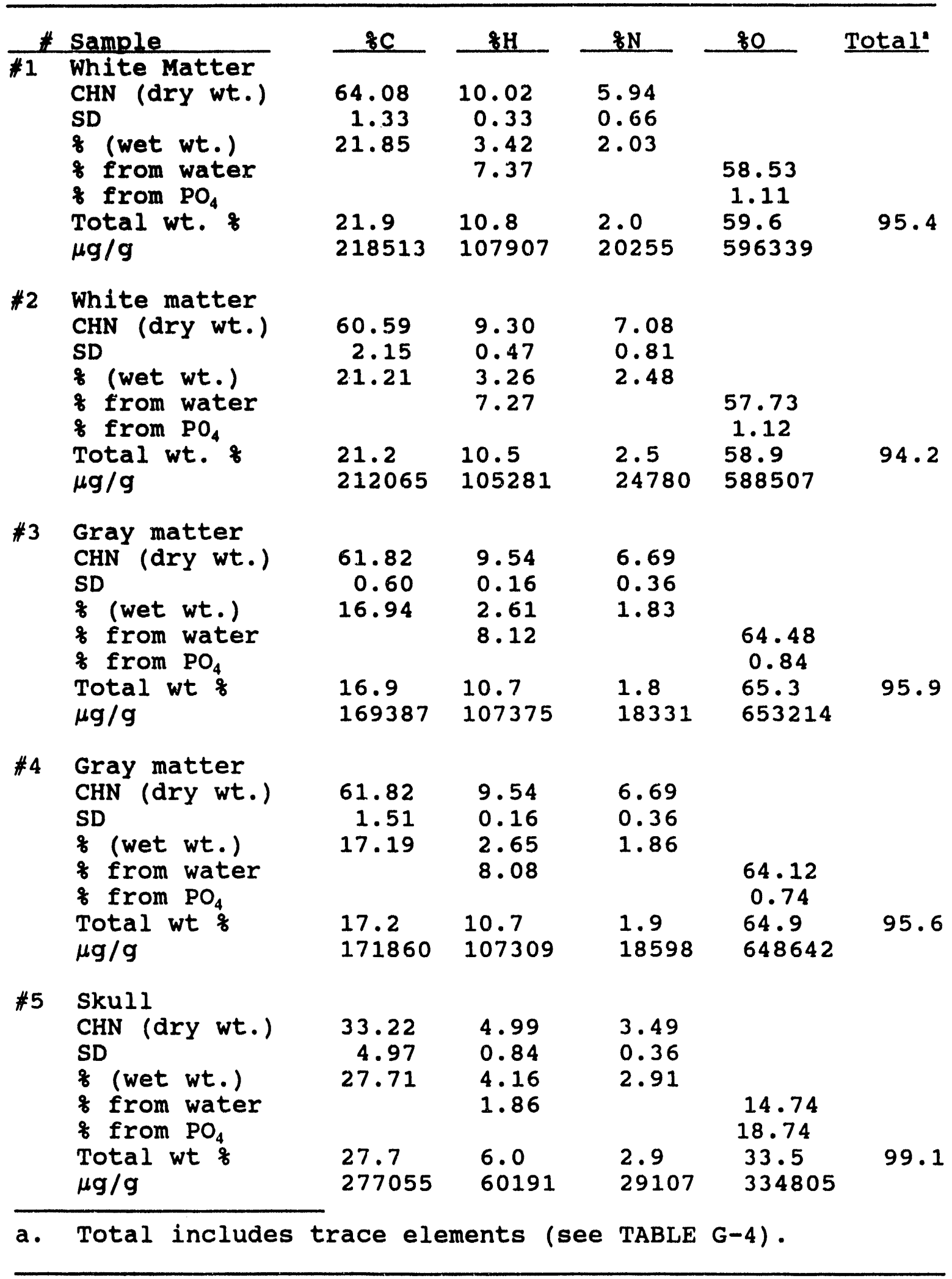


TABLE G-3. CONTINUED

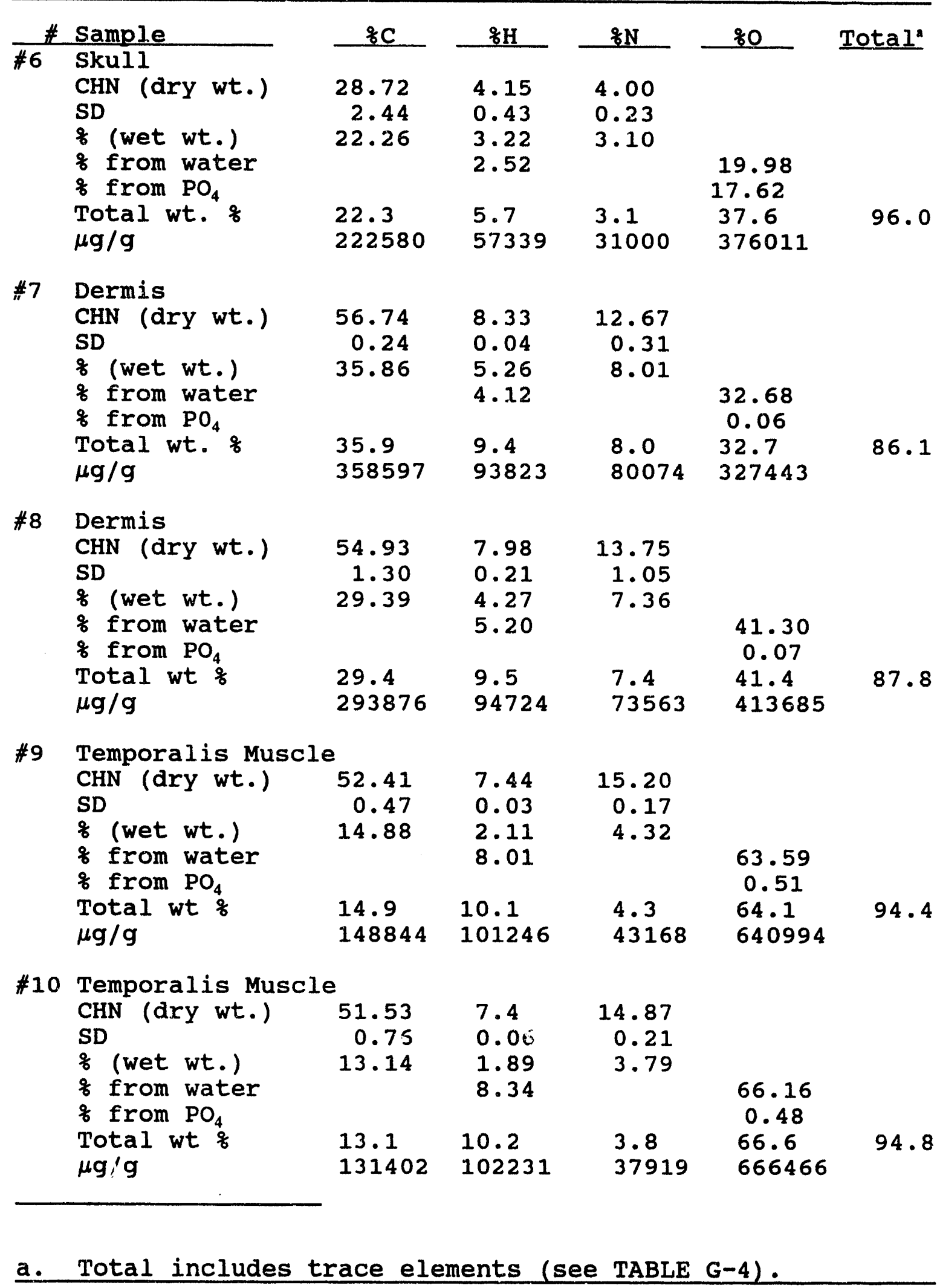


TABLE G-3. CONTINUED

\begin{tabular}{|c|c|c|c|c|c|c|}
\hline \multirow{3}{*}{$\frac{\#}{\# 11}$} & \multirow{2}{*}{$\frac{\text { Sample }}{\text { Epidermis }}$} & \multirow{2}{*}{ \&C } & \multirow[t]{2}{*}{$\% \mathrm{H}$} & $8 \mathrm{~N}$ & \multirow[t]{2}{*}{80} & \multirow[t]{2}{*}{ Totala } \\
\hline & & & & & & \\
\hline & CHN (dry wt.) & 51.57 & 10.13 & 12.77 & & \\
\hline & SD & 0.20 & 3.85 & 3.87 & & \\
\hline & \% (wet wt.) & 23.46 & 4.61 & 5.81 & & \\
\hline & of from water & & 6.10 & & 48.40 & \\
\hline & 8 from $\mathrm{PO}_{4}$ & & & & 0.10 & \\
\hline & $\begin{array}{l}\text { Total wt. \% } \\
\mu \mathrm{g} / \mathrm{g}\end{array}$ & $\begin{array}{l}23.5 \\
234644\end{array}$ & $\begin{array}{l}10.7 \\
107074\end{array}$ & $\begin{array}{l}5.8 \\
58104\end{array}$ & $\begin{array}{l}48.5 \\
485038\end{array}$ & 89. \\
\hline$\# 12$ & Epidermis & & & & & \\
\hline & CHN (dry wt.) & 51.15 & 7.28 & 15.75 & & \\
\hline & & 0.17 & 0.04 & 0.05 & & \\
\hline & \% (wet wt.) & 20.87 & 2.97 & 6.43 & & \\
\hline & $\begin{array}{l}\text { Irom water } \\
\text { \& from } \mathrm{PO}_{4}\end{array}$ & & 6.62 & & $\begin{array}{c}52.58 \\
0.10\end{array}$ & \\
\hline & $\begin{array}{l}\text { Total wt. } \% \\
\mu \mathrm{g} / \mathrm{g}\end{array}$ & $\begin{array}{l}20.9 \\
208692\end{array}$ & $\begin{array}{l}9.6 \\
95944\end{array}$ & $\begin{array}{l}6.4 \\
64260\end{array}$ & $\begin{array}{l}52.7 \\
526712\end{array}$ & \\
\hline
\end{tabular}

\#13 Ethmoid Labyrinth

$\begin{array}{llllll}\text { CHN (dry wt.) } & 27.92 & 4.09 & 6.94 & & \\ \text { SD (wet wt.) } & 4.78 & 0.62 & 0.92 & & \\ \text { \& (wet } & 6.76 & 0.99 & 1.68 & 67.32 & \\ \text { \& from water } & & 8.48 & & 3.26 & \\ \text { of from PO } & & & & 70.6 & 93.5 \\ \text { Total wt \% } & 6.8 & 9.5 & 1.7 & 70.6 \\ \mu \text { g } / g & 67566 & 94714 & 16795 & 705736 & \end{array}$

\#14 Ethmoid Labyrinth

$\begin{array}{llllll}\text { CHN (dry wt.) } & 35.42 & 5.16 & 8.33 & & \\ \text { SD (wet wt.) } & 3.66 & 0.53 & 0.48 & & \\ \text { \& (wet) } & 8.57 & 1.25 & 2.02 & 67.32 & \\ \text { \& from water } & & 8.48 & & 3.16 & \\ \text { \& from PO } & & & & 70.5 & 95.6 \\ \text { Total wt \& } & 8.6 & 9.7 & 2.0 & 70.5 \\ \mu \mathrm{g} / \mathrm{g} & 85716 & 97303 & 20159 & 704784 & \end{array}$

\#15 no sample

\#16 no sample

a. Total includes trace elements (see TABLE G-4). 
TABLE G-3. CONTINUED

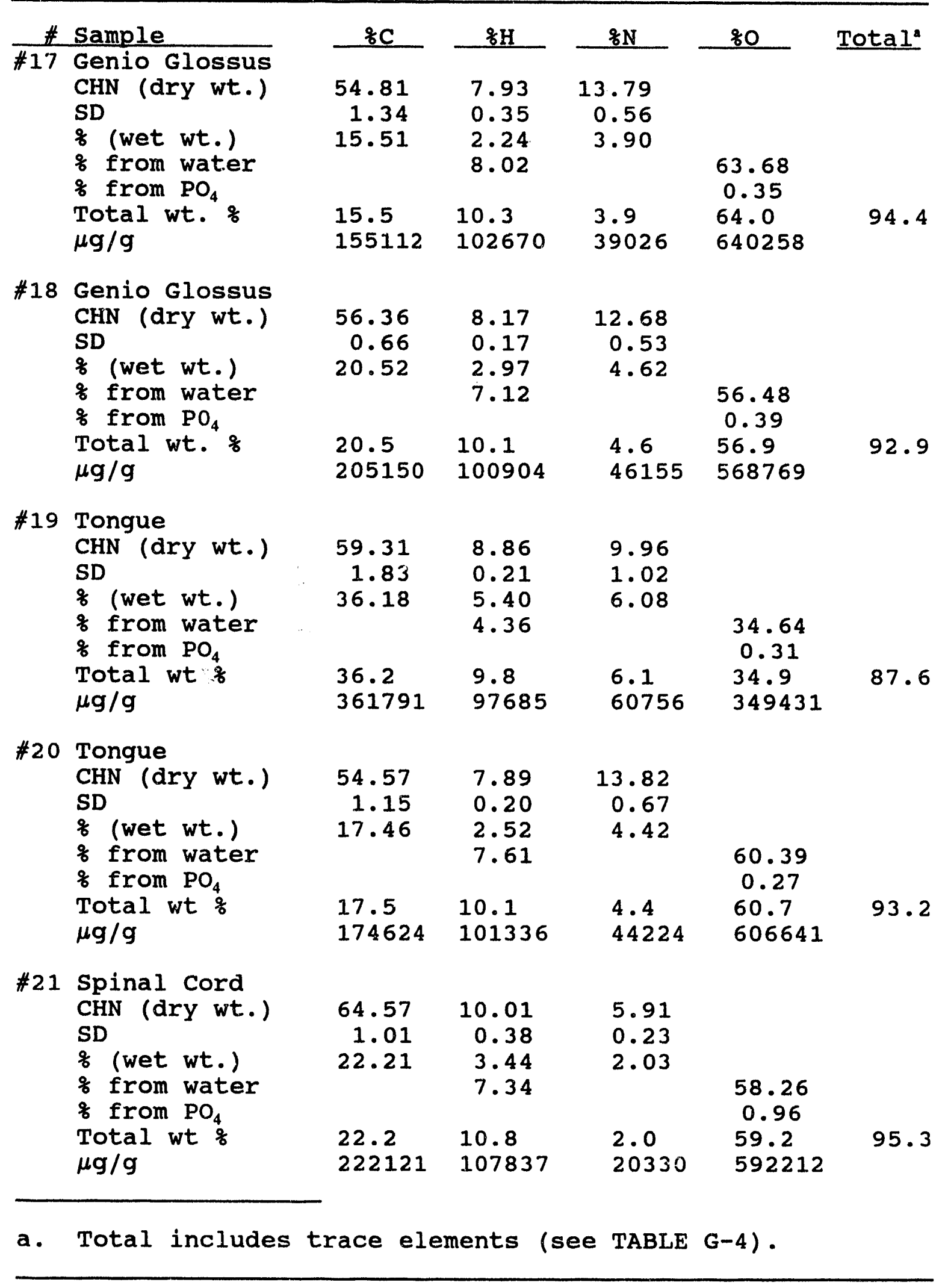


TABLE G-3. CONTINUED

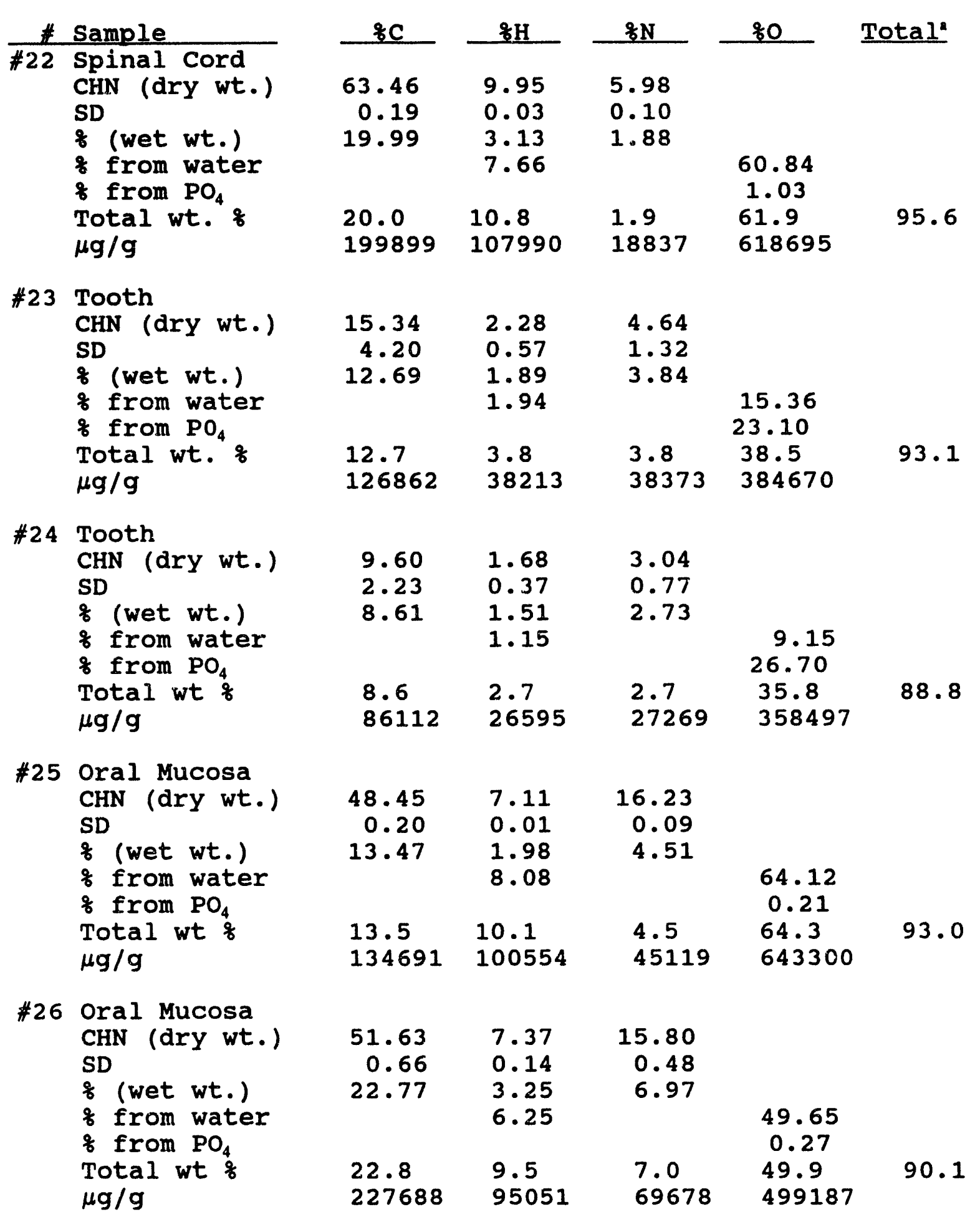

a. Total includes trace elements (see TABLE G-4). 
TABLE G-3. CONTINUED

\begin{tabular}{|c|c|c|c|c|c|c|}
\hline \multirow{2}{*}{$\frac{\#}{\# 27}$} & Sample & $8 \mathrm{C}$ & $8 \mathrm{H}$ & $\frac{8}{8} \mathrm{~N}$ & 80 & \multirow[b]{2}{*}{$\begin{array}{r}\text { Tota }{ }^{*} \\
96.0\end{array}$} \\
\hline & $\begin{array}{l}\text { Eye } \\
\text { CHN (dry wt.) } \\
\text { SD } \\
\text { \& (wet wt.) } \\
\text { \& from water } \\
\& \text { from } \mathrm{PO}_{4} \\
\text { Total wt. \& } \\
\mu \mathrm{g} / \mathrm{g}\end{array}$ & $\begin{array}{c}57.00 \\
0.40 \\
10.94 \\
\\
10.9 \\
109440\end{array}$ & $\begin{array}{l}8.37 \\
0.06 \\
1.61 \\
9.04 \\
10.6 \\
106481\end{array}$ & $\begin{array}{r}10.89 \\
0.40 \\
2.09\end{array}$ & $\begin{array}{c}71.76 \\
0.15 \\
71.9 \\
719093\end{array}$ & \\
\hline$\# 28$ & $\begin{array}{l}\text { Eye } \\
\text { CHN (dry wt.) } \\
\text { SD } \\
\text { \& (wet wt.) } \\
\text { \& from water } \\
\text { \% from } \mathrm{PO}_{4} \\
\text { Total wt. } \% \\
\mu \mathrm{g} / \mathrm{g}\end{array}$ & $\begin{array}{r}55.32 \\
1.00 \\
16.10\end{array}$ & $\begin{array}{l}7.83 \\
0.40 \\
2.28 \\
7.93 \\
10.2 \\
102118\end{array}$ & $\begin{array}{r}13.45 \\
1.09 \\
3.91\end{array}$ & $\begin{array}{l}62.97 \\
0.09 \\
63.1 \\
630517\end{array}$ & 93.8 \\
\hline$\bullet$ & tal & ace e & & Fig & $G-4)$ & \\
\hline
\end{tabular}


TABLE G-4. SUMMARY OF TRACE ELEMENT CONTENT IN DOG 22 SAMPLES

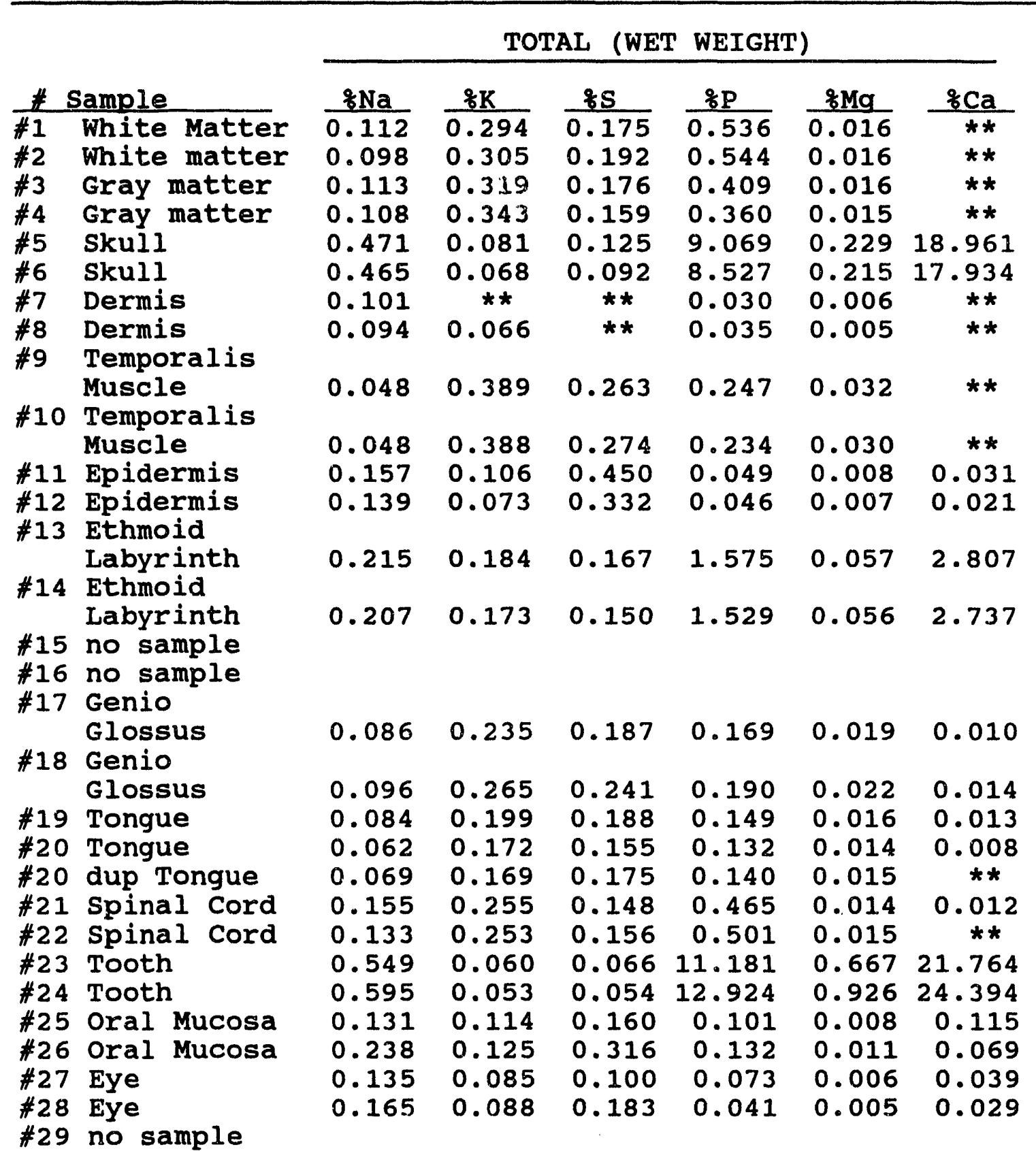

** Sample was below detection limits. 

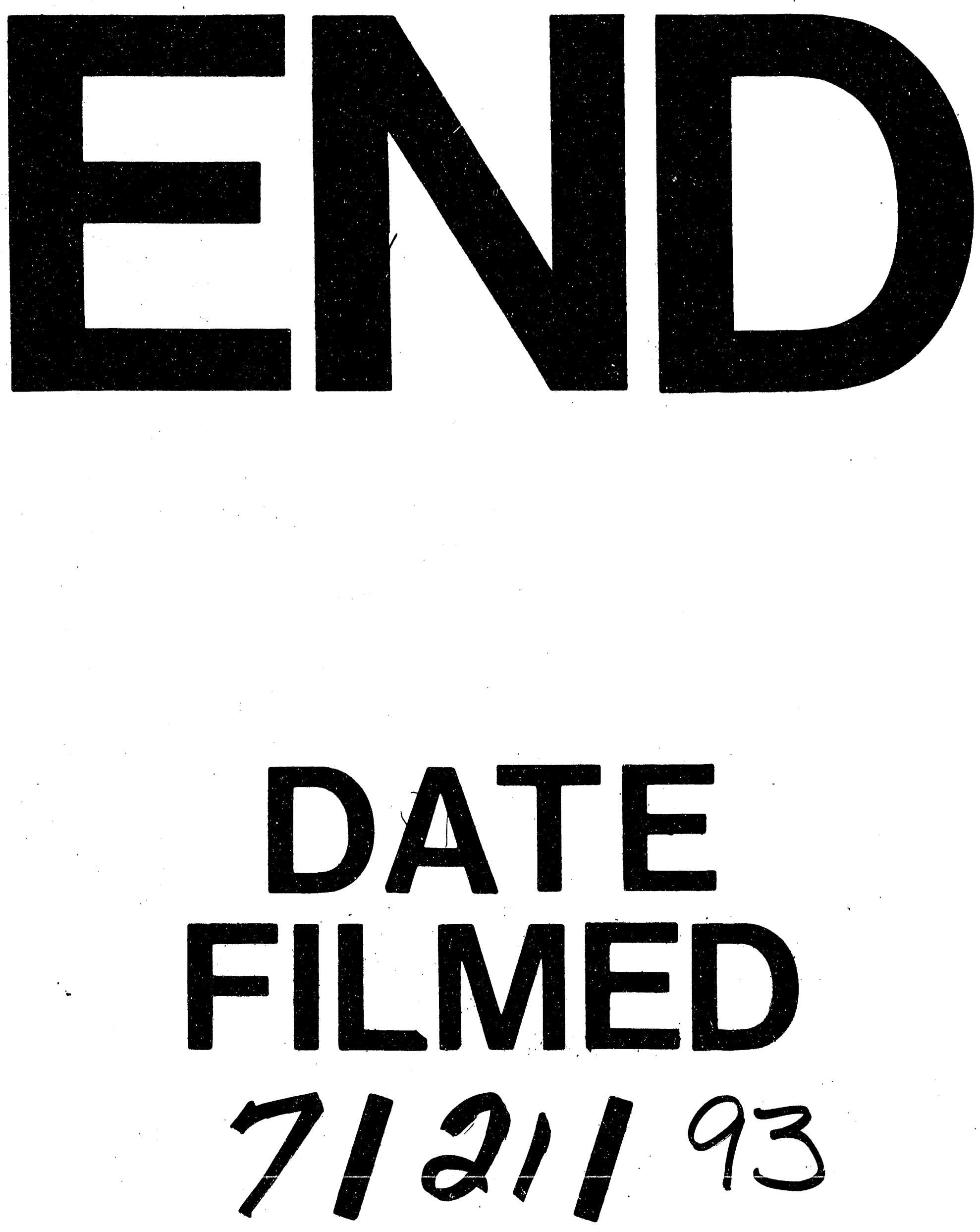

1 
